Evelyn Moser

\title{
Postsowjetische Transformationen in der Weltgesellschaft
}

Politische Dezentralisierung

und wirtschaftliche Differenzierung im ländlichen Russland 
Evelyn Moser

Postsowjetische Transformationen in der Weltgesellschaft

Global Studies \& Theory of Society | Band 2 Herausgegeben von David Kaldewey, Rudolf Stichweh und Tobias Werron 
Evelyn Moser (Dr. phil.) forscht am Forum Internationale Wissenschaft der Universität Bonn. Ihre Schwerpunkte liegen in der politischen Soziologie und der Wirtschaftssoziologie. 
Evelyn Moser

\section{Postsowjetische Transformationen in der Weltgesellschaft}

Politische Dezentralisierung

und wirtschaftliche Differenzierung

im Iändlichen Russland

[transcript] 
Die Arbeit wurde mit einem Promotionsstipendium der Graduate School of Humanities and Social Sciences der Universität Luzern gefördert.

\section{(2) $(1) \Theta \Theta$}

Dieses Werk ist lizenziert unter der Creative Commons Attribution-NonCommercial-NoDerivs 4.0 Lizenz (BY-NC-ND). Diese Lizenz erlaubt die private Nutzung, gestattet aber keine Bearbeitung und keine kommerzielle Nutzung. Weitere Informationen finden Sie unter https://creativecommons.org/licenses/by-nc-nd/4.o/deed.de/. Um Genehmigungen für Adaptionen, Übersetzungen, Derivate oder Wiederverwendung zu kommerziellen Zwecken einzuholen, wenden Sie sich bitte an rights@transcript-verlag.de

\section{(c) 2015 transcript Verlag, Bielefeld}

Die Verwertung der Texte und Bilder ist ohne Zustimmung des Verlages urheberrechtswidrig und strafbar. Das gilt auch für Vervielfältigungen, Übersetzungen, Mikroverfilmungen und für die Verarbeitung mit elektronischen Systemen.

\section{Bibliografische Information der Deutschen Nationalbibliothek}

Die Deutsche Nationalbibliothek verzeichnet diese Publikation in der Deutschen Nationalbibliografie; detaillierte bibliografische Daten sind im Internet über http://dnb.d-nb.de abrufbar.

Umschlaggestaltung: Kordula Röckenhaus, Bielefeld Druck: Majuskel Medienproduktion GmbH, Wetzlar Print-ISBN 978-3-8376-3101-2 PDF-ISBN 978-3-8394-3101-6

Gedruckt auf alterungsbeständigem Papier mit chlorfrei gebleichtem Zellstoff. Besuchen Sie uns im Internet: http://www.transcript-verlag.de Bitte fordern Sie unser Gesamtverzeichnis und andere Broschüren an unter: info@transcript-verlag.de 


\section{Inhalt}

Abbildungs- und Tabellenverzeichnis |7

\section{Postsowjetische Transformationen und gesellschaftliche Komplexität | 9}

Die Logik der sowjetischen Organisationsgesellschaft | 33

Sozialismus als Programm und das Programm des Sozialismus | 35

Die Organisationen der Organisationsgesellschaft $\mid 50$

Die Organisationsgesellschaft im sowjetischen Dorf $\mid 70$

Reichweite und Grenzen der sowjetischen Organisationsgesellschaft | 100

\section{Lokale Selbstverwaltung als Form interner Differenzierung} der Politik | 105

Beobachtungsvarianten $\mid 107$

Eine differenzierungstheoretische Annäherung | 121

Lokale Selbstverwaltung in Russland: Der formale Rahmen | 133

Leitaspekte für die Beobachtung lokaler Selbstverwaltung

in der postsowjetischen Transformationsgesellschaft | 157

\section{Das postsowjetische Dorf als (verschwindende) Nische} in der Weltgesellschaft $\mid 163$

Prolog: Methodologische Vorbemerkungen | 165

Aufregung um ein Flugblatt - oder: Formen der Kommunikation zwischen Kolchos und Gemeinde | 185

Der Anschluss an das Gasnetz - oder: Die »Gasifizierung des ganzen Landes« als Einfallstor der Weltgesellschaft $\mid 214$

Das Auftauchen der Agroholding - oder: Neue Selbstverständnisse und fortgeführte Strukturen $\mid 246$

Die Verteidigung des Dorfes - oder: Wie Veränderungssorgen

Individualisierung und funktionale Differenzierung befördern | 277

Epilog: Formen, Foren und Fixpunkte der dörflichen Kommunikation | 303

Von der Organisations- zur Transformationsgesellschaft | 321

Literatur | 351

Dank | 389 



\section{Abbildungs- und Tabellenverzeichnis}

Abbildung 1: Gliederung subnationaler Verwaltungseinheiten vor und nach der Kommunalreform von 2003 | 143

Tabelle 1: Personelle Struktur der sowjetischen Lokalverwaltung (1985) | 61 Tabelle 2: Anteile privater Produktion in der sowjetischen Landwirtschaft |91 Tabelle 3: Anteil privater Viehhaltung am sowjetischen Viehbestand |91 Tabelle 4: Kommunale Aufgabenverteilung gemäß Gesetz Nr. 131 | 151 Tabelle 5: Kommunale und anteilige föderale Steuern (ab 2006) | 155 Tabelle 6: Budget der Gemeinde Andreevka (2007) | 156 Tabelle 7: Beschäftigungsstruktur in der Gemeinde Andreevka (2008) | 174 Tabelle 8: Produktionsstruktur der russischen Landwirtschaft (1992-2011) | 175 



\section{Postsowjetische Transformationen und gesellschaftliche Komplexität}

Von den Regimewechselprozessen in den sozialistischen Staaten des ehemaligen Ostblocks wurden nicht nur die betroffenen Regierungen selbst, sondern auch die >westlichen < Sozialwissenschaften förmlich überrannt. Zurückzuführen ist dies zum einen auf das schlagartige Eintreten, die weitgehend friktionslose Ausbreitung und den schnellen Verlauf der Ereignisse, zum anderen dürfte der Überraschungseffekt aber auch eine Folge der verwendeten wissenschaftlichen Beobachtungsmittel gewesen sein. Eine Fußnote Luhmanns ist sicher nicht allein für die Soziologie gültig:

»Dass die Soziologie dies [den Zusammenbruch der Sowjetunion, E.M.] nicht vorausgesehen hat, wird viel beklagt und ist nicht zuletzt ihrem Theoriedefizit zuzuschreiben. Andererseits wäre, wie Chaostheorie und Evolutionstheorie zeigen, eine konkrete Voraussicht ohnehin nicht möglich gewesen, sondern allenfalls eine Beschreibung der Situation, die erkennen lässt, dass jetzt irgendwelche Zufälle, Personen oder Ereignisse genügen könnten, um die Destabilisierung einzuleiten.«(Luhmann 2000a: 385, Fn. 7)

Mit Blick auf die Ereignisse in Osteuropa attestierte der Politikwissenschaftler Adam Przeworski seiner Disziplin angesichts ihrer mangelnden Prognosefähigkeit klägliches Versagen (1991: 1) und Klaus von Beyme bezeichnete den Zusammenbruch des Realsozialismus als »>schwarzen Freitag< der Sozialwissenschaften. Sie haben überwiegend nicht >vorhergesagt<, wie es ihr methodischer Anspruch ist, sondern >nachhergesagt« (1994a: 35). ${ }^{1}$

Nach der Überraschung und der Überwindung des ersten Schwindels Mommsen (2013: 126) spricht rückblickend gar von einer »Schockstarre« -,

1 Für eine Diskussion der Prognosefähigkeit der Sozialwissenschaften mit Blick auf die postsozialistischen Regimewechsel und der daran anschließenden gesellschaftlichen Erwartungen vgl. Sharman (1998). 
konstituierte sich mit der Transformationsforschung ein Forschungsfeld, das sich auf die Beobachtung der einsetzenden Wandlungsprozesse in Osteuropa und auf dem Territorium der zerfallenden Sowjetunion spezialisierte. Zunächst in Westeuropa und den USA, später zunehmend auch in den postsozialistischen Staaten kam es zur Gründung von wissenschaftlichen Instituten, Forschungsabteilungen in Unternehmen und nicht-wirtschaftlichen Organisationen, spezialisierten Studiengängen, Tagungsreihen und Fachzeitschriften, die sich bis in die Gegenwart am Label postsozialistischer Transformationsforschung orientieren. Doch nicht nur die organisatorische Verankerung weitete sich aus: Mit den Publikationen, die in den vergangenen rund zwanzig Jahren in diesem Kontext entstanden sind, floss eine mittlerweise unüberschaubare Anzahl an Kommunikationsangeboten in das Wissenschaftssystem ein. Angesichts deren disziplinärer, inhaltlicher, methodischer und theoretischer Diversität und angesichts eines Forschungsgegenstandes, der sich zeitlich und sachlich kontinuierlich ausweitet, drängt sich jedoch die Frage auf, ob das ursprünglich interdisziplinär angelegte Etikett der Transformationsforschung überhaupt noch ein zusammenhängendes Forschungsfeld bezeichnet, in dem wechselseitig aneinander angeschlossen wird und über die Form dieser internen Verknüpfungen Grenzen zur Umwelt - anderen Forschungsfeldern - hervortreten. Oder ob sich >die< Transformationsforschung nicht längst in ein bestenfalls lose gekoppeltes Netzwerk einzelner, weitgehend unabhängig voneinander operierender Kommunikationszusammenhänge differenziert hat, die sich gegenseitig höchstens noch aus den Augenwinkeln beobachten und deren disziplinär, theoretisch und/oder methodisch bedingte Unterschiede das ursprünglich geteilte Erkenntnisinteresse längst überlagern. ${ }^{2}$ Eine Antwort auf diese Frage ließe sich möglicherweise mit den Mitteln der Netzwerkanalyse finden, was an dieser Stelle jedoch nicht weiter verfolgt werden soll. ${ }^{3}$

Im Hinblick auf die vorliegende Studie sind diese Überlegungen ohnehin auf eine andere Weise relevant: Denn unabhängig davon, wie diese Antwort ausfallen würde, sehen sich Analysen wie die vorliegende, die sich auf dem weitläufigen Feld der Transformationsforschung verorten, mit einem gestiegenen Begründungsaufwand konfrontiert. Sie stehen vor der Herausforderung, sich mit ihrem Erkenntnisinteresse in einem zunehmend disparaten Forschungs- und Kommunikationszu-

2 Ein Effekt dieses Differenzierungsprozesses ist, dass weithin sichtbare und breit akzeptierte >Autoritäten< auf diesem Feld verschwinden oder an Bedeutung verlieren. In diesem Kontext ist möglicherweise auch die Debatte über den Niedergang der Russlandexpertise in Deutschland zu verstehen, die jüngst in der Zeitschrift »Osteuropa « angestoßen wurde (vgl. Götz 2012; Sapper 2012; als Gegenposition Segbers 2013).

3 Für ein solches Vorgehen am Beispiel der Humangeographie vgl. Steinbrink et al. (2012), am Beispiel der Physik vgl. Palla et al. (2005). 
sammenhang zu verorten, den für sie relevanten Wissensstand plausibel herauszufiltern und den eigenen Beitrag dazu nicht nur zu positionieren, sondern in möglichst viele Richtungen Anschlussmöglichkeiten aufzuzeigen.

\section{Thema, Problem und erste Orientierungspunkte}

Das Thema der vorliegenden Studie befindet sich an einer der Schnittstellen zwischen sogenannter politischer und wirtschaftlicher Transformation: Im Fokus stehen politische Dezentralisierungs- und wirtschaftliche Privatisierungsprozesse im ländlichen Raum Russlands, die am Beispiel zweier Gemeinden empirisch nachgezeichnet werden. Problematisiert werden dabei Strukturen und Ordnungen, die sich im Kontext dieser Transformationsbereiche und speziell bei der Einführung kommunaler Selbstverwaltung herausbilden. Besonderes Augenmerk liegt dabei auf der jüngsten Kommunalreform und ihren Effekten, dem Gesetz Nr. $131 »$ Über allgemeine Prinzipien der Organisation lokaler Selbstverwaltung in der Russischen Föderation«, das seit Anfang 2006 (mit einer Übergangsfrist bis Anfang 2009) in Kraft ist und von der Politik als Neuanfang im Prozess der Einführung lokaler Selbstverwaltung dargestellt wird. Gesellschaftstheoretisch lassen sich solche Reformmaßnahmen allerdings kaum isoliert betrachten, sondern sind stets als Teil eines Geflechts von (politischen) Maßnahmen - in diesem Fall Privatisierung und Kommunalreformen - zu analysieren, mit denen ländliche Gemeinden seit dem Zusammenbruch des sowjetischen Regimes in verschiedenen Reformwellen konfrontiert wurden und werden und die ein kontinuierliches Irritationspotenzial für die dörflichen Kommunikationszusammenhänge bereithalten.

Mit Blick auf den inhaltlichen Rahmen dieser Studie erscheint somit eine erste Orientierung an den beiden >großen< Bereichen der Transformationsforschung Wirtschaft und Politik naheliegend. Obwohl sämtliche gesellschaftliche Bereiche sowohl vom sozialistischen Programm als auch von den einsetzenden Transformationsprozessen betroffen waren, kam diesen beiden Bereichen auf dem Feld der Transformationsforschung stets besondere Aufmerksamkeit zu: im Fall der Wirtschaft bedeutete dies den Fokus auf den Übergang von der Plan- zur Marktwirtschaft und im Fall der Politik von autokratischen zu demokratischen Strukturen. Der innere und äußere Druck, die Umstrukturierungsprozesse gerade in diesen beiden Bereichen möglichst schnell und reibungslos voranzutreiben, war in den postsozialistischen Transformationsstaaten zweifellos hoch - schien doch die Existenz und Stabilität der (teilweise neugegründeten) Nationalstaaten unmittelbar nach den Regimewechseln vor allem davon abzuhängen, dass innerhalb kürzester Zeit handlungsfähige und möglichst demokratisch legitimierte politische Entscheidungsträger gefunden und die materielle Lebensgrundlage der Bevölkerung gesichert wurden. In der Folge stiegen Beratungsaktivitäten schlagartig an, die sich sowohl an die po- 
litischen Akteure in den unmittelbar betroffenen Staaten richteten als auch an internationale Organisationen (allen voran die Weltbank, die Europäische Bank für Wiederaufbau und Entwicklung und der Internationale Währungsfonds), die in vielen Ländern maßgeblich an der Konzeption und Umsetzung politischer Transformationsmaßnahmen beteiligt waren. Beratungsbedarf meldeten auch die scheinbar unbeteiligten Regierungen vor allem der westlichen Nachbarstaaten des ehemaligen Ostblocks an, für die es nun galt, sich angesichts der neuen (außen-)politischen Lage neu zu orientieren und mit den sich kontinuierlich und tiefgreifend verändernden Strukturen umzugehen. Diesen vermeintlichen Beratungsbedarf nahmen vor allem die Wirtschafts- und Politikwissenschaften dankend auf, sehen sie sich doch in unmittelbarer >Verantwortung < für die entsprechenden Funktionssysteme (vgl. von Beyme 1999: 292). Weil beiden Disziplinen zudem auch von außen entsprechende Zuständigkeiten zugeschrieben werden, erlangten sie auf dem Feld der Transformationsforschung eine dominierende Position. Diese blieb bis in die späten 1990er Jahre nahezu unangefochten, auch wenn parallel dazu Teilaspekte der postsozialistischen Transformationsprozesse seit Beginn der Regimewechsel auch in anderen sozialwissenschaftlichen Disziplinen thematisiert wurden. Ernsthaft zu bröckeln begann die politik- und wirtschaftswissenschaftliche Dominanz erst um die Jahrtausendwende. Neben einem von ihnen dominierten >ersten< Strang der Transformationsforschung etablierte sich sukzessive ein >zweiter $<$ Strang, an dem die >übrigen $<$ sozialwissenschaftlichen Disziplinen maßgeblich beteiligt waren. Ein zentraler Abstoßpunkt dieser Entwicklung waren aufkommende Zweifel am bisher verwendeten Transformationsbegriff.

Diese Zweifel und den Wandel des Transformationsverständnisses möchte ich zum Ausgangspunkt nehmen, um im Folgenden sowohl die angedeuteten Entwicklungen auf dem Feld der Transformationsforschung nachzuzeichnen als auch auf ausgewählte Implikationen für die Reichweite wissenschaftlicher Beobachtung zu verweisen, die sich daraus ergeben. Im Anschluss daran soll das Erkenntnispotenzial eines systemtheoretischen Blicks ausgelotet werden, das sich nicht allein aus der bisher vernachlässigten Thematisierung von postsozialistischen Transformationsprozessen in der Systemtheorie ergibt (zu den wenigen Ausnahmen gehören Hayoz 1995, 1997; Pollack 1990, 1994). ${ }^{4}$ Stattdessen, so mein Argument, resultiert das systemtheoretische Potenzial für diesen spezifischen Forschungsbereich aus den

4 Ungeachtet dessen erwähnt interessanterweise Merkel (2010: 67) in seinem Kompendium zur Systemtransformation die Systemtheorien nach Parsons und Luhmann als eigenständige Transformationstheorien (neben Struktur-, Kultur- und Akteurstheorien). Als einziges Werk, das sich im Hinblick auf konkrete Transformationsprozesse diesem Theoriebereich zuordnen lässt, zitiert er dann jedoch einen Aufsatz von Pollack (1990) zum Zusammenbruch der DDR. 
sparsamen, aber sehr präzisen theoretischen Grundannahmen sowie einem differenzlosen Verständnis der modernen Gesellschaft als Weltgesellschaft. Darauf aufbauend hält die Systemtheorie hinreichend komplexe Instrumentarien bereit, die es ermöglichen, Prozesse sozialen Wandels zu beobachten, d.h. zu unterscheiden und zu bezeichnen, verschiedene Transformationsbereiche mit ihren divergierenden Logiken in ihrer wechselseitigen Bedingtheit zu erfassen und ohne teleologischen Imperativ aufeinander zu beziehen, sowie dem Phänomen postsowjetischer Transformation an sich einen logischen Ort im Horizont der Weltgesellschaft zuzuweisen.

\section{Geschlossener Transformationsbegriff: ,Von dort nach hier}

Der erste Strang der Transformationsforschung, der als unmittelbare Reaktion auf die Regimewechsel in den frühen 1990er Jahren ein- und ansetzte und der von seinen Vertreterinnen und Vertretern und Kritikerinnen und Kritikern gleichermaßen als Transitologie bezeichnet wird (Müller 2001: 5f; vgl. auch die Zusammenfassung in Steimann 2010: 11ff), ist geprägt von einem - nach kybernetischem Verständnis - geschlossenen Transformationsbegriff. Dieser Transformationsbegriff basiert auf der Annahme definierbarer Ausgangs- und Endzustände des Transformationsprozesses. Entsprechend kann jedes Element der klar bestimmbaren Ausgangsituation durch geeignete Operationen (sprich: Transformationsmaßnahmen) in ein Element des ex ante determinierten Endzustandes versetzt werden (Ashby 1974: 27ff; auch Baecker 1998 [1995]: 39). Zwar garantiert die Benennbarkeit nicht den Erfolg der Transformationsmaßnahmen. Im Falle des Scheiterns lässt sich jedoch zumindest eine Referenz angeben, um den Grad des Misserfolgs zu beurteilen, nach Möglichkeit zu korrigieren oder als Anomalie einzustufen.

An ein solches Verständnis schließen Kennzahlen und Indizes zur Messung spezifischer gesellschaftlicher Wandlungsprozesse unmittelbar an. Sie kommen vor allem dann zum Einsatz, wenn Forschungsergebnisse als Expertenwissen in die Gestaltung und Umsetzung politischer Maßnahmen einfließen und im Hinblick darauf handhabbar gemacht werden sollen. Die Kennzahlen und Indizes sorgen für eine Komplexitätsreduktion, indem sie es erlauben, politische Regime und wirtschaftliche Strukturen von Staaten allein auf die Unterscheidung von Ausgangs- und Endzustand hin zu beobachten - Demokratie|Autokratie als Varianten der inneren Ordnung der Politik und Marktwirtschaft|Planwirtschaft für die Wirtschaft - und damit die Transformationsvariablen in den Blick zu nehmen, die für die Konzeption spezifischer Maßnahmen als relevant erachtet werden.

Mit Blick auf wirtschaftliche Transformationsprozesse, auf denen von Beginn an ein großer Teil der wissenschaftlichen und politischen Aufmerksamkeit lag, rankte sich die entsprechende Diskussion in der Transformationsforschung seit den frühen 1990er Jahren primär um die Frage, wie der Übergang »vom Plan zum 
Markt « möglichst zügig und reibungslos gestaltet werden könnte (vgl. dazu programmatisch und oft zitiert The World Bank 1997). Bald dominierten verschiedene Transformationsstrategien die Debatte, wobei sich Vertreterinnen und Vertreter der sogenannten Schocktherapie, die vor allem durch den amerikanischen Ökonomen Jeffrey Sachs bekannt wurde (vgl. z.B. De Melo/Denizer/Gelb 1996; Sachs 1996), und Befürworterinnen und Befürworter gradualistischer Strategien gegenüberstanden: Die >Schocktherapeutinnen und -therapeuten < argumentierten, dass sich mit dem Zerfall der sozialistischen Regime und der anfänglichen $>$ Reformbegeisterung< ein begrenztes Zeitfenster öffne, das es mit der schnellen und gleichzeitigen Implementierung umfassender Liberalisierungsmaßnahmen in möglichst allen wirtschaftlichen Bereichen notfalls auch gegen den Willen der Bevölkerung zu nutzen gelte. Die Irreversibilität der gewählten Maßnahmen wiederum erzwinge sodann einen zwar schmerzhaften, aber zügigen Anpassungsprozess an die neuen Strukturen, wodurch Transformationskosten wie Arbeitslosigkeit, Inflation und Wachstumseinbußen zwar nicht vermieden, aber in einem vor allem zeitlich überschaubaren Rahmen gehalten werden könnten. Befürworterinnen und Befürworter einer gradualistischen Vorgehensweise hielten dagegen, dass sich die einzelnen Maßnahmen nicht substitutiv, sondern komplementär zueinander verhalten würden und auf Grund von Wechsel- und Abhängigkeitsbeziehungen sowohl die sachliche Reihenfolge als auch die zeitliche Gestaltung des Implementationsprozesses ausschlaggebend für den Erfolg der Transformation sei. Durch erste Erfolge, die auf diese Weise bereits zu einem frühen Zeitpunkt sichtbar würden, könne zudem die gesellschaftliche Unterstützung für die jeweils nächste Reformstufe gesichert werden (vgl. z.B. Popov 2000; Roland 2002; Schneider 1992; Staehr 2003). Bei der Beobachtung der Transformationsmaßnahmen wurden Indizes wie beispielsweise die »transition indicators « der Europäischen Bank für Wiederaufbau und Entwicklung (EBRD) ${ }^{5}$ (Staehr 2003) oder der Transformationsindex der Bertelsmann-Stif-

5 Die EBRD beobachtet die postsozialistischen Reformen in acht Bereichen: (1) Privatisierung von Klein- und (2) Großunternehmen, (3) Preisliberalisierung, (4) Handels- und Wechselkursliberalisierung sowie Reformen (5) im Bankensystem, (6) in der Wettbewerbspolitik, (7) der Unternehmensumstrukturierung und (8) des Wertpapiermarktes. Beurteilt wird der Reformfortschritt in allen Bereichen auf einer Ordinalskala von 1,00 (»1«) bis 4,33 (»4+«), wobei der niedrigste Wert für keine nennenswerte Abweichung vom System zentraler Planung steht und der höchste der vollständigen Implementierung marktwirtschaftlicher Prinzipien entspricht (vgl. zur Methodologie http://www.ebrd.com/ $\mathrm{cs} /$ Satellite $\mathrm{c}=$ Content $\& \mathrm{cid}=1395237866249 \&$ pagename $=$ EBRD $\% 2$ FContent $\% 2$ FConte ntLayout [zuletzt eingesehen am 30.4.2015]). Die ersten Bewertungen nahm die EBRD im Jahr 1994 vor. Im Jahr 2000 wurden sie für den Zeitraum von 1989 bis 1993 rückblickend ergänzt. Individuelle Bewertungen der postsozialistischen Transformationsstaaten 
tung herangezogen. ${ }^{6}$ Beide beobachten und klassifizieren Transformationsfortschritte in einzelnen Bereichen anhand einer Ordinalskala und versuchen sie auf diese Weise länderübergreifend zu vergleichen. Als Erfolgsindikatoren werden in entsprechenden Analysen, die an diese und ähnliche Indikatoren anknüpfen, in erster Linie makroökonomische Kennzahlen wie das Wirtschaftswachstum, die Inflation oder die Arbeitslosigkeit verwendet (Blanchard 1996; Blanchard/Kremer 1997; Fischer/Sahay/Végh 1996; Selowsky/Martin 1997). Resultate sind meist mehr oder weniger konkrete Handlungsempfehlungen für die >Transformationspraxis $<$.

So wenig, wie nach dem Zusammenbruch der sozialistischen Regime die Ablösung planwirtschaftlicher durch marktwirtschaftliche Strukturen als Ziel in Frage gestellt wurde, wurde in den frühen 1990er Jahren für den politischen Transformationsprozess bezweifelt, dass die ehemals sozialistischen Staaten nach den ersten Umbruchswirren in einen Demokratisierungsprozess eintreten würden (Carothers 2002: 6f). Demokratie, so die Erwartung, würde sich als das »only >rightful< game in town« etablieren (Sartori 1991: 448), für dessen Spielregeln nach einer Übergangsphase mit umfassender gesellschaftlicher Akzeptanz zu rechnen sei. Auf dieser Überzeugung fußt Huntingtons $(1991,1993)$ vielzitierte These der Demokratisierungswellen, die die postsozialistischen Transformationsprozesse einer dritten Welle zuordnet. ${ }^{7}$ Ähnlich argumentieren Phasenmodelle des Regimewechsels, die auch, aber nicht nur mit Blick auf Osteuropa - mit leicht variierenden Abgrenzun-

nach diesem Schema finden sich bis in die Gegenwart in den jährlich erscheinenden »Transition Reports« der Bank (vgl. zuletzt EBRD 2014).

6 Der Bertelsmann-Transformationsindex (BTI) beobachtet Transformationen in den Bereichen Demokratie und Marktwirtschaft gleichermaßen und umfasst entsprechend politische Indikatoren (z.B. Rechtsstaatlichkeit, politische Partizipation, Stabilität demokratischer Institutionen) und wirtschaftliche Indikatoren (z.B. Währungs- und Preisstabilität, Privateigentum, Wirtschaftsentwicklung). Er bezieht sich auf 128 Staaten, für die alle zwei Jahre ein Ranking erstellt wird (Informationen zur Indexmethodologie sowie Ländergutachten finden sich unter http://www.bti-project.de [zuletzt eingesehen am 30.4.2015]).

7 Huntingtons These zufolge zeichnen sich Demokratisierungsprozesse durch eine globale Wellendynamik aus. Zwar folgen auf Demokratisierungswellen auch immer wieder Gegenbewegungen, gemessen an der Anzahl demokratischer Regierungen sei insgesamt jedoch ein eindeutiger Trend in Richtung Demokratie zu erkennen. Die erste Welle vollzog sich von 1828 bis 1926 in Folge der Revolutionen in den Vereinigten Staaten von Amerika und in Frankreich, die zweite Welle von 1943 bis 1962 wurde durch das Ende von Mussolinis Herrschaft in Italien ausgelöst und die dritte Welle, die auch über Osteuropa und die Sowjetunion >hereinbrachく, setzte 1974 mit dem Ende der Militärdiktatur in Portugal ein (Huntington 1993: 13ff). 
gen und Bezeichnungen die Phasen der Liberalisierung, die das Ende des autokratischen Regimes einläutet, der Demokratisierung und schließlich der Konsolidierung der neuen Ordnung unterscheiden (siehe z.B. Merkel 2010: 93ff; O'Donnell/ Schmitter 1991; von Beyme 1994b; auch Fuchs/Roller 2006). Folgt man dieser Sichtweise, so lässt sich der Demokratisierungsgrad messen, der Auskunft darüber gibt, an welcher Stelle sich ein Staat auf dem Weg zu einer demokratischen Ordnung des politischen Systems befindet. Beispielhaft für ein solches Vorgehen sind breit und auf globale Ländervergleiche angelegte Indizes $^{8}$ wie - erneut - der Transformationsindex der Bertelsmann-Stiftung, Vanhanens Demokratieindex, der auf Partizipation und Wettbewerbsgrad als zentrale Dimensionen von Demokratie abstellt (Vanhanen 2006: insb. Kap. 3; vgl. auch Schmidt 2010: 374ff), oder individuelle Messversuche einzelner Autorinnen und Autoren. So konstruiert beispielsweise Haerpfer (2002) auf der Basis von Umfragedaten zur Akzeptanz demokratischer Werte einen eigenen Konsolidierungsindex und Diamond (1999: 68) sieht in einer Zustimmungsrate zum demokratischen Regime von 70 Prozent respektive einer maximalen Unterstützung autoritärer Regierungsformen von 15 Prozent die Schwellenwerte für eine erfolgreiche demokratische Konsolidierung.

Angesichts der wirtschaftlichen und politischen Entwicklungen in Osteuropa und den postsowjetischen Staaten, die teilweise deutlich von den Annahmen und Prognosen der Transitologie abwichen, wuchsen gegen Ende der 1990er Jahre die Zweifel an der wissenschaftlichen Stimmigkeit der Beobachtungen respektive an dem zugrundeliegenden Transformationsverständnis. Dabei erstaunt es wenig, dass die Kritik nicht in den Politik- und Wirtschaftswissenschaften, welche das Feld der Transformationsforschung bis dahin dominiert hatten, einsetzte, sondern primär in der Soziologie, der Ethnologie, den Kulturwissenschaften, der Humangeographie und verwandten sozialwissenschaftlichen Disziplinen geäußert wurde. Diese Entwicklung ist nicht zufällig, zeichnen sich die nun verstärkt zu diesem Forschungsfeld hinzustoßenden Disziplinen doch erstens dadurch aus, dass sie sich in der Anfangsphase der postsozialistischen Regimewechsel vergleichsweise zurückhielten,

8 Für wirtschaftliche und politische Transformation gilt gleichermaßen: Vor allem dann, wenn die in diesen Analysen und im Rahmen von Modellbildungen generierten Indizes und Kennzahlen nicht nur als wissenschaftliche Beobachtungsinstrumente zum Einsatz kommen, sondern auch darüber hinaus und insbesondere in der Politik für Irritationen sorgen, lässt sich die Frage anschließen, welche Konsequenzen für die Selbst- und Fremdbeobachtung von Staaten im Hinblick auf die Herausbildung globaler Konkurrenzen mit dieser Art des Vergleichs von >Transformationsleistungen< und der darin impliziten Temporalisierung von Unterschieden verbunden sein könnten (vgl. dazu die Überlegungen in Werron 2010; auch die Bemerkungen zum Entwicklungsbegriff in Stichweh 2012). 
und zweitens, und vermutlich entscheidender, sich nicht a priori für spezifische gesellschaftliche Funktionssysteme zuständig erklären. Zielscheibe der Kritik war in erster Linie die binäre Logik des geschlossenen Transformationsbegriffs, der Transformation als »a simple transition from >there< to >here<« verstand (Altvater 1998: 595) und die Operationen - politische Maßnahmen - für einen möglichst reibungslosen Übergang in den Fokus rückte:

»During the decade following the collapse of socialism, transformation processes in East and Central Europe (ECE) and in the former Soviet Union (Commonwealth of Independent States [CIS]) have been analysed as a transition from a planned economy and politically authoritarian (or totalitarian) one-party system to a free market economy with a pluralistic and democratic political system, i.e. from >bad boys < socialism to > good girls < capitalism. « (Altvater 1998: 591)

Eine erfolgreiche Transformation, so der Vorwurf, imitiere nach einem solchen Verständnis möglichst originalgetreu die (westlichen) Vorbilder und führe unweigerlich dazu, dass die Gegenwart nur noch im Hinblick auf die Eigenschaften idealtypischer Zukunftsvorstellungen hin bewertet werde: Der Weg zum Erfolg führe unweigerlich über die >Blaupausen< westlicher Marktinstitutionen und er tue dies ungeachtet regionaler Besonderheiten, länderspezifischer Ausgangszustände, Pfadabhängigkeiten und ähnlichem. Der Komplexität der sozialen Realität trage ein solcher Blickwinkel jedoch kaum angemessen Rechnung (Stark/Bruszt 1998: 5, 2001: 1130f; auch Baecker 1998 [1995]: 41). Ignoriert würden notwendigerweise sämtliche Zustände, die sich der Kontextur Ausgangs-|Endzustand entziehen, wie etwa die politische und wirtschaftliche Grauzone, in der sich die meisten postsozialistischen > Transformationsstaaten < in den 1990er Jahren faktisch befanden (Carothers 2002: 9). Auch die Nuancierung der analytischen Kategorien etwa durch die Einführung der »Demokratien mit Adjektiven« (Collier/Levitsky 1997) löste dieses Problem nur scheinbar, da empirisch wiederum nur eine kleine und spezifische Gruppe von Staaten erfasst wurde (Carothers 2002: 10). Theoretisch definieren sich die neuen Demokratietypen stets in Abweichung vom Modell der liberalen Demokratie als Idealtypus und basieren damit letztlich ebenfalls auf der Unterscheidung von Demokratie und Autokratie - also implizit auf einem geschlossenen Transformationsverständnis. ${ }^{9}$ Das Resultat ist eine zunehmend unüberschaubare Anzahl an analytischen Kategorien mit kaum steigender Erklärungskraft (für eine ähnliche Kritik vgl. auch Mommsen 2013: 132).

9 Dieser Logik folgen zum Beispiel die verschiedenen Typen »defekter Demokratien « in Merkel et al. (2003) oder die »hybriden Systeme« bei Knobloch (2006). 
Um die Entwicklungen im postsozialistischen Raum verstehen zu können, so die neue Forderung, sei eine grundlegende Anpassung des Transformationsverständnisses vonnöten. Als erster Schritt in diese Richtung sei die bislang gängige Bezeichnung Transition durch den Begriff der Transformation zu ersetzen (vgl. z.B. Altvater 1998: 594f; Burawoy/Verdery 1999: 14f; Müller 2001: 10; Stark/Bruszt 1998: 80ff):

»Thus, in place of transition (with the emphasis on destination) we analyze transformations (with the emphasis on actual processes) in which the introduction of new elements takes place most typically in combination with adaptations, rearrangements, permutations, and reconfigurations of already existing institutional forms.«(Stark/Bruszt 1998: 83)

Ein solches Transformationsverständnis sei in der Lage zu berücksichtigen, dass neue Strukturen letztlich nicht auf, sondern mit den Ruinen der sozialistischen Ordnung errichtet würden (Stark 1996: 995), wobei die postsozialistischen Gesellschaften das Material zur Errichtung neuer Ordnungen in ebenjenen Ruinen fänden (Stark/Bruszt 1998: 82). Zu vermeiden sei jedoch auch, ins andere Extrem zu verfallen, also Pfadabhängigkeiten übermäßig zu betonen und die Gegenwart als schlichte Fortschreibung der Vergangenheit zu betrachten (Stark/Bruszt 1998: 5f). ${ }^{10}$

\section{Offener Transformationsbegriff: Unordnung und »fuzziness «}

Wiederum kybernetisch gewendet verbirgt sich hinter dieser Kritik und dem postulierten Richtungswechsel die Forderung nach einem offenen Transformationsverständnis (Baecker 1998 [1995]: 45). Im Gegensatz zum geschlossenen Transformationsbegriff geht dieses weder von fixen Ausgangs- und Endzuständen noch von benennbaren und abgrenzbaren Operationen aus, die Erstere in Letztere überführen. Stattdessen erkennt es explizit die Möglichkeit, dass zwar bestimmte Elemente des Ausgangszustands >planmäßig< übersetzt werden, andere jedoch ausgegrenzt werden und >ins Leere laufen $<$. Ebenso ist es denkbar, dass im Verlauf des

10 Eine solche Sichtweise, die Stark und Bruszt (1998) vor allem in der kritischen Transformationsforschung ausmachen, bezeichnen sie als »involution« (im Gegensatz zu »imitation«): »Whereas neoliberalism sees blueprints for the imitation of market institutions as the road to progress, the contrary view perceives the weight of the socialist past as so heavy that attempts at marketization and democratization become the path to retrogression. One advocate of this view, Michael Burawoy, for example, identifies the postsocialist epoch as a period not of imitation but of sinvolution<. [...] Burawoy's prescriptions follow directly from the idea that the postsocialist present is condemned by its past.« (Stark/Bruszt 1998: 5f) 
Transformationsprozesses selbst neue Elemente entstehen, für die es keine Entsprechung im Ausgangszustand gibt und die somit auch nicht das Resultat einer Übersetzung sein können, sondern als emergente Produkte des eigendynamischen Übersetzungsvorgangs selbst zu verstehen sind. Transformation ist als »in hohem Maße selektiv « zu begreifen (Baecker 1998 [1995]: 46) oder - um geläufigere Begriffe der Transformationsforschung zu verwenden - als ein nicht linearer Prozess, »but a combined and uneven one having multiple trajectories. [...] That is, policies combine with preexisting circumstances in different ways to produce different outcomes and reactions « (Burawoy/Verdery 1999: 14f). Ein solcher Prozess lässt sich kaum noch auf vollständig oder partiell erreichte Ziele hin beobachten, sondern ist durch Unsicherheit, Vielschichtigkeit, Unordnung, Simultaneität, Verschwommenheit und dergleichen mehr gekennzeichnet (vgl. den Überblick in Steimann 2010: 22ff).

Die Kritik am geschlossenen Transformationsverständnis und den daran anschließenden Arbeiten mündete in einen zweiten Strang der Transformationsforschung, der um die Jahrtausendwende einsetzte. Bemerkenswert ist, dass sich dabei ungeachtet der disziplinären Vielfalt sowohl hinsichtlich der Forschungszugänge als auch mit Blick auf die spezifischen Gegenstände bald Ähnlichkeiten und gemeinsame Schwerpunkte herauskristallisierten. ${ }^{11}$ Eine erste Gemeinsamkeit ist das Verlassen der > Vogelperspektive < auf den Untersuchungsgegenstand: An die Stelle statistischer Auswertungen von Makrodaten und der Analyse formal-gesetzlicher Regelungen (nationaler Verfassungen, einzelner gesetzlicher Regelungen und ähnliches) trat die Beobachtung lokaler Kommunikation durch Fallstudien und qualitative Forschungsdesigns - nun regelmäßig auftauchende Bezeichnungen wie >lokale Arrangements< oder >individuelle Praktiken< deuten dies an. Zweitens liegt ein inhaltlicher Schwerpunkt auf wirtschaftlicher Transformation und dabei vor allem auf Privatisierungsprozessen. Möglicherweise ist dies darauf zurückzuführen, dass das Scheitern respektive die Unzulänglichkeiten der frühen Transformationsmaßnahmen in diesem Bereich anhand ökonomischer Kennzahlen besonders deutlich beobachtbar waren und gleichzeitig die Befürworterinnen und Befürworter der zugrundeliegenden Modelle mit ihrem selbstbewussten Auftreten eine Gegenbewegung geradezu provozierten. Ein drittes einendes Moment ist schließlich die Suche nach alternativen Konzepten zu den Modellen der ersten Stunde, die sich insbesondere an der analytischen Kategorie des Privateigentums entzündeten. Die Kritik zielt zum einen auf die >westliche< oder >europäische< Dichotomie von privatem und kollektivem Eigentum, die alternative Eigentumsformen ebenso ignoriere wie die Einbettung von Eigentum in eine Vielzahl sozialer (wirtschaftlicher und nicht-

11 Im Folgenden geht es um das Nachzeichnen von allgemeinen Forschungstrends. Die Darstellung impliziert nicht, dass keine Arbeiten entstanden sind, die von den genannten Merkmalen abweichen. 
wirtschaftlicher) Beziehungen. Zweifel richten sich zum anderen auf den vor allem in neoklassischen Modellen postulierten Zusammenhang zwischen klar definierten (privaten) Eigentumsrechten und wirtschaftlichem Wachstum und Wohlstand. Und bemängelt wird schließlich die ideologische Komponente, die mit einem solchen Eigentumsverständnis verbunden sei und die nicht zuletzt auch in den Privatisierungsmaßnahmen im postsozialistischen Kontext zum Tragen kommt (für diese Gemeinsamkeiten und Kritikpunkte vgl. z.B. Blomley 2005; Blomley/Sturgeon 2009; Hann 1998, 2006: Kap. 2; Humphrey/Verdery 2004; Sturgeon/Sikor 2004; Wolford 2007).

An diese Bedenken anschließend gruppieren sich die Arbeiten lose um drei alternative Eigentumskonzepte. Gleichwohl diese Konzepte in der Diskussion nicht scharf abgegrenzt werden, stellen sie je Unterschiedliches ins Zentrum: Dies ist erstens die Kontrastierung des westlichen eigentumsbasierten Regimes mit der sozialistischen Ordnung auf Grundlage von Zugangsrechten (»property-based« vs. »access-based regimes«) (vgl. Amelina 2001: 44ff). Das zugangsrechtsbasierte Regime habe auch über den Zusammenbruch der Sowjetunion hinaus deutliche Spuren hinterlassen und sei folglich bei der Analyse postsozialistischer Eigentumsregime zu berïcksichtigen: »In assessing the failures and successes of any transition, it is important to analyze the differences in the goals and set of skills that the managers and the administrators need in order to succeed in an access-based distribution system as opposed to an ownership-based one« (Amelina 2001: 46). In einem ähnlichen Zusammenhang zitiert Humphrey (2002: 144f) einen Berater einer Regionalverwaltung zur Landwirtschaftsumstrukturierung mit den Worten: »We have no tradition of private property. People don't even feel it. [...] In our society, everything depends on your post and your power-authority. You feel yourself an owner because of your powerful position, not because of your legal rights «. Ein zweiter Begriff, der in der Debatte Prominenz erlangt hat und mit leicht verschobenem Fokus an die Logik der Zugangsrechte anschließt, ist »fuzziness « (vgl. den Überblick in Sturgeon/Sikor 2004). Richtungweisend waren dabei vor allem die Arbeiten der Ethnologin Katherine Verdery, die den Begriff auf Grundlage ihrer ethnographischen Studien zum Dekollektivierungsprozess im ländlichen Rumänien geprägt hat (Verdery 1998, 1999, 2003). Erfasst werden soll die Ambiguität postsozialistischer Eigentumsregime, die sich den klaren Kategorien des neoklassischen Eigentumskonzepts entziehen (Verdery 1999: 54f): »[P] ost-socialist property rights are >fuzzy< in the sense that they lack clarity of borders, owners and exclusion« (Sturgeon/Sikor 2004: 3). An diese Beschreibung schließt drittens wiederum der Begriff des »recombinant property« an, der auf wirtschaftssoziologische Arbeiten David Starks zum Privatisierungsprozess ungarischer Unternehmen zurückgeht (Stark 1996). Die im postsozialistischen Kontext emergierenden >gemischten< Eigentumsformen, so Stark (1996: 997), »blur (1) the boundaries of public and pri- 
vate, (2) the organizational boundaries of enterprises, and (3) the boundedness of justificatory principles«. Auch hier liegen die Reibungspunkte zum neoklassischen Eigentumsbegriff auf der Hand.

Während der Transitologie mit Blick auf wirtschaftliche Transformationsprozesse scharfe Kritik entgegenschlug und daraus ein recht klar konturierter Diskussionszusammenhang erwuchs, ist ähnliches mit Blick auf die Transformation des Politischen weitgehend ausgeblieben. Dies gilt ungeachtet der Beobachtung, dass vor allem die Annahmen liberaldemokratischer Konzepte, die Phasenmodelle des Regimewechsels und die quantitativen Zugänge und Messversuche immer wieder kritisch beäugt wurden (für Kritik an der >Konsolidologie<vgl. z.B. von Beyme 1999: 291ff; auch den Überblick in Mommsen 2013). Alternativkonzepte werden jedoch nicht oder zumindest nicht mit derselben Entschiedenheit angestrebt wie es im Rahmen der Analyse wirtschaftlicher Umstrukturierungsprozesse zu beobachten ist. Obwohl gerade in Arbeiten zum ländlichen Raum regelmäßig betont wird, dass wirtschaftliche und politische Transformationsmaßnahmen für das Verständnis der Wandlungsprozesse gerade in kleinen Gemeinden gleichermaßen relevant seien, stehen Phänomene wie Demokratisierung, Dezentralisierung und Verwaltungsreformen auf der kommunalen Ebene wenn überhaupt am Rande der Untersuchung und finden nur dann besondere Beachtung, wenn sie für den Verlauf der Privatisierungsprozesse unmittelbar relevant erscheinen (so z.B. zu sehen bei Allina-Pisano 2004; Lindner 2008; Verdery 2002).

Stehen Dezentralisierungsprozesse und kommunale Verwaltung als Reformbereiche hingegen explizit im Fokus, scheint die Art der Beobachtung und der Zugang zu diesen Phänomenen von den skizzierten Wandlungsprozessen in der Transformationsforschung wenig tangiert zu sein: Beobachtet werden im Wesentlichen Gesetze und Reformmaßnahmen unter Gesichtspunkten wie lokale Partizipation, Eigenständigkeit der Verwaltungsorgane oder formale Gewaltenteilung (z.B. Campbell 1995, 2006; Gelman 2007; Lankina 2005; Ross 2006, 2009; Wollmann/Gritsenko 2009). Der Blick liegt damit mehr oder weniger ausschließlich auf Operationen (Transformationsmaßnahmen) und nicht, wie es ein offenes und »nichttriviales« Transformationsverständnis (Baecker 1998 [1995]) nahelegen würde, auf dem Operanden, also der Gesellschaft, in der und auf die bestimmte Maßnahmen und Strategien angewendet werden und die diese Operationen zu bewältigen hat (Baecker 1998 [1995]: 48). Die Umsetzung der formalen Akte und die dadurch induzierten gesellschaftlichen Strukturänderungen bleiben folglich weitgehend außen vor. 


\section{Postsowjetische Transformationen in der Weltgesellschaft: Zum Potenzial eines systemtheoretischen Blicks}

Die beiden Stränge der Transformationsforschung - die Transitologie und ihre Gegenbewegung - werden ungeachtet ihrer betont zur Schau gestellten Gegensätzlichkeit durch eine Leitdifferenz zusammengehalten, an der sie sich aus konträren Richtungen kontinuierlich abarbeiten und gerade dadurch auch reproduzieren: Der Gegensatz zwischen $>$ Ost $<$ und $>$ West $<$ respektive die (latent) unterstellte grundsätzliche Andersartigkeit postsozialistischer Gesellschaften im Vergleich mit westlichen Gesellschaften fungiert in beiden Strängen gleichermaßen als Explanans und Fluchtpunkt der Forschung. In der Transitologie kommt diese Differenz in der Unterscheidung von Ausgangszuständen (Planwirtschaft und Autokratie) und Endzuständen (Marktwirtschaft und Demokratie) zum Ausdruck. Mit geeigneten Maßnahmen sollen die >östlichen< postsozialistischen Gesellschaften dazu gebracht werden, von der einen auf die andere Seite zu wechseln, und den Gegensatz zum $>$ Westen< auf diese Weise in ihrem eigenen Interesse überwinden. Werden die angestrebten Endzustände verfehlt, lässt sich die Andersartigkeit sowohl zur Begründung des Scheiterns als auch als Orientierungspunkt für die Rekonzeption der entsprechenden Maßnahmen ins Feld führen. Deutlich wird der Gegensatz von >Ost< und >West< aber auch mit Blick auf die Gegenbewegung zur Transitologie: Die Andersartigkeit der postsozialistischen Gesellschaften ist hier sowohl Ausgangspunkt für die Kritik an konkreten transformationspolitischen Maßnahmen als auch für die Beschreibung abweichender Entwicklungswege und die Ableitung alternativer Konzepte. Im Unterschied zur Transitologie steht dahinter jedoch die Annahme der prinzipiellen Unüberwindbarkeit des Gegensatzes zwischen > westlichen< und >postsozialistischen<Gesellschaften, was letztlich dazu führt, dass gängige Transformationsmaßnahmen ebenso wie > westliche< Modelle und Theorien per se als unangemessen gelten.

Die Transitologie neigt dazu, gesellschaftliche Komplexität in ihren Modellen und deren Anwendung zu unterdrücken. Demgegenüber tendieren die Vertreterinnen und Vertreter der Gegenbewegung dazu, gesellschaftliche Komplexität in ihren Alternativkonzepten lediglich zu spiegeln: Meist stützen sich die Analysen vornehmlich auf vermeintlich erwartungsfreie dichte Beschreibungen der beobachteten Phänomene und versuchen, sich durch diesen Zugang zur Empirie von Konzepten, die sich an abstrakten Modellen orientieren, betont zu distanzieren. Mit den wirtschaftswissenschaftlichen Kategorien wird dabei jedoch nicht selten gleich jegliche Theorie oder Anwendung von >Großkategorien` mehr oder weniger strikt abgelehnt. So liegt beispielsweise zwar allen drei oben skizzierten Eigentumskonzepten im Kern die Forderung nach einem soziologischen Eigentumsbegriff zugrunde. Dieser müsste im Unterschied zur wirtschaftswissenschaftlichen Lesart anerkennen, 
dass Eigentum zwar immer auch auf bestimmte Objekte verweist, sich seine gesellschaftliche Relevanz jedoch daraus ergibt, dass Eigentumsbeziehungen in erster Linie soziale Beziehungen zwischen Personen - Eigentümern und Nicht-Eigentümern - sind: » $[\mathrm{P}]$ roperty< is about social relations, relations among persons rather than between persons and things « (Verdery 1999: 75; auch Hann 2006: 19). In (empirischen) Arbeiten, die an diese Feststellung anschließen und sie teilweise explizit aufgreifen, wird dieses Soziologisierungspostulat jedoch nicht konsequent weiterverfolgt. Soziologische Eigentumskonzepte (vgl. z.B. Carruthers/Ariovich 2004; Fligstein 2001; klassisch Weber 1964) kommen in diesem Bereich der Transformationsforschung faktisch nicht vor oder bleiben - sofern sie dennoch erwähnt werden - folgenlos.

Einen Eigentumsbegriff, der sich an dieser Stelle anschließen lässt, hält beispielsweise die Systemtheorie bereit. Nach ihrem Verständnis bildet sich Eigentum, wenn der Zugriff auf knappe Mengen Positionen des Habens und des Nicht-Habens kondensiert. Eigentum ist die Erstcodierung von Knappheit und dabei stets exklusiv: Wenn die einen etwas (in Form von Verfügungsrechten) besitzen, sind alle anderen vom Gebrauch ausgeschlossen (Luhmann 1988: 187f; Baecker 2006: 52f). Beide Seiten der Unterscheidung Eigentum|Nicht-Eigentum bieten Ansatzpunkte für (wirtschaftliche) Anschlusskommunikation, die sich in der modernen Gesellschaft üblicherweise im Medium des Geldes vollzieht. Zahlungen als Zweitcodierung von Knappheit respektive das Medium des Geldes machen das knappgehaltene Eigentum liquide und sorgen für die Reproduktion der Wirtschaft, indem mit Hilfe von Geld die Knappheitsparadoxie bearbeitet wird. Geld ermöglicht das Erleben der Knappheitsreduktion anderer, welche ohne Zahlungen Handeln wahrscheinlich machen würde. Die Wahrscheinlichkeit, dass Zahlungen an Zahlungen anschließen, steigt - auch weil Geld fast beliebig transformierbar ist (Baecker 2006: 48ff; Deutschmann 2009a; Esposito 2008: 126).

An dieses Eigentumsverständnis lassen sich zwei Überlegungen anschließen, die auch im Hinblick auf den postsozialistischen Transformationsbegriff relevant sind: Die erste zielt auf die innere Ordnung von Wirtschaft und die Ausgestaltung von Knappheitskommunikation. In den Fokus rücken dabei zum einen das Verhältnis und die relative Reichweite von Markt als lose und Hierarchie als fest gekoppelte Formen der Knappheitskommunikation (vgl. Baecker 2006: 121ff, 2008: 114). Zum anderen lässt sich in diesem Zusammenhang nach den Inklusions- und Exklusionsformen fragen, mit denen Eigentum stets verbunden ist und bei deren Gestaltung und Verschiebung diverse gesellschaftliche Kontexte zum Tragen kommen können. Neben formal-rechtlichen Kontexten, denen in westlichen Gesellschaften meist eine hervorgehobene Bedeutung zukommt, wirken sich Aspekte wie die Zugehörigkeit zu einer Gruppe, der Verweis auf Traditionen, religiöse Strukturen und ähnliches potenziell auf das jeweils konkrete Erscheinungsbild von Eigentum aus. 
Die zweite Überlegung dreht sich weniger um die innere Verfasstheit von Wirtschaft als vielmehr um die Grenzen des Wirtschaftssystems gegenüber seinen Umwelten. Zu fragen ist dann, an welchen Stellen Knappheitskommunikation in andere Kommunikationsformen umschlägt, was überhaupt für knapp gehalten und potenziell zum Gegenstand wirtschaftlicher Kommunikation gemacht wird und auf welche Weise sich dabei Verschiebungen beobachten lassen. Das Erkenntnisinteresse von vielen der genannten Arbeiten über postsozialistische Transformations- und speziell Privatisierungsprozesse lässt sich einer oder beiden dieser Richtungen zuordnen, auch wenn dies in den Arbeiten selbst in der Regel nicht auf diese Weise expliziert wird. Nicht ohne Grund, denn offensichtlich fehlen die Mittel, um das, was beobachtet wird und in seiner inhaltlichen Substanz überwiegend gar nicht bestritten werden soll, theoretisch einzuordnen und handhabbar zu machen.

Doch auch über den speziellen Transformationsbereich der wirtschaftlichen Privatisierung mit seinen spezifischen Problemlagen hinaus finden sich gute Gründe für einen systemtheoretischen Blick auf die sozialen Wandlungsprozesse im postsowjetischen Raum. In erster Linie ist dabei von Belang, dass die Systemtheorie nach Luhmann von einem operativen Gesellschaftsbegriff ausgeht, dem zufolge sich Gesellschaft über Kommunikation - und nur über Kommunikation - als basales Element vollzieht. Die Grenzen der Gesellschaft ergeben sich damit über die Verknüpfungsfähigkeit von Kommunikationen, sodass im Umkehrschluss von einem autonom operierenden Gesellschaftssystem nur dann die Rede sein kann, wenn keine kommunikativen Verknüpfungen mit anderen Gesellschaften existieren respektive auf ein Minimum (beispielsweise die bloße wechselseitige Wahrnehmung) reduziert sind (Stichweh 2006: 239; auch 2000 [1999]). Gleichzeitig gilt die Gesellschaft in der Systemtheorie als das umfassendste Sozialsystem (oder das Sozialsystem höchster Ordnung), das sich zwar intern differenziert, ohne dass die jeweiligen kommunikativen Strukturen und Prozesse aber aus der Gesellschaft herausfallen würden (Luhmann 1997: 145ff; mit Bezug auf Parsons: Stichweh 1995: 32). Daraus folgt unter anderem und mit einer bei anderen Theorien nicht erkennbaren Radikalität, dass die Trennung oder gar Opposition von Gesellschaft und ihren Teilsystemen - etwa der Wirtschaft - in etwa so gehaltvoll ist wie die Unterscheidung von Tieren und Katzen: »Alles wirtschaftliche Handeln ist soziales Handeln, daher ist alle Wirtschaft immer auch Vollzug von Gesellschaft« (Luhmann 1988: 8).

Für die moderne Gesellschaft ist vor dem Hintergrund dieser Überlegungen davon auszugehen, dass nur die Weltgesellschaft als selbstgenügsames Sozialsystem und damit differenzlos operiert (Luhmann 1997: 145ff; Stichweh 2006: 239f). Die Weltgesellschaft weist, so argumentiert Stichweh (2006), eine Reihe sogenannter Eigenstrukturen auf, also Strukturen, die mit dem Phänomen der Weltgesellschaft in einem Verhältnis wechselseitiger Intensivierung stehen, indem sie »die Herausbildung der Weltgesellschaft in dem Maße [befördern], in dem sie selbst ihr 
Profil immer deutlicher gewinnen. Umgekehrt werden sie durch das vorhandene System der Weltgesellschaft privilegiert, weil sie sich als kompatible Muster erweisen« (Stichweh 2006: 241). Eine zentrale Bedeutung kommt dabei den Funktionssystemen $\mathrm{zu}$, die sich als primäre Differenzierungsform der Weltgesellschaft in Form globaler Kommunikationszusammenhänge herausgebildet haben. Als naheliegende Kandidaten für weitere Eigenstrukturen erscheinen darüber hinaus (formale) Organisationen, Netzwerke, epistemische Gemeinschaften, Weltereignisse und Märkte.

Aus diesen Annahmen folgt wohlgemerkt nicht, dass sich regionale Besonderheiten zu Gunsten weltgesellschaftlicher Strukturen homogenisieren und einebnen oder andere Differenzierungsformen notwendigerweise und zwingend verschwinden (zu Unterschieden und Ungleichzeitigkeiten in der Weltgesellschaft vgl. Stichweh 1995: 33). Zwar wohnt gerade Funktionssystemen eine erhebliche Penetrationskraft inne, die auf die Reproduktion über binäre Codes und die Verwendung symbolisch generalisierter Kommunikationsmedien zurückzuführen ist und die sich (auch) auf die autonomen »Regionalkulturen der Welt« (Stichweh 2006) richtet. Geht man dabei jedoch - erneut mit Stichweh - davon aus, dass die Bildung sozialer Strukturen kumulativ erfolgt, dass neue Strukturen die alten nicht ersetzen und zum Verschwinden bringen, sondern sie überlagern, mit ihnen koexistieren und gegebenenfalls zu neuen Formen verschmelzen, dann müsste sich die Reproduktion regionaler Idiosynkrasien über die spezifischen Bedingungen des Aufeinandertreffens und die spezifische Gestaltung der Koexistenz regionaler Differenzierungsformen und weltgesellschaftlicher Eigenstrukturen erklären lassen.

Die Überlegungen zu den Strukturen der Weltgesellschaft und zu regionalen Besonderheiten lenken den Blick nicht allein auf postsowjetische Transformationsprozesse, sondern erweitern sehr grundsätzlich die Perspektive auf innergesellschaftliche Diversität. Zu denken ist hier an Beschreibungen wie >kapitalistische<, $>$ demokratische< oder eben auch >(post-)sozialistische< Gesellschaften. Insofern die Systemtheorie über ihren Gesellschaftsbegriff zunächst die grundsätzliche Einheit von Gesellschaft betont, die sich über Kommunikation und das Anschließen von Kommunikation an Kommunikation reproduziert, müssen sowohl der Sozialismus als auch der Kapitalismus als innere Variationen dieser Weltgesellschaft gesehen werden. ${ }^{12}$ Dies gilt ungeachtet ihrer signifikanten strukturellen Unterschiede und trotz des hermetisch anmutenden »Eisernen Vorhangs«. Analytisch sind sie weder

12 Wenn im weiteren Verlauf dieser Arbeit dennoch vereinzelt die Rede von der >sowjetischen Gesellschaft< ist, dann trägt dies allein der besseren Lesbarkeit Rechnung und geschieht im Wissen um die damit verbundenen theoretischen Unschärfen. Theoretisch korrekt, aber sprachlich ungleich sperriger wäre beispielsweise die Bezeichnung als >sowjetisches Segment der Weltgesellschaft . 
als unterschiedliche, noch als einander ablösende Gesellschaftstypen mit verschiedenen Operationsweisen zu begreifen und damit voneinander zu trennen (Baecker 1998 [1995]: 54). Ohnehin und ganz praktisch sind sie auch empirisch nicht als voneinander autonom operierende und in diesem Sinne >eigenständige< Gesellschaften zu beobachten. Daraus folgt, dass sich

»auch das Jahr $1989^{13}$ nicht als Jahr des Zusammenbruchs einer Gesellschaftsformation beschreiben [lässt], sondern vielmehr als ein Datum, mit dem unübersehbar wurde, daß die unter dem Programm des Sozialismus laufende Reproduktionsform der Gesellschaft an den Grenzen ihrer Leistungsfähigkeit angelangt war.« (Baecker 1998 [1995]: 56f)

Diese Überlegung lässt sich mit einer Implikation des bereits skizzierten offenen oder nichttrivialen Verständnisses von Transformation ergänzen, die darauf verweist, dass alle Bestandteile des geschlossenen Transformationsprozesses - Anfangs- und Endzustand, Operationen und Operand - nun nicht mehr als exogene Faktoren in den Prozess einfließen, sondern endogenisiert werden (Baecker 1998 [1995]: 50ff). Alle Elemente, die den Verlauf des Transformationsprozesses bestimmen, werden im Prozess selbst hervorgebracht, sodass Transformation zu einem Spezialfall von Rekursivität wird: »Die Transformation unterliegt selbst der Transformation und kann für die Variation der Elemente der Transformation auf nichts anderes als auf Transformation verweisen« (Baecker 1998 [1995]: 51). Durch den ständig mitlaufenden Verweis auf sich selbst übersetzt Transformation nicht nur Zustände, sondern führt Veränderung herbei, die Übersetzung wünschbar machen, ermöglichen oder auch verhindern. Kontinuierlich verändert sie damit nichts weniger als ihre eigenen Voraussetzungen und dies sowohl in positiver als auch in negativer Hinsicht (Baecker 1998 [1995]: 54). An die Stelle definierbarer Ausgangs- und Endzustände tritt der »offene Prozeß einer sich selbst mitverändernden Transformation« (Baecker 1998 [1997]-b: 104), deren Verlauf sich aus dem Zusammenwirken des Dualismus von Steuerung und Intentionalität (auf der Innenseite) und Prozessualität im Sinne einer Offenheit gegenüber gesellschaftlicher Komplexität (auf der Außenseite) ergibt (Baecker 1998 [1995]: 49). Die Beobachtung von Transformation - und dies gilt auch für den postsowjetischen Fall - ist dann die Beobachtung einer Transformationsgesellschaft, »die bestimmte Zustände ihrer selbst als >sozialistisch<, andere als >kapitalistisch< bezeichnet und auf der Suche nach Übersetzungsregeln ist, die die einen in die anderen zu transformieren erlaubt« (Baecker 1998 [1995]: 54).

»There is no theory of transition« (vgl. Steimann 2010: 19) - diese in der Transformationsforschung regelmäßig und je nach Autorin oder Autor und Adressatin

13 Baecker bezieht sich hier auf den Zusammenbruch der DDR. 
oder Adressaten mal resignierend, mal belehrend und mal triumphierend geäußerte Behauptung mag vor diesem Hintergrund für den postsowjetischen Fall durchaus zutreffend sein, sofern damit das Postulat der theoretischen Herleitung eines gesellschaftlichen Zielzustandes samt entsprechender Kausalitäten und Gesetzmäßigkeiten gemeint ist. Sie steht allerdings nicht im Widerspruch zu einem theoretisch informierten Blick auf die postsowjetische Transformationsgesellschaft. Ein Blick, der geboten ist, um die Besonderheiten dieser Region in der Weltgesellschaft und deren Ordnung als Ordnung der Kommunikation (Baecker 2007: 105) nicht nur zu beschreiben, sondern auch zu verstehen. Der systemtheoretische Zugang ist eine Möglichkeit, dabei gesellschaftliche Komplexität weder zu unterdrücken noch zu spiegeln, sondern durch theorieinternen Komplexitätsaufbau zu reduzieren respektive aufzuheben ${ }^{14}$ (Luhmann 1995 [1984]: 1) und entlang der skizzierten theoretischen Kategorien systemintern zu strukturieren.

\section{Erkenntnisinteresse und Argumentationsverlauf}

Die vorliegende Studie bewegt sich thematisch im Kontext politischer Dezentralisierungs- und wirtschaftlicher Privatisierungsprozesse, wobei der jüngste Reformversuch im Bereich kommunaler Verwaltung, das seit Anfang 2006 geltende Gesetz Nr. 131 »Über allgemeine Prinzipien der Organisation lokaler Selbstverwaltung in der Russischen Föderation«, den primären Orientierungspunkt für die empirische Argumentation setzt. Konkret geht es um die Frage, welche Strukturen und Ordnungen sich in ländlichen Gemeinden Russlands mit Blick auf diesen Reformbereich und die daraus hervorgehenden Irritationen für die lokalen Kommunikationszusammenhänge herausbilden. Erklärungsbedürftig sind dabei Wandlungsprozesse ebenso wie Kontinuitäten, die sich mit Blick auf die spezifischen Strukturmerkmale des ländlichen Raums unter dem sowjetischen Regime beobachten lassen. Vor dem Hintergrund der einleitenden Überlegungen zum Stand der Transformationsforschung und zum Potenzial einer systemtheoretischen Perspektive lässt sich dieses Erkenntnisinteresse in zwei Kontexte einordnen: Der Beitrag zur Transformationsforschung ergibt sich daraus, dass mit Kommunalreformen und den in ihrem Kontext stattfindenden Prozessen sozialen Wandels in den ländlichen Gemeinden ein Gegenstand beleuchtet wird, der in zweifacher Hinsicht bislang ein Schattendasein führte. Zum einen ist die Anzahl der Arbeiten zu dieser spezifischen Thematik überschaubar, zum anderen liegt der Fokus bisheriger Analysen meist auf dem In-

$14 \mathrm{Zu}$ dieser Form der Komplexitätsreduktion bemerkt Luhmann (1995 [1984]: 1): »Thus the presentation of theory itself practices what it preaches: the reduction of complexity. Yet for it, reduced complexity is not excluded complexity, but rather >sublated [aufgehobene] complexity.« 
halt von Formalstrukturen und gesetzlichen Regelungen, die hier bewusst nicht in den Vordergrund gerückt werden. Gleichzeitig versteht sich die vorliegende Studie als Beitrag zur soziologischen - und speziell: systemtheoretischen - Diskussion um den Begriff und die Strukturen der Weltgesellschaft, indem mit Blick auf zwei russische Gemeinden das Aufeinandertreffen und Zusammenspiel regionaler und globaler Strukturen detailliert analysiert und der Prozess der Herausbildung einer spezifischen regionalen Ordnung in der modernen Weltgesellschaft exemplarisch nachgezeichnet wird.

Im Hinblick auf diese Interessen und Ansprüche gliedert sich die Analyse in drei Hauptteile: Im ersten Argumentationsschritt fokussiere ich die Vergangenheit der ländlichen Gemeinden und frage nach der Variante gesellschaftlicher Differenzierung, die sich im sowjetischen Regime herausgebildet hat. Den wesentlichen theoretischen Schlüssel zum Verständnis dieser Variante bilden zum einen die Unterscheidung von Code und Programm gesellschaftlicher Funktionssysteme sowie zum anderen die Merkmale von Organisationen, auf welche die Prozesse gesellschaftlicher Reproduktion in der Sowjetunion kontinuierlich verweisen. Sozialismus (dies gilt zumindest für die sowjetische Lesart) lässt sich entsprechend als ein Gesellschaftsprogramm mit umfassendem Geltungsanspruch beschreiben, anhand dessen die Gesellschaft unter der Führung der Einheitspartei entlang von zwei Leitunterscheidungen eingerichtet und strukturiert werden sollte: Erstens die Unterscheidung von (Voll-)Inklusion im Sinne einer umfassenden politischen Adressierbarkeit und Exklusion, zweitens die Unterscheidung von Hierarchie (oder Planwirtschaft) und Markt respektive von Kollektiv- und Privateigentum. Das sozialistische Programm beanspruchte, im Rahmen dieser Unterscheidungen jeweils die erstgenannte Seite zu markieren und die letztgenannte als >kapitalistisches Anderes $<$ mitlaufen zu lassen. Das wesentliche Instrument zur Programmumsetzung waren Organisationen. Sämtliche gesellschaftliche Operationen sollten in einen von zwei Organisationstypen integriert werden: Entweder in die Partei, die sich an die Spitze der Gesellschaft setzte und die interne Entdifferenzierung des politischen Systems bewirkte, oder in einer Vielzahl von Massenorganisationen in allen gesellschaftlichen Bereichen, die für die umfassende Inklusion aller zuständig waren.

Die Identifikation von zwei Leitunterscheidungen und einem zentralen Implementationsmechanismus bedeutet keineswegs, dass die sozialistische Programmierung der Gesellschaft in dieser intendierten umfassenden Weise gelang. Aber sie fungieren in der Selbst- wie auch in der Fremdbeobachtung des sowjetischen Regimes als Referenzpunkte, über die sich sowohl die Konformität mit als auch die Abweichung von den politischen Idealen und den daraus abgeleiteten Maßnahmen erschließen. Beispielhaft verdeutlichen lässt sich dies mit Blick auf die Strukturmerkmale sowjetischer ländlicher Gemeinden: An den landwirtschaftlichen Kollektivbetrieben als dörfliche Massenorganisation lässt sich illustrieren, wie sowohl 
die Hierarchisierung wirtschaftlicher Kommunikation und die Eigentumskollektivierung als auch die umfassende Inklusion der Dorfbevölkerung in Organisationsstrukturen wirkungsvoll umgesetzt wurden. Der Kolchos generierte sich innerhalb des Dorfes als Machtinstanz, wohingegen der Gemeindeverwaltung eine untergeordnete Position zukam. Gleichzeitig führte die Dominanz der Kollektivbetriebe aber dazu, dass sich die ländlichen Gemeinden als weitgehend abgeschlossene Kommunikationszusammenhänge innerhalb der staatlich gelenkten Hierarchie etablierten, in denen sich bemerkenswerte Spielräume für abweichende >regimefremde $<$ Kommunikationsformen - beispielsweise in Form privater Hoflandwirtschaften und wirtschaftlicher Kommunikation - eröffneten. Es lässt sich zeigen, dass regimekonforme und -abweichende Strukturen in den Gemeinden nicht nur koexistierten, sondern sich durch kontinuierliche Verweise aufeinander reproduzierten und zunehmend ineinander verzahnten. Die ländlichen Gemeinden stehen damit nicht nur beispielhaft für die Funktionslogik der sowjetischen Gesellschaft, indem hier Reichweite und Bruchstellen des sozialistischen Programms hervortreten. Sie sind auch ein instruktiver Ansatzpunkt, um durch den Regimewechsel induzierte Wandlungsprozesse zu beobachten, im Zuge derer speziell Privatisierungsund Dezentralisierungsmaßnahmen die Kontrapunkte zu den zentralen Strukturmerkmalen des sowjetischen Dorfes bildeten.

An den Maßnahmen politischer Dezentralisierung und der Einführung kommunaler Selbstverwaltung sowie deren wissenschaftlicher Beobachtung setzt der zweite Argumentationsschritt an. Mit Blick auf die Entwicklungspolitik und -forschung lässt sich zeigen, dass dieser Reformbereich in einen allgemeinen Trend einzuordnen ist, der in Richtung des >Lokalen< weist und von der Abkehr vom sogenannten Washington Consensus als entwicklungspolitisches Leitbild geprägt ist. Im Zuge dieser Verschiebung gewannen unter anderem drei Argumentationslinien (zum Teil erneut) an Aufschwung: Politische Dezentralisierung und kommunale Selbstverwaltung werden im Horizont von (i) wirtschaftlicher Effizienz, (ii) der Einführung und Stärkung demokratischer Strukturen und (iii) der Stärkung respektive Ermächtigung (»empowerment«) lokaler Gemeinschaften beobachtet. Alle drei Argumentationslinien sind, wenngleich je unterschiedlich, an politische Zielsetzungen und normative Vorstellungen darüber gekoppelt, wie Gesellschaft strukturiert sein sollte. Diese enge Kopplung erschwert oder verhindert gar eine wissenschaftliche Reflexion, verstellen die einschlägigen Begriffe doch eher den Blick auf das Phänomen der Dezentralisierung und der kommunalen Verwaltung an sich.

Verzichtet man hingegen auf derartige politische und/oder normative Setzungen und nähert sich aus einer soziologischen Perspektive, die nach Kommunikationsund Beobachtungsformen im Zusammenhang mit Dezentralisierung und kommunaler Selbstverwaltung fragt, dann treten sie als eine Form der internen Differenzie- 
rung des politischen Systems hervor, im Zuge derer sich Organisationen bilden und etablieren und Inklusionsmodi verschieben respektive vervielfältigen. Vor dem Hintergrund der Überlegungen zur sowjetischen Organisationsgesellschaft erlaubt ein auf diese Weise geschärfter Blick am Beispiel der jüngsten Kommunalreform in Russland potenzielle Bruchstellen der sowjetisch geprägten Strukturen ländlicher Gemeinden zu identifizieren. In den Fokus rückt dabei unter anderem das Verhältnis von Gemeindeverwaltung und ehemaligen Kollektivbetrieben. Für die nachfolgende empirische Analyse lassen sich aus diesen Überlegungen vier Leitaspekte für die Beobachtung von Kommunikation in diesem Transformationsbereich ableiten: (i) Formen der Inklusion und Exklusion, (ii) Entscheidungen und Lernprozesse von Organisationen, (iii) der Umgang mit Formalität und Informalität sowie (iv) räumliche Integration und weltgesellschaftliche Strukturen.

Im dritten Argumentationsschritt tritt meine eigene Empirie auf die Bühne. Sie wird auf die vier zuletzt genannten Leitaspekte hin beobachtet. Die Themen der dörflichen Kommunikation, auf die ich während meiner drei Feldaufenthalte stieß, legen eine Gliederung in vier Episoden nahe, die auf jeweils unterschiedliche Weise Einsichten zu den genannten Aspekten bieten. In der ersten Episode geht es um Formen der Kooperation zwischen Gemeindeverwaltung und ehemaligem Kolchos: Hier führte ein kolchoskritisches Flugblatt, das im Zusammenhang mit der Kandidatur des Kolchosvorsitzenden für das Bezirksparlament verteilt wurde, zu einer Diskussion über die Rolle des ehemaligen Kollektivbetriebs im Dorf und speziell gegenüber der Gemeindeverwaltung. Es lenkte den Blick sowohl auf die Erwartungen, die sich an den Betrieb richten und ihn auf einem Spektrum zwischen Wirtschafts- und Wohlfahrtsorganisation verorten, als auch auf den Klärungsanspruch, der diesbezüglich von der jüngsten Kommunalreform erhoben wurde. Deutlich wird, dass sich die >sowjetischen< Kooperationsstrukturen zwar reproduzieren, dabei jedoch zunehmend von Normalitätserwartungen irritiert werden, die sich in Folge der jüngsten Kommunalreform zu verschieben beginnen.

Die zweite Episode dreht sich um den Anschluss der Gemeinden an das Gasnetz. Es kollidieren die Beobachtungen der Gemeindeverwaltung, für die sich die sogenannte Gasifizierung als ein Steuerungsproblem darstellt, mit den Beobachtungen der Gemeindemitglieder, die sich mit einer individuell folgenreichen wirtschaftlichen Entscheidung konfrontiert sehen. Verdeutlichen lassen sich an diesem Beispiel nicht nur die Funktion lokalpolitischer Kommunikationsforen und die Grenzen lokaler Machtkommunikation, sondern auch die Art und Weise, wie wirtschaftliche Strukturen über Eigentum, Zahlungen und Kredite in den Dorfkontext hineinzuwirken beginnen und diesen umstrukturieren.

Die dritte Episode thematisiert, auf welche Weise sich eine Agroholding als ein für den russischen ländlichen Raum neuartiger Betriebstyp in einer Gemeinde etabliert. Mit Blick auf die Anpassungsprozesse und Widerstände, die das Auftreten 
dieser Organisation im Dorf auslösen, lässt sich nachzeichnen, wie sich einerseits vermeintlich tief verankerte Erwartungsstrukturen, die sich um das kollektive Eigentum an Agrarland ranken, innerhalb kurzer Zeit grundlegend wandeln und sich im Dorf umfangreiche Formalisierungs- und Monetarisierungsprozesse vollziehen. Andererseits verweist vor allem das Verhältnis des Unternehmens zur Gemeindeverwaltung unübersehbar auf >sowjetische< Strukturen, die sich ungeachtet dieser Brüche durchsetzen.

Der Ausgangspunkt der vierten Episode ist ein Kaufangebot, das im Zuge der Expansionspläne ebenjener Agroholding den Anteilseignerinnen und -eignern des ehemaligen Kolchos im Nachbardorf unterbreitet wurde. Die Absicht der Agroholding, die Eigentumszertifikate und damit die Rechte auf das Land des ehemaligen Kolchos zu erwerben, wurde in dieser Gemeinde als Angriff auf die tradierten dörflichen Strukturen interpretiert, den es abzuwehren galt. Nachdem politische Appelle der Gemeindeverwaltung und Verweise auf die dörfliche Moral verhallten, wurden unter der Federführung der Bürgermeisterin Formalisierungsprozesse angestoßen und Zahlungen eingeführt, die umfassende Änderungsprozesse in ebenjenen Bereichen anstießen, die ursprünglich in ihrer tradierten Form hätten bewahrt werden sollen.

Das Fazit fasst die zentralen Ergebnisse der drei Argumentationsschritte zusammen und bezieht sie abschließend aufeinander. Speziell die dabei herausgearbeiteten Aspekte des Wandels der lokalen Kommunikationszusammenhänge in den Gemeinden werden in den Kontext jener Muster und Mechanismen postsowjetischer Transformationen eingeordnet, die in bisherigen empirischen Studien zum ländlichen Russland aufscheinen, und auf Anknüpfungspunkte hin beobachtet. 



\section{Die Logik der sowjetischen Organisationsgesellschaft}

Die Sowjetunion lässt sich als umfassender Versuch beobachten, eine beträchtliche Region innerhalb der modernen Weltgesellschaft abzugrenzen, indem die gesellschaftlichen Strukturen im sowjetischen Teil der Welt an marxistisch-leninistischen Prinzipien ausgerichtet und die Verbindungen mit der >kapitalistischen $<$ Umwelt unterbrochen werden sollten. Aus systemtheoretischer Perspektive wurde wiederholt darauf hingewiesen, dass ein entscheidender Grund für den Zusammenbruch der sowjetischen Gesellschaftsordnung gerade in ihrem scharfen Kontrast zur modernen Weltgesellschaft lag (vgl. z.B. Hayoz 1997; Luhmann 1997: 809f, 2000a: 384f; Willke 2005: 46ff). Ungeachtet des letztlichen Scheiterns des sowjetischen Versuchs war es jedoch gelungen, über rund 70 Jahre eigene und aus westlicher Perspektive hochgradig intransparente Strukturen zu etablieren und zu reproduzieren, die im sogenannten postsowjetischen Raum auch mehr als 20 Jahre nach dem Zusammenbruch des Regimes noch deutlich erkennbare Spuren hinterlassen haben und nach wie vor Bindungskraft entfalten. Um zu verstehen, welche gesellschaftlichen Strukturen in den ehemals sowjetischen Staaten im Transformationskontext entstehen, ist es wichtig, zunächst die Logiken dieser sowjetischen Variante gesellschaftlicher Differenzierung nachzuvollziehen - denn letztlich gilt: Die neue Ordnung wird nicht auf, sondern mit den Ruinen des Kommunismus errichtet (Stark 1996: 995). Der Konstruktionsweise des Gebäudes, auf das die bis in die Gegenwart verbliebenen >Ruinen< verweisen, möchte ich mich im folgenden ersten Teil dieser Studie schrittweise widmen.

Als Beschreibungs- und Beobachtungskategorien bietet sich dabei das systemtheoretische Instrumentarium an, insbesondere die Logik von und Beziehungen zwischen Funktionssystemen und Organisationen. Dies gilt ungeachtet der betont betriebenen hermetischen Abgrenzung der Sowjetunion gegenüber dem Rest der (nicht-sozialistischen) Welt und den daraus resultierenden unbestreitbaren strukturellen Diskrepanzen zwischen >Ost $<$ und >West $<$. Gerade die spezifischen Bedingungen gesellschaftlicher Reproduktion in der Sowjetunion lassen sich auf diese Weise 
nicht nur sichtbar machen, sondern trotz ihrer Fremdheit und scheinbaren Widersprüchlichkeit als Variation innerhalb und damit Teil der Weltgesellschaft greifbar machen. Diese Sichtweise steht quer zu einer in weiten Teilen der Transformationsforschung eingeübten Perspektive, die die Sowjetunion implizit in Opposition zur modernen Gesellschaft als theoretisch kaum greifbaren Sonderfall mit einer grundsätzlich distinkten Logik einordnet: »Wir richten den Blick immer gleich auf unterschiedliche Gesellschaftsformen und übersehen dabei, daß auch der Sozialismus nicht die Gesellschaft, sondern nur das Verhältnis von Organisation und Gesellschaft variiert hat « (Baecker 1998 [1997]-b: 108). Oder im Sinne Pollacks (2003: 11): Eine eigene Theorie des Staatssozialismus führt notwendigerweise in eine analytische Sackgasse, denn »[w]er lediglich eine Gesellschaft studiert, versteht keine«. Ein solches gesellschaftstheoretisches Aufschließen sowjetischer Idiosynkrasien verspricht insbesondere in zwei Richtungen Anschlussfähigkeit: Empirisch bereitet es den Boden für die Beschreibung der postsowjetischen Transformationsgesellschaft (ebenfalls) als Teil der Weltgesellschaft, theoretisch eröffnet es Anknüpfungs- und Reibungspunkte für soziologische Theoriebildung und -weiterentwicklung. ${ }^{1}$

Um nun einerseits ein hinreichend allgemeines Bild der sowjetischen Variante gesellschaftlicher Differenzierung zu zeichnen, andererseits aber den Fluchtpunkt meiner eigenen empirischen Analyse der postsowjetischen Transformationsgesellschaft nicht aus den Augen zu verlieren, argumentiere ich im Folgenden in drei Schritten, die sich auf den ländlichen Raum und das sowjetische Dorf hin konkretisieren: Im ersten Schritt werden die ideologischen Prämissen des Sozialismus und der daraus resultierende umfassende Interventionsanspruch mit systemtheoretischen Kategorien beschrieben und eingeordnet. Der zweite Argumentationsschritt fokussiert die Form der Umsetzung des sozialistischen Gesellschaftsprogramms in der Sowjetunion, die letztlich darin bestand, die Gesellschaft möglichst umfassend in organisierte Strukturen zu überführen. Im dritten Schritt ziehe ich schließlich ausgewählte Strukturmerkmale des sowjetischen Dorfes und der sowjetischen Landwirtschaft heran, um mit Blick auf konkrete gesellschaftliche Operationen eine Reihe von Widersprüchlichkeiten innerhalb des Regimes deutlich zu machen, die wiederum exemplarisch instruktiv für das Gesamtphänomen der sowjetischen Organisationsgesellschaft sind.

1 In ähnlicher Weise fordert Pollack (2003: 10f) für die DDR-Forschung eine stärkere Orientierung an soziologischen Theorien, womit sie ihre gleich mehrfache Entkopplung von der Osteuropaforschung, aber auch von historischen, politikwissenschaftlichen und soziologischen Debatten überwinden könne. 


\section{Sozialismus als Programm Und das Programm des Sozialismus}

Wenn die deutschsprachige Soziologie und Sozialgeschichte sozialistische Gesellschaftsordnungen behandelt, steht meist die DDR im Fokus. Dabei schwingt der Begriff der Entdifferenzierung mal leise mit, mal wird er prominent gestellt um zu erörtern, ob es sich bei der DDR um eine moderne Gesellschaft handelte. ${ }^{2}$ Trotz seiner augenscheinlich zentralen Stellung in den Diskussionen und Kontroversen, die sich an ihm entzünden (und auf die ich, da sie sich ausschließlich um den Gegenstand der DDR drehen, an dieser Stelle nicht näher eingehen möchte), bleibt der Begriff der Entdifferenzierung jedoch überraschend unscharf: ${ }^{3}$ Die Verwendung ist entweder schwammig, theoretisch inkohärent oder übermäßig simplifizierend. Schwammig ist sie, weil üblicherweise weder der theoretische Rahmen noch die Differenzierungsform, die im jeweiligen Fall den Abstoßpunkt von Entdifferenzierung bilden, geklärt werden. Inkohärent ist sie etwa wenn system- und akteurstheoretische Aspekte vermischt und aufeinander bezogen werden. Und übermäßig simplifizierend ist sie, weil die Begriffsklärung meist mit der Gleichsetzung von Entdifferenzierung und fehlender Differenzierung endet und die Frage nach zugrundeliegenden Mechanismen ebenso ausgeblendet bleibt wie die Unterscheidung von Interaktion, Organisation und Funktionssystemen als Ebenen sozialer Systembildung. Die Operationalisierung erfolgt in solchen Fällen meist eindimensional über die Frage, inwieweit beispielsweise in der DDR Parteizugehörigkeit in professionellen Rekrutierungsverfahren eine Rolle gespielt hat - was zweifellos ein relevanter Aspekt ist, das Phänomen jedoch nicht in umfassender Weise erfasst (die genannten Argumente betreffen in unterschiedlichem Maße z.B. Ettrich 2003; Gebauer 2003; Meuschel 1992, 1993; Pollack 1990, 1998, 2000; für die UdSSR: Hayoz 1995, 1997).

Zwar geht es im Folgenden nicht um die DDR, sondern um Russland respektive das Staatengebilde der Sowjetunion, weshalb bei vorschnellen Gleichsetzungen und Analogieschlüssen Vorsicht geboten ist. Ungeachtet der zum Teil erheblichen strukturellen Diskrepanzen verschiedener sozialistischer Staaten erscheint mir die

2 In Analysen der sozialistischen Regime und postsozialistischen Transformationsprozesse in Osteuropa und den Staaten der ehemaligen Sowjetunion kommt der Begriff der Entdifferenzierung hingegen interessanterweise faktisch nicht vor - und dies gilt sowohl für deutschsprachige als auch für nicht-deutschsprachige Autorinnen und Autoren, zu den Ausnahmen gehören die Arbeiten von Hayoz (1995, 1997).

3 Die mangelnde theoretische Konturiertheit und den fehlenden Blick für das Auflösevermögen systemtheoretischer Begrifflichkeiten sieht Nassehi (2012) als ein generelles Problem in der Rezeption der Luhmannschen Systemtheorie in der Soziologie. 
Annahme einer Familienähnlichkeit sozialistischer Regime jedoch plausibel (vgl. für diese Sichtweise z.B. Kornai 2000; Verdery 1996: 19f) und auch ich nehme vor diesem Hintergrund den Begriff der Entdifferenzierung als Ausgangspunkt meiner Beschreibung, die mit einer Begriffsklärung beginnt. Mit Luhmann erschließt sich gesellschaftliche Entdifferenzierung im Kontext der modernen, d.h. funktional differenzierten Gesellschaft über Codes und Programme. Eine solche theoretische Grundlage erlaubt es, Sozialismus (wie er in der Sowjetunion umgesetzt wurde) als Programm zu kategorisieren, das sich durch seinen universellen Geltungsanspruch von den Programmen einzelner gesellschaftlicher Funktionssysteme unterscheidet. Im Anschluss daran lässt sich nachzeichnen, auf welche Weise im sowjetischen Regime Programmelemente aus ideologischen Prinzipien abgeleitet und in konkrete gesellschaftliche Interventionsansprüche transformiert wurden.

\section{Theoretische Vorüberlegungen: Code, Programm und Entdifferenzierung}

In den Funktionssystemen der modernen Gesellschaft kommen Programme potenziell auf zwei Ebenen vor: Auf der Codeebene ergänzen sie den funktionssystemspezifischen Code und auf der Inhaltsebene beeinflussen sie die Frage, auf welche Objekte sich die Kommunikation beziehen kann oder soll. Die Logik der Codeebene erschließt sich über den Differenzierungs- und Abgrenzungsmodus von Funktionssystemen gegenüber ihrer (innergesellschaftlichen) Umwelt: Funktionssysteme entwickeln dazu systemspezifische Kommunikationsformen, die häufig, wenn auch nicht zwingend die Form binärer Codes annehmen (also beispielsweise Zahlung|Nicht-Zahlung im Wirtschaftssystem, wahr|unwahr in der Wissenschaft, Recht|Unrecht im Rechtssystem). Programme übernehmen in diesem Kontext die Aufgabe, Regeln und Kriterien für die Zuweisung der Codewerte zu konkreten Operationen festzulegen (Luhmann 1997: 362ff u. 750, 2008 [1986]: 59f). Sie stellen damit notwendige Ergänzungen der Binärcodes dar, die als solche »zunächst nur hochabstrakte Schematismen [sind], die gegeneinander differenziert sind, aber damit noch nicht begreiflich machen, wie die Operationen der Gesellschaft tatsächlich reguliert werden« (Luhmann 2008 [1986]: 59). Mit anderen Worten: Die Frage, unter welchen Umständen eine Aussage als (wissenschaftlich) wahr gilt oder eine Operation als (rechtlich) unrecht erachtet wird, kann durch den Code allein nicht geklärt werden. Programme sind folglich stets codespezifisch und können nicht zwischen verschiedenen symbolisch generalisierten Kommunikationsmedien fluktuieren (Beispiele sind Theorien und Methoden für das Medium der Wahrheit oder Gesetze für das Medium des Rechts, vgl. Luhmann 1997: 377). Während der Code nicht geändert werden kann, ohne gleichzeitig die Identität des Systems zu ändern, führen Programme die Möglichkeit der Variabilität in das System ein: »[W]ährend 
die Codes Einfachheit und Invarianz erreichen, wird ihr Programmbereich, gleichsam als Supplement dazu, mit Komplexität und Veränderlichkeit aufgeladen« (Luhmann 1997: 362). Programme erlauben den Funktionssystemen, das Spektrum möglicher Umweltbezüge, das durch den Code zunächst begrenzt wird, auszuweiten und auf diese Weise ihre Anpassungs- und Lernfähigkeit zu steigern, ohne die eigene Identität zu verlieren (Luhmann 1997: 364f, 2008 [1986]: 60).

Zusätzlich zur Codeebene können Programme auch auf der Inhaltsebene auftreten. Dort regulieren sie, was als Objekt bestimmter Kommunikationen in Frage kommt, also worauf der systemspezifische Code potenziell angewendet werden kann. Auf diese Weise kann über das Programm zunächst Ausgeschlossenes wieder in das System eingeschlossen werden, wie Luhmann anhand des Kunstsystems illustriert: »Ein Kunstwerk muß dem eigenen Code stimmig/unstimmig oder, traditionell gesprochen: schön/häßlich genügen. Aber in der Wahl des sujets kann man >politisieren< oder auf Verkaufsmöglichkeiten achten« (Luhmann 1997: 378). Im Unterschied zur Codeebene, auf der Programme notwendige Ergänzungen des systemspezifischen Codes darstellen und für das Verständnis der Logik des Funktionssystems essentiell sind (Luhmann 2008 [1986]: 59f), sind Programme auf der Inhaltsebene optional. Das Kunstsystem - um beim Beispiel zu bleiben - operiert und reproduziert sich unabhängig davon, ob seine Sujets aufgrund entsprechender Inhaltsprogramme >politisiert<, >ökonomisiert< oder nach anderen Kriterien ausgewählt werden. Relevant für seine Funktionsfähigkeit und Schließung gegenüber der Umwelt ist allein, dass die Codeebene davon unberührt bleibt und hier Anschlussmöglichkeiten für weitere systemspezifische Kommunikationen generiert werden.

Darüber hinaus unterscheiden sich die beiden Programmebenen in ihrem Umgang mit Umweltkomplexität: Kriterien für die Zuweisung von Codewerten sind üblicherweise selbstreferentiell. Taucht dabei dennoch Fremdreferenz auf, muss sie in systemeigene Operationen überführt werden und latent bleiben - also als Selbstreferenz getarnt werden - um Teil des Programms sein zu können. Beispielsweise wird in der Wissenschaft der wissenschaftliche Wert einer Aussage untergraben, wenn bei ihrer Qualifikation als >wahr< explizit auf die gegenwärtigen politischen Machtverhältnisse verwiesen wird. Stattdessen müssen es die (wissenschaftsintern konstituierten) Theorien und Methoden übernehmen, geeignete Kriterien für die Zuweisung der beiden Seiten des Wahrheitscodes zu formulieren - auch wenn dabei faktisch politische Überlegungen eine wesentliche Rolle spielen (vgl. zu diesem Beispiel Kneer/Nassehi 2000: 133f).

Diese Latenzanforderung an Fremdreferenz gilt hingegen nicht für die Inhaltsebene des Programms: Ausgeschlossenes kann hier wieder eingeschlossen und dieser Prozess kann auch als solcher gekennzeichnet werden. Beispielsweise steht der wissenschaftliche Wert eines Forschungsprojekts nicht notwendigerweise deshalb in Frage, weil das Projekt unter Verweis auf seine Finanzierbarkeit oder seine poli- 
tische Bedeutung gerechtfertigt wird. Probleme für die wissenschaftliche Kommunikation entstehen allerdings, sobald nur diejenigen Ergebnisse und Aussagen als wahr akzeptiert werden, die politisch erwünscht oder ökonomisch nützlich sind. An diesem Punkt wird der Übergang zur Entdifferenzierung deutlich: Entdifferenzierung liegt vor, wenn Fremdreferenz auf die Codeebene eines Funktionssystems durchdringt, wenn also die Kriterien für die Vergabe der Codewerte nicht mehr aus dem System selbst heraus entstehen, sondern in seiner Umwelt festgelegt werden. Die systemspezifische Kommunikation wird aus der Umwelt heraus okkupiert und die Codewerte - selbst wenn sie semantisch weiterhin Bestand haben - werden unterminiert.

Systemtheoretische Darstellungen haben darauf hingewiesen, dass Programme in Organisationen expliziert werden und damit dort besonders deutlich zum Vorschein kommen. Organisationen übernehmen es auch, Sequenzen für den Umgang mit Codes und Kommunikation einzurichten, den Umgang mit diesen Sequenzen in der Gesellschaft zu beobachten (also Erfolg oder Misserfolg festzustellen), daraus zu lernen und gegebenenfalls über Änderungen zu entscheiden (Baecker 2006: 123). Letztlich zielt die einsetzende Organisation dabei darauf, die Gesellschaft in ihrer Gesamtheit oder zumindest einzelne Funktionssysteme im Hinblick auf bestimmte Vorstellungen zu gestalten und so die Kontingenz der weiteren Entwicklung einzuschränken (Baecker 2006: 125). Ein möglicher Effekt der Implementierung von Programmen, deren Geltungsbereich in der Regel räumlich, sachlich oder sozial begrenzt ist, besteht darin, regionale Unterschiede in der Weltgesellschaft zu verstärken oder sogar zu begründen. Durch das Aufeinandertreffen unterschiedlicher Programme in einer der genannten Dimension lässt sich die Diffusion bestimmter Kommunikationen wie sie der Neoinstitutionalismus beobachtet (klassisch DiMaggio/Powell 1983) oder aus systemtheoretischer Perspektive das für die Genese der Weltgesellschaft konstitutive »Und-so-weiter« (Stichweh 1995: 36) verzögern, in ihrer Form ändern oder sogar ganz unterbrechen. ${ }^{4}$

4 In der »Und-so-weiter«-Hypothese setzt Stichweh (1995: 36) einzelne Interaktionen in Relation zum Horizont der Weltgesellschaft: »Die >Und so weiter<-Hypothese besagt, daß der für die Theorie der Weltgesellschaft entscheidende Sachverhalt nicht ist, daß die einzelne Interaktion enorme räumliche oder zeitliche Distanzen überspannt, daß es vielmehr darum geht, daß in jeder einzelnen Interaktion ein >Und so weiter $<$ anderer Kontakte der Teilnehmer präsent ist und dies die Möglichkeit weltweiter Verflechtungen eröffnet, eine Möglichkeit, die wiederum als Selektivitätsbewußtsein in der einzelnen Interaktion relevant wird und auf diese Weise in die Interaktionssteuerung eingreift.« 


\section{Sozialismus als Gesellschaftsprogramm: Ideologische Grundprinzipien}

Vor dem Hintergrund der skizzierten theoretischen Kategorien und als eine erste Annäherung lässt sich der Sozialismus in der Form, wie er in den Staaten der ehemaligen Sowjetunion als Grundprinzip gesellschaftlicher Ordnung etabliert wurde, als Programm begreifen. Als ein solches war er jedoch nicht auf ein einzelnes Funktionssystem begrenzt, sondern beanspruchte, die Bedingungen gesellschaftlicher Reproduktion generell entlang marxistisch-leninistischer Prinzipien auszurichten (Baecker 2006: 124; auch Koenen 2012: 83). Das ideologische Selbstverständnis des Sozialismus speist sich zu einem erheblichen Teil aus der Beobachtung - und Ablehnung - des Kapitalismus, die den Ausgangspunkt für das Gefühl der eigenen Überlegenheit bildet. Dabei werden die dem Kapitalismus zugeschriebenen Defizite und Ineffizienzen primär auf die zentralen Prinzipien Privateigentum, Wettbewerb und Markt zurückgeführt. Diese Prinzipien lassen sich, so die sozialistische Überzeugung, durch die Einführung einer planwirtschaftlichen Ordnung beseitigen (Kornai 1992: 50f), so dass auch die Defizite verschwinden.

Im Kern dieser Idee steht der Antagonismus von Arbeit und Kapital. In Anlehnung an Lockes Arbeitswertlehre wird Arbeit als der zentrale Faktor zur Erzeugung von Wohlstand und damit zur Überwindung der Differenz von arm und reich bzw. Haben und Nichthaben betrachtet (Luhmann 1988: 159f). Als die aus der Unterscheidung von Haben und Nichthaben ausgeschlossene Dritte (oder bei Luhmann als Parasit im Sinne von Serres) wird Arbeit in einer Gesellschaft mit Geldwirtschaft zur Möglichkeit, Eigentum zu erlangen, das sich aus kondensierten Positionen des Habens oder Nichthabens und damit als Erstcodierung von Knappheit ergibt (Luhmann 1988: 187f). Arbeit wird demnach durch die Differenz von Haben und Nichthaben respektive von Eigentum und Nichteigentum stimuliert (Luhmann 1988: 212).

Der Eigentumscode verweist unmittelbar auf Exklusion (Luhmann 1988: 189; auch Baecker 2006: 52f): Jeder ist im Hinblick auf alle eigentumsfähigen Güter entweder Eigentümer oder Nichteigentümer, eine dritte Möglichkeit - etwa beides gleichzeitig in Bezug auf ein und dasselbe Gut - ist nicht denkbar: »Jedes Eigentum des einen ist das Nichteigentum aller anderen« (Luhmann 1988: 189). Exklusion von der Nutzung eines Gutes - Nichteigentum - ist dabei jedoch theorielogisch und auch in der Praxis nicht gleichzusetzen mit Exklusion aus wirtschaftlicher Kommunikation. Die Inklusion in das Wirtschaftssystem erfolgt gerade nicht allein durch den positiven Codewert, sondern durch die Differenz beider Codewerte - und in diesem Sinne bietet Eigentum im Sinne von Nutzungsrechten und deren Verteilung 
in der Gesellschaft einen ersten Anhaltspunkt, an dem sich Knappheitskommunikation »entzünden« kann $^{5}$ (Baecker 2006: 52; vgl. dazu auch Polanyi 1978 [1944]).

Letztlich ist hier auch der Grund für den evolutionär unwahrscheinlichen Fall zu suchen, dass die exkludierende Codierung von Knappheit in der Regel akzeptiert wird: Die aktuellen Nichteigentümer »akzeptieren ihren Ausschluss von bestimmtem Eigentum, weil das ihre Inklusion in die Wirtschaft bewirkt. Wirtschaft kann deshalb nur evoluieren, wenn für diese Form der Inklusion hinreichende Motive bereitgestellt werden können« (Luhmann 1988: 189). Diese Inklusionsmotive wiederum ergeben sich aus der Zweitcodierung von Knappheit durch Geld und Zahlungen, d.h. der Güterknappheit wird die Knappheit des Geldes gegenübergestellt und die Knappheit wird auf diese Weise selbst codiert (Luhmann 1988: 197):

»Während Eigentum noch uninteressant sein kann - was soll ich mit einem Garten mit zwanzig Apfelbäumen -, wird durch das Medium Geld sowohl Knappheit als auch Interesse universalisiert. [...] Geld dient als Medium der Beobachtung von Knappheit, und Zahlungen sind Formen, die das Medium operationalisieren.«(Luhmann 1997: 349)

Erst durch die Möglichkeit der Zahlung und den offenen Verwendungszweck von Geld wird Eigentum universell transformierbar und im selben Maße universalisieren sich die Motive für die Teilnahme an wirtschaftlicher Kommunikation. Geld wird in der modernen Gesellschaft zum symbolisch generalisierten Kommunikationsmedium, das eine Überbrückungsfunktion in allen drei Sinndimensionen ausübt (Baecker 1998 [1997]-a: 83ff; Luhmann 1997: 319): Differenzen in der Sozialdimension werden überbrückt, wenn Alter bereit ist, Egos Geld im Tausch gegen Leistungen anzunehmen; in der Zeitdimension überbrückt Geld die Differenz zwischen heute und morgen, indem es »gegenwärtig Möglichkeiten [sichert], auch morgen auf knappe Güter und Dienstleistungen zugreifen zu können« (Baecker 1998 [1997]-a: 83); sachliche Differenzen werden durch die Erwartung überbrückt, dass alles, »was eine Gesellschaft als Bedürfnis definiert, auch als Bedürfnis akzeptiert bleibt« (Baecker 1998 [1997]-a: 85) und Bedürfnisse sich mit Geld befriedigen lassen. Auf diese Weise transformiert sich die Unwahrscheinlichkeit von Zahlungen in Wahrscheinlichkeit (Esposito 2008: 126). Solange Zahlungen fließen, kann Alter Egos Knappheitsreduktion erleben und sieht sich nicht zum Handeln gezwungen. Zwar steigt durch den Zugriff eines einzelnen immer noch die (Güter-)Knappheit

5 Aus systemtheoretischer Sichtweise rückt so im Hinblick auf Eigentum die Sozialdimension und nicht die Sachdimension in den Vordergrund. Letztere bezieht sich auf die dyadische Beziehung zwischen einem Eigentümer und einem Gegenstand oder einer Ressource, bei der sowohl die Wirtschaftswissenschaften als auch das gängige Alltagsverständnis von Eigentum ausgehen (Carruthers/Ariovich 2004: 23). 
aller anderen, gleichzeitig sinkt jedoch deren Knappheit an Geld, mit dem andere Knappheiten heute oder in der Zukunft verringert werden können und das somit zwingend auf neue Zahlungsmöglichkeiten verweist (Baecker 2006: 48ff): »Das Medium Geld stellt dann sicher, daß der Erlebende akzeptiert, daß andere mit ihrem Geld sich das beschaffen, was sie möchten« (Luhmann 1997: 348). Mit Weber ist Wirtschaften dann die »friedliche Ausübung von Verfügungsgewalt « (Weber 1964: 43). Unter der Bedingung der zweifachen Knappheitscodierung findet Inklusion in das Wirtschaftssystem nicht nur über den positiven Codewert statt (also Zahlung). ${ }^{6}$ Stattdessen generiert die Unterscheidung der beiden Codewerte und die Option des »crossings « unterschiedliche Anschlussmöglichkeiten für weitere Operationen (also Zahlungsmöglichkeiten) auf beiden Seiten der Unterscheidung (Luhmann 1988: 189): Zahlungen und damit die Weitergabe von Zahlungsfähigkeit auf der positiven (markierten) Seite und die Wiederherstellung von Zahlungsfähigkeit auf der negativen (unmarkierten) Seite (Baecker 2002: 73ff).

Der Zusammenhang von Arbeit, Wohlstand und (potenzieller) Inklusion eröffnet jedoch, so stellt Luhmann fest, Spielraum für Ambivalenz und abweichende Beobachtungsvarianten. Gerade Geld als Kommunikationsmedium, das die Zweitcodierung von Knappheit in Form von Zahlung und Nichtzahlung erst ermöglicht, lässt sowohl Raum für symbolisches Erleben - also den Blick auf das, was verbindet - als auch für diabolisches Erleben, das auf die trennenden Aspekte des Mediums abstellt (Luhmann 1988: 258f). Mit dem »symbolon« ist für Luhmann gleichzeitig das »diabolon« gesetzt, »die Einheit der Differenz kann in Richtung auf das Zusammen des Unterschiedenen, aber auch in Richtung auf das Auseinander artikuliert werden« (Luhmann 1988: 258). Letztere Lesart macht sich der Sozialismus zu eigen: Für ihn begünstigt die Differenz von arm und reich bzw. von Haben und Nichthaben primär die Machtakkumulation auf Seiten des Kapitals und führt zur Ausbeutung der Arbeiterinnen und Arbeiter als deren notwendige Konsequenz (Luhmann 1985: 122f): Das Resultat ist Exklusion statt Inklusion. An die Stelle von (wirtschaftlicher) Dynamik, die der erstgenannten Argumentation zugrunde liegt, tritt die Moral - jedoch, so Luhmann mit Blick auf die sozialistische Ideologie, »offenbar ohne Kontakt mit der eigentümlichen strukturellen Dynamik der modernen Geldwirtschaft« (Luhmann 1988: 160). Der stufenreiche Gegensatz zwischen arm

6 Deutschmann (2009a, 2009b) geht mit Blick auf Inklusionen und Exklusionen, die durch Geld induziert werden, noch einen Schritt weiter: Er sieht Geld als dominantes Inklusionsmedium in der modernen Gesellschaft, das seinem Besitzer universale Freiheitsgrade eröffnet. Entsprechend sind Wahrheit, Recht oder Liebe zwar nicht käuflich, stehen aber unter Finanzierungsvorbehalt (2009a: 155). Mit Simmel ist Geld für ihn »nicht nur Kaufkraft, sondern >Vermögen $<$. Wer über es verfügt, >hat $<$ nicht nur etwas, sondern $>$ kann etwas« (2009b: 227). 
und reich respektive zwischen Haben und Nichthaben, denn auch hier sind Abstufungen denkbar, wird ersetzt durch eine Dichotomie, die sich wiederum leicht in die gesellschaftlichen Klassen der Arbeiter und der Kapitalisten übersetzen lässt (Luhmann 1988: 161): »Unsere Epoche, die Epoche der Bourgeoisie, zeichnet sich jedoch dadurch aus, dass sie die Klassengegensätze vereinfacht hat. Die ganze Gesellschaft spaltet sich mehr und mehr in zwei große feindliche Lager: Bourgeoisie und Proletariat« (Marx/Engels 1997 [1848]: 20). Der Klassenunterschied wird zum Gegensatz, »transformiert die Ordnungssemantik in eine Kampfsemantik« (Luhmann 1985: 124) und bringt eine Änderungserwartung ins Spiel, deren Angelpunkt die als kontingent beobachteten Produktionsverhältnisse sind (Luhmann 1985: 123). Letztendlich führt dieser Konflikt, so Marx, zum Sieg des Proletariats über die Bourgeoisie, zur Abschaffung von Eigentum und damit zur Auflösung der gesellschaftlichen Klassen selbst (Marx/Engels 1997 [1848]: 35).

Aus dieser knappen Darstellung der sozialistischen Idee lassen sich zwei Leitunterscheidungen ableiten, anhand derer sich der Sozialismus als Gesellschaftsprogramm von seiner Umwelt abzugrenzen versuchte und sich die interne Logik des sozialistischen Programms verstehen lässt: Erstens die Unterscheidung von Inklusion und Exklusion, zweitens und daraus abgeleitet die von Hierarchie und Markt respektive und komplementär dazu von Kollektiv- und Privateigentum. Diese Leitunterscheidungen und die damit notwendigerweise einhergehenden Reduktionen beanspruchen nicht, alle Facetten der sozialistischen Regime ${ }^{7}$ in den ehemaligen Ostblockstaaten beschreiben zu können, doch sie erscheinen mir mit Blick auf meine Fragestellung ausreichend geeignet, um die spezifischen Bedingungen gesellschaftlicher Reproduktion unter dem Programm des Sozialismus im Hinblick auf den Transformationsprozess zu ordnen und zu verstehen.

Dreh- und Angelpunkt dieser Leitunterscheidungen ist wiederum das Eigentum. Sowohl der Sozialismus als auch der Kapitalismus, so lassen sich die vorhergehenden Gedanken zur sozialistischen Ideologie zusammenfassen, nehmen dessen Exklusionspotenzial wahr, beobachten es jedoch im Hinblick auf sich ergebende Anschlussmöglichkeiten unterschiedlich: Das kapitalistische (Wirtschafts-)Programm richtet seinen Blick auf das Inklusionspotenzial der Differenz von Haben und Nichthaben, das durch Monetarisierung und den Marktmechanismus in Gang ge-

7 Um eine doppelte Verwendung des Systembegriffs zu vermeiden, verwende ich die Bezeichnung >System< ausschließlich im systemtheoretischen Sinn. Für >politische Systeme< im Sinne der politischen Ordnung von Staaten verwende ich im Folgenden den Begriff des Regimes, der sich aus politikwissenschaftlicher Sicht auf »eine Lebensweise, Ordnungs- oder Regierungsform, also ein institutionalisiertes Set von Prinzipien, Normen und Regeln, das die Umgangsweise der Akteure in einem gegebenen Handlungszusammenhang grundlegend regelt«, bezieht (Zürn 2002: 798). 
setzt wird. Die wirtschaftliche Inklusion aller Gesellschaftsmitglieder ist möglich, kann jedoch aufgrund der per se unkontrollierbaren Evolution von Wirtschaft nicht umfassend sichergestellt werden: ${ }^{8} \gg$ Wer zahlt, bekommt, was er wünscht. Wer nicht zahlt, muß dies beobachten. [...] Es gibt Leute, die nicht zahlen können. Und wie bei allen Funktionssystemen gilt auch hier: Die Inklusion ist zugleich Exklusion« (Luhmann 1988: 267). Exklusion ist aufgrund der Logik und strukturellen Realität einer funktional differenzierten Gesellschaft kaum zu vermeiden (und andersherum ist Vollinklusion sehr unwahrscheinlich). Die moderne Gesellschaft muss also mit Exklusion rechnen und damit auch mit Exklusionsverkettungen, da das Herausfallen aus wirtschaftlichen Kommunikationszusammenhängen unter der Bedingung funktionaler Differenzierung potenziell weitere Ausschlüsse nach sich ziehen kann (Stichweh 2000c: 96ff). Dies wiederum kann dazu führen, dass die Bedürfnisse bestimmter Gesellschafsmitglieder nicht zur Geltung kommen (Baecker 2006: 124), weil ihnen regelhaft der Zugang zu bestimmten materiellen Ressourcen und damit potenziell auch $\mathrm{zu}$ anderen zentralen gesellschaftlichen Bereichen wie Bildung oder Kunst verwehrt wird. ${ }^{9}$ Weder das Wissen um mögliche noch die Beobachtung aktueller Exklusion bedeuten jedoch, dass Exklusion für eine funktional differenzierte Gesellschaft grundsätzlich legitim wäre - im Gegenteil: In der modernen Gesellschaft wird Exklusion zunehmend nur noch dann als zulässig gewertet, wenn sie in die Form von Inklusion gebracht wird, was in der Regel (aber nicht ausschließlich) Organisationen übernehmen ${ }^{10}$ (Stichweh 2009c: 37f). So wird die drohende Exklusion aus wirtschaftlichen Kommunikationszusammenhängen in vielen kapitalistischen Regimen beispielsweise durch Transferzahlungen und andere wohlfahrtsstaatliche Maßnahmen zu korrigieren versucht ${ }^{11}$ (Deutschmann

8 Insbesondere durch die Differenzierung von Leistungs- und Publikumsrollen ist in der modernen Gesellschaft die Vollinklusion aller Gesellschaftsmitglieder in alle Funktionssysteme möglich - aber nicht unbedingt wahrscheinlich (Stichweh 2000: 89, 2009: 33f) und Exklusion wird damit in allen sozialen Bereichen zu einer Möglichkeit, auf die sich die Gesellschaft einstellen muss. In welcher Form Exklusionen miteinander verkettet sind, ergibt sich aus dem Grad der Differenzierung bzw. den Modi der strukturellen Kopplung der Funktionssysteme (Stichweh 2000: 97ff).

9 Vgl. dazu zum Beispiel auch Kronauer (2000), Buhr und Leibfried (2009) sowie Häussermann und Kronauer (2009).

10 Organisationen zur Umsetzung >inkludierender Exklusion< sind beispielsweise Psychiatrien oder Gefängnisse.

11 Die historische Entwicklung verschiedener Formen der Geldinklusion in der ständischen Gesellschaft, der Klassengesellschaft und der wohlfahrtsstaatlichen Gesellschaft erläutert Deutschmann (2009b: 231ff). Zu Mechanismen der Inklusion und Exklusion auf der Grundlage des Geldmediums siehe außerdem Bohn (2009). 
2009b: 232f; Stichweh 2000c: 91ff). Gleichzeitig tendieren Regime, die sich für symbolisches Erleben von Geld entschieden haben, gerade aufgrund dieser Zuschreibungspraxis dazu, die Exklusionsproblematik nicht als Effekt der Strukturen des Wirtschaftssystems zu beobachten - und unterscheiden sich dadurch von der sozialistischen Perspektive:

»Eine Geldtheorie, die nur den Tausch im Blick hat, wird die hier angedeuteten Phänomene also gar nicht registrieren. Sie wird sie als >soziale Probleme< für Hilfsaktionen beiseite schieben. Daß es soziale Probleme sind, ist natürlich nicht zu bestreiten, es wären aber keine sozialen Probleme, wenn es keine wirtschaftlichen Probleme wären, wenn also Normalbeteiligung an Wirtschaft für alle sichergestellt wäre.«(Luhmann 1988: 262f)

An diesem Punkt wird eine Asymmetrie zwischen dem sozialistischem und dem kapitalistischen Programm deutlich: Die Abgrenzung beider über die Unterscheidung von Vollinklusion und Exklusion erscheint allein aus der sozialistischen Beobachterposition heraus angemessen und nicht als Selbstbeschreibung des Kapitalismus, der sich kaum als in erster Linie exkludierendes Unterfangen beobachtet. Die Selbstbeobachtung des Kapitalismus fokussiert hingegen das Ziel, durch die Umsetzung seiner Prinzipien wirtschaftliches Wachstum zu induzieren und ein höheres (ökonomisches) Wohlstandsniveau zu schaffen. Bedeutende Parameter sind entsprechend Daten und Indikatoren zum Einkommen, zu Gewinnen, zur Arbeitslosigkeit und zum Wirtschaftswachstum (in der Regel bezogen auf regionale Segmente). Kapitalismus ist seinem Selbstverständnis nach ein Wirtschaftsprogramm, das bestimmte Kriterien für wirtschaftliche Kommunikation (also Zahlungen) bereithält und durchzusetzen versucht, aus sich heraus jedoch keine Umstrukturierungen über das Wirtschaftssystem hinaus beansprucht. Wohlgemerkt schließt dies nicht aus, dass sich nicht andere Funktionssysteme mehr oder weniger stark an der Wirtschaft und ihrer Expertise auszurichten beginnen (beispielsweise das Erziehungssystem, die Wissenschaft oder die Politik) und dies Ergebnisse zeitigt, die in der Gesellschaft und vor allem in den jeweiligen Funktionssystemen selbst auf Kritik stoßen. Dass dem Kapitalismus ungeachtet dessen vor allem, aber nicht nur von seinen Kritikerinnen und Kritikern der Anspruch gesellschaftsweiter Geltung unterstellt wird (in Analogie zum Sozialismus) und umgangssprachlich oft von >kapitalistischen Gesellschaften< die Rede ist, ist letztlich vermutlich im Wesentlichen auf solche >externen Effekte< zurückzuführen. Entsprechende Beobachtungen aus der Umwelt des Wirtschaftssystems heraus - allen voran aus der Politik, die aus unterschiedlichen Perspektiven mit dem kapitalistischen Programm hantiert, aber beispielsweise auch (meist kritisch) aus der Kunst oder der Wissenschaft - tendieren wahlweise dazu, den Kapitalismus zu verteufeln oder ihn als Sinnbild gesamtgesellschaftlicher >Heilsversprechen $<\mathrm{zu}$ sehen. Die moderne Gesellschaft, so stellt 
Baecker (2003: 7) mit Blick auf Walter Benjamin fest, glaube an den Kapitalismus, der für sie alternativlos geworden sei und in ihr Züge einer »Kultreligion« annehme (Benjamin 2009 [1985]). Im kapitalistischen Programm selbst fehlt hingegen dieser umfassende Anspruch. ${ }^{12}$

Zwar beriefen sich in der Vergangenheit auch kapitalistische, liberaldemokratische Regime auf eine klare Grenze zum Sozialismus, diese war jedoch zum einen historisch durch die Konfrontation beider Regime während des Kalten Krieges bedingt. Zum anderen gingen Abgrenzungsbestrebungen wohl aus der Beobachtung des >Westens $<$ hervor (dessen Selbstverständnis im Übrigen neben einer kapitalistischen Wirtschaftsweise auch Kategorien wie Demokratie, Freiheit, Rechtsstaatlichkeit und die Achtung der Menschenrechte umfasst ${ }^{13}$ ), dass in seiner unmittelbaren Nachbarschaft Menschen in Unfreiheit leben - was seinem liberalen Selbstverständnis fundamental widerspricht. Irritationen dieser Art und allgemein der Blick auf das sozialistische >Gegenprogramm< sind für kapitalistische (Wirtschafts-)Regime jedoch in wesentlich geringerem Maße identitätsstiftend, als es umgekehrt aus sozialistischer Perspektive der Fall ist. Dort nimmt die Unterscheidung von Sozialismus und Kapitalismus als konkurrierende Gesellschaftsordnungen oder -programme innerhalb der eigenen ideologischen Selbstbeschreibung eine hochgradig identitätsstiftende Funktion ein. Selznick (1952: 11) spricht gar von einer »Negativideologie «, die auf ein Feindbild oder zumindest eine mehr oder weniger diffus wahrgenommene Bedrohung angewiesen ist, um sich $\mathrm{zu}$ definieren und $\mathrm{zu}$ legitimieren. ${ }^{14}$ In diesem Sinne kommt dem angestrebten >Sieg über den Kapitalis-

12 Ergänzen ließe sich an dieser Stelle noch, dass sich auch in einer vermeintlich >kapitalistischen Gesellschaft< die nicht-wirtschaftlichen Funktionssysteme kaum selbst als kapitalistische beobachten und beschreiben würden. Folgt man (auch) den entsprechenden Selbstbeschreibungen, gab es im Unterschied dazu jedoch durchaus sozialistische Kunst, sozialistischen Sport oder sozialistische Wissenschaft.

13 Deutlich wird dies z.B. an der sogenannten Trias des Europarats: Demokratie, Rechtsstaatlichkeit und Menschenrechte (zum Europarat allgemein vgl. z.B. Brummer 2008; für eine Analyse ausgewählter Aspekte des Verhältnisses des Europarats und Russlands vgl. Melzer 2008, 2012). Auch wenn dieser umfassende Anspruch nicht immer eingelöst wird, ist er doch ein Bezugspunkt, um den die Mitgliedsstaaten kaum herumkommen und sich bei Verletzung dieser Prinzipien erheblichem Rechtfertigungsdruck aussetzen.

14 So heißt es beispielsweise in der »Deklaration über die Gründung der Union der Sozialistischen Sowjetrepubliken« in der Verfassung der UdSSR vom 6. Juli 1923: »Seit der Bildung der Sowjetrepubliken zerfielen die Staaten der Welt in zwei feindliche Lager: in das Lager des Kapitalismus und in das Lager des Sozialismus. Dort, im Lager des Kapitalismus, herrschen nationale Feindseligkeiten und Ungleichheit, koloniales Sklaventum, Chauvinismus, nationaler Haß und Pogrome, imperialistische Grausamkeit und Kriege - 
mus s sowie der eigenen Profilierung durch den Vergleich mit ausgewählten Parametern kapitalistischer Gesellschaften eine hervorgehobene Bedeutung zu. Mit Luhmann, für den die Etablierung von Gegenbegriffen auf Beobachterinteressen verweist - »und wie immer, wenn Bezeichnungen durch Gegenbegriffe bestimmt sind, verrät das die Präsenz eines Beobachters, nach dessen Interessen man fragen kann« (Luhmann 1997: 519) - lässt sich zusammenfassen: Während das sozialistische Programm ein vitales Eigeninteresse daran hat, den Kapitalismus als Gegenbegriff und ideologischen Kontrapunkt zu setzen, erscheint es aus kapitalistischer Perspektive weniger zwingend, den Sozialismus zur Bildung und Absicherung der eigenen Identität in vergleichbarer Weise prominent zu stellen.

Wie aber versucht der Sozialismus die beobachteten Defizite des kapitalistischen Programms zu überwinden? An die Stelle kapitalistischer Exklusion sollte die Vollinklusion aller Gesellschaftsmitglieder treten. Jeder sollte in den Aufbau der neuen Gesellschaftsordnung eingebunden sein und keiner sollte (und durfte) sich entziehen (es sei denn, er erwies sich als der Teilnahme unwürdig und wurde entsprechend >zwangsexkludiert<). Durch eine grundlegende Umgestaltung der Wirtschaftsweise, der Abschaffung von Markt und Privateigentum sowie der Einführung planwirtschaftlicher Mechanismen, so die Erwartung, werde es möglich, »ökonomisch effektiver, besser und sozial gerechter als die kapitalistischen Gesellschaften die Bedürfnisse der Menschen zu befriedigen« (Koch 2002: 130). Und dabei bleibt es nicht: Aus der Überzeugung, die marktbedingten Defizite des Kapitalismus kompensieren zu können, schließt der Sozialismus nicht nur auf die eigene ökonomische Überlegenheit, sondern ergänzt diese mit einer ethisch-moralischen Komponente. Die überdeutlich postulierten positiven Effekte der sozialistischen Wirtschaftsweise, vor allem Gleichheit und soziale Gerechtigkeit, stellen demnach nicht nur das Regime als solches moralisch über den Kapitalismus, sondern bewirken darüber hinaus einen Wandel der Gesellschaftsmitglieder selbst. Eigenschaften wie Egoismus und Gewinnstreben würden sich quasi von selbst dem Streben nach dem Wohl des sozialistischen Kollektivs unterordnen (Kornai 1992: 52). Das anvisierte Ziel ist damit nicht mehr allein die Änderung gesellschaftlicher Strukturen, sondern »das Umerziehen der in ihr lebenden Menschen und das Herausbilden eines >neuen Menschen««(Koch 2002: 119):

»A transformation of the physical world was not, however, the only item on the Bolshevik agenda. It was a cultural revolution that they sought, the creation of a new person. [...] The

hier, im Lager des Sozialismus, gegenseitiges Vertrauen und Friede, nationale Freiheit und Gleichheit, friedliches Zusammenleben und brüderliches Zusammenarbeiten der Völker.« (Zitiert nach Altrichter 1986: 161f) 
>new man< - the Bolshevik specialist, engineer, or functionary - came to represent a new code of social ethics, which was sometimes simply called kultura.« (Scott 1998: 195)

In einem ko-konstitutiven Prozess stellt dieser neue Typ Mensch oder »Sowjetmensch ${ }^{15}$ (Mehnert 1971; von Zsolnay 1968) wiederum sicher, dass sich die ökonomische Überlegenheit des Sozialismus entfalten kann. Und auf diesem Wege lassen sich auch politische Eingriffe zur Beschleunigung dieses Umerziehungsprozesses rechtfertigen, denn »the profound change in human nature is an important factor for ensuring the economic superiority of socialism« (Kornai 1992: 52).

\section{Vollinklusion als politische Adressierbarkeit}

Das Erreichen des Ziels der Vollinklusion aller Gesellschaftsmitglieder verlangt nach umfassenden gesellschaftlichen Steuerungsmöglichkeiten und prinzipieller Planbarkeit. Ein Wunsch, der aus systemtheoretischer Perspektive im scharfen Widerspruch zur Logik komplexer und selbstreferentieller Sozialsysteme steht, da diese ihre Strukturen nur durch eigene Operationen ändern können (zur Steuerungsproblematik vgl. Willke 2005: insb. Kap. 2). Alle (politischen) Steuerungsversuche sind aus Sicht der Funktionssysteme Irritationen oder Impulse aus der Umwelt, die das System aufnehmen kann oder auch nicht. Steuerung - oder im Sinne Luhmanns Differenzminderung (1989: 5) - ist damit immer nur im und durch das System selbst möglich (Luhmann 1988: 331). Im Kontext funktionaler Differenzierung kann die Politik zwar beispielsweise mit Hilfe von Programmen versuchen, Einfluss darauf zu nehmen, wer in ein Funktionssystem (zum Beispiel die Wirtschaft) inkludiert wird und wer ausgeschlossen bleibt (Luhmann 1988: 346), und diese Versuche werden mit einer gewissen Wahrscheinlichkeit vom jeweiligen

$15 \gg$ Der neue Mensch lebt nicht für sich, sondern für die Gesellschaft. Er arbeitet nicht für seine persönliche Bereicherung oder für sein persönliches Glück, sondern für das Wohl der Gesellschaft. Er denkt in internationalen Klassenkategorien und nimmt bei jeder Stellungnahme einen Klassenstandpunkt ein. Alles, was in der Welt geschieht, beobachtet er durch das Prisma der klassengebundenen Lehren des Marxismus-Leninismus. [...] >Der neue Mensch, der vom Kollektivgeist durchdrungen ist, entwickelt sich nicht automatisch, sondern in einem ständigen erbitterten Kampf gegen die Überreste der Vergangenheit $<$, unter denen der Individualismus einen vorrangigen Platz einnimmt. Der Mensch müsse speziell dazu erzogen werden, sich der Gesellschaft und den Interessen der Gemeinschaft freiwillig unterzuordnen.« (von Zsolnay 1968: 20ff) 
Adressaten auch registriert. Inklusion determinieren kann sie jedoch nicht, sondern sie nur als mehr oder weniger zufällig beobachten ${ }^{16}$ (Stichweh 2000c: $91 \mathrm{ff}$ ):

»Das politische System kann also nur sich selbst steuern mit Hilfe einer spezifischen politischen Konstruktion der Differenz von System und Umwelt. Daß dies geschieht und wie dies geschieht, hat ohne Zweifel gewaltige Auswirkungen auf die Gesellschaft, weil es Differenzen erzeugt, an denen sich andere Funktionssysteme dann ihrerseits orientieren müssen. Aber dieser Effekt ist schon nicht mehr Steuerung und auch nicht steuerbar, weil er davon abhängt, was im Kontext anderer Systeme als Differenz konstruiert wird und unter die dort praktizierten Steuerungsprogramme fällt.«(Luhmann 1988: 337)

Um Inklusion nicht dem Zufall zu überlassen - und dies ist aus sozialistischer Perspektive ein zentraler Reibungspunkt mit dem Kapitalismus - muss die Gesellschaft in eine Form gebracht werden, die ebenjene Kontrolle und Steuerung ermöglicht oder doch wenigstens wahrscheinlicher macht. Das sozialistische Programm sucht die Lösung für dieses Problem in dem Versuch, die gesellschaftliche Gemeinschaft als Organisation einzurichten (Pollack 1990; Baecker 1998 [1997]-b: 128).

Im Unterschied zu Funktionssystemen sind Organisationen in der Lage, in allen drei Sinndimensionen Kontrolle auszuüben: Kontrollfähigkeit in der Sachdimension ergibt sich aus Entscheidungen als basalen, d.h. nicht weiter auflösbaren Elementen von Organisationen (Luhmann 2009 [1978]: 395). So wie sich Gesellschaft über Anschlusskommunikation reproduziert, verknüpfen Organisationen Entscheidungen miteinander: »Man entscheidet, weil entschieden worden ist oder damit entschieden werden wird « (Luhmann 2009 [1978]: 398). Auf der Grundlage von Entscheidungen bindet die Organisation sich einschließlich ihrer Mitglieder selbst, indem sie sich auf einige wenige Möglichkeiten festlegt und andere durch die Verwendung von Hierarchien, Routinen oder Zielvorgaben ausschließt (Baecker 2007: 116f). In der Zeitdimension erweisen sich Organisationen als die einzigen Sozialsysteme, die sich Ziele setzen und auf diese Weise die eigene Zukunft bestimmen können, »[a]lle anderen Ordnungen der Gesellschaft müssen stattdessen die Zukunft als offen behandeln« (Baecker 2007: 118). Nicht zuletzt aus dieser Fähigkeit und den damit einhergehenden Möglichkeiten der Unsicherheitsreduktion resultiert ihre Attraktivität sowohl für die eigenen Mitglieder als auch für die Gesellschaft, die die Organisation beobachtet (Baecker 2007: 118f). Um ihre Grenze zur Umwelt zu beobachten, unterscheiden Organisationen zwischen Mitgliedern und Nicht-Mitglie-

16 Versuche der gezielten Einflussnahme auf Inklusion und Exklusion im Wirtschaftssystem sind die vielfältigen Aktivitäten des Wohlfahrtsstaates, deren Wirkung jedoch, wie Stichweh (2000: 89ff) argumentiert, angesichts der zunehmenden Globalisierung der Funktionssysteme immer weniger planbar und steuerbar wird. 
dern (Luhmann 1997: 826ff). Anders als den gesellschaftlichen Funktionssystemen ist es Organisationen möglich, Inklusion und Exklusion gezielt zu moderieren, also die Sozialdimension zu kontrollieren. Die Entscheidungen in diesen drei Dimensionen sind zugleich die konstitutiven Elemente der Organisation selbst.

An Organisationsmitglieder - das Personal - lassen sich vor diesem Hintergrund bestimmte Verhaltenserwartungen richten, die außerhalb der Organisation nicht zum Tragen kommen. ${ }^{17}$ Durch den Beitritt richtet sich an die Mitglieder die Erwartung, dass die Mitgliedschaftsregeln, der Bestandszweck und das Programm der Organisation weitgehend unabhängig von individuellen Interessen akzeptiert und respektiert werden. Je nach dem Verhältnis von Organisationszweck und Mitgliedschaftsmotivation lassen sich in diesem Kontext zwei Organisationstypen unterscheiden: In sogenannten »conjoint authority systems« (beispielsweise Vereine oder Gewerkschaften) sind beide kongruent respektive kann die Organisation Übereinstimmung unterstellen. In »disjoint authority systems« (zum Beispiel Unternehmen) fallen sie hingegen auseinander respektive muss die Organisation mit Diskrepanz rechnen (Coleman 1994: 72ff; Stichweh 2000b: 25):

»Für klassische Organisationen gelten >disjoint authority systems $<$, d.h. man tritt in der Organisation das Recht auf die Wahl der eigenen Handlung im Tausch gegen Ressourcen ab. [...] Vereine werden demgegenüber durch >conjoint authority systems $<$ gekennzeichnet. Auch hier erfolgt ein Transfer von Rechten, da man sich durch andere Mitglieder des Vereins oder den Vorstand in der Handlungswahl bestimmen oder durch sie vertreten läßt, aber man kann auf eine tauschförmige Gegenleistung verzichten, weil unterstellt wird, daß die abgetretenen Rechte im Sinn eigener Interessen genutzt werden.« (Stichweh 2000b: 25)

»Disjoint authority systems « sind aus diesem Grund darauf angewiesen, durch entsprechende Kompensation der Mitglieder (in der Regel in Form von monetären Ressourcen, also Gehaltszahlungen, Aufwandsentschädigungen und ähnlichem) eine Indifferenzzone zu schaffen. Innerhalb dieser Zone kann den Mitgliedern aufgrund des intraorganisationalen Tauschs die Bereitschaft abverlangt werden, weitgehend unabhängig von den eigenen Interessen an der Verfolgung des Organisationszwecks mitzuwirken, ohne dass dafür explizite Anweisungen notwendig wären und ohne dass dabei Autoritätsstrukturen regelhaft in Frage gestellt würden (Barnard 1971 [1938]: 168f; unter Verweis auf Barnard auch Luhmann 2000a: 19). In jedem Fall - mit oder ohne Indifferenzzone - führt die Mitgliedschaft jedoch

17 Eine Ausnahme stellen hier normative Organisationen (nach der Typologie von Etzioni) dar, die für ihre Normen einen Geltungsanspruch erheben, der die eigenen Grenzen explizit überschreitet (Etzioni 1975: 40; vgl. auch Bonazzi 2008: 273ff). 
dazu, dass das Recht der eigenen Handlungswahl in einem bestimmten Bereich abgetreten wird (Stichweh 2000b: 25).

Diese grundlegenden Eigenschaften von Organisationen finden sich in der Struktur sozialistischer Regime deutlich wieder. Sie greifen auf die Organisationsform zurück, um ihre Ideologie möglichst in allen gesellschaftlichen Bereichen umzusetzen. Selznick (1952: 2) verwendet mit Blick auf die sozialistische Einheitspartei der Sowjetunion gar den Begriff der »organizational weapon« als einen aus Sicht des Regimes selbst unverzichtbaren Bestandteil der Gesellschaftsordnung:

»For Lenin, organization was an indispensable adjunct to ideology. He did not believe that he could win power by propaganda alone. Rather, he urged the need to forge a group which, beginning with an ideological commitment, would use whatever means available to influence decision in society.« (Selznick 1952: 8)

Alle Gesellschaftsmitglieder werden - so zumindest die Idee - zu Organisationsmitgliedern. Das Ziel der Vollinklusion nahm auf diese Weise die Form umfassender politischer Adressierbarkeit an: Alle sollten ständig für politische Kommunikation erreichbar sein und jede kommunikative Operation sollte als politische Kommunikation interpretierbar und zurechenbar gemacht werden.

\section{Die Organisationen der Organisationsgesellschaft}

Wie gestaltete sich die Umsetzung des sozialistischen Gesellschaftsprogramms und seiner ideologischen Ideale in der Praxis und welche strukturellen Konsequenzen waren damit verbunden? Mit Blick auf die umfassende >Programmierung< der sowjetischen Gesellschaft griff die politische Führung auf zwei Organisationstypen zurück, die sich in diesem Fall weniger durch organisationsinterne Merkmale, sondern vielmehr durch ihre externe Funktionalität unterschieden: Die Einheitspartei an der Spitze der gesellschaftlichen und politischen Hierarchie, die auf verschiedene Weise die Autonomie der staatlichen Verwaltung unterlief und auf eine interne Entdifferenzierung des politischen Systems hinwirkte, sowie Massenorganisationen, die durch ihre Omnipräsenz Mitgliedschaft unumgänglich machten und für einen hohen Organisationsgrad - und damit politische Kontrollierbarkeit - der meisten gesellschaftlichen Bereiche sorgten.

\section{Partei und Staat}

Die Kommunistische Partei der Sowjetunion (KPdSU, im Folgenden auch Einheitspartei) setzte sich selbst an die Spitze der sowjetischen Organisationsgesellschaft. 
Ihren exklusiven und umfassenden Führungsanspruch versuchte die Partei zu legitimieren, indem sie sich selbst den Alleinvertretungsanspruch der Arbeiterklasse zuschrieb:

»Unerläßliche Voraussetzung dieser sozialen Revolution ist die Diktatur des Proletariats [...]. Die internationale Kommunistische Partei, die sich die Aufgabe stellt, das Proletariat zu seiner großen historischen Mission zu befähigen, organisiert es in einer selbständigen, allen bürgerlichen Parteien entgegengesetzten politischen Partei, leitet alle Äußerungen seines Klassenkampfes, enthüllt ihm den unversöhnlichen Gegensatz zwischen den Interessen der Ausbeuter und denen der Ausgebeuteten und macht ihm die historische Bedeutung und die notwendigen Voraussetzungen der bevorstehenden sozialen Revolution klar.« (aus dem zweiten Parteiprogramm der KPdSU von 1919, zitiert nach Meissner 1965: 122f)

Ein organisches Gesellschaftsbild, dem zufolge die Interessen jedes Gesellschaftsmitglieds unauflöslich mit den Interessen des Kollektivs verknüpft sind, bildete die Grundlage für das »ideologische Monopol« der Einheitspartei und für die Unterdrückung von politischem Pluralismus: »Because there can only be one common interest - the creation of a communist society - there can only be one political party « (Hahn 1988: 81; auch Kornai 1992: 55). Alle übrigen Elemente des politischen Systems, darunter sowohl staatliche als auch nicht-staatliche, sind damit an die Partei und ihre Vorgaben gebunden, so dass sich auch in der Architektur der politisch-administrativen Struktur des Regimes das organische Gesellschaftsverständnis widerspiegelt: »The party itself is regarded as the leading social organization and the vanguard of all other social organizations as well as of state organs. Party, soviets and social organizations are all concerned with building communism « (Churchward 1983: 38). Die Partei selbst übernahm die Federführung, wenn es darum ging, alles, was in der Gesellschaft geschah, auf Konsistenz mit dem sozialistischen Programm zu überprüfen und vermeintliche Abweichungen zu kriminalisieren. Wer sich widersetzte, geriet nicht nur in Opposition zur politischen Führung, sondern wurde als >Klassenfeind< per definitionem zum Gegner der gesamten Gesellschaft (Pollack 1990: 294).

»Er [der Sozialismus, E.M.] machte die Gesellschaft zur Organisation, die sich die vollständige Inklusion zur Aufgabe gab und jeden, der sich diesem Inklusionsangebot entzog, nur als Abweichenden unschädlich zu machen versuchen konnte. Wer abwich, war undankbar.« (Baecker 1998 [1997]-b: 128)

Dass es hierfür eines gigantischen bürokratischen Apparats bedurfte, liegt auf der Hand und damit auch, dass die Kontrollkosten zur Aufrechterhaltung dieser Ord- 
nung schnell ins Unermessliche stiegen, sobald sich Abweichungstendenzen unter den Mitgliedern auch nur ansatzweise als Normalität zu etablieren begannen.

Innerhalb des politischen Systems fand der gesellschaftliche Führungsanspruch der Partei seinen Ausdruck in einer zweidimensionalen Hierarchie, die sich von der obersten (nationalen) Ebene bis hinunter auf die lokale Ebene der Dörfer erstreckte. In der vertikalen Dimension setzte sich diese Hierarchie aus drei Strängen zusammen: Den ersten Strang bildete die Partei selbst mit dem Zentralkomitee (bzw. dem Politbüro) an ihrer Spitze, von der ausgehend sie sich in einzelne Unterabteilungen und Subkomitees bis auf die unterste (lokale) Ebene untergliederte (Gill 1988). Daneben standen als zweiter und dritter Strang die Hierarchie der »Sowjets« (Räte) und der daraus hervorgehenden Exekutivkomitees (»izpolnitel'nyi komitet « oder kurz »izpolkom«) als Organe der Lokalverwaltung, ${ }^{18}$ wobei Erstere offiziell als Repräsentativ- und Legislativorgane fungieren sollten und Letztere für das organisatorische und administrative Tagesgeschäft verantwortlich waren ${ }^{19}$ (Campbell 1995: 149f; Hahn 1988: 117). Den groben formalen Rahmen der Funktionen der Lokalverwaltung steckte die dritte und letzte Verfassung der Sowjetunion von 1977 $\mathrm{ab}$ :

»Die örtlichen Sowjets der Volksdeputierten entscheiden alle Fragen von örtlicher Bedeutung [...]. Die örtlichen Sowjets der Volksdeputierten leiten auf ihrem Territorium den staatlichen, wirtschaftlichen sowie sozial-kulturellen Aufbau; sie bestätigen die Pläne für die ökonomische und soziale Entwicklung sowie den örtlichen Haushalt; sie verwirklichen die Leitung der ihnen unterstehenden Organe, Betriebe, Einrichtungen und Organisationen [...].« (Verfassung der UdSSR von 1977, Art. 146, zitiert nach Henke/Wirantaprawira 1981: 194)

18 Gemäß der sowjetischen Verfassung umfasst der Begriff Lokalverwaltung oder - nach der gängigen Übersetzung - örtliche Verwaltung alle Verwaltungsorgane unterhalb der obersten, zentralstaatlichen Ebene (Hahn 1988: 83). Innerhalb der Lokalverwaltung wurde wiederum ein breites Spektrum an Verwaltungseinheiten unterschieden, von denen die größeren wie Gaue (»krai«), (autonome) Gebiete (»oblast'«) und bedeutende Städte direkt dem Zentralstaat untergeordnet waren, während Dörfer (»posëlok «) und ländliche Siedlungen (»sel'skij naselennyj punkt«) am unteren Ende der Hierarchie angesiedelt waren (Jacobs 1983: 4ff). Eine mehrstufige Ebenendifferenzierung in national, regional und lokal wurde erst mit dem postsowjetischen System kommunaler Verwaltung eingeführt (vgl. dazu S. 135ff).

19 Dies umfasste beispielsweise die Implementierung der Wirtschaftspläne, Personalentscheidungen, die Überwachung der Unternehmen, Entscheidungen über Angelegenheiten lokaler Bedeutung sowie die Bereitstellung kommunaler Dienstleistungen. 
»Im Rahmen ihrer Kompetenzen gewährleisten die örtlichen Sowjets der Volksdeputierten die komplexe ökonomische und soziale Entwicklung auf ihrem Territorium, und kontrollieren die Einhaltung der Gesetzgebung durch die übergeordneten Organen unterstehenden Betriebe, Einrichtungen und Organisationen auf ihrem Territorium; sie koordinieren und kontrollieren deren Tätigkeit hinsichtlich der Bodennutzung, des Naturschutzes, des Bauwesens, der Nutzung des Arbeitsvermögens, der Konsumgüterproduktion, der sozial-kulturellen Betreuung, der Dienstleistungen und anderer Dienste für die Bevölkerung.« (Verfassung der UdSSR von 1977, Art. 147, zitiert nach Henke/Wirantaprawira 1981: 194f)

Aus diesen allgemein gehaltenen Vorgaben ließ sich eine Reihe konkreter Aufgaben ableiten: Zum einen sollten die lokalen Verwaltungsorgane über die Bereitstellung diverser kommunaler Güter und Dienstleistungen entscheiden, worunter beispielsweise medizinische Versorgungseinrichtungen, Wohnungsbau, der Unterhalt kultureller Einrichtungen (in den Dörfern vor allem die sogenannten Kulturhäuser) sowie die Bereitstellung und Instandhaltung lokaler technischer Infrastruktur (StraBen und Wege, öffentlicher Transport, Wasserversorgung und ähnliches) fielen (Jacobs 1983: 3). Zum anderen war die Lokalverwaltung als Kontrollinstanz der Unternehmen auf dem Verwaltungsterritorium vorgesehen, um die plangemäße Produktion zu überwachen und gegebenenfalls durchzusetzen (Ross 2009: 32f). Auf der formalen Ebene wurden den lokalen Verwaltungsorganen damit bis auf die unterste Ebene vor allem mit Blick auf die unmittelbaren Bedürfnisse der Bevölkerung, auf die reagiert und für die Lösungen erarbeitet werden mussten, überraschend umfassende Zuständigkeiten und Entscheidungskompetenzen eingeräumt (Hahn 1988: 90f): »With such a broad range of responsibilities, the potential is there for local soviets to become dynamic forces for economic and social development in their areas« (Jacobs 1983: 3). Dieser Eindruck täuscht jedoch.

Die politische Führung stand angesichts dieser Formalstrukturen vor einem (Partizipations-)Dilemma, das es in ihrem eigenen Interesse zu lösen galt: Einerseits konnte sie aus ideologischen wie aus praktischen Gründen kaum auf die formalen Strukturen der Massenpartizipation verzichten. Nach offizieller Lesart des sozialistischen Programms sollte insbesondere die Institution der (lokalen) Räte Stalin zufolge die »Massenorganisation der Werktätigen« (Meissner 1985: 65) dazu dienen, die Regierung und Staatsverwaltung den Bürgerinnen und Bürgern durch umfassende Einbindung anzunähern. Auch hier greift wieder das Exklusionsmoment als identitätsstiftendes Prinzip: Durch Massenpartizipation in der staatlichen Verwaltung sollte das Ideal eines >Arbeiter- und Bauernstaates< verwirklicht werden, das offiziell als Kontrapunkt zu >kapitalistischen $<$ und >bourgeoisen $<$ Regierungsformen präsentiert wurde. Dort, so gab man vor, werde die Mehrheit der Bevölkerung systematisch exkludiert und unter dem Deckmantel der Demokratie faktisch von einer schmalen Elite regiert (Churchward 1983: 35): »Die Formen der 
bürgerlichen Staaten sind außerordentlich mannigfaltig, ihr Wesen ist aber ein und dasselbe: Alle diese Staaten sind so oder so, aber in letzter Konsequenz unbedingt eine Diktatur der Bourgeoisie« (Lenin 1970 [1917]: 425). Massenpartizipation wurde entsprechend zum Mittel der Wahl, um höhere gesellschaftliche Entwicklungsstufen auf dem Weg zum Kommunismus zu erreichen: »[T]he Soviet Union was passing out of the dictatorship of the proletariat into mature socialism when the state would become the instrument of the entire society mobilized to achieve the rapid transition to communism« (Churchward 1983: 36). Als Teil des formalen Staatsaufbaus genoss die sowjetische Lokalverwaltung damit auch und gerade unter Legitimitätsgesichtspunkten seitens der Politik eine Art >Bestandsschutz $<$, denn ganz ohne ein Mindestmaß an Akzeptanz in der Bevölkerung kommen selbst totalitäre Regime nicht aus (vgl. Luhmann 2000a: 385). Andererseits impliziert jede Form der Publikumseinbindung - also Demokratisierung - die Dekomposition von Entscheidungen in der Sozialdimension, die über direkte oder indirekte Zustimmung in Subentscheidungen zerlegt werden (Luhmann 2009 [1978]: 401). Das damit einhergehende Moment der Kontingenz, struktureller Unbestimmtheit und sinkender Kontrollmöglichkeiten birgt jedoch stets die Möglichkeit, von der offiziellen Linie der Einheitspartei abzuweichen. Da solche »grundsätzlichen Risse in der Zielstruktur von >Partei und Staat « in Einparteiensystemen nur schwer zu akzeptieren sind (Luhmann 2007 [1971]: 75), waren Mechanismen zur Einhegung dieser Kontingenz gefragt, ohne dabei die Formalstrukturen der Lokalverwaltung als solche ideologisch in Frage zu stellen.

Eine Methode bestand darin, die Einbindung des Publikums im Moment ihres Vollzugs - also im Wesentlichen bei den Wahlen der Volksdeputierten - ins Leere laufen zu lassen. Zwar waren die Sowjets als zentrale Verwaltungsorgane tatsächlich vordergründig an demokratische Legitimation geknüpft - Hahn (1988: 92) bemerkt mit sarkastischer Note: »Soviet specialists on government insist that elections in the Soviet system are the most democratic in the world «-, der vermeintliche Einfluss des Publikums wurde jedoch von den (de jure machtlosen) Parteifunktionären überrollt (Jacobs 1983: 6f; Kornai 1992: 36): »[P]arty bodies were theoretically meant to provide only ideological guidance, and were expressly prohibited from directly interfering in the work of local councils, in practice party substitution (podmena) of local soviets was widespread « (Ross 2009: 30). Massenpartizipation wurde zu einem wesentlichen Bestandteil der Fassade oder scheinheiligen »Schauseite « des Regimes ${ }^{20}$ und bediente die Fiktion demokratischer

20 Diesen Aspekt der Fassade der DDR beschrieb Walter Ulbricht mit den Worten: »Es muß demokratisch aussehen, aber wir müssen alles in der Hand haben « (überliefert von Wolfgang Leonhard und zitiert nach Mählert 2002: 106). 
Legitimation (Hahn 1988: 93; zum Begriff der Fassade von Organisationen vgl. Kühl 2011: 136). Strukturell wurde sie jedoch in Massenkontrolle umgemünzt:

»Under such a system of superiority and subordination, the distinction between elected officers and appointed officials counts for little. Collectively, the elected (though full-time) party leaders and the appointed party officials are known in common parlance as the party apparatus.« (Kornai 1992: 36)

In der Praxis hatte die horizontale Unterordnung des Exekutivkomitees sowohl unter ihr Pendant auf der nächsthöheren Hierarchieebene als auch unter den Sowjet derselben Ebene (das Prinzip der sogenannten dualen Subordination) wohl bestenfalls Fassadencharakter (Ross 2009: 29ff) und nebenbei den Effekt, den Umfang der Bürokratie ins Unermessliche zu steigern (Hahn 1988: 88f; Jacobs 1983: 6f):

»In reality, however, dual subordination ensures the bureaucratic centralization of the local government system. For a local decision to become final, it usually has to go right up the administrative hierarchy, causing much paperwork and delay. Because of democratic centralism and dual subordination, the lower organs historically have found it difficult to become policy initiators.«(Jacobs 1983: 7)

Campbell (1995: 149) bezeichnet die gewählten Räte vor diesem Hintergrund als »dekorative« Versammlungen ohne nennenswertes Mitspracherecht, die den Exekutivkomitees Legitimität verschaffen und deren Entscheidungen abnicken sollten:

»The ispolkom is empowered to make legally binding desicions and functions as the executive and administrative organ of government between sessions of the soviet. The role of the deputies at the subsequent sessions is largely to confirm the decisions made by this body [...].« (Hahn 1988: 114)

Innerhalb des Kreislaufs von Politik, Verwaltung und Publikum, den Luhmann in demokratischen Regimen beobachtet - Wähler (als Publikum) beeinflussen mit ihren Äußerungen die Entscheidungsprämissen der Politik, die Politik nimmt Einfluss auf die Verwaltung, die Verwaltung auf das Publikum und das Publikum wiederum reagiert in der politischen Wahl darauf, wie es von der Verwaltung behandelt wird (Luhmann 2000b: 257, 2010: 139) - kehrt sich damit in sozialistischen Regimen an der Schnittstelle zwischen Politik und Publikum um. Rückkopplungsmechanismen in Richtung der Politik werden unterdrückt und der politische Code Regierung|Opposition wird unterlaufen, indem in der autokratischen Version die Mög- 


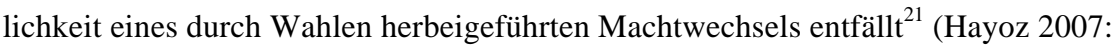
167; vgl. auch Luhmann 2000b: 98).

Aus liberal-demokratischer Perspektive ist eine solche Wendung zweifellos scharf zu kritisieren. Jedoch: Die fehlende Rückkopplung zwischen Publikum und Politik beeinträchtigt zwar vermutlich die Fähigkeit des politischen Systems zur Informationsverarbeitung (Luhmann 2000b: 253ff). Seine Funktion, Kapazität bereitzuhalten um in der Gesellschaft und für die Gesellschaft kollektiv bindende Entscheidungen zu treffen, wird dadurch aber nicht zwingend außer Kraft gesetzt. Im Hinblick darauf ist neben den undemokratischen Implikationen der sowjetischen Ordnung noch ein weiterer Aspekt relevant, der die Schnittstelle von Politik und Verwaltung betrifft: Hier wurden Strukturen etabliert, die der Partei einen direkten Zugriff auf die Verwaltung ermöglichten und die Beschneidung der Verwaltungsautonomie bis hin zur internen Entdifferenzierung des politischen Systems bewirkten - kurz die vertikale Dimension der politischen Hierarchie wurde um eine horizontale Dimension ergänzt.

\section{Die interne Entdifferenzierung des politischen Systems}

Das Phänomen der internen Entdifferenzierung eines Systems, dem eine Schlüsselrolle zukommt, um die Strukturen des sowjetischen Regimes zu verstehen, erschließt sich über die Autonomie seiner Teilsysteme. Autonomie ist mit Luhmann und Stichweh - in expliziter Abgrenzung zu Unabhängigkeit - zu verstehen als die »Pluralisierung der Abhängigkeiten eines Systems« (Stichweh 2009a: 44). Das System kann zu gegebenen Zeitpunkten seine Abhängigkeiten nach eigenem Ermessen und im Hinblick auf seine jeweils akuten Interessen variieren und unterschiedlich gewichten und schafft sich so Freiräume (Stichweh 2009a: 44). Es ist demnach in der Lage, seine eigenen Entscheidungsprämissen innerhalb jener Grenzen zu bestimmen, die durch die Operationen anderer (Sub-)Systeme gesetzt werden (Luhmann 2010: 141). Während innerhalb der Hierarchie des politischen Systems die Kapazität der Spitze - hier der politischen Führung - zur Komplexitätsverarbeitung stets begrenzt ist, kann diese Begrenzung durch die Toleranz autonomer

21 Einer vergleichbaren Logik folgt Offes (2007: 528) Charakterisierung der (liberalen) Demokratie als Regime, in dem »die Ergebnisse der Herrschaftsausübung [durch das Volk, E.M.] ungewiss sind, die Prozeduren dagegen feststehen. Wenn Wahlen >fair < sind, kann man erst nach der Auszählung der Stimmen mit Sicherheit sagen, wer gewonnen hat «. In sozialistischen (und allgemein in autokratischen) Regimen kehrt sich dieses Verhältnis um: Das Resultat, d.h. der Wahlsieger, steht bereits im Vorhinein fest, welche Prozeduren (beispielsweise Wahlfälschung oder Zwang) zu diesem Ergebnis führen, ist hingegen ungewiss. 
Teilsysteme aufgefangen werden: »[U]nbewältigte Komplexität [wird] von der Ebene des Gesamtsystems auf die seiner Teile abgewälzt« und damit für das Gesamtsystem handhabbar gemacht (Luhmann 2010: 141). Die Eigenkomplexität des Gesamtsystems erhöht sich und damit steigen auch seine Fähigkeiten im Umgang mit (Umwelt-)Komplexität.

Die Aufgabenteilung, die sich zwischen den Teilsystemen eines binnendifferenzierten politischen Systems ergibt, besteht für Luhmann im Auseinanderziehen der Entscheidungsvorbereitung durch die Politik (im engeren Sinne, d.h. als Subsystem des politischen Systems) und der Ausführung durch die Verwaltung: Politisches Handeln dient dazu, »die Komplexität der gesellschaftlichen Möglichkeiten so weit zu reduzieren, dass verbindlich und ohne das Risiko erheblichen Widerstandes entschieden werden kann« (Luhmann 2010: 126). Die Verwaltung übernimmt die Ausführung »des politisch Möglichen und Notwendigen« (Luhmann 2010: 126) in Form der Herstellung bindender Problementscheidungen nach Maßgabe von Plänen und Programmen (Luhmann 2007 [1971]: 66):

»[Sie] befaßt sich mit dem Spielraum, den die Ausführung formaler Weisungen läßt, mit schriftlicher Dokumentation (Aktenführung) und mit der Frage, was und was nicht in die Akten kommt. Sie erörtert Rechtsfragen und Fragen der Möglichkeiten, budgetierte Gelder zu verwenden; und natürlich sind Personalfragen nur zum geringen Teil politische Fragen.« (Luhmann 2000b: 255)

Zwar war auch die sowjetische Verwaltung mit derartigen Aufgaben betraut, ihre Autonomie wurde dabei allerdings auf mehreren Wegen unterlaufen und damit eine horizontale Hierarchiedimension eingeführt, in der die Parteihierarchie die beiden anderen Hierarchiestränge der Sowjets und der Exekutivkomitees faktisch dominierte. Diese Dominanz basierte maßgeblich auf drei Mechanismen, die darauf zielten, die Verwaltungsautonomie vor allem in der Sach- und der Sozialdimension einzuhegen:

- Budgetkontrolle: Die Entscheidung über die Vergabe finanzieller Mittel an die lokalen Verwaltungsorgane war Teil des zentralen Planungsprozesses, der von der nationalen Ebene aus koordiniert wurde (dazu ausführlich Ross 1987: Kap. 2 und 3). Deren Entscheidungen spiegelten selten die Bedürfnisse der Kommunen wider, sondern basierten auf zentralen Bedarfsschätzungen und reflektierten primär die Präferenzen der politischen Führung. ${ }^{22}$ Dass die Interessen beider Seiten

22 Neben Zuweisungen aus dem Staatshaushalt und zweckgebundenen staatlichen Subventionen bildeten Steuerzahlungen der im Verwaltungsgebiet ansässigen Unterneh- 
gelegentlich übereinstimmten, war damit nicht ausgeschlossen, aber eben weder garantiert noch strukturell anvisiert (Lewis 1983: 56). Auch über die Mittelverwendung wurde grundsätzlich auf übergeordneten Verwaltungsebenen entschieden. An die Stelle einer lokalen Budgetautonomie trat die sachliche Abhängigkeit vom politischen Zentrum bzw. der Hierarchiespitze und der faktische Entscheidungsspielraum der Kommunen war entsprechend streng limitiert (Hahn 1988: 128; Lewis 1983: 51ff). Die Zentralisierung der Mittelvergabe in der Sachdimension ermöglichte es der politischen Führung, den örtlichen Verwaltungsorganen generelle Entscheidungskompetenzen zuzugestehen, die dann auf der Ebene der konkreten Entscheidung und Umsetzung finanziell wieder eingefangen und in kontrollierte Bahnen gelenkt wurden. Die Lokalverwaltung war ausführendes, nicht aber entscheidendes Organ - denn nicht vorhandene oder zweckgebundene Mittel lassen schwerlich Entscheidungen zu: »These norms and indices, in conjunction with the lack of substantial discretion over local budgets, lead to the conclusion that local soviets may indeed be implementing distributional choices, but they cannot be said to be making them« (Lewis 1983: 56).

- Einrichtung einer politischen Parallelhierarchie: Darüber hinaus wurden die gewählten Amtsinhaberinnen und Amtsinhaber durch ernannte Funktionärinnen und Funktionäre ergänzt und damit innerhalb der Einheitspartei eine Parallelstruktur zur Verwaltung eingerichtet, die die Konformität von Verwaltungsentscheidungen mit dem sozialistischen Programm auf allen Ebenen überwachen und gegebenenfalls korrigieren konnte. Luhmann zufolge ist dies ein bewährtes Mittel von Einparteienregimen, um Schwächen und Unzulänglichkeiten der Programmierung zu kompensieren (vgl. Luhmann 2007 [1971]: 62). Und auch über die Ernennung, Beförderung und Entlassung von Verwaltungsmitgliedern hatte stets die Partei das letzte Wort und spielte dementsprechend eine Schlüsselrolle bei Mitgliedschaftsentscheidungen ${ }^{23}$ (Kornai 1992: 37f): »Thus, according to Article 150 of the 1977 USSR Constitution: >Executive committees of local Soviets of People's Deputies shall be directly accountable both to the council that elected them and to their executive administrative body.< But, the councils' rights to elect their executive committees was also a sham. The party's powers of appointment (nomenklatura) over all key posts meant that, in practice, city executive committees were nominated by party officials at the regional (oblast) level (or higher), and then rubber stamped by deputies of the city council« (Ross 2009:

men eine zweite Einnahmequelle, die für die ländlichen Gemeinden aufgrund ihrer geringen Höhe jedoch zu vernachlässigen war (Lewis 1983: 52; Ross 2009: 34f).

23 So wurden beispielsweise die Mitglieder der Stadtverwaltung zunächst auf Aufforderung der Partei von der regionalen Exekutive ernannt und im zweiten Schritt vom städtischen Sowjet zwar formal gewählt, faktisch aber lediglich >abgenickt< (Campbell 1995: 149f). 
29). Ineinander verzahnte Karrierewege von Parteifunktionären und Verwaltungsbeamten »with officials regularly moving from one administrative hierarchy to another « (Ross 2009: 31) waren vor diesem Hintergrund nicht ungewöhnlich.

- Parteimitgliedschaft als Kontrollinstrument: Schließlich fungierten Zwänge, die aus der Mitgliedschaft in der Partei erwuchsen, als kontinuierlich mitlaufende Kontrolle von Verwaltungsentscheidungen. Zwar reichte der Anteil der Parteimitglieder in den dörflichen Verwaltungsorganen nicht an die Parteidominanz in Organen höherer Verwaltungsebenen heran, war aber dennoch beachtlich: Im Jahr 1985 gehörten rund 40 Prozent der Abgeordneten lokaler Sowjets und 67 Prozent der Mitglieder der Exekutivkomitees (im Durchschnitt fünf von sieben) der Einheitspartei an, während sich die Exekutivkomitees auf der Ebene des Rajons oder des Bezirks bereits fast vollständig aus Parteimitgliedern zusammensetzten (vgl. Tabelle 1). Parteimitglieder waren auch abseits konkreter Anweisungen verpflichtet, den parteilichen Prinzipien Folge zu leisten. Anfallende Entscheidungen wurden damit im Sinne Luhmanns gesteuert ${ }^{24}$ - nicht indem die Lokalverwaltung selbst, wohl aber ihre Mitglieder durch die Parteibindung gehalten waren, die Differenz der eigenen (Verwaltungs-)Entscheidungen zur Parteiideologie zu minimieren. In diesem Sinne lässt sich die Partei als normative und in hohem Maße durchdringende (»pervasive«) Organisation beschreiben, deren Anspruch auf universelle Gültigkeit von und Bekenntnis zu marxistisch-leninistischen Prinzipien die eigenen Grenzen transzendierte und sich auf alle Lebensbereiche der Mitglieder erstreckte ${ }^{25}$ (vgl. zu diesem Organisationstyp Etzioni 1975: 264ff; auch: Bonazzi 2008: 275f).

In Gestalt der Einheitspartei, so lässt sich zusammenfassen, gelang es der Politik, Verwaltungsautonomie effektiv zu beschneiden, indem sie sich selbst als überrelevante innere Umwelt des politischen Systems generierte. Der Einfluss der Politik konnte von der Verwaltung gerade nicht nach eigenen Maßgaben hervorgehoben oder zurückgedrängt werden, sondern war in jedem Fall unumgänglich: In der Sozialdimension wurde mit Blick auf die Verwaltungsmitglieder das autonomiekonsti-

24 »Nun ist Steuerung aber nicht einfach Beobachtung, nicht einfach Verwendung einer Unterscheidung zur Bezeichnung der einen (und nicht der anderen) Seite. Als Steuerung bezeichnet man vielmehr eine ganz spezifische Verwendung von Unterscheidungen, nämlich das Bemühen um eine Verringerung der Differenz." (Luhmann 1988: 328)

25 Einen vergleichbaren Anspruch erheben in der Regel religiöse Organisationen gegenüber ihren Mitgliedern - nicht umsonst betitelt Lewytzkyi (1967) seine Analyse der Kommunistischen Partei der Sowjetunion als »Porträt eines Ordens« -, worin eine Ursache für die strikte Inkompatibilität von Sozialismus und Religion (respektive Partei/Sowjetmacht und Kirche) zu suchen ist. 
tuierende Prinzip der Rollentrennung umgekehrt in eine explizite Rollenkopplung (vgl. dazu Luhmann 2010: 174f). Die Verwaltung konnte es sich nicht leisten, von den politischen Rollen ihrer Mitglieder (d.h. der Beamtinnen und Beamten) zu abstrahieren, sondern war gezwungen, diese explizit in die eigenen Operationen einzubeziehen (die bereits erwähnten »interlocking career structures« weisen darauf hin, vgl. Ross 2009: 31; vgl. zum Prinzip der Rollentrennung in diesem Zusammenhang Luhmann 2010: 177). In der Sachdimension sorgte die Politik auf direktem und indirektem Wege für eine »durchgreifende Ideologisierung allen öffentlichen Handelns« (Luhmann 2010: 128f). Die sowjetische Verwaltung wurde damit ausschließlich über ihre Grenze zur Politik beherrscht und letztlich »zu einem bloßen Annex, zu einem verlängerten Arm« (Luhmann 2010: 174) Letzterer reduziert:

»The intricacies of the hierarchical stratification, the principles of democratic centralism and dual subordination, and the overlapping and duplication of responsibilities are there for a purpose: to incorporate local government into the total bureaucratic system that runs the country, and to ensure that the center's will is enforced in the localities.«(Jacobs 1983: 17)

Oder ideologisch gewendet:

»[E]ach local soviet must strive to ensure that what it is doing fits harmoniously with the greater good of Soviet society as a whole. [...] Ideally, the role of the deputy as representative in this system is to merge dialectically the specific interests of constituents with the general interests of society.«(Hahn 1988: 81f)

Erkauft wurde die weitreichende Kontrolle der Einheitspartei sowohl über den eigenen Apparat als auch über die Entscheidungen der staatlichen Verwaltung jedoch mit sehr begrenzten Möglichkeiten zum Aufbau von Eigenkomplexität und damit mit beschränkten Fähigkeiten zur Verarbeitung von Umweltkomplexität. Das auf die Einheitspartei fixierte politische System war nicht mehr in der Lage, mit Kontroversen und widersprüchlichen Handlungsorientierungen umzugehen, ohne seinen eigenen Bestand zu gefährden (Luhmann 2010: 127). In der Folge war es darauf angewiesen, seine Umweltbeobachtung auf Übereinstimmung mit oder Abweichung von den ideologischen Grundsätzen des sozialistischen Programms zu reduzieren. Im System selbst wurde dies zwar reflektiert. Es zog daraus jedoch nicht die Konsequenz, ein höheres Maß an interner Differenzierung und Teilsystemautonomie zuzulassen und damit Eigenkomplexität aufzubauen, denn dies hätte sich notwendigerweise negativ auf seine Kontrollfähigkeit ausgewirkt. 
Tabelle 1: Personelle Struktur der sowjetischen Lokalverwaltung (1985)

\begin{tabular}{l|c|c|c|c} 
& \multicolumn{2}{|c|}{ Lokale Räte } & \multicolumn{2}{c}{ Lokale Exekutivkomitees } \\
$\begin{array}{l}\text { Verwaltungs- } \\
\text { einheit }\end{array}$ & $\begin{array}{c}\text { Mitglieder } \\
\text { der KPdSU }\end{array}$ & $\begin{array}{c}\text { Mitglieder d. } \\
\text { Komsomol }\end{array}$ & $\begin{array}{c}\text { Mitglieder } \\
\text { der KPdSU }\end{array}$ & $\begin{array}{c}\text { Mitglieder d. } \\
\text { Komsomol }^{*}\end{array}$ \\
\hline $\begin{array}{l}\text { Gebiet, Gau, } \\
\text { Distrikt }\end{array}$ & $54,6 \%$ & $26,5 \%$ & $90,4 \%$ & $5,6 \%$ \\
\hline Rajon & $49,0 \%$ & $24,6 \%$ & $88,1 \%$ & $6,8 \%$ \\
\hline Stadt & $46,3 \%$ & $25,1 \%$ & $85,7 \%$ & $6,3 \%$ \\
\hline Stadtbezirk & $46,8 \%$ & $25,0 \%$ & $88,2 \%$ & $6,2 \%$ \\
\hline Siedlung & $41,1 \%$ & $22,1 \%$ & $73,0 \%$ & $7,4 \%$ \\
\hline Dorf & $40,5 \%$ & $21,0 \%$ & $67,2 \%$ & $8,7 \%$ \\
\hline $\begin{array}{l}\text { Anteil aller } \\
\text { Abgeordneter }\end{array}$ & $42,8 \%$ & $22,3 \%$ & $71,1 \%$ & $8,2 \%$ \\
\hline $\begin{array}{l}\text { Anteil an der } \\
\text { Bevölkerung }\end{array}$ & $6,5 \%{ }^{* *}$ & $15,4 \%$ & $6,5 \%{ }^{2}$ & k.A. \\
\hline
\end{tabular}

* Jugendorganisation der KPdSU

** Dieser Anteil entspricht etwa 18,5 Millionen Parteimitgliedern

Quelle: Hahn (1988: 110), Kornai (1992: 35).

Zwar gab es im Bereich der sowjetischen Lokalverwaltung hin und wieder zaghafte Dezentralisierungsbestrebungen. Beispielsweise ließen sich mit Chruschtschows Amtsantritt nach dem Tod Stalins leichte Tendenzen in Richtung einer Verschiebung der politischen Macht beobachten, im Rahmen derer die Räte als Partizipationsorgane reaktiviert und die Mitsprache der lokalen Ebene in Entscheidungsprozessen gesteigert werden sollten (Campbell 1995: 148ff; Hill 1983: 21ff). Derartige Reformmaßnahmen blieben jedoch in der Regel entweder ohne nennenswerte Effekte oder führten zu einem undurchschaubaren Netz an verwaltungsinternen Rechenschaftsverpflichtungen ohne klar erkennbare Autorität (Campbell 1995: 150). Denn sobald das Regime durch die Schaffung neuer Stellen auf unteren Hierarchieebenen die Möglichkeit dezentraler Entscheidung schuf, welche der eigenen Logik jedoch stets zuwider lief, sah es sich im Bewusstsein dieses Widerspruchs gezwungen, mit einer Ausweitung des bürokratischen Apparats zu reagieren. Dies wiederum führte zu bekannten Struktureffekten übermäßig wachsender Organisationen: Unüberschaubarkeit, Trägheit und steigende Kosten der Kontrolle und eigenen Aufrechterhaltung. Die Partei initiierte Maßnahmen, deren Effekte sie hinterrücks wieder einfangen musste, um ihre eigene Existenz nicht zu 
gefährden $^{26}$ (Campbell 1995: 149), und war damit buchstäblich gefangen in den eigenen Prinzipien:

»It is true that all the positive changes of the past three decades have been sponsored by the party. And yet the party as an institution insists on keeping local government firmly under control, issuing binding directives, influencing appointments and directly interfering in the work of state employees. [...] Soviet local government today still seems trapped by the cultural legacy of the past, and by the ideological underpinnings of the system.« (Hill 1983: 33)

Um die eigene Reproduktionsfähigkeit unter diesen Bedingungen zu sichern, war das politische System - respektive die Partei - darauf angewiesen, auf seine Umwelt durchzugreifen und diese auf eine Weise zu strukturieren, die die Wahrscheinlichkeit von Abweichungen reduzierte und deren Beobachtbarkeit anhand der eigenen Unterscheidungen ermöglichte. Dabei musste die Umwelt jedoch Umwelt bleiben. Was banal klingt, macht deutlich, dass die Partei allein das Ziel der Vollinklusion nicht bewerkstelligen konnte. Die Integration der Umwelt durch die Ausweitung der eigenen Grenzen mit dem Ziel der Kontrolle - alle werden Parteimitglieder und dadurch organisational gebunden - entfiel aufgrund des gesellschaftlichen Führungsanspruchs der Partei: Wollte sie diesen Führungsanspruch behaupten, musste Mitgliedschaft ein Privileg bleiben - und dies galt selbst für eine Massenpartei wie die $\mathrm{KPdSU}^{27}$ deren Rekrutierungspolitik auf der Idee basierte »that the party represented the cream of Soviet society« (Sakwa 1998: 84; zum aufwendigen und anspruchsvollen Aufnahmeprozess in die Partei vgl. auch Hough/Fainsod 1982: 320ff). Stattdessen versuchte sie, Umweltkomplexität - also gesellschaftliche Komplexität - so weit zu reduzieren, dass sie den eigenen Verarbeitungskapazitäten entsprach. Weicks (1979) Beobachtung - »Organisationen malen ihr eigenes Bühnen-

26 Die ersten ernstzunehmenden Reformen in Richtung politischer Dezentralisierung wurden in den späten 1980er Jahren auf Gorbatschows Initiative in Angriff genommen, wobei es im Kern darum ging, die Machtposition der Partei zugunsten anderer Institutionen und vor allem gegenüber den Sowjets zu schwächen (Hahn 1989; Ross 2009: 38ff). Zu den sichtbarsten Maßnahmen gehörte die Einführung zumindest ansatzweise kompetitiver Wahlen in einigen Bezirken, bei denen mehr als ein Kandidat zugelassen wurde (Ross 2009: 41f). Historisch kamen Gorbatschows Reformversuche jedoch zu spät, als dass sie den Zusammenbruch des Regimes hätten verhindern können, so dass aus heutiger Sicht mehr die zugrundeliegende Intention als die tatsächlichen Effekte beurteilt werden können (vgl. dazu ausführlich S. 135ff).

27 Im Jahr 1986 umfasste die KPdSU 19,04 Millionen Mitglieder, was einem Anteil von 9,7 Prozent der erwachsenen Bevölkerung oder 6,8 Prozent der Gesamtbevölkerung entsprach (Sakwa 1998: 84f). 
bild, betrachten es durch das Opernglas und versuchen, ihren Weg durch die Bühnenlandschaft zu finden « (Tom Lodahl zitiert nach Weick 1995 [1979]: 198) - trifft demnach nicht nur auch auf die KPdSU zu, sondern entfaltete aufgrund der hervorgehobenen Stellung Letzterer innerhalb der sowjetischen Gesellschaft besondere Durchschlagskraft. Das zentrale Instrument im Hinblick auf dieses Vorhaben stellten Massenorganisationen dar, die sich als Versuch beobachten lassen, die Funktionslogik der ganzen Gesellschaft auf die Unterscheidung von Akzeptanz und NichtAkzeptanz des sozialistischen Programms auszurichten und damit für das (unterkomplexe) politische System handhabbar machen.

\section{Massenorganisationen}

Ideologisch und in den Worten Lenins fungierten Massenorganisationen als »Transmissionsriemen « für die Vermittlung der marxistisch-leninistischen Ideologie an die breite Masse der Bevölkerung. Letztere wurde meist in Form klar definierter gesellschaftlicher Zielgruppen adressiert, wie etwa Arbeiterinnen und Arbeiter, Jugendliche, Frauen, Kulturschaffende und andere (Kornai 1992: 40; vgl. dazu auch Selznick 1952: 8ff). Beispiele für Massenorganisationen, die in vergleichbarer Form nicht nur in der Sowjetunion, sondern in allen Staaten des ehemaligen Ostblocks existierten, waren dementsprechend Einheitsgewerkschaften, Genossenschaften, die Sowjets (Räte), Jugendorganisationen, Kultur- und Sportverbände etc. Über ihre ideologische Transmissionsfunktion hinaus kamen Massenorganisationen aber auch mit Blick auf ganz praktische Ziele zum Einsatz: Sie wurden für Kampagnen mobilisiert, die Defizite vor allem ökonomischer Art kompensieren sollten (Planerfüllung, Energiesparen, die Steigerung schulischer oder universitärer Leistungen und dergleichen mehr) (Mählert 2002: 110).

Ein augenfälliges Merkmal sozialistischer Massenorganisationen war ihr Anspruch auf ein Funktionsmonopol für einen bestimmten gesellschaftlichen Bereich. ${ }^{28}$ Der überwiegende Teil von ihnen wurde dabei entlang gesellschaftlicher Bereiche oder Problemlagen eingerichtet, die in einer funktional differenzierten Gesellschaft für gewöhnlich Funktionssysteme ausfüllen. Bestimmte Operationen (respektive Kommunikationen) sollten auf diese Weise möglichst umfassend in organisierte Strukturen überführt und damit kontrollierbar gemacht werden (Kornai 1992: 39; für das Beispiel der DDR auch Mählert 2002: 105f). So beanspruchte der Kulturbund »von den Briefmarkensammlern bis zu den Schriftstellern alle >Kultur-

28 Ungeachtet ihrer Omnipräsenz im sowjetischen Alltag führen Massenorganisationen in Analysen sowjetischen Regimes bislang weitgehend ein Schattendasein. Unter den wenigen Ausnahmen sind die Studie von Kornai (1992: v.a. 39f) und für die DDR die Beiträge in Stephan et al. (2002). 
schaffenden< in seinen Reihen [zu] vereinen« (Mählert 2002: 105). Ähnliche Ziele verfolgten die Einheitsgewerkschaften, Genossenschaften, Jugendorganisationen oder Sportverbände. Ein besonders prominentes Beispiel für den Versuch der umfassenden Organisation eines gesellschaftlichen Bereichs ist sicher das Knappheitsproblem, das über die Steuerung des Produktions- und Distributionsprozesses durch den Zentralstaat gelöst werden sollte (vgl. für den Bereich der Landwirtschaft ausführlich S. 70ff). Die defizitären Konsequenzen dieses Vorhabens traten in Form der chronischen sowjetischen Mangelwirtschaft besonders deutlich zu Tage. Vergleichbare Organisationsmonopole finden sich aber auch für die Erziehung, das Recht, die Kunst und andere.

$\mathrm{Zu}$ beachten ist im Gegenzug allerdings auch, dass in sozialistischen Regimen nicht für alle >klassischen< Bereiche moderner Gesellschaften Organisationen eingerichtet wurden. Vor allem diejenigen gesellschaftlichen Bereiche, in denen die politische Führung keine Möglichkeit sah, sie durch entsprechende Organisation in Einklang mit der sozialistischen Ideologie zu bringen, wurden oft kurzerhand nicht zugelassen oder zu illegalisieren versucht. Ein bekanntes Beispiel hierfür ist die Religion bzw. die Kirche als Organisation, die die sozialistischen Regime offensichtlich nie umfassend in den Griff bekamen, was auch am Fehlen einer spezialisierten Massenorganisation zu erkennen ist. Interessanterweise spielte ausgerechnet die Kirche während der Revolutionen in vielen sozialistischen Staaten eine bedeutende Rolle (für die DDR vgl. Pollack 1990, 1994) und die Bindung an diese Institution entwickelte sich zu einer besonders klar erkennbaren Möglichkeit, individueller Opposition zum politischen Regime Ausdruck zu verleihen.

Indem die Massenorganisationen der Partei untergeordnet waren und den parteilichen Anweisungen und Vorgaben bezüglich ihrer Entscheidungen und Organisationszwecke Folge zu leisten hatten (was in der Regel wiederum durch die Parteimitgliedschaft der jeweiligen Führungsriege sichergestellt werden sollte), konnte die Partei potenziell auf alle gesellschaftlichen Bereiche durchgreifen und über Mitgliedschaftsentscheidungen den Zugang einzelner Personen zu diesen Bereichen beeinflussen. Gleichzeitig bestand im Verhältnis der Massenorganisationen zur Partei eine klare Trennung: Die Mitgliedschaften in beiden Organisationstypen bedingten sich weder wechselseitig noch schlossen sie sich aus - Massenorganisationen und Partei waren füreinander Umwelt.

Mit Blick auf das sowjetische Vorhaben der Vollinklusion aller Gesellschaftsmitglieder leisteten die Massenorganisationen einen für das Regime unverzichtbaren Beitrag. Die Omnipräsenz organisationaler Strukturen in allen Bereichen des alltäglichen Lebens machte es faktisch unmöglich, die Mitgliedschaft vollständig zu umgehen. Ob im Hinblick auf den Beruf, auf Freizeitaktivitäten oder auf Bildung und Erziehung, stets war der Beitritt zur entsprechenden Organisation die Voraussetzung für die Adressierbarkeit im jeweiligen gesellschaftlichen Bereich. Das Re- 
sultat war ein gesellschaftsweit umfassender Organisationsgrad ${ }^{29}$ unter staatlicher Kontrolle.

Verstärkt wurde der Mitgliedschaftsdruck durch Sanktionen: Positive Sanktionen bestanden im Gewähren persönlicher Vorteile, die für (Noch-)Nicht-Mitglieder der Massenorganisationen Beitrittsanreize darstellten und Mitglieder zu stärkerem Engagement innerhalb der Organisation anspornen sollten. So spielten beispielsweise wirtschaftliche Überlegungen für den Beitritt zu Konsumgenossenschaften eine zentrale Rolle und Gewerkschaften wurden zu wichtigen Anbietern von Urlaubsreisen und Kulturveranstaltungen (Mählert 2002: 112). Nachdem im Bereich der organisierten Produktion (also in Staatsunternehmen und Genossenschaften) das Medium Geld aus ideologischen Gründen nur begrenzt als Anreiz zur Produktivitätssteigerung zur Verfügung stand ${ }^{30}$ (Luhmann 1997: 830), diente der Einsatz von Auszeichnungen und Privilegien diesem Zweck. Auf Organisationsstrukturen wurde dabei bei konkreten Motivations- und Stimulierungsmaßnahmen zurückgegriffen. So kam beispielsweise betriebsintern der Vergabe von Prämien eine hervorgehobene Bedeutung zu (Berliner 1957, 1952) (vgl. dazu auch S. 83ff). Für die Landwirtschaft sah das dritte Musterstatut, ${ }^{31}$ ein standardisiertes Regelwerk für die

29 Genaue Mitgliedschaftszahlen für die Massenorganisationen der Sowjetunion lassen sich nicht ermitteln. Als Maßstab für den Organisationsgrad eignet sich jedoch das Beispiel der DDR, deren politisches Regime in dieser Hinsicht ähnlich strukturiert war. Im Jahr 1988 umfasste der Freie Deutsche Gewerkschaftsbund (FDGB), die Einheitsgewerkschaft der DDR, 9,6 Millionen Mitglieder (60 Prozent der Bevölkerung), die Gesellschaft für Deutsch-Sowjetische Freundschaft 6,3 Millionen (rund 40 Prozent der Bevölkerung), die Freie Deutsche Jugend (FDJ) und die Pioniere 4,3 Millionen und der Deutsche Turnund Sportbund (DTSB) 3,7 Millionen (Zahlen aus Stephan et al. 2002).

30 Ganz konnte das Regime jedoch offensichtlich nicht auf monetäre Anreize verzichten: Beispielsweise beschreibt Figes (2012: 193ff), wie seit 1950 selbst in den Arbeitslagern des Gulag, die eigentlich der sozialistischen Umerziehung ihrer Insassen dienen sollten, teilweise Lohnzahlungen als Anreiz für die Häftlinge eingeführt wurden, nachdem deren Produktivität und damit ihr Beitrag zur sowjetischen Wirtschaft nach Ansicht der politischen Führung zu stark gesunken war.

31 Die Musterstatute für die Kollektivbetriebe waren standardisierte Regelwerke, die den Status von Gesetzen hatten und die generellen Prinzipien für die interne Struktur und die Aufgaben und Verantwortlichkeiten der Betriebe festlegten. In der Geschichte der Sowjetunion waren vier Versionen des Musterstatuts in Kraft, die in den Jahren 1930, 1935, 1969 und 1988 implementiert wurden (Altrichter 1984: 195; Lindner 2008: 72). Die vierte Version von 1988 hatte jedoch aufgrund des Regimewechsels keine praktische Relevanz mehr, so dass hier ausschließlich das dritte Musterstatut von 1969 von Bedeutung ist (weitere Details dazu folgen auf S. 72ff). 
Struktur landwirtschaftlicher Kollektivbetriebe, ein ganzes Bündel an »Maßnahmen zur Stimulierung der Kolchosmitglieder« vor, deren Einsatz der Entscheidung der Betriebsleitung unterlag: Belobigungen, Prämien, die Auszeichnung mit Geschenken oder Ehrenurkunden, ein Eintrag auf der Ehrentafel oder in das Ehrenbuch oder die Verleihung der Titel »Verdientes Kolchosmitglied « oder »Ehrenmitglied des Kolchos« (Drittes Musterstatut, Art. 34, zitiert nach Brunner/Westen 1970: 155; siehe auch Humphrey 1998: 111). Einer vergleichbaren Logik folgten die sogenannten >sozialistischen Wettbewerbe< (»sozsorevnovanie«), die zwischen einzelnen Betriebsangehörigen oder ganzen Abteilungen sowohl innerhalb als auch zwischen Unternehmen veranstaltet wurden. Den Siegerinnen und Siegern winkten ebenfalls Prämien, die Gewährung individueller Vorteile und Vergünstigungen sowie - selbstredend - sozialistisches Prestige, das beispielsweise durch die Verleihung des »roten Banners « sichtbar gemacht wurde (Berliner 1957: 26; Humphrey 1998: 111).

Negative Sanktionierungen bestanden zum einen schlicht in unterlassenen positiven Sanktionen, beispielsweise der expliziten Nicht-Gewährung von persönlichen Vorteilen, die mit der Mitgliedschaft und besonderem Engagement in den Massenorganisationen verbunden waren, sie konnten aber auch eigene Formen annehmen. Wiederum am Beispiel landwirtschaftlicher Kollektivbetriebe nennt Humphrey (1998: 111) das Erteilen von Verweisen, Verwarnungen und strengen Verwarnungen, die zeitlich begrenzte Herabstufung auf eine schlechter bezahlte Stelle innerhalb der Organisation und im Extremfall die Entlassung aus dem Betrieb.

In positiver wie in negativer Richtung verstärkte das Außer-Kraft-Setzen von Konvertibilitätssperren den Effekt der Sanktionen: Unter der Bedingung funktionaler Differenzierung bewirken Konvertibilitätssperren, dass Erfolge aus einem Funktionssystem (z.B. ein in einem Teilsystem erworbener Status einer Person) nicht reibungslos in andere Funktionssysteme übermittelt werden können (Stichweh 2005: 175). Zwar wird diese theoretisch postulierte Rigidität von Konvertibilitätssperren in der Praxis häufig nicht eingelöst - wohlgemerkt auch nicht in sogenannten westlichen Gesellschaften -, sobald jedoch ein gewisses Maß an Durchlässigkeit offenkundig wird, entsteht zumindest Rechtfertigungsdruck. Ein »gesamtgesellschaftlicher Status und eine gesamtgesellschaftliche Sichtbarkeit« einzelner Personen oder Familien sind in der modernen Gesellschaft kaum denkbar (Stichweh 2005: 177). Im Gegensatz dazu war die fast universelle Transformierbarkeit von politischem Kapital - um auf Bourdieus Terminologie zurückzugreifen - ein verbreitetes und erwartbares Phänomen und organisationsübergreifende Karrieren waren, um ein Beispiel zu nennen, keine Seltenheit (Kornai 1992: 38f). Andersherum steigerte die Abwesenheit von Konvertibilitätssperren die Wahrscheinlichkeit von Exklusionsverkettungen: Innerhalb der umfassenden und omnipräsenten Strukturen der Massenorganisationen konnte nicht nur glaubwürdig mit dem Ausschluss aus 
einer Organisation gedroht werden, sondern die Exklusion aus einer Organisation (und damit faktisch: aus einem gesellschaftlichen Bereich) zog über die entsprechenden Organisationen potenziell Exklusionen aus weiteren gesellschaftlichen Bereichen nach sich. Eine derartige Verkettung wurde beispielsweise durch das sogenannte Arbeitsbuch (»trudovaja knižka«) ermöglicht. Es wurde für alle Beschäftigten ausgestellt und dokumentierte Auszeichnungen ebenso wie Strafen und Vergehen. Diese Historie beeinflusste nicht allein die Beförderungsmöglichkeiten innerhalb des eigenen Unternehmens, sondern wirkte sich auch auf Aufstiegsmöglichkeiten innerhalb der Parteihierarchie oder auf potenzielle Stellenwechsel aus:

»Sometimes a fine is imposed, but more serious is the fact of documentary evidence of misdemeanours in a person's work record (trudovaya knizhka) - somewhat like the endorsement of a driving license in Britain - since this will affect promotion, success in the Party, and possibilities of transferring to other jobs.« (Humphrey 1998: 111)

Massenorganisation, dies legen die skizzierten Merkmale nahe, stellten innerhalb der sowjetischen Organisationsgesellschaft Strukturen bereit, die es der Partei ermöglichten, das Problem des eigenen Machterhalts zu bearbeiten, indem den Gesellschaftsmitgliedern persönliche Freiheitsgrade innerhalb politisch kontrollierbarer Strukturen geboten wurden. Dabei lässt sich eine Aufgabenteilung zwischen beiden Organisationstypen beobachten: Die Einheitspartei konnte sich auf eine isolierte und klar begrenzte Position zurückziehen, was ihrem gesellschaftlichen Führungsanspruch entsprach; die >unordentliche< Integration und Kontrolle der Bevölkerung wurde den Massenorganisationen überlassen, in die jeder eintreten durfte (und sollte), deren Führung sich jedoch immer wieder auf ihre Stellung innerhalb der gesellschaftlichen Hierarchie und damit auf die Vorgaben der Partei zurückziehen und Partizipation auf diese Weise beschneiden konnte. Sowohl der Verlust an individueller Autonomie als auch mangelnde Partizipationsmöglichkeiten wurden dabei nicht nur durch das breite Spektrum an wohlfahrtsstaatlichen Leistungen kompensiert, die der sowjetische Staat seinen Bürgerinnen und Bürgern bot (für analoge Mechanismen in der DDR vgl. Pollack 1998: 117f). Organisationsmitgliedschaft wurde darüber hinaus zur entscheidenden Voraussetzung für faktisch alle individuellen Handlungsmöglichkeiten ${ }^{32}$ - Zaslavsky (1982: 142ff) verweist in die-

32 Mit dem empirischen Zusammenhang von Systemloyalität mit sozialer Mobilität und individuellen Karriereverläufen am Beispiel der DDR beschäftigt sich beispielsweise Solga (1994; vgl. auch Gebauer 2003). Die enge Verzahnung von wissenschaftlichen und politisch-administrativen Karrieren beschreibt Bikbov (2005: 315ff) am Beispiel zweier sowjetischer Soziologen. 
sem Zusammenhang auf Strategien zur Herstellung von »organisiertem Massenkonsens «:

»Nachdem es einmal beschlossene Sache war, unabhängige Organisationen oder Interessensverbände nicht zuzulassen, mußte das Regime den Bürgern Raum lassen für individuelle Initiativen zur Verbesserung ihrer wirtschaftlichen Lage und für andere Formen sozialen Aufstiegs.« (Zaslavsky 1982: 142)

Umgekehrt mussten diejenigen, die sich den politischen Ansprüchen widersetzten, damit rechnen, »disqualifiziert und an den Rand der Gesellschaft gedrängt zu werden« (Pollack 1990: 294).

Das koordinative Verzahnen von Mitgliedschaftsmotivation und Organisationszweck - ein für Organisationen mit Blick auf das Gewinnen und Halten von Mitgliedern üblicherweise essentielles Unterfangen - konnte aus Sicht der politischen Führung unter diesen Bedingungen weitgehend ignoriert werden. Stattdessen konnte sie sich zumindest in der offiziellen Außendarstellung mit der Illusion von Freiwilligkeit als zentraler Mitgliedschaftsmotivation umgeben (zu verschiedenen Formen von Compliance in Organisationen vgl. Etzioni 1975: 9ff). Dies wiederum verweist auf eine weitere Dimension von Vollinklusion, auf die Massenorganisationen ebenfalls hinwirkten. Das Ziel der Vollinklusion beinhaltete nicht bloß den Anspruch der umfassenden Adressierbarkeit aller, sondern darüber hinaus der Inklusion der entsprechenden Personen in toto. Zum Ausdruck kam dies insbesondere in einem gigantischen Umerziehungsprojekt, das mit dem sozialistischen Gesellschaftsprogramm verbunden war und dem die Idee der Schaffung eines »Sowjetmenschen« als neuen Menschentyp zugrunde lag. Ergebnisse und Erfolge dieses Unterfangens - also die Internalisierung sozialistischer Prinzipien als psychischer Prozess - blieben für die politische Führung respektive für die Partei jedoch unbeobachtbar. Zwar sind Organisationen über Personen strukturell an psychische Systeme gekoppelt (Luhmann 2008 [1991]: 146). Letztere sind dabei jedoch stets Teil der Umwelt von Sozialsystemen und können folglich auch nur als solche wahrgenommen und behandelt werden (dasselbe gilt vice versa). Ein direkter Zugang zu geschweige denn die Steuerung von - Umweltsystemen ist nicht möglich (Baraldi/Corsi/Esposito 1997: 129).

Mit der Möglichkeit, dass ihre umfassende Inklusionsabsicht unbeobachtet ins Leere laufen könnte, konnte sich die Einheitspartei angesichts ihres umfassenden Kontroll- und Führungsanspruchs jedoch kaum abfinden. Gefragt waren Indikatoren, um die Effekte des Umerziehungsvorhabens (vermeintlich) beobachtbar zu machen. Erneut kommen hier die Massenorganisationen ins Spiel: Ihre Strukturen wurden herangezogen, um die entscheidende, aber gesellschaftlich unbeobachtbare Unterscheidung Akzeptanz|Nicht-Akzeptanz sozialistischer Prinzipien und Werte in 
die beobachtbare Unterscheidung Mitgliedschaft|Nicht-Mitgliedschaft in politisch kontrollierten Organisationen zu überführen, die auf beiden Seiten Anschlussmöglichkeiten für weitere Kommunikation eröffnete. Ungeachtet der damit verbundenen Selbsttäuschung, die dem Regime durchaus bewusst war, konnte die politische Führung auf Grundlage dieser Unterscheidung und unter Verweis auf den hohen Organisationsgrad der sowjetischen Gesellschaft nach innen und außen die Illusion ihrer vollständigen und bedingungslosen Unterstützung durch die Mitglieder der sozialistischen Volksgemeinschaft konstruieren und sich zudem einreden, diese stehe unter ihrer Kontrolle.

Im Zweifelsfall waren die Strukturen der Massenorganisationen unter diesen Umständen für die Partei eine Grundlage, um die Unterstützung des eigenen Programms einzufordern und jedes Gesellschaftsmitglied auf sozialistische Prinzipien festzulegen - genau dazu hatten sich die Mitglieder durch ihren Beitritt schließlich offiziell verpflichtet. Auch bei der Identifikation - oder genauer: Zuschreibung von abweichendem oder systemkritischem Verhalten konnte die politische Führung stets auf die Massenorganisationen zurückgreifen, indem Letztere einen Rahmen boten, innerhalb dessen jegliches Verhalten als Entscheidung zu deuten und zuzurechnen war, auf die man sich in anschließenden Entscheidungen beziehen konnte: »Organisationen sind insofern soziale Systeme, die sich erlauben, menschliches Verhalten so zu behandeln, als ob es ein Entscheiden wäre« (Luhmann 2009 [1978]: 410; vgl. dazu auch Baecker 1999: 144f). In der sowjetischen Organisationsgesellschaft kam dies in der Repression von Dissidentinnen und Dissidenten ebenso zum Ausdruck wie in der Politisierung von Alltagsphänomenen wie einem >westlichen < Kleidungsstil oder dem Konsum von >kapitalistischer $<\mathrm{Mu}$ sik und Literatur, die unumwunden mit dem Widerstand gegen das Regime gleichgesetzt wurden (Koch 2002: 135). Aus Sicht der Mitglieder entfiel damit die Möglichkeit, sich nicht zu entscheiden - und zwar völlig unabhängig davon, ob ihnen dies bewusst war oder nicht:

»Es mag durchaus sein (und kommt nicht gerade selten vor), daß ein Funktionär gar nicht merkt, daß er entscheidet; und erst recht, daß er nicht alle Alternativen sieht, die er mit einer Entscheidung ausscheidet. Ausschlaggebend ist, dass eine Kommunikation früher oder später als (geplante, vermiedene, vollzogene) Entscheidung gelesen werden kann.« (Luhmann 2000b: 234)

Paradoxerweise und ungeachtet der daraus resultierenden Unwägbarkeiten politischer Entscheidungen - beispielsweise war >Klassenfeind < eine offene Kategorie, die willkürlich mit Inhalt gefüllt wurde, so dass man nie sicher sein konnte, für vermeintliche Vergehen angeklagt und verurteilt zu werden (vgl. Applebaum 2003: 47 ) - entlastete die Gleichsetzung von Organisationsmitgliedschaft mit Programm- 
akzeptanz und der Internalisierung sozialistischer Werte nicht nur die politische Führung selbst. Auch >normalen< Bürgerinnen und Bürgern bot sie die Möglichkeit, weitgehend unabhängig von ihren individuellen Überzeugungen nach außen Konformität zu demonstrieren, wovon nicht unerhebliche Anreize ausgingen, einer oder gleich mehrerer Organisationen beizutreten - konnte man auf diese Weise doch unangenehmen Nachfragen entgehen, auf welcher Seite man denn stehe (Mählert 2002: 113).

Gegen eine solche Darstellung der Massenorganisationen im Sozialismus lässt sich einwenden, dass auch in liberaldemokratischen Staaten Massenorganisationen in Form von Parteien, Verbänden oder Vereinen existieren. Im Unterschied zum sowjetischen Regime entstehen sie dort jedoch nicht als Produkt zentralisierter Planung, sondern in einem evolutionären Prozess, bei dem stets die Möglichkeit gegeben ist, dass Organisationen gerade nicht eindeutig funktional zuzuordnen sind (vgl. dazu Tacke 2001). Überdeutlich wird das Inkongruenzverhältnis von Funktionssystemen und Organisationen bei Verbänden. Sie können als Instrumente der strukturellen Kopplung zwischen Politik und anderen Teilsystemen gesehen werden, die sich aus Letzteren heraus entwickeln, so dass mit Konflikten oder widersprüchlichen Interessen zumindest gerechnet werden muss (Brodocz 1996). Mit Blick auf die Gesellschaft in ihrer Gesamtheit ist es aufgrund dessen faktisch ausgeschlossen, jenseits der konstitutiven Merkmale von Organisationen als Systemtyp ein gesellschaftsweit einheitliches Organisationsprinzip zu identifizieren. Im Gegensatz dazu sind Massenorganisationen in totalitären Regimen stets mit der Organisationsgesellschaft als Ganzes gleichgeschaltet und spiegeln als Fraktale ${ }^{33}$ deren Struktur wider, so dass eine dominante Funktionslogik sichtbar wird und sich durch den hohen Organisationsgrad in ihrer Durchsetzungskraft verstärkt. Zusammengenommen erwiesen sich Massenorganisationen als wirkungsvolles Argument, um funktionale Differenzierung zwar - wie ich im Folgenden zeigen möchte - nicht vollständig auszuschalten, aber doch unter erheblichen politischen Druck zu setzen.

\section{Die Organisationsgesellschaft IM SOWJETISCHEN DORF}

Aufgrund von zwei Lücken wird die bisherige Beschreibung der sowjetischen Organisationsgesellschaft ihrem Gegenstand noch nicht vollständig gerecht. Erstens lag der Fokus bislang im Wesentlichen auf Organisationen, die mit ihren Merkma-

33 Darunter versteht Baecker (1999: 235) »einen Strukturwert, der unabhängig von der jeweils gewählten Ebene wieder erkennbar ist und daher als $>$ Fraktal $<$, das heißt als $>$ Selbstähnlichkeit<, verstanden werden kann«. 
len und Logiken im sowjetischen Kontext überdominant waren. Die Ebene der Funktionssysteme und die Auswirkungen der dargestellten Strukturen auf basale funktionssystemspezifische Kommunikationsformen blieben bis hierhin weitgehend ausgeklammert. Unbeantwortet ist damit die Frage, was das dichte Netz an politisch kontrollierten Organisationen für funktionale Differenzierung impliziert. Lässt sich der Befund einer umfassenden politischen Adressierbarkeit (und Kontrollierbarkeit) von faktisch jeder und jedem innerhalb organisierter Strukturen mit der Schlussfolgerung gleichsetzen, dass auch funktionssystemspezifische Kommunikationsformen politisch okkupiert wurden - wie es der Absicht der Einheitspartei entsprach?

Zweitens beschränkte sich die bisherige Darstellung weitgehend auf die Formalstrukturen der Organisationen. Im Fokus standen also diejenigen Verhaltenserwartungen, die Bestandteil der Mitgliedsrolle sind und die ein Mitglied folglich anerkennen muss, möchte es seine Mitgliedschaft nicht riskieren - $\gg[w] e r$ abweicht, muß damit rechnen allein zu bleiben« (Luhmann 1964: 69). Gleichzeitig stellt der Rückzug auf die Formalstrukturen für die Mitglieder eine Möglichkeit dar, sich organisationsintern der Kritik zu entziehen und unangreifbar zu machen (Holzer 2006: 269; Kühl 2007: 272). Um den Reproduktionsmodus einer Organisation nachzuvollziehen, ist die Formalstruktur insoweit relevant, als dass sie die »tägliche Produktion und Reproduktion [der Organisation, E.M.] in bestimmte Bahnen lenkt, bestimmte Kommunikationen entmutigt, andere ermutigt, bestimmte Kommunikationen rechenschaftspflichtig macht, andere nicht, und auf diese Weise konditioniert, was möglich ist « (Baecker 1999: 136). Jede Formalstruktur wäre jedoch überlastet, wollte sie sämtliche organisationsinternen Kommunikationen erfassen und regeln, denn $»[v]$ on all den faktischen Erwartungen, die ein soziales Handlungssystem orientieren und sinnhaft bestimmen, ist nur ein geringer Teil formalisiert « (Luhmann 1964: 268). Folglich ist es kaum denkbar, dass eine Organisation sich allein mittels ihrer Formalstrukturen reproduziert.

Die nicht-formalisierten Erwartungen können sowohl informaler als auch illegaler Art sein: Informale Erwartungszusammenhänge unterscheiden sich von der Formalstruktur, stehen jedoch nicht zwingend im Widerspruch zu ihr. Sie dienen dazu, die Formalstruktur auszufüllen, sie oftmals sogar zu stabilisieren und generell den Umgang mit formalen Vorgaben zu regeln. Informal wird beispielsweise festgelegt, in welchen Fällen ein Rückzug auf die formale Ordnung geboten respektive möglich ist und wann man sie ignorieren kann oder sogar muss. Während die formalen Rechte und Pflichten einer Mitgliedsrolle eindeutig bestimmt sind, ist weniger eindeutig - und gerade für neue Mitglieder und >Uneingeweihte< auch wesentlich schwieriger in Erfahrung zu bringen - was über die formale Rollenbeschreibung hinaus personenbezogen (und nicht rollenbezogen!) von einem Mitglied erwartet wird (Holzer 2006: 268f). Hinzu kommt, dass die Mitglieder bei informellen Handlungen nicht auf entsprechende formale Erwartungen, d.h. die Befolgung von 
Anweisungen, verweisen können und damit angreifbar werden (Kühl 2007: 273). Im Unterschied zu Informalität entbehrt illegale Kommunikation nicht nur der Absicherung durch die Formalstruktur der Organisation, sondern verletzt formale Erwartungen (Kühl 2007: 237; Luhmann 1964: 304). Während Informalität in Organisationen stillschweigend akzeptiert werden kann, erfordert Illegalität - sofern sie registriert wird - eine Reaktion (die auch in der Duldung bestehen kann, was aber selbst wiederum als illegal zu bewerten ist) (Kühl 2007: 273). Erst über das Zusammenspiel von formalen, informalen und gegebenenfalls auch illegalen Erwartungsstrukturen, so lässt sich diese kurze Skizze zusammenfassen, erschließt sich die spezifische Praxis einer Organisation (vgl. Luhmann 2000a: 24) - und dies galt ungeachtet des umfassenden Formalisierungs- und Kontrollanspruchs der politischen Führung auch für die sowjetische Organisationsgesellschaft.

Mit Blick auf diese beiden noch bestehenden Argumentationslücken lässt sich am Beispiel des sowjetischen Dorfes beobachten, auf welche Weise die politische Führung über die Formalstrukturen funktionssystemspezifische Kommunikation zwar beiseite drängte - also Entdifferenzierung betrieb. Gleichzeitig wird jedoch auch deutlich, auf welche Weise sich funktionssystemspezifische Kommunikationsformen gleichsam >unter der Hand <, in informalen und illegalen Nischen, durchsetzten und unter kontinuierlich mitlaufendem Verweis auf die Formalstrukturen reproduzierten. Die Beobachtung, dass Funktionssysteme im Sozialismus nicht vorkommen (Baecker 1998 [1997]-b: 128), ist zwar mit Blick auf die Formalstrukturen, die Außendarstellung und die Selbstbeschreibungen des sowjetischen Regimes plausibel. Sie lässt aber offen, ob und wie die informalen und illegalen Strukturen diese Fassade stützen oder hintergehen. Vor diesem Hintergrund zeigt das Beispiel des sowjetischen Dorfes und der sowjetischen Landwirtschaft nicht nur instruktiv, wie die skizzierten Organisationstypen real zusammenwirkten und gesellschaftliche Bezugsprobleme (hier vor allem Knappheit) bearbeiteten, sondern auch, auf welche Weise organisierte (d.h. politisierte) und unorganisierte (respektive unorganisierbare) Strukturen koexistierten und sich miteinander verzahnten. Die Möglichkeitsbedingungen für solche widersprüchlichen Strukturen wurden innerhalb des Regimes selbst geschaffen, indem die von der Partei dominierte politisch-administrative Hierarchie in den Dörfern unerwartet konterkariert wurde. Genau hier kommen die landwirtschaftlichen Kollektivbetriebe ins Spiel.

\section{Landwirtschaftliche Kollektivbetriebe als lokale Machtinstanz}

Die landwirtschaftlichen Kollektiv- und Staatsbetriebe (Kolchose und Sovchose) waren aus den Massenkollektivierungen der späten 1920er und frühen 1930er Jahre hervorgegangen - alternativ mit den Worten Verderys (2003: 40) formuliert »the greatest theft in history«. Die Kollektivierungen entsprachen der ideologischen For- 
derung nach Abschaffung allen Privateigentums an Produktionsmitteln und sollten den Bereich der Produktion in organisierte und kontrollierbare Strukturen überführen. Die Landwirtschaft, deren Kollektivierung auf dem 16. Parteitag der Kommunistischen Partei im November 1929 eingeleitet wurde, nahm dabei ideologisch eine hervorgehobene Stellung ein. Rückblickend heißt es dazu im dritten Parteiprogramm der KPdSU von 1961:

»In einem Land wie der UdSSR hing die Zukunft des Sozialismus wesentlich von der Lösung eines überaus schwierigen Problems ab: der Überführung der zersplitterten bäuerlichen Kleinbetriebe auf den Weg der sozialistischen Vergenossenschaftlichung. [...] Die Kollektivierung erlöste das Dorf für alle Zeiten von der Knechtung durch die Großbauern, der Klassendifferenzierung, dem Ruin und dem Elend.« (Zitiert nach Meissner 1965: 150f)

Die offizielle Begründung der Massenkollektivierung schloss zwei ideologische Dimensionen ein: Zum einen sollte die »Kulakengefahr « gebannt werden, die Stalin erstmals auf ebenjenem Parteikongress erwähnte und in diesem Zusammenhang den »dörflichen Klassenkampf« heraufbeschwor (Goehrke 2005: 31ff; Stökl 1997: 716ff). Als Kulaken (oder Großbauern) galten nach marxistischer Klassifikation jene bäuerlichen Betriebe, die nicht nur Familienmitglieder, sondern auch Außenstehende beschäftigten, und dadurch eine kapitalistische und regimefeindliche Produktionsweise verkörperten (Kornai 1992: 77). Zum anderen waren die Kollektivierungsmaßnahmen inhärenter Bestandteil des Projekts der Industrialisierung landwirtschaftlicher Produktion. Unter Verwendung der »Entkulakisierung « als Drohmittel (Goehrke 2005: 44) wurden kleine und offiziell als ineffizient bewertete Familienunternehmen, die im vorrevolutionären Russland die landwirtschaftliche Produktion prägten, ${ }^{34}$ mit ihrem Besitz in genossenschaftliche Großbetriebe überführt - »provided with machines, equipped with the data of science, and capable of producing the greatest quantity of grain for the market« (Scott 1998: 209). Der politische Führungsblick richtete sich dabei stets auch auf die (kapitalistische) Umwelt, von der das eigene >Reich< strukturell entkoppelt werden sollte: Die von den Kollektivierungsmaßnahmen erhofften Effizienzsteigerungen sollten nicht zuletzt auch die Überlegenheit einer sozialistischen Wirtschaftsweise beweisen:

»Warum sollte man annehmen, dass Stalin sich selbst nicht glaubte, als er im Winter 1929 programmatisch davon sprach, dass die Sowjetunion nach der Kollektivierung und Motorisie-

34 Wurden 1928, am Vorabend der Kollektivierung, noch 96 Prozent der landwirtschaftlich nutzbaren Fläche von Kleinbauern bearbeitet, war ihr Anteil im Jahr 1940, als faktisch alle ländlichen Haushalte >kollektiviert< waren, auf 4 Prozent gesunken (Altrichter/Haumann 1986: 532; Ioffe/Nefedova/Zaslavski 2006: 20). 
rung der Landwirtschaft binnen zwei, drei Jahren das getreidereichste Land der Welt werden würde, und auf dieser Basis dann auch zu einer militärisch-industriellen Weltmacht ersten Ranges?«(Koenen 2012: 85)

Doch die Ambitionen liefen schnell ins Leere: Die Hoffnungen auf (land-)wirtschaftliche Prosperität blieben unerfüllt und auch die Appelle an das Klassenbewusstsein der Landbevölkerung scheiterten am fehlenden Realitätsbezug. Der Dorfalltag hatte mit der propagandistisch beschworenen »Klassenkampfsituation« wenig zu tun (Stökl 1997: 717), so dass die Erwartung einer freiwilligen Kollektivierung >von unten< unerfüllt blieb. Die politische Führung ließ sich davon jedoch nicht irritieren und ging schließlich dazu über, die neuen Eigentums- und Produktionsformen gewaltsam umzusetzen. Vermeintliche Großbauern - und diese Bezeichnung wurde in der Praxis sehr flexibel verwendet, denn alle, die sich gegen das Regime stellten, liefen Gefahr, als »Kulak« denunziert zu werden (Applebaum 2003: 89) wurden enteignet, deportiert oder exekutiert (Kornai 1992: 77f; Stökl 1997: 718). Der Zwangsprozess der Massenkollektivierung führte in ein ökonomisches Desaster und eine Versorgungskrise mit dramatischen Auswirkungen: In Folge der geringen Getreideernte im Jahr 1932 und der daran anschließenden staatlichen Verteilungspolitik brach vor allem in der Ukraine und im Nordkaukasus eine Hungersnot aus, die circa sechs bis sieben Millionen Todesopfer forderte (Davies/Wheatcroft 2002; Mark/Simon 2004). Die Regierung reagierte auf die Getreideknappheit mit einer Verschärfung der Repressionen gegenüber der Landbevölkerung und vor allem gegenüber den damals noch existierenden Einzelbauern (Merl 1990: 223ff). ${ }^{35}$

Eine kontroverse wissenschaftliche Diskussion im Zusammenhang mit der Kollektivierung rankt sich um die Frage nach der Kontinuität oder dem Wandel der Eigentumsformen. Das Kontinuitätsargument vertritt beispielsweise aus historischer Perspektive Figes (1986) und verweist darauf, dass bereits in den Landkommunen vor 1917 kollektive Produktionsformen existiert hatten. Tatsächlich wurden im 19. Jahrhundert in zahlreichen Provinzen des russischen Reiches sogenannte Gemeinschaftsäcker (»obščestvennye zapaški«) betrieben, die zum Teil auf bäuerliche Traditionen zurückgingen und zum Teil per Dekret eingeführt worden waren. Im

35 In jüngerer Vergangenheit wird vor allem die Frage diskutiert, ob es sich beim sogenannten »holodomor« (wörtl.: Tötung durch Hunger) um eine >organisierte< Hungersnot gehalten habe, die von der politischen Führung als Substitut für die Deportation vermeintlicher Regimegegner bewusst herbeigeführt oder zumindest toleriert wurde (vgl. für diese Sichtweise z.B. Ellman 2005, 2007; Jahn 2004; Simon 2004; für die Gegenposition Davies/Wheatcroft 2002). Daran schließt sich die vor allem rechtlich und politisch bedeutsame Frage an, ob der »holodomor« als Genozid, also als gezielte Vernichtung des ukrainischen Volkes, anzuerkennen ist (vgl. z.B. Bilinsky 1999; Kul'čyc'kyj 2004). 
letzteren Fall dienten sie vor allem dem Ziel, die Zahlung kommunaler Steuern durch die Gemeinden sicherzustellen. In ähnlicher Weise berufen sich aus geographischer Sicht auch Ioffe und Nefedova auf die Kontinuität hierarchisch-patriarchalischer Strukturen und sehen »ein klares, auf Affinität beruhendes Nachfolgeverhältnis zwischen Kolchozen und traditionellen Bauernkommunen « (Ioffe/Nefedova 1997: 60; zitiert nach Lindner 2008: 32; vgl. auch Ioffe/Nefedova/Zaslavski 2006: 13). Dagegen lässt sich argumentieren, dass diese Formen kollektiver Produktion im Unterschied zu den sozialistischen Kollektivbetrieben die private Produktion der bäuerlichen Familienbetriebe nicht ersetzten, sondern sie lediglich ergänzten. Zwar wurden damit durchaus kollektive Elemente (wie das Bewusstsein gegenseitiger Verantwortung, die Notwendigkeit gerechter Arbeitsteilung, die Selbstwahrnehmung als Produktionsgemeinschaft und ähnliches) in die landwirtschaftliche Produktionstätigkeit eingeführt, sie dürften jedoch nicht den Rang eines grundlegenden Prinzips erlangt haben, so dass die Kollektivierungsmaßnahmen ungeachtet dessen als drastischer Umbruch wahrgenommen wurden (vgl. zu dieser Sichtweise Goehrke 2005: 29ff).

Die beiden Typen sowjetischer Landwirtschaftsbetriebe - Kolchose und Sovchose -, die sich letztlich etablierten, ${ }^{36}$ unterschieden sich primär durch die formalen Eigentumsverhältnisse und ihre Position in der wirtschaftlichen Planungshierarchie: Kollektivbetriebe (Kolchose) befanden sich offiziell als Zusammenschluss kleinbäuerlicher Betriebe im Eigentum ihrer Mitglieder; die trotz ihres ideologisch höheren Stellenwertes (Humphrey 1998: 74f; Lewytzkyi 1967: 119) zahlenmäßig geringer vertretenen Staatsbetriebe (Sovchose) waren staatliches Eigentum ${ }^{37}$ (Altrichter/Haumann 1986: 531; Schinke/Wädekin 1985: 36). Relevant für die weitere Argumentation ist allein der Betriebstyp der Kolchose.

Für die Kollektivbetriebe waren im sowjetischen Organisationsgefüge drei Zwecke vorgesehen, die formal im ersten Abschnitt des dritten Musterstatuts von 1969 umrissen wurden: Erstens sollten die Kolchose als Landwirtschaftsbetriebe

36 In der Anfangszeit der Kollektivierung hatte man mit verschiedenen anderen Betriebsformen experimentiert, darunter die Kommune, das »artel« oder das »TOZ« (»tovariščestvo po sovmestnoj obrabotke zemli«, wörtl.: Genossenschaft für die gemeinschaftliche Bearbeitung von Land), die sich jeweils durch unterschiedliche Vergesellschaftungsgrade von Boden und technischen Produktionsmitteln unterschieden (Hofmann 1983: 293).

37 Für die Praxis der landwirtschaftlichen Produktion war die Unterscheidung zwischen Staats- und Kollektiveigentum zu vernachlässigen. Ein bedeutsamer Unterschied bestand allerdings darin, dass Mitglieder von Kollektivbetrieben (im Unterschied zu Angestellten von Staatsbetrieben) die Möglichkeit hatten, neben ihrer Arbeit im Betrieb in begrenztem Umfang eigene Hoflandwirtschaften zu betreiben (zur Bedeutung dieser faktisch privaten Produktionsform im Schatten der Kollektivbetriebe siehe S. 90ff). 
vor allem durch die Steigerung der Arbeitsproduktivität und Produktionseffizienz die »gesellschaftliche Wirtschaft nach Kräften [.] festigen « und die Produktion von landwirtschaftlichen Erzeugnissen und deren Verkauf an den Staat vorantreiben. Zweitens sollten sie an der Erziehung der Kolchosmitglieder im Sinne des kommunistischen Ideals mitwirken, galt doch der Kolchos als »Schule des Kommunismus für die Bauernschaft« (Lindner 2008: 75f). Die Funktion der Kollektivbetriebe entsprach in dieser zweiten Hinsicht der bereits erwähnten Transmissionsfunktion der Massenorganisationen, die über die umfassende Inklusion der ländlichen Bevölkerung eine ideologische Brücke zu Letzterer schlagen sollten (Meissner 1985: 65): $»[\mathrm{~T}]$ he $>$ dark $<$ narod, the peasants who were perhaps the great remaining, intractable threat to the Bolshevik state, were to be replaced by rational, industrious, de-Christianized, progressive-thinking kolkhoz workers « (Scott 1998: 211). Drittens sollten die Kollektivbetriebe »die Dörfer allmählich in moderne, mit allen sozialen Erfordernissen ausgestaltete Siedlungen [.] verwandeln « und dabei die materiellen und kulturellen Bedürfnisse der Kolchosmitglieder befriedigen (Drittes Musterstatut, Art. 2, zitiert nach Brunner/Westen 1970: 148). Die Bereitstellung kommunaler Dienstleistungen und lokaler Infrastruktur war somit formal nicht allein die Aufgabe der Lokalverwaltung. Auch die Betriebe hatten sich zu beteiligen, solange die entsprechenden Tätigkeiten zweckgebunden im Hinblick auf die Ziele der Genossenschaft erfolgten. Im dritten Musterstatut heißt es dazu:

\section{»Zu diesem Zweck}

- baut der Kolchos Klubs, Bibliotheken und andere Kultur- und Bildungsstätten sowie Sportanlagen und [...] Kinderbetreuungsstätten;

- wirkt der Kolchos mit Elternhaus und Schule bei der richtigen Erziehung der Kinder zusammen, unterhält er enge Verbindung zur Schule [...];

- organisiert der Kolchos im Bedarfsfall Gemeinschaftsverpflegung [...];

- erweist er den Organen des Gesundheitsweisens Hilfe bei ärztlichen und vorbeugenden Maßnahmen im Kolchos, stellt er Kolchosmitgliedern unentgeltlich und vordringlich Transportmittel zur Einlieferung von Kranken in medizinische Einrichtungen zur Verfügung;

- sorgt der Kolchos für die siedlungstechnische Ausgestaltung der Kolchosortschaften, für den Anschluß der Ortschaften und der Häuser der Kolchosmitglieder an das Stromnetz [...], fördert er die Organisierung von Dienstleistungen für die Kolchosmitglieder und erweist ihnen nach den im Kolchos geltenden Bestimmungen Hilfe beim Bau und bei der Reparatur von Wohnhäusern [...].

- Der Kolchos sorgt für die verbesserte berufliche Fortbildung sowie für die Hebung des kulturellen und technischen Niveaus der Kolchosmitglieder.«

(Drittes Musterstatut, Art. 41, zitiert nach Brunner/Westen 1970: 158) 
Zwar spricht die rechtlich-formale und territoriale Kongruenz ihrer Zuständigkeitsbereiche für ein gemeinsames Interesse von Kollektivbetrieben und Gemeindeverwaltungen (Lindner 2008: 82). Doch was auf eine (gleichberechtigte) Kooperation beider Institutionen schließen lassen könnte, entpuppte sich in der Praxis als zunehmende funktionale Abhängigkeit der Gemeindeverwaltungen von den Kollektivbetrieben (während der Massenkollektivierung, mit deren Umsetzung sie betraut waren, hatten erstere zumindest ansatzweise noch eine bedeutende Rolle gespielt, vgl. Wädekin 1969: 301). Zwar handelte es sich bei dem im Musterstatut genannten Aufgabenkatalog für den Bereich lokaler Infrastruktur weniger um verbindliche Vorgaben (wie sie beispielsweise die Wirtschaftspläne enthielten), sondern eher um eine Orientierung (Lindner 2008: 81). Nichtsdestotrotz erfüllten die Kollektivbetriebe in der Regel aber ein breites Spektrum infrastruktureller Aufgaben: Sie errichteten Kindergärten, Schulen und Bibliotheken, kümmerten sich um die Instandhaltung der Dorfwege, unterhielten Einrichtungen zur medizinischen Versorgung und ähnliches (vgl. dazu z.B. Lerman 2002: 43f; Ross 2009: 33), wozu die personellen und finanziellen Ressourcen der Gemeindeverwaltung nicht reichten. Zwar wurde die betriebliche Abhängigkeit von der staatlichen Zuteilungspolitik durchaus registriert, doch der Kolchos galt im Dorf dennoch als umfassende Organisation, als Garant für eine funktionierende Infrastruktur und letztlich auch als Vermittler staatlicher Fürsorge, der als solcher durchaus ein Gefühl der Sicherheit vermittelte - wie sich auch Gemeindemitglieder aus meinem Untersuchungsgebiet erinnern:

»Es gab eine Periode, in der der Kolchos viel gebaut hat [...], viele Gebäude im sozial-kulturellen Bereich wurden errichtet und damals wurde auch für die Wasserleitung Geld gegeben und für Wohnraum, den Kindergarten, die Schule - all das hat der Kolchos gebaut. Staatliche Mittel wurden dafür zugeteilt. [...] In der sowjetischen Zeit gab es mehr Geld als heute - aber dass man selbst etwas gebaut hat, das war nicht notwendig. Heute gibt es keine großen Bauaktivitäten mehr, aber wenn vereinzelt doch was gebaut wird, dann wird das mit eigenem Geld finanziert.«(Bürgermeisterin, 8.8.2008) ${ }^{38}$

»Wir [Kolchosarbeiter, E.M.] haben 16 Häuser im Jahr gebaut. Alles hat der Kolchos gebaut. [...] Der einzelne Mensch hat damals den Einfluss des Staates auf sich viel stärker gespürt. Und nicht nur wie heute, wenn jemand 200 Rubel Sozialhilfe bekommt, die sowieso unsichtbar sind - damals war das ganz konkret: einem wurde eine Wohnung gebaut. Das Kulturhaus, dort der Kindergarten - das alles hat der Kolchos von seinem eigenen Geld gebaut. Natürlich hat ihm der Staat geholfen, Hilfe vom Chef sozusagen. Aber heute gibt es keine Hilfe vom

38 Der Verweis auf eigene Interviews erfolgt mit der (in der Regel beruflichen) Tätigkeit der oder des Befragten und dem Datum des Interviews. Erläuterungen zum Datenmaterial und zu den Erhebungs- und Auswertungsmethoden folgen auf Seite 165ff. 
Chef, keine Hilfe vom Staat - es gibt überhaupt keine Hilfe mehr.« (Gemeinderat und ehemaliger Kolchosangestellter, 13.8.2008)

Die entsprechenden Entscheidungen in diesen Angelegenheiten traf im sowjetischen Dorf die Kolchosleitung. Die Gemeindeverwaltung war bestenfalls noch ausführendes Organ mit mehr Pflichten als Rechten. Daneben war es üblich, die Bürgermeisterinnen und Bürgermeister respektive die Vorsitzenden der dörflichen Sowjets (»sel'sovet«) in die Kolchosleitung aufzunehmen, allerdings dort in untergeordneter Position gegenüber den Kolchosvorsitzenden. Oftmals ergänzten die Kollektivbetriebe die örtliche Verwaltung damit nicht nur, sondern verschmolzen mit ihr in funktionaler wie in personeller Hinsicht oder ersetzten sie vollständig (Wädekin 1969: 300ff; Lindner 2008: 80ff).

Der Bedeutungsverlust der Kommunen und insbesondere der örtlichen Sowjets, deren Entscheidungs- und Handlungsmöglichkeiten ohnedies bereits minimal und deren politisches Prestige denkbar gering war, wurde durch diese Konstellation zusätzlich verstärkt (Wädekin 1969: 301): »Der Vorsitz im Dorfsowjet wurde oft zu einem Abstellgleis, einer kleinen Sinekure für unfähige oder kompromittierte Funktionäre, die, da einmal in der >Nomenklatur< stehend, irgendeinen Posten erhalten sollten« (Wädekin 1969: 303). Dass die lokalen Verwaltungsorgane vor diesem Hintergrund weder ein machtvolles Gegengewicht zur Leitung der Großbetriebe bilden konnten (Ross 2009: 32) noch ihre Kontrollfunktion gegenüber den Großbetrieben effektiv wahrnahmen (Wädekin 1969: 191), erscheint kaum noch erwähnenswert.

Kolchosintern gegenüber den Mitgliedern und damit faktisch gegenüber allen Dorfbewohnerinnen und -bewohnern verfügte die Betriebsleitung ohnedies über fast uneingeschränkte Macht. Betriebsinterne Mitbestimmungsrechte als Teil der offiziell proklamierten »Kolchosdemokratie« - laut den Vorgaben des dritten Musterstatuts erfolgt die Leitung des Kolchos »auf der Grundlage einer breiten Demokratie und der aktiven Teilnahme der Kolchosmitglieder an der Lösung aller Fragen des Kolchoslebens« (Drittes Musterstatut, Art. 45, zitiert nach Brunner/Westen 1970: 160) - entpuppen sich bei genauerem Hinsehen schnell als Farce: Zwar war formal die Vollversammlung das höchste Leitungsorgan, die die Kolchosleitung wählte, welche wiederum den Kolchosvorsitzenden bestimmte (Humphrey 1998: 103). Die Wahlen standen jedoch unter dem faktischen Einfluss der Parteikomitees der Bezirksebene (Wädekin 1969: 179), für den Vorsitz kandidierte häufig nur eine einzige Person und die öffentliche Wahl per Handzeichen machte »abweichendes Verhalten« unwahrscheinlich (Humphrey 1998: 331). Im Zweifelsfall konnte die Betriebsleitung zudem einzelne Personen von der Teilnahme an der Vollversammlung ausschließen (Humphrey 1998: 104). Wurden trotz dieser Gleichschaltungs- 
mechanismen Vorsitzende erfolgreich abgelehnt, war dahinter das stillschweigende Plazet der Bezirksverwaltung zu vermuten (Wädekin 1969: 180).

Ohnedies waren die Kompetenzen der Kolchosleitung gegenüber der Vollversammlung im Musterstatut nur sehr ungenau beschrieben, wodurch sich Auslegungsspielräume eröffneten, die im Zweifel dazu genutzt wurden, die dominante Position der oder des Vorsitzenden zusätzlich zu stärken (Wädekin 1969: 188ff):

»Wenn aus dem Musterstatut beispielsweise nicht eindeutig hervorging, für welche Fragen die Vollversammlung zuständig war, dann war ebenso wenig klar, was der Vorsitzende des Kolchoz alleine entscheiden durfte und was nicht. Derartige Unbestimmtheiten waren ganz offensichtlich beabsichtigt und öffneten willkürlichen Entscheidungen beziehungsweise einer starken Machtkonzentration in den Händen des Vorsitzenden Tür und Tor.« (Lindner 2008: 78)

Die einzige annähernd unabhängige kolchosinterne Institution war die Revisionskommission, die ebenfalls von der Vollversammlung bestimmt wurde und deren Aufgabe in der Überprüfung der Wirtschafts- und Finanztätigkeit der Betriebsleitung bestand (Humphrey 1998: 104; Lindner 2008: 77), ihre faktische Bedeutung als Kontrollorgan war über finanzielle und wirtschaftliche Fragen hinaus jedoch zu vernachlässigen (Wädekin 1969: 191f). Wie bereits im Fall der Lokalverwaltung beruhte der Einfluss der Partei auf die Kollektivbetriebe weniger auf formalen Entscheidungskompetenzen im Hinblick auf die Belange des Unternehmens, sondern ergab sich in der Sozialdimension über das Personal. Über 90 Prozent aller Kolchosvorsitzenden waren Parteimitglieder, was sie faktisch unangreifbar machte (Lindner 2008: 79; Wädekin 1969: 192).

Die Machtposition der Kolchosleitung im Dorf, so lässt sich zusammenfassen, speiste sich aus zwei Quellen: Über die organisationsinternen Strukturen waren die Kolchosmitglieder - und damit die überwiegende Mehrheit der Dorfbewohnerinnen und Dorfbewohner - der Kolchosleitung untergeordnet und verfügten über keine nennenswerten Mitbestimmungsmöglichkeiten; organisationsextern resultierte die dominante Position der Kolchosleitung gegenüber der formalen Autorität des »sel'sovet « (der Gemeindeverwaltung) aus der umfassenden und für das Dorf alternativlosen Funktion des Kollektivbetriebs im Bereich lokaler Infrastruktur. Der Kollektivbetrieb als »Massenorganisation der Bauernschaft« (Meissner 1985: 65) war auf diese Weise im Dorfkontext kaum zu umgehen und in praktisch allen Bereichen des alltäglichen Lebens handlungs- und entscheidungsrelevant. Die Kolchosleitung nahm eine Doppelrolle »sowohl als Organisator der Produktion als auch als Führer der Gesellschaft« ein (Janov zitiert nach Wädekin 1969: 198), in deren Händen mehr oder weniger sämtliche lokalen Fäden zusammenliefen: »[C]ollective farm administration does not discriminate between economic, politi- 
cal, and legal power. Any official holds all these powers in some degree« (Humphrey 1998: 108; vgl. auch Ross 2009: 33).

Zwar befand sich die Kolchosleitung selbst innerhalb der Hierarchie der Organisationsgesellschaft in untergeordneter Stellung, im Dorf war jedoch ungeachtet dessen der direkte Einfluss der Partei zweitrangig, zumal das zuständige Parteikomitee in der Regel nicht im Dorf selbst, sondern auf höheren Verwaltungsebenen verankert war (Merl 1985: 98). Die resultierenden Machtverhältnisse beschreibt Wädekin als Kombination von »Ohnmacht nach oben« (1969: 178) und »Machtfülle nach unten« (1969: 188). Humphrey (1998: 328) betont mit Blick die Inversion der dörflichen Machtstrukturen im Vergleich mit der nächsthöheren Ebene des Bezirks: »Therefore we can summarise the relations of power at the level of the district as a whole as: Party $\rightarrow$ enterprise $\rightarrow$ Soviets, while at the locality the pattern is more likely to be: enterprise $\rightarrow$ Party $\rightarrow$ sel'sovet «. In identischer Weise berichtet eine Bürgermeisterin aus meinem Forschungsgebiet über die sowjetische Vergangenheit:

»Zu sowjetischer Zeit hatte der Vorsitzende des Sel'sovet [der Gemeindeverwaltung, E.M.] überhaupt keine Macht. Er war formal zwar eine Autorität, aber er hatte keine Macht. Der Vorsitzende des Kolchos war die einflussreichste Instanz auf dem Territorium. Nach ihm kam der Sekretär der lokalen Parteiorganisation.« (Bürgermeisterin, 8.8.2008)

Zusammengenommen lässt sich diese Situation des sowjetischen Dorfes als eine (begrenzte) Entkopplung innerhalb der politisch-administrativen Hierarchie beschreiben. Sie ist als Beispiel dafür zu lesen, wie sich innerhalb der Formalstrukturen des Regimes Spielräume für widersprüchliche Kommunikationsformen eröffneten. Um diese sichtbar zu machen, werfe ich im Folgenden zunächst einen Blick auf die andere Seite der Unterscheidung, die dieser Beobachtung zugrunde liegt: auf die nicht-widersprüchlichen, vom Regime vorgesehenen organisierten Strukturen landwirtschaftlicher Produktion. Mit Blick auf die wirtschaftliche Funktion der Kollektivbetriebe lässt sich dabei illustrieren, auf welche Weise die politische Führung über das sozialistische Programm nicht nur auf die Inhaltsebene wirtschaftlicher Kommunikation zugriff, indem die Art und der Umfang der Produktion über verbindliche Planziele vorgegeben wurden, sondern darüber hinaus auch die Codeebene okkupierte und den Gehalt von Zahlungen unterminierte.

\section{Hierarchisierte Knappheitskommunikation}

Ungeachtet variierender Bezeichnungen als Planwirtschaft, Zentralverwaltungswirtschaft oder Kommandowirtschaft stimmen Beschreibungen der sowjetischen Wirtschaftsordnung darin überein, dass der Versuch zentralistischer Planung sämtlicher 
ökonomischer Aktivitäten als ihr Herzstück betrachtet wird. Aus systemtheoretischer Perspektive ist Planen die »Festlegung von Entscheidungsprämissen für künftige Entscheidungen, oder kürzer formuliert: Planen heißt über Entscheidungen entscheiden« (Luhmann 2007 [1971]: 67) - und dies im Hinblick auf ein bestimmtes Ziel. Dabei werden künftige Entscheidungen nicht vorweggenommen, denn in der Planung geht es um die »Definition eines Entscheidungsproblems «, nicht »um die Festlegung der Bedingungen seiner Lösung« (Luhmann 2007 [1971]: 68). Ein Planungsprozess ist ohne Entscheidungen nicht denkbar und als planende Sozialsysteme kommen somit allein Organisationen in Frage. Für die Planung wirtschaftlicher Kommunikation wurde die Formalstruktur des sowjetischen Wirtschaftssystems als strikte Hierarchie eingerichtet (respektive einzurichten versucht), indem System-Umwelt-Grenzen durch die Bildung von Subsystemen in das System hineingeholt wurden (Luhmann 1964: 307). Suborganisationen waren Betriebe, Behörden und Parteikomitees, die sowohl intern als auch im Verhältnis zueinander hierarchisch geordnet wurden: Übergeordnete Ebenen gaben unteren Ebenen die Entscheidungsprämissen vor und untergeordnete Ebenen waren nach oben rechenschaftspflichtig. Wiederum nach Art der Fraktale spiegelte sich auf diese Weise die (Formal-)Struktur des Gesamtsystems in seinen Teilen und sollte mit Blick auf den Planungsprozess maximale Kontrollierbarkeit aller Ebenen durch die politische Führung sicherstellen.

Oberstes Planungsorgan war die zentrale Planungsbehörde »Gosplan«. Sie arbeitete unter der Kontrolle und mit Zustimmung der Partei (respektive des Zentralkomitees) und weiterer staatlicher Institutionen Wirtschaftspläne für Zeiträume von einem und fünf Jahren aus. Während die Fünfjahrespläne generelle wirtschaftspolitische Zielsetzungen absteckten und als Entscheidungsprämissen im Produktionsprozess von nachrangiger Bedeutung waren (Kornai 1992: 111), enthielten die Einjahrespläne detaillierte Vorgaben über Produktionsmengen, -qualitäten und -zusammensetzungen sowie den Einsatz von Arbeitskräften, ${ }^{39}$ regelten die Abwicklung des Verkaufs an den Staat sowie die Verwendung der Überschussproduktion ${ }^{40}$ (Humphrey 1998: 95). Die Planvorgaben verstanden sich als Anweisungen - »Our plans are not forecast-plans, not guess-work plans, but directive plans« (Stalin zitiert nach Kornai 1992: 113) -, die entlang von Weisungsketten von der Hierar-

39 Die Einjahrespläne wiesen folgende einheitliche Struktur auf: (1) Produktion; (2) Verwendung der Produktion; (3) Arbeit; (4) Investitionen; (5) Ziele für die technische Entwicklung; (6) Außenhandel und internationale Wirtschaftsbeziehungen; (7) Finanzen (Kornai 1992: 111ff).

40 Landwirtschaftliche Kollektivbetriebe waren beispielsweise verpflichtet, mindestens 35 Prozent der Überschussproduktion an Getreide und rund 10 Prozent der Überschussproduktion an Vieh an den Staat zu verkaufen (Humphrey 1998: 95). 
chiespitze nach unten weitergegeben und auf diese Weise sukzessive disaggregiert wurden. Ausgerichtet an den Jahresplänen wurden betriebsintern Vierteljahres- und Monatspläne erstellt und tägliche Planziele für jede Abteilung und sogar für einzelne Arbeiterinnen und Arbeiter und Angestellte abgeleitet. Die Disaggregation der Planziele bewirkte in erster Linie eine Bindung der umfangreichen und detaillierten Planvorgaben an identifizierbare Adressen innerhalb des Organisationengefüges. Wurde ein (Teil-)Ziel nicht erfüllt oder kam es zu Verzögerungen, konnte dies unmittelbar der entsprechenden Stelle innerhalb der Hierarchie zugerechnet und die Stelleninhaberin oder der Stelleninhaber zur Verantwortung gezogen werden: »Each inanimate figure in the plan is matched by a live bureaucratic institution with a responsible (and accountable) head « (Kornai 1992: 113). Die Freiheitsgrade der ausführenden Suborganisationen wurden auf diese Weise auf ein Minimum beschränkt und die größtmögliche Sichtbarkeit von Abweichungen hergestellt.

Die Kollektivierung von Eigentum an Land, Vieh und technischen Produktionsmitteln lässt sich vor diesem Hintergrund als eine wesentliche Voraussetzung für die Organisation und Hierarchisierung der landwirtschaftlichen Produktion beobachten. Scott (1998: Kap. 6) beschreibt den Kollektivierungsprozess als Großprojekt mit dem Ziel, den ungeordneten und aus Sicht der politischen Führung »unleserlichen« (»illegible«) ländlichen Raum des vorrevolutionären Russlands »lesbar« und damit kontrollierbar zu machen (eine ähnliche Sichtweise findet sich u.a. auch bei Kornai 1992: 82; Stökl 1997: 713). Kolchose und Sovchose waren in diesem Sinne Instrumente zur (vermeintlichen) Komplexitätsreduktion, indem die dezentrale und unübersichtliche kleinbetriebliche Produktion durch zentral gelenkte Großbetriebe ersetzt wurde: »In place, therefore, of an agriculture in which planting, harvesting, and marketing decisions were in the hands of individual households, the party-state had built a rural economy where all these decisions would be made centrally« (Scott 1998: 214). Es entstanden beobachtbare und gezielt adressierbare Einheiten, die sich im Unterschied zur Vielzahl der Familienbetriebe als Suborganisationen der Wirtschaft einrichten ließen. Als solche wurde ihnen zwar ein gewisser Handlungsspielraum eingeräumt, jedoch gleichzeitig ein fester Platz in der Hierarchie zugewiesen: Die staatlichen Sovchose unterstanden dem Landwirtschaftsministerium; die Kolchose galten formal als eigenständige Genossenschaften und waren als solche den Parteikomitees auf der Bezirksebene (»rajon«) unterstellt. Die Kolchose waren damit zwar in die Planungshierarchie eingebunden und hatten vorgegebene Produktionsziele zu erfüllen, für betriebsinterne Entscheidungen konnten jedoch seitens der Politik keine direkten Anweisungen ausgesprochen werden, wohl aber Empfehlungen (Wädekin 1969: 156ff).

Ungeachtet dieser begrenzten Entscheidungsfreiheit waren die Kolchose ebenso wie andere sowjetische Betriebe hinsichtlich ihrer Reproduktionsfähigkeit fast vollständig von den übergeordneten Hierarchieebenen abhängig, die über Planvorgaben 
und die Zuteilung von Produktionsfaktoren und damit buchstäblich über »Leben oder Tod « eines Betriebs entschieden (Kornai 1992: 115). Entscheidungsgrundlage waren meist - aus Betriebssicht - undurchschaubare Kriterien: »Firms with losses were about as likely to obtain resources as firms with profits« (Olson 2000: 147) und unrentable Unternehmen galten häufig sogar als erfolgreich (Birman 1978: 159). Aus wirtschaftswissenschaftlicher Perspektive werden diese Praktiken in Kontrastierung mit der Logik des Marktes meist als > weiche< Budgetbeschränkungen beschrieben, die weitgehend profitunabhängigen, politischen oder schlicht willkürlichen Kriterien unterlagen (Kornai 1986). Alle ökonomischen Parameter wurden auf diese Weise vertikal, d.h. mit höheren Hierarchieebenen, verhandelbar ${ }^{41}$ (Kornai 2000: 30, 1992: 140ff). Die Unterstützung wirtschaftlich schwacher Betriebe durch eine entsprechende Investitionspolitik, so stellt Wegren (1998: 25) mit Blick auf die Landwirtschaft fest, wurde zum »unique feature of soviet-style agriculture«. Zum wesentlichen Parameter für Erfolg oder Misserfolg eines Unternehmens entwickelten sich vor diesem Hintergrund die Position und der Einfluss der Betriebsleitung in der Planungshierarchie, »ihre Beziehungen nach >oben«« (Wädekin 1969: 181; auch Humphrey 1998: 94ff): »Ultimately, [...] the income of an enterprise is not given by its contribution to the achievement of the Politburo's objectives or by the revenue from its sales but by its political administrative power « (Olson 2000: 147). Ohnedies waren die Anreize Profit zu erzielen für die Unternehmen denkbar gering: Kapitalbesitz war ihnen nur in sehr geringem Umfang gestattet und Gewinne waren ab einem bestimmten Schwellenwert zu verstaatlichen. Zur freien Verwendung für kleinere Investitionen blieben lediglich >informelle< Gewinne, die an den Kontrollbehörden vorbeigeschleust wurden (Berliner 1952: 351f). Die Reproduktionsfähigkeit eines Betriebs wurde auf diese Weise effektiv von seiner Zahlungsfähigkeit entkoppelt. ${ }^{42}$

Ein bedeutendes Instrument, um im Rahmen dieses Entkopplungsprozesses konkrete unternehmensinterne Entscheidungen auf die politischen Vorgaben auszurichten und mit dem Planungsprozess gleichzuschalten, waren Prämien als leis-

41 Kornai (1992: 141f) nennt beispielsweise >weiche< Subventionen, Besteuerung, Kreditvergabe oder Preissetzung.

42 In ähnlicher Weise verwies das Geldvermögen eines Haushaltes nur vage auf den Zugang zu Konsumgütern und die Möglichkeit der Bearbeitung individueller Knappheiten. Die Ursache hierfür war in erster Linie die chronische Mangelsituation als Konsequenz der umfassenden Organisation des Wirtschaftssystems. An die Stelle generalisierter Kauferwartungen (vgl. dazu Kasuga 1987: 552) traten Zugangsrechte (als Nebeneffekt von Mitgliedschaft) und personalisierte Netzwerke. 
tungsabhängige, monetäre oder nicht-monetäre ${ }^{43}$ Ergänzungen zum Arbeitslohn: »The salary is paid for doing the job, the premium for doing it well« (Berliner 1957: 46). Über die Prämienvergabe entschieden teils die Ministerien, teils aber auch die Betriebsleitungen selbst. Betriebsintern hatte dies den Effekt, dass sich bereits in den Anfangsjahren der Sowjetunion sämtliche Entscheidungen über alle Ebenen hinweg maßgeblich an den Prämien orientierten und diese letztlich zu einem zentralen Fluchtpunkt unternehmensinterner Kommunikation avancierten: »Since the striving for premia is uppermost in the minds of management, and since premia are paid according to certain formalized criteria, these criteria become effective determinants of managerial decisions « (Berliner 1952: 349). Die (zumindest vordergründige) Planerfüllung, an die die Prämien direkt oder indirekt gekoppelt waren, wurde so zum obersten Organisationsziel, auf das sich ein ganzes Spektrum informaler Managementprinzipien ausrichtete. Diese hatten zwar allesamt positive Effekte auf die Reproduktionsfähigkeit der Betriebe innerhalb der Planungshierarchie und vor allem auf die individuelle wirtschaftliche Lage der Mitglieder der Betriebsleitungen, wirkten sich jedoch tendenziell negativ auf die Wirtschaftsleistung (gemessen an Indikatoren wie Produktionsmenge, Qualität und Bedarfsorientierung) aus:

- Aushandlung günstiger Planvorgaben: Um für den Betrieb möglichst günstige Ausgangsbedingungen zu schaffen, wurde der Planungsprozess durch die Aushandlung > weicher <, also geringer und leicht zu erreichender Planziele und durch die Übertreibung des eigenen Bedarfs an Produktionsfaktoren $\mathrm{zu}$ beeinflussen versucht (Berliner 1952: 353ff; für das Beispiel landwirtschaftlicher Kollektivbetriebe Wädekin 1969: 177f).

- Horten von Ressourcen: Das Aufsparen oder Horten zugeteilter Ressourcen verschaffte den Betrieben immerhin ein begrenztes Maß an Flexibilität und Entscheidungsspielraum, da auf diese Weise eine Reserve an Produktionsmitteln verfügbar war, die sie nach eigenem Ermessen einsetzen konnten. Zudem bildeten diese Vorräte die Grundlage für ein netzwerkförmiges Tauschsystem zwischen den Betrieben (Berliner 1957: 182ff; Verdery 1996: 20ff), das sich in einer Grauzone zwischen Informalität und Illegalität auf sämtliche Arten reziproker >Gefälligkeiten< zur Aufrechterhaltung des Produktionsprozesses bezog und unter der Bezeichnung »blat « bekannt war. ${ }^{44}$ Gegenstand von »blat « waren neben dem direkten Tausch von Inputfaktoren zwischen Betrieben auch die bevorzugte Be-

43 Nicht-monetäre Prämien waren beispielsweise zusätzliche Urlaubstage, der Aufenthalt in einem Ferienlager und dergleichen (Berliner 1957: 27ff).

44 Für eine umfassende Darstellung von »blat« als netzwerkförmige Struktur informalen Austauschs im sowjetischen und postsowjetischen Kontext vgl. Ledeneva (1998, 1999). 
handlung auf Wartelisten für bestimmte Lieferungen oder generell die Umgehung bürokratischer Hindernisse im Produktionsprozess.

- Simulierte Planerfüllung: Neben Versuchen, die tatsächliche Produktionsleistung im Hinblick auf die Erfüllung der Planvorgaben zu beeinflussen, kam eine Reihe von Täuschungsmanövern zum Einsatz, um die Planerfüllung zu simulieren und die eigene Leistung in bestmöglichem Licht darzustellen (Berliner 1952: 355ff): Die Outputqualität wurde zu Gunsten einer größeren Produktionsmenge herabgesetzt, gegenüber den Behörden wurden falsche Angaben über die im Produktionsprozess befindlichen - und damit kaum kontrollierbaren - Mengen gemacht, finanzielle Mittel wurden zweckentfremdet, fiktive und tatsächliche Kosten bei der Berechnung der planungsrelevanten Selbstkosten eines Produkts mit Blick auf das strategisch erwünschte Resultat beliebig umgeschichtet (für Letzteres vgl. Kitching 2001) ${ }^{45}$ oder die Zusammensetzung der Produktpalette eigenmächtig geändert »by shifting factors of production away from the less advantageous planned products into the overfulfillment of the targets for the more advantageous products. Thus the firm simulates plan overfulfillment by producing an unplanned product-mix« (Berliner 1952: 355f).

An die Stelle von Wirtschaft, so lässt sich resümieren, trat eine (politisch kontrollierte) Hierarchie, innerhalb derer sich die Betriebe primär an Macht und nicht an Zahlungen (in Form von Profit und Zahlungsfähigkeit) orientierten. Primärorientierung heißt, dass die Organisation ihren Zweck auf die Funktion eines Funktionssystems ausrichtet und ihre Entscheidungskriterien ebenso wie ihre Kriterien »der Hoffnungslosigkeit des Weitermachens « (Luhmann 2009: 471) aus diesem Funktionssystem als relevanter (jedoch nicht einzig relevanter!) Umwelt bezieht. ${ }^{46}$ Richtet sich die Primärorientierung einer Organisation auf die Wirtschaft (wie es bei Unternehmen üblicherweise der Fall ist), ist die Stoppregel verhältnismäßig simpel und

45 Kitching (2001) bezieht sich in seiner Studie auf das Beispiel landwirtschaftlicher Kollektivbetriebe, die gegenüber den Planungsbehörden im jährlichen Rechenschaftsbericht für jedes Produkt die Selbstkosten anzugeben hatten.

46 Instruktiv sind in diesem Zusammenhang die Überlegungen von Tacke (2001) zum Verhältnis von Organisationen und Funktionssystemen. Die Zuordnung von Organisationen zu Funktionssystemen sei, so Tacke, eine typologische Simplifikation. Dies ist solange unproblematisch, wie die empirische Zuordnung der Organisation in der Gesellschaft eindeutig und unbestritten ist (was im hier dargestellten Beispiel der Fall ist). Sobald sich hinsichtlich der Zuordnung jedoch empirische oder theoretische Inkonsistenzen ergeben, indem beispielsweise Selbst- und Fremdzuordnung einer Organisation auseinanderfallen, ist eine Auflösung dieser Simplifikation angebracht (Tacke 2001: 148). Letzteres wird im Kontext postsowjetischer Transformation relevant. 
verhindert ein zu starkes Abweichen von der Orientierung am Knappheitsproblem: ${ }^{47}$ Die Organisation gilt als gescheitert, wenn sie (dauerhaft) zahlungsunfähig ist (Luhmann 2009: 263f). Wohlgemerkt impliziert diese wirtschaftliche Stoppregel nicht, dass sämtliche organisationsinternen Entscheidungen durchweg ökonomisch rational sind. Aber um Profit oder zumindest die glaubwürdige Kommunikation von Gewinnerwartungen kommt ein Unternehmen im Interesse der eigenen Existenz zumindest langfristig nicht herum. Gleichzeitig verweist der Gewinn von Wirtschaftsorganisationen stets auf die Wirtschaft selbst, während über Löhne und Steuern Geld an die Umwelt der Wirtschaftsorganisation und an nicht-wirtschaftliche Teilsysteme überführt wird - das allerdings indirekt auch wieder an die Wirtschaft gebunden ist, indem es hier letztlich seine Verwendung findet (Luhmann 2009: 466ff).

Zwar wurden auch in sowjetischen Betrieben Gewinne und Verluste berechnet, die Resultate blieben jedoch weitgehend folgenlos für das Weitermachen der Organisation. Und auch in sowjetischen Betrieben wurden Entscheidungen über die Gestaltung des Produktionsprozesses getroffen, die Entscheidungskriterien ergaben sich jedoch aus den Planzielen, die unabhängig von ihrer ökonomischen Rationalität, d.h. ihrem Beitrag zur Bearbeitung des Knappheitsproblems, zum Selbstzweck wurden (Birman 1978: 158). Gewinnorientierung oder der Blick auf die (Wieder-)Herstellung der betrieblichen Zahlungsfähigkeit gewannen bestenfalls Bedeutung, wenn der Profit in Zusammenhang mit der Planerfüllung und Prämienzahlung stand, wie Berliner (1952: 350) die Beschreibungen seiner Interviewpartner zusammenfasst: ${ }^{48} \gg$ Profits were desirable $>$ if the [output, E.M.] plan was fulfilled $<$, and then the informant proceeded to list not the direct benefits of profit plan fulfillment, but the direct benefits of output plan fulfillment «. Zwar mochte sich die politische Leistungsbewertung in Einzelfällen mit ökonomischen Kriterien überschneiden (beispielsweise wenn Prämien für die Entwicklung von Maßnahmen zur Optimierung des Produktionsprozesses, für Kosteneinsparungen oder für technische

47 Im Gegensatz dazu geben andere Funktionssysteme weniger klare Stoppregeln vor: Wann gilt eine Organisation, die primär auf das Bildungs-, das Wissenschafts- oder das Gesundheitssystem hin ausgerichtet ist, als gescheitert?

48 Von Berliner $(1952,1957)$ stammt eine der umfangreichsten und vor allem eine der frühesten Studien über die Organisationsprinzipien und Funktionslogiken sowjetischer Unternehmen. Die Arbeit basiert auf Interviews, die der Autor mit aus der Sowjetunion emigrierten ehemaligen Führungspersönlichkeiten aus verschiedenen Betrieben geführt hatte (Berliner 1957: 4ff). Auch wenn sich die Studie auf eine sehr frühe Phase der Sowjetunion bezieht, finden sich (bestätigende) Verweise auf die von Berliner skizzierten Phänomene regelmäßig auch in späteren Texten anderer Autorinnen und Autoren zur sowjetischen Wirtschaft (beispielsweise Grossman 1977, 1981). 
Innovationen vergeben wurden) und auf diese Weise zwischen wirtschaftlicher Leistungsabhängigkeit und politischem Gehorsam oszillieren, ${ }^{49}$ in jedem Fall verwies das System jedoch immer wieder auf sich selbst und das heißt: auf die Hierarchie der Politik. Produziert wurde nicht mit Blick auf die Frage, was in der Gesellschaft für knapp gehalten wurde, sondern entsprechend der politischen Knappheitsvorgaben.

Mit Blick auf das Knappheitsproblem wäre ein solches Vorgehen dann unproblematisch, wenn die Kongruenz beider Knappheitskonzepte sichergestellt ist. Angesichts der chronischen Komplexitätsüberlastung der Spitze der Planungshierarchie war der Grad der Übereinstimmung jedoch regelhaft eher gering: Für die Erstellung und Kontrolle der Planziele galt es, eine kontinuierlich steigende Menge an Informationen zu sammeln und zu verarbeiten, um der gesellschaftlichen Komplexität zumindest ansatzweise gerecht zu werden (Kornai 1992: 127). Mit Blick auf die regional unterschiedlichen Produktionsbedingungen wurden beispielsweise für Getreide im Jahr 1965 zunächst acht Preiszonen festgelegt und diese Anfang der 1970er Jahre auf 35 Zonen und Subzonen ausgeweitet. Im Jahr 1978 schließlich existierten bereits 199 Preiszonen allein für Weizen. Ähnliche Entwicklungen lassen sich auch für andere Produkte beobachten (Wegren 1998: 29f). Schätzungen zufolge war die Anzahl der Einzelpreise Ende der 1970er Jahre auf mindestens zehn Millionen gestiegen. Die Preise wurden auf Listen dokumentiert und in einem zunehmend undurchschaubaren Prozess vom Staatlichen Preiskomitee, dessen Abteilungen in den Sowjetrepubliken und unter Mitwirkung von Ministerien, lokalen Preisbehörden sowie teilweise einzelnen Betrieben festgelegt, revidiert und auf ihre Umsetzung hin überprüft (Bornstein 1978).

Für die Verarbeitung dieser Informationen standen vergleichsweise begrenzte Kapazitäten zur Verfügung: Gemäß den Programmvorgaben bündelten sich die relevanten Entscheidungskompetenzen an der Hierarchiespitze, deren Entscheidungen wiederum für sämtliche untergeordneten Ebenen Prämissencharakter hatten. Für eine einzelne Koordinationsinstanz wie die zentrale Planungsbehörde war dies eine kaum zu bewältigende Aufgabe. Letztlich markiert dieser »Engpaß des Hierarchiemodells« in Form des begrenzten »Informationsverarbeitungs- und Kommuni-

49 Die Meinungen, die dazu in der Literatur zu finden sind, divergieren: Beispielsweise betont Berliner auf Grundlage seiner Analyse die wirtschaftliche Leistungsabhängigkeit der Prämien als »a very real incentive« (1957: 28) für talentierte Angestellte, die selbst aus eingefleischten sowjetischen Managern den Homo oeconomicus herauskitzelten (1957: 49f). Kornai (1992: 116f) und Humphrey (1998: 111) rücken hingegen das Moment politischer Disziplinierung in den Vordergrund: »[B]onuses and penalties are not directly linked with performance, but clearly intended to stiffen discipline and obedience to superiors (Kornai 1992: 116f). 
kationspotential[s] der Spitze« (Luhmann 2007 [1971]: 70) den zentralen Gegensatz zur Koordination von Knappheitskommunikation über den Markt, dessen dezentrale und polykontexturale Struktur es erlaubt, die zu verarbeitende Komplexität auf viele Beobachter zu verteilen und damit zu absorbieren (vgl. dazu Goeke/Moser 2011: 232).

Um dennoch Pläne erstellen und ihre Umsetzung koordinieren und kontrollieren zu können, kamen zwei Strategien zum Einsatz: Erstens wurde versucht, die zu verarbeitende Komplexität zu reduzieren, indem beispielsweise große Teile der verfügbaren Informationen bei Entscheidungen mehr oder weniger gezielt unberücksichtigt blieben. ${ }^{50}$ Zweitens sollte die Komplexitätsverarbeitungskapazität der Planungshierarchie durch eine kontinuierliche Ausweitung des Verwaltungsapparates gesteigert werden, indem immer mehr Institutionen in den Planungsprozess eingebunden und intern zusätzliche Stellen geschaffen wurden. Da auf diese Weise auf jede unregulierte oder unerfasste >Lücke $<$ mit neuen Regulierungen und einer Ausweitung und Verdichtung des bürokratischen Netzes reagiert werden musste (Kornai 1992: 128f), geriet die sowjetische Bürokratie unter einen ständigen Expansionsdruck, bis schließlich fast alle Institutionen des Regimes in den Planungsprozess eingebunden waren:

»Thousands upon thousands of functionaries in the party apparatus, the state administration, the firm and cooperative managements, and the mass organizations negotiate, calculate, renegotiate, and recalculate before the millions of planning commands finally emerge at all levels.«(Kornai 1992: 114)

Dies wiederum führte in erster Linie dazu, dass das kommunikative Rauschen im System lauter wurde und die Anzahl derjenigen Interaktionen zunahm, die für die Bearbeitung der eigentlichen Aufgabe folgenlos blieben, weil sie allein der Kontrolle dienten. Ohne Entscheidungskompetenzen blieb den unteren Hierarchieebe-

50 In diesem Kontext identifiziert Birman (1978) die Vorgehensweise »from the achieved level« als dominierendes Prinzip der Planerstellung, die im Wesentlichen darin besteht, die Produktion vergangener Perioden mit einer Wachstumsrate zu versehen: »The well known >from the achieved level< denote that the plan indicators are derived by means of adding to the relevant ex post figures a certain percentage of growth. That is the foundation of all the technique, all the methodology of Soviet planning (1978: 161). Auf der Grundlage dieser Berechnung wurde ein erster Entwurf erstellt, auf den Aushandlungen mit den untergeordneten Ebenen (vor allem den Betrieben) folgten. Die faktische Relevanz solcher Rückmeldungsschleifen für Planänderungen, die oftmals auch noch im Verlauf der Implementierung vorgenommen wurden, war jedoch fraglich (vgl. dazu Berliner 1957: 18; Birman 1978: 163). 
nen gar nichts anderes übrig als unbearbeitete Komplexität zusammen mit Verantwortung entlang der bürokratischen Hierarchie nach oben weiterzureichen. Dies kostet Zeit (vgl. Luhmann 2010: 187) und Geld und beeinträchtigt die Fähigkeit des Systems, sich zeitnah an Veränderungen anzupassen. Die Planungshierarchie reproduzierte sich unter diesen Bedingungen zwar selbst, indem sie kontinuierlich Entscheidungen traf und Anordnungen weitergab, die Bearbeitung des Knappheitsproblems geriet dabei jedoch aus dem Blick.

Ungeachtet dessen fanden Zahlungen auch unter dem sowjetischen Wirtschaftsprogramm statt: Haushalte zahlten für Konsumgüter und erhielten Löhne, Unternehmen verkauften ihre Produktion an den Staat und erzielten im Produktionsprozess Gewinne oder Verluste und mit jeder Zahlung wurde Zahlungsfähigkeit weitergegeben. Das Knappheitsproblem blieb davon jedoch weitgehend unberührt, indem die Überbrückungsfunktion, die Geld als symbolisch generalisiertes Kommunikationsmedium für gewöhnlich erfüllt (Baecker 1998 [1997]-a: 83ff; Luhmann 1997: 319), politisch durchbrochen wurde. Grossman (1962: 212) beschreibt diesen Effekt als partielle Entmonetarisierung der sowjetischen Wirtschaft: »[M]oney must be deprived of $>$ moneyness $<$, and prices must be kept $>$ passive $<-$ mere accounting and measurement units « (mit Verweis auf Grossman: Ericson 2006: 57). Mit der Sinnentleerung von Geld verschwinden auch die Freiheitsgrade, die üblicherweise mit Geldbesitz verbunden sind (vgl. dazu mit Verweis auf Simmel Deutschmann 2009b: 227), woraus der politischen Führung zusätzliche Kontrolleffekte erwuchsen. Ebenso unterlagen die administrierten Preise der Entscheidung der Planungsbehörde und fungierten als Kontrollinstrumente im Planungsprozess (Bornstein 1962: 65), nicht jedoch - wie im Marktkontext - als Instrument der Beobachtung zweiter Ordnung und als Spiegel von Erwartungserwartungen der anderen Marktteilnehmer (Luhmann 1988: 18; vgl. auch Goeke/Moser 2011: 234). Administrierte Preise im Sozialismus ähnelten vor diesem Hintergrund eher einer Form von Steuern, bei denen staatliche Organisationen im Moment der Entscheidung davon ausgehen können, dass die entsprechenden Zahlungen stattfinden (oder im Notfall gewaltsam erwirkt werden) (Humphrey 1998: 96), und in denen sich die politischideologisch motivierten Knappheits- und Verteilungsentscheidungen der politischen Führung respektive der zentralen Planungsbehörde widerspiegelten. ${ }^{51}$

51 Die konkrete Ausgestaltung des sowjetischen Preissystems führte zum Teil zu grotesken Effekten (Humphrey 1998: 96): Beispielsweise erhielten die Betriebe für den Verkauf von Überschussproduktion (in Relation zu den Planvorgaben) höhere Preise als für den Absatz derjenigen Produktion, die unterhalb des Plansolls lag. Dies führte erstens zu erheblichen Kalkulationsproblemen, da Unsicherheit darüber bestand, von welchen Preisen auszugehen war. Zweitens hatte das Preissystem den Effekt, dass der Gewinn, den ein Betrieb erzielte, bei identischer Menge und Qualität der Produktion von den ausgehan- 
In einem Substitutionsprozess, so lassen sich die vorhergehenden Überlegungen zusammenfassen, wurde innerhalb der Formalstrukturen der sowjetischen Wirtschaft Macht an die Stelle von Geld gesetzt und in der Folge durch Zahlungen nicht Knappheit kommuniziert, sondern politische Entscheidungen. In diese Hierarchie organisierter Produktion gliederten sich auch die landwirtschaftlichen Kollektivbetriebe ein. Jedoch: Die oben beschriebene Konterkarierung der Machtverhältnisse im Dorfkontext schuf einen Möglichkeitsraum, in dem sich neben den Kollektivbetrieben, die > von oben $<$ fest in diese politisch organisierten Strukturen eingebunden waren, kleinbäuerliche und als solche >regimefremde< Strukturen landwirtschaftlicher Produktion in Form privater Hoflandwirtschaften entwickelten, auf der Außenseite der Planungshierarchie reproduzierten und deren funktionale Defizite zumindest für einen begrenzten Bereich kompensierten.

\section{Knappheitskommunikation sunter der Hand}

Den offiziellen Beteuerungen zum Trotz wurden private Produktionsformen in der sowjetischen Landwirtschaft nie vollständig abgeschafft. Schätzungen gehen davon aus, dass spätestens seit den 1970er Jahren rund ein Viertel der Lebensmittelproduktion auf den privaten Sektor entfiel, obwohl dieser nur etwa zwei Prozent der landwirtschaftlich nutzbaren Flächen bearbeitete (vgl. Tabelle 2). Die Einkünfte aus privater Produktion machten mindestens die Hälfte des Monatseinkommens eines Kolchosbauern aus ${ }^{52}$ (Wädekin 1973: 190ff) und auch ein erheblicher Anteil des Viehbestands befand sich in privatem Besitz (vgl. Tabelle 3).

Die formale Grundlage für die sogenannten persönlichen Hoflandwirtschaften (»ličnoe podsobnoe chozjajstvo«) geht auf die Frühphase der Massenkollektivierung zurück: Die Zwangskollektivierung zielte ursprünglich darauf ab, das Privateigentum der bäuerlichen Haushalte vollständig in Großbetriebe zu überführen. Nachdem jedoch die erste Kollektivierungswelle, mit deren Umsetzung die örtlichen Verwaltungsorgane betraut waren, auf unerwartet heftige Gegenreaktionen gestoßen war, entschloss sich die politische Führung, kleine private Nebenerwerbswirtschaften zu dulden.

delten Planzielen - und damit seiner Verhandlungsmacht innerhalb der Planungshierarchie - abhing. Drittens stieg auf diese Weise das Preisniveau mit der Produktionsmenge an, so dass beispielsweise in der Landwirtschaft in guten Erntejahren höhere Preise erzielt wurden als in schlechten Perioden.

52 Schätzungsweise stammte das monatliche Einkommen eines Kolchosbauern im Jahr 1964 zu 43,4 Prozent aus dem Kolchoslohn (einschließlich Naturalzahlungen), zu 43,9 Prozent aus privater Produktion und zu 12,8 Prozent aus anderen Quellen (Wädekin 1973: 190f) 
Tabelle 2: Anteile privater Produktion in der sowjetischen Landwirtschaft

\begin{tabular}{l|c|c|c|c|c} 
Anteil an: & $\mathbf{1 9 4 0}$ & $\mathbf{1 9 6 0}$ & $\mathbf{1 9 7 0}$ & $\mathbf{1 9 8 0}$ & $\mathbf{1 9 9 0}$ \\
\hline $\begin{array}{l}\text { Landwirtschaftlicher } \\
\text { Fläche gesamt }\end{array}$ & k.A. & $1 \%$ & $2 \%$ & $2 \%$ & $2 \%$ \\
\hline $\begin{array}{l}\text { Landwirtschaftlicher } \\
\text { Produktion gesamt }\end{array}$ & k.A. & k.A. & $31 \%$ & $29 \%$ & $26 \%$ \\
\hline Getreide & $1 \%$ & $1 \%$ & $0 \%$ & $0 \%$ & $0 \%$ \\
\hline Kartoffeln & $54 \%$ & $63 \%$ & $65 \%$ & $65 \%$ & $66 \%$ \\
\hline Gemüse & $45 \%$ & $48 \%$ & $41 \%$ & $33 \%$ & $30 \%$ \\
\hline Fleisch & $65 \%$ & $41 \%$ & $33 \%$ & $30 \%$ & $25 \%$ \\
\hline Milch & $70 \%$ & $48 \%$ & $34 \%$ & $27 \%$ & $24 \%$
\end{tabular}

Quelle: Berechnung auf Grundlage von Ioffe/Nefedova/Zaslavski (2006: 21).

Tabelle 3: Anteil privater Viehhaltung am sowjetischen Viehbestand

\begin{tabular}{l|c|c|c|c} 
& Kühe & Schweine & Schafe & Ziegen \\
\hline 1950 & $66 \%$ & $35 \%$ & $16 \%$ & $53 \%$ \\
\hline 1960 & $47 \%$ & $26 \%$ & $21 \%$ & $82 \%$ \\
\hline 1969 & $39 \%$ & $25 \%$ & $21 \%$ & $82 \%$
\end{tabular}

Quelle: Wädekin (1973: 52ff).

Dieser »Sieg der russischen Bäuerinnen über den Sozialismus« erwies sich als folgenreich für das gesamte Kolchossystem (Merl 1990: 257f). Nach anfänglichen Formalisierungsschwierigkeiten dieser Produktionsform (vgl. dazu Merl 1990: 259) wurden die Hoflandwirtschaften schließlich im dritten Musterstatut aus dem Jahr 1969 berücksichtigt. ${ }^{53}$ Das Statut gewährte jedem Kolchosmitglied das Recht, ein Stück Hofland privat zu nutzen (maximal 0,5 Hektar, die formal weiterhin Kolchosund damit Gemeinschaftseigentum waren), eine begrenzte Menge an Nutzvieh zu halten (vor allem Kleinvieh wie Schweine, Schafe, Ziegen und Hühner) und die daraus hervorgehende Produktion auf eigene Rechnung zu verkaufen. Der Status des Kolchosmitglieds war dabei sehr weit gefasst: Die Möglichkeit einer privaten Hoflandwirtschaft stand nicht nur Kolchosbäuerinnen und -bauern offen, sondern auch Lehrerinnen und Lehrern, medizinischem Personal, Verwaltungsangestellten und anderen Fachkräften im Dorf. Auch Rentnerinnen und Rentner durften nach ihrem formalen Ausscheiden aus dem Betrieb ihre Hoflandwirtschaft weiterführen (Drittes Musterstatut, Art. 42 bis 44, zitiert nach Brunner/Westen 1970: 158ff).

53 Vergleichbare Regelungen fanden sich bereits im Musterstatut des landwirtschaftlichen »artel«, einem Vorläufer des Kolchos, aus dem Jahr 1935 (Goehrke 2005: 70). 
Die Kollektivbetriebe unterstützten die Hoflandwirtschaften, indem sie technisches Gerät für private Zwecke zur Verfügung stellten und Produktionsmittel wie Futter, Düngemittel oder Saatgut zu vergünstigten Preisen verkauften oder teilweise sogar kostenlos als Kompensation für ausbleibende Lohnzahlungen abgaben. Entsprechende formale Regelungen dazu fanden sich ebenfalls ansatzweise im Musterstatut, das nicht nur das Recht der Kolchosmitglieder festlegte, »Kolchosweiden, Kolchoseigenes Arbeitsvieh und Kolchoseigene Transportmittel für persönliche Zwecke nach den im Kolchos geltenden Bestimmungen zu nutzen « (Drittes Musterstatut, Art. 4, zitiert nach Brunner/Westen 1970: 149), sondern auch Vorgaben enthielt, zu welchen Leistungen der Betrieb gegenüber den Hoflandwirtschaften verpflichtet war. Ohne diese Leistungen wären diese wiederum kaum in der Lage gewesen, sich zu reproduzieren, da sie weder über die entsprechenden technischen Geräte verfügten noch aus eigener Kraft Zugang zu den notwendigen Produktionsmitteln hatten (Nove 1980: 127; Osteuropa-Archiv 1989: A 284).

Die übliche Praxis dieser Unterstützungsleistungen überstieg jedoch regelmäßig den formalen Rahmen und spielte sich in einer Grauzone zwischen Informalität und Illegalität ab: So gehörte es zum Beispiel unter Kolchosangehörigen zum Repertoire legitimer Verhaltensweisen, sich an Produktionsmitteln zu nehmen - respektive zu stehlen -, was ihnen nach eigenem Ermessen zustand, sie aber nicht erhielten, weil die vorgegebenen Mengen zu gering waren, der Kolchos die Herausgabe verweigerte oder Lohnzahlungen in Naturalien erfolgten (Goehrke 2005: 96; Grossman 1977: 26; Wädekin 1973: 232). Ausschlaggebend waren dabei Zugangsmöglichkeiten, die an die diversen formalen Mitgliedsrollen im Betrieb gekoppelt waren und auf deren Grundlage sich teilweise rege Tauschaktivitäten innerhalb des Dorfes entwickelten:

»Die Melkerinnen und Stallarbeiter hatten Zugang zu Milch, der Aufseher der Tankstelle konnte Benzin besorgen, der Traktorist Brennholz transportieren und die Verfügungsgewalt eines Vorsitzenden erstreckte sich im Grunde auf alle in einem Betrieb vorhandenen Güter und Betriebsmittel.« (Lindner 2008: 143)

Dass diese Praktiken und die daran geknüpften Legitimitätsvorstellungen mit dem Zusammenbruch des sowjetischen Regimes keinesfalls endeten, illustriert anekdotisch eine Umfrage aus dem Jahr 2000: 857 Bewohner ländlicher Gemeinden wurden dabei auf ihre Akzeptanz bestimmter Verhaltensweisen - darunter auch Diebstahl - und ihre Beobachtung von privatem und kollektivem Eigentum hin befragt (Efendiev/Bolotina 2002: 105; auch Ioffe/Nefedova/Zaslavski 2006: 99). Ging es um das Entwenden von Eigentum des ehemaligen Kolchos - eine Melkerin hat Milch gestohlen, um über die Runden zu kommen, ein Dorfbewohner hat für seine Kuh Heu und Futter entwendet -, beurteilte die Mehrheit dieses Verhalten zwar als 
unerwünscht, war jedoch nach eigenem Bekunden bereit ein Auge zuzudrücken. Ging es hingegen um das Eigentum der Dorfbewohnerinnen und -bewohner - von einem Dorbewohner wird Geld oder das bereits gemähte Heu gestohlen -, wurde der Sachverhalt durchweg als inakzeptabel beurteilt. ${ }^{54}$ War der Zugriff auf ein Gut durch entsprechende Zugangsrechte >gedeckt<, so könnte man sowohl mit Blick auf die Umfrageergebnisse als auch auf das sowjetische Dorf schlussfolgern, treten Eigentumsrechte und die damit verbundenen Mechanismen der Inklusion und Exklusion demgegenüber in den Hintergrund (zur Unterscheidung von Zugangs- und Eigentumsrechten im sowjetischen und postsowjetischen Kontext vgl. ausführlich Amelina 2000: 486ff, 2001: 44ff).

Aus rechtlicher Perspektive wäre im sowjetischen Regime in den beschriebenen Fällen der Tatbestand des Diebstahls kollektiven oder staatlichen Eigentums zweifellos erfüllt (vgl. dazu das Interview mit einem Vertreter des Innenministeriums in Osteuropa-Archiv 1989: A 284ff) und die Kolchosleitung in Kenntnis dieser illegalen Erwartungsstrukturen zum Handeln gezwungen gewesen, um sich nicht ebenfalls schuldig zu machen. Genau dies geschah jedoch selten und selbst offenkundiger Diebstahl wurde bisweilen von der Betriebsleitung ignoriert: »In other words: If the kolkhoznik is not given what he vitally needs, then he is forced to steal it; many in authority who perceive this desperate situation tolerate it more or less tacitly « (Wädekin 1973: 232). Im selben Zusammenhang zitiert Ledeneva (1998: 136) einen Kolchosarbeiter, der von seinem Vorgesetzten explizit darum gebeten wurde, nicht zu große Mengen aus dem Betrieb zu entwenden, um ihn nicht zu zwingen, den Vorfall aktenkundig werden zu lassen. Beschränkten sich die Arbeiterinnen

54 Auszug aus den Umfrageergebnissen (Efendiev/Bolotina 2002: 105):

\begin{tabular}{l|c|c|c} 
& $\begin{array}{c}\text { Völlig in- } \\
\text { akzeptabel }\end{array}$ & $\begin{array}{c}\text { Unerwünscht, } \\
\text { aber akzeptiert }\end{array}$ & $\begin{array}{c}\text { Völlig } \\
\text { akzeptabel }\end{array}$ \\
\hline $\begin{array}{l}\text { Melkerin stiehlt beim Kolchos Milch, } \\
\text { um über die Runden zu kommen. }\end{array}$ & $20,6 \%$ & $54,3 \%$ & $24,8 \%$ \\
\hline $\begin{array}{l}\text { Traktorist gräbt jemandem den Ge- } \\
\text { müsegarten um. }\end{array}$ & $77,6 \%$ & $18,6 \%$ & $3,7 \%$ \\
\hline $\begin{array}{l}\text { Diebstahl von Geld aus einem priva- } \\
\text { ten Haushalt. }\end{array}$ & $95,3 \%$ & $3,9 \%$ & $0,9 \%$ \\
\hline $\begin{array}{l}\text { Dorfbewohner stiehlt für seine Hof- } \\
\text { landwirtschaft beim Kolchos Heu und } \\
\text { Futter. }\end{array}$ & $33,4 \%$ & $51,9 \%$ & $14,7 \%$ \\
\hline $\begin{array}{l}\text { Dorfbewohner stiehlt einem anderen } \\
\text { die Heuernte. }\end{array}$ & $93,8 \%$ & $4,7 \%$ & $1,5 \%$
\end{tabular}


und Arbeiter dagegen auf den Diebstahl überschaubarer Mengen, so wurde dieses Verhalten als kompensatorische Maßnahme für ausbleibende Lohnzahlungen stillschweigend hingenommen. Goehrke (2005: 96) beschreibt, wie ein Kolchosvorsitzender gegenüber einer Gruppe von Kolchosbäuerinnen, die Korn gestohlen hatten, auf seinen eigenen Entscheidungsspielraum verwies. Er könne sie dafür einsperren lassen, entscheid sich aber letztlich, über das Vergehen hinwegzusehen (angeblich wohl wissend, dass diese Drohung nicht umzusetzen gewesen wäre, da er auf ihre Arbeitskraft nicht hätte verzichten können). Auch die politische Führung wusste von diesen Praktiken, ihre Reaktionsmöglichkeiten waren jedoch angesichts der Position und faktischen Entscheidungsspielräume der Kolchosleitungen in den Dörfern begrenzt, wie implizit eingeräumt wurde. So stellte ein hochrangiger Beamter des Innenministeriums zum verbreiteten Problem des Futterdiebstahls fest: »Es ist klar, daß vieles von den Betriebsleitern abhängt. Manchmal begünstigen sie geradezu die Verstöße gegen das Gesetz« (Osteuropa-Archiv 1989: A 286).

Angesichts dieser Situation und der Machtposition der Kolchosleitung im Dorf steht zu vermuten, dass es sich für die Kollektivbetriebe bei alldem um »brauchbare Illegalität« handelte (vgl. zu diesem Begriff Luhmann 1964: 304). Illegalität, also organisationsinterne Kommunikation, die explizit im Widerspruch zu den formalen Erwartungsstrukturen steht (siehe oben und Kühl 2007: 237; Luhmann 1964: 304), erweist sich aus Organisationssicht immer dann als brauchbar, wenn die Verstöße gegen die Formalstruktur entweder zum Aufbau informaler Strukturen führen, welche die Formalstruktur auf indirektem Wege stärken, oder Raum für schöpferisches Verhalten schaffen, das die Fähigkeit der Organisation zur Umweltanpassung erhöht. Ungeachtet dessen muss Illegalität jedoch stets latent bleiben - und dies gilt für das illegale Verhalten selbst genauso wie für seine Funktion (Luhmann 1964: 313). Die Kolchosleitung löste diese Latenzanforderung, indem sie ihre einflussreiche Position nutzte, um im Bereich der Illegalität die Unterscheidung zwischen Informalität und Illegalität erneut einzuführen. Die beiden Seiten dieser zusätzlichen Unterscheidung wurden markiert durch die (akzeptable) großzügige Interpretation der formalen Regeln durch die Kolchosmitglieder (>informale Illegalität $<$ ) und den (inakzeptablen) Diebstahl von Kollektiveigentum (>illegale Illegalität $<$ ). Auf diese Weise eröffneten sich auf der Seite der >informalen Illegalität< Anschlussmöglichkeiten, die sowohl der Kolchosleitung als auch den Mitgliedern gelegen kamen. Um von der einen auf die andere Seite zu wechseln, diente wiederum die untergeordnete Stellung des Betriebs in der Hierarchie als strategisch einsetzbares Mittel: Während sich die Kolchosleitung bei Restriktionen oder (der Androhung von) Sanktionen auf die Formalstruktur zurückziehen und glaubwürdig auf ihre »Ohnmacht nach oben« (Wädekin 1969: 178) verweisen konnte, wurden Zugeständnisse stets als eigene Entscheidung ausgewiesen. 
Worin bestand aber die >Brauchbarkeit< dieser illegalen Erwartungsstrukturen für die Organisation Kolchos? Ein wesentlicher Grund für die vermeintliche Großzügigkeit der Betriebsleitung gegenüber den Mitgliedern lag in der symbiotischen Beziehung, über die beide Produktionsformen - Hoflandwirtschaften und Kollektivbetrieb - unauflöslich aneinander gekoppelt waren. Erstens stellten die Hoflandwirtschaften eine bedeutsame Einkommensquelle der ländlichen Haushalte dar und trugen damit erheblich zur Sicherung der materiellen Lebensgrundlage der Kolchosmitglieder bei, was den Betrieb entlastete und damit auch in seinem Interesse war. Selbst unter sowjetischen Beobachterinnen und Beobachtern bestand Einigkeit darüber, dass die Kolchose erheblich mehr hätten produzieren müssen, wenn sie selbst für die Versorgung ihrer Mitglieder verantwortlich gewesen wären (mit Verweis auf die sowjetische Ökonomin Tatjana Zaslavskaia, die für diese hypothetische Zusatzproduktion konkrete Zahlen nennt, vgl. Wädekin 1973: 189). Zweitens und darüber hinaus spielten die Hoflandwirtschaften eine entscheidende Rolle bei der Erfüllung der Planvorgaben. Da die Kollektivbetriebe die Wirtschaftspläne häufig nicht aus eigener Kraft, d.h. mittels der ihnen zugewiesenen Produktionsmittel, erfüllen konnten, griffen sie zur Verbesserung des Betriebsergebnisses auf die private Produktion ihrer Mitglieder zurück (Goehrke 2005: 97f; Wädekin 1973: 185f). Das Ausmaß und der Grad der Freiwilligkeit dieser Praxis und die zugrundeliegenden Arrangements zwischen dem Kollektivbetrieb und den Hoflandwirtschaften variierten beträchtlich: Während es in extremen Fällen vorkam, dass die Großbetriebe lediglich als Schirm für private Produktion fungierten und sämtliche Kolchoserzeugnisse tatsächlich aus den Hoflandwirtschaften stammten, griffen andere Betriebe nur in Krisenzeiten oder zur Übererfüllung der Pläne auf die private Produktion zurück und teilten in diesem Fall die Bonuszahlungen mit den Haushalten (Grossman 1977: 31; Nove 1980: 128). Die Art der Arrangements deckte dabei das gesamte Spektrum von freiwilligen Vereinbarungen bis hin zu forcierten Abgaben $\mathrm{ab}^{55}$ (Humphrey 1998: 169f; Lindner 2008: 86). Formal waren die Kollektivbetriebe dabei zwar verpflichtet, den von den Hoflandwirtschaften erworbenen Teil der Produktion in ihrer Buchhaltung auszuweisen. Da die kolchoseigene Produktion allein häufig die Planvorgaben nicht erfüllt hätte und in diesem Fall mit Sanktionen zu rechnen war, geschah dies in den meisten Fällen jedoch nicht (Wädekin 1973: 236f). Die Produktion aus den privaten Hoflandwirt-

55 Humphrey (1998: 169f) beschreibt zum Beispiel, wie das Prinzip des >sozialistischen Wettbewerbs < zum Teil auch auf die Hoflandwirtschaften ausgeweitet wurde, so dass sich einzelne Haushalte trotz propagierter Freiwilligkeit faktisch kaum entziehen konnten: » [S]ocialist competition< can invade even the private sector of production: people may > voluntarily< take on the obligation to provide specific products from their own household economies towards the plan.« 
schaften wurde entweder direkt als die eigene ausgegeben oder entsprechend $>$ verschleiert<, indem beispielsweise Vieh aus privater Produktion zunächst für ein Jahr im Kolchos behalten wurde, bevor man das Fleisch als vermeintlich betriebseigenes Erzeugnis an den Staat verkaufte (Wädekin 1973: 237). Solche Strategien waren den Kontrollorganen und Planungsbehörden nicht unbekannt, wurden jedoch meist stillschweigend akzeptiert. ${ }^{56}$

Aus Sicht der Hoflandwirtschaften waren die Arrangements mit den Kollektivbetrieben teils vorteilhaft, teils mangels Alternativen unumgänglich: Sofern die eigene Produktion nicht der Selbstversorgung diente, hatten sie zwar die Möglichkeit, ihre Erzeugnisse auch außerhalb des Dorfes zu verkaufen. Eine Absatzmöglichkeit waren die städtischen Kolchosmärkte, die ursprünglich für den Verkauf der überschüssigen Produktion der Kollektivbetriebe eingerichtet worden waren. Die verhältnismäßig hohen Preise, die auf diesen Märkten erzielt werden konnten, machten diese Möglichkeit auch sehr attraktiv. Aufgewogen wurde dieser Vorteil jedoch durch die großen Entfernungen, denn die Märkte waren für viele Privatproduzenten schlicht schwer zu erreichen (Schinke 1989: 6f; Wädekin 1973: 127ff; auch Der Spiegel 1984). Und weil die Dorfbewohnerinnen und Dorfbewohner untereinander nur selten handelten, blieb letztendlich doch nur eigene Betrieb als Abnehmer übrig. In jedem Fall, daran lassen weder zeitgenössische noch rückblickende Beobachtungen einen Zweifel, waren die Kollektivbetriebe - und damit der offizielle oder organisierte Teil landwirtschaftlicher Produktion in der Sowjetunion - und private, faktisch kleinbäuerliche Produktionsformen in hohem Maße voneinander abhängig.

Bemerkenswert an dieser wechselseitigen Abhängigkeit und engen Verzahnung beider Produktionsformen ist, dass das Musterstatut die Hoflandwirtschaften zwar in Grundzügen in der Formalstruktur des Regimes verankerte, diese dabei aber in mindestens zweifacher Hinsicht den sozialistischen Prinzipien widersprachen und für beträchtliche Reibung sorgten: Erstens operierten die Hoflandwirtschaften nach der Logik (privaten) Eigentums, auch wenn das von ihnen bearbeitete Agrarland formal dem Kolchos gehörte und nur temporär zur Verfügung gestellt wurde. Ungeachtet dieser rechtlichen Eigentumsverhältnisse boten sich damit klare Anhalts-

56 Interessant ist in diesem Zusammenhang die von Wädekin (1973: 237) skizzierte Diskussion aus den frühen 1960er Jahren, die sich um die Frage drehte, inwieweit sich solche Praktiken noch innerhalb des rechtlichen Rahmens bewegten oder bereits als illegale Manipulation einzustufen seien. Anstoß für die Diskussion war ein im Jahr 1961 erschienener Bericht in einer der führenden sowjetischen Fachzeitschriften für Statistik, der entsprechende Daten enthielt. In dem Beitrag wurden auch verschiedene konkrete Möglichkeiten für den Umgang mit den fraglichen Praktiken vorgeschlagen, unter anderem der Vorschlag, nur die Gewichtsdifferenz der Tiere der Kolchosproduktion zuzurechnen. 
punkte für Knappheitskommunikation, die das sozialistische Programm mit den Kollektivierungsmaßnahmen eigentlich verschleiern wollte: Das >Kollektiv $<$ war kommunikativ kaum greifbar, Eigentum damit nicht (oder nur diffus) zurechenbar und die Frage, wer im konkreten Fall zu welchem Zugriff berechtigt war, in vielen Fällen nicht mehr zu beantworten. Für die Kolchose galt damit ebenso wie für alle sowjetischen Staatsbetriebe: »[T]here is no individual, family, or small group of partners to whom one can point as owners. [...] [P]roperty in this sense is not only depersonalized, but eliminated. State property belongs to all and to none « (Kornai 1992: 75). Wohlgemerkt bleibt unbestritten, dass Eigentum als Rechtskategorie durchaus existierte und >auf dem Papier zugeordnet werden konnte. Für die Codierung von Knappheit blieben diese Kategorien jedoch folgenlos. Mit Blick auf die Knappheitscodierung wurde die Grenze zwischen Eigentum und Nicht-Eigentum stattdessen durch eine Grauzone ersetzt und unbeobachtbar. Anschlussmöglichkeiten auf beiden Seiten wurden entsprechend verwässert und Knappheit somit nicht mehr kommunizierbar: »Die Abschaffung allen Eigentums gilt daher als Voraussetzung für die Abwehr allen Übels. Unklar bleibt dabei allerdings, wie man wirtschaften soll, wenn es nicht möglich ist, Knappheit zu kommunizieren und dabei Eigentum vorauszusetzen« (Baecker 2006: 55).

Eine Folge der diffusen Zurechenbarkeit waren Probleme, die in den Wirtschaftswissenschaften allgemein als Effekte öffentlicher Güter oder unter dem Titel »Tragik der Allmende« diskutiert werden (klassisch: Hardin 1968; auch Ostrom 1990): Die Übernutzung und Tendenz zur Verschwendung von Ressourcen infolge unklarer Verantwortlichkeiten und fehlender Sanktionsmöglichkeiten. ${ }^{57}$ In diesem Sinne liest sich auch die Beschreibung eines Kolchosbrigadiers:

»Zwei Jahre nach der Kollektivierung hatten sie alles zerbrochen, nichts war mehr funktionstüchtig, weder Wagen, noch Schlitten. Jedermann verhielt sich zum Kolchoseigentum wie zu etwas Fremdem und nicht wie früher zu etwas, das einem gehört.« (Goehrke 2005: 96)

Im Falle der privaten Hoflandwirtschaften lässt sich hingegen beobachten, dass die Grenze zwischen Eigentum und Nicht-Eigentum an Kontur gewann: Die Frage, wer im Hinblick auf den Zugriff auf und die Nutzung von einem privat bearbeiteten Stück Land, privat gehaltenem Vieh und ähnlichem in- oder exkludiert war und welche Pflichten und Verantwortlichkeiten dies auf beiden Seiten der Unterscheidung einschloss, stand weitgehend außer Frage. Sogar der Einfluss machtvoller dörflicher Instanzen wie dem Kolchos brach sich offensichtlich an dieser Grenze

57 Als Erfolgsstrategie bei der Bewirtschaftung sogenannter Allmendgüter ermittelt Ostrom (1999: 241) auf Grundlage ihrer empirischen Fallstudien unter anderem auch die Einführung von Zurechnungsmechanismen. 
zwischen Eigentum und Nicht-Eigentum: In aller Regel griff er auf private Produktion nicht einfach zu, sondern handelte mit den Hoflandwirtschaften entsprechende Vereinbarungen aus (die mangels Alternative auf Seiten der Hoflandwirtschaften allerdings häufig zu seinem Vorteil ausfielen). Die Anhaltspunkte für Knappheitskommunikation, die sich daraus ergaben, gerieten in scharfen Widerspruch zu den unklaren Eigentumsstrukturen der Kolchose und damit zu den Grundprinzipien des sozialistischen Programms.

Widersprüchlichkeit wird darüber hinaus noch in einer zweiten, daran anschließenden Hinsicht deutlich: Zwar waren die Hoflandwirtschaften über die Kolchosmitgliedschaft mindestens eines Haushaltsmitglieds formal an die Kolchose gebunden, im Unterschied zu den Kollektivbetrieben bezogen sie ihre Entscheidungskriterien jedoch bestenfalls indirekt aus der Planungshierarchie. Stattdessen waren die Verfügbarkeit von und die Preise für Inputfaktoren sowie - neben der Selbstversorgung - die Absatzmöglichkeiten über die Kollektivbetriebe und auf den Kolchosmärkten ausschlaggebend für die Produktionsentscheidungen dieser landwirtschaftlichen Kleinstorganisationen. Sicher wäre es unangemessenen und übertrieben, die Hoflandwirtschaften angesichts dessen als kapitalistische Kleinunternehmen innerhalb des sowjetischen Regimes zu bezeichnen. Und dennoch: Im Hinblick auf ihre eigene Reproduktionsfähigkeit war für sie Knappheit maßgeblich und politische Macht nachrangig (vgl. auch Merl 1990: 454). Die Hoflandwirtschaften verwiesen somit zwar einerseits über die Bindung an den Kolchos immer wieder auf die Formalstruktur des Regimes, mäandrierten gleichzeitig jedoch kontinuierlich über deren Grenzen hinweg. Die Entscheidungen von Kolchos und Hoflandwirtschaften bezogen sich aufeinander, waren füreinander jedoch nicht bindend. Letztere entzogen sich auf diese Weise der direkten Adressierbarkeit in der politischen (Planungs-)Hierarchie und entglitten der unmittelbaren Kontrolle durch die politische Führung, waren aber gleichzeitig in funktionaler Hinsicht für die sowjetische Landwirtschaft unverzichtbar.

Die reine Existenz privater Produktionsstrukturen innerhalb des sowjetischen Regimes - ganz zu schweigen von ihrer ökonomischen Bedeutung - stürzte die politische Führung in erhebliche Schwierigkeiten. Sie musste dieses Phänomen legitimieren und »propagandistisch [.] bemänteln« (Pollack 1998: 121, Fn. 32). Mit anderen Worten: Es galt, die ideologische Anschlussfähigkeit innerhalb des sozialistischen Programms herzustellen. Zwar waren die Hoflandwirtschaften über das Musterstatut der Kollektivbetriebe zumindest in rudimentärer Form formalisiert. Es war jedoch kaum möglich, darüber hinaus und im Hinblick auf ihre tatsächliche Funktion formale Entscheidungsprämissen zu formulieren und verbindlich festzulegen, ohne den ideologischen Kern des Regimes zu delegitimieren und damit seine funktionalen Defizite (hier: mit Blick auf das Knappheitsproblem) offen einzugestehen. In dieser Hinsicht zeigten die vorsichtigen Formalisierungsschritte und die 
zögerliche politische Liberalisierung gegenüber dieser von der Parteiideologie abweichenden Produktionsform seit den späten 1970er Jahren bereits erste desintegrative Tendenzen in der sowjetischen Gesellschaftsordnung an (Wädekin 1989b: 547, 1989a: 384ff; Osteuropa-Archiv 1989: A 284). Als einzige gangbare Möglichkeit des offiziellen Umgangs mit den Hoflandwirtschaften erschien letztlich die Strategie des Latenthaltens - und allein dies erforderte beträchtliche ideologische Volten: Die Hoflandwirtschaften wurden gezielt entideologisiert, bagatellisiert ${ }^{58}$ und als Übergangsphänomen auf dem Weg zur vollständigen Kollektivierung abgetan (Merl 1990: 258; Wädekin 1973: 2). Darüber hinaus wurde im offiziellen Sprachgebrauch die Bezeichnung >privat< (»častnyj«) in Zusammenhang mit den Hoflandwirtschaften durch >persönlich $(» l i c ̌ n y j \ll)$ ersetzt und damit versucht, eine vermeintlich klare Trennlinie gegenüber kapitalistischen Produktionsformen zu ziehen und die ideologische Kluft zumindest semantisch zu überbrücken ${ }^{59}$ (Wädekin 1973: 10).

Im Transformationskontext erlebten die Hoflandwirtschaften als vermeintliche Keimzelle privatwirtschaftlicher Produktion eine späte Konjunktur: Bereits Gorbatschow hatte - in der Hoffnung auf Effizienzsteigerungen in der Landwirtschaft deutliche Schritte in Richtung einer Liberalisierung unternommen, indem 1989 privatbäuerliche Betriebe legalisiert und Möglichkeiten geschaffen wurden, Land unabhängig vom Kolchos zu pachten (nicht jedoch privat zu besitzen). Die private Produktion wurde so von der Mitgliedschaft im Kolchos entkoppelt und bis 1992 entstanden daraufhin knapp 50.000 privatbäuerliche Betriebe (Wegren 1998: 36f). Der damalige Landwirtschaftsminister Viktor Chlystun prognostizierte angesichts dieser Entwicklung im Jahr 1993 die Entstehung von 600.000 bis 650.000 privatbäuerlichen Betrieben bis Ende 1995, die ein Fünftel der landwirtschaftlich genutzten Flächen Russlands bearbeiten sollten (Craumer 1994: 339; zitiert nach Lindner 2003: 20f). Ein ambitioniertes Ziel, das jedoch um Längen verfehlt wurde: Im Jahr 2005, knapp anderthalb Jahrzehnte nach der Auflösung der Sowjetunion, waren offiziell 257.400 privatbäuerliche Betriebe registriert (Rosstat 2006).

Ich möchte das Beispiel der sowjetischen Landwirtschaft nicht überstrapazieren und ende an dieser Stelle mit meiner Darstellung, die sich sicher an dem einen oder anderen Punkt vertiefen und mit weiteren Details füllen ließe. Ich möchte auch

58 So äußerte sich beispielsweise Stalin offiziell mit folgender Beschreibung der Lage: »Gewiß, es hat zwischen der Sowjetmacht und den Kollektivbäuerinnen vor nicht langer Zeit ein kleines Mißverständnis gegeben. Es handelte sich um die Kuh. Jetzt aber ist die Sache mit der Kuh geregelt und das Mißverständnis beseitigt« (Zitiert nach Merl 1990: 258).

$59 \mathrm{Ob}$ diese beiden Vorhaben geglückt sind, kann an dieser Stelle nicht beurteilt werden, die Bezeichnung >persönliche Hoflandwirtschaft (oder Nebenwirtschaft)< (»ličnoe podsobnoe chozjajstvo«) hat sich jedoch bis heute im alltäglichen Sprachgebrauch erhalten. 
nicht behaupten, dass die Strukturen des sowjetischen Dorfes, wie sie hier beschrieben wurden, der alleinige Schlüssel zum Verständnis der Operationsweise der sowjetischen Organisationsgesellschaft in all ihren empirischen Idiosynkrasien sind. Jedoch: Mit Blick auf die eingangs zu diesem Kapitel dargestellten theoretischen Überlegungen zu Programmen, Codes und Organisationen erlaubt der Blick ins sowjetische Dorf erstens exemplarische Rückschlüsse auf die Reichweite und die Grenzen des sowjetischen Gesellschaftsprojekts und eignet sich zur Visibilisierung ausgewählter Mechanismen, die dabei zum Tragen kamen. Zweitens verdeutlicht die obige Beschreibung eine Reihe von Strukturmerkmalen des sowjetischen Dorfes, die für die nachfolgende Analyse der Transformationsgesellschaft am Beispiel des ländlichen Raums und speziell mit Blick auf politische Dezentralisierungsprozesse bedeutsam sind. Auf beide Aspekte möchte ich in einem abschließenden Fazit zu diesem Kapitel eingehen.

\section{REICHWEITE UND GRENZEN DER SOWJETISCHEN ORGANISATIONSGESELLSCHAFT}

Die sowjetische Gesellschaftsordnung war das Ergebnis des Versuchs, gesellschaftliche Reproduktion nach den Vorgaben des sozialistischen Programms zu steuern. Eingesetzt und umgesetzt wurde das Programm von der sozialistischen Einheitspartei, die dazu eine moderne oder zumindest sich modernisierende Gesellschaft $^{60}$ in eine organisierte Hierarchie unter politischer Führung verwandelte. Das Problem der vergleichsweise geringen Kapazität organisierter Kommunikation zur Verarbeitung von Umweltkomplexität war gleichermaßen Ursache und Effekt dieses Vorhabens: Indem die politische Führung versuchte, das Problem ihrer eigenen geringen Komplexitätsverarbeitungskapazität handhabbar zu machen, übertrug sie es auf das gesamte Regime. Fluchtpunkte der politisch angestrebten Strukturänderungen waren zum einen die vollständige Inklusion aller Gesellschaftsmitglieder in Form einer umfassenden politischen Adressierbarkeit und zum anderen mit Blick auf die ideologischen Prämissen des Marxismus-Leninismus eine grundlegende Umstellung wirtschaftlicher Kommunikation durch die Transformation von Privat-

60 Die Diskussion, inwieweit es sich beim vorrevolutionären Russland um eine stratifizierte oder um eine moderne, d.h. funktional differenzierte Gesellschaft handelte, würde den Rahmen dieser Studie übersteigen. Spätestens seit der Abschaffung der Leibeigenschaft 1856 und den anschließenden einschneidenden Reformen im Bereich der Kommunalverwaltung, der Bodennutzungsrechte, der Justiz und des Steuersystems lassen sich in Russland jedoch deutliche Differenzierungs- und Modernisierungstendenzen beobachten (vgl. dazu z.B. Stökl 1997: 536ff). 
in Kollektiveigentum und die Unterdrückung respektive Verdrängung von Markt durch Hierarchie.

Die Formalstruktur des sowjetischen Regimes zeichnete sich durch die Kombination aus der Einheitspartei an der Spitze der gesellschaftlichen Hierarchie und den ihr untergeordneten Massenorganisationen aus. Letzteren konnten (und sollten) alle Gesellschaftsmitglieder beitreten und wurden mit ihrem Beitritt politisch adressierbar. Über die Massenorganisationen sollten darüber hinaus politisch kontrollierte >Funktionsmonopole` für möglichst alle gesellschaftlichen Bereiche eingerichtet und damit Strukturen etabliert werden, die die vollständige Umgehung einer Mitgliedschaft faktisch unmöglich machten. Der Entzug des Mitgliedschaftsstatus bedeutete unter diesen Bedingungen den Entzug der Adressierbarkeit in einer oder mehreren sozialen Sphären.

Das Ergebnis dieses Umbauprozesses war ein gesellschaftsweit umfassender Organisationsgrad, der es der Einheitspartei erlaubte, über die entsprechenden Organisationen nicht nur auf die Inhaltsebene, sondern auch auf die Codeebene der gesellschaftlichen Funktionssysteme zuzugreifen. Die gesellschaftsinterne Umwelt, in der die Funktionssysteme operierten, war in einer Weise konstruiert, die es den teilnehmenden Systemen unmöglich machte, die Politik (respektive die Partei) zu umgehen. Wie sich am Beispiel landwirtschaftlicher Kollektivbetriebe und der Hierarchisierung von Knappheitskommunikation im Bereich der Agrarproduktion zeigen lässt, ging diese Strategie zumindest in begrenztem Maße auch auf. Die Dominanz der Einheitspartei resultierte dabei als eine Art Eigenwert des sowjetischen Regimes: Zum einen schritt sie entsprechend der formalen Hierarchie der Struktur des sowjetischen Regimes voraus, zum anderen verwies jede Operation innerhalb des Regimes mindestens indirekt auf die Einheitspartei und das von ihr etablierte Gesellschaftsprogramm, so dass ihre Position durch den Vollzug von Gesellschaft kontinuierlich bestätigt und gestärkt wurde. Die Partei stellte sicher, dass nichts ohne sie, aber alles mit ihr möglich war.

Die Annahme, dass die Vollinklusion aller Gesellschaftsmitglieder in organisierte Strukturen mehr oder weniger automatisch dazu führte, dass die gesellschaftlichen Funktionssysteme ausgeschaltet wurden und Kommunikation vollständig kontrollierbar wurde, verkennt jedoch das Ausmaß gesellschaftlicher Komplexität, das weiterhin mit im Spiel war. Schließlich war immer noch Gesellschaft (und nicht Organisation!) die Umwelt, mit der die Organisationen umgehen mussten - und dies auch dann, wenn sie unter politischer Kontrolle standen. Diese Feststellung, so lässt sich berechtigt einwenden, ist aus systemtheoretischer Perspektive wenig überraschend und wurde auch an anderen Stellen und in anderen Kontexten bereits getroffen. Interessant ist in diesem Zusammenhang allerdings die Frage nach den Ansatzpunkten, über die sich widersprüchliche, regimefremde Erwartungsstrukturen innerhalb der sowjetischen Organisationsgesellschaft etablierten. 
In dieser Hinsicht bietet das sowjetische Dorf ein instruktives Beispiel: Aufgrund des Unvermögens der lokalen Verwaltungsorgane, die ihnen zugewiesenen Funktionen zu erfüllen, etablierten sich die landwirtschaftlichen Kollektivbetriebe als umfassende Organisationen und die Betriebsleitung als Machtinstanz in den Dörfern. Die formale Hierarchie, an deren Spitze auf allen Ebenen die Partei vorgesehen war, verkehrte sich im Dorfkontext in ihr Gegenteil um. Die Kolchosleitung fungierte strukturell als Kommunikationsunterbrechung, die entlang der Hierarchie weder von oben noch von unten ohne weiteres zu durchdringen war. Dies wiederum bewirkte, dass sich die Dörfer innerhalb des Regimes als Kommunikationszusammenhang entkoppeln, schließen und einen begrenzten Grad an Autonomie erlangen konnten. Zwar wäre es vermessen, in diesem Zusammenhang von einem einheitlichen Welthorizont zu sprechen (vgl. dazu Bohn 2005: 53ff), aber zumindest richtete sich die lokale Kommunikation an einem Horizont aus, der sich nicht auf die offiziellen ideologischen Prinzipien reduzieren ließ, sondern deutlich darüber hinaus ging. Die auf diese Weise entstehenden Freiheitsgrade ermöglichten, dass (politisch) organisierte (entdifferenzierte) Kommunikationsformen und - aus der Perspektive des sozialistischen Programms - deviante Erwartungsstrukturen im Dorf nicht nur koexistierten, sondern eng miteinander verzahnt und wechselseitig voneinander abhängig waren. Dabei deutet vieles darauf hin, dass sich derartige Nischen funktionssystemspezifischer Kommunikationsformen nicht nur ungeachtet aller Kontroll- und Organisationsansprüche der politischen Führung Bahn brachen, sondern den Operationsmodus ebenso wie die Reproduktionsfähigkeit der sowjetischen Gesellschaft mitbestimmten.

Zwei solcher Anhaltspunkte für funktionale Differenzierung, auf die meine Argumentation verweist, möchte ich abschließend noch einmal auf den Punkt bringen: Ein erster Grund für die Anschlussfähigkeit ideologisch devianter Kommunikationsformen lag zweifellos in der Komplexitätsüberfrachtung des Partei- und Verwaltungsapparats als eine Konsequenz des Versuchs, funktionssystemspezifische Kommunikationsformen unter politische Kontrolle $\mathrm{zu}$ bringen und dort $\mathrm{zu}$ halten. Die politische Führung erwies sich als unfähig, die entsprechenden Bezugsprobleme, die sich gerade nicht politisch ausschalten lassen, in einer Weise zu bearbeiten, die gesellschaftliche Reproduktion ermöglichte. In der Folge entzog sich die Kommunikation zunehmend den Formalstrukturen des Regimes, um Lösungen in davon abweichenden Formen zu suchen - und das bedeutete: in der Logik der Funktionssysteme. Private Produktion in der Landwirtschaft ist (mit allen genannten Einschränkungen) ein Beispiel für diesen Mechanismus. Aber auch andere Bereiche der sogenannten sowjetischen Schattenwirtschaft (ausführlich dazu Simis 1982; auch Ericson 2006; Grossman 1977, 1981) oder die verschiedenen Formen von Untergrundkunst (wie beispielsweise das sogenannte »samizdat « [wörtl.: Selbstverlag], ein weitläufiges System für die Verbreitung regimekritischer Literatur, vgl. 
z.B. Joo 2004; Komaromi 2004) sind Evidenz dafür, dass sich funktionssystemspezifische Kommunikationsformen in von der Politik unbeobachteten oder stillschweigend akzeptierten Nischen herausbildeten, nach eigenen Maßgaben reproduzierten und Anschlussfähigkeit generierten.

Ein zweiter Anhaltspunkt für widersprüchliche Strukturen innerhalb des sowjetischen Regimes, der in meiner Darstellung nur am Rande tangiert wurde, ging von den Strukturen der Weltgesellschaft aus: Ungeachtet aller Konfrontationsrhetorik gegenüber dem >Westen $<$ war das sozialistische Programm ohne ständig mitlaufenden Verweis auf die Weltgesellschaft kaum denkbar, denn letztlich vollzog sich die durch das Programm induzierte Entkopplung und Abgrenzung immer auch im Modus der Konkurrenz im Horizont der Weltgesellschaft. Komplementär dazu baute sich im Inneren des Regimes Erfolgsdruck auf: »The Soviet Union was ostensibly committed to increasing production, to churning out all those tractors, all that pig iron, and to >catching up and surpassing < the West in the economic sphere « (Clark 2011: 9). Massenkollektivierung und die Etablierung industrieller Produktionsformen in der Landwirtschaft zielten vor diesem Hintergrund immer auch darauf, die Überlegenheit der Sowjetunion gegenüber der restlichen (kapitalistischen) Welt zur Schau zu stellen. Einem vergleichbaren Erfolgsdruck war nicht nur die Wirtschaft ausgesetzt, sondern auch der sowjetische Sport, die sowjetische Wissenschaft und andere. Um die vermeintliche eigene Überlegenheit nachzuweisen, war die Teilnahme - und damit Adressierbarkeit - in weltgesellschaftlichen Kommunikationskontexten unumgänglich. Diese Form globaler Anschlussfähigkeit war wiederum nur zu generieren, indem man sich auf die Logik von Funktionssystemen als einer zentralen Eigenstruktur der Weltgesellschaft (Stichweh 2006: 241ff) einstellte. In dieser Hinsicht wurden beispielsweise in den sowjetischen Natur- und Ingenieurswissenschaften nicht nur international sichtbare Erfolge erzielt, sondern beide waren auch in Kommunikationszusammenhänge außerhalb der Grenzen des sowjetischen Regimes eingebunden (beispielsweise in Form von Zitationen oder der Teilnahme an Konferenzen). ${ }^{61}$ Die entsprechenden Wissenschaftlerinnen und Wissen-

61 In den Sozialwissenschaften als ideologisch hochsensibles Unterfangen, um ein Gegenbeispiel zu nennen, griff das sozialistische Programm hingegen explizit auf die Codeebene der Kommunikation durch, indem wissenschaftliche Anschlussfähigkeit an ideologische Konformität gekoppelt wurde (zur Frage der Autonomie der sowjetischen und postsowjetischen [russischen] Soziologie vgl. Bikbov 2005). In diesem Sinne forderte der »Berufskodex des Soziologen« beispielsweise »das Prinzip der Parteilichkeit der Wissenschaft« ebenso ein wie »einen klaren Klassenstandpunkt« ihrer Vertreterinnen und Vertreter (Sovetskaja sociologiceskaja associacija [Sowjetischer Soziologenverband] 1989 [1988]). Die anerkanntesten Soziologinnen und Soziologen hatten Positionen sowohl im Sowjetischen Soziologenverband als auch in der Einheitspartei inne, »was in 
schaftler schützte dies regimeintern allerdings nicht vor politischer Verfolgung (Graham 1994: 173ff). Ähnliche Beispiele lassen sich auch für andere Funktionssysteme finden, wie beispielsweise den Sport, wo sowjetische Athletinnen und Athleten in vielen Disziplinen zur Weltspitze gehörten, oder - mit Einschränkungen - auch für die Kunst.

Die katalysatorische Wirkung in Richtung funktionaler Differenzierung, die sowohl von den funktionalen Defiziten als auch von der Weltgesellschaft als Horizont sowjetischer Selbstbeobachtung ausging, kreierte eine Eigendynamik, die kaum umfassend politisch einzuhegen war, wollte das Regime weder seine eigene Stabilität noch seine Position in der Weltgesellschaft aufs Spiel setzen. Stattdessen endeten die Reaktionsmöglichkeiten der politischen Führung in der Regel mit der nachträglichen Korrektur der Symptome, ${ }^{62}$ wie beispielsweise dem Absetzen einzelner Personen von entscheidenden Stellen in der Organisationshierarchie oder der Verhaftung und damit Exklusion einzelner Personen aus bestimmten Kommunikationszusammenhängen. ${ }^{63}$ Darüber hinaus konnte lediglich versucht werden, ideologische Semantiken an offensichtliche Strukturen anzupassen (wie das Beispiel der Hoflandwirtschaften zeigt) - nota bene nicht andersherum -, wobei für die politische Führung allerdings stets das Risiko mitschwang, sich zunehmend in Paradoxien zu verstricken.

entscheidender Weise die thematischen und theoretischen Prioritäten der sowjetischen Soziologie bestimmte (Bikbov 2005: 314). Derartige explizite Politisierungen leisteten wiederum einer fast hermetischen Schließung der entsprechenden Kommunikationszusammenhänge gegenüber dem nicht-sozialistischen Ausland Vorschub.

62 Die Intensität solcher Eingriffe variierte dabei nicht nur über verschiedene gesellschaftliche Bereiche hinweg, sondern auch in der Zeitdimension: Dies zeigen Beispiele wie die wechselvolle Politik gegenüber den Hoflandwirtschaften (vgl. Wegren 1998: Kap. 2), aber auch generelle Tendenzen wie der Kontrast zwischen dem strikten Regime unter Stalin und der sogenannten Tauwetterperiode unter Chruschtschow, als über alle gesellschaftlichen Bereiche hinweg, vor allem aber in der Kunst, ein temporärer Rückzug des Programms auf die Inhaltsebene zu beobachten war (vgl. Sakwa 1998: 55f).

63 Um Missverständnissen vorzubeugen: Die Zwangsmaßnahmen, die von der politischen Führung ausgingen und sich gegen einzelne Personen oder Personengruppen richteten, nahmen zum Teil drastische Ausmaße an, die durch die hier gewählte Form der Analyse und Darstellung keinesfalls verharmlost werden sollen. 


\section{Lokale Selbstverwaltung als Form interner Differenzierung der Politik}

Seit dem Zusammenbruch des sowjetischen Regimes wurde und wird die ehemals sowjetische Gesellschaft mit einer Vielzahl an politischen Transformationsmaßnahmen konfrontiert, die bis heute nahezu alle gesellschaftlichen Bereiche berühren. Im Hinblick auf die beschriebenen Strukturmerkmale des sowjetischen Dorfes erweist sich neben der Privatisierung der ehemaligen Kollektivbetriebe vor allem das Reformthema »Einführung einer kommunalen Selbstverwaltung « als bedeutsam - allerdings wurde diesem Thema (auch) mit Blick auf Russland in der wissenschaftlichen Beobachtung bislang verhältnismäßig wenig Aufmerksamkeit zuteil. Die Einführung kommunaler Selbstverwaltung zielt im Kern auf den Status der dörflichen Verwaltung sowohl im Institutionengefüge der russischen Staatsverwaltung als auch in Relation zu den nach wie vor dominanten ehemaligen Kollektivbetrieben. Der jüngste Reformschritt in diesem Bereich, das Gesetz Nr. 131 »Über allgemeine Prinzipien der Organisation lokaler Selbstverwaltung in der Russischen Föderation«, das seit Anfang 2006 (mit einer Übergangsfrist bis 2009) in Kraft ist und seither die zentrale formal-rechtliche Grundlage kommunaler Selbstverwaltung bildet, sieht dazu Neuregelungen in fünf Bereichen vor. Im Fokus stehen:

- Die territoriale Gliederung der kommunalen Verwaltungseinheiten,

- die Struktur kommunaler Verwaltungsorgane,

- die Partizipationsmechanismen in den Gemeinden,

- die kommunalen Aufgaben im Bereich der lokalen Infrastruktur,

- das Gemeindebudget und das kommunale Eigentum.

Während die Zentralregierung und die Gesetzesautorinnen und -autoren - im Wesentlichen die eigens für dessen Ausarbeitung eingesetzte »Kozak-Kommission« nicht müde werden, die Reform als Neuanfang im Prozess der Einführung und Stärkung kommunaler Selbstverwaltung hervorzuheben, betonen Kritikerinnen und Kritiker vor allem die unterschwellig rezentralisierenden Effekte des Gesetzes. 
Doch nicht allein in Russland selbst wurde der jüngsten Kommunalreform Aufmerksamkeit zuteil: Ungeachtet der kontroversen Diskussion öffnet sich zum Beispiel aus der Sicht der Weltbank mit dem In-Kraft-Treten der Reform ein Zeitfenster, um gerade die Position kleiner ländlicher Gemeinden im Verhältnis zum Zentralstaat zu stärken und mit der Zivilgesellschaft auch die wirtschaftliche Entwicklung im ländlichen Raum zu fördern. Entsprechend begleitete und unterstützte die Weltbank die Einführung von Gesetz Nr. 131 in den Jahren 2004 bis 2007 in ausgewählten Pilotregionen und -gemeinden - darunter auch in zwei Gemeinden meines Untersuchungsgebiets - mit dem Projekt »Local Governance and Civic Engagement in Rural Russia «.

Auf die Bestandteile der jüngsten Reformbestrebungen in Russland und ihren historischen Kontext gehe ich im Folgenden im Einzelnen ein und formuliere auf dieser Grundlage eine Reihe von Leitaspekten für die empirische Analyse. Zuvor trete ich jedoch einen Schritt vom russischen Fall zurück und ordne ihn sowohl in einen globalen Zusammenhang als auch theoretisch ein: Die Inhalte der jüngsten Kommunalreform ebenso wie deren Beobachtung aus russischer und internationaler Perspektive folgen nicht nur einer globalen Tendenz in Richtung politischer Dezentralisierung, die seit der Mitte des 20. Jahrhunderts anhält, sondern verweisen explizit auch auf einen umfassenden Trend, der seit den späten 1990er Jahren in der Entwicklungsforschung und -politik zu beobachten ist und zunehmend an Konturen gewinnt. Ein augenfälliges Merkmal dieses Trends ist die Abkehr vom entwicklungspolitischen Leitbild des sogenannten »Washington Consensus « und eine Hinwendung zum >Lokalen $<$. Bei diesem Wendemanöver erlangten Begriffe und Konzepte wie »good governance « oder partizipatorische Entwicklung Prominenz in der allgemeinen Debatte. Mit Blick auf lokale Selbstverwaltung und politische Dezentralisierungsprozesse wurden im Kontext dieser Entwicklung verschiedene wissenschaftliche Beobachtungsvarianten teils im Anschluss an ältere Debatten wiederbelebt, teils neu induziert, die sich mit den Schlagworten Effizienz, Demokratie und »empowerment « etikettieren lassen und die ich nachfolgend im ersten Schritt skizziere. Die Erkenntnisse der zahlreichen Analysen theoretischer und empirischer Art, die - teils überlappend - im Kontext dieser drei Schlagworte entstanden sind und überwiegend eng an die dahinterstehenden politischen Diskurse anknüpfen, wurden und werden vor allem in der Entwicklungspolitik bei der Konzeption konkreter Beratungsprojekte und politischer Maßnahmen genutzt und sollen hier nicht grundsätzlich in Frage gestellt werden. Bei näherem Hinsehen weisen sie jedoch >blinde Flecken< - diese Bezeichnung ist hier weniger streng theoretisch als vielmehr metaphorisch zu verstehen - auf, die zum einen darauf zurückzuführen sind, dass Dezentralisierung und Lokalverwaltung stets im Horizont von Kausalbeziehungen analysiert werden. Zum anderen rühren sie daher, dass es sich bei den genannten Theoretisierungen und daran anschließenden empirischen Analysen im 
Kern um Reflexionstheorien der Politik handelt, die in ihren Semantiken und verwendeten Unterscheidungen im politischen System selbst anschlussfähig sind. Diese blinden Flecken möchte ich aufzeigen und anschließend unabhängig von politischen Zielvorgaben und normativen Setzungen einen differenzierungstheoretischen Annäherungsversuch unternehmen, der auch die Grundlage für die Darstellung der russischen Reformmaßnahmen bildet. Richtungweisend ist dabei die Frauge, um welche Kommunikationsformen, Erwartungsstrukturen und Unterscheidungen es geht, wenn die Rede von politischer Dezentralisierung und kommunaler Selbstverwaltung ist.

\section{BEOBACHTUNGSVARIANTEN}

Der Begriff des »Washington Consensus« wurde im Jahr 1990 mit Blick auf Wirtschaftsreformen im lateinamerikanischen Kontext von dem US-amerikanischen Ökonomen John Williamson geprägt (1990; auch 2004: 1f) und avancierte sodann zum entwicklungspolitischen Leitbild internationaler Organisationen, allen voran des Internationalen Währungsfonds (IWF) und der Weltbank. Er steht für Strukturanpassungsprogramme und einige darin enthaltene Grundprinzipien, die ganz allgemein auf die Herstellung von wirtschaftlicher Stabilität und Wachstum zielen. Orientierungspunkt sind neoklassisch fundierte, universalistische Modelle über die Wirkung von Marktmechanismen, monetären Zusammenhängen und fiskalpolitischen Maßnahmen. Daraus abgeleitete Reformmaßnahmen richten sich auf wirtschaftliche Liberalisierung, weitgehende Deregulierung und den Rückzug des Staates aus der Wirtschaft, fiskalische Disziplin und umfassende Privatisierung als Garanten für ökonomische Prosperität (Kolodko 1999: 236ff; Müller 2001: 6f).

Nicht zuletzt aufgrund von ausbleibenden Erfolgen der Reformagenden von IWF und Weltbank in den ehemals sozialistischen Staaten Mittel- und Osteuropas stießen die neoliberalen Prinzipien des Washington Consensus jedoch zunehmend auf Kritik. Wurden Zweifel zu Beginn der 1990er Jahre noch vereinzelt und zögerlich geäußert (Kolodko 1999; Steimann 2010: 19), brachen sie sich ab Mitte der 1990er Jahre sukzessive Bahn und gewannen vor allem in Folge der Wirtschaftskrisen in Mexiko (die sogenannte Tequila-Krise) und Südostasien, deren Verläufe und Erscheinungsformen mit der Stoßrichtung des Washington Consensus unvereinbar schienen, an Kontur und Durchschlagskraft (Kolodko 1999: 243; Pender 2001: 402). Einen entscheidenden Wendepunkt in der Debatte markierte der Richtungswechsel ausgerechnet jener Ökonominnen und Ökonomen, welche die Prinzipien des Washington Consensus einst propagiert und damit in hohem Maße zur seiner Etablierung als Entwicklungs- und Reformleitbild beigetragen hatten - allen voran Jeffrey Sachs und John Williamson (Müller 2001: 9; Evidenz für diesen Richtungs- 
wechsel findet sich z.B. in Gelb 1997; Rodrik 2000; Sachs/Woo/Yang 2000). In der Folge gerieten nach und nach faktisch sämtliche Merkmale des Leitbilds in die Kritik, darunter insbesondere der Marktfundamentalismus und das >blinde< Zurückdrängen des Staates, das rigide Durchsetzen von Austeritätsprogrammen, die mangelnde Berücksichtigung von Institutionen, die Anwendung universeller Rezepte ohne Sensibilität für spezifische regionale Kontexte und nicht zuletzt der Topdown-Ansatz der Reformen, die im Namen des Washington Consensus mehrheitlich von Weltbank und IWF auch im postsozialistischen Raum gefordert und umgesetzt wurden (mit Blick auf Russland und Osteuropa findet sich diese Kritik beispielsweise in Stiglitz 2002: Kapitel 5; Kolodko 1999: 234f).

Die Kritik stieß einen politischen Umorientierungsprozess an, der schließlich zum »Comprehensive Development Framework« der Weltbank führte und das neoliberale Leitbild des Washington Consensus formal ablöste (vgl. Wolfensohn 1998: 10ff; Pender 2001: 407). In Konzepten wie »good governance« oder »participatory development « fand nun eine stärker holistisch geprägte Denkrichtung ihren programmatischen Ausdruck, die nicht nur dem Staat beim Aufbau > guter < Institutionen fortan eine konstruktive Rolle zuwies, sondern auch die Bedeutung regionaler Kontexte und lokalen Wissens (z.B. Rodrik 2000) sowie die Notwendigkeit eines administrativen und politischen Gegengewichts zum Zentralstaat betonte (Kolodko 1999: 257). Auf diese Weise entstand zunehmend Raum für eine Aufwertung des >Lokalen< und Dezentralisierungsprozesse rückten ebenso wie Formen lokaler Selbstverwaltung in den Fokus (entwicklungs-)politischer Aufmerksamkeit (vgl. den Überblick in Oyugi 2000; für die deutsche Entwicklungszusammenarbeit vgl. Coly/Brecker 2004; für die Weltbank respektive die US-amerikanische Entwicklungszusammenarbeit vgl. Shah 2006b, 2007c, 2007b; USAID 2000).

In der anwendungsbezogenen Entwicklungsforschung findet die politische Umorientierung zum >Lokalen < in einem »new regionalism « und dem »global trend towards devolution« ihren Ausdruck (Pike/Tomaney 2004: 2091; Rodríguez-Pose/ Gill 2003: 333; auch Rodríquez-Pose/Sandall 2008). In der Schriftenreihe der Weltbank mit dem Titel »Public Sector Governance and Accountability« heißt es beispielsweise programmatisch:

»Although the concept of local governance is as old as the history of humanity, only recently has it entered the broad discourse in the academic and practice literature. Globalization and the information revolution are forcing a reexamination of citizen-state relations and roles and the relationships of various orders of government with entities beyond government - and thereby an enhanced focus on local governance.« (Shah 2006c: 2)

Der Kern dessen, was üblicherweise unter Dezentralisierung und lokaler Selbstverwaltung verstanden wird, lässt sich ungeachtet der Klagen über Begriffswirrwarr 
und eine Überzahl an Definitionen (z.B. Dubois/Fattore 2009; Oyugi 2000) schnell umreißen: Dezentralisierung meint allgemein Prozesse der Aufwertung subnationaler administrativer und/oder politischer Ebenen gegenüber der nationalen Ebene im Hinblick auf ihre Entscheidungskompetenzen (Dubois/Fattore 2009: 710f; Pratchett 2004: 362). An diese Definition schließen weitere Klassifikationen bezüglich verschiedener Dezentralisierungsbereiche, -formen oder -reichweiten an (vgl. die Übersicht in Dubois/Fattore 2009), von denen ich zwei beispielhaft nennen möchte. Gängig ist zum einen die Unterscheidung von politischer, administrativer und fiskalischer Dezentralisierung (vgl. z.B. Hooghe/Marks/Schakel 2010: 7ff; Rodden 2004: 482ff; Treisman 2007: 21ff):

- Politische Dezentralisierung kommt in der (direkten) Wahl subnationaler Amtsinhaberinnen und -inhaber durch die Bevölkerung zum Ausdruck, die an die Stelle der Ernennung durch übergeordnete Ebenen tritt;

- administrative Dezentralisierung erhöht die Kompetenz subnationaler Verwaltungsebenen Entscheidungen zu treffen, über die sich übergeordnete (meist nationale) Ebenen formal nicht ohne Weiteres hinwegsetzen können;

- fiskalische Dezentralisierung bezieht sich auf die Verteilung von Ausgaben und Einnahmen zwischen den Verwaltungsebenen sowie den Grad subnationaler Budgetautonomie.

Zum anderen findet sich mit Blick auf die Reichweite der Dezentralisierungsmaßnahmen in der Literatur die Trias von Dekonzentration, Delegation und Devolution (ursprünglich Rondinelli 1981: 137f; Rondinelli/Nellis/Cheema 1983: 13ff):

- Dekonzentration bezeichnet die schwächste Dezentralisierungsform, bei der lediglich die Ausführung bestimmter Funktionen an untergeordnete Ebenen weitergegeben wird, ohne dass damit ein Transfer von Entscheidungskompetenzen verbunden wäre;

- die Steigerungsform ist Delegation, d.h. die Gewährung subnationaler Entscheidungskompetenzen für spezifische Funktionen unter der Kontrolle des Zentralstaats;

- mit Devolution wird schließlich die weitreichendste Dezentralisierungsform bezeichnet, also »the strengthening or creation of independent levels and units of government « auf regionaler und lokaler Ebene (Rondinelli 1981: 138).

- In gewisser Weise außerhalb dieser Klassifikation steht die Privatisierung, d.h. die Ausgliederung einzelner Leistungen und der entsprechenden Entscheidungen aus der Verwaltung (meist) in die Wirtschaft. 
Die gängige Definition von lokaler Selbstverwaltung schließt an dieses Verständnis an und setzt weitreichende Dezentralisierung insbesondere im Bereich der Verwaltung voraus, wodurch unabhängige kommunale Verwaltungseinheiten mit umfassender Entscheidungskompetenz für sämtliche Angelegenheiten von lokaler Bedeutung geschaffen werden (in erster Linie die Bereitstellung kommunaler Dienstleistungen und die Verwaltung des kommunalen Budgets). Die Europäische Charta der Kommunalen Selbstverwaltung des Europarats definiert lokale Selbstverwaltung als »das Recht und die tatsächliche Fähigkeit der kommunalen Gebietskörperschaften, im Rahmen der Gesetze einen wesentlichen Teil der öffentlichen Angelegenheiten in eigener Verantwortung zum Wohl ihrer Einwohner zu regeln und zu gestalten« (Europarat 1985: Art. 3, Abs. 1).

\section{Dezentralisierungsdiskurse}

Auf welche Weise aber schlägt sich die Hinwendung zum >Lokalen $<$ als ein politisch gewollter Prozess der Aufmerksamkeitsverschiebung in der wissenschaftlichen Beobachtung von lokaler Selbstverwaltung und Dezentralisierungsprozessen nieder? Zunächst einmal darf der quantitative Anstieg einschlägiger Arbeiten zu diesem Thema nicht darüber hinwegtäuschen, dass weder das Phänomen an sich noch die theoretischen Kontexte und wissenschaftlichen Problematisierungen so neu sind, wie die politische Aufregung suggeriert: Ein kontinuierlicher und weltweiter Trend in Richtung politischer Dezentralisierung und Regionalisierung lässt sich spätestens seit der Mitte des 20. Jahrhunderts empirisch robust beobachten (vgl. Hooghe/Marks/Schakel 2010; auch Cunningham 2014). Die Forschungsarbeiten dazu lassen sich mehrheitlich grob drei Argumentationslinien zuordnen, deren Anfänge zum Teil mehrere Jahrzehnte zurückreichen. Diese drei Stränge sind nicht streng voneinander getrennt, verweisen zum Teil wechselseitig aufeinander und werden gerade in entwicklungspolitischen Projekten meist kombiniert. Abgrenzen lassen sie sich jedoch im Hinblick darauf, welcher Beobachtungsaspekt für sie jeweils unhintergehbar ist und welche Rationalitäten unterstellt werden: Wirtschaftliche Effizienz, die Stärkung eines demokratischen Staatsaufbaus oder die Ermächtigung (»empowerment«) lokaler Gemeinschaften im Sinne der Stärkung von Eigenverantwortung und Selbstbestimmungsrechten.

\section{Politische Dezentralisierung und Effizienz}

Wesentliches Merkmal der ersten Argumentationslinie sind die explizite Marktanalogie und der inhaltliche Fokus auf die Bereitstellung öffentlicher Güter und Dienstleistungen. Dezentrale Verwaltungsstrukturen sollen ermöglichen, dass das Angebot von und die Nachfrage nach öffentlichen Gütern und Dienstleistungen füreinander sichtbar werden und entsprechend aufeinander reagieren können. Dezen- 
tralisierung ist dann »a situation in which public goods and services are provided primarily through the revealed preferences of individuals by market mechanisms « (Rondinelli/McCullough/Johnson 1989: 59). Auf kommunalen »Quasi-Märkten« oder »Markt-Surrogaten«, so die Idee, agieren die lokalen Verwaltungsinstitutionen als Anbieter öffentlicher Güter und Dienstleistungen, deren Beobachtung und Handeln sich an den Präferenzen der Bürgerinnen und Bürger ausrichten, die als »consumer-voter « in die Rolle der Nachfrager schlüpfen (Tiebout 1956: 418; Bartlett/Le Grand 1993). Je besser das Angebot den Präferenzen der Nachfrageseite entspricht, desto höher die Wahrscheinlichkeit, dass die Bürgerinnen und Bürger der Kommune mit ihren Steuerzahlungen erhalten bleiben (anstatt den Wohnort zu wechseln - »voting by feet «) und den Amtsinhaberinnen und -inhabern auch bei der nächsten Wahl ihre Stimme geben.

Das Konzept des Wettbewerbs wird auf diese Weise auf die öffentliche Verwaltung übertragen. Kommunen konkurrieren untereinander um Steuerzahlungen und verschiedene politische Fraktionen innerhalb einer Kommune um Wählerstimmen (vgl. z.B. Frey/Eichenberger 1996, 2005). Resultat ist im Idealfall eine nach wirtschaftlichen Gesichtspunkten möglichst effiziente Bereitstellung von öffentlichen Gütern und Dienstleistungen bei einer im Vergleich zur zentralstaatlich koordinierten Variante stärkeren Nachfrageorientierung: »A decentralized form of government therefore offers the promise of increasing economic efficiency by providing a range of outputs of certain public goods that corresponds more closely to the differing tastes of groups of consumers « (Oates 1972: 12). Die Rolle des Staates beschränkt sich im Idealfall auf die Bereitstellung finanzieller Mittel, die Ausführung der Dienstleistungen selbst wird privatisiert und im gewünschten Umfang von den Kommunen gekauft: »[T]he intention is for the state to stop being both the funder and the provider of services. Instead it is to become primarily a funder, purchasing services from a variety of private, voluntary and public providers, all operating in competition with one another « (Le Grand 1991: 1257).

Die Debatte um Marktmechanismen im Bereich kommunaler Verwaltung geht auf eine vorrangig wirtschaftswissenschaftliche Diskussion zurück, die unter dem Schlagwort des »fiscal federalism« bereits in den 1950er Jahren begann (vgl. Tiebout 1956; Musgrave 1959; Olson 1969; Oates 1972) und seitdem immer wieder zu Modellbildungen und -modifikationen vor allem spieltheoretischer oder institutionenökonomischer Art angeregt hat (vgl. z.B. Inman/Rubinfeld 1997; Oates 1981, 1999; Treisman 2006; Weingast 1995). Die explizite Verknüpfung der Modelle mit der Verwaltungspraxis kam in den 1980er Jahren zunächst mit Blick auf die Reform (westlicher) wohlfahrtsstaatlicher Systeme im Konzept des »New Public Management« zum Ausdruck (für einen Überblick vgl. z.B. Schedler/Proeller 2009; Thom/Ritz 2008; die Beiträge in Le Grand/Bartlett 1993; systemtheoretisch auch Kegelmann 2007), im Rahmen dessen Richter (2012: 105) eine »Entsoziologisie- 
rung der Diskurse um öffentliche Verwaltungen« beobachtet. Die Verwaltungswissenschaft, so das Argument, habe (zumindest in Deutschland) in der Debatte längst die Deutungshoheit übernommen und wirtschaftswissenschaftliche Perspektiven Transaktionskostentheorie, Neue Politische Ökonomie und ähnliche - fungierten als dominanter Orientierungsrahmen.

In der jüngeren Vergangenheit lässt sich unter Stichworten wie Nachhaltigkeit und nachhaltige Ressourcennutzung eine ähnliche Tendenz auch im Bereich der Entwicklungsforschung beobachten. Ein entscheidender Katalysator waren und sind dabei die Programme internationaler Organisationen, die auf Dezentralisierungsmaßnahmen und die Einführung respektive Stärkung lokaler Selbstverwaltung zielen und wirtschaftliche Überlegungen dabei explizit zum Ausgangspunkt nehmen (vgl. für viele Bahl 1999, 2006; Faguet 2003, 2008; die Beiträge in Shah 2006b; vor allem die Einleitung in Treisman 2007). »[I]t has been the economic realm which has guided the involvement of international organisations at subnational level« (Rodríquez-Pose/Sandall 2008: 65) und entsprechend ziehen sich Marktanalogien und der Fokus auf ökonomische (Effizienz-)Argumente als roter Faden durch die Selbstbeschreibungen einschlägiger Projekte. ${ }^{1}$ Dezentralisierung soll helfen, Marktund Staatsversagen im öffentlichen Sektor zu korrigieren und lokalen Gemeinschaften im Sinne einer effizienteren und nachhaltigen Nutzung die Kontrolle über ihre Ressourcen zurückzugeben (z.B. Shah 2007a: 15; vgl. auch die zahlreichen Beiträge in Shah 2006b, 2007c; The World Bank 2007a). Die Frage >Why local governance? $<$ beantwortet die Weltbank auf ihrer Website mit dem Dreiklang aus gesteigerter Allokationseffizienz, verstärkter Rechenschaftspflicht der örtlichen Verwaltungsorgane sowie verschärftem Wettbewerb. ${ }^{2}$ Dezentralisierung, so wird betont, »creates the possibility of exerting stronger pressures on government per-

$1 \mathrm{Zu}$ beobachten ist dies unter anderem am Beispiel des »Online Sourcebook on Decentralization and Local Development«, einem gemeinsamen Informationsportal der Weltbank, dem Entwicklungsprogramm der Vereinten Nationen (UNDP), der Ernährungs- und Landwirtschaftsorganisation der Vereinten Nationen (FAO), der deutschen Gesellschaft für technische Zusammenarbeit (GTZ, inzwischen GIZ) und der schweizerischen Direktion für Entwicklung und Zusammenarbeit (SDC/DEZA): http://www.ciesin. org/decentralization/ (zuletzt eingesehen am 14.4.2015).

2 Vgl. dazu die Themenseite der Weltbank zu »Local Governance« unter http://www. worldbank.org/ (zuletzt eingesehen am 30.4.2015). Speziell mit Blick auf die Einführung kommunaler Selbstverwaltung in Russland finden sich diese Argumente in der Dokumentation des Projekts »Local Governance and Civic Engagement in Rural Russia«, das in den Jahren 2004 bis 2007 den Aufbau lokaler Verwaltungsstrukturen unter anderem in zwei Gemeinden meines Forschungsgebiets unterstützte (vgl. The World Bank 2003, 2007). 
formance both from below (the demand side) and from above (the supply side) « (Yilmaz/Yakup/Serrano-Berthet 2008: 1; auch The World Bank 2007b: viiff).

Empirisch getestet wird das Effizienzargument in wissenschaftlichen Studien in aller Regel in Form von quantitativen Modellen. Modelliert wird der Zusammenhang von Dezentralisierung (d.h. meist die Verteilung finanzieller Ressourcen auf die verschiedenen Verwaltungsebenen) und verschiedenen makroökonomischen Indikatoren wie Wirtschaftswachstum (Cai/Treisman 2006), Inflation (Treisman 2000), dem Umfang öffentlicher Investitionen (Faguet 2001, 2008), dem Ausmaß regionaler Ungleichheiten (Martinez-Vazquez/Timofeev 2008) oder dem Grad öffentlicher Verschuldung (Rodden 2002) - um nur einige Beispiele zu nennen.

Am Kern dieser ersten Argumentationslinie, der wirtschaftlichen Konnotation und den expliziten Marktanalogien, entzündete sich zum Teil heftige Kritik. Das Konzept der kommunalen >Quasi-Märkte<, so der zentrale Vorwurf, führe zu einer Entpolitisierung der Debatte um die Ausgestaltung lokaler Selbstverwaltung. Unter Berufung auf das Marktprinzip würden soziale Fragen zu ausschließlich technischen Problemen umgedeutet: »The market then seemed to capture the high ground of democracy, and accountability was reformulated as something that should take place outside the political process « (Phillips 1996: 28; auch Mohan/Stokke 2008: 548ff; Slater 1989: 521; Entpolitisierung im generellen Kontext der Entwicklungszusammenarbeit thematisieren und kritisieren z.B. Ferguson 1990; Harriss 2002). Der Effekt sei letztlich das Heraufziehen eines technokratischen »Managerstaates « (Clarke/Newman 1997; auch Desai/Imrie 1998), in dem die Kommunen nicht mehr als »mutually dependent moral and political communit[ies]« betrachtet würden, sondern als rationale »quasi-individuals, obliged to find their own ways to economic vitality« (Lovering 2011: 584) - alle (vornehmlich negativen) Konsequenzen dieser Entwicklung inklusive. Diese Kritik, so lässt sich paraphrasieren, ist letztlich getrieben von der Sorge, dass ausgehend von der Einführung wirtschaftlicher Logiken in den Bereich der Kommunalverwaltung die Eigenlogik des Politischen ökonomisch unterlaufen wird. Ein solche Ökonomisierung läge dann vor, wenn nicht nur allgemein »Strukturen, Prozesse, Orientierungen und Effekte, die man gemeinhin mit einer modernen kapitalistischen Wirtschaft verbindet, gesellschaftlich wirkmächtiger werden« (Schimank/Volkmann 2008: 382), sondern Evidenz dafür auftritt, dass der wirtschaftliche Code Zahlung|Nicht-Zahlung außerhalb der Wirtschaft gezielt als Nebencode anderer Funktionssysteme zu wirken beginnt und deren Eigendynamik okkupiert ${ }^{3}$ (Schimank/Volkmann 2008: 385f; ähnliche Überlegungen zur Entdifferenzierung respektive Ökonomisierung mit Blick auf Kommunalverwaltung finden auch in Pelizzari 2001; Richter 2009). Die Frage, ob und wo dies in der modernen Gesellschaft zu beobachten ist, stellt im Hinblick auf

3 Stichweh (2005: 194) beschreibt dieses Phänomen als korrupte strukturelle Kopplung. 
mein Forschungsinteresse jedoch ein Nebengleis dar, das ich an dieser Stelle nicht weiter verfolgen möchte.

\section{Politische Dezentralisierung und Demokratie}

Eine zweite Argumentationslinie erachtet die Aufwertung lokaler Selbstverwaltung durch Dezentralisierungsprozesse als unverzichtbares Fundament für einen demokratischen Staatsaufbau - »one of the cornerstones on which subsequent participation develops « (Phillips 1996: 26). Die zugrundeliegende Argumentation beinhaltet zwei Dimensionen: Zum einen, so wird unter Verweis auf Klassiker der Demokratietheorie wie Tocqueville (2006 [1835]) oder Mill (2010 [1861]) argumentiert, sei Mitbestimmung auf der lokalen Ebene ein inhärenter Bestandteil demokratischer Strukturen. Sie sorge für Machtverteilung innerhalb des Staates und schütze damit vor Autoritarismus. Sie begünstige lokale Diversität und Varietät, beispielsweise im Hinblick auf die Leistungen des Staates gegenüber seinen Bürgerinnen und Bürgern, und setze damit einen Kontrapunkt zur uniformen nationalen Politik, die unterschiedslos für alle gilt. Und sie erhöhe die unmittelbare Verantwortlichkeit und Reaktionsfähigkeit demokratischer Institutionen im Hinblick auf lokale Bedürfnisse (vgl. John 2001: 2f; Phillips 1996: 23; Pratchett 2004: 359f). Zum anderen - und dieses Argument war und ist vor allem im postsozialistischen Transformationskontext von Bedeutung - stelle gerade die Bürgerbeteiligung an lokalen und damit besonders und unmittelbar sichtbaren politischen Entscheidungen ein wichtiges Instrument der politischen Erziehung dar. Sie fördere und stärke das Bewusstsein für demokratische Werte und die eigene >politische Kompetenz< und sei damit die Voraussetzung für eine umfassende, über die Beteiligung an Wahlen hinausgehende Form der politischen Inklusion. Eine nationale demokratische Kultur, so das Argument, generiere sich schließlich nicht allein auf Grundlage nationaler Wahlen im Mehr-Jahres-Zyklus (Phillips 1996: 22f; Pratchett 2004: 360f; Sisk 2001: 11ff; mit Blick auf lokale Selbstverwaltung im postsozialistischen Raum respektive in Russland vgl. z.B. Reynolds 2005; Ross 2009: 1ff; Soós/Zentai 2005).

Die beiden Dimensionen des Arguments zum Stellenwert lokaler Demokratie und Selbstverwaltung spiegeln sich unter anderem in der »Europäischen Charta der kommunalen Selbstverwaltung « des Europarats wider (Europarat 1985), die mittlerweile alle Mitgliedstaaten und damit auch die Mehrheit der postsozialistischen Transformationsstaaten unterzeichnet haben. Darüber hinaus taucht ähnlich wie das oben skizzierte Konzept der kommunalen Quasi-Märkte auch die liberaldemokratische Argumentation zu lokaler Demokratie regelmäßig in der explizit praxisorientierten Literatur auf (z.B. Sisk 2001; USAID 2000). Beide Ansätze stehen dabei nicht per se im Widerspruch zueinander, sondern stellen - je nach argumentativer Stoßrichtung hin zu Demokratie oder Effizienz - füreinander durchaus erwünschte Nebeneffekte dar, welche die empfohlenen Maßnahmen zusätzlich legi- 
timieren: Quasi-Märkte und Effizienz implizieren die (demokratische) Mitbestimmung durch die Bürgerinnen und Bürger, lokale Demokratie führt zur stärkeren Berücksichtigung der Bürgerinteressen und damit zu einer besseren Allokation öffentlicher Leistungen (mit Blick auf die Unterstützung lokaler Selbstverwaltung in Russland vgl. erneut The World Bank 2003, 2007a). Letztlich entsprechen beide Perspektiven Hirschmans Unterscheidung von »exit« als gängige Äußerungsform in ökonomischen Kontexten und »voice« als primär politische Reaktions- und Mitbestimmungsmöglichkeit (Hirschman 1970: 15ff; $\mathrm{zu}$ »exit« und »voice« im politischen Kontext am Beispiel der DDR vgl. auch Hirschman 1993).

Wenn konkrete Dezentralisierungsprozesse auf ihr Demokratiepotenzial hin beobachtet werden, stehen meist deren formal-rechtliche Grundlagen im Fokus der Analyse. Die entsprechenden Gesetze und Reformen werden nach Kriterien wie der formalen Autonomie kommunaler Behörden oder lokalen Partizipationsmöglichkeiten abgeklopft und bewertet (so z.B. Blair 2000). Einem solchen Ansatz folgen mehrheitlich auch diejenigen Arbeiten, die sich mit lokaler Selbstverwaltung in Russland, darunter auch die jüngste Kommunalreform, auseinandersetzen (z.B. Campbell 1995, 2006; Gelman 2007; Lankina 2005; Ross 2006, 2009; Wollmann/ Gritsenko 2009). Demokratieüberlegungen spielen ebenfalls eine Rolle, wenn das Zustandekommen institutioneller Designs, darin enthalten auch Gesetze und Reformprogramme zu Dezentralisierung und lokaler Selbstverwaltung, Gegenstand der Forschung ist. Beobachtet werden dabei weniger Gesetzes- und Reforminhalte, als vielmehr die Aushandlungsprozesse der politischen Eliten, die dazu führen, dass bestimmte Vorschläge umgesetzt und andere abgelehnt werden (vgl. für viele Linz/ Stepan 1996; Przeworski 1991; für Russland Campbell 2009; Gelman 2004; Gel man 2009).

Doch auch die liberaldemokratischen Grundannahmen, die diesen Analysen zugrunde liegen, sind nicht unumstritten, wenngleich die Kritik daran weniger reflexhaft ausfällt als im Fall des Effizienzarguments. Ihren stärksten Ausdruck findet die Kritik im Konzept der deliberativen Demokratie, das lokaler Partizipation ebenfalls eine prominente Position zuweist, sie jedoch grundsätzlich anders begründet. Das Gemeinwohl (»common good«) als Zielgröße des demokratischen Prozesses, das es zu erreichen respektive dem es sich anzunähern gilt, so die Idee, ergibt sich nicht aus der Aggregation präexistenter individueller Präferenzen, sondern formiert sich im Verlauf öffentlicher Debatten (»public reasoning«). Die inhaltliche Definition des Gemeinwohls wird damit zum Produkt von Interaktionen, die es ermöglichen, die eigenen Interessen im Spiegel der Interessen anderer zu beobachten - und vice versa: »[T]he institutional designs of modern democracy must be based on the >principle of reciprocity « (Held 2006: 233). Die zentrale Institution - und hier liegt ein wesentlicher praktischer Anknüpfungspunkt zur Idee der lokalen Selbstverwaltung - ist das Forum, in dem in lokalen Aushandlungsprozessen legitime und ratio- 
nale Entscheidungen herbeigeführt werden sollen (vgl. theoretisch Held 2006: 232ff; Stoker 1996: 198f; auch Benhabib 1996; Bohman/Rehg 1997; Elster 1997; Habermas 1997; als empirisches Beispiel aus dem Kontext der Entwicklungsforschung z.B. Schouten/Leroy/Glasbergen 2012).

\section{Politische Dezentralisierung und "empowerment"}

Sowohl das Effizienz- als auch das Demokratieargument betonen im Zusammenhang mit Dezentralisierungsprozessen und der Etablierung lokaler Selbstverwaltung die Entwicklung von Strukturen, welche die Artikulation und den Ausgleich individueller und potenziell divergierender Interessen ermöglichen sollen - im ersten Fall über den Markt, im zweiten über Partizipation. Eine dritte Perspektive unterscheidet sich von diesen beiden Argumentationssträngen in erster Linie durch die Radikalität ihrer Forderungen, indem sie bereits die Grundintention - das Schaffen von allgemein verbindlichen (Formal-)Strukturen - in Frage stellt. Stattdessen stellt sie lokale Aushandlungsprozesse und die eigenständige und autonome Verwaltung lokaler Gemeinschaften in den Mittelpunkt und knüpft damit zum Teil unmittelbar an die theoretischen Grundannahmen des Konzepts der deliberativen Demokratie an. Dreh- und Angelpunkt des Ansatzes, der sich unter dem Begriff des »empowerment « (oder der radikalen Demokratie) zusammenfassen lässt, im Kontext der kritischen Sozialwissenschaften anzusiedeln ist und große Resonanz im Umfeld sozialer Bewegungen findet, sind die entmächtigenden und unterdrückenden Strukturen von Staat und Markt: ${ }^{4}$ Regiert werde, so der Vorwurf, »im Medium der Freiheit« (vgl. z.B. Lessenich 2008: 83) und »at a distance« (Rose/Miller 1992: 180). >Neoliberale $<$ Dezentralisierungsprojekte, die vor allem mit weitreichenden Privatisierungsprozessen assoziiert werden, würden unter dem Deckmantel von Partizipation und Effizienzversprechen letztlich den Interessen des Privatsektors dienen und die staatlich initiierten Dezentralisierungsmaßnahmen seien primär »a Trojan horse that brings the power of private-sector interests into public decision-making « (Miraftab 2008: 21).

Angesichts dieser starken Kritik wird nach Möglichkeiten gesucht, um die beobachteten Machtstrukturen zu durchbrechen. Lokale Selbstverwaltung wird dabei als ein Mechanismus verstanden, der es insbesondere marginalisierten Bevölkerungsgruppen ermöglichen soll, eigene angepasste Entwicklungswege und Partizipationsformen zu finden (Gaventa 2002: 35, 2004: 20). Nicht von Zentralstaaten

4 Der Begriff des »empowerment« kommt auch in Projektbeschreibungen internationaler Organisationen vor, ist dort jedoch nicht kritisch konnotiert, sondern bezieht sich auf den formalen Transfer von Entscheidungskompetenzen und die Ausweitung von Partizipationsmöglichkeiten auf der lokalen Ebene, meist im Kontext von wirtschaftlichen Überlegungen und Armutsbekämpfung. 
oder internationalen Organisationen entworfene Top-down-Programme, sondern die Förderung ergebnisoffener Bottom-up-Initiativen - als Gegengewicht zu >Staat< und >Markt< - wird vor diesem Hintergrund als zentrales entwicklungspolitisches Instrument angesehen (Craig/Mayo 1995; Friedmann 1996). Lokale Foren und Aushandlungsprozesse werden gerade nicht als Unterbau staatlicher Institutionen betrachtet. Stattdessen werden sie als von oben vorgegebene Strukturen unter dem Schlagwort der radikalen Demokratie per se abgelehnt: »Empowerment is a matter of collective mobilisation of marginalised groups against the disempowering activities of both the state and the market « (Mohan/Stokke 2000: 248). Dem marktliberalen Effizienzargument mit der ihm inhärenten Tendenz zu global einheitlichen institutionellen Konfigurationen auf der lokalen Ebene wird das Konzept der Zivilgesellschaft entgegengesetzt, in dem, so Mohan und Stokke (2008: 545), vielfältige Formen von Identitätspolitik ihren Ausdruck finden und die Hegemonie des globalen ökonomischen Liberalismus und der zugehörigen politischen Institutionen herausfordern.

Während theoretische Argumentationen, die sich dieser radikaldemokratischen Perspektive zuordnen, meist den Charakter von Forderungen haben und normative Setzungen darüber vornehmen, wie die Lokalverwaltung aussehen sollte, nehmen die überwiegend qualitativ konzipierten empirischen Arbeiten in der Regel einen der zwei folgenden Foki ein: Entweder zielen empirische Studien darauf, Missstände im Kontext konventioneller - und das heißt: top-down initiierter und staatlicher gelenkter - Dezentralisierungsprozesse aufzuzeigen, die dann meist auf die bestehenden (globalen) Machtverhältnisse zurückgeführt werden (für das Fallbeispiel der Müllentsorgung in Kapstadt vgl. Miraftab 2004). Oder es werden Beispiele für die Ermächtigung lokaler Gemeinschaften und Prozesse der Strukturanpassung in Folge lokaler (Bottom-up-)Aushandlungen nachgezeichnet. Die Beobachtung reicht dabei sowohl über den politisch-administrativen Bezugsrahmen als auch über ein vordefiniertes Set an (formalen) Institutionen hinaus und schließt potenziell eine große Bandbreite an Akteuren und gesellschaftlichen Bereichen ein. Neben >klassischen< Verwaltungsaspekten geraten auf diese Weise auch Aushandlungsprozesse über Produktionsformen, die Verteilung von Verfügungsrechten über Ressourcen, Regime der Gesundheitsversorgung oder Bildung und andere in den Fokus (vgl. für viele die Beispiele in Fung/Wright 2001; García-López/Arizpe 2010; Heller 2001; Jaglin/Repussard/Belbéoch 2011).

\section{Was wird gesehen - und was nicht?}

Diese Tour d'Horizon ließe sich an verschiedenen Stellen erweitern und vertiefen, soll jedoch in dieser Form genügen, um das Perspektivenspektrum auf Dezentralisierung und lokale Selbstverwaltung zu skizzieren. Die Berechtigung jedes einzel- 
nen dieser Ansätze möchte ich ebenso wenig in Frage stellen wie den Erkenntnisgewinn, den sie jeweils mit Blick auf ihre spezifische Problemstellung ermöglichen. Dessen ungeachtet möchte ich aber abschließend aus einer soziologischen Perspektive nicht nur auf die Reichweiten der verschiedenen Zugänge blicken, sondern auch ihre Grenzen ausloten und auf einige Aspekte verweisen, die ungesehen bleiben.

Die beiden erstgenannten Ansätze, das Effizienz- und das Demokratieargument, bilden vor allem im Hinblick auf die durch sie inspirierten empirischen Studien den quantitativen Löwenanteil der wissenschaftlichen Beobachtungen zum Thema. Unabhängig von ihrer unterschiedlichen disziplinären Verortung - Effizienzargumente sind in der Regel mit einer wirtschaftswissenschaftlichen, Demokratieargumente mit einer politikwissenschaftlichen Perspektive verknüpft - weisen beide Ansätze eine architektonische Gemeinsamkeit auf: Politische Dezentralisierung und lokale Selbstverwaltung werden in beiden Fällen im Horizont von Zweck-Mittel-Beziehungen beobachtet. Jeweils für sich genommen oder komplementär zueinander dienen sie dazu, die Allokationseffizienz öffentlicher Dienstleistungen respektive die Verwendungseffizienz öffentlicher Mittel zu steigern oder demokratische Strukturen zu festigen respektive Demokratiedefizite sichtbar zu machen. Die jeweiligen Zwecke sind dabei stets unhintergehbar und dies kontrolliert unweigerlich den Blick der empirischen Analysen. Mit Blick auf wirtschaftliche Überlegungen geht es letztlich um die Analyse des Zusammenhangs von administrativen Formalstrukturen und ökonomischen Kennzahlen; im Rahmen des Demokratiearguments erfolgt in erster Linie ein Abklopfen auf die (formale) Übernahme bestimmter Standards vor dem Hintergrund von Demokratiemodellen und daraus abgeleiteten Verfahrensweisen (beispielsweise für das Zustandekommen spezifischer institutioneller Designs). Grundlegend ist dabei ein Verständnis von Dezentralisierung und lokaler Selbstverwaltung, das in erster Linie politisch-administrative Selbstbeschreibungen spiegelt. Zu erkennen ist dies zum Beispiel daran, dass die Begrifflichkeiten kaum umstritten sind und bestenfalls die Vielzahl an Abgrenzungen und Kategorien beklagt wird, die sich an dieses Grundverständnis anschließen, sich bei näherem Hinsehen jedoch oft nur in Nuancen unterscheiden. Auch die verwendeten ZweckMittel-Beziehungen folgen den gängigen politischen Argumenten, die zur Begründung und Legitimation von Maßnahmen zur Dezentralisierung und Stärkung lokaler Selbstverwaltung ins Feld geführt werden. So beobachten beispielsweise Brunsson und Olsen (1997) am Beispiel des öffentlichen Sektors in Norwegen und Schweden, dass Reformen in den 1970er Jahren mit dem Verweis auf Demokratisierung durchgeführt wurden, während seit den späten 1980er Jahren zunehmend Effizienzsteigerung als Begründung fungierte (vgl. auch Hasse/Krücken 2005: 39).

Die Form der Komplexitätsreduktion, die auf diese Weise im Rahmen der wissenschaftlichen Beobachtung vorgenommen wird, entspricht derjenigen der Politik 
und diese Kopplung der Beobachtungsrahmen markiert folglich den potenziellen Erkenntnisgewinn, aber auch die Grenzen des damit Sichtbaren: Für die politische Praxis, die ein Großteil der Analysen, darunter viele explizite Implementationsstudien, im Blick hat, erscheinen die skizzierten Vorgehensweisen durchaus angemessen, um das Erreichen politischer Ziele zu überprüfen - die Übernahme formaler Standards zur Einrichtung demokratischer Strukturen respektive die Steigerung von Effizienz im öffentlichen Sektor. In diesem Sinne spiegeln, überprüfen oder kritisieren die Analysen die entsprechenden Leitbilder, Modelle und Legitimationsstrategien. Daran anschließend lässt sich beispielsweise fragen, unter welchen Bedingungen bestimmte Formen von Dezentralisierung in einzelnen nationalen Kontexten Berücksichtigung gefunden haben, ob und auf welche Weise internationale Organisationen und deren Forschungsabteilungen als Katalysatoren für die Verbreitung dieser Leitbilder wirken oder inwieweit die unterstellten Zweck-MittelBeziehungen Ausdruck globaler Rationalisierungsbestrebungen sind, die letztlich zu einer formal-strukturellen Angleichung von Nationalstaaten führen (vgl. dazu Meyer et al. 2005 [1997]).

Aus dem zugrundeliegenden Verständnis von Dezentralisierung und lokaler Selbstverwaltung und aus den Kausalzusammenhängen, in die beide Phänomene eingebettet werden, folgt aber auch, dass die Beobachtung auf das politisch Anvisierte begrenzt wird. Denkbares Resultat der Analysen ist die Bestätigung oder die Kritik (möglicherweise im Hinblick auf Modifikationen) der politischen Ziele. Letztere selbst sind für diese Art der Beobachtung jedoch unhintergehbar. Schlussendlich handelt es sich damit bei den skizzierten Ansätzen um Selbstbeschreibungsvarianten des politischen Systems, die sich durch ihre Anschlussfähigkeit an die im System verwendeten Semantiken als solche ausweisen (zu einem solchen Befund in ähnlichem Kontext vgl. Japp/Kusche 2004: 513ff). Auf eine dazu inkongruente Perspektive in Form einer (soziologischen) Fremdbeschreibung, wie Luhmann (1984: 88) sie als Voraussetzung für wissenschaftliche Erkenntnisse über die Gesellschaft einfordert, wird verzichtet. Im Unterschied zu Selbstbeschreibungen lösen sich Fremdbeschreibungen von den systemeigenen Begrifflichkeiten und Kategorien ab und bewirken eine Distanzierung vom System, welche wiederum gerade durch diese Abweichung »für dieses überraschende, irritierende und für sein Operieren gerade nicht anschlussfähige Aussagen erlaubt« (Japp/Kusche 2004: 514).

Ein zweiter Aspekt bleibt aufgrund der in beiden Perspektiven jeweils gängigen Konzeptionen empirischer Studien ungesehen: Dezentralisierung und lokale Selbstverwaltung werden in der Regel über Indikatoren operationalisiert, die auf formalen (Gesetzes-)Regelungen basieren. Für die Möglichkeit der Entkopplung von formalen Regelungen und Praktiken, die für Meyer et al. (2005 [1997]: 100) Teil der Logik des Kopierens von (globalen) Modellen ist und die auf Schlagworte wie »Fas- 
sadenmodernisierung« oder die »Hinterbühne der Weltgesellschaft« verweist (vgl. dazu Holzer 2006), bleibt die Mehrzahl der Studien auf diese Weise blind. Derartige Entkopplungsprozesse sprechen beispielsweise Dubois und Fattore (2009: $717 \mathrm{ff})$ mit ihrem Konzept der »silent decentralization « an, wobei sie davon ausgehen, dass sich die Dezentralisierung von Entscheidungskompetenzen durchaus auch abseits formaler Regelungen oder sogar als deren unbeabsichtigter Effekt vollziehen kann. Diese Spur verfolgen die beiden Autoren jedoch weder theoretisch noch empirisch weiter. Ohne das - intendierte oder unintendierte - Abweichen der Praktiken von den formalen Regelungen von vornherein unterstellen zu wollen, lässt sich vor diesem Hintergrund festhalten, dass die meisten empirischen Studien nur wenig darüber aussagen, welche Strukturänderungen und Irritationen in den Kommunikationszusammenhängen der Gemeinden infolge von Dezentralisierungsprozessen und Kommunalreformen faktisch induziert werden (oder auch nicht). Und auch die Umwelt lokaler Selbstverwaltung bleibt nicht nur theoretisch unberücksichtig, sondern gerät (dadurch) empirisch gar nicht erst in den Fokus. Erhellen lässt sich dieser Aspekt nur durch entsprechend konzipierte empirische Forschungsdesigns. Die Anzahl an Arbeiten, die um eine entsprechend erweiterte Fragestellung und empirische Perspektive zu den Effekten von Verwaltungsdezentralisierung bemüht sind, ist jedoch überschaubar. Regional am stärksten vertreten sind hier Arbeiten zu Lateinamerika, von wo auch eines der prominentesten Beispiele überhaupt stammt, die Einführung einer partizipativen Budgetplanung in der brasilianischen Stadt Porto Alegre (vgl. dazu z.B. Abers 1998; Avritzer 2002; Gaventa 2004; UNDESA 2008: 75ff; Wampler 2007). Russland respektive die Staaten der ehemaligen Sowjetunion blieben bislang weitgehend unbeachtet, obwohl auch diese seit dem Zusammenbruch der kommunistischen Regime auf einen zum Teil sehr wechselvollen Prozess formal-rechtlicher De- und Rezentralisierung zurückblicken können (vgl. S. 133ff).

Das Verständnis von Dezentralisierung und lokaler Selbstverwaltung und die Einbettung in Zweck-Mittel-Beziehungen, auf die das Effizienz- ebenso wie das Demokratieargument verweisen, so lässt sich resümieren, verstellen allzu leicht den Blick auf die Phänomene an sich. Für eine Annäherung an Letztere wären Dezentralisierung und lokale Selbstverwaltung aus den üblichen Beobachtungsrahmen herauszunehmen. Die wissenschaftliche Beobachtung müsste hinter die in der politischen Praxis unhintergehbaren Zwecke zurücktreten und einen Blickwinkel quer zu den politisch-administrativen Kategorien einnehmen. Ansatzweise geschieht dies in Arbeiten, die sich der dritten der skizzierten Perspektiven zuordnen, dem (radikaldemokratischen) »empowerment «-Argument. Im Vergleich mit den beiden erstgenannten Perspektiven wird hier der Fokus in mehrfacher Hinsicht ausgeweitet: Strukturbildungs- und Strukturanpassungsprozesse als Resultat lokaler Aushandlungen werden nicht im Horizont ex ante festgelegter Indikatoren, sondern betont 
ergebnisoffen beobachtet und über Politik und Verwaltung hinaus gerät potenziell eine Vielzahl an gesellschaftlichen Bereichen in den Blick. Wieder eingefangen wird diese Offenheit allerdings durch eine explizit normative Grundhaltung, in deren Kontext die Distanzierung von politisch-administrativen Kategorien in eine Ablehnung übergeordneter Strukturen per se umschlägt - was auch den Blick auf das empirische Material prägt. Der Fokus liegt stets auf Herrschafts- und Ausbeutungsverhältnissen, die es zu durchbrechen gilt, und die Lage ist zu kritisieren, sobald Markt und Staat mit im Spiel sind. Im Kern findet sich also auch hier eine teleologische Überzeugung, wenn auch auf vollkommen andere Art als im Falle des Effizienz- und des Demokratiearguments.

\section{EINE DIFFERENZIERUNGSTHEORETISCHE ANNÄHERUNG}

Auf explizite oder implizite Zielvorstellungen und normative Setzungen darüber, wie politische Strukturen gestaltet sein sollen, möchte ich im Folgenden verzichten und gleichzeitig Gesellschaft - und das heißt hier: Politik und Verwaltung und ihre Umwelten - im Blick behalten. Dezentralisierungsprozesse und die Einführung lokaler Selbstverwaltung sind dann, wenn auch von spezifischen politischen Maßnahmen angestoßen und mit spezifischen Selbstbeschreibungen verknüpft, Teil gesellschaftlicher Evolutionsprozesse. Aber was und woraufhin wird beobachtet, wenn sowohl politische Zielvorstellungen wie Demokratie und Effizienz als auch kritisch inspirierte normative Setzungen über die Gestaltung und die Effekte lokaler Selbstverwaltung als Anhaltspunkte entfallen? Mit anderen Worten: Mit welchen Kommunikations- und Beobachtungsformen haben wir es zu tun, wenn von lokaler Selbstverwaltung die Rede ist und welche strukturellen Änderungen werden unterstellt, wenn sich Dezentralisierungsprozesse vollziehen oder eingefordert werden? Im Hinblick auf diese Überlegungen möchte ich mich im Folgenden den Kernkonzepten politische Dezentralisierung und lokale Selbstverwaltung aus soziologischer Perspektive annähern und ihren Gehalt aus der Eigenlogik des politischen Systems ableiten. Mit Hilfe des differenzierungstheoretischen Instrumentariums sollen auf diese Weise möglichst präzise und abstrakte Begriffe formuliert werden, aus denen sich abschließend Leitaspekte für die empirische Analyse ableiten lassen.

\section{Autonomiekonzessionen im politischen System}

Einen ersten Anhaltspunkt für eine differenzierungstheoretische Annäherung an die Phänomene politische Dezentralisierung und lokale Selbstverwaltung bieten Luhmanns Überlegungen zum politischen System und seinen internen Differenzierungen. Neben Nationalstaaten als Segmente des politischen Systems auf globaler Ebe- 
ne benennt Luhmann zwei weitere Differenzierungsformen: Funktional differenziert sich das politische System erstens in die Teilsysteme Politik und Verwaltung, die sich im Hinblick auf ihren Beitrag zur Funktion des politischen Systems, dem Bereithalten von Kapazität zu kollektiv bindendem Entscheiden, unterscheiden lassen (siehe auch S. 56ff). In der Politik werden Entscheidungen vorbereitet, d.h. »[p]olitisches Handeln dient im wesentlichen dazu, die Komplexität der gesellschaftlichen Möglichkeiten so weit zu reduzieren, daß verbindlich und ohne das Risiko erheblichen Widerstands entschieden werden kann« (Luhmann 2010: 126). In der Verwaltung geht es um die Durchführung und Umsetzung dieser Entscheidungen, sie »befaßt sich mit der Ausführung des politisch Möglichen und Notwendigen durch Ausarbeitung verbindlicher Entscheidungen nach Maßgabe schon festliegender (heute im allgemeinen positiv entschiedener) Entscheidungsprämissen « (Luhmann 2010: 126). Eine zweite interne Differenzierungsform des politischen Systems ist inhaltlich in weiten Teilen deckungsgleich mit der Differenzierung in die Teilsysteme Politik und Verwaltung. Gemeint ist die Differenzierung von politischem Zentrum und Peripherie. Luhmann führt sie auf die Frage zurück, wo im System kollektiv bindende Entscheidungen getroffen werden - und dies geschieht ausschließlich im Zentrum, während in der Peripherie die Vorbereitung der Entscheidungen unter anderem in Form »des Austestens von Unterstützung für noch unverbindliche Themen und Beiträge « stattfindet (Japp/Kusche 2004: 518; auch Luhmann 2000b: 246). Alle Bereiche des politischen Systems, Verwaltung und Politik respektive Zentrum und Peripherie, sind auf Organisationen angewiesen: Im Zentrum befinden sich üblicherweise die Staatsorgane (Regierung, Parlament, Ministerialbürokratie), die als Orientierungspunkt aller sonstigen politischen Organisationen fungieren und »für die alle anderen politischen Organisationen dann Zulieferungsdienste erbringen« (Luhmann 2000b: 245). Und auch politisch gemeinte Kommunikation, die größtenteils in der Peripherie respektive der Politik stattfindet, ist nicht zwingend, faktisch aber meist an Organisationen gebunden, darunter Parteien, Interessengruppen, Bürgerverbände und ähnliches.

Der Vorteil dieser spezifischen Form interner Differenzierung liegt »gerade darin, daß sie im Zentrum andere Formen der Differenzierung ermöglicht als in der Peripherie und darauf verzichten kann, diese unterschiedlichen Differenzierungsformen über ein eindeutiges Rangverhältnis miteinander zu koppeln« (Luhmann 2000b: 251). Das Zentrum respektive die Verwaltung ist hierarchisch strukturiert die Staatsorgane sind über Weisungs- und Machtbeziehungen miteinander verknüpft -, was notwendigerweise mit einem begrenzten Maß an Komplexitätsverarbeitungskapazität einhergeht. Die Hierarchie des Zentrums ist deshalb nur aufgrund der segmentären Struktur der Peripherie aufrecht zu erhalten, wodurch die »Wahrung höherer Komplexität und Unkoordiniertheit« (Luhmann 2000b: 251) ermöglicht wird: Es gibt stets mehrere politische Parteien, mehrere Interessenorganisatio- 
nen, mehrere Bürgerinitiativen und ähnliches, die dafür sorgen, dass das Zentrum im Hinblick auf die zu treffenden Entscheidungen mit vorreduzierter Komplexität versorgt wird - was jedoch wohlgemerkt nicht mit Eindeutigkeit gleichzusetzen ist: Das Zentrum wird »aus der Peripherie mit einer Fülle von inkonsistenten Entscheidungsanforderungen überschüttet « (Luhmann 2000b: 247), die es dann in kollektiv bindende Entscheidungen zu überführen gilt und für deren Umsetzung gesorgt werden muss.

Dezentralisierungsprozesse setzen an diesen Formen interner Differenzierung des politischen Systems an, indem zunächst durch die territoriale Segmentierung innerhalb von Nationalstaaten Verwaltungseinheiten neu geschaffen respektive bereits existierende, historisch gewachsene Einheiten wie Provinzen, Regionen, Gemeinden und ähnliche aufgewertet werden, die sich - wie auch Nationalstaaten untereinander - über ihre räumlichen Grenzen von anderen Verwaltungseinheiten abgrenzen. Innerhalb des nationalen politischen Systems werden durch den Transfer entsprechender Entscheidungskompetenzen für diese Einheiten als eine Art Abbild der oben skizzierten Strukturen >im Kleinen< lokale Verwaltungen eingerichtet respektive Zentren konstituiert. Bezogen auf ein spezifisches Territorium und mit Blick auf bestimmte sachliche Bereiche werden die dort anfallenden Probleme eigenständig bearbeitet und nach eigenem Ermessen kollektiv bindende Entscheidungen getroffen.

Indem politische Entscheidungskompetenzen von der nationalstaatlichen Ebene an die lokalen Verwaltungsinstitutionen übertragen werden, wird die hierarchische Struktur des politischen Zentrums aus sich heraus durchbrochen (Dubois/Fattore 2009: 710; Pratchett 2004: 362). Ein Clou lokaler Selbstverwaltung besteht zumindest dem Anspruch nach - gerade darin, die Sphäre kommunaler Verwaltung als einen von der staatlichen Verwaltung distinkten Bereich einzurichten, indem der Zentralstaat Autonomie konzediert (zum Autonomiebegriff vgl. Stichweh 2009a: 44; auch Willke 1987: 341). Diese Autonomie bemisst sich unter anderem danach, inwieweit die lokalen Institutionen bei konkreten Sach- oder Personalfragen vom Einfluss der verwaltungsinternen Umwelt absehen können respektive inwieweit sie unterschiedliche Umweltabhängigkeiten pluralisieren und ihnen nach eigenem Ermessen Bedeutung für die eigenen Operationen zuweisen können. Das ungebrochene, unmittelbare oder mittelbare Hineinwirken zentralstaatlicher Institutionen in lokale Prozesse kollektiv bindenden Entscheidens ist ausgeschlossen. Entscheidungen der Lokalverwaltung können nicht mehr ohne weiteres von anderen administrativen Ebenen überrollt werden und das politische Zentrum beschränkt sich bestenfalls auf deren Ratifikation, fungiert jedoch nicht als übergeordnete Letztentscheidungsinstanz. Die hierarchiekonstitutive Differenzierung von oben und unten (Baecker 1999: 200f) wird so für einzelne Teilbereiche gelockert oder sogar vollständig aufgelöst. Als Resultat von Dezentralisierungsprozessen sind lokale Verwaltungsorga- 
ne nicht mehr vollständig in hierarchische Strukturen eingegliedert, sondern einem zunehmend kleinen Teil ihrer verwaltungsinternen Umwelt untergeordnet, dessen Bedeutung im Verhältnis $\mathrm{zu}$ anderen Umweltbereichen gesunken ist (Brunsson/Sahlin-Andersson 2000: 723). Bezeichnungen wie national, regional und lokal verweisen in diesen Bereichen dann nur noch auf unterschiedliche Zuständigkeiten, jedoch nicht mehr auf Weisungsverhältnisse.

Zumindest ihrem formalen Anspruch nach impliziert die Einführung lokaler Selbstverwaltung, dass sich die neu geschaffenen lokalen politischen Zentren als Kommunikationszusammenhänge schließen und damit als solche überhaupt erst in Erscheinung treten: Lokale Machtkommunikation schließt an lokale Machtkommunikation an und die Entscheidungen lokaler Verwaltungsorgane werden zu den primären Prämissen für lokale Anschlussentscheidungen. Für die internen Strukturen des politischen Systems bedeutet dies, dass sich durch Dezentralisierungsprozesse und die Etablierung oder Stärkung lokaler Selbstverwaltung die Struktur des Zentrums sukzessive in Richtung eines Netzwerks wandelt. Eine Entwicklung, die die Politikwissenschaft als Übergang von »government« zu »governance« bezeichnet (vgl. z.B. Sørensen/Torfing 2005). Der Staat wird zu einem zunehmend polyzentrischen Gebilde aus verschiedenen Teilorganisationen, die nicht mehr ausschließlich über Weisungsverhältnisse aneinander gekoppelt sind, sondern miteinander in Form von Wettbewerb, Beratung, Kooperation oder wirtschaftlichen Transaktionen in Verbindung treten (Brunsson/Sahlin-Andersson 2000: 730). Eine solche Strukturänderung haben letztlich auch die Verwaltungswissenschaften im Blick, wenn von der Trennung von kommunaler und staatlicher Verwaltung die Rede ist oder diese bei der Analyse von Reformen des öffentlichen Sektors mit unterschiedlicher Vehemenz eingefordert wird (vgl. z.B. die Diskussion um Verstaatlichungstendenzen im deutschen System kommunaler Verwaltung in Wollmann 2010, 2013: 179ff; für entsprechende Bedenken im Hinblick auf Russland vgl. z.B. Lankina 2005; Ross 2006, 2009; Wollmann/Gritsenko 2009).

\section{Organisationsbildung zwischen Agent und Akteur}

Ein wesentlicher Aspekt von Dezentralisierungsprozessen und der Einführung lokaler Selbstverwaltung ist die Bildung von Organisationen. Entscheidungen über Angelegenheiten lokaler Bedeutung werden den lokalen Verwaltungsorganen wie Bürgermeisterinnen und Bürgermeistern, Gemeinderäten oder Gemeindeversammlungen übertragen, die zu diesem Zweck und im Hinblick auf ihre Aufgaben entweder neu eingerichtet oder auf die veränderten und erweiterten Entscheidungskompetenzen ausgerichtet werden müssen. Die (Teil-)Organe lokaler Verwaltung werden mit eigenen Identitäten ausgestattet, mit Rationalitätszuschreibungen konfrontiert und es werden interne Koordinationsmechanismen etabliert (Brunsson/Sahlin-Anders- 
son 2000: 723ff). Indem sie sich über Entscheidungen reproduzieren, die an eigene Entscheidungen anschließen, einen Zweck haben und ihre Grenze über die Unterscheidung von Mitgliedern und Nicht-Mitgliedern beobachten, werden die einzelnen Verwaltungsorgane schließlich als Organisationssysteme abgrenzbar - oder kurz: »The [public sector, E.M.] reforms can thus be described as a way of turning public services into organizations or at least into something closer to this than before « (Brunsson/Sahlin-Andersson 2000: 723).

Subnationalen (regionalen oder lokalen) Ebenen werden die Entscheidungskompetenzen dabei stets von >oben<, also von der Regierung und durch entsprechende Gesetze und Rechtsakte zugesprochen und können ihnen potenziell auf demselben Wege auch wieder entzogen werden. ${ }^{5}$ Aus nationalstaatlicher Sicht werden Dezentralisierungsprozesse vor diesem Hintergrund stets auch vom Risiko des Zerfalls der eigenen Einheit begleitet, wecken einmal gewährte Selbstbestimmungsrechte doch potenziell immer weitergehende Sonderstatus- und im Extremfall Sezessionsforderungen subnationaler Kollektive (vgl. empirisch z.B. Cunningham 2014). Zwar können für Rechenschaftsverpflichtungen der lokalen und regionalen Ebenen, Interventionsmöglichkeiten des Zentralstaats und ähnliches, die im Hinblick auf die Begrenzung subnationaler Autonomie etabliert werden, unterschiedliche Abstraktionsgrade gewählt und Freiheitsgrade entsprechend zugestanden werden, die Abgabe von Entscheidungskompetenzen an sich bleibt jedoch aus der Logik politischer Dezentralisierung heraus unverzichtbar.

Lokalverwaltungen lassen sich in diesem Zusammenhang und je nach Reichweite und inhaltlicher Ausgestaltung von Dezentralisierungsmaßnahmen als Hybride zwischen Akteurs- und Agentenorganisationen beschreiben (für die öffentliche Verwaltung und in Anlehnung an Weber vgl. Brunsson/Sahlin-Andersson 2000: 731ff; zur Unterscheidung von Akteuren und Agenten in der modernen Gesellschaft auch Meyer/Boli/Thomas 2005 [1987]; Meyer/Jepperson 2005 [2000]): Ebenso wie individuellen Akteuren werden Akteursorganisationen Unabhängigkeit und Souveränität zugeschrieben sowie die Fähigkeit, eigene Ziele zu setzen und diese anhand rationaler Mittel und durch den Einsatz selbst kontrollierter Ressourcen eigenverantwortlich zu verfolgen - kurz: »An actor is able to act «(Brunsson/Sahlin-Andersson 2000: 731). Demgegenüber sind Agentenorganisationen in erster Linie ausführende Organe, deren Zwecke und Aufgaben von außen vorgegeben werden. Indem bestimmte Umweltabhängigkeiten auf diese Weise für sie unhintergehbar sind, verfügen sie über geringere Autonomie als die Akteursorganisationen. Zudem wird

5 Interessanterweise scheint einmal gewährte subnationale Autonomie aber nur in Ausnahmefällen wieder zurückgenommen zu werden, so dass sich ein globaler Trend in Richtung einer stärkeren Regionalisierung der Politik relativ robust beobachten lässt (siehe z.B. Hooghe/Marks/Schakel 2010). 
den Agenten im Unterschied zu den Akteuren keine Existenzlegitimation >aus sich heraus « und unabhängig von den ihnen zugeteilten Aufgaben zugeschrieben: »The survival of an agent is not a legitimate interest « (Brunsson/Sahlin-Andersson 2000: 733). Das bedeutet: Stoppregeln für das Operieren von Agenten sind Entscheidungen, die in der Umwelt getroffen werden und deren Umsetzung erfolgt, ohne dass eigene Entscheidungen dazwischen geschaltet wären.

Die Agentschaft lokaler Verwaltungen besteht in diesem Zusammenhang vor allem darin, dass der Organisationszweck, d.h. die zu erfüllenden Aufgaben, grundsätzlich vorgegeben sind - wenn auch Freiheitsgrade offen bleiben - und die Existenz der kommunalen Verwaltungsorgane daran gekoppelt ist. Gleichzeitig besteht die Idee lokaler Selbstverwaltung jedoch gerade darin, dass innerhalb dieses Rahmens Akteursqualitäten zum Tragen kommen (beispielsweise in Form sogenannter kommunaler Hoheitsrechte): Die Kommunen verfügen über Entscheidungsfreiräume bei der Ausführung spezifischer Aufgaben, was (begrenzte) Kontrolle über die Verwendung ihrer Ressourcen oder die Gestaltung ihrer internen Struktur einschließt, beispielsweise im Hinblick auf die Einrichtung von Verwaltungsstellen. Sie handeln dabei eigenverantwortlich und ihnen wird Rationalität im Hinblick auf ihre Zwecke unterstellt (und in diesem Sinne irrationale Entscheidungen erfordern in der Regel gesonderte Rechtfertigung). Darüber hinaus sind auch Personalentscheidungen über die Besetzung der Stellen eine lokale Angelegenheit, sei es in Form von Wahlen oder von Personalentscheidungen der lokalen Behörden.

\section{Warum Dezentralisierung?}

Aus welchen Dynamiken des politischen Systems heraus lässt sich erklären, dass sich lokale Selbstverwaltung in der Gegenwart als Standardmerkmal moderner Nationalstaaten etabliert hat und Dezentralisierungsprozesse einen kaum mehr wegzudenkenden Schwerpunkt internationaler Entwicklungszusammenarbeit bilden? Aus systemtheoretischer Perspektive erscheinen zwei Beweggründe naheliegend, die erstens auf das Gemeinwohl als Konstruktion und Legitimationsformel der Politik und zweitens auf einen gesellschaftsweiten Drang in Leistungsrollen und damit auf die soziale Umwelt des politischen Systems verweisen.

Über die Bindung an das Gemeinwohl, das politisch von seinem Gegenbegriff, dem Privatinteresse, abzugrenzen ist, wird die Herrschaft eines Kollektivs über den Einzelnen legitimiert und der kontinuierliche Bedarf an kollektiv bindenden Entscheidungen - und damit an Politik - gerechtfertigt (Luhmann 2000b: 120ff; auch Fuhse 2005: 73f). In demokratischen Regimen schwingt in der Gemeinwohlorientierung der Verweis auf die Wählerschaft, also das Publikum des politischen Systems, mit, denn letztlich geht es hier in besonderem Maße um die öffentliche Darstellbarkeit der politischen Grenzziehung zwischen Gemeinwohl und Privatinteres- 
se sowie der Präferenzen, die die Politik als inhaltliche Grundlage ihrer Programme daraus ableitet. Will sich das politische System besser und das heißt: mit zunehmend höherer Auflösung an sein Publikum anpassen und steigt, wofür in der modernen Gesellschaft vieles spricht, parallel dazu noch die Komplexität jener Umwelt, auf die sich die politischen Steuerungsansprüche beziehen, folgt daraus nicht nur ein kontinuierlicher, sondern auch ein kontinuierlich steigender Bedarf an Entscheidungen. Sofern dieser Bedarf nicht unterdrückt wird (wie es in der sowjetischen Organisationsgesellschaft teilweise geschah), ist dies für die hierarchisch strukturierte Verwaltung respektive den Staat gleichbedeutend mit internem Wachstumsdruck - schließlich basieren Organisationssysteme auf Entscheidungen, die nun vermehrt anfallen.

Wachstum wiederum bedeutet die Steigerung der Komplexität und die Verschärfung der Selektivität der Organisation (Luhmann 2009 [1978]: 399). Dezentralisierung und lokale Selbstverwaltung lassen sich vor diesem Hintergrund nicht nur als Wachstumsformen beobachten, die auf den Imperativ der Gemeinwohlorientierung reagieren, sondern gleichzeitig als Strategien, um die steigende interne Komplexität der Verwaltung handhabbar zu machen. Durch die Einrichtung von autonomen Einheiten - im weitestgehenden Fall eine kommunale Verwaltung, die von staatlichen Verwaltungsstrukturen (weitgehend) losgelöst ist - innerhalb der Verwaltungshierarchie werden die Hierarchiespitze wie auch das System insgesamt entlastet und seine Komplexitätsverbeitungskapazität gesteigert.

Als normative Wachstumsregeln identifiziert Luhmann (2009 [1978]: 400f) in modernen Organisationen Rationalisierung und Demokratisierung: Rationalisierung meint dabei die Dekomposition von Entscheidungen in sachlicher Hinsicht. Entscheidungsprobleme werden in Einzelschritte zerlegt, um die Transparenz im Hinblick auf Alternativen zu erhöhen. Demokratisierung umfasst die Aufgliederung von Entscheidungen in der Sozialdimension. Es werden mehr Personen in den Entscheidungsprozess einbezogen, indem Entscheidungen über direkte oder indirekte Zustimmungsanforderungen in Subentscheidungen zerlegt werden. Beide Strategien, so Luhmanns Kritik, laufen auf weiteres Wachstum durch eine steigende Anzahl von Entscheidungen und damit auf weitere Komplexitätssteigerung hinaus, ohne dass die damit verbundenen Strukturprobleme mitrationalisiert respektive mitdemokratisiert werden. Vor allem Letzteres trifft auch auf die oben skizzierten Dezentralisierungsdiskurse zu: Beide Wachstumsregeln finden sich hier als dominierende Prinzipien wieder, ohne dass dies weiter reflektiert werden würde.

Politische Dezentralisierung und die Konzession von Autonomie an lokale Verwaltungsinstitutionen lässt sich darüber hinaus als Reaktion auf ein Umweltphänomen deuten, das eng mit dem Prozess der Ausdifferenzierung von Funktionssystemen verknüpft ist und das folglich nicht allein die Politik, sondern alle gesellschaftlichen Funktionssysteme in ähnlicher Weise betrifft. Die Ausdifferenzierung 
gesellschaftlicher Funktionssysteme erfolgt typischerweise als dreiteilige Sequenz: Zunächst vereinzelt anfallende Situationen spezialisierter Kommunikation werden zum Ansatzpunkt für die Entstehung spezialisierter (Leistungs-)Rollen, die jeweils auf spezifische Bezugsprobleme verweisen und entsprechende Kommunikationszusammenhänge stabilisieren. Komplementär zu den Leistungsrollen etablieren sich schließlich Publikumsrollen, die Vollinklusion - die Inklusion aller in alle Funktionssysteme - erwarten lassen, dadurch die soziale Relevanz der Funktionssysteme sichern und sie in Weltsysteme transformieren (Stichweh 2005 [1988], 2005 [1998]). Auch wenn sich Leistungs- und Publikumsrollen wechselseitig bedingen, implizieren die mit dieser Unterscheidung einhergehenden Rollenerwartungen eine Asymmetrie, die die Leistungsrollen im Hinblick auf Handlungs- und Aktivitätschancen begünstigt, während vom Publikum tendenziell Erleben erwartet wird (es beobachtet oder wird betreut).

Diese Asymmetrie kollidiert jedoch zunehmend mit dem »kontemplationsskeptischen, aktivistischen Wertmuster der Moderne« (Stichweh 2005 [1988]: 34), das Selbstbestimmung zum Zentralwert erhebt und die Partizipation an Handlungszusammenhängen gegenüber der Beobachtung bevorzugt. Der »Aufstand des Publikums « (Gerhards 2001) und sein Drang in Leistungsrollen gewinnt in den 1960er Jahren an Dynamik und setzt auch in der Politik Anpassungsprozesse in Gang: Auf der Output-Seite etabliert sich die Idee des aktiven Bürgers, an dessen Interessen sich vor allem die Verwaltung zu orientieren hat; auf der Input-Seite etablieren sich unkonventionelle Partizipationsformen wie die Teilnahme an Demonstrationen oder Unterschriftenlisten, während beispielsweise die Wahlbeteiligung als konventionelle Partizipationsform tendenziell sinkt (Gerhards 2001: 175f). Diese neuen Partizipationsformen mögen momenthaft, spontan und unverbindlich sein (vgl. Blühdorn 2013: 190ff; Stoker 2006: 87ff), lassen aber auch den Wunsch nach unmittelbarer Beteiligung in politischen Entscheidungsprozessen erkennen.

Kommunale Selbstverwaltung ist vor diesem Hintergrund als eine Strukturform zu erkennen, die diese Funktion erfüllt, indem auf der lokalen Ebene zusätzliche und tendenziell niederschwellig zugängliche Leistungsrollen geschaffen werden (in Form von Bezirksparlamenten, Lokalverwaltungen, Gemeinderäten u.ä.), die dem Partizipationsdrang des Publikums ein Ventil bieten. Hinzu kommen lokale Modi der Publikumsinklusion, die es der Politik ermöglichen, Publikumsäußerung nuancierter zu registrieren und prozessieren, als dies auf der nationalen Ebene möglich wäre, ohne das System zu überlasten (siehe unten). Im Ergebnis führt Dezentralisierung zur Ausweitung von politischen Handlungsmöglichkeiten, vervielfältigt die Formen politischer Inklusion und steigert die Fähigkeit der Politik zur Komplexitätsverarbeitung. 


\section{Die lokale Politik und ihr Publikum}

Die skizzierten politikinternen Dynamiken, die auf Dezentralisierung und die Einrichtung lokaler Selbstverwaltung hinwirken, verweisen zwingend auf das Publikum der Politik: Staatlich initiierte Dezentralisierungsprozesse und ihre wissenschaftliche Beobachtung fokussieren in erster Linie die Formalstrukturen der lokalen Verwaltung und damit das (lokal-)politische Zentrum. Eine ähnliche Perspektive nimmt auch die Beobachtung von Dezentralisierung und der Einführung lokaler Selbstverwaltung als Prozess der Organisationsbildung ein, die darauf hinausläuft, dass sich kommunale Verwaltung respektive lokale Politik - mit Blick auf ein spezifisches Territorium und spezifische Inhalte - als geschlossener Kommunikationszusammenhang etabliert, ihre Umweltabhängigkeiten pluralisiert und es selbst übernimmt, den verschiedenen Anlehnungskontexten und Leistungserwartungen bei konkreten Entscheidungen Bedeutung zuzuschreiben. Gleichzeitig ist aber die Ausweitung von Partizipationsmöglichkeiten und die Forderung nach höherer Reaktionsfähigkeit und -bereitschaft der Verwaltungsorgane im Hinblick auf die Bürgerpräferenzen und nach gesteigerter Verantwortlichkeit gegenüber der lokalen Öffentlichkeit - kurz: die Aufwertung des Publikums als spezifisches Umweltsegment - wesentlicher Bestandteil von Dezentralisierungsprozessen gerade auch im entwicklungspolitischen Kontext (vgl. z.B. Shah 2006a).

In systemtheoretischen Beschreibungen des politischen Systems kommt das Publikum und seine Einbindung in politische Kommunikationen in vierfacher Weise zur Sprache: Stichweh (2005: 78) beobachtet zwei direkte Formen der politischen Publikumsinklusion, die letztlich »im Konzept der Staatsbürgerschaft homogenisiert und kodifiziert « werden. Publikumsinklusion vollzieht sich demnach erstens über das aktive und passive Wahlrecht. Die Tatsache, dass auf diese Weise das Publikum sowohl in die Leistungs- als auch in die Komplementärrollen der Politik inkludiert werden kann, ist eine Besonderheit des politischen Systems, die Stichweh darauf zurückführt, dass der Zugang zu politischen Leistungsrollen meist keine Professionalisierung voraussetzt, sondern Professionalisierung erst mit der Übernahme eines Amtes oder einer politischen Funktion einsetzt (Stichweh 2005: 75; zum Wahlrecht auch Luhmann 2000b: 253). Das passive Wahlrecht zeichnet sich dadurch aus, dass diese Beteiligungsform an der politischen Kommunikation im System nicht als individueller Äußerungsakt verstanden wird, sondern ausschließlich in aggregierter Form (also beispielsweise in Form von Wahlergebnissen) registriert und gedeutet wird. Für Politikerinnen und Politiker und Beamtinnen und Beamte als Leistungsrollen des Systems sind im Hinblick auf Wahlen allein die Mehrheitsverhältnisse relevant und beobachtbar, die dann bestimmte Anschlussmöglichkeiten eröffnen und andere unterbinden (vgl. Stichweh 2005: 23). Darüber hinaus benennt Stichweh (2005: 75f) noch eine zweite Form der politischen Publi- 
kumsinklusion, die auf der Zweitinterpretation moderner Demokratien als Sozialoder Wohlfahrtsstaaten basiert: Komplementär zu den staatlichen Leistungsversprechen und der Leistungsverwaltung vollzieht sich Inklusion über die Rolle des Leistungsempfängers. Kommuniziert werden entsprechend Leistungsansprüche und -zusagen respektive die Leistungen selbst. Ansatzpunkte dieser beiden Inklusionsmodi ist im ersten Fall der Input - das, was in Form von Bürgerbeteiligung als Entscheidungsprämissen in das System hineingegeben wird - und im zweiten Fall der Output des Systems - in Form von Leistungen an die Bürgerinnen und Bürger als Resultat politischer Entscheidungen.

Drittens lässt sich ein indirekter und gering formalisierter Modus politischer Publikumsinklusion beobachten: Intermediäre Organisationen wie Parteien, Bürgerinitiativen und ähnliche, die in den Kommunikationszusammenhang der Peripherie eingeschlossen sind, übernehmen es, auf Grundlage von - tatsächlichen oder vermuteten - Interessen und Präferenzen des Publikums Themen zu bündeln und auf Entscheidungsmöglichkeiten hin zu verdichten. Diese werden dann von der Verwaltung in kondensierter Form aufgegriffen und in Entscheidungsprozesse überführt (Fuhse 2005: 86).

Viertens schließlich ist die Politik um eine möglichst detaillierte Publikumsbeobachtung bemüht, wozu sie die öffentliche Meinung als Medium der Beobachtung zweiter Ordnung heranzieht (Luhmann 2000b: 287). Die öffentliche Meinung erfüllt damit zum einen eine Komplementärfunktion zu den politischen Wahlen: »Da diese nur mit erheblichen Zeitabständen erfolgen können, regiert die öffentliche Meinung in der Zwischenzeit und ermöglicht zugleich eine (laufend korrigierbare) Vorausschau auf die Ergebnisse künftiger Wahlen« (Luhmann 2000b: 281) und damit auf die künftige Zuordnung der Codewerte Regierung und Opposition. Zum anderen - und hier wird die öffentliche Meinung zu einem Spiegel (Luhmann 2000b: 286) - dient sie auch zur Selbstbeobachtung der Politik. Über die öffentliche Meinung beobachten Politikerinnen und Politiker, die miteinander im Konkurrenzverhältnis stehen, »sich selbst und andere im Hinblick auf das, was von einem Handeln $\mathrm{zu}$ halten ist, das sich dem Beobachtetwerden aussetzt« (Luhmann 2000b: 292). Maßgeblich an der Produktion der öffentlichen Meinung beteiligt sind die Massenmedien, die »die Welt in der Gesellschaft für die Gesellschaft « repräsentieren (Luhmann 2000b: 304), indem sie Weltbeschreibungen anfertigen und sich dabei des Codes Information|Nicht-Information bedienen. Für die Politik bedeutet das: »Was immer die Massenmedien an aufregend neuen Umweltinformationen in die öffentliche Meinung hineinpublizieren, für die Politik spiegelt sich darin maßgeblich die eigene Durchsetzungsfähigkeit, die eigene Thematisierungschance und die eigene Unterstützung « (Japp/Kusche 2004: 519).

Diese Funktion der Massenmedien führt wiederum dazu, dass sich innerhalb des politischen Systems eigens Aktivitäten auf die Vermittlung von Politik aus- 
richten, also darum bemüht sind, über entsprechende Darstellungen in den Massenmedien Legitimität in der öffentlichen Meinung herzustellen. Dieser Darstellungsaspekt der Politik, so die Symmetriethese von Japp und Kusche (2004: 519ff), erlangt neben dem Herstellungsaspekt, also den politischen Aushandlungs- und Entscheidungsprozessen, in der politischen Kommunikation eine durchaus gleichrangige Position. Das Publikum selbst wird im Verhältnis von Politik und Massenmedien zum ausgeschlossenen Dritten (Fuhse 2003: 136ff).

Auch im lokalpolitischen Kontext spielen diese vier Formen der Publikumsbeobachtung und -inklusion eine wichtige Rolle und sind teilweise expliziter Bestandteil formaler Dezentralisierungsprozesse. Mit besonderer Deutlichkeit gilt dies für die beiden erstgenannten Inklusionsmodi: Die Einführung oder Ausweitung von aktivem und passivem Wahlrecht bei der Besetzung lokaler Ämter und der Schutz der Wahlergebnisse gegen Eingriffe von übergeordneten Verwaltungsebenen machen den Kern dessen aus, was üblicherweise unter politischer Dezentralisierung (in Abgrenzung von administrativer und fiskalischer Dezentralisierung) verstanden wird. Auf die Inklusion des Publikums als Leistungsempfänger beziehen sich sämtliche formalen Regelungen, die den kommunalen Verwaltungsorganen die Verantwortung für Angelegenheiten von lokaler Bedeutung übertragen, worunter überwiegend Aufgaben und Leistungen im Bereich der sozialen und technischen Infrastruktur fallen, die sich an die Gemeindemitglieder richten und auf deren Bereitstellung Letztere Einfluss nehmen sollen.

Eine kategoriale Erweiterung der genannten Inklusionsmodi besteht im Fall lokaler Selbstverwaltung darin, dass eine ganze Bandbreite mehr oder weniger kontinuierlicher Eingabe- (aus Sicht des Publikum) respektive Beobachtungsmöglichkeiten (aus Sicht der Politik) geschaffen wird. In formaler Hinsicht gilt dies im Speziellen für den Fall der jüngsten russischen Kommunalreform, für die Reformbeobachter ein auch im Vergleich mit westlichen Demokratien erstaunliches Spektrum gesetzlicher Mitbestimmungsmöglichkeiten feststellen (Lankina 2005: 166; Wollmann/Gritsenko 2009: 235). Auch in anderen nationalen Kontexten dürfte diese Form der Mitbestimmung aber eine Rolle spielen. Über die kommunalen Wahlen hinaus findet - zumindest der Idee nach - Partizipation in Foren wie Gemeindeversammlungen und öffentlichen Anhörungen, über Referenden oder Bürgersprechstunden statt. Hirschmans (1970: 17) Feststellung angesichts des Beispiels von Reformen des öffentlichen Schulsystems gilt in diesem Sinne auch für lokale Selbstverwaltung generell: »[D]ecentralization has been advocated and undertaken as a means of making the channel of communication between members and management $[. .$.$] less >cumbrous <$ than heretofore «. Voice als »any attempt at all to change, rather than to escape from, an objectionable state of affairs « oder allgemeiner als Interessenartikulation (Hirschman 1970: 30) weitet sich aus und differenziert sich in seinen Erscheinungsformen, die teilweise deutliche Züge von Leis- 
tungsrollen annehmen (zu »exit« und »voice« als Form der Publikumsinklusion vgl. auch Stichweh 2005: 22f).

Abseits der formalen Regelungen kommt hinzu, dass die politischen Leistungsrollen sich durch die Einführung lokaler Selbstverwaltung nicht nur pluralisieren, sondern sich auch die Distanz zum Publikum in zum Teil sehr materieller Form verringert. Persönlichen Kontakten zu den Trägerinnen und Trägern lokalpolitischer Leistungsrollen fällt gerade in kleinen Gemeinden, um die es im empirischen Teil gehen wird, eine vergleichsweise große Bedeutung zu, da diese unmittelbar in die alltagsweltlichen Kommunikationsstrukturen eingebunden sind und sie mit ihrem Publikum teilen. Dies ist zum einen durch die räumliche Nähe bedingt, dürfte aber auch darauf zurückzuführen sein, dass (abgesehen von wenigen Ausnahmen) auf der lokalen Ebene Berufspolitikerinnen und -politiker in der Minderheit sind. Die meisten lokalpolitischen Leistungsrollen sind somit an andere (professionelle) Rollen gekoppelt, was die Berührungspunkte mit dem Publikum auch außerhalb politischer Kontexte erhöht.

$\mathrm{Zu}$ vermuten ist darüber hinaus insbesondere für kleine Gemeinden, dass in diesen die Gemeindeöffentlichkeit der Politik vergleichsweise direkt zugänglich ist. Dies gilt sowohl im Hinblick auf die Produktion als auch auf die Beobachtung der öffentlichen Meinung. Dadurch werden Interaktionen, die sich grundsätzlich vom Umgang mit Kommunikationsangeboten der Massenmedien unterscheiden, stark aufgewertet: Massenmedien werden vom Publikum weitgehend passiv >konsumiert<. Dies deshalb, weil Massenmedien im Kommunikationsprozess Information und Mitteilung übernehmen und dem Publikum in der Regel nur das Verstehen bleibt. Unter diesen Bedingungen fällt es relativ leicht, massenmediale Kommunikationsangebote zu ignorieren oder abzulehnen. Anders verhält es sich im Fall von Interaktionen, bei denen Information, Mitteilung und Verstehen zusammenfallen, die Ablehnungswahrscheinlichkeit gering ist und für Widersprüche und potenzielle Konflikte Umgangsformen gefunden werden müssen. Die Verarbeitung von Sinnangeboten und die daran anschließende Herausbildung politischer Orientierungen vollziehen sich im Interaktionsfall unter sozialer Kontrolle, während sie im Kontext massenmedialer Vermittlung davon weitgehend unberührt bleiben (vgl. zu dieser Gegenüberstellung Fuhse 2003: 139ff).

Inhaltlich beschränken sich diese unmittelbaren Formen lokalpolitischer Publikumsäußerungen nicht mehr in erster Linie auf Personalfragen, über die in politischen Wahlen entschieden wird, sondern weiten sich auch auf (ausgewählte) Sachfragen aus. Es geht also nicht mehr >nur $<$ um die demokratische Legitimation der politischen Entscheidungsträgerinnen und -träger durch Wahlen, die dann auf dieser Grundlage weiter entscheiden, ohne das Publikum direkt einzubeziehen, sondern auch um Möglichkeiten der direkten und kontinuierlichen Einflussnahme auf den Entscheidungsprozess selbst. Die Publikumsäußerungen lösen sich dabei zuneh- 
mend aus der binären Form (ja|nein in Abstimmungen) und sind in wesentlich höherem Maße diskretionär möglich (durch das vergleichsweise niederschwellige Einbringen von Themen und ähnliches), als dies auf der nationalen Ebene praktiziert werden kann.

Ungeachtet der Freiheit, nicht zu partizipieren und sich passiv zu verhalten (Stichweh 2005: 74), ist eine Ausweitung der Reaktionsmöglichkeiten immer auch mit neuen Herausforderungen für das Publikum verbunden: Sie wird den Bürgerinnen und Bürgern nicht nur »geboten«, sondern »abverlangt« (Luhmann 2010: 373). Eine Herausforderung für die Politik besteht dann darin, im System oder genauer: im Teilsystem der Verwaltung entsprechende Resonanzstrukturen anzuschließen, um die formalen und informalen Publikumsäußerungen zu registrieren, zu interpretieren und auf die eigenen Entscheidungen zu beziehen, ohne dabei den Zweck die Erfüllung der eigenen Aufgaben - aus den Augen zu verlieren. Sofern dies gelingt, steigt aus der Perspektive des politischen Systems die Auflösbarkeit des Publikums, dessen Äußerungen nun nicht mehr ausschließlich in aggregierter und quantitativer Form im System interpretationsrelevant sind (vgl. Stichweh 2005: 23), sondern stärker individualisiert beobachtet und interpretiert werden können. Mit welchen Implikationen dies letztlich für die Strukturen der lokalen politischen Peripherie, die wechselseitige Beobachtung von Politik und Publikum und die Ausgestaltung der Grenzen der politischen Kommunikationszusammenhänge verbunden ist, bleibt empirisch zu klären.

\section{Lokale SelbstVerwaltung in Russland: DER FORMALE RAHMEN}

Die Konzession lokaler Autonomie, die Bildung von Organisationen und die Inklusion des Publikums: Im Kern geht es um diese Prozesse, wenn die Rede von lokaler Selbstverwaltung und Dezentralisierung ist. Auf welche Weise dies formal im russischen Kontext umgesetzt wurde und welche Aspekte dabei besonders hervortreten, möchte ich im Folgenden am Beispiel der jüngsten Kommunalreform erörtern. Ihre Regelungen zielen auch und gerade auf die Strukturen ländlicher Gemeinden. Begonnen wurde mit der Konzeption einer entsprechenden gesetzlichen Reformgrundlage, nachdem sich die Aufmerksamkeit der russischen Regierung kurz nach dem ersten Amtsantritt von Präsident Vladimir Putin im Jahr 2000 generell auf die Regionen und Gemeinden zu richten begann: Im Kontext der offiziell proklamierten Stärkung der >Machtvertikalen< wurde noch im Jahr 2000 eine Reform des föderalen Systems initiiert, die den Einfluss der Regionen auf der nationalen Ebene er- 
heblich schwächen sollte ${ }^{6}$ (vgl. dazu Gel'man 2009; Young/Wilson 2007: 1075). Im Folgejahr beauftragte Putin eine Kommission unter der Leitung seines damaligen stellvertretenden Stabschefs Dmitrij Kozak (die sogenannte >Kozak-Kommission ${ }^{7}$ ), das existierende System kommunaler Selbstverwaltung zu überarbeiten und dabei Regelungen für die klare Abgrenzung der Aufgaben und Funktionen der föderalen, regionalen und kommunalen Verwaltungsebene zu finden (Lankina 2003, 2005: 145). Dieser Prozess mündete in das föderale Gesetz Nr. 131 »Über allgemeine Prinzipien der Organisation lokaler Selbstverwaltung in der Russischen Föderation «, das 2003 vom Parlament verabschiedet wurde, seit Januar 2006 - mit einer Übergangsfrist bis Januar 2009 - in Kraft ist und gegenwärtig die relevante formal-rechtliche Grundlage für die Gemeindeverwaltungen darstellt.

Bereits vor ihrem In-Kraft-Treten und während der Übergangsphase wurden die Reform und ihre Prinzipien innerhalb und außerhalb Russlands kontrovers diskutiert. Ihre Kritikerinnen und Kritiker werden nicht müde, die unterschwellig rezentralisierenden Effekte zu beklagen (vgl. z.B. Gelman 2007; Gel'man 2009; Wollmann/Gritsenko 2009: 240f) und sehen in dem Gesetz »the victory of >local government< over >local self-government<« (Ross 2009: 67). Die Reformautorinnen und -autoren und ihre Befürworterinnen und Befürworter zeigen sich hingegen überzeugt, dass das Gesetz einen Neuanfang in Sachen lokaler Selbstverwaltung darstelle, der in vollem Umfang den Prinzipien der »Europäischen Charta der Kommunalen Selbstverwaltung « des Europarats gerecht werde. Diese Charta hatte Russland 1996 unterzeichnet und sie war 1998 nach der Ratifizierung durch das

6 Die Föderalreform des Jahres 2000 bestand aus drei Kernelementen, die zusammen darauf zielten, die föderale und regionale Gesetzgebung zu harmonisieren (Schröder 2003: 21) und sowohl die Einheitlichkeit des Staates als auch die »exekutive Machtvertikale« (Perovic 2001: 153) zu stärken: (1) Das Dekret »Über die Bevollmächtigten Vertreter des Präsidenten der Russländischen Föderation in den Föderalen Bezirken«, das das Staatsgebiet in sieben föderale Bezirke aufteilte und zu deren Verwaltung Vertreter des Präsidenten einsetzte (Mangott 2002: 260f; Perovic 2001: 142); (2) das Gesetz »Über das Verfahren zur Bildung des Föderationsrats der Föderalen Versammlung der Russischen Föderation «, das für die Mitglieder des Föderationsrats die Trennung zwischen ihrem Amt auf regionaler Ebene und dem Sitz im Föderationsrat einführte (Schneider 2001: 86ff); sowie (3) das Gesetz »Über die Einführung von Änderungen und Ergänzungen in das Föderale Gesetz >Über die allgemeinen Prinzipien der Organisation der gesetzgebenden und exekutiven Organe der Staatsmacht der Föderationssubjekte der Russischen Föderation « (Schneider 2001: 165).

7 Die offizielle Bezeichnung lautete »Präsidiale Kommission für die Machtabgrenzung zwischen der föderalen, regionalen und lokalen Regierungsebene (Young/Wilson 2007: 1077, Fn. 5). 
Parlament in Kraft getreten. In einem Interview mit der russischen Tageszeitung »Rossijskaja Gazeta« betonte Dmitrij Kozak, dass das Gesetz aus »objektiver « Sicht eine »substantielle, ja sogar radikale Dezentralisierung der Macht « bewirke ${ }^{8}$ (Rossijskaja Gazeta 2003: 1). Das Jahr 2003, in dem das Gesetz verabschiedet wurde, so die generelle Einschätzung in der russischen Politik, markiere einen »Wendepunkt« in der Entwicklung lokaler Selbstverwaltung in Russland (Shipov 2003: 6) und auch aus Sicht der Weltbank bietet die Reform »an opportunity to approach rural development in a comprehensive bottom up manner «(Georgieva 2007: 5). Weder der ostentativ proklamierte Neuanfang noch die vehemente Kritik sollten allerdings darüber hinwegtäuschen, dass es auch in Russland eine Tradition lokaler Selbstverwaltung gibt - wie überschaubar diese auch sein mag.

\section{Ein Blick zurück: Lokale Selbstverwaltung in Russland vor 2006}

Zur Legitimation der jüngsten Kommunalreform, aber auch bereits mit Blick auf vorhergehende Versuche der Einführung lokaler Selbstverwaltung nach dem Zusammenbruch der Sowjetunion wurde und wird in Russland regelmäßig auf die Tradition der Semstwo (»zemstvo«) verwiesen (Evans 2004: 71ff; für die jüngste Reform z.B. Shipov 2003: 6; sowie die Beiträge in The World Bank 2007a). Die Bezeichnung Semstwo verweist auf das russische Wort »zemlja« für Boden und wurde über mehrere Jahrhunderte als Oberbegriff für unterschiedliche Formen lokaler (Selbst-)Verwaltung im Russischen Reich verwendet ${ }^{9}$ (Porter/Seregny 2004: 20). Wenn im heutigen Russland von Semstwo die Rede ist, geht es in aller Regel um das Regime ländlicher Selbstverwaltung, das unter Zar Alexander II nach der Bauernbefreiung im Jahr 1864 eingeführt wurde und das bis zur Oktoberrevolution Bestand hatte. Mit der sogenannten Semstworeform wurden Selbstverwaltungsorgane auf der Ebene der Provinzen (»gubernija«) und Bezirke (»uezd) eingerichtet, die auf beiden Ebenen jeweils aus der gewählten Semstwoversammlung (»zemskoe sobranie $\ll^{10}$ ) und der daraus hervorgehenden Semstwoverwaltung (»zemskaja uprava «) bestanden (McKenzie 1982: 31; Stökl 1997: 546). Das (aktive und passive) Wahlrecht für diese Institutionen erhielt, wer Land besaß (Atkinson 1982: 79). Die

8 »An objective analysis of rights of subjects and municipalities that actually exist in comparison with those proposed by the drafts should unavoidably lead to the conclusion that the reform presupposes a significant, I would even say a radical, decentralization of power [...].« (Dmitrij Kozak in Rossijskaja Gazeta 2003: 1; englische Übersetzung: Rossijskaja Gazeta 2005 [2003]: 9).

9 Die Bezeichnung »lokale Selbstverwaltung « (»mestnoe samoupravlenie«) tauchte hingegen im 20. Jahrhundert erstmals auf (siehe unten).

10 Dieser Begriff hat sich bis heute als Bezeichnung für die Bezirksparlamente erhalten. 
Kompetenzen und Aufgaben der Organe der Semstwo bezogen sich im Wesentlichen auf Bereiche wie das Schulwesen, die medizinische Versorgung, den öffentlichen Transport, die Instandhaltung von Straßen und Brücken sowie die Förderung der lokalen wirtschaftlichen Entwicklung in Industrie, Handel und Landwirtschaft (Stökl 1997: 546).

Die Autonomie, die der Semstwo bei der Erfüllung ihrer Aufgaben während ihres rund 50jährigen Bestands vom Zentralstaat zugestanden wurde, variierte jedoch. Ähnlich wie in Bezug auf das gegenwärtige Regime lokaler Selbstverwaltung in Russland immer wieder kritisch hervorgehoben wird, sieht die Geschichtswissenschaft auch in der Semstwo einen Ausdruck des Widerspruchs zwischen einer im Kern zentralistischen Staatsauffassung und gleichzeitigen Dezentralisierungsbestrebungen (Porter/Seregny 2004: 20). Einigkeit besteht in der historischen Betrachtung darüber, dass die Institution der Semstwo im Rahmen der Integration des Russischen Reiches als Kontrollmechanismus vor allem mit Blick auf die von der damaligen Hauptstadt Sankt Petersburg weit entfernten ländlichen Regionen fungierte. Als ein Indikator dafür gilt die Beobachtung, dass das Semstworegime keinesfalls auf Druck der ländlichen Bevölkerung hin eingesetzt wurde, sondern auf Initiative der Regierung. Zudem finden sich im nebulös formulierten Semstwostatut von 1864 zahlreiche Regelungen, die die vordergründig gewährte Autonomie der ländlichen Gemeinden hinterrücks wieder einfingen, dem politischen Zentrum weitreichende Kontroll- und Interventionsbefugnisse zusicherten und die Gemeinden somit nicht nur in Abhängigkeit von Letzterem versetzten, sondern ihnen für viele Bereiche lediglich den Status eines ausführenden Organs von Regierungsweisungen zusprachen. Darüber hinaus wurde ab 1889 unter den Zaren Alexander III und später unter Nikolas II eine Reihe von Gegenreformen durchgesetzt, die darauf zielten, die ohnedies beschränkte Autonomie zusätzlich einzugrenzen und die Kontrolle des Zentralstaats weiter zu stärken (Porter/Seregny 2004: 20ff; vgl. auch Thompson Manning 1982).

$\mathrm{Zu}$ behaupten, dass sich die Bedeutung der Semstwo in ihrer Kontrollfunktion erschöpfte, wäre jedoch verkürzt, denn »even these restrictions could not diminish the inherent contradiction embodied in the establishment of local self-government its incompatibility with the very idea of autocracy« (Porter/Seregny 2004: 21). Nicht zuletzt in Folge der Abschaffung der Leibeigenschaft und dem Verschwinden der Feudalherren samt ihren Leistungen gegenüber den Leibeigenen im wirtschaftlichen und sozialen Bereich war die Zentralregierung auf Unterstützung bei der Verwaltung der ländlichen Regionen angewiesen (Atkinson 1982: 80). Die Semstwo als gewählte Institution entwickelte in dieser Situation eine wenn auch beschränkte, aber in ihrem Wesen vermutlich unvorhergesehene Eigendynamik und erwies sich als Katalysator für die Herausbildung zivilgesellschaftlicher Strukturen: »[I]t ran counter to Russia's autocratic traditions and served as a wellspring for 
constitutionalist aspirations. This was the single most important aspect of the zemstvo - the fact that the elective principle had been inaugurated « (Porter/Seregny 2004: 22). Zwar dominierte auch nach der Einführung des Wahlrechts der Adel in faktisch allen Regionen sowohl die Semstwoversammlung als auch die aus ihr hervorgehende Semstwoverwaltung. Unter anderem in Folge der Einführung von Quoten, die die Anteile der verschiedenen Statusgruppen regulierten und begrenzten (Atkinson 1982: 85; Stökl 1997: 547), hielt jedoch auch die Bauernschaft sukzessive Einzug in die gewählten Organe (Porter/Seregny 2004: 22).

Hinzu kam, dass sich der politische Wind zu Beginn des 20. Jahrhunderts erneut drehte: Auf Druck, der um die Jahrhundertwende aus »einer regelrechten Zemstvobewegung « erwuchs (Stökl 1997: 547), wurden die Autonomie und der Kompetenzbereich der Semstwo seit 1905 ausgeweitet. Im Ergebnis wandelte sich das Verhältnis zur Zentralregierung von Weisungsabhängigkeit in Richtung Kooperation. Federführend bei diesen Änderungen war Peter Stolypin, der von 1906 bis zu seiner Ermordung 1911 Premierminister des Russischen Reiches war. Stolypin erkannte die brüchige soziale Untermauerung des Zarenregimes und versuchte, diese durch eine breitere Einbindung der Bauernschaft in die lokale Verwaltung zu stärken. Er initiierte eine Reihe von Reformmaßnahmen, die auf die Demokratisierung der Wahlen, auf die Begrenzung des Einflusses des Adels sowie auf Änderungen der bestehenden Organe zielten und agierte dabei stets, so das rückblickende Urteil, auch im Horizont der Ausweitung zivilgesellschaftlicher Strukturen auf ländliche Gebiete und der Schaffung eines >Bürgerbewusstseins` in der bäuerlichen Bevölkerung (Porter/Seregny 2004: 27f): »All of Stolypin's reforms (and especially that of local government) were connected to other projects - most notably, dismantling the rural commune and achieve universal schooling - designed to break down peasant particularism and make peasants into citizens« (Porter/Seregny 2004: 30).

Die Bewertung der Semstwo in der russischen (Geschichts-)Wissenschaft und Politik hat sich mit dem Zusammenbruch des sowjetischen Regimes radikal gewandelt: Im sowjetischen Regime nahm die vorherrschende Beobachtungsweise und Interpretation einen klaren Klassenstandpunkt ein, fokussierte die suppressiven Intentionen und schob die Funktion der Semstwo als Instrument zum Machterhalt des Adels in den Vordergrund:

»The standard Soviet interpretation of the zemstvo reform was as an attempt to adapt the tsarist autocracy to the demands of the growth of capitalism in Russia, while striving to assure that the operation of government on all levels would continue to serve the interests of the landed gentry.«(Evans 2004: 70)

Im Kontext der maßgeblich von Gorbatschow initiierten Politik der Perestroika in den späten 1980er Jahren begann sich diese Sichtweise zu ändern. Parallel zur 
wachsenden Kritik am sowjetischen Regime, die nun zunehmend offen geäußert wurde und sich insbesondere auf die zentralistischen Strukturen und das Fehlen von demokratisch gewählten, repräsentativen Staatsorganen richtete, stieg auch die rückblickende Wertschätzung der Semstwo als eine Art Gegenmodell (Evans 2004: 69). In den 1990er Jahren nahm die Bewertung schließlich nicht nur mehrheitlich positive, sondern bisweilen auch idealisierende Züge an und wurde längst nicht mehr nur von Historikerinnen und Historikern, sondern mehr und mehr auch in der Politik geäußert. Die Semstwo galt als Model für eine unabhängige und demokratisch gewählte Lokalverwaltung und wurde erstmals als »lokale Selbstverwaltung « begrifflich etikettiert. Zudem galt ihre Existenz als Beleg dafür, dass eine eigene russische Tradition in diesem Bereich existierte und man nicht allein auf $>$ westliche< Erfahrungen und Ratschläge als Reformgrundlage angewiesen war (Evans 2004: 72). Mit anderen Worten: Mit der Semstwo hatte man eine eigene Tradition wiederentdeckt, die sich im Hinblick auf die Legitimation anstehender Reformmaßnahmen aktualisieren und an die sich anschließen ließ, nachdem man sich von ihr zuvor ebenso betont distanziert und mit ihr zu brechen versucht hatte.

Dass einer solchen euphorischen Sichtweise nicht notwendigerweise auch Taten folgten, die zahlreichen Versuche der Einführung lokaler Selbstverwaltung in Russland seit dem Zusammenbruch des sowjetischen Regimes oftmals halbherzig verliefen und durch ein Wechselspiel aus De- und Rezentralisierung gekennzeichnet waren, zeigt ein Blick auf die jüngere Vergangenheit der 1990er Jahre. Die unmittelbaren Vorläufer von Gesetz Nr. 131 lassen sich drei Reformphasen oder -wellen zuordnen:

- Lokale Selbstverwaltung in der Endphase der Sowjetunion: Erste Schritte in Richtung einer formalen Stärkung der Gemeinden wurden noch zu Sowjetzeiten unternommen. Kurz vor dem Zusammenbruch des sowjetischen Regimes trat im Jahr 1990 das Gesetz »Über allgemeine Prinzipien lokaler Selbstverwaltung und lokaler Wirtschaft der UdSSR « vom 9. April 1990 in Kraft, in dem auch der Begriff »lokale Selbstverwaltung « erstmals in einem Gesetzestext auftauchte (vorher galt die Bezeichnung »örtliche Organe der staatlichen Macht und Leitung«, vgl. die Verfassung der UdSSR von 1977, Kap. 19, zitiert nach Henke/Wirantaprawira 1981: 194). Das Gesetz beendete formal die Praxis der dualen Subordination der örtlichen Exekutivkomitees sowohl gegenüber dem Sowjet derselben Ebene als auch gegenüber dem Exekutivkomitee der nächsthöheren Verwaltungsebene (dazu ausführlich S. 50ff). Darüber hinaus ermöglichte es die Direktwahl der lokalen Sowjets. Die Bürgermeisterinnen und Bürgermeister wurden mit Ausnahme von Moskau und Leningrad jedoch weiterhin ernannt (Ross 2009: 42f, 2006: 640f). Das erste Gesetz der Russischen Republik (RSFSR), das lokale Selbstverwaltung fokussierte, Gesetz Nr. 1550-1 »Über die kommunale Selbst- 
verwaltung in der Russischen Sowjetischen Föderalen Sozialistischen Republik«, wurde am 6. Juli 1991 ratifiziert und trat noch im selben Jahr in Kraft. Insbesondere aufgrund der vorgesehenen (direkt-)demokratischen Elemente stieß das Gesetz in der Literatur überwiegend auf ein positives Echo, seine effektive Umsetzung wurde jedoch vom einsetzenden politischen Regimewechsel überrollt (vgl. Bjalkina 2001: 16; Campbell 1995: 157ff; Ross 2006: 641, 2009: 43ff; Young/ Wilson 2007: 1073).

- Die Verankerung lokaler Selbstverwaltung in der russischen Verfassung: Die russische Verfassung von 1993 garantiert die lokale Selbstverwaltung der Gemeinden als grundlegendes Prinzip des Staatsaufbaus und betont ihre Autonomie gegenüber dem Bereich staatlicher Verwaltung (Art. 12), was in Form verschiedener Garantien für die kommunale Verwaltungsebene konkretisiert wird (Kap. 8, Art. 130-133) (Bjalkina 2001: 17; Kourliandskaja/Nikolayenko/Golovanova 2001: 172; Wollmann/Gritsenko 2009: 228). So spricht die Verfassung beispielsweise den Kommunen das Recht zu, Angelegenheiten von lokaler Bedeutung eigenständig zu klären (welche dies im Einzelnen sind, bleibt jedoch unspezifiziert). Regionale Autoritäten müssen die Präferenzen der Kommunen berücksichtigen, wenn die Grenzen von Verwaltungsbezirken festgelegt werden. Die Lokalverwaltungen erhalten begrenzte finanzielle Autonomie und ihnen wird die Übernahme der Kosten bei der Ausführung föderaler Aufgaben zugesagt. Die Bewertung dieser Regelungen fällt in der wissenschaftlichen Literatur verhalten positiv aus: »However, the Federal Constitution of 1993 provided LSG with surprisingly progressive rights (Wollmann/Gritsenko 2009: 228).

- Das föderale Gesetz »Über die grundlegenden Prinzipien der Organisation kommunaler Selbstverwaltung « von 1995: Im Jahr 1995 folgte das föderale Gesetz »Über die grundlegenden Prinzipien der Organisation kommunaler Selbstverwaltung «(Föderales Gesetz Nr. 154 vom 28. August 1995), das in erster Linie allgemeine Prinzipien kommunaler Selbstverwaltung beinhaltete. Sie umfasst demnach vor allem Aufgaben der Gemeinden im Bereich lokaler Infrastruktur und wohlfahrtsstaatlicher Leistungen, darunter den Unterhalt von Schulen und medizinischen Einrichtungen, die Strom-, Gas- und Wasserversorgung, Stadtplanung und Kontrolle der Landnutzung, öffentlichen Transport, Brandschutz und ähnliches (vgl. Ross 2009: 57). Darüber hinaus wird für die kommunale Ebene jedoch kein konkreter Katalog an Verwaltungsinstitutionen festgelegt, sondern den Regionen werden verhältnismäßig weitreichende Kompetenzen bei der Ausgestaltung der lokalen Selbstverwaltung auf ihrem jeweiligen Territorium eingeräumt (Kourliandskaja/Nikolayenko/Golovanova 2001: 172; Wollmann/Gritsenko 2009: 228). Als Kommune und damit als eigenständige Verwaltungseinheit definiert das Gesetz »any populated territory (city, town, township or any combination of these on a contiguous territory) which is self-governed and possesses 
municipal property, a budget and an elected local government body« (Kourliandskaja/Nikolayenko/Golovanova 2001: 172). Das Recht auf Selbstverwaltung ist damit nicht mehr an eine Mindesteinwohnerzahl gebunden, sondern steht prinzipiell jedem Gemeinwesen offen: »No settlement, regardless of its population, shall be deprived of the right to establish local self-government « (Art. 13 zitiert nach Ross 2009: 51). Während bis zum Jahr 1995 zwischen den verschiedenen subregionalen Verwaltungseinheiten je nach Größe, Bevölkerungsdichte, administrativem und territorialem Status eine strikte Hierarchie bestanden hatte (Kourliandskaja/Nikolayenko/Golovanova 2001: 178f), wird diese nun abgeschafft: Alle Einheiten kommunaler Selbstverwaltung erhalten denselben rechtlichen Status und geschaffen wird ein System einander nicht-überlappender Verwaltungsbezirke (Kourliandskaja/Nikolayenko/Golovanova 2001: 172f).

Inwieweit die Reformversuche der 1990er Jahre tatsächlich eine größere Eigenständigkeit der Gemeinden bewirkten, ist angesichts der verfügbaren Analysen, die sich überwiegend auf die Bewertung der formalen Regelungen beschränken, schwer zu beurteilen. Zumindest schienen die Kommunalwahlen seit dem Ende der Sowjetunion keine reinen Akklamationsrituale mehr zu sein: In jedem der drei kommunalen Wahlzyklen bis zum Jahr 2005 (1994-1996, 1998-2001, 2002-2005) wurde etwa die Hälfte der Verwaltungschefs in den Städten und Bezirken ausgetauscht und die Mitglieder der sowjetischen Nomenklatur, die nach dem ersten Wahlzyklus noch beinahe sämtliche Ämter in der lokalen Verwaltung innehatten, wurden bis zum Ende des dritten Wahlzyklus im Jahr 2005 vollständig aus der Kommunalverwaltung verdrängt (Selzer 2009: 7ff).

Jedoch: Obwohl der Regierung in dieser Phase ernsthafte Bemühungen um lokale Selbstverwaltung und die Schaffung einer gesetzlichen Basis für die Autonomie der Gemeinden gegenüber dem Zentralstaat zugestanden werden, wird das Vorhaben unter dem Strich einhellig als gescheitert beurteilt (vgl. z.B. Gel'man 2002; Wollmann/Gritsenko 2009; Young/Wilson 2007: 1075f): »[T]he perception of local self-government - especially local self-government outside the largest cities - was that it had for the most part ceased to function « (Reynolds 2005: 4). Die unbefriedigende Lage Ende der 1990er Jahre wird dabei vor allem auf drei Ursachen zurückgeführt: Erstens sei die territoriale Struktur und die Verknüpfung der verschiedenen Verwaltungsebenen komplex und unüberschaubar, da die gesetzliche Grundlage drei verschiedene Varianten vorsah, wie die regionalen und lokalen Ebenen einzurichten und miteinander in Beziehung zu setzen seien (vgl. Abbildung 1). Die Entscheidung, welche der drei Varianten implementiert wird, oblag den re- 
gionalen Verwaltungsorganen der 83 Subjekte der Russischen Föderation. ${ }^{11}$ Das Resultat waren Strukturen kommunaler Selbstverwaltung, die von Region zu Region variierten und mit jeweils unterschiedlichen Organen, Aufgaben und Kompetenzen ausgestattet waren (Fitch Ratings 2006; Kourliandskaja/Nikolayenko/Golovanova 2001: 178ff; Wollmann/Gritsenko 2009: 230ff). Zweitens und unter anderem als Folge der diffusen territorialen und administrativen Abgrenzung der Verwaltungseinheiten wird die Zuweisung der Verantwortlichkeiten kritisiert: Die Aufgaben der kommunalen Verwaltungsorgane bezogen sich primär auf den Erhalt bestehender öffentlicher Infrastruktur, wohingegen die effiziente Bereitstellung kommunaler Dienstleistungen eine sekundäre Rolle spielte: »Many of the responsibilities are stated as the obligation to maintain infrastructure facilities that were transferred to municipal ownership, rather than the obligation to perform local functions « (Kourliandskaja/Nikolayenko/Golovanova 2001: 173). Wenn beispielsweise auf dem Territorium einer Gemeinde ein Krankenhaus existierte, war die Gemeinde für dessen Unterhalt zuständig. Befand sich hingegen kein Krankenhaus oder eine vergleichbare medizinische Einrichtung auf ihrem Territorium, fiel die medizinische Versorgung auch nicht in ihren Verantwortungsbereich und die Bürgerinnen und Bürger hatten entsprechend keinen oder nur erschwerten Zugang zu den entsprechenden Dienstleistungen. In der Praxis führte diese Regelung zu beachtlichen Ungleichgewichten sowohl unter den Gemeinden als auch zwischen den Gemeinden und übergeordneten Verwaltungsebenen (Center for Fiscal Policy 2008: 6f), da die Verteilung der Verantwortlichkeiten zwischen regionalen und lokalen Behörden in hohem Maße von der Eigentumszuordnung zwischen den Ebenen abhing (Kourliandskaja/Nikolayenko/Golovanova 2001: 173). Vor allem für die Bürgerinnen und Bürger kleinerer Gemeinden waren die zuständigen Behörden oftmals allein räumlich kaum zu erreichen (Reynolds 2005: 4). Drittens wird die finanzielle Grundlage der Gemeinden unisono als inadäquat für ein tragfähiges System lokaler Selbstverwaltung eingeschätzt: Die rechtliche Basis für die kommunalen Finanzen wurde mit dem Gesetz »Über die finanziellen Grundlagen kommunaler Selbstverwaltung « (Föderales Gesetz Nr. 126 vom 25. September 1997) gelegt. Die vorgesehenen Einkommensquellen der Kommunen deckten faktisch jedoch kaum mehr als

11 (Föderations-)Subjekt ist die Bezeichnung für die 83 föderalen Territorialeinheiten der Russischen Föderation, die auch im Föderationsrat, dem russischen Oberhaus, vertreten sind. Nach interner Verfasstheit und Grad an Autonomie werden dabei sechs Kategorien von Subjekten unterschieden: Es gibt 21 Republiken (»respublik«), neun Regionen (»kraj«), 46 Gebiete (»oblast'«), ein Autonomes Gebiet (»avtonomnaja oblast'«), vier Autonome Kreise (»avtonomnyj okrug«) und zwei Städte mit Subjektstatus (Moskau und Sankt Petersburg) (CIA World Factbook, www.cia.gov, zuletzt eingesehen am 14.4.2015). 
20 Prozent der Ausgaben und die Finanzierungslücke wurde mit Transferzahlungen von höheren Verwaltungsebenen gefüllt, so dass die offiziell vorgesehene finanzielle Autonomie der kommunalen Verwaltung durch diese Abhängigkeiten von vornherein untergraben wurde (Kourliandskaja/Nikolayenko/Golovanova 2001: 173; für eine detaillierte Analyse kommunaler Finanzen vgl. auch Chernyavsky/Vartapetov 2004). Zudem senkte die verbreitete Praxis der ungedeckten Mandate, also staatliche Aufgaben, deren Ausführungen den Gemeinden übertragen wurde, ohne dass entsprechende finanzielle Mittel dafür bereitgestellt wurden, die Unterstützung und das Vertrauen der Bevölkerung in die lokalen Verwaltungsorgane: $»[\mathrm{~L}]$ ocal governments became effective buffers, allowing a regional governor or federal ministry to make empty promises concerning pensions or subsidies without ever transferring the necessary resources to the local administrations « (Young/Wilson 2007: 1074).

\section{Ein Blick nach vorn: \\ Gesetz Nr. 131 »Über allgemeine Prinzipien der Organisation lokaler Selbstverwaltung in der Russischen Föderation«}

Das föderale Gesetz Nr. 131 »Über allgemeine Prinzipien der Organisation lokaler Selbstverwaltung in der Russischen Föderation«, das im Jahr 2003 vom Parlament verabschiedet wurde und seit Januar 2006 (mit einer Übergangsfrist bis Januar 2009) in Kraft ist, sollte in dieser Situation Abhilfe schaffen. Die erneute Reform zielt darauf, das System kommunaler Verwaltung in Russland strukturell einheitlich, transparenter und effizienter zu gestalten. Konkret sind dafür Neuregelungen in fünf Bereichen vorgesehen: ${ }^{12}$

- Die territoriale Gliederung der kommunalen Verwaltungseinheiten,

- die Struktur kommunaler Verwaltungsorgane,

- die Partizipationsmechanismen in den Gemeinden,

12 Die Grundlage der folgenden Darstellung ist Gesetz Nr. 131 »Über die allgemeinen Prinzipien der Organisation lokaler Selbstverwaltung in der Russischen Föderation « vom 6. Oktober 2003 (offizielle Bezeichnung auf Russisch: »Federal'nyj zakon Rossijskoj Federacii ot 6 oktjabrja 2003 g. N 131-FZ >Ob obščich principach organizacii mestnogo samoupravlenija v Rossijskoj Federacii « $)$, das von der Rossijskaja Gazeta in einer Sonderbeilage der Ausgabe Nr. 3316 vom 8.10.2003 veröffentlicht wurde (http://www.rg.ru/ 2003/10/08/zakonsamouprav.html [zuletzt eingesehen am 2.4.2012]). Bei sprachlichen Unklarheiten wurde zusätzlich eine englische Übersetzung des Gesetzestexts herangezogen (Föderales Gesetz 1312005 [2003]). 
- die kommunalen Aufgaben im Bereich der lokalen Infrastruktur,

- das Gemeindebudget und das kommunale Eigentum.

\section{Territoriale Verwaltungsgliederung: Kontrolle des Raums}

Im Hinblick auf die territoriale Gliederung der administrativen Einheiten führt das Gesetz (Kap. 2, Art. 10 bis 13) ein verbindliches zweigliedriges System ein, das zwei kommunale Verwaltungsebenen vorgibt: Bezirke (»rajon«) auf der oberen und städtische und ländliche Gemeinden (»selskoe/gorodskoe poselenie«) auf der unteren lokalen Ebene (vgl. Abbildung 1). Mit Ausnahme der großen Städte respektive städtischen Kreise (»gorodskij okrug«), in denen beide kommunalen Ebenen zusammenfallen, wird so das gesamte russische Territorium in Siedlungen unterteilt, die wiederum zu Bezirken zusammengefasst sind (Reynolds 2005: 5).

Abbildung 1: Gliederung subnationaler Verwaltungseinheiten vor und nach der Kommunalreform von 2003

Vor der Reform:

Drei Varianten der Organisation subnationaler Verwaltung
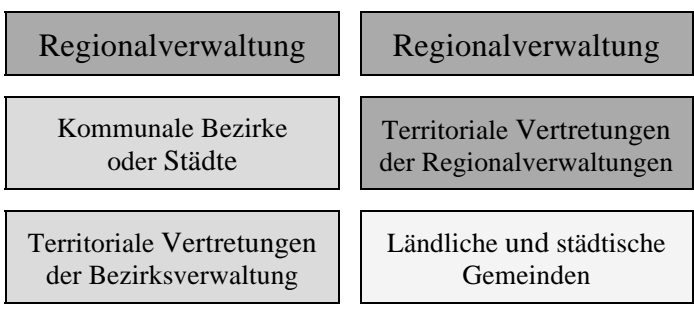

Territoriale Vertretungen der Regionalverwaltungen Regionalverwaltung

Ländliche und städtische Gemeinden

Kommunale Bezirke oder Städte

Ländliche und städtische Gemeinden

\section{Nach der Reform:}

Einheitliche Struktur subnationaler Verwaltung

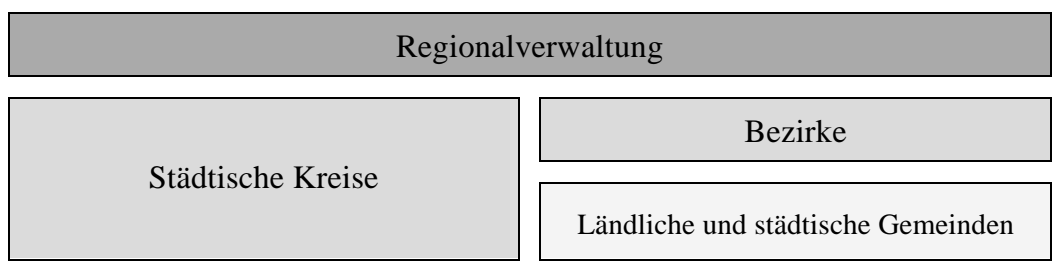

Quelle: Gesetz Nr. 131 (Kapitel 2, Artikel 10 bis 13), Fitch Ratings (2006).

Die Bevölkerungsuntergrenze für ländliche Siedlungen als kleinste administrative Einheit, um die es im Hinblick auf meine empirischen Beispiele im Folgenden vor- 
rangig gehen soll, wird bei 1.000 Einwohnern festgelegt. In Abhängigkeit von der Bevölkerungsdichte sind allerdings auch Ausnahmen vorgesehen, die diese Zahl unterschreiten. Beschränkungen bezüglich räumlicher Distanzen basieren auf der Erreichbarkeit der kommunalen Behörden für die Gemeindemitglieder (und damit auch auf der lokalen Verkehrsinfrastruktur): So soll der Hin- und Rückweg zum Verwaltungszentrum einer Siedlung für jedes Gemeindemitglied innerhalb eines Arbeitstages zu Fuß zu bewältigen sein, das Verwaltungszentrum eines Bezirks soll in derselben Zeit über öffentliche Verkehrsmittel erreicht werden können. Durch die Neuregelung wurde das alte und unüberschaubare dreigliedrige System abgelöst, in dem es der Entscheidung der regionalen Behörden unterlag, welche von drei möglichen Varianten kommunaler Verwaltungsstrukturen auf ihrem Territorium umgesetzt wurde und im Ergebnis alle drei parallel existierten.

Mit Blick auf die Funktionslogik des politischen Systems lässt sich der Reformbereich der territorialen Verwaltungsgliederung als ein Schritt in Richtung Kontrolle des Raums beobachten, wobei Kontrolle mit Stichweh (2003: 95) und in Anlehnung an die kybernetische Kontrollhierarchie und die dort zugrundeliegende Differenz von Energie und Information heißt, »dass man einen Sachverhalt in seiner Eigenständigkeit akzeptiert, aber Operationen anschließt, die den Sachverhalt den Imperativen eines anderen Systems zu unterwerfen versuchen«. In der Politik vollzieht sich die Kontrolle des Raums klassischerweise und im Wesentlichen über das Territorialitätsprinzip, was dazu führt, dass unter den gesellschaftlichen Funktionssystemen der Raumbezug hier mit am deutlichsten zum Ausdruck kommt. Territorialität wiederum meint, dass sich der Eigenraum der Politik über »die Ordnung der Machtverhältnisse auf einem durch Grenzen so genau wie irgendwie möglich markierten Raum« definiert (Stichweh 2008: 160). In den Blick gerät damit primär das Prinzip des souveränen Nationalstaats, das den Machtanspruch eines Staates auf ein Territorium festlegt und räumlich begrenzt: Das staatliche Gewaltmonopol bezieht sich zunächst auf jeden, der sich auf einem bestimmten Territorium aufhält. Daran anschließend ist zu beobachten, dass die meisten Staaten sich nicht nur gegenüber anderen Staaten abgrenzen, sondern auch intern räumlich segmentiert und dabei um größtmögliche Eindeutigkeit bemüht sind. Für Scott (1998: Kap. 2) ist eine solche Einführung standardisierter Einheiten Teil einer Strategie, mit der Staaten ihr Territorium »lesbar« machen. Diese und ähnliche schematisierte Kategorien wie Volkszählungen, Karten oder Katasterpläne beobachtet Scott (1998: 77) als Techniken »for grasping a large and complex reality; in order for officials to be able to comprehend aspects of the ensemble«. Derartige Erfassungsmechanismen einer komplexen Realität seien unverzichtbare Voraussetzung für Staatlichkeit an sich. Die verschiedenen Verwaltungsebenen und ihre Abgrenzung voneinander ergeben sich wiederum aus der Möglichkeit, das Staatsgebiet anhand solcher Mechanismen mit unterschiedlicher Auflösung zu beobachten. Im 
Rahmen staatlicher Verwaltung sind dann einzelne Segmente (z.B. eine Gemeinde) isoliert oder als Teil eines Aggregats (z.B. eines Bezirks oder einer Region) adressierbar und Zuständigkeiten und Rechenschaftsverpflichtungen können eindeutig an spezifizierte Einheiten vergeben werden.

Auch im vorliegenden russischen Fall zielte die offizielle Begründung für die durch die Reform eingeführte territoriale Neuordnung auf derartige Kontrollaspekte (Lankina 2005: 155). Ein besonders eindeutig quantifizierbarer Effekt der Neuordnung und Vereinheitlichung der lokalen Verwaltungseinheiten spiegelt sich dabei in ihrer Anzahl wider: In Folge der Reform verdoppelte sich die Anzahl der lokalen Verwaltungseinheiten (also Städte, Bezirke sowie ländliche und städtische Gemeinden) von 11.957 Kommunen im Jahr 2003 auf 24.208 zum Zeitpunkt der Einführung der Reform Anfang 2006 (Ross 2009: 68; vergleichbare Zahlen finden sich auch in Gelman 2007: 4). Die deutlichste Steigerung ist dabei im ländlichen Raum zu beobachten: Etwa 10.700 der rund 12.000 neuen Kommunen zählen zur Kategorie der ländlichen Gemeinden (Ross 2009: 68) - darunter auch die Dörfer meines Forschungsgebiets.

\section{Organe, Ämter und Stellen kommunaler Selbstverwaltung: Leistungsrollen und Fixpunkte lokaler Machtkommunikation}

Mit dem In-Kraft-Treten der Reform galt es, in den formal neugeschaffenen Gemeinden entsprechend der gesetzlichen Vorgaben Verwaltungsorgane und im Zuge dessen Ämter und Stellen - politische Leistungsrollen - zu schaffen, personell zu besetzen und sie zur Umsetzung der Reformmaßnahmen zu befähigen. Politische Ämter als »friedliche Form der Präsentation und Ausübung von Macht « (Luhmann 2000b: 91) sind innerhalb des politischen Systems Fixpunkte der Kommunikation, über die sich der spezifisch politische Machtgebrauch von anderen Formen der Machtausübung in der Gesellschaft unterscheiden lässt (Luhmann 2000b: 73). Durch die Form des Amtes lässt sich der Machtcode (machtüberlegen|machtunterlegen) »definieren als Differenz von Amtsträger und Amtsunterworfenem, von Regierung und Volk« (Luhmann 2000b: 91). Zudem kann die an politische Ämter geknüpfte Macht unter der Bedingung funktionaler Differenzierung allein politisch legitimiert sein, also nicht religiös, dynastisch oder auf andere nichtpolitische Weise begründet. Zusammengenommen sorgen Identifizierbarkeit und politische Legitimation ämtergebundener Machtkommunikation dafür, dass sich das politische System schließen und rekursiv auf seine Operationen beziehen kann (Luhmann 2000b: 75): Sofern Machtkommunikation von politischen Ämtern ausgeht, kann politisch daran angeschlossen werden.

Die »organisatorische Implikation« von politischen Ämtern sind Stellen als »Formen für die Variation von Inhalten« (Luhmann 2000b: 92). Diese Variationen können sich als Personalwechsel, Programmänderungen oder Änderungen der 
Kompetenzen oder des Gehalts einer Stelle vollziehen. Solange nur einzelne dieser Parameter geändert werden, bleibt die Identität einer Stelle davon unbeeindruckt (Luhmann 2000b: 240f). Das Identitätsprinzip der Stelle als formalisierte Mitgliedschaftsrolle sichert die Kontinuität politischer Herrschaft, indem der »Machtcode als Macht der Stellen« im Verhältnis zu allen anderen Ämtern und Stellen fixiert und damit geregelt wird, welche Entscheidungen anderer als Prämissen in die Entscheidungen eines Stelleninhabers oder einer Stelleninhaberin übernommen werden müssen (Luhmann 2000b: 93).

Mit Blick auf die interne Struktur der dörflichen Lokalverwaltung sieht Gesetz Nr. 131 zwei solcher Fixpunkte der Machtkommunikation vor: Den Gemeinderat (»sovet deputatov«) und das Amt des Bürgermeisters oder der Bürgermeisterin (»glava municipal'nogo obrazovanija«). Je nach Variante lokaler Selbstverwaltung existierten beide unter dieser Bezeichnung bereits vor der Reform, ihre formalen Grundlagen wurden jedoch erst durch Gesetz Nr. 131 konkretisiert und beide neu zueinander in Beziehung gesetzt. Der Gemeinderat (Art. 35) als kommunales Repräsentativorgan setzt sich aus mindestens sieben (in Gemeinden mit weniger als 1.000 Einwohnern) und maximal 35 Mitgliedern (in Gemeinden mit mehr als 500.000 Einwohnern) zusammen, die alle fünf Jahre von den Gemeindemitgliedern gewählt werden. Jedes Gemeinderatsmitglied ist für einen bestimmten Gemeindebezirk verantwortlich und soll den Einwohnerinnen und Einwohnern als direkte Ansprechperson dienen. Die exklusiven Kompetenzen des Gemeinderats umfassen die Verabschiedung der obligatorischen Gemeindecharta (»ustav municipal'nogo obrazovanija«), die Bestätigung des kommunalen Budgets und des Berichts über die Budgetverwendung, die Einführung und Änderung lokaler Steuern und Gebühren, die Verabschiedung von Programmen zur wirtschaftlichen Entwicklung der Kommune, Entscheidungen bezüglich der Verwaltung kommunalen Eigentums und kommunaler Unternehmen sowie allgemein die Überwachung der kommunalen Exekutivorgane. Zumindest formal, so die Einschätzung von Wollmann und Gritsenko (2009: 235f), verfügen die Gemeinderäte damit über eine unerwartet beachtliche Fülle an Kontroll- und Entscheidungskompetenzen, auch und gerade im Vergleich mit westeuropäischen Systemen kommunaler Selbstverwaltung.

Der Bürgermeister oder die Bürgermeisterin (Art. 36) ist formal das höchste Amt in den Gemeinden und Teil der lokalen Exekutive, die sich mit der Umsetzung der kommunalen Aufgaben befasst. Die Wahl erfolgt entweder direkt durch die Gemeindemitglieder oder indirekt durch den Gemeinderat. Vor allem in kleinen Gemeinden leitet der Bürgermeister oder die Bürgermeisterin zugleich die kommunale Administration (»mestnaja administracija «, umgangsspr. »sel'sovet «) (Art. 37) ${ }^{13}$ als

13 Dies gilt vor allem für ländliche Siedlungen und ist insgesamt in knapp der Hälfte aller Gemeinden der Fall (Wollmann/Gritsenko 2009: 237f). In Gemeinden, in denen die bei- 
dritte obligatorische Institution und ernennt deren Personal. Letzteres besteht aus Sachbearbeiterinnen und Sachbearbeitern für Bereiche wie Budgetplanung, Buchführung, Eigentumsfragen oder allgemeine Angelegenheiten lokaler Selbstverwaltung.

Die Autorinnen und Autoren und die (politischen) Befürworterinnen und Befürworter des Gesetzes sehen gerade in den Mechanismen der Personalauswahl und Stellenbesetzung, die formal nur in Ausnahmefällen Einflussmöglichkeiten seitens des Bezirks oder der Region einräumen, einen wesentlichen Garanten für lokale Autonomie und die Trennung von staatlicher und kommunaler Verwaltung. Hinzu kommt, dass die Bürgermeisterin oder der Bürgermeister als zentrale Stelle in der Gemeinde sowohl von der Bevölkerung als auch vom Gemeinderat überwacht wird und gegenüber beiden rechenschaftspflichtig ist (Art. 36, Abs. 5). Unter den (wissenschaftlichen) Beobachterinnen und Beobachtern der jüngsten Kommunalreform herrscht mit Blick auf diese Kerninstitutionen lokaler Selbstverwaltung verhaltener Optimismus: Die Trennung zwischen dem Gemeinderat und der lokalen Exekutive kann laut Wollmann und Gritsenko (2009: 235f) mit einiger Vorsicht als lokale Variante der Gewaltenteilung und Einführung von »checks and balances « interpretiert werden. Zudem sorge der obligatorische Charakter der beschriebenen Verwaltungsinstitutionen dafür, so urteilt Lankina (2005: 155), dass alle Gemeinden zumindest formal-organisatorisch mit den neuen Prinzipien kommunaler Verwaltung durchdrungen würden: »The rigid >requirement< for setting up the above bodies is aimed at ensuring that LG [local government, E.M.] will now be present at all territorial levels «.

\section{Lokale Partizipationsmechanismen: Publikumsinklusionen}

Über die Wahl von Bürgermeisterin oder Bürgermeister und Gemeinderat hinaus sieht Gesetz Nr. 131 ein verhältnismäßig breites Spektrum an Formen der unmittelbaren Publikumsinklusion in politisch-administrative Entscheidungsprozesse vor: Mit obligatorischen Gemeindeversammlungen (»schod«) und öffentlichen Anhörungen (»publičnoe slušanie«) sollen Schnittstellen zwischen Politik und Publikum geschaffen werden, die es der Bevölkerung ermöglichen, Lokalpolitik aktiv mitzugestalten (Kap. 5, Art. 22 bis 33). ${ }^{14}$ Die Versammlungen finden in regelmäßigen Abständen statt und können auf Initiative des Bürgermeisters oder der Bürgermeisterin, des Gemeinderats oder von Gemeindemitgliedern selbst einberufen werden. Sie dienen der Informationsverbreitung, der Diskussion von >Fragen lokaler Bedeu-

den Stellen von verschiedenen Personen besetzt werden, sind die Gemeinderäte für die Auswahl der Verwaltungsvorsitzenden zuständig.

14 Das Gesetz sieht noch eine Reihe weiterer ähnlicher Mitbestimmungsformen vor, für ländliche Gemeinden sind jedoch vor allem die beiden genannten von Bedeutung. 
tung < (Art. 29, Abs. 1) und die Amtsinhaberinnen und Amtsinhaber müssen sich in diesem Rahmen gegenüber ihren Wählerinnen und Wählern regelmäßig und direkt für ihre Entscheidungen - beispielsweise hinsichtlich des Budgets oder kommunaler Rechtsakte - verantworten.

Mit diesen Regelungen werden im Bereich der lokalen Partizipation sowohl Neuerungen eingeführt wie auch bereits existierende Mitbestimmungsformen erweitert und auf eine gesetzliche Grundlage gestellt, die zumindest formal wiederum eine vergleichsweise große Reichweite aufweisen (Lankina 2005: 166): »In fact, judging by the formal letter of the law the repertoire of local citizen rights is more extensive than in many (West) European countries « (Wollmann/Gritsenko 2009: 235). Bedenken werden dennoch geäußert: Zum einen falle die praktische Umsetzung der Partizipation in vielen Kommunen deutlich hinter dem formalen Anspruch zurück (Wollmann/Gritsenko 2009: 235). Zum anderen wird an der Sinnhaftigkeit der exzessiven Regulierung der Partizipationsmöglichkeiten gezweifelt und es werden - auch und gerade mit Blick auf die offiziellen Absichtsbekundungen - diesbezüglich kontraproduktive Effekte antizipiert (vgl. für viele Ross 2009: 70):

»Such minute detail is bound to stifle, rather than encourage, local popular initiative, as failure to comply with some trivial detail could be deemed illegal. [...] Excessive regulation of local democratic procedure is also at odds with one of the main principles on which the logic of decentralization rests, namely allowing for and encouraging diversity and initiative.« (Lankina 2005: 166)

Neben derartigen Bedenken zur Umsetzung der gesetzlichen Bestimmungen, deren Berechtigung letztlich empirisch zu überprüfen ist, ist im Hinblick auf die formal eingeräumten Partizipationsmöglichkeiten aus systemtheoretischer Perspektive ein weiterer Aspekt bemerkenswert: Durch die verpflichtende Einführung von Gemeindeversammlungen, öffentlichen Anhörungen und ähnlichem stellt das politische System die Kommunikation an der Schnittstelle zum Publikum auf Interaktion um, mit deren Eigenlogik fortan $\mathrm{zu}$ rechnen ist. Wie jedes Interaktionssystem grenzt sich die Gemeindeversammlung über die Unterscheidung Anwesenheit|Abwesenheit gegenüber ihrer innergesellschaftlichen Umwelt ab. Nur wer anwesend ist, kann adressiert werden, »[w]er nicht anwesend ist, der kann an der Interaktion auch nicht mitwirken, wie immer mächtig er auch sonst sein mag« (Kieserling 1999: 17). Das Interaktionssystem zerfällt, wenn die Anwesenheit der Teilnehmenden endet. Interaktionen können bestenfalls wiederholt werden, sich jedoch als Systeme nicht gegenüber der Anwesenheit der Teilnehmenden verselbstständigen und darüber hinaus andauern (Kieserling 1999: 17). Als Folge der interaktionskonstitutiven wechselseitigen Wahrnehmung der Teilnehmenden wohnt Interaktionssystemen ein Kommunikationszwang inne: Die Möglichkeit nicht zu kommunizieren entfällt, 
»man muß Abwesenheit wählen, wenn man Kommunikation vermeiden will« (Luhmann 1984: 562). In der Interaktion kann ausgewählt werden, »was ins Zentrum der gemeinsamen Aufmerksamkeit gelangt und was unbeobachtet bleibt « (Luhmann 1984: 565) - und dies gilt stets und undifferenziert für alle Teilnehmenden. Im Unterschied zu Funktionssystemen und Organisationen sind Interaktionen nicht in der Lage, sich intern zu differenzieren (also Subsysteme zu bilden), sondern leisten undifferenzierte Inklusion:

»Was jeweils im Zentrum der Aufmerksamkeit geschieht, das ist allen sofort zugänglich, ohne daß Boten oder Vermittler erforderlich würden. Kommunikation in der Interaktion ist automatisch interaktionsöffentliche Kommunikation. In der Interaktion gibt es keine Geheimnisse.« (Kieserling 1999: 48)

Daraus resultiert nicht nur der Zwang zur Serialität und die hervorgehobene Bedeutung von Themen und Sprecherrollen - es kann stets nur ein Thema gleichzeitig behandelt werden und nur wer das Wort hat, kann ein Thema setzen (Kieserling 1999: 37f). Auch die Negierfähigkeit mit Blick auf die gewählten Inhalte ist gering - oder andersherum: die Sicherheit eines gemeinsamen Informationsbesitzes unter den Anwesenden ist hoch (Luhmann 1984: 561). Wer teilnimmt, kann nicht auf die eigene Unwissenheit verweisen.

Als »vorübergehende Verselbstständigung einer kleinen Sozialordnung inmitten der großen Sozialordnung « (Kieserling 2008: 130) ist eine Interaktion in der Lage, mit diversen »Absonderlichkeiten« $\mathrm{zu} »$ experimentieren« (Luhmann 1997: 478f). So leistet sich die Interaktion »eine merkwürdig selektive Ordnung der Relevanz« (Kieserling 1999: 17) - von speziellen Beziehungen zwischen einzelnen Interaktionspartnern kann in der interaktionsinternen Öffentlichkeit abstrahiert werden und auch soziale Statusmerkmale einzelner Teilnehmerinnen oder Teilnehmer kommen möglicherweise nur selektiv in Abhängigkeit von der konkreten Situation zum Zug - und erlangt damit Autonomie gegenüber anderen Systemebenen. Dabei ist jedoch das, was in der Interaktion geschieht, für die Gesellschaft und ihre Strukturen nicht zwingend von Belang (Luhmann 1997: 478f). Interaktionssysteme sind aus sich heraus nicht in der Lage, über sich selbst herauszureichen und beispielsweise darauf Einfluss zu nehmen, wie sich der in ihnen erzeugte Sinn nach ihrem Zerfall fortsetzt (oder auch nicht). In der Art und Weise, wie Interaktionssysteme in Form von öffentlichen Versammlungen gezielt als Instrumente zur politischen Publikumsinklusion eingesetzt werden, ist diese Wirkung über sich selbst hinaus jedoch ein entscheidender Punkt - andernfalls könnte schwerlich von effektiver Mitbestimmung die Rede sein. Um eine solche Wirkung auf die Organe der Lokalverwaltung und ihre Entscheidungen sicherzustellen, greifen teilweise und für klar definierte Bereiche formal-gesetzliche Regelungen (beispielsweise darüber, in welchen Fällen Ab- 
stimmungen durchzuführen sind und wie mit deren Ergebnissen weiter zu verfahren ist). Eine solche übergreifende Wirkung bestimmt sich jedoch nicht aus der Interaktion selbst heraus, sondern sie wird spezifischen Interaktionen, die sich erst als solche ausweisen müssen oder extern als solche definiert werden, aus dem politischen System heraus zugewiesen. Ob und inwieweit die Kommunikation derartigen Formalvorgaben gehorcht und auf welche Weise abseits des formalen Rahmens, standardisierter Prozesse und quantifizierbarer Resultate Wirkung über die einzelne Interaktion hinaus entfaltet wird und sich gegebenenfalls entsprechende Erwartungsstrukturen herausbilden, ist empirisch zu klären.

\section{Kommunale Aufgabenbereiche: Funktionale Klärungsansprüche und die formale Entflechtung von Kolchos und Gemeinde}

Auch mit Blick auf die Zuständigkeiten der Lokalverwaltung führt die jüngste Reform eine entscheidende Neuerung ein: Blieben frühere Regelungen bezüglich der Zuteilung von Verantwortlichkeiten oft im Vagen und bezogen sich primär auf den Erhalt bereits bestehender öffentlicher Infrastruktur, was letztlich zu starken Ungleichgewichten zwischen den Gemeinden geführt hatte, zielt Gesetz Nr. 131 auf eine Verbesserung der Lage vor allem in den kleineren Gemeinden des ländlichen Raums. Den Gemeinden werden erstmals genau festgelegte Zuständigkeiten im Hinblick auf die Bereitstellung von kommunalen Dienstleistungen übertragen, d.h. die »Angelegenheiten lokaler Bedeutung «, die üblicher Bestandteil von Definitionen lokaler Selbstverwaltung sind und in der Regel die verschiedenen Bereiche der sogenannten Daseinsvorsorge umfassen, werden spezifiziert und in konkrete Aufgaben überführt (Kap. 3, Art. 14 bis 18). Im Gegensatz zu den vorhergehenden gesetzlichen Regelungen sind spezifische kommunale Aufgaben von nun an unabhängig von der Existenz entsprechender Einrichtungen zu erfüllen, wodurch Versorgungsungleichheiten im ländlichen Raum vermieden werden sollen. Zwischen beiden kommunalen Ebenen besteht dabei Arbeitsteilung: Während sich die Aufgaben der Gemeinden auf ihr jeweils eigenes Territorium beschränken, sind die Bezirke für übergeordnete Leistungen zuständig (vgl. Tabelle 4).

Vor dem Hintergrund der Strukturen des sowjetischen Dorfes, die sich durch die 1990er Jahre hindurch in vielen Gemeinden praktisch unverändert erhalten haben, stellt gerade dieser Reformbereich einen wichtigen Einschnitt dar. Während der Handlungs- und Entscheidungsspielraum der Gemeindeverwaltungen im sowjetischen Regime auf ein Minimum begrenzt war, waren die landwirtschaftlichen Großbetriebe formal, d.h. vor allem per Musterstatut, für eine Vielzahl von Aufgaben vorgesehen: Sie galten gleichermaßen als landwirtschaftliche Produktionseinheiten, sollten für die (politische) Erziehung ihrer Mitglieder sorgen und sich um die Einrichtung und den Unterhalt der öffentlichen Infrastruktur in den Dörfern bemühen, solange dies - so die Vorgabe - mit den Zielen der Genossenschaft im Ein- 
klang stand. Zusammengenommen führte dieses breite Aufgabenspektrum dazu, dass die Betriebe in den Dörfern zu umfassenden Organisationen wurden, zu denen die Gemeindeverwaltungen in Abhängigkeit standen respektive von denen sie faktisch vollständig übernommen wurden. Da sich die Politik ohnedies als überrelevante Umwelt der Kolchose aufführte und auch Kommunikation in Form von Zahlungen nur scheinbar auf das Knappheitsproblem verwies, letztlich aber stets politisch war, generierte die Multifunktionalität der Betriebe in allen Richtungen Anschlussmöglichkeiten. Klärungsbedarf hinsichtlich der funktionalen Zuordnung blieb hingegen latent (zu funktionaler Differenzierung als Beobachtungsschema von Organisationen vgl. Tacke 2001).

Tabelle 4: Kommunale Aufgabenverteilung gemäß Gesetz Nr. 131

\section{Obere kommunale Ebene: Aufgaben der Bezirke}

- Unterhalt von Kindergärten und Schulen

- medizinische Versorgung in Krankenhäusern und Ambulanzen

- Polizei

- Umweltschutz

- Abfallentsorgung

- Unterhalt der Bezirksbibliotheken

- Organisation von Kultur-, Freizeit- und Sportereignissen

- Strom- und Gasversorgung

- Instandhaltung der Straßen und Wege zwischen den Gemeinden

- Bereitstellung öffentlicher Verkehrsmittel zwischen den Gemeinden

\section{Untere kommunale Ebene: Aufgaben ländlicher Gemeinden}

- Bereitstellung und Unterhalt technischer Infrastruktur für die Strom-, Wasserund Gasversorgung, Straßenbeleuchtung und Abfallentsorgung (innerhalb der Gemeinden)

- Bereitstellung von Wohnraum für einkommensschwache Familien

- Brandschutz

- Instandhaltung von Friedhöfen, Parkanlagen und öffentlichen Gärten

- Unterhalt der Gemeindebibliotheken

- Organisation von Kultur-, Sport- und Freizeitveranstaltungen und Aktivitäten für Kinder und Jugendliche

- Instandhaltung der Straßen und Wege im Dorf

- Bereitstellung öffentlicher Verkehrsmittel innerhalb der Gemeinden

Quelle: Gesetz Nr. 131 (Art. 14 und 15), Young/Wilson (2007), Center for Fiscal Policy (2008). 
Bereits die Privatisierungsmaßnahmen, die Anfang der 1990er Jahre mit Blick auf die Etablierung marktwirtschaftlicher Strukturen in der Landwirtschaft eingeleitet wurden, hatten in das geräuschlose Funktionieren des zugrundeliegenden »Kolchos-Skripts « indirekt eingegriffen und Klärung erfordert (zur postsowjetischen Privatisierungspolitik allgemein vgl. The World Bank 1992: Kap. 5). In einer Art »Privatisierungsultimatum « (Lindner 2003: 20), das der damalige Präsident Jelzin im Jahr 1991 per Regierungserlass stellte, hieß es dazu: »Die Kolchoze und Sovchoze sind verpflichtet, sich bis zum 1. Januar $1993 \mathrm{zu}$ reorganisieren und ihren rechtlichen Status in Einklang mit dem Gesetz der Russischen Föderation >Über Unternehmen und unternehmerische Tätigkeit< $\mathrm{zu}$ bringen « (Regierungserlass Nr. 86 vom 29. Dezember 1991, zitiert nach Lindner 2008: 149). Letzteres wiederum definierte gleich im ersten Artikel unternehmerische Tätigkeit als »eigenständige Initiative eines einzelnen Bürgers oder mehrerer Bürger, die auf die Erzielung von Gewinn ausgerichtet ist « (Gesetz der RSFSR Nr. 445-1 vom 25. Dezember $1990 »$ Über Unternehmen und unternehmerische Tätigkeit«, Art. 1, Abs. 1). ${ }^{15}$ Organisationszweck (auch) der ehemaligen Kollektivbetriebe sollte fortan allein die möglichst effiziente Produktion landwirtschaftlicher Erzeugnisse sein. Die Funktion der Betriebe wurde damit wirtschaftlich, d.h. im Hinblick auf Zahlungen und Zahlungsfähigkeit definiert und nicht mehr - wie im sowjetischen Regime - als umfassende politische Kontrolle der Mitglieder festgelegt (zur Unterscheidung von Zweck und Funktion vgl. Luhmann 1995: 222f). Zum Fluchtpunkt politischer Steuerungsversuche wurde entsprechend die Frage, wie der Kolchos zum Unternehmen wird (Amelina 2002) respektive sich gleich vollständig in kleinbäuerliche Produktionsstrukturen überführen lässt (für einen Überblick über die entsprechenden politischen Maßnahmen vgl. z.B. Lerman 2001; Wegren 2009b: Kap. 3 und 4). Jegliches >soziale< Engagement der Betriebe, das früher im Einklang mit den Formalstrukturen stand, geriet dazu in Widerspruch und galt (und gilt) als wesentliches Hindernis für die effektive Privatisierung der ehemaligen Kollektivbetriebe (vgl. z.B. Davydova/Franks 2006; Lerman 2002). Deren zwar nicht formal, aber faktisch starke Einbindung in öffentliche Aufgaben, so das Argument, verhindere nicht nur eine effizientere Wirtschaftsweise, sondern stehe auch dem Herauslösen der Unternehmen aus dem lokalen Dorfkontext und der Orientierung an globalen Marktstrukturen im Wege:

»LSEs [large-scale enterprises, E.M.] have four roles: commercial producers of food, employers, providers of inputs to workers' and to villagers' household plots, and as providers of

15 Der russische Gesetzestext ist online verfügbar unter http://www.ru-90.ru/25-12-1990-2№-44 (zuletzt eingesehen am 30.4.2015). 
social services to the village community. The first role links them to the wider market economy, the remainder to their local (village) community.« (Davydova/Franks 2006: 52)

»Freed from central planning and stripped of traditional financial support, farm enterprises remain burdened by paternalistic responsibilities, including lifetime employment and social services in the villages.«(Lerman 2002: 46)

Sämtliche Operationen, die direkt oder indirekt einer wirtschaftlichen Primärorientierung der Betriebe widersprachen und nicht unmittelbar auf die (Wieder-)Herstellung der Zahlungsfähigkeit der Organisation ausgerichtet waren, so der Anspruch, sollten mindestens in den Hintergrund gedrängt, im besten Fall jedoch ganz unterlassen werden. Kommunikation galt es in einer Weise umzustrukturieren, dass die Wirtschaft (und nicht mehr die Politik) zur relevanten Umwelt wurde, an der sich die Organisation orientiert - und sich auf diese Weise auch die Frage ihrer funktionalen Zuordnung klärt.

Die Festlegung eines klar umrissenen kommunalen Aufgabenbereichs durch Gesetz Nr. 131, das diesbezüglich in seinem Konkretisierungsgrad deutlich tiefer greift als vorhergehende Regelungen, verhält sich in diesem Sinne komplementär zum Privatisierungsprozess (vgl. dazu auch die Darstellungen in Lindner/Moser 2009b; Moser/Lindner 2011): Es untermauert die Forderung nach einer Entflechtung und funktionalen Klärung beider Organisationen - Kolchos und Kommune und im Zuge dessen nach einer Stärkung der Gemeindeverwaltung (auch) in Relation zu den ehemaligen Kollektivbetrieben. Konkret forcieren die Regelungen zum einen nochmals den in den Privatisierungsmaßnahmen bereits angelegten Klärungsanspruch, indem sie formal die Lücke zu schließen versuchen, die durch den Rückzug der Kollektivbetriebe aus dem Bereich der öffentlichen Infrastruktur entstehen würde - und vereinzelt bereits entstanden ist. Während die Privatisierungsmaßnahmen die Landwirtschaftsbetriebe implizit von bestimmten Aufgaben zu entbinden versuchen, greift die Kommunalreform an dieser Stelle ein und weist die entsprechenden Aufgaben den Gemeinden zu. Dabei entsprach es bis weit in die 1990er Jahre hinein eher der Regel als der Ausnahme, dass die ehemaligen Kollektivbetriebe den Großteil der lokalen Infrastruktur in den Dörfern trugen. ${ }^{16}$ In den wenigen Fällen, in denen sich die Betriebe tatsächlich aus ihren >sozialen< Leistungen zurückgezogen hatten, versetzten die formalen Rahmenbedingungen zur kommunalen Selbstverwaltung die Kommunen kaum in die Lage dies auszugleichen. Unweigerliches Resultat waren eine geringere Verfügbarkeit und schlechtere Qualität

16 Aus Sicht der Gemeindeverwaltung ergaben sich aus dieser Konstellation gute Gründe, die Privatisierungsmaßnahmen aktiv zu blockieren (vgl. dazu empirisch Allina-Pisano 2004, 2008). 
der Leistungen in den entsprechenden Dörfern (vgl. die empirischen Befunde in Lerman 2002: 43ff). Zum anderen sind die formal konsequentere Aufgabenteilung und ein erweiterter Entscheidungs- und Einflussbereich der lokalen Behörden wesentliche Ansatzpunkte, um das Abhängigkeitsverhältnis zu den Kollektivbetrieben $\mathrm{zu}$ durchbrechen und die damit einhergehende Marginalisierung der Gemeindeverwaltungen aufzuheben (Lerman 2002: 45ff; auch Norsworthy/Paluba 2000), die ebenfalls meist über den Regimewechsel hinaus Bestand hatten. Die formale Konkretisierung von Aufgaben und Entscheidungskompetenzen allein ist dabei jedoch kaum ausreichend, um diesen Anspruch umzusetzen und tradierte Abhängigkeiten aufzubrechen. Erforderlich ist darüber hinaus eine adäquate Ressourcenausstattung als Grundlage kommunaler Autonomie, womit ein weiterer Reformbereich in den Blick gerät: Budget und Eigentum.

\section{Kommunales Budget und Eigentum: Fragliche Akteure}

Eine tiefgreifende und kontrovers diskutierte Änderung im Kontext der jüngsten Kommunalreform betrifft die kommunalen Finanzen. Durch das neue Gesetz und die etwa zeitgleich angepasste Finanzgesetzgebung der Russischen Föderation ${ }^{17}$ erhalten die Kommunen erstmals ein eigenes Budget, für das sie auch selbst die Verantwortung tragen. Hauptbestandteile des Gemeindebudgets sind neben den Transferzahlungen von höheren Verwaltungsebenen Einnahmen aus lokalen Steuern sowie außersteuerliche Einnahmen. Mit der Grund- und der Vermögenssteuer fließen dabei erstmals zwei Arten von Steuern direkt und vollständig den Kommunen zu: Die Steuer auf persönliches Eigentum und die Steuer auf Land (vgl. Tabelle 5). Zudem erhalten die Gemeinden einen festgelegten Anteil bestimmter föderaler Steuern, was insbesondere in Kombination mit dem von nun an klar definierten Aufgabenbereich die Grundlage für eine längerfristige und nachhaltigere Finanzplanung schaffen soll. Gemäß den vorherigen Regelungen waren die Transferzahlungen ständigen Änderungen unterworfen, so dass die finanzielle Lage der Kommunen größtenteils vom Ermessen der übergeordneten regionalen Behörden abhängig war (Lankina 2005: 163). Zudem steht hinter den Neuregelungen die Absicht, kommunale Aufgaben und deren Finanzierung stärker aufeinander abzustimmen. Vor allem die Praxis der sogenannten ungedeckten Mandate sollte durch die Reform abgeschafft werden, also Verantwortlichkeiten, die den Kommunen zwar von übergeordneten Ebenen übertragen wurden, für die aber von Vornherein keine adäquaten Mittel zur Verfügung standen (Gelman 2007: 4; Lankina 2005: 163; Young/Wilson 2007: 1074f).

17 Die Regelungen zu den kommunalen Steuereinnahmen sind nicht Teil der Reform lokaler Selbstverwaltung selbst, sondern gehen aus der zeitgleich stattfindenden Reform der russischen Haushaltsordnung (»Bjudžetnyj kodeks Rossijskoj Federacij«) hervor. 
Diese Absichten werden in Beurteilungen der Reform zwar durchweg begrüßt, im selben Atemzug wird jedoch auch auf die faktische Aussichtslosigkeit des Unterfangens verwiesen: Zum einen seien die kommunalen Steuern diejenigen, die voraussichtlich verhältnismäßig wenig Einkommen generierten (Lankina 2005: 165). Zum anderen wird das Erheben und Eintreiben der Grundsteuer als problematisch eingeschätzt, da nur dasjenige Land besteuert werden könne, für das eine offizielle Bewertung durch die örtlichen Katasterämter vorliege. Der Bewertungsprozess verliefe jedoch ausgesprochen zögerlich und entziehe sich vollständig dem Einfluss der Gemeinden, so dass gerade in den ersten Jahren nach In-Kraft-Treten der Reform mit Einnahmeeinbußen im Vergleich zur formal vorgesehenen kommunalen Steuerbasis zu rechnen sei (Center for Fiscal Policy 2008: 11).

Tabelle 5: Kommunale und anteilige föderale Steuern (ab 2006)

\begin{tabular}{l|l|l|l|l|l|} 
& Föderale & Regionale & \multicolumn{3}{|c|}{ Lokale Ebene } \\
& Ebene & Ebene & Städte & Bezirke & Gemeinden \\
\hline Kommunale Steuern & & $100 \%$ & & $100 \%$ \\
\hline $\begin{array}{l}\text { Vermögens- } \\
\text { steuer }\end{array}$ & & & $100 \%$ & & $100 \%$ \\
\hline $\begin{array}{l}\text { Steuer auf Grund } \\
\text { und Boden }\end{array}$ & & & & & \\
\hline $\begin{array}{l}\text { Anteile föderaler Steuern } \\
\text { Steuer auf geld- } \\
\text { werte Vorteile }\end{array}$ & $10 \%$ & & $90 \%$ & $90 \%$ & \\
\hline $\begin{array}{l}\text { Steuer auf Agrar- } \\
\text { unternehmen }\end{array}$ & $10 \%$ & $30 \%$ & $60 \%$ & $30 \%$ & $30 \%$ \\
\hline $\begin{array}{l}\text { Einkommens- } \\
\text { steuer }\end{array}$ & & $70 \%$ & $30 \%$ & $20 \%$ & $10 \%$ \\
\hline
\end{tabular}

* Steuer auf das Vermögen natürlicher Personen

Quelle: Fitch Ratings (2006), Center for Fiscal Policy (2008).

Ergänzt werden die Regelungen zum Gemeindebudget durch Vorgaben zum kommunalen Eigentum: Die Gemeinden dürfen gemäß der Reform alles besitzen, was unmittelbar der Bereitstellung öffentlicher Dienstleistungen dient, also beispielsweise Strom-, Wasser- und Gasleitungen, die Straßen und Wege im Dorf, Gebäude für sozialen Wohnraum, Fahrzeuge für den öffentlichen Transport, Bibliotheken und ähnliches (Kap. 8, Art. 49 bis 51). Eigentum, das explizit nicht in Zusammenhang mit den kommunalen Aufgaben steht, ist hingegen zu privatisieren oder in 
Staatsbesitz zu übergeben. Potenzielle Mieteinnahmen gehen den Gemeinden damit verloren (Center for Fiscal Policy 2008: 11).

Vor dem Hintergrund der zu erwartenden Probleme und faktischen Einschränkungen sei absehbar, so eine häufig geäußerte Sorge, dass vor allem kleine Gemeinden in hohem Maße von Transferzahlungen abhängen werden, um ihre Aufgaben erfüllen zu können - wie das Beispiel der Gemeinde Andreevka illustriert, deren Einnahmen und Ausgaben zugleich einen Eindruck vermitteln, um welche Beträge es dabei in ländlichen Gemeinden üblicherweise geht (vgl. Tabelle 6): Im Jahr 2007, also im zweiten Jahr nach Einführung der Reform, bestanden die Einnahmen der Gemeinde, die zur Deckung der kommunalen Ausgaben notwendig waren, zu 94 Prozent und damit fast vollständig aus Transferzahlungen übergeordneter Ebenen (Daten aus Center for Fiscal Policy 2008).

Tabelle 6: Budget der Gemeinde Andreevka (2007)

\begin{tabular}{l|c|c} 
& Rubel pro Kopf & Prozent \\
\hline Eigene Einnahmen (Steuern u.a.) & 144 & $6 \%$ \\
Transferzahlungen & 2159 & $94 \%$ \\
Einnahmen gesamt & $\mathbf{2 3 0 3}$ & $\mathbf{1 0 0} \%$ \\
\hline Allgemeine kommunale Dienstleistungen & 1005 & $43 \%$ \\
Kultur, Massenmedien, Jugend & 765 & $33 \%$ \\
Transferzahlungen & 252 & $11 \%$ \\
Wohnungsbau und Haustechnik & 241 & $10 \%$ \\
Sicherheit & 18 & $1 \%$ \\
Sozialpolitik & 18 & $1 \%$ \\
Sport & 15 & $1 \%$ \\
Ausgaben gesamt & $\mathbf{2 3 5 3}$ & $\mathbf{1 0 0} \%$
\end{tabular}

* 1 Euro entsprach im Jahr 2007 etwa 35 Rubel. Das Gemeindebudget betrug also knapp 66 Euro pro Kopf oder rund 65.800 Euro insgesamt.

Quelle: Center for Fiscal Policy (2008).

Die hohe finanzielle Abhängigkeit der Gemeinden, so die Befürchtung, bildet nicht nur ein mögliches Einfallstor für den Einfluss übergeordneter Ebenen, sondern schränkt den Gestaltungsspielraum, der durch die Fülle an Partizipationsmöglichkeiten formal gewährt wird, hinterrücks wieder ein. Ungeachtet der formalen Einschränkungen würden die Gemeinden entmutigt, sich als eigenständige Akteure im föderalen Institutionengefüge $\mathrm{zu}$ inszenieren und insbesondere die Partizipationsmöglichkeiten der Bürgerinnen und Bürger würden auf diese Weise faktisch ad absurdum geführt: 
»While some of the law's finance provisions introduce a more sound system, the thrust of the legislation further strengthens local government's dependence on both regional and federal levels of authority. Not only is such dependence likely to lead to greater local inefficiencies, it will also discourage municipalities from acting as autonomous political players in the federation's intergovernmental system.«(Lankina 2005: 162)

Ergänzend zu diesen Bedenken erheben Reformbeobachter regelmäßig den Vorwurf einer »Verstaatlichung« lokaler Selbstverwaltung, da die Möglichkeit bestünde, dass der oberen lokalen Ebene zusätzlich zu den eigenen Aufgaben die Ausführung von Staatsaufgaben übertragen wird (Gelman 2007: 4; Ross 2009: 80f; Wollmann/Gritsenko 2009: 234): »Municipalities will now have fewer functions to finance, but will continue to perform many tasks delegated from above, funded through subsidies and other federal or regional transfers«(Lankina 2005: 164f). Zwar sollte dabei der Staat auch die damit verbundenen Kosten vollständig übernehmen, so dass auf der lokalen Ebene keine zusätzliche finanzielle Belastung entsteht. In der Praxis wird dies jedoch oft nicht oder nur unzuverlässig umgesetzt (Wollmann/Gritsenko 2009: 240). Eine Mitsprache lokaler Verwaltungsorgane bei der Ausführung dieser delegierten Funktionen ist nicht vorgesehen. Ländliche Gemeinden, um die es in den folgenden Fallbeispielen geht, sind von diesen delegierten Funktionen nicht unmittelbar betroffen, die Sorge einer unterschwelligen »Rezentralisierung « schließt jedoch auch sie mit ein.

\section{LEITASPEKTE FÜR DIE BEOBACHTUNG LOKALER SELBSTVERWALTUNG IN DER POSTSOWJETISCHEN TRANSFORMATIONSGESELLSCHAFT}

Worauf ist nun vor diesem Hintergrund - d.h. den Ausführungen zu den Merkmalen des sowjetischen Dorfes, zu einem soziologischen Verständnis von lokaler Selbstverwaltung und zur jüngsten Kommunalreform in Russland - zu schauen, wenn man Transformationsprozesse in russischen ländlichen Gemeinden unter dem Gesichtspunkt lokaler Selbstverwaltung beobachten und verstehen will? Welche Engpässe hat die Kommunikation angesichts von Dezentralisierungsprozessen und der Umstellung auf lokale Selbstverwaltung potenziell zu überwinden? Im Folgenden möchte ich aus der bisherigen Argumentation eine Reihe von Leitaspekten ableiten. Diese wurden teilweise im Argumentationsverlauf bereits implizit tangiert, teilweise auch schon explizit genannt. Sie sollen an dieser Stelle im Hinblick auf die nachfolgende empirische Analyse noch einmal aufgegriffen und präzisiert werden. Wohlgemerkt geht es dabei nicht um die Formulierung theoriegeleiteter Hypothesen, die dann empirisch überprüft und für zutreffend oder unzutreffend erklärt 
werden. Stattdessen soll der Blick auf das empirische Material theoretisch geschärft, dabei aber gleichzeitig für Irritationen aus dem >Feld< offen gehalten werden.

Eine erste grobe Eingrenzung auf zwei theoretische Themenfelder folgt aus dem skizzierten soziologischen Verständnis von Dezentralisierungsprozessen und lokaler Selbstverwaltung: Es geht um die Gewährung lokaler Autonomie, um die Bildung von Organisationen und um die Herausbildung von Inklusionsmodi vor allem auch mit Blick auf das Publikum der Politik und die spezifische Strukturierung der lokalen politischen Peripherie. Für die ländlichen Gemeinden in Russland, um die es empirisch gehen soll, spiegeln sich diese Bereiche formal in der jüngsten Kommunalreform, Gesetz Nr. 131 »Über allgemeine Prinzipien der Organisation lokaler Selbstverwaltung in der Russischen Föderation«, das seit seinem In-Kraft-Treten im Jahr 2006 die verbindliche rechtliche Grundlage für die Selbstverwaltung (auch) kleiner ländlicher Gemeinden darstellt. Im Hinblick auf den Aspekt der Organisationsbildung beinhaltet das Gesetz unter anderem die formale Grundlage für die Schaffung von Stellen im kommunalpolitischen Kontext und deren Ausstattung mit Entscheidungskompetenzen und legt den Zweck lokaler Verwaltung in Form eines konkreten Aufgabenspektrums fest. Durch Regelungen zu den kommunalen Finanzen werden diese Aufgaben nicht nur wirtschaftlich untermauert, sondern auch der Entscheidungsspielraum der Kommunalverwaltungen spezifiziert. Im Hinblick auf lokalpolitische Inklusionsmodi werden nicht nur neue politische Leistungsrollen formal etabliert, sondern die Reform umfasst auch eine Reihe detaillierter Regelungen zu lokalen Partizipationsformen, die von der Wahl der Amtsinhaber über die Durchführung von Volksentscheiden bis hin zur Ausgestaltung von Foren wie Gemeindeversammlungen und öffentlichen Anhörungen reichen, den Gemeindemitgliedern in diesem Rahmen Möglichkeiten zur direkten Einflussnahme bieten und die Verwaltungsmitglieder mit Rechenschaftspflichten versehen.

Angesichts der Strukturmerkmale des sowjetischen Dorfes sind die Bereiche, die durch die Festlegung kommunaler Aufgaben primär tangiert werden, überwiegend nicht neu: Entscheidungen, die im Zusammenhang mit der Bereitstellung und dem Unterhalt der sozialen und technischen Infrastruktur in den Gemeinden anfallen und deren Transfer auf die lokale Ebene einen Reformschwerpunkt darstellt, wurden bereits unter dem sowjetischen Regime in den Dörfern getroffen. Dabei spielten jedoch nicht die Gemeindeverwaltungen, die gleichwohl formal existierten, sondern die landwirtschaftlichen Kollektivbetriebe respektive deren Vorsitzende eine dominierende Rolle. Gemäß der Logik des sowjetischen Regimes sollten über die Kolchose sowohl das allgemeine Ziel der Vollinklusion ihrer Mitglieder im Sinne einer umfassenden politischen Adressierbarkeit als auch die Kollektivierung von Privateigentum und die hierarchische Koordination wirtschaftlicher Produktion im ländlichen Kontext umgesetzt werden. Die Betriebe wurden im Hinblick darauf 
als umfassende Organisationen in den Dörfern eingerichtet, denen sowohl die Produktion landwirtschaftlicher Erzeugnisse als auch die sozialistische Erziehung ihrer Mitglieder und die Bereitstellung der lokalen technischen und sozialen Infrastruktur oblag. Vor dem Hintergrund dieser Formalvorgaben erlangten die Leitungspersonen der Betriebe in den Dörfern eine faktisch fast uneingeschränkte Machtposition, über die sich die Logik der sowjetischen Dörfer zu einem erheblichen Teil erschloss. Diese Position speiste sich aus zwei Quellen: Organisationsextern folgte aus den umfangreichen Leistungen der Betriebe, dass die Kolchose die Gemeindeverwaltungen (als politische Organe) in der Regel nicht nur ergänzend unterstützten, sondern faktisch vollständig übernahmen. Organisationsintern waren aufgrund der sozialen Kongruenz von Betrieb und Gemeinde - sämtliche Gemeindemitglieder waren in der Regel Kolchosangehörige oder eng mit dem Betrieb assoziiert sämtliche relevanten Entscheidungen im Dorf Kolchosentscheidungen und verwiesen auf die Betriebshierarchie.

Die Dörfer generierten sich auf diese Weise als weitgehend geschlossene Kommunikationszusammenhänge innerhalb der sowjetischen Hierarchie, wobei die Kolchosleitung als eine Art >Flaschenhals< in Richtung Umwelt fungierte. Innerhalb der Gemeinden entstand Raum für den diskretionären Umgang mit den Formalstrukturen des sowjetischen Regimes und für die Reproduktion informaler und illegaler Strukturen, so dass die begrenzte Reichweite des sozialistischen Gesellschaftsprogramms hier beispielhaft zum Ausdruck kam: Während sich die Kollektivbetriebe formal als Beispiel für die Hierarchisierung der landwirtschaftlichen Produktion beobachten ließen, basierte diese >Schauseite< oder >Fassade< letztlich darauf, dass sich buchstäblich unter der Hand und im Schatten der starken Kolchose mit den Hoflandwirtschaften informale und teils illegale Produktionsformen reproduzierten. Als faktisch privatbäuerliche Kleinstorganisationen operierten Letztere nach >regimefremden< Prinzipien, erwiesen sich für die Reproduktionsfähigkeit der sowjetischen Organisationsgesellschaft gerade dadurch aber nicht nur als >brauchbar , sondern vielmehr als überlebensnotwendig. Und auch wenn sich die Grenze der Illegalität mit dem Regimewechsel verschob und die Hoflandwirtschaften als erhoffte Keimzellen privatbäuerlicher Betriebe von nun an offiziell erwünscht waren, hatte sich trotz zahlreicher Reformversuche zumindest an der dominanten Position der Kollektivbetriebe und an der untergeordneten Stellung der Gemeindeverwaltungen bis in die späten 1990er Jahre wenig geändert.

Führt man die Überlegungen zu allen drei Aspekten - dem sowjetischen Dorf, dem soziologischen Verständnis von lokaler Selbstverwaltung und der Stoßrichtung der jüngsten Kommunalreform - zusammen, so treten potenzielle Engpässe hervor, die in diesem spezifischen Bereich postsowjetischer Transformation zu bewältigen und für die empirische Analyse bedeutungsvoll sind. Den Rahmen bildet dabei die allgemeine Frage nach dem Zusammenwirken von Organisations- und Funktions- 
systemen im postsowjetischen Dorf, die sich mit Blick auf die bisherige Argumentation in verschiedene theoretische Teilaspekte und empirische Foki aufschließen lässt.

Dies ist erstens die Frage nach verschiedenen Formen der Exklusion und Inklusion und ihrer jeweiligen Bedeutung im dörflichen Kontext. Während die Inklusion in Organisationssysteme über Mitgliedschaft stattfindet, vollzieht sich die Adressierbarkeit in gesellschaftlichen Funktionssystemen entweder durch die Übernahme von Leistungs- und Publikumsrollen oder über den Zugang zu symbolisch generalisierten Kommunikationsmedien (vgl. dazu Stichweh 2009c: 33f). Im sowjetischen Regime und darüber hinaus war die Mitgliedschaft im landwirtschaftlichen Kollektivbetrieb als umfassender dörflicher Organisation nicht nur mit der Zugehörigkeit zur Gemeinde gleichzusetzen, sondern determinierte darüber hinaus für jedes einzelne Mitglied, was in faktisch allen Lebensbereichen kategorial möglich war (und was nicht). Die Transformationsmaßnahmen zur Privatisierung der Kollektivbetriebe und zur Einführung kommunaler Selbstverwaltung führen an dieser Stelle Änderungsdruck ein, indem sie Inklusionsmechanismen ins Spiel bringen und forcieren, deren Bedeutung im Dorfkontext zuvor gering war: Adressierbarkeit für wirtschaftliche Kommunikation durch die Einführung von Eigentum und dessen Transformierbarkeit in Zahlungen sowie die Inklusion in politische Kommunikationszusammenhänge durch die formale Einführung und Stärkung (lokal-)politischer Leistungs- und Publikumsrollen. Aufgeworfen ist damit die Frage, wie sich diese funktionssystemspezifischen Mechanismen der Inklusion und Exklusion im Dorfkontext entfalten und Anschlussfähigkeit generieren, wie sie sich aneinander ausrichten und gegebenenfalls Reibung erzeugen, in welches Verhältnis sie zum vormals dominanten Mechanismus der Organisationsmitgliedschaft treten und welche Konsequenzen daraus für die dörflichen Strukturen resultieren. Im Dorfkontext lässt sich dazu eine Vielzahl konkreter Inklusions- und Exklusionsprozesse empirisch beobachten: Wird die Kolchosmitgliedschaft durch eine neue dominante Inklusionsform abgelöst und wenn ja: durch welche? Welche spezifischen Leistungs- und Publikumsrollen bilden sich im lokalpolitischen Kontext heraus und welche Erwartungen werden daran geknüpft (und: welche Rolle übernimmt die Kolchosleitung)? Welche Funktion erfüllen in diesem Zusammenhang die Gemeindeversammlungen und ähnliche öffentliche Foren, die durch die Reform formal eingerichtet und aufgewertet werden? Auf welche Weise konstituiert sich eine lokalpolitische Peripherie, die die Bündelung von Publikumsinteressen und die Vorreduktion von Komplexität im Hinblick auf die Entscheidungen des Zentrums übernimmt? Welche Bedeutung kommt dabei der Alltagskommunikation abseits formaler Strukturen und Kanäle $\mathrm{zu}$ ?

Zweitens rücken Organisationen mit ihren Entscheidungen und Lern- und Anpassungsprozessen in den Fokus. Insbesondere die Gemeindeverwaltung und der 
ehemalige Kollektivbetrieb sind hier von Bedeutung. Mit der Festlegung eines kommunalen Aufgabenkatalogs tangiert die jüngste Kommunalreform eine Reihe von Problemlagen, über die bereits vor der Reform im Dorfkontext kollektiv bindend entschieden wurde. Die Forderung nach einer Stärkung der dörflichen Verwaltungsorgane kommt einer >Umleitung< von Entscheidungskommunikationen gleich: Entscheidungskompetenzen sollen in der Lokalverwaltung gebündelt und die entsprechenden Verwaltungsstellen für spezifische Bereiche als (neue) Fixpunkte dörflicher Kommunikation eingerichtet werden. Der ehemalige Kolchos hingegen hat die entsprechenden Kompetenzen, die ihm vor der Reform teils formal, teils informal zugeschrieben wurden, aufzugeben und soll sich, so die Idee, aus den zugehörigen Aufgabenbereichen zurückziehen. Implizit eingefordert wird damit die Entflechtung beider Organisationen und die Primärorientierung an unterschiedlichen gesellschaftlichen Funktionssystemen. Der Verlauf dieses Umstellungsprozesses lässt sich mit Blick auf verschiedene Bereiche der dörflichen Kommunikation nachvollziehen: Lässt sich eine solche Entflechtung von Gemeindeverwaltung und ehemaligem Kollektivbetrieb beobachten und wenn ja: auf welche Weise vollzieht sie sich? Welche Bindungen werden gekappt, welche eingerichtet? Wie beobachten beide Organisationen ihre Grenzen sowohl zueinander als auch gegenüber anderen Umweltbereichen? An welchen Rationalitätskriterien richten sie ihr Handeln und ihre Entscheidungen aus? Welche Entscheidungen trifft die Gemeindeverwaltung, welche der ehemalige Kolchos? Welche Zuschreibungsprozesse im Hinblick auf Entscheidungen und Entscheidungskompetenzen lassen sich beobachten und welche Verschiebungen finden statt? Wer setzt für wen Entscheidungsprämissen und wie wird daran angeschlossen?

Ein dritter Bereich, an dem sich die nachfolgende Analyse orientiert, ist der Umgang mit Formalität und Informalität. Ausgehend von rechtlichen Formalstrukturen - und damit auch: von den normativen Reformregelungen - ungebrochen auf die Faktizität gesellschaftlicher Strukturen zu schließen, wäre zweifellos naiv. Ähnlich unangemessen wäre es jedoch, die Bedeutung und das Irritationspotenzial von Formalvorgaben auf Erwartungsstrukturen, Beobachtungsmodi und Sinnzuschreibungen per se zu negieren. Auch wenn gerade im Kontext postsowjetischer Transformationsprozesse gesellschaftlicher Wandel häufig und vielleicht sogar meistens nicht den formal vorgegebenen Pfaden zu folgen schien und scheint, ist dennoch zu vermuten, dass formal-rechtliche Regelungen Möglichkeitsräume neu abstecken, so dass sich Sinnhorizonte potenziell verschieben, an deren Grenzen sich Kommunikation reibt und gegebenenfalls Konflikte entzünden, außerhalb derer die Kommunikation zumindest einem gesteigerten Rechtfertigungsdruck ausgesetzt ist und von denen sich informale und illegale Strukturen nicht nur abstoßen, sondern als solche überhaupt erst unterscheidbar werden. Im Hinblick auf die Implementierung der jüngsten Kommunalreform, aber auch auf die tradierte Stellung der Kollektivbetrie- 
be im Dorf lässt sich fragen, wie sich informale Erwartungsstrukturen (beispielsweise über bestimmte Mitgliedschaftsrollen) entwickeln, wenn sich die formalen Rahmenbedingungen ändern? An welchen Punkten lassen sich dorfinterne Formalisierungsprozesse im Sinne von »Entscheidungen über Entscheidungen« (Kühl 2007: 281) beobachten, an welchen Stellen wird auf Formalisierungen verzichtet mit welchen Folgen? Welche Varianten des Zusammenspiels von formalen und informalen Erwartungen entstehen und generieren Anschlussfähigkeit und was sind die Konsequenzen für die dörflichen Strukturen (vgl. dazu in anderen empirischen Kontexten Holzer 2006; auch Japp 2007, 2010; Kühl 2007)?

Viertens geht es schließlich um die räumliche Integration der Gemeinden und um weltgesellschaftliche Strukturen. Auch hier implizieren die tradierten sowjetischen Strukturen und die Stoßrichtung der Reformmaßnahmen gegenläufige Tendenzen: Generierten sich die sowjetischen Dörfer als weitgehend geschlossene Kommunikationszusammenhänge innerhalb der politisch kontrollierten Hierarchie, drängen vor allem die Privatisierungsmaßnahmen, aber auch die Reformen im Bereich kommunaler Verwaltung auf Öffnung und Auflösung der maßgeblich durch den Kolchos gesetzten Dorfgrenzen. Inwieweit sind die lokalen Bedingungen, die »Stelle im Raum, an der sie [die Systeme, E.M.] operieren«, maßgeblich für die Freiheitsgrade, »also die Menge der Möglichkeiten, die sie realisieren können « (Luhmann 1997: 314)? An welchen Stellen löst Kommunikation sich faktisch und nicht nur potenziell aus dem lokalen Kontext heraus und beginnt, sich an einem »einheitlichen Welthorizont« (Bohn 2005: 54) auszurichten, wo wird sie durch die territorialen Grenzen des Dorfes unterbrochen und so Komplexität reduziert? Wie beginnen Eigenstrukturen der Weltgesellschaft - und dabei geht es auch, aber bei Weitem nicht ausschließlich um gesellschaftliche Funktionssysteme (vgl. Stichweh 2006) - in die dörfliche Kommunikation hineinzuwirken? An welchen Stellen bewirken sie eine erhöhte Durchlässigkeit der Dorfgrenzen, wo wird Anschlussfähigkeit verweigert? Inwieweit werden diese Entwicklungen zum Gegenstand politischer Entscheidungen (zu erwarten ist dies insbesondere im Zusammenhang mit kommunalen Aufgaben) und wie gestalten sich die daran anschließenden Entscheidungsprozesse? 


\section{Das postsowjetische Dorf als (verschwindende) Nische in der Weltgesellschaft}

Das Erkenntnisinteresse der vorliegenden Studie richtet sich auf Strukturen und Ordnungen der Kommunikation, die sich in ländlichen Gemeinden Russlands im Kontext politischer und wirtschaftlicher Transformationsmaßnahmen und speziell der jüngsten Reform kommunaler Verwaltung aus dem Jahr 2006 herausbilden. Im ersten Argumentationsschritt wurde mit der Analyse der Strukturmerkmale des sowjetischen Dorfes nicht nur der theoretische und inhaltliche Boden bereitet, um dieses Erkenntnisinteresse mit Blick auf die gegenwärtige postsowjetische Transformationsgesellschaft weiter zu verfolgen, sondern auch die Relevanz ländlicher Gemeinden als Beobachtungsgegenstand hergeleitet. In ihnen, so ließ sich zeigen, spiegelt sich exemplarisch die Funktionslogik der sowjetischen Organisationsgesellschaft, kommen doch beim Zusammenspiel von landwirtschaftlichen Kollektivbetrieben, Gemeindeverwaltungen und privaten Hoflandwirtschaften die Reichweiten und Grenzen des sozialistischen Gesellschaftsprogramms zum Ausdruck. Wirtschaftliche Privatisierungs- und politische Dezentralisierungsmaßnahmen stellen einen wesentlichen Kontrapunkt zu diesen Strukturen dar. Das von ihnen ausgehende Irritationspotenzial verspricht daher Einsichten in die Logiken postsowjetischer dörflicher Kommunikationszusammenhänge. Woraufhin dabei zu beobachten ist, wurde im zweiten Argumentationsschritt vor dem doppelten Hintergrund einer differenzierungstheoretischen Perspektive auf die Phänomene politischer Dezentralisierung und kommunaler Selbstverwaltung einerseits und den Inhalten des Gesetzes Nr. 131 »Über allgemeine Prinzipien der Organisation lokaler Selbstverwaltung in der Russischen Föderation« als jüngstem Reformschritt in diesem Bereich andererseits konkretisiert. Ergebnis dieser Überlegungen sind vier Leitaspekte - Formen der Inklusion und Exklusion, Entscheidungen sowie Lern- und Anpassungsprozesse von Organisationen, der Umgang mit Formalität und Informalität sowie räumliche Integration und weltgesellschaftliche Strukturen -, auf die hin das empirische Material nun $\mathrm{zu}$ beobachten ist. Letzteres umfasst rund 58 Stunden aufgenommene mündliche Kommunikation (darunter im Wesentlichen Interviews, aber auch Mit- 
schnitte von Versammlungen, Sitzungen und ähnlichem), offizielle Protokolle von Gemeindeversammlungen und Gemeinderatssitzungen einer ländlichen Gemeinde für die Jahre 2005 bis 2009, thematisch selektierte Auszüge einer Lokalzeitung für den Zeitraum von 2002 bis Anfang 2010, Statistiken, Bild- und Filmmaterial sowie knapp 100 Seiten Feldtagebuch, die im Rahmen von drei mehrwöchigen Feldaufenthalten entstanden und zusammengetragen worden sind. Angesichts der empirischen Materialfülle und -diversität stellt sich für die Auswertung nicht nur die Frage des methodischen Zugangs, sondern auch das Problem der Auswahl und Gliederung.

Auf die Methodenfrage gehe ich im folgenden ersten Teilkapitel ein, das in Form eines Prologs an den Implikationen ansetzt, die sich aus der Systemtheorie als empirischem Forschungsprogramm ergeben, und davon ausgehend die Form der methodischen Umsetzung begründet und erläutert, die ich für die vorliegende Studie gewählt habe. Schließlich bietet das Kapitel auch einen ersten Einblick in >mein Feld<, ebenjene ländlichen Gemeinden, auf die sich die empirische Analyse bezieht.

Meine Lösungsvariante des Selektions- und Gliederungsproblems spiegelt sich in den vier daran anschließenden Teilkapiteln: Bereits zu Beginn meines ersten Feldaufenthalts hatte ich die Erfahrung gemacht, dass Fragen, die sich unmittelbar auf Aspekte kommunaler Selbstverwaltung richteten, bei meinen Interviewpartnerinnen und -partnern kaum >zündeten « und oftmals nur ein Schulterzucken oder und dies gilt vor allem für Mitglieder der Gemeindeverwaltungen - lediglich weitgehend belanglose Floskeln hervorriefen. Als eine Reaktion darauf änderte ich meine Strategie: Anstatt >meine< Themen und theoretischen Kategorien explizit an mein Forschungsfeld heranzutragen, ließ ich die Interviews, informellen Gespräche und meine Beobachtungen inhaltlich von jenen Ereignissen leiten, die während meiner Feldaufenthalte in den Gemeinden Diskussionsthemen waren und als solche mit unterschiedlicher Intensität für Aufregung sorgten. Auf diese Weise kristallisierten sich vier Episoden heraus, die auf den ersten Blick sehr unterschiedlich wirken, bei näherem Hinsehen und vor dem Hintergrund der oben genannten Leitaspekte jedoch eine Reihe von Verknüpfungen aufweisen und sich im Hinblick auf mein Erkenntnisinteresse zu einem Gesamtbild zusammenfügen lassen: In der ersten Episode, die durch ein Flugblatt ausgelöst wurde, das im Zusammenhang mit der Kandidatur des Kolchosvorsitzenden für ein politisches Amt im Dorf verteilt wurde, geht es um Kommunikations- und Kooperationsformen zwischen Gemeindeverwaltung und ehemaligem Kollektivbetrieb primär im Bereich der lokalen Infrastruktur. Ansatzpunkt für die zweite Episode ist der Prozess des Anschlusses der Gemeinden an das Gasnetz, der sich während meiner Forschungsaufenthalte in meinem Forschungsgebiet vollzog. Im Fokus der dritten Episode steht eine Agroholding als für das ländliche Russland neuartiger Betriebstyp, die sich ebenfalls während meiner Aufenthalte in einem der Dörfer zu etablieren begann. 
Die vierte Episode dreht sich schließlich um eine Reihe von Ereignissen, die durch das Kaufinteresse ebenjener Agroholding an Agrarland ausgelöst wurden. Gesetz Nr. 131, dessen Inhalte bereits dargestellt wurden, dient mir dabei nicht per se als Untersuchungsgegenstand, sondern spannt zum einen den allgemeinen Hintergrund meiner Beobachtungen auf und fungiert zum anderen als Instrument der Aufmerksamkeitssteuerung, das den Blick immer wieder auf Anknüpfungspunkte der beobachteten Kommunikation an formale Gesetzesregelungen lenkt.

Die Gegenstandsnähe dieser vier Episoden, in denen theoretische Kategorien zwar durch die empirische Analyse hindurch gelegt werden, sich jedoch tendenziell im Hintergrund halten, fängt der abschließende Epilog wieder auf: Die genannten Leitaspekte kommen hier noch einmal explizit zum Tragen und werden zum Ausgangspunkt, um von den gegenständlichen Beschreibungen zu abstrahieren und die zentralen Erkenntnisse daraus in den breiteren Kontext der Studie einzuordnen.

\section{Prolog: Methodologische Vorbemerkungen}

Hartnäckig haftet der Systemtheorie der Ruf mangelnder Empiriefähigkeit an. Leider wird dieser Ruf nur selten expliziert und noch seltener begründet, so dass sich der Verdacht aufdrängt, dass die Kritik vielfach nur auf oberflächlicher Textlektüre basiert (vgl. als eine der wenigen Ausnahmen Knorr Cetina 1992; als Reaktion darauf Nassehi 2004). Einer von vielen Gründen für die Vorbehalte mag sein, dass beispielsweise Vertreterinnen und Vertreter ethnographischer Forschung, wie Lee (2007) freimütig einräumt, angesichts von Luhmanns zur Schau gestelltem »Antihumanismus« bereits nach wenigen Seiten das Interesse an der Lektüre systemtheoretischer Texte verlieren - und notabene trifft Lee diese Anmerkung aus einer generell wohlwollenden Haltung gegenüber der Systemtheorie heraus und im Kontext eines überzeugenden Versuchs, die Systemtheorie für qualitativ-empirisches Forschen in den Sozialwissenschaften und speziell für die Ethnographie fruchtbar zu machen:

»Some may have lost interest as early as page 35, immediately after Luhmann announces that his project intends to develop a >radically antihumanistic < concept of society. Ethnographers have trained one another to observe actors; why would they read a 1200 page book outlining a sociology that excludes people from society?« (Lee 2007: 457)

Doch nicht nur die Skepsis darüber, dass die Systemtheorie Menschen - den vermeintlich wichtigsten Gegenstand jeder sozialwissenschaftlichen Forschung - in die Umwelt sozialer Systeme platziert, ist in Disziplinen wie der Ethnologie oder der Humangeographie, die Menschen in ihren Bezügen zur Kultur oder zum Raum 
zentral stellen, verbreitet anzutreffen (vgl. z.B. Gershon 2005; auch die Verweise in Lee 2007). Mit Blick auf die empirische Eignung der Systemtheorie wird darüber hinaus regelmäßig pauschal auf den Abstraktionsgrad und den technischen Charakter systemtheoretischer Begriffe verwiesen: »Luhmann's theory can be seen as part of the age-old dream to give life to the machine, in this case the machinelike system. [...] I take these intellectual and cultural phenomena as further signs of the capitulation of autonomous life to the automaton « (Halton 1992: 35; vgl. auch Lee 2007: 460). Ein korrespondierender Vorwurf lautet, dass die Systemtheorie sich wie andere Theorien hoher Komplexität der empirischen Überprüfbarkeit generell entziehe (z.B. Amann/Hirschauer 1997: 11; vgl. auch Egner 2010: 11).

Bei diesen und anderen Vorbehalten fällt auf, dass die eingespielte Trennung zwischen Theorie und Empirie so stark gemacht wird, dass die Gemeinsamkeiten beider Seiten und ihr ko-konstitutiver Bezug regelhaft aus dem Blick geraten (Nassehi/Saake 2002: 69ff; Nassehi 2008: 79ff): Versteht man Theorie als das Handhaben von Unterscheidungen, dann drängt sich die Frage nach dem Bezug dieser Unterscheidungen förmlich auf. Und dieser Bezug weist in der Regel über die Theorie hinaus auf eine ihr äußerliche Wirklichkeit. Gleichwohl das auch eine andere Theorie sein kann, wird deutlich, dass gute Theorie - also das kritische Operieren mit Unterscheidungen und Begriffen - sich niemals selbst genügt oder sich Selbstzweck sein kann. In diesem Sinne geht es auch der Systemtheorie, die sich weniger als homogener Block denn als ein lose gekoppeltes Theorienetzwerk verstehen lässt, ${ }^{1}$ um das Verstehen und Erklären gesellschaftlicher Wirklichkeiten (Stichweh 2010: 21ff).

Wenn es also das Ziel ist, Gesellschaft besser zu verstehen und neue Einsichten $\mathrm{zu}$ vermitteln, sind Theorien Instrumente der Beobachtung von Gesellschaft und damit stets >empirisch <. Eine solche Perspektive wird auch bei Luhmann deutlich, dessen Systemtheorie die Distanz zwischen der Gesellschaft und ihrem wissenschaftlichen Beobachter einebnet (Stichweh 2010: 18). Wissenschaft vollzieht sich als gesellschaftliche Operation und (sozial-)wissenschaftliche Beobachtungen sind damit stets Selbstbeobachtungen der Gesellschaft. Die Systemtheorie ist in diesem Sinne von vornherein (auch) als Methode angelegt, die auf den systematischen Vergleich funktionaler Äquivalente im Hinblick auf gesellschaftliche Strukturbildungen zielt (Stichweh 2010: 17; zum Äquivalenzfunktionalismus vgl. Luhmann 2009 [1962], 2009 [1964]).

1 Stichweh (2010: 21ff) hebt vier Elemente dieses Netzwerk hervor: (1) Die allgemeine Theorie sozialer Systeme, (2) die Systemtheorie als Gesellschaftstheorie, die wiederum die Differenzierungstheorie, die Theorie soziokultureller Evolution, die Kommunikationstheorie und die Theorie der Selbstbeobachtung von Systemen umfasst, (3) die Interaktionstheorie und (4) die Organisationstheorie. 


\section{Die Systemtheorie als empirisches Forschungsprogramm und die ethnographische Perspektive}

Die grundlegende und zentrale methodologische Vorgabe der Systemtheorie lässt sich in zwei an das empirische Material zu richtende Fragen zusammenfassen: Wie wird beobachtet? Welche Anschlussmöglichkeiten werden eröffnet oder restringiert, welche realisiert oder unterlassen? Es geht mit anderen Worten darum, Beobachter auf die von ihnen getroffenen Unterscheidungen hin zu beobachten und dabei (Un-)Regelmäßigkeiten auszumachen. Beobachtungsgegenstand ist Kommunikation respektive das Anschließen von kommunikativen Operationen in Form des »echtzeitlichen Nacheinanders von Ereignissen« (Nassehi 2008: 88). Dabei gilt die Grundannahme, dass jede Aussage - ob theoretisch, empirisch oder methodisch stets beobachterabhängig ist und auch vermeintlich >harte< Daten eine Form von Beobachtungen sind, die das, was sie sehen (nicht aber die gesellschaftliche Wirklichkeit an sich), selbst erzeugen. Soziologie ist damit immer auch Gegenstandskonstitution, so dass sich die Frage aufdrängt, »wie der wissenschaftliche Beobachter seinen Gegenstand durch Beobachtung konstituiert« (Nassehi/Saake 2002: 70). Lösen lässt sich dieser Zirkel auch durch qualitative Forschungsdesigns nicht, aber mit Hilfe von Methoden lässt sich zumindest kontrolliert beobachten und Kontingenz durch Regeln reduzieren (wobei die Art und Weise dieser Kontingenzreduktion dann wiederum (methodologisch) beobachtet werden kann) (Nassehi 1997: 156, 2002: 70).

In der empirischen Forschung geht es vor diesem Hintergrund um zweierlei: Erstens stellt die wissenschaftliche Beobachtung darauf ab etwas zu sehen, was den Handelnden in ihrer Praxis selbst in der Regel nicht bewusst ist (Nassehi 2008: 89), indem sie eine zu den beobachteten Systemen inkongruente Perspektive einnimmt (Luhmann 1984: 88). Eine solche inkongruente Perspektive, das »anders beobachten«, ist keinesfalls mit »besser wissen « gleichzusetzen oder zu verwechseln. An dieser Stelle lässt sich Karl Mannheims Unterscheidung zwischen dem reflexiven oder theoretischen Wissen der Akteure einerseits und ihrem handlungspraktischen, inkorporierten oder atheoretischen Wissen andererseits aufgreifen: ${ }^{2}$

»Die sozialwissenschaftlichen Interpret(inn)en im Sinne der Mannheim'schen Wissenssoziologie gehen also nicht davon aus, dass sie mehr wissen als die Akteure oder Akteurinnen, sondern davon, dass letztere selbst nicht wissen, was sie da eigentlich alles wissen, somit also über ein implizites Wissen verfügen, welches ihnen reflexiv nicht so ohne weiteres zugänglich ist.«(Bohnsack/Nentwig-Gesemann/Nohl 2007b: 11)

2 Eine alternative Bezeichnung unterscheidet zwischen kommunikativ generalisiertem und handlungspraktischem Wissen (Przyborski/Wohlrab-Sahr 2009: 275). 
Nimmt man diese Unterscheidung von zwei Wissensformen zum Ausgangspunkt, besteht die Aufgabe sozialwissenschaftlicher Beobachtung darin, dieses implizite oder atheoretische Wissen der Akteure zu explizieren. Sofern dies gelingt, wird es möglich, latente Strukturen zu »entbergen« (Nassehi 1997: 154), also diejenigen Unterscheidungen $\mathrm{zu}$ visibilisieren, die der Kommunikation zugrunde liegen und für die sie selbst blind ist, denn »[1]etztlich sucht Sozialforschung stets nach Unsichtbarem - nach Unsichtbarem in dem Sinne, daß es nicht das Offensichtliche allein ist, das den sozialen Sinn eines Geschehens ausmacht« (Nassehi 2008: 89).

Zweitens kann davon ausgegangen werden, dass beobachtbare Kommunikationen Lösungen oder Bearbeitungsmodi für grundsätzlich benennbare Probleme darstellen. Ziel der Analyse ist daher nicht allein die Explikation des Praxiswissens, sondern auch das Aufzeigen des Verhältnisses von Problem und Lösung, wobei beide - also Probleme und Lösungen - kontingent gesetzt werden. Den Übergang von ex ante festgelegten, der Empirie vor- und übergeordneten Bezugsproblemen (im Sinne Parsons) hin zu einem Verständnis von Problemen und Lösungen als Produkten der Kommunikation, die sich erst im Vollzug der Kommunikation konstituieren, bringt Nassehi (vgl. v.a. 2008) als neue Lesart des Funktionalismus ins Spiel, den er methodisch fruchtbar zu machen versucht. Angelegt ist diese Wendung der funktionalen Analyse allerdings bereits bei Luhmann (vgl. z.B. 2009 [1964]: 49ff), der im Kontext seiner Ausführungen zum Äquivalenzfunktionalismus von Ursachen und Wirkungen (anstelle von Problemen und Lösungen) spricht. Auch bei Merton (1995 [1949]) finden sich vergleichbare Überlegungen. Beide, Luhmann und Merton, gehen ebenfalls nicht von vorempirischen Bezugsproblemen aus, die es zu lösen gilt, sondern beobachten (variable) Probleme und Lösungen im Horizont der Stabilität sozialer Systeme und der Art der Gestaltung der SystemUmweltbeziehungen:

»Das Problem mag subjektiv formuliert sein als Unkenntnis der Lösung. Man kann aber auch in Gegenrichtung vorgehen und zu einem bekannten Sachverhalt das Problem suchen, das diesen Sachverhalt als Lösung erscheinen läßt und eventuell die Suche nach anderen Problemlösungen stimulieren kann.« (Luhmann 1990: 421)

Lösungen vollziehen sich immer dann, wenn Kommunikation Anschlussfähigkeit generiert, also im Kommunikationsprozess soziales oder operatives Verstehen erfolgt (Nassehi 1997: 143, 2008: 95). Ausgehend von der Beobachtung von Lösungen lässt sich dann nach den zugrundeliegenden Problemkontexten fragen und daran anschließend nach Folgeproblemen, die sich ergeben und für die wiederum Lösungen gefunden werden müssen (vgl. die »Problemstufenforschung « bei Luhmann 2009 [1962]: 28): 
»The ordered realty of a social system may be sociologically described from the perspective of operative functionalism. What can we learn about a system if we imagine that its operations create and solve a particular problem with a particular solution? What can we gain by comparing and contrasting different problems and solutions? With this strategy, the ethnographer documents the system as an imaginary unity of different operations that self-describes its recurrent problem and accounts for its historical operations as if they were contingent but meaningful (functional) solutions.« (Lee 2007: 464)

Gleichermaßen Voraussetzung wie Ziel dieses Unterfangens ist ein Sinnverstehen, welches über die reine Beschreibung - wie >dicht< sie auch sein mag - der beobachteten Kommunikation hinaus geht. (Sinn-)Verstehen im Rahmen empirischer Forschung ist für Lee und Brosziewski (2007: 259) das Beobachten von Selbstreferenz (der beobachteten Systeme). Unter Rückgriff auf die Unterscheidung von Medium und Form (vgl. Luhmann 1997: 195ff), d.h. die Differenz zwischen lose und fest gekoppelten Elementen, setzt Sinnverstehen voraus, dass der soziologische Beobachter in der Lage ist, die aktuelle Kommunikation (Operation) als eine Form zu beobachten, die auf andere potenzielle Formen verweist, die aus demselben Medium emergieren können:

»Sinn ist das Medium, das mit der Differenz von Aktualität und Potenzialität arbeitet, und zwar mit der Differenz, mit der Unterscheidung in dem Sinne, dass die Einheit der Unterscheidung immer mitspielt, dass man also immer in dem, was man sieht, Möglichkeitsperspektiven hat und umgekehrt Möglichkeiten nicht thematisieren kann, nicht denken kann, auch gar nicht kommunikativ nutzen kann, wenn man dies nicht aktuell tut.« (Luhmann 2004: 233)

Folgt man den Prämissen der Systemtheorie, so folgt daraus für empirisches Forschen respektive für die Ethnographie, auf die Lee und Brosziewski sich hier beziehen: »[E]thnographers should explain how symbolically generalized forms are actualized within a generally irritating - and thus perceptible - medium « (Lee 2007: 461). Dies ist wiederum nur möglich, wenn der soziologische Beobachter selbst mit den vorhergehenden Operationen, an die angeschlossen wird, und mit den möglichen Sinnhorizonten der beobachteten Kommunikation vertraut ist - mit anderen Worten: wenn er Zugang zum Vorrat der Sinnformen des beobachteten Systems hat und damit zum Medium, aus dem die beobachteten Formen hervorgehen: »To observe in the form of understanding, the ethnographer must gain access to the memory of a social system, to its own imaginary history « (Lee/Brosziewski 2007: 260).

Einen möglichen (wohlgemerkt aber nicht den einzigen) Zugang zu beiden Seiten der Unterscheidung Medium|Form bietet die Ethnographie als empirische For- 
schungsstrategie, die darauf ausgerichtet ist, über das Beschreiben von »kleinen Lebenswelten « (Lüders 2008: 389) eine Antwort auf die Frage zu finden, »wie die jeweiligen Wirklichkeiten praktisch >erzeugt< werden; es geht also um die situativ eingesetzten Mittel zur Konstitution sozialer Phänomene aus der teilnehmenden Perspektive« (Lüders 2008: 390). Konstitutiv für das Selbstverständnis ethnographischer Forschung ist das »Entdecken« als Erkenntnisstil und Fremdheit und Vertrautheit als die der Ethnologie entlehnte Leitdifferenz (Amann/Hirschauer 1997: 8ff): Das Fremde ist vertraut zu machen, wobei das Kriterium der Unbekanntheit jener Lebenswelten, die es zu entdecken und (wissenschaftlich) zu verstehen gilt, nicht notwendigerweise räumliche Ferne impliziert. Auch vermeintlich Vertrautes lässt sich methodisch auf Distanz zum Beobachter bringen und betrachten »als sei es fremd «(Amann/Hirschauer 1997: 12). Ein wesentlicher Bestandteil und das Alleinstellungsmerkmal des ethnographischen Zugangs ist dementsprechend das Eintauchen der Forscherin oder des Forschers als »professional stranger « (Agar 1996) in fremde Lebenswelten. Im Einklang mit den oben skizzierten Prinzipien eines systemtheoretischen Forschungsprogramms setzt auch die Ethnographie dabei auf Beobachtungen, die sich inkongruent zur alltäglichen oder bereits gewussten Perspektive der Teilnehmenden selbst verhalten und gerade dadurch (wissenschaftlich) erkenntnisversprechend sind. Auf eine Beobachtungstheorie, so monieren Lee und Brosziewski (2007), wird seitens der Ethnographie dabei allerdings in der Regel verzichtet.

Methodisch zeichnet sich ethnographische Feldforschung in erster Linie durch Pluralität aus $^{3}$ (Lüders 2008: 389). Abgearbeitet wird nicht ein festgelegter Methodenkatalog, forschungsleitend ist stattdessen ein »methodischer Pragmatismus « (Flick 2009: 302), bei dem verschiedene Methoden im Forschungsprozess flexibel gehandhabt und gegebenenfalls der Situation im Feld angepasst werden (Amann/Hirschauer 1997: 16ff; Flick 2009: 298ff; Lüders 2008: 393ff). Erlaubt ist, was zum Erkenntnisgewinn beiträgt, den methodischen Schwerpunkt bildet jedoch üblicherweise die »Beobachtung im Rahmen einer ausgedehnten Teilnahme am Geschehen« (Flick 2009: 298), die bedarfsweise durch Interviews und Dokumentenanalysen ergänzt wird:

»In terms of data collection, ethnography usually involves the researcher participating, overtly or covertly, in people's daily live for an extended period of time, watching what hap-

3 Dieses Merkmal der Ethnographie deckt sich mit einer Forderung, die Stichweh (2010: 20) für systemtheoretisches empirisches Forschen erhebt: Die Systemtheorie solle sich weder bei der Auswahl ihrer Beobachter einschränken lassen noch möge sie der vielfach geäußerten Aufforderung Folge leisten, sich mit ihren Beobachtungen »primär im institutionellen Bereich der >empirischen Sozialforschung « zu verorten. 
pens, listening to what is said, and/or asking questions through informal and formal interviews, collecting documents and artefacts - in fact, gathering whatever data are available to throw light on the issues that are the emerging focus of inquiry. (Hammersley/Atkinson 2007: 3)

Das Resultat ethnographischer Feldforschung sind unstrukturierte und im Vergleich $\mathrm{zu}$ anderen, stärker extern kontrollierten und kontrollierbaren Methoden empirischer Sozialforschung >anarchisch< anmutende Daten (Amann/Hirschauer 1997: 16ff). Die Datenerhebung folgt nicht einem festgelegten Untersuchungsdesign und spiegelt entsprechend auch keine Kategorien wider, die ex ante an das Feld herangetragen werden (beispielsweise in Form von Fragebögen, statistischen Variablen oder ähnlichem). Stattdessen ist die Art der Daten selbst ein Produkt des Erhebungs- und Reflexionsprozesses im Feld (Hammersley/Atkinson 2007: 3), der wiederum selbst stets nur ansatzweise planbar und zahlreichen Unwägbarkeiten unterworfen ist (Flick 2009: 297f).

\section{Einblicke: Merkmale meines Forschungsfeldes}

Die ländlichen Gemeinden Andreevka und Beljaevka, auf denen der Schwerpunkt meiner Feldforschung lag und in denen ich drei jeweils rund einmonatige Feldaufenthalte verbrachte (August 2008, März 2009 und Februar/März 2010), befinden sich in der Region Perm. Die Nachbargemeinden liegen rund zehn Kilometer voneinander entfernt und bewegen sich mit knapp 1.000 Einwohnern an der numerischen Untergrenze, die erforderlich ist, um im russischen System lokaler Selbstverwaltung als eigenständige Verwaltungseinheit gelten zu können. Eine Zusammenlegung der beiden Gemeinden wurde in den vergangenen Jahren wiederholt als mögliches Szenario diskutiert, die Umsetzung dieser Pläne zeichnete sich aber während meiner Forschungsaufenthalte nicht ab. An das knapp 40 respektive 50 Kilometer entfernte Bezirkszentrum sind beide Gemeinden mit einem zweimal täglich verkehrenden Bus angeschlossen. Vom Bezirkszentrum aus besteht eine Busverbindung in die rund 120 Kilometer entfernte Regionshauptstadt Perm. Der gesamte Bezirk besteht aus sieben ländlichen Gemeinden sowie dem Bezirkszentrum und umfasst rund 17.000 Einwohner. Sowohl hinsichtlich der Bevölkerungszahl als auch mit Blick auf seine Fläche zählt er damit zu den kleineren der insgesamt 42 Bezirke der Region Perm.

Die Variante lokaler Selbstverwaltung, die in der Region Perm vor der Vereinheitlichung durch die jüngste Kommunalreform Geltung hatte, sah eine eigenständige Verwaltung der Bezirke und Städte vor, nicht aber der ländlichen Gemeinden (Center for Fiscal Policy 2008: 5). Die ländlichen Gemeinden wurden bis Ende 
2005 von territorialen Vertretungen der Bezirksverwaltung verwaltet ${ }^{4}$ und erst mit dem In-Kraft-Treten des Gesetzes Nr. 131 Anfang 2006 zu eigenständigen administrativen Einheiten. Die Gemeindeverwaltung - der sogenannte »sel'sovet « - in Andreevka und Beljaevka besteht neben den Bürgermeisterinnen aus drei Sachbearbeiterinnen und Sachbearbeitern, die für die Bereiche Wirtschaft und Finanzen, Eigentumsfragen und Landnutzung, Buchhaltung und Rechnungslegung sowie allgemeine Fragen kommunaler Verwaltung zuständig sind. Sie dienen den Gemeindemitgliedern als erste Anlaufstelle für die meisten administrativen Angelegenheiten, so dass ein Besuch der Behörden im Bezirkszentrum nur selten und in außergewöhnlichen Fällen notwendig ist (beispielsweise für die Registrierung von Landeigentum, von der noch die Rede sein wird). Die siebenköpfigen Gemeinderäte setzen sich in beiden Gemeinden aus Lehrerinnen und Lehrern, den Leitungspersonen von Schule, Kindergarten und Krankenstation sowie aus Vertreterinnen und Vertretern der landwirtschaftlichen Großbetriebe zusammen und spiegeln damit die Struktur der dörflichen >Elite<.

Die dörfliche Infrastruktur, in ihrer gegenwärtigen Form ein vollständiges Relikt aus sowjetischen Zeiten und repräsentativ für ländliche Gemeinden vergleichbarer Größe, umfasst in beiden Dörfern eine Schule, ${ }^{5}$ eine Krankenstation, eine Postfiliale, zwei bis drei kleinere Geschäfte für Produkte des täglichen Bedarfs, das sogenannte Kulturhaus (»dom kultury«) mit angeschlossener Bibliothek sowie eine Bankfiliale (in Beljaevka) und ein Heimatmuseum (in Andreevka). Das öffentliche Leben in den Gemeinden - und auch dies ist typisch für russische Dörfer - konzentriert sich auf das Kulturhaus (vgl. dazu auch die Beiträge in Donahoe/Habeck 2011): Hier finden die Gemeindeversammlungen, kulturelle Veranstaltungen und Feierlichkeiten statt und es gibt Räumlichkeiten für Freizeitaktivitäten (Chor, Kindergruppen, eine Art Jugendclub, an den Wochenenden stattfindende Diskotheken und ähnliches). Eine Dorfkneipe oder ein Dorfplatz als allgemeiner Treffpunkt existiert in russischen Dörfern üblicherweise nicht.

Zum Zeitpunkt des Zusammenbruchs des sowjetischen Regimes gab es in Andreevka und in Beljaevka je einen landwirtschaftlichen Kollektivbetrieb. Beide Betriebe wurden in den frühen 1990er Jahren formal privatisiert, indem Anteilsscheine (»paj«) auf Land- und Sacheigentum des ehemaligen Kolchos an alle Be-

4 Dies entspricht der linken Spalte im oberen Teil von Abbildung 1.

5 In beiden Gemeinden war zur Zeit meiner Forschungsaufenthalte noch der Schulbesuch bis einschließlich zur 11. Klasse und damit bis zur russischen Hochschulreife möglich. Zumindest in Andreevka stand aber aufgrund der geringen Schülerzahlen die Schließung der beiden oberen Klassen (entspricht der deutschen Sekundarstufe II) in der nahen Zukunft bereits fest, was allgemein als signifikanter Verlust der dörflichen Autonomie beobachtet wurde. 
triebsangehörigen, Rentnerinnen und Rentner sowie die Beschäftigten lokaler sozialer Einrichtungen wie der Schule, dem Kindergarten oder der Krankenstation, die allesamt faktisch an die Kollektivbetriebe angegliedert waren, ausgegeben wurden. Entgegen den politischen Erwartungen entschieden sich die neuen Anteilseignerinnen und Anteilseigner der Betriebe jedoch nur vereinzelt dazu, ihre Eigentumsrechte durch eine Herauslösung der Parzellen aus dem Kolchos geltend zu machen und sie einer anderen Verwendung zuzuführen, beispielsweise als Grundlage für den Aufbau eines eigenen privatbäuerlichen Betriebs. Die überwiegende Mehrheit der Dorfbewohnerinnen und -bewohner in beiden Gemeinden überließ ihre Landanteile auch nach dem Regimewechsel dem ehemaligen Kolchos zur Pacht, so dass das Unternehmen zwar formal seine Rechtsform änderte (meist wurden die Kolchose in genossenschaftliche Betriebe überführt), faktisch aber in sowjetischer Form fortbestand.

Gegen Mitte der 1990er Jahre begann die Entwicklung der landwirtschaftlichen Großbetriebe in Andreevka und Beljaevka deutlich zu divergieren: In Andreevka existierte der auf Milchwirtschaft spezialisierte ehemalige Kolchos - wenn auch mit Hilfe staatlicher Subventionen - relativ stabil und ist bis heute der größte Arbeitgeber im Dorf. Im Jahr 2008 beschäftigte er 173 Personen und damit laut der Gemeindestatistik etwa die Hälfte der Gemeindemitglieder, die im Dorf eine Arbeitsstelle haben (Tabelle 7). Die nächstgrößeren lokalen Arbeitgeber waren zu diesem Zeitpunkt die Schule (58 Angestellte), die Krankenstation mit Apotheke (neun Angestellte), das Kulturhaus und die Bibliothek (acht Angestellte), die Gemeindeverwaltung (sechs Angestellte) sowie der Einzelhandel (drei Geschäfte mit insgesamt 16 Beschäftigen). Rund 130 Gemeindemitglieder arbeiteten außerhalb des Dorfes (meist in Perm), 180 waren in Rente.

In Beljaevka hingegen wurde der ehemalige Kolchos von den wirtschaftlichen Schwierigkeiten, die sich im Anschluss an den Regimewechsel stellten, voll erfasst. Bereits seit Mitte der 1990er Jahre konnte er keine Gehälter mehr auszahlen und musste seine Beschäftigten in Naturalien entlohnen, was seitens der Angestellten nur mangels Alternativen zähneknirschend hingenommen wurde. Im Jahr 2006 stellte er seine Produktion vollständig ein. Die Insolvenzmasse wurde von einer privaten Investorengruppe übernommen, die sich auch darum bemühte, den Anteilseignern des zahlungsunfähigen Betriebs die Eigentumszertifikate abzukaufen (vgl. dazu ausführlich S. 246ff). Das Nachfolgeunternehmen des ehemaligen Kolchos, eine Agroholding, die sich auf Kartoffelanbau spezialisierte, wurde anfangs sowohl in Beljaevka als auch aus Andreevka skeptisch beäugt, konnte jedoch nach kurzer Zeit erste wirtschaftliche Erfolge aufweisen - gerade auch im Vergleich zum Kolchos im Nachbardorf: Die durchschnittlichen Monatslöhne des Betriebs in Beljaevka lagen im Jahr 2010 mit 10.000 Rubel (etwa 285 Euro) deutlich über dem Durchschnittslohn des ehemaligen Kolchos in Andreevka von 6.300 Rubel (etwa 
180 Euro), wobei sich Letzterer mit seinen Lohnzahlungen zudem mehrere Monate im Rückstand befand. Zum Vergleich: Im Jahr 2008 betrug der Durchschnittslohn in der russischen Landwirtschaft insgesamt 8.000 Rubel (etwa 230 Euro) (Kalugina/Fadeeva 2009: 104). Der Landwirtschaftsbetrieb in Beljaevka beschäftigte rund 80 Angestellte, wovon allerdings im Unterschied zum ehemaligen Kolchos gerade die besser bezahlten Führungs- und Fachkräfte primär außerhalb des Dorfes rekrutiert wurden.

Tabelle 7: Beschäftigungsstruktur in der Gemeinde Andreevka (2008)

\begin{tabular}{l|c|c} 
Art der Beschäftigung & Anzahl & Prozent \\
\hline Erwerbstätige mit Beschäftigung im Dorf & 329 & $33 \%$ \\
Renterinnen und Rentner & 179 & $18 \%$ \\
Erwerbstätige mit Beschäftigung außerhalb des Dorfes ${ }^{*}$ & 130 & $13 \%$ \\
Schülerinnen und Schüler & 106 & $11 \%$ \\
Arbeitslose/arbeitsunfähige Personen & 87 & $9 \%$ \\
Studium und Wehrdienst & 76 & $7 \%$ \\
Kinder im Kindergarten & 50 & $5 \%$ \\
Kinder außerhalb von Betreuungseinrichtungen & 40 & $4 \%$ \\
\hline Gemeindemitglieder gesamt & 997 & $100 \%$
\end{tabular}

*Bei rund einem Fünftel der Gemeindemitglieder (Beschäftigte außerhalb des Dorfes, Studierende, Wehrdienstleistende) ist davon auszugehen, dass sie dauerhaft außerhalb des Dorfes leben.

Quelle: Interne Statistik der Gemeindeverwaltung Andreevka.

Kleinbäuerliche Produktionsformen haben sich in beiden Gemeinden seit dem Regimewechsel nur sehr zögerlich entwickelt: In Andreevka gibt es eine, in Beljaevka zwei Familien, die zwar nicht offiziell als kleinbäuerliche Betriebe registriert sind, jedoch eine Hoflandwirtschaft führen, deren Erträge den Lebensunterhalt decken. Kleinere Hoflandwirtschaften als Nebenverdienst unterhält in beiden Dörfern zwar eine Reihe von Haushalten, doch deren Anzahl und Umfang sind rückläufig. Mit der Kombination aus landwirtschaftlichem Großbetrieb, privaten Hoflandwirtschaften und einer geringen Anzahl privatbäuerlicher Betriebe sind die beiden Gemeinden repräsentativ für die gegenwärtige Produktionsstruktur der russischen Landwirtschaft insgesamt (Tabelle 8). 
Tabelle 8: Produktionsstruktur der russischen Landwirtschaft (1992-2011)

\begin{tabular}{|c|c|c|c|c|c|c|}
\hline & 1992 & 1995 & 2000 & 2005 & 2010 & 2011 \\
\hline Großbetriebe & $67 \%$ & $50 \%$ & $45 \%$ & $45 \%$ & $45 \%$ & $48 \%$ \\
\hline $\begin{array}{l}\text { Hoflandwirt- } \\
\text { schaften }^{*}\end{array}$ & $32 \%$ & $48 \%$ & $52 \%$ & $49 \%$ & $48 \%$ & $43 \%$ \\
\hline $\begin{array}{l}\text { Privatbäuerliche } \\
\text { Betriebe }^{*}\end{array}$ & $1 \%$ & $2 \%$ & $3 \%$ & $6 \%$ & $7 \%$ & $9 \%$ \\
\hline
\end{tabular}

"Die Situation in Andreevka und Beljaevka legt die Vermutung nahe, dass ein nicht unerheblicher Teil privater Hoflandwirtschaften faktisch als privatbäuerlicher Betrieb agiert (d.h. gewinnorientiert und über den eigenen Bedarf hinaus produziert), jedoch formal nicht als solcher registriert ist. Die Grenzen zwischen diesen beiden Kategorien dürften entsprechend fließend sein.

Quelle: Rosstat (2012).

Sowohl in Andreevka als auch in Beljaevka war ich während meiner Feldaufenthalte in privaten Haushalten untergebracht: In Andreevka wohnte ich jeweils zu Beginn meiner Aufenthalte für einige Tage bei der Bürgermeisterin selbst und später bei einer alleinstehenden Rentnerin, in Beljaevka bei einer jungen Lehrerin. Auf diese Weise bot sich mir die Gelegenheit, in umfassender Weise am Dorfalltag teilzunehmen und auch über die Interviews und unmittelbaren Forschungsaktivitäten hinaus Kontakte zu Gemeindemitgliedern zu knüpfen.

Während das empirische Material, das ich in diesen beiden Dörfern erhoben habe, den Schwerpunkt meiner Analyse bildet, fließen ergänzend dazu Daten aus zwei weiteren Gemeinden des Bezirks ein, aus der ländlichen Gemeinde Dubrovo und dem Bezirkszentrum. Dort habe ich jeweils eine Reihe von Interviews geführt und auch einigen offiziellen Veranstaltungen beigewohnt, meine Anwesenheit beschränkte sich jedoch auf kurze Besuche zu ebendiesen Zwecken, so dass meine Einblicke in diese beiden Gemeinden notwendigerweise oberflächlicher ausfielen als es bei den erstgenannten der Fall war. Auf eine detaillierte Darstellung der beiden Gemeinden möchte ich deshalb verzichten und lediglich die Gründe für deren Auswahl darlegen.

Die Gemeinde Dubrovo, die mit insgesamt 2.000 Einwohnern zwar größer als Andreevka und Beljaevka ist, sich aber in ihren sozioökonomischen Merkmalen kaum unterscheidet, war für meine Forschung insbesondere deshalb interessant, weil der Anschluss an das Gasnetz, auf den ich in der nachfolgenden Analyse eingehen werde, dort recht weit fortgeschritten war. Während die Gasifizierung in Andreevka und Beljaevka während meiner Feldforschung erst begann, stand der Prozess in Dubrovo bei meinem letzten Aufenthalt bereits kurz vor dem Abschluss, 
so dass gerade der Vergleich der drei Gemeinden wertvolle Einblicke in verschiedene Phasen und Aspekte des Gasifizierungsprozesses bot.

Im Bezirkszentrum, das in die Kategorie der städtischen Siedlungen fällt, leben rund 5.000 Einwohner. Hier befinden sich nicht nur die Organe der Bezirksverwaltung und die Vertretungen staatlicher Behörden, sondern auch eine Reihe größerer Geschäfte, Restaurants und Cafés sowie ein Wochenmarkt. Für meine Forschung war das Bezirkszentrum vor allem aufgrund der Einblicke in die obere kommunale Verwaltungsebene bedeutsam, die ich in Gesprächen mit Mitgliedern der städtischen und der Bezirksverwaltung gewann. Darüber hinaus fanden in der Bezirksverwaltung wöchentliche Treffen der Bürgermeisterinnen und Bürgermeister der Gemeinden des Bezirks statt, auf denen Probleme diskutiert und offizielle Mitteilungen gemacht wurden. Bei diesen Veranstaltungen konnte ich nicht nur Kontakte zu anderen Gemeinden knüpfen, sondern erhielt darüber hinaus - wenn auch schlaglichtartige - Einblicke in deren Verwaltung.

\section{Feldzugänge: Problemzentrierte Interviews und teilnehmende Beobachtungen}

Um methodischer Beliebigkeit vorzubeugen, grenzte ich die Methoden, an denen ich mich bei meiner Feldforschung orientiert habe, auf zwei Foki ein: Zur Anwendung kamen primär teilnehmende Beobachtungen und problemzentrierte Interviews, wobei im Unterschied zur üblichen ethnographischen Vorgehensweise (vgl. dazu Flick 2009: 296ff) die Interviews und nicht die teilnehmenden Beobachtungen den Schwerpunkt sowohl der Datenerhebung als auch der Auswertung des empirischen Materials darstellten. Gemeinsam ist beiden Erhebungsmethoden, dass Kommunikationen beobachtet werden, unterscheiden lassen sie sich über die Merkmale der jeweiligen sozialen Situation und meine Rolle als Beobachterin darin.

Das Interview generiert durch die wechselseitige Wahrnehmung von Interviewerin oder Interviewer und Befragten ein Interaktionssystem, in dem die Kommunikation, die den Gegenstand der wissenschaftlichen Beobachtung darstellt, durch die Interviewsituation selbst erst hervorgerufen wird. Das Interaktionssystem Interview ist dabei - so heben Nassehi und Saake hervor - von einer asymmetrischen Zurechenbarkeit der kommunikativen Anschlussfähigkeit gekennzeichnet: Für den Befragten oder die Befragte entfällt das Risiko der Ablehnung von Kommunikation, »das Problem der Selektion von Anschlüssen ist [...] durch die selektionsbegründende Thematik gelöst« (Nassehi/Saake 2002: 74). Für den Interviewer oder die Interviewerin ergibt sich daraus das Problem, dass die »Interaktion mit Ablehnungsverzicht « (Nassehi/Saake 2002: 74) potenziell (aber nicht notwendigerweise) bestimmte Formen der Selbstbeschreibung seitens der Befragten begünstigt, andere 
hingegen unwahrscheinlich macht: So ist zum Beispiel zu erwarten, dass >Heldengeschichten< gegenüber vermeintlich banalen Erzählungen bevorzugt werden, hingegen als eigenes Fehlverhalten bewertetes Verhalten eher nicht zur Sprache kommt. Diesen Effekten angemessen Rechnung $\mathrm{zu}$ tragen oder sie gar zu kontrollieren, ist während des Interviews kaum möglich. Einhegen lassen sie sich jedoch, indem das Interview durch andere Beobachtungsformen ergänzt wird, so dass sich unterschiedliche Datentypen am Ende wechselseitig interpretieren und kontrollieren (Amann/Hirschauer 1997: 16). In meinem Fall übernahmen diese Funktion die teilnehmende Beobachtung sowie in Einzelfällen die Dokumentenanalyse. Begrenzen lassen sich die unerwünschten Effekte auch, indem eine Vielzahl von Interviews im Hinblick auf denselben Gegenstand geführt werden und damit unterschiedliche Beobachtungen in die Analyse einfließen, die sich in Relation zueinander einordnen lassen.

Bei der Auswahl meiner Interviewpartnerinnen und Interviewpartner bemühte ich mich entsprechend um ein möglichst breites Spektrum von Beobachtungsperspektiven. Die erste Kontaktaufnahme fand meist über die Bürgermeisterinnen und Bürgermeister statt, die während meiner Feldaufenthalte auch durchweg zentrale Ansprechpartnerinnen und Ansprechpartner blieben. Von ihnen ausgehend - dies gilt zumindest für die Gemeinden Andreevka und Beljaevka - konnte ich das Feld meiner Gesprächspartnerinnen und Gesprächspartner sukzessive in zwei Richtungen erweitern: Erstens ermöglichten die Bürgermeisterinnen und Bürgermeister mir den Zugang zu den Mitgliedern der jeweils eigenen Gemeindeverwaltung. Die Gespräche mit den Mitarbeitenden dieser unteren Hierarchieebenen fanden in der Regel in der Gemeindeverwaltung selbst am Arbeitsplatz der oder des Befragten statt. Interviews mit Mitgliedern des Gemeinderats als zentrales gewähltes Organ lokaler Selbstverwaltung führte ich teils in den Räumlichkeiten der Gemeindeverwaltung, teils bei den Befragten zuhause. Zweitens vermittelten mir die Bürgermeisterinnen in Andreevka und Beljaevka Kontakte zu Leitungspersonen von anderen Organisationen, also zu Krankenstation, Schule, Kindergarten, Kulturhäusern und landwirtschaftlichen Großbetrieben. Bei Bedarf - relevant war dies für mich vor allem in den Landwirtschaftsbetrieben - ließen sich dort dann weitere Gesprächspartnerinnen und Gesprächspartner unterer Hierarchieebenen gewinnen.

Ein zweiter wichtiger Ansatzpunkt für die Kontaktaufnahme zu Interviewpartnerinnen und Interviewpartnern abseits der organisationalen Formalstrukturen waren meine Gastgeberinnen. Über deren Verwandten- und Bekanntenkreise ergaben sich Gelegenheiten für zahlreiche Gespräche sowohl in Form formaler Interviews als auch im Rahmen von Einladungen zum Abendessen, zum Tee oder in die Banja, die russische Sauna. Ein Anruf meiner Gastgeberin bei den entsprechenden Personen nahm dabei nicht selten die anfängliche Scheu vor einem Gespräch mit >der Deutschen< und mit der Zeit wurde es sogar zu einer Art Statussymbol, mich einmal 
als Gast zuhause begrüßt zu haben. Viele dieser Kontakte nutzte ich nicht nur einmalig, sondern besuchte dieselben Familien zum Teil bei jedem meiner drei Feldaufenthalte, wobei die Offenheit und Intensität der Gespräche in der Regel mit jedem Treffen zunahmen.

Für die Gespräche wählte ich die Form des problemzentrierten Interviews (vgl. dazu Witzel 1982: 66ff). Problemzentrierung »kennzeichnet dabei zunächst den Ausgangspunkt einer vom Forscher wahrgenommenen gesellschaftlichen Problemstellung« (Witzel 1982: 67). Das Interview findet vor dem Hintergrund theoretischer Annahmen und sachlichen Vorwissens seitens der Interviewerin oder des Interviewers statt, Annahmen und Vorwissen sind dabei jedoch keinesfalls starr (beispielsweise in Form zu überprüfender Hypothesen), sondern irritierbar und modifizierbar im Hinblick auf Erkenntnisse, die aus dem Interview gewonnen werden (Lamnek 2005: 363ff). Diese Anforderung knüpft unmittelbar an die Prinzipien systemtheoretisch informierter empirischer Forschung an: Mit ihrem Fokus auf gesellschaftlicher Evolution vertritt die Systemtheorie ein explizit nicht-teleologisches Gesellschaftsverständnis, stellt jedoch gleichzeitig ein breites Instrumentarium präziser theoretischer Kategorien zur Beobachtung von Gesellschaft bereit: »The theory is closed to itself, to its own concepts and ways of relating constructs; but it does so in order to open itself to the scientific study of society. Society is emergent and reproduces itself without a teleological imperative « (Lee/Brosziewski 2007: 258).

Diese grundsätzliche Haltung spiegelt sich auch in der Art der Fragen wider: Zwar wird im problemzentrierten Interview zielorientiert und mit Blick auf den Forschungsgegenstand gefragt, der Charakter der Fragen ist jedoch offen und lässt somit möglichst viele Antwortmöglichkeiten zu. Den Schwerpunkt des Interviews bilden die Erzählungen der Befragten (Lamnek 2005: 364ff; Witzel 1982: 92ff). Leitfäden setzte ich in den Interviews als Orientierung ein, sie enthielten jedoch keinen festgefügten Fragenkatalog, sondern dienten in erster Linie der inhaltlichen Strukturierung des Hintergrundwissens und der anzusprechenden Themen (vgl. dazu Witzel 1982: 90). Welche Themen dies im Einzelfall waren, variierte je nach Interviewpartnerin oder Interviewpartner, so dass sich der typische Verlauf eines Interviews nur grob skizzieren lässt. Um die Befragten zum Erzählen zu ermutigen, wählte ich insbesondere beim jeweils ersten Treffen als Gesprächseinstieg meist eine Frage nach der eigenen Biographie, aus der heraus sich Verflechtungen mit und Einblicke in die dörflichen Strukturen häufig von selbst ergaben. Faktisch alle Gemeindemitglieder hatten im Laufe ihres Lebens beim Kolchos oder einer der angegliederten Organisationen gearbeitet oder waren, sofern sie nicht aus dem Dorf stammten, wegen der Anstellung beim Kolchos in die Gemeinde gezogen. Entsprechend war zwar nicht jede Person, aber doch mindestens jeder Haushalt auch unmittelbar vom Privatisierungsprozess und den damit verbundenen Reformen betrof- 
fen und hatte eine Meinung dazu. Diese und ähnliche Verbindungen von individuellen Biographien und dörflichen Strukturen boten Ansatzpunkte für die Vertiefung einzelner Aspekte, beispielsweise durch Fragen nach den individuellen Motiven für spezifische Entscheidungen (»Warum haben Sie Ihre Hoflandwirtschaft aufgegeben?«), persönlichen Einschätzungen zu allgemeinen Entwicklungen im Dorf (»Welche Rolle spielt der Kolchosvorsitzende in der Gemeinde?«) oder Vergleichen im Zeithorizont (»Was sind die wesentlichen Probleme im Dorf heute, welche waren es früher?«). Ergänzend dazu stellte ich meist Verständnisfragen (»Was verstehen Sie unter lokaler Selbstverwaltung?«, »Wie lief der Prozess der Kolchosprivatisierung $a b$ ? $)$, wobei meine Interviewpartnerinnen und Interviewpartner durchaus auch als Expertinnen und Experten fungierten, und konfrontierte die Befragten mit meiner Beobachtung spezifischer Situationen und Problemlagen im Dorf (»Ich habe gehört, dass im Dorf dieses und jenes Problem existiert - wie denken Sie darüber?«).

Die Datenerfassung erfolgte bei den Interviews in Form von Tonaufnahmen, wobei ich dafür stets vor dem Interview explizit die Zustimmung der Befragten einholte. Von insgesamt 63 Aufnahmen wurden letztendlich 46 vollständig verschriftlicht. ${ }^{6}$ Einige wenige Interviews erwiesen sich bei nochmaligem Anhören als (überwiegend) unbrauchbar, so dass ich auf eine Transkription verzichtet habe. In den wenigen Fällen, in denen die Befragten die Aufnahme des Interviews ablehnten, wurde im Anschluss an das Gespräch ein möglichst umfassendes Gedächtnisprotokoll angefertigt. Ergänzend dazu verfasste ich in der Regel zu jedem Interview eine Notiz im Feldtagebuch, in der die Merkmale der Interviewsituation und der Befragten beschrieben und gegebenenfalls Informationen über die Gespräche, die sich im Anschluss an das Interview ergeben hatten, festgehalten wurden (dies entspricht dem Postskriptum bei Witzel 1982: 91f).

Ergänzend zu den Interviews führte ich im Rahmen meiner Feldforschung teilnehmende Beobachtungen durch. ${ }^{7}$ Im Unterschied zu den Interviews verstehen sich

6 Darunter fallen neben den Interviews auch Mitschnitte von Gemeindeversammlungen, Gemeinderatssitzungen und ähnlichem. Die Transkription der Tonaufnahmen übernahmen studentische Hilfskräfte der Moscow School of Social and Economic Sciences unter der Leitung von Aleksandr Nikulin. Sämtliche Übersetzungen von Interviewpassagen aus dem Russischen, die in der nachfolgenden empirischen Analyse auftauchen, stammen von mir.

7 Das Verhältnis von Ethnographie und teilnehmender Beobachtung geht aus der Methodenliteratur nicht eindeutig hervor. Einigkeit scheint zwar darüber zu bestehen, dass die Ethnographie eine generelle Forschungsstrategie und die teilnehmende Beobachtung eine konkrete (und im Rahmen ethnographischer Forschung die zentrale) Methode darstellt (vgl. z.B. Flick 2009: 296). Die Definitionen beider Konzepte sind jedoch 
teilnehmende Beobachtungen als Beobachtungen derjenigen Kommunikation, für die gute Gründe vorlagen, dass sie in unveränderter Form auch ohne mich stattgefunden hätte und durch mich nicht in entscheidender Weise beeinflusst wurde. Dies war zweifellos bei Veranstaltungen wie Gemeindeversammlungen oder Gemeinderatssitzungen der Fall, aber auch bei Beobachtungen des Alltagsgeschehens in der Gemeindeverwaltung (Bürgersprechstunden und ähnliches). Meine Teilnahme an den jeweiligen Situationen beschränkte sich auf Anwesenheit und passive Beobachtung (Lamnek 2005: 562). Eine Interaktion mit den Beteiligten fand nicht oder nur minimal statt, so dass meine Rolle je nach Situation zwischen dem »Teilnehmer als Beobachter« und dem »Beobachter als Teilnehmer« wechselte (vgl. dazu Flick 2009: 283; Lamnek 2005: 575ff). Meine Beobachtungen waren dabei jedoch offen, das heißt die Beteiligten waren sowohl über meine Anwesenheit als auch über meine Motive informiert. Eine Ausnahme davon stellten größere öffentliche Veranstaltungen wie Gemeindeversammlungen dar, bei denen nicht alle Beteiligten, aber zumindest immer die Bürgermeisterinnen und Bürgermeister Bescheid wussten, auf deren Einladung hin ich an den Veranstaltungen teilnahm. Meine Beobachtungen waren darüber hinaus unsystematisch in dem Sinne, dass kein standardisiertes Beobachtungsschema zum Einsatz kam (zu den Merkmalen teilnehmender Beobachtung vgl. Flick 2009: 282ff; Lamnek 2005: 556ff). Unabhängig davon kristallisierten sich mit der Zeit jedoch Beobachtungsschwerpunkte heraus, und ich ging im Verlauf meiner drei Forschungsaufenthalte zunehmend von einer deskriptiven Beobachtung als erster Orientierung zu einer stärker fokussierten Beobachtung der für meine Fragestellung relevanten Prozesse und Verhaltensweisen über (vgl. dazu Flick 2009: 288). Dokumentiert wurden die teilnehmenden Beobachtungen teilweise ebenfalls durch Tonaufnahmen (beispielsweise von Gemeindeversammlungen und Gemeinderatssitzungen), überwiegend jedoch durch Aufzeichnungen im Feldtagebuch (Beobachtung von Alltagsgeschehen).

Neben Interviews und der passiven teilnehmenden Beobachtung war ich während meiner Forschungsaufenthalte zudem mit einem dritten Typus sozialer Situationen konfrontiert, der sich im Hinblick auf meine Rolle als Beobachterin als $\mathrm{Hyb}$ rid beschreiben lässt: Bei privaten oder auch halb-offiziellen Einladungen zum Abendessen oder zum Tee war meine Anwesenheit zwar zweifellos der Anlass für die jeweilige Interaktion, zumal sich Situationen dieser Art häufig im Anschluss an ein Interview ergaben. Beispielsweise beendete die Aufforderung »Lassen Sie uns Tee trinken« in der Regel nicht nur den >offiziellen< Teil eines Interviews, sondern stellte oftmals gleichzeitig die Einladung zum weiterführenden Gespräch unter ver-

meist redundant, da sich in der Regel beide über die Trias aus Beobachtung, Interviews und Dokumentenanalyse bestimmen (z.B. Denzin 2009 [1970]: 185f; Lamnek 2005: 548). 
änderten Bedingungen dar: Das Aufnahmegerät wurde ausgeschaltet, meist fand ein räumlicher Wechsel vom Wohnzimmer, wohin ich für das Interview gebeten worden war, in die Küche statt und weitere Familienmitglieder oder Nachbarn gesellten sich hinzu. Ich stellte nicht mehr in erster Linie Fragen, sondern wurde gefragt nach meiner Arbeit, meiner Familie, dem Leben in Deutschland. Nicht selten kam das Gespräch dabei jedoch auch auf Umwegen nochmals auf die Interviewthemen zurück, Vergessenes oder Vertrauliches wurde ergänzt und potenzielle oder tatsächliche Missverständnisse wurden zu klären versucht. Unabhängig davon, dass meine Anwesenheit im Haushalt die Interaktion ausgelöst hatte, ließ sich dabei meist eine Verselbstständigung des Gesprächs beobachten, wenn die Anwesenden sich beispielsweise über Probleme im Dorf echauffierten oder Meinungen austauschten und meine Anwesenheit dabei zunehmend bedeutungslos $\mathrm{zu}$ werden schien. Situationen dieser Art, die meist wertvolle Ergänzungen der Interviews darstellten, wurden ebenfalls im Nachhinein möglichst detailliert im Feldtagebuch protokolliert.

Neben Interviews und der Dokumentation von Beobachtungen im Feldtagebuch hatte ich die Möglichkeit, als weitere Kategorie empirischer Daten eine Reihe von Dokumenten zu sammeln: In der Gemeinde Andreevka gewährte mir die Bürgermeisterin umfassenden Einblick in zahlreiche Akten der Gemeindeverwaltung, die seit dem In-Kraft-Treten der jüngsten Kommunalreform im Januar 2006 erstellt worden waren. In Budgetpläne sowie die Protokolle von Gemeindeversammlungen, Gemeinderatssitzungen und öffentlichen Anhörungen durfte ich nicht nur vor Ort Einsicht nehmen, sondern darüber hinaus von sämtlichen Dokumenten Kopien erstellen. Auch zum Redaktionsarchiv der Lokalzeitung »Ochanskaja Storona« erhielt ich unbeschränkten Zugang und die Möglichkeit, Kopien von thematisch relevanten Artikeln zu erstellen (abgedeckt wurde dabei der Zeitraum von 2002 bis Anfang 2010).

\section{Datenauswertung zwischen (methodologischem) Anspruch und (Forschungs-)Wirklichkeit}

Methodologisch am deutlichsten ausgearbeitet wurden die oben dargestellten Prinzipien systemtheoretischer empirischer Forschung - die Explikation impliziten Wissens über Beobachtungen zweiter Ordnung und die Beobachtung von Kommunikation auf Lösungen und die zugrundeliegenden Probleme hin - unter dem Label der dokumentarischen Methode (Bohnsack 2003, 2008, 2010; Bohnsack/NentwigGesemann/Nohl 2007a; für die Auswertung von Interviews vgl. Nohl 2009; für die explizit systemtheoretische Anwendung und deren Dokumentation vgl. Vogd 2007, 2009, 2010). Als Form der rekonstruktiven Sozialforschung sucht die dokumentarische Methode nach den tieferliegenden Bezugsproblemen der beobachteten Kom- 
munikation und will aufzeigen, wie bestimmte Antworten auf diese Probleme ihre »eigenen Sachzwänge erzeugen, die dann bestimmte Lösungswege zu mehr oder weniger stabilen Orientierungen verfestigen lassen« (Vogd 2010: 124). Relativ dauerhafte und stabile Kommunikationsmuster, so die dahinterstehende Annahme, sind als evolutionäre Antworten auf benennbare Probleme zu verstehen. Beobachtbar sind diese Antworten oder Problembearbeitungsmuster im Einbringen von Themen oder in der positiven wie negativen Selektion von Kommunikationsanschlüssen. Dabei gilt es, die grundlegende Kontingenz der Lösungen respektive der Relation von Lösungen und Problemen zu beachten. Da Gesellschaft in der Systemtheorie aus guten Gründen und im Anschluss an Heinz von Foerster als nicht-triviale Maschine verstanden wird, können Problemlösungen - also der Output einer Situation - neue Probleme provozieren, für die wiederum neue Lösungen gefunden werden müssen. Der Fokus liegt dabei nicht auf der Frage »nach dem Was der gesellschaftlichen Realität«, sondern auf der Frage »nach dem Wie ihrer Herstellung « (Bohnsack/Nentwig-Gesemann/Nohl 2007b: 13; auch Przyborski/Wohlrab-Sahr 2009: 275). Es findet ein Übergang von der Beobachtung erster zur Beobachtung zweiter Ordnung statt. Darüber hinaus - und auch dies spricht für die Anwendung auf meine empirischen Daten - zeichnet sich die dokumentarische Methode durch einen breiten Anwendungsbereich aus: Ursprünglich konzipiert für die Interpretation von Gruppendiskussionen, wurde ihr Anwendungsbereich mittlerweile auf verschiedene Formen von Interviews, auf Feldforschungsprotokolle über teilnehmende Beobachtungen, auf Bilder oder auch auf Videomaterial ausgeweitet (Bohnsack 2008: 31f; Nohl 2009: 14).

Für die Auswertung von empirischem Material sieht die dokumentarische Methode drei Analyseschritte vor: die formulierende und die reflektierende Interpretation sowie die Typenbildung, wobei sich in den ersten beiden ebenjener Wechsel von der Beobachtung erster zur Beobachtung zweiter Ordnung widerspiegelt (Bohnsack 2003: 552; Vogd 2010: 123f). Insbesondere die ersten beiden Schritte, dies möchte ich vorwegnehmen, rahmten auch die Auswertung meiner Daten, vor allem der transkribierten Interviews. Unter Berücksichtigung des Erkenntnisinteresses meiner Arbeit und der Materialfülle erschien es mir jedoch unzweckmäßig, die Vorgaben der dokumentarischen Methode in allen Details sklavisch zu befolgen. Stattdessen - um auf eine treffende Formulierung von Hard (2003 [1987]: 315) zurückzugreifen - verortete ich meine Vorgehensweise in angemessener Distanz sowohl zu den methodischen »Bischöfen« als auch zu den »Menfoutisten« und war dabei bemüht, meine persönliche Neigung zu Letzteren in kontrollierten Bahnen zu halten und den Anschluss an Erstere nicht zu verlieren.

Für die Interviewauswertung bedeutet dies, dass die erste systematische Annäherung an das Material analog zum ersten Analyseschritt der dokumentarischen Methode in Form einer formulierenden Interpretation stattfand (Bohnsack 
2008: 134f; Nohl 2009: 46f). Die formulierende Interpretation (oder Beobachtung erster Ordnung) zielt auf die Explikation des immanenten Sinns der beobachteten Kommunikation (Vogd 2010: 128ff). Das empirische Material wird dazu zunächst inhaltsanalytisch ausgewertet: Es werden ein Überblick über den Gesprächsverlauf in Form einer schematischen Gliederung in Ober- und Unterthemen sowie kurze Inhaltsangaben zu den einzelnen Passagen erstellt. Abschnitte, die sich im Hinblick auf das Erkenntnisinteresse als relevant erweisen, werden auf dieser Grundlage ausgewählt und detaillierter interpretiert (Bohnsack 2008: 135; Przyborski/WohlrabSahr 2009: 286). Fragen, die im Rahmen der formulierenden Interpretation an das empirische Material gerichtet werden, sind beispielsweise:

- Wo und wie setzt ein Thema ein?

- Wie entfaltet es sich?

- Auf welche Weise verschwindet es wieder?

- Lassen sich Verästelungen in Unterthemen beobachten?

- Durch welches Thema wird ein Thema abgelöst?

Wesentlich ist, dass die Interpretation bei diesem ersten Analyseschritt auf den Rahmen des Beobachteten beschränkt bleibt (Bohnsack 2008: 134). Kontextwissen der Forscherin oder des Forschers und die Zurechnung von Motiven bleiben zunächst ausgeklammert - ein Vorgehen, das im Vergleich zum gewöhnlichen Alltagsverstehen »eine gewisse methodologische Disziplin« erfordert (Vogd 2010: 128).

Der anschließende zweite Analyseschritt der reflektierenden Interpretation fokussiert nicht mehr den immanenten, sondern den dokumentarischen Sinngehalt der beobachteten Kommunikation, der sich nicht über deren Inhalte, sondern über den Herstellungsprozess der Kommunikation erschließt (Bohnsack 2008: 135ff; Nohl 2009: 47ff; Vogd 2010: 130ff). Dabei sollen Relationen, Prozessdynamiken und Zurechnungsvorgänge, die unterhalb der Inhalte der Kommunikation liegen, rekonstruiert und Kontexturen wie Interaktion, Organisation und die Reproduktion gesellschaftlicher Funktionssysteme sowie deren Beziehung zueinander sichtbar gemacht werden. Die Analyse tritt jetzt explizit aus dem Deutungsrahmen der interviewten oder beobachteten Personen hinaus und rekonstruiert, »wie ein Thema oder eine Problemstellung verarbeitet, d.h. in welchem Orientierungsrahmen ein Thema oder eine Problemstellung abgehandelt wird « (Nohl 2009: 9). An diesem Punkt des Auswertungsprozesses kommen Kontextwissen, Motive und ähnliches ins Spiel, die im ersten Interpretationsschritt ausgeklammert wurden.

Beobachtet habe ich das Interviewmaterial im Rahmen der reflektierenden Interpretation in Anlehnung an die methodologischen Vorgaben und Beispielinter- 
pretationen von Bohnsack (2008), Nohl (2009) und Vogd (2009, 2010) auf Aspekte wie

- negative und positive Gegenhorizonte, die im Erzählungsverlauf aufgespannt werden (Wovon grenzt man sich ab? Woran orientiert man sich?),

- potenzielle und aktualisierte Kommunikationsanschlüsse,

- das Verhältnis zwischen den Beteiligten, wie es in der Kommunikation zum Tragen kommt (Auf welche Weise werden Hierarchien, Geschlechterdifferenzen oder ähnliches reproduziert?),

- den (funktionalen) Rahmen des Gesprächs,

- dramaturgische Steigerungen und Höhepunkte im Gesprächsverlauf,

- die Frage, >als was< das Gespräch vollzogen wird (Geht es um die Stabilisierung von Machtverhältnissen, wird ein Fachgespräch geführt, eine pädagogische Lektion erteilt oder ähnliches?),

- die Art der Lösungen, die in der Kommunikation gefunden werden (Lassen sich Verweise auf Sachzwänge beobachten? Wird die Erfüllung von Funktionen in den Fokus gerückt? Geht es um die Schließung der Organisation?).

Aufzeichnungen aus dem Feldtagebuch als Dokumentation der teilnehmenden Beobachtung wurden dabei zur Rekonstruktion sowohl des unmittelbaren als auch des erweiterten Kontexts herangezogen.

Als dritten Auswertungsschritt sieht die dokumentarische Methode die Typenbildung oder komparative Analyse vor, also die Beobachtung einzelner Fälle im Horizont vergleichbarer Fälle (Bohnsack 2008: 141ff; Nohl 2009: 13f). Unterschieden wird dabei zwischen sinn- und soziogenetischer Typenbildung. Erstere beobachtet unterschiedliche Typen von Orientierungsrahmen der Kommunikation, Letztere die Genese dieser Orientierungsrahmen über die dahinterstehenden sozialen Zusammenhänge (Nohl 2009: 57ff). Vergleiche und Typenbildung auf der Grundlage von regelmäßig auftauchenden Kommunikationsmustern fanden auch im Rahmen meiner empirischen Analyse statt. Da mein Erkenntnisinteresse jedoch auf das Verstehen von Einzelfällen - Strukturänderungen in den von mir beobachteten Dörfern - ausgerichtet ist und nicht primär auf die Generalisierung meiner empirischen Ergebnisse zielt, verzichte ich darauf, den Vergleich explizit als eigenständigen Analyseschritt auszuflaggen, und beschränke mich stattdessen auf den impliziten, fallbezogenen Vollzug.

Während mir die dokumentarische Methode als Orientierungsrahmen bei der Analyse des Interviewmaterials und der Aufzeichnungen aus meinem Feldtagebuch diente und sich die nachfolgende Darstellung meiner empirischen Ergebnisse primär auf diese beiden Datenkategorien stützt, nimmt die Dokumentenanalyse sowohl in der Auswertung als auch in der Ergebnisdarstellung einen nachrangigen Stellen- 
wert ein. Aus den Budgetplänen und Protokollen von Gemeindeversammlungen, Gemeinderatssitzungen und öffentlichen Anhörungen, die mir aus der Gemeinde Andreevka für die Jahre 2005 bis 2009 vorliegen, habe ich zwar mehrfach Sachinformationen entnommen (beispielsweise über die Höhe getätigter Ausgaben, die Inhalte gefasster Beschlüsse und ähnliches). Eine detaillierte Auswertung und Interpretation dieser schriftlichen Dokumentation von Verwaltungskommunikation erwiesen sich jedoch sowohl angesichts der inhaltlichen Qualität der Texte - die Protokolle fassen die jeweiligen Veranstaltungen meist nur bruchstückhaft zusammen, so dass zwar Ergebnisse, nicht aber der Verlauf der Kommunikation rekonstruierbar sind - als auch im Hinblick auf das Erkenntnisinteresse meiner Arbeit als nicht sinnvoll. In analoger Weise wurde mit den Beiträgen aus der Lokalzeitung »Ochanskaja Storona« verfahren, die mir zu relevanten Themen für den Zeitraum von 2002 bis Anfang 2010 vorliegen. Auch sie dienten mir in erster Linie als Quelle für Sachinformationen und an wenigen Stellen als Belege für ein generelles Stimmungsbild, eine detaillierte und umfassende Auswertung der Artikel erfolgte hingegen nicht.

\section{AUfregung Um EIN Flugblatt - ODER: Formen DER KOMMUNIKATION ZWISCHEN KOLCHOS UND GEMEINDE}

Im Februar 2009 sorgte in der Gemeinde Andreevka ein Flugblatt für Aufregung. Es war anlässlich der Kandidatur des Kolchosvorsitzenden ${ }^{8}$ für einen Sitz im Bezirksparlament, der »zemskoe sobranie«, kurz vor der Wahl an die Haushalte verteilt worden. Unter der Überschrift »Die Wahrheit über [Name des Betriebs]« wurde dort eine Reihe von Vorwürfen gegen den Kolchosvorsitzenden erhoben. Er habe, so die Stoßrichtung der Argumentation, den Betrieb heruntergewirtschaftet (wobei als Vergleich ein wirtschaftlich besser gestellter Betrieb aus einer Nachbargemeinde herangezogen wurde) und nehme über Subventionen unnötig und übermäßig viele Steuergelder in Anspruch. Stets unter dem Deckmantel der Wohltätigkeit operierend, füge er dem Dorf und seinen Bewohnern letztlich Schaden zu. Unterzeichnet war das Flugblatt lediglich mit »Die Bürger von Andreevka« (»žiteli Andreevki «). Niemand im Dorf konnte (oder wollte) sich zur Autorenschaft äu-

8 Die Verwendung der Bezeichnung Kolchos folgt dem dörflichen Sprachgebrauch, entspricht aber nicht der gegenwärtigen formalen Rechtsform des landwirtschaftlichen Großbetriebs. Die meisten Kollektivbetriebe wurden Anfang der 1990er Jahre in Genossenschaften umgewandelt. 
Bern. ${ }^{9}$ Weil es zudem nach Ablauf der Frist für Wahlwerbung und damit illegal verteilt worden war, waren die Gemüter zusätzlich erregt.

Bedeutsam an diesem Fall ist nicht so sehr das Flugblatt an sich - also die Tatsache, dass in dieser Form Wahlwerbung betrieben wurde -, sondern die Kommunikation, die sich an ihm entzündete. Obwohl die geäußerte Kritik gar nicht auf die Gemeindeverwaltung zielte, sah sich die Bürgermeisterin zu einer Stellungnahme genötigt. Von den Vorwürfen, die im Dorf hinter vorgehaltener Hand durchaus hier und da auf Zustimmung stießen, distanzierte sie sich nicht nur explizit, sondern war darüber hinaus bemüht, die Haltlosigkeit der vorgebrachten Argumente vorzuführen. Um ihrem Widerspruch eine sichtbare Form zu verleihen, wurde unter ihrer Federführung sogleich ein Gegenflugblatt lanciert, auf dem es unter anderem hieß: ${ }^{10}$

»Im Bezirk [.] kennt man den Leiter des [ehemaligen Kolchos], [Name des Kolchosvorsitzenden], gut. Er ist geboren und aufgewachsen im Gebiet Orenburg. [...] In der sowjetischen Zeit war man nach Abschluss des Studiums zu einer dreijährigen Dienstzeit verpflichtet. Hätte er anschließend nach Hause zu seinen Eltern und Brüdern zurückkehren können, die ihn lieben und schätzen? Natürlich, das hätte er gekonnt. Aber Andreevka zog ihn wie ein Magnet an und wurde für ihn zur Heimat. Zu Fuß lief [der Kolchosvorsitzende] alle Felder ab, kannte die Qualität jeder Saatfläche. [...] Niemals unterteilte er die Bevölkerung in >seineく und >Fremdeく. Für alle Feierlichkeiten stellte er finanzielle Mittel bereit. Seinen Traktor stellt er zur Verfügung, um Gemüsegärten umzugraben und Futter herzustellen. Nach seinen Möglichkeiten liefert er Rentnern Brennholz. [...] An die Menschen zu denken und sich um sie zu sorgen steht für ihn im Vordergrund. [...] [Der Kolchosvorsitzende] hat sich angewöhnt, nicht für sich selbst zu leben. Sein Lebensziel ist der Dienst an den Bürgern im Bezirk [.].«

Dieses >Gegenflugblatt< greift insofern die Kontextur des >oppositionellen< Flugblatts auf, als dass der Kolchosvorsitzende ebenfalls auf die Unterscheidung guter|schlechter Mensch beobachtet wird. Der Beobachtungsrahmen der Wirtschaftlichkeit bleibt dabei jedoch ausgeklammert. In den Vordergrund gerückt werden stattdessen die Wohltätigkeit und Großzügigkeit des Kolchosvorsitzenden als Person ebenso wie die Bedeutung des Betriebs für die dörfliche Infrastruktur.

9 Zwar wurde mir das Flugblatt mehrfach zum Lesen gegeben, eine Kopie anfertigen oder gar ein Exemplar mitnehmen durfte ich jedoch nicht. Die inhaltliche Darstellung basiert deshalb aus Aufzeichnungen aus dem Feldtagebuch, die unmittelbar nach der Lektüre des Flugblatts erstellt wurden.

10 Das Flugblatt der Bürgermeisterin wurde mir bereitwillig in die Hand gedrückt, so dass auch direkte Zitate daraus möglich sind. 
Der weitere Verlauf der >Flugblattaffäre < - im engeren Sinne - ist schnell erzählt: An den Wahlen zum Bezirksparlament nahm die Dorfbevölkerung mit ungewöhnlich hoher Aktivität teil, ${ }^{11}$ der Kolchosvorsitzende erhielt die überwältigende Mehrheit der Stimmen und zog ins Parlament ein. Noch ein Jahr später verbuchte die Bürgermeisterin die Episode auf einer Gemeindeversammlung explizit als Sieg für das Dorf und verband damit auch eine Warnung mit Blick auf zukünftige Wahlen:

»Da gab es vor einem Jahr diese Flugblätter und wir haben alle gemeinsam erlebt, wie sie am Vorabend der Wahlen verteilt wurden. Sie waren unterzeichnet von den Bürgern aus Andreevka, aber niemand von uns hat diesen Text geschrieben, niemand von uns hat ihn gedruckt. Ein Teil der Flugblätter wurde nicht von unseren Dorfbewohnern verteilt, ein Teil schon. Und unsere Bürger haben dann an den Wahlen teilgenommen [und für den Kolchosvorsitzenden gestimmt, E.M.], weil alles hätte passieren können.« (Bürgermeisterin auf einer Gemeindeversammlung, 18.2.2010)

Die >Flugblatt-Affäre < lenkt den Blick auf drei Aspekte, die aus soziologischer Perspektive und für das Erkenntnisinteresse der Studie relevant sind: Erstens wird deutlich, dass der ehemalige Kollektivbetrieb und insbesondere die Person seines Vorsitzenden auch im postsowjetischen Dorf eine bedeutende Rolle spielen, an der sich Diskussionen und Konflikte entzünden. Mit der >Kolchosfrage < scheint ein neuralgischer Punkt der Dorfstrukturen tangiert zu sein, der (auch) - darauf deutet die hastige Reaktion der Bürgermeisterin hin - mit Blick auf die Selbstverwaltung der Gemeinde relevant ist. Zweitens markieren Flugblatt und Gegenflugblatt bildhaft zwei Enden des Spektrums an Erwartungen, die sich auf den ehemaligen Kollektivbetrieb richten und sich zwischen Wohlfahrts- und Wirtschaftsorganisation bewegen. Mit den beiden Flugblättern wurde versucht, jeweils eines der beiden Enden nicht nur als alleiniges Beobachtungsschema, sondern darüber hinaus als Maßstab für die Beurteilung der Leistungsfähigkeit des Betriebs festzulegen. Und drittens lässt sich die Episode als ein Beispiel dafür analysieren, auf welche Weise die jüngste Kommunalreform in der dörflichen Kommunikation trotz oberflächlicher Kontinuitäten einen Wandel von Beobachtungsformen und Erwartungsstrukturen induziert und Klärungsansprüche hinsichtlich der Positionierung von Gemeindeverwaltung und ehemaligem Kolchos forciert.

11 Die Mitglieder des Komitees, das mit der Durchführung der Wahlen in der Gemeinde und mit der Stimmenauszählung befasst war, gaben eine Wahlbeteiligung von 60 Prozent an. Üblich sei eine Wahlbeteiligung von rund 30 Prozent. 


\section{»Ohne den Kolchos kommen wir nirgendwohin«}

Fragt man in der Gemeinde nach den Leistungen und der Rolle des Kolchos, erhält man als Antwort meist einhellige Relevanzbekundungen. Der Betrieb wird als »dorfkonstituierend « (»seloobrazujuščij«) beschrieben und seine Leistungen gelten mit Blick auf die Reproduktionsfähigkeit des Dorfes als unverzichtbar. Zum Tragen kommt dies in drei Tätigkeitsbereichen: Erstens sei der ehemalige Kollektivbetrieb der zentrale Arbeitgeber im Dorf (im Jahr 2008 beschäftigte er 173 Dorfbewohner und damit gut 50 Prozent derjenigen, die sich in einem festen Arbeitsverhältnis innerhalb der Gemeinde befanden), zweitens unterstütze er die nach wie vor existierenden Hoflandwirtschaften der Haushalte und drittens erbringe er eine Reihe von Leistungen im Bereich der öffentlichen Infrastruktur und kommunalen Dienstleistungen:

»[Privatbauer]: Der Kolchos ist als Unternehmen dorfkonstituierend. Ein großer Teil der Bevölkerung arbeitet beim Kolchos. Und im sozialen Bereich sowieso - wie ich schon gesagt habe - befindet sich die Wasserleitung auf seiner Bilanz, das heißt, er versorgt die Bevölkerung mit Wasser. Und auch die öffentlichen Institutionen: der Kindergarten, die Schule - deren Wasserversorgung liegt vollständig auf den Schultern des Kolchos. Jetzt im Winter räumt er die Wege, damit man sich einigermaßen normal bewegen kann. Die Privaten haben keine geeignete Technik dafür, um die Wege frei zu halten. Und auch was sonst die Dienstleistungen betrifft: wer eine Hoflandwirtschaft hat - wir sind ja nicht die einzigen, die Kühe halten , für alle wird das Heu mit Kolchostraktoren gemäht. Von den Privaten hat nur einer eine Mähmaschine, soweit ich weiß. Einer oder zwei. Die Gemüsegärten gräbt der Kolchos um. So ist das.

[Privatbäuerin]: Ohne den Kolchos kommen wir nirgendwohin.

[Privatbauer]: Ja, ohne den Kolchos kommen wir nirgendwohin.

[Privatbäuerin]: Und dann noch die Arbeitsplätze.«

(privatbäuerliches Ehepaar, 21.2.2010)

Auch wenn sich das Engagement des Betriebs vor allem im Bereich der lokalen Infrastruktur zum Teil aus schwer benennbaren Einzelleistungen zusammensetzt, die sich erst in der Summe bemerkbar machen, so betonen zwei Mitglieder des Gemeinderats, käme die Gemeinde ohne den Betrieb kaum über die Runden:

»[Gemeinderat ${ }_{1}$ ]: Er stellt Transportmöglichkeiten bereit, Rentnern liefert er Brennholz. [...] Und auch wenn eine Beerdigung ansteht, braucht man Transportmöglichkeiten. Und schon wieder ist dann der Kolchos zur Stelle. 
[Gemeinderat ${ }_{2}$ ]: Das sind alles Dienstleistungen. Irgendwie bemerkt man sie kaum, aber ohne sie wären wir aufgeschmissen.«

(Mitglieder des Gemeinderats, 13.8.2008)

Letztlich verbiete es die Macht der Gewohnheit, Alternativen zu den Kolchosleistungen - beispielsweise in Form privatbäuerlicher Betriebe, die aus technischer Sicht zu denselben Leistungen in der Lage wären - überhaupt in Betracht zu ziehen und die Abhängigkeiten vom Betrieb dadurch aufzubrechen:

»Alle haben einen Gemüsegarten, der mit dem Kolchostraktor bearbeitet wird. Die Preise dafür sind niedrig, für die Bevölkerung ist das vorteilhaft - umso mehr, wenn man einen Landanteil [beim Kolchos, E.M.] besitzt. Über den Boden sind wir alle abhängig [vom Kolchos, E.M.]. Wenn man etwas braucht, wenn jemand Probleme hat - als erstes gehen sie [zum Kolchosvorsitzenden]. [...] Wir sind aneinander gebunden. Das ist die Macht der Gewohnheit, dass man zu ihm geht.«(Bürgermeisterin, 11.8.2008)

Aus der Vielzahl von Leistungen, die in den Interviews angesprochen und dem ehemaligen Kollektivbetrieb zugeschrieben wurden, fanden zwei besonders häufig und nachdrücklich Erwähnung: Die Wasserleitungen und die Instandhaltung der Dorfwege.

Die dorfinternen Wasserleitungen stammen in Andreevka - wie in den meisten ländlichen Gemeinden Russlands - aus der Zeit des sowjetischen Regimes: Im Kontext der politisch vorangetriebenen Industrialisierung landwirtschaftlicher Produktion hatte der sowjetische Staat fast alle Gemeinden im ländlichen Raum an die Wasserversorgung angeschlossen (OECD 2011: 29ff). Für den Bau der Hauptleitungen innerhalb des Dorfes waren dabei in der Regel die landwirtschaftlichen Großbetriebe zuständig. Auf diese Weise wurden öffentliche Gebäude (wie Gemeindeverwaltung, Schule, Kindergarten, Kulturhaus) und ein mehr oder weniger großer Teil der Wohnhäuser (in Andreevka etwa 40 Prozent) mit eigenen Wasseranschlüssen versorgt, die bis heute genutzt werden. Die übrigen Haushalte - und das ist meist die Mehrheit - haben über öffentliche Hydranten, die über das Dorf verteilt sind, zwar Zugang zu fließendem Wasser, die Wohnhäuser selbst sind jedoch weder an die Wasserleitung noch an die Kanalisation angeschlossen, einige verfügen über einen eigenen Brunnen auf ihrem Grundstück. In Andreevka sind die Hauptleitungen bis heute Eigentum des ehemaligen Kollektivbetriebs und werden von ihm unterhalten. ${ }^{12}$ Dies erfordert vor allem laufend anfallende Reparatur- und

12 Nach Aussage der Bürgermeisterin ist diese Lage kein Einzelfall, sondern verhält sich in zwei weiteren Gemeinden im Bezirk ebenso - und damit in allen Gemeinden, in denen der ehemalige Kolchos noch in >sowjetischer Form< existiert. 
Instandhaltungsarbeiten an den mittlerweile maroden Leitungen, für die sowohl entsprechende Ausgaben in der betriebsinternen Finanzplanung berücksichtigt werden müssen als auch Fachpersonal bereitgestellt wird, das Teil der Kolchosbelegschaft ist:

$»\left[\right.$ Frage $\left.^{13}\right]$ : Sie haben den sozial-kulturellen Bereich erwähnt. Unterstützen Sie die Gemeinde bis heute in diesem Bereich?

[Kolchosvorsitzender]: Natürlich helfe ich. Die Wasserleitung ist noch auf unserer Bilanz, wir unterhalten sie mehr schlecht als recht.

[Frage]: Und der Dienst, der die Wasserleitung repariert - gehört der zu Ihnen?

[Kolchosvorsitzender]: Ja, das ist unserer.«

(Kolchosvorsitzender, 11.8.2008)

»Im Bereich kommunaler Dienstleistungen, natürlich, da macht er [der ehemalige Kolchos, E.M.] einiges. Erstens läuft über ihn das Abpumpen der Kanalisation. Wir setzen uns mit den Brigadeleitern in Verbindung und sie schicken dafür einen Traktor. Die Wasserleitung versuchen wir zur Zeit zu erneuern. Der Plan ist, einen Teil der alten Wasserleitung gegen eine neue auszutauschen, damit sie nicht bricht.« (Kolchosangestellte, 22.2.2010)

Trotz der für viele Gemeindemitglieder unkomfortablen Zugangsbedingungen wird die Sicherung der Existenz und die prinzipielle Erreichbarkeit von Wasserleitungen und Kanalisation als wesentlicher Beitrag zur Lebensqualität im Dorf bewertet und dieses Verdienst dem ehemaligen Kolchos zugeschrieben:

»Sowieso scheint mir, dass der größte Teil der kommunalen Wirtschaft über den Kolchos läuft. Die Wasserleitung ist dabei essentiell für unser Leben [lacht]. [...] Wenn es sie nicht gäbe, hätten wir es sehr schwer.« (Kolchosangestellte, 22.2.2010)

Ein zweiter und - wie die Bürgermeisterin und verschiedene Gemeindemitglieder betonen - nicht weniger relevanter Bereich der Kooperation zwischen ehemaligem Kolchos und Gemeinde betrifft das Instandhalten der Wege im Dorf. Da die Gemeinde über keine eigenen technischen Gerätschaften verfügt, stellt der Betrieb regelmäßig einen Traktor nebst Fahrer bereit, der sich um die Säuberung und Reparaturen kümmert und während der Wintermonate den Schnee räumt:

13 An einigen Interviews waren außer mir selbst noch Maria Amelina (Weltbank), Peter Lindner (Institut für Humangeographie, Goethe-Universität Frankfurt) sowie Aleksandr Nikulin (Moscow School of Social and Economic Sciences) beteiligt, weshalb ich Äußerungen der Interviewerinnen und Interviewer aus Gründen der besseren Lesbarkeit nicht namentlich, sondern einheitlich als $>$ Frage $<$ kennzeichne. 
»Für das Säubern der Wege haben wir eine Vereinbarung mit [dem Kolchosvorsitzenden]. Er stellt uns den Traktor zur Verfügung, wir zahlen den Lohn des Traktorfahrers und den Treibstoff, aber wir zahlen keine Miete für den Traktor. Für uns ist es existentiell, dass im Winter die Wege geräumt werden, und der Winter ist bei uns lang.«(Bürgermeisterin, 8.8.2008)

Was auf den ersten Blick wie ein altbekanntes Arrangement aussieht, vollzieht sich indes vor der Kulisse eines massiven Strukturwandels. Zwar deuten die beschriebenen Leistungen des Betriebs oberflächlich Kontinuität an, denn mit Blick auf die Praktiken des sowjetischen Dorfes scheint sich an den Tätigkeitsbereichen des Betriebs wenig geändert zu haben: Unverändert und unermüdlich übernimmt er Aufgaben, die abseits des Bereichs landwirtschaftlicher Produktion liegen und sich auf die Infrastruktur der Gemeinde richten. Die formalen Rahmenbedingungen, die die Rolle der landwirtschaftlichen Großbetriebe in den Dörfern und ihr Verhältnis zur Gemeindeverwaltung umgeben, haben sich im Zuge der Transformation jedoch gewandelt: Während die Privatisierungsmaßnahmen implizit die Landwirtschaftsbetriebe von bestimmten Aufgaben zu entbinden versuchen, weist die Kommunalreform ebenjene Aufgaben den Gemeinden zu. Angesichts der Tatsache, dass sich in Andreevka wie in zahlreichen anderen ländlichen Gemeinden die tradierte Aufgabenteilung zwischen Gemeindeverwaltung und ehemaligem Kollektivbetrieb bis zum In-Kraft-Treten der jüngsten Kommunalreform erhalten hatte, ist diese Verschiebung der Normen durchaus mit Reibung verbunden - worauf auch der Kolchosvorsitzende verweist:

»Früher, ganz am Anfang meiner Tätigkeit entfielen alle Leistungen im sozial-kulturellen Bereich auf den Kolchos. Das war Anfang der 80er Jahre. 86 habe ich begonnen, als Direktor [des Kolchos, E.M.] zu arbeiten. Und ganz am Anfang gehörte das alles zu uns - auch die Schule war auf der Bilanz des Kolchos, auch der Klub. Später dann in den 90er Jahren kam es zu einer Teilung, die soziale Sphäre und die Produktion sollten getrennt sein. Aber trotzdem leben wir immer noch hier - und das Dorf ist so oder so unseres.« (Kolchosvorsitzender, 20.2.2010)

Im Hinblick auf die Frage nach der Neuausgestaltung des Verhältnisses von ehemaligem Kolchos und Gemeindeverwaltung, die das Justieren von Grenzen zwischen beiden Organisationen ebenso betrifft wie die Klärung von Anschlussmöglichkeiten und die Herausbildung neuer Kooperationsformen, treten zwei Bereiche der jüngsten Kommunalreform besonders hervor: Die kommunalen Finanzen und das Steuersystem, über das die Gemeindeverwaltung und der Betrieb finanziell miteinander verflochten sind, sowie die Regelungen zur kommunalen Infrastruktur, durch die den Gemeinden erstmals genau festgelegte Zuständigkeiten im Hinblick auf die Bereitstellung von kommunalen Dienstleistungen auf ihrem Territorium 
übertragen werden. $\mathrm{Zu}$ diesem zweiten Bereich zählen auch die bereits skizzierten Leistungen: Die Instandhaltung der Wasserleitungen und der Dorfwege.

\section{Wirtschaftliche Selbstinszenierungen}

Der aus den Transformationsmaßnahmen erwachsende Anspruch einer wirtschaftlichen Primärorientierung der ehemaligen Kollektivbetriebe spiegelt sich überdeutlich in den Selbstbeschreibungen wider, die vor allem von Mitgliedern der Betriebsleitung vorgetragen werden. Um die Umorientierung seines Betriebs zum Ausdruck zu bringen und sich vom >sowjetischen $<$ Kolchos abzugrenzen, beschreibt der Vorsitzende sein Unternehmen als >Business $<$ : Marktorientierung, Preise und Zahlungen sind dominierende Parameter der Selbstdarstellung und die eigene Leistung wird mit den Betriebsergebnissen anderer Landwirtschaftsbetriebe in der Region verglichen. Entsprechend verkündet der Kolchosvorsitzende gleich zu Beginn eines Interviews mit sichtlichem Stolz und fast euphorisch das Betriebsergebnis für das vergangene Jahr - ohne dass ich danach gefragt hätte:

»2009 war im Hinblick auf die Produktion für uns ein erfolgreiches Jahr, unsere Produktion ist um 22 Prozent gestiegen. Das ist sehr gut. Wir haben im Jahr 200960 Stück Vieh gekauft. Und Milch ist für uns gegenwärtig der profitabelste Produktionsbereich.« (Kolchosvorsitzender, 20.2.2010)

Aufgrund der guten Zahlen, so betont er, sei das Unternehmen in einem Ranking der Regionalverwaltung, das als Orientierung bei der Vergabe staatlich subventionierter Kredite dienen soll, der obersten von drei Gruppen zugeordnet worden wenn auch verbunden mit einer Reihe von Auflagen und Verpflichtungen. Es gehöre damit der Gruppe derjenigen Unternehmen an, die nach Einschätzung der Politik »darauf ausgerichtet sind, Landwirtschaft als Business zu betreiben« (Kolchosvorsitzender, 20.2.2010) und als entsprechend förderungswürdig gelten. Der zweiten und dritten Gruppe dieser Klassifikation würden hingegen Betriebe zugeordnet, die lediglich zur Sicherung von Arbeitsplätzen aufrechterhalten werden sollen oder deren Zerfall und Überführung in Kleinbetriebe politisch forciert werden soll. Beinahe lehrbuchartig stellt der Kolchosvorsitzende vor diesem Hintergrund seine Maxime für eine erfolgreiche Geschäftstätigkeit dar:

»Der erste Aspekt ist - das habe ich Ihnen ja schon gesagt - die Auswahl effizienter Bereiche, die sich am Markt orientieren. Der zweite Aspekt ist die Senkung der Produktionskosten. Das heißt, die eigene Produktion muss teurer verkauft werden, gleichzeitig muss man günstiger produzieren und dann wächst auch der Gewinn. Das ist der Weg, den wir verfolgen.« (Kolchosvorsitzender, 20.2.2010) 
Auf der operativen Ebene schließen an diese Selbstdarstellung des Betriebs eine Reihe von Formalisierungsversuchen an, die (auch) auf seine >sozialen< Leistungen im Dorf zielen. Die Kooperationen mit der Gemeindeverwaltung und den Hoflandwirtschaften, so die generelle Stoßrichtung, sollen in die wirtschaftliche Selbstbeobachtung integriert werden. Organisationsintern geschieht dies vor allem über die Berücksichtigung der entsprechenden Operationen in der Buchhaltung und Personalplanung, wodurch deren Beobachtbarkeit im Medium des Geldes sichergestellt und Auswirkungen auf die Zahlungsfähigkeit des Betriebs sichtbar gemacht werden sollen. Beispielsweise gehen die Kosten für Reparaturen an der Wasserleitung offiziell in die Unternehmensbuchhaltung ein und sind als eigener Geschäftsbereich des Betriebs Teil seiner Finanzplanung. Über jede erbrachte Leistung sowohl gegenüber der Kommune als auch gegenüber einzelnen Haushalten - so hebt die leitende Ökonomin des Betriebs hervor - wird offiziell Buch geführt und Arbeitskräfte werden entsprechend eingeteilt:

»[Ökonomin]: Das gehört alles uns [mit Blick auf die Wasserleitung, E.M.]. Dem Landwirtschaftsbetrieb. Wir übernehmen alle Überprüfungen und Reparaturen, sie ist vollständig unsere. [...] Das war schon immer so. Sie gehörte immer uns, wir - der Kolchos - haben sie gebaut. Der Kolchos hat sie selbst gebaut, wer die Projektdokumentation erstellt hat, weiß ich nicht. Vermutlich auch der Kolchos. So läuft das. [...] Das ist sehr kostspielig. Für die Bevölkerung wird das schwierig. Die Leistungen der Wasserversorgung, die Entsorgung - das ist sehr teuer.

[Frage]: Befassen Sie sich als Ökonomin auch mit diesen Ausgaben?

[Ökonomin]: Ja. Ich plane sie. Wir planen, so um die 200.000 für die Wasserleitung auszugeben. Für die Reparatur, Ersatzteile, Lohn, Steuern, Amortisation der Kosten.

[Frage]: Gibt es noch andere Leistungen, bei denen der Kolchos die Gemeindeverwaltung unterstützt?

[Ökonomin]: Nicht nur die Gemeindeverwaltung, sondern die ganze Bevölkerung. Für die Gemeindeverwaltung - Transportleistungen für die Instandhaltung der Wege. Für die Bevölkerung alle anderen Dienstleistungen - alle. Das Bearbeiten der Gemüsegärten, die Lieferung von Brennholz, die Futterherstellung, Heu für persönliche Hoflandwirtschaften. Wir haben eine Mahlmaschine, mit der wir das Getreide mahlen, um damit das Vieh zu füttern. Das ist auch eine Dienstleistung, die Lieferung von Futter. Alle diese Leistungen erbringt der Kolchos gegenüber der Bevölkerung gegen minimale Bezahlung.

$[\ldots]$

[Frage]: Werden für diese Dienstleistungen auch Pläne erstellt?

[Ökonomin]: Unbedingt. Das hier ist unser Geschäftsplan. Hier werden zum Beispiel alle Ausgaben für 2010 berechnet. Die Kosten für Heu, Silage, die verschiedenen Futterarten. Getreide, Milch, Fleisch - wie viel das alles kosten soll. Wir planen das alles, planen unsere 
Einkünfte, und dann am Ende des Jahres, im Januar 2011, schauen wir, was wir tatsächlich erhalten haben und inwiefern wir mit unseren Plänen recht behalten haben.«

(Kolchosökonomin, 19.2.2010)

Extern, also über die Außengrenzen der Organisation hinweg, lassen sich Formalisierungen sowohl gegenüber den privaten Haushalten (vgl. dazu ausführlich S. 290ff), als auch gegenüber der Gemeindeverwaltung beobachten. Die Zusammenarbeit mit Letzterer, darauf pochen der Kolchosvorsitzende ebenso wie Mitglieder der Kolchosverwaltung, basiert auf Verträgen. Der Betrieb nimmt konkrete und klar abgegrenzte Aufträge von der Gemeinde entgegen und wird dafür bezahlt:

»Zur Zeit helfen wir [der Kolchos, E.M.] mit verschiedenen Dienstleistungen - unser Traktor säubert die Wege, die Wasserleitung ist unsere. Viele wenden sich an uns, wir arbeiten miteinander zusammen - sei es mit der Schule, sei es mit dem Sel'sovet, sei es mit dem Klub. Wir schließen Verträge ab über gemeinschaftliche Dienstleistungen und auf Grundlage dieser Verträge arbeiten wir. Sie bezahlen uns für die Dienstleistungen. Wir nehmen ein bisschen was - und sie bezahlen.« (Kolchosvorsitzender, 20.2.2010)

»[Frage]: Und wissen Sie, wie das läuft, wenn der Kolchos diese Leistungen erbringt - ist das immer formal und auf vertraglicher Basis oder auch informal? Dass er einfach so hilft?

[Kolchosangestellte]: Sowohl mit Verträgen als auch einfach so, aber im Wesentlichen, so scheint mir, auf formaler Grundlage. Sie schließen Verträge ab und leisten Unterstützung, im Wesentlichen auf Grundlage von Verträgen, natürlich.«

(Kolchosangestellte, 22.2.2010)

Deutlich werden hier die Verschiebungen der Erwartungsstrukturen: Entsprach Informalität, also Kommunikation abseits vertraglicher Vereinbarungen und unter Umgehung der betrieblichen Buchhaltung, bei Unterstützungsleistungen gegenüber der Gemeindeverwaltung oder auch gegenüber einzelnen Haushalten im sowjetischen Regime durchaus den Normalitätserwartungen, vollzieht sich nun eine Umstellung. Im Betrieb werden die besagten Leistungen zunehmend primär als entgangene Zahlung und damit als Umsatzeinbuße beobachtet und unter Verweis auf wirtschaftliche Rationalitäten $\mathrm{zu}$ vermeiden versucht. Um $\mathrm{zu}$ verhindern, dass dem Betrieb auf informalem Wege Einnahmen entgehen, wurde ein spezielles Verrechnungssystem für erbrachte Dienstleistungen entwickelt, das sich eng an die tradierten (ebenfalls informalen) Praktiken anlehnt und so versucht, die Schwelle zur Formalisierung möglichst niedrig zu halten. Neben der Möglichkeit, bestimmte Leistungen über die Kolchosverwaltung zu bestellen, für die dann entsprechend Arbeitskräfte und Technik bereitgestellt werden, haben die Arbeiter (dies betrifft im 
Wesentlichen die Traktoristen) die Möglichkeit, kleinere Dienstleistungen (wie beispielsweise das Umgraben eines Gemüsegartens) auch weiterhin spontan zu erbringen. Die Zahlungen der Dorfbewohnerinnen und -bewohner nehmen sie in diesem Fall direkt entgegen. Sie sind jedoch angehalten, die Art und den zeitlichen Umfang der erbrachten Leistung auf einer mitgeführten Liste zu vermerken, so dass die entsprechenden Zusatzeinnahmen mit ihrem Lohn verrechnet werden können:

»[Frage]: Und kommt es nicht vor, dass ein Traktorist einfach so bestimmte Arbeiten ausführt?

[Ökonomin]: Das kommt vor. Aber prinzipiell versuchen wir, die Dienstleistungen zu erfassen, denn für uns ist das echtes Geld. Wir haben zwei Brigaden. Wir haben zwei Mitarbeiter im Rechnungswesen und sie machen das mit ihren Traktoristen aus. Sie stellen einen Auftrag aus und tragen ein, wer welche Leistung bestellt hat und mit welchem Zeitumfang. Beim Bestellen eines Gemüsegartens werden die Hektar gezählt, anschließend in eine Liste eingetragen und die Buchhaltung rechnet das mit ihm [dem Traktoristen, E.M.] ab, zum Beispiel mit dem Lohn. Für den einzelnen Arbeiter ist das angenehm. Das wird mit dem Lohn verrechnet, das heißt, er muss das Geld nicht in Bar abgeben, es wird ihm vom Lohn abgezogen.«

(Kolchosökonomin, 19.2.2010)

Zentrale Parameter der vertraglichen Vereinbarungen sind Preise, an deren Zustandekommen - dies gilt zumindest für kommunale Dienstleistungen - der Betrieb und die Gemeindeverwaltung gleichberechtigt beteiligt sind. Über den Inhalt der entsprechenden Verhandlungen geben die Protokolle von zwei Gemeinderatssitzungen im Mai und Juni 2007 beispielhaft Aufschluss, auf denen Preiserhöhungen für die Wasserversorgung thematisiert wurden: Für die Bereitstellung und Instandhaltung der Wasserleitung erhebt der Kolchos über die Gemeinde unterschiedliche Tarife ein höherer Tarif gilt für Organisationen (also Gemeindeverwaltung, Schule, Kindergarten und die drei Lebensmittelgeschäfte) und ein etwas geringerer für Privathaushalte. In seiner Sitzung vom 29. Mai 2007 thematisierte der Gemeinderat den Vorschlag des Betriebs, Ersteren um 28 Prozent zu anzuheben (von 10 auf 12,80 Rubel pro Kubikmeter). Um seine Forderung zu begründen, hatte der Betrieb dem Gemeinderat eine detaillierte Aufstellung über die erwarteten Reparaturkosten vorgelegt, die im laufenden Jahr anstehen würden (115.114 Rubel, etwa 3.200 Euro). Die Tariferhöhung traf bei den Gemeinderatsmitgliedern durchweg auf Zustimmung und wurde entsprechend beschlossen. Unter stärkeren Vorbehalten diskutierte das Gremium rund einen Monat später, im Rahmen der Sitzung am 25. Juni 2007, einen weiteren Vorschlag des Betriebs, der vorsah, die Wassertarife für Privathaushalte in Zukunft über Pauschalbeträge pro Kopf und nicht mehr wie 
bislang pro Haushalt zu berechnen. ${ }^{14}$ Als Berechnungsgrundlage sollte das $»$ pochozjajstvennaja kniga«, das dörfliche Haushaltsregister, ${ }^{15}$ dienen, das für jeden Haushalt auch Angaben zur Anzahl der zugehörigen Personen enthält. Tatsächlich, so der Einwand, würden im Register jedoch oftmals Personen als Haushaltsmitglieder geführt, die die meiste Zeit gar nicht im Dorf lebten, so dass die auf diese Weise berechneten Zahlungen kaum die tatsächlichen Verhältnisse spiegelten. Nach kürzerer Diskussion, deren genauer Verlauf nicht aus dem Protokoll hervorgeht, wurde die Änderung allerdings trotz dieser Bedenken ebenfalls verabschiedet.

Welche Schlüsse lassen sich aus den skizzierten Formalisierungsbestrebungen mit Blick auf die Erwartungsstrukturen im Dorf und insbesondere auf die Beziehung zwischen ehemaligem Kollektivbetrieb und Gemeinde ziehen? Mit Luhmann lassen sich Verträge als ein Instrument verstehen, um Kommunikation zu binden und Kontingenz zu reduzieren, indem sich Selektionen durch den Vertrag »wechselseitig aufeinander einlassen, einander benutzen, einander aufbauen, Alternativen verwerfen« (Luhmann 1984: 176). Zwar entspringen Verträge der Interaktion, die durch sie festgelegte Beziehung löst und verselbständigt sich jedoch von der Interaktion: Die Identiät der Beziehung wird durch den Vertrag unabhängig von der Anwesenheit der Beteiligten auf Dauer gestellt und ihr weiterer Verlauf wird nicht durch den Verlauf der Interaktion bestimmt, sondern durch Vertragsregelungen (Teubner 1992: 195f). Über den Vertrag legen Betrieb und Gemeindeverwaltung die Konditionen ihrer Zusammenarbeit bindend fest. Kontinuierliche Aushandlungen zwischen Betriebsleitung und Bürgermeisterin werden damit ebenso überflüssig wie mitlaufende Selbstvergewisserungen darüber, $>$ woran man denn nun sei $<$.

Mit Blick auf das Fallbeispiel ist noch eine weitere, daran anschließende Überlegung relevant. Insofern Verträge wie im vorliegenden Fall zwischen Organisationen geschlossen werden, verweisen sie stets auch auf deren Grenzen, d.h. hier konkret auf den Prozess der organisationalen Entflechtung von Gemeindeverwaltung

14 Diese Art der pauschalen Tarifberechnung ist prinzipiell notwendig, weil es in den Gebäuden keine Wasserzähler gibt. Je nach Art des Zugangs zur Wasserleitung werden drei Gruppen unterschieden: In die unterste Preiskategorie fallen Haushalte, die nur über die öffentlichen Hydranten Zugang zu Wasser haben (6 Rubel pro Kopf), höhere Pauschalbeträge werden für Haushalte mit eigenem Wasseranschluss (15 Rubel) respektive mit Wasser- und Kanalisationsanschluss erhoben (23 Rubel). In die höchste Tarifgruppe fallen Haushalte, die zusätzlich zu Wasseranschluss und Kanalisation noch eine Badewanne besitzen (30 Rubel).

15 Das Haushaltsregister wurde bereits zu sowjetischer Zeit von den Gemeindeverwaltungen geführt und enthält neben Informationen über die zum Haushalt zugehörigen Personen auch Angaben über die Größe von Wohnhaus und Garten sowie über das Eigentum an Vieh, technischen Geräten und ähnlichem. 
und ehemaligem Kollektivbetrieb. Verträge, die der ehemalige Kolchos mit der Gemeindeverwaltung oder mit anderen Einrichtungen und Haushalten im Dorf schließt, binden nicht allein die Kommunikation, sondern verkoppeln die beteiligten Sozialsysteme auf spezifische Weise, bei der stets der Verweis auf die jeweilige Umwelt mitschwingt: Die Vertragspartner sind füreinander gerade nicht Teil des eigenen Systems, sondern explizit voneinander distinkt. Teubner (1992: 195) bezieht sich in diesem Zusammenhang auf unterschiedliche Typen sozialer Beziehungen, die der Organisation und dem Vertrag zugrunde liegen: »Organisationen sind Formalisierung von sozialen Kooperationsbeziehungen - Verträge sind Formalisierung von sozialen Tauschbeziehungen «. Eine vermeintlich identische Leistung, etwa das Pflügen des Gemüsegartens, findet durch die vertragliche Rahmung in gänzlich anderen Kontexten statt: Im sowjetischen Dorf waren bestimmte Leistungen Teil des (durch die politische Führung bestimmten) Organisationszwecks der Kollektivbetriebe und unterlagen damit organisationsinternen Entscheidungen. Die Grenze zur Gemeindeverwaltung wurde jedoch nicht nur in der Sachdimension verwischt, sondern auch in der Sozialdimension, indem die Vorsitzenden der ländlichen Sowjets in die Kolchosleitung aufgenommen wurden und sich dort gegenüber dem Kolchosvorsitzenden in untergeordneter Position befanden. Der Kolchos war damit nicht nur im Hinblick auf seine Leistungen eine umfassende Organisation, sondern auch im Hinblick darauf, dass faktisch alles, was im Dorf entschieden wurde, Teil seiner Binnenkommunikation war. Diese Strukturen reproduzierten sich vielfach über den Regimewechsel hinaus. Die Umstellung auf Verträge verweist vor diesem Hintergrund auf einen strukturellen Wandel: Unabhängig davon, wie eng sich die Kooperation gestaltet oder ob Kolchos und Kommune von außen als Einheit wahrgenommen werden, lässt sich das Abschließen von Verträgen als ein Modus beobachten, in dem sich die Entflechtung von Gemeindeverwaltung und ehemaligem Kollektivbetrieb vollzieht. Damit einher geht das zumindest partielle Auflösen der >sowjetischen< Hierarchie: Die Kommunikation mit Blick auf die Kolchosleistungen ist nicht mehr hierarchisch strukturiert, kanalisiert sich folglich nicht mehr über die Kolchosleitung an der Hierarchiespitze, sondern findet dezentral in den vertraglich verkoppelten Organisationen Kolchos und Gemeindeverwaltung statt.

\section{Das Dorf als renitente lokale Umwelt}

Das soeben Beschriebene sagt viel über die Selbstdarstellung des ehemaligen Kolchos und über sein Bemühen aus, operative Strukturen an sein Selbstbild anzupassen. Die Umweltperspektive - insbesondere die Beobachtung durch die Gemeindeverwaltung - wurde bislang jedoch vernachlässigt, so dass das Bild unvollständig bleibt. Zwar werden Verträge geschlossen und nehmen ebenso wie die dargestellten organisationsinternen Formalisierungen einen hervorgehobenen Stellenwert in Be- 
schreibungen der entsprechenden Arrangements ein. Die Umstellung der Kommunikation auf eine formale Grundlage vollzieht sich jedoch nur unvollständig. Auch stößt die angestrebte wirtschaftliche Primärorientierung des ehemaligen Kollektivbetriebs in seiner Umwelt auf Widerstände.

Ein erster Hinweis auf solche Widerstände findet sich in der Diskussion auf einem Treffen der Bürgermeisterinnen und Bürgermeister der ländlichen Gemeinden des Bezirks, das in regelmäßigen Abständen in der Bezirksverwaltung stattfindet. Die Zusammenarbeit der Gemeinden mit den ehemaligen Kolchosen, so die dort herrschende einhellige Meinung, zeichne sich gerade durch ihren informalen Charakter aus. Darüber hinaus spiele die Wirtschaftlichkeit der Arrangements im Unterschied zu den >Privaten< (also beispielsweise kleinbäuerlichen Betrieben oder dem lokalen Einzelhandel) für die ehemaligen Kollektivbetriebe meist eine nachrangige Rolle. Ungeachtet der praktischen Vorteile, die den Gemeinden nach eigenem Bekunden gerade aus der Kooperation mit Letzteren erwachsen, wird die Klarheit formaler Regelungen jedoch durchaus geschätzt:

»[Frage]: Aber das sind dann stärker formale Beziehungen [mit den Privatunternehmen, E.M.] als mit den ehemaligen Kollektivbetrieben?

[Bürgermeister ${ }_{1}$ ]: Ich würde sagen, mit den Privaten sind das sogar die besseren Beziehungen. Denn der Leiter eines Privatunternehmens lässt nicht zu, dass ein Arrangement für ihn verlustbringend ist und führt sein Unternehmen auf eine gute Weise.

[Bürgermeister ${ }_{2}$ ]: Mit denen haben wir ein vertraglich klar geregeltes Verhältnis. Was die Instandhaltung der Wege betrifft, zum Beispiel.

[Bürgermeister ${ }_{1}$ ]: Mit den Privaten. [...] Über Verträge, das läuft alles über das Budget. Für diese Art von Tätigkeiten bezahlen wir sie, aber dass sie ihr eigenes Geld dafür hergeben das würden sie nicht tun.«

(Bürgermeister auf einem Treffen in der Bezirksverwaltung, 11.8.2008)

Dass die Formalstrukturen unterlaufen werden, lässt sich auch in der Praxis beobachten (respektive entsprechenden Beschreibungen entnehmen). In diesem Sinne beschreibt beispielsweise die Leiterin der ambulanten Krankenstation in Andreevka zunächst mit großer Überzeugung, dass sämtliche Unterstützungsleistungen durch den ehemaligen Kolchos auf formaler Ebene und gegen Bezahlung abgewickelt und über die Buchhaltung verrechnet werden (wobei sie die Preise als durchaus hoch beobachtet). Auf mehrfaches Nachfragen hin räumt sie jedoch ein, dass daneben auch kostenlose Leistungen erbracht werden, für die entsprechende Vereinbarungen an der Betriebsleitung vorbei direkt mit den entsprechenden Arbeitseinheiten, den sogenannten Brigaden, getroffen werden: 
»[Frage]: Erhalten Sie in irgendeiner Form Hilfe aus dem Dorf? Gibt es irgendeine Form der Zusammenarbeit?

[Krankenhausleiterin]: Nein. Wir haben zum Beispiel Bretter beim Kolchos bestellt, solche Leisten. Wissen Sie, wir haben sehr viel dafür bezahlt. Für zwei Kubikmeter haben wir ich weiß nicht wie viel Geld bezahlt, das lief alles über unsere Buchhaltung. Kostenlos gibt es absolut nichts mehr. Früher hat der Kolchos geholfen, als es bei ihm so einigermaßen lief. Als der Kolchos noch sicher auf den Beinen stand. [Der Kolchosvorsitzende] hat geholfen. Ekaterina Nikolaevna, so hat er gesagt, sag mir, wie ich dir helfen kann. Aber jetzt laufen die Geschäfte bei ihm selbst nicht mehr gut.

[Frage]: Aber trotzdem wenden Sie sich noch an ihn, wenn Sie etwas brauchen?

[Krankenhausleiterin]: Ja. Aber er sagt Nein und auch wenn er nicht Nein sagt, gibt es nichts kostenlos. Er kann sich das auch nicht leisten, er kann es sich nicht leisten.

[Frage]: Wann hat sich das geändert?

[Krankenhausleiterin]: So in den letzten drei bis vier Jahren, als es mit dem Kolchos immer schlechter und schlechter wurde. [...]

[Frage]: Mich interessiert trotzdem, wie die Situation mit dem Kolchos genau ist. Unterstuitzt er Sie gar nicht mehr oder gibt es doch Beispiele? Auch wenn es kleine Dinge sind?

[Krankenhausleiterin]: Er hat uns kostenlos eine Müllgrube ausgehoben. Das stimmt schon. Und auch beim Transport hilft er. Wenn wir den Traktor für irgendwas brauchen oder um den Müll wegzufahren. Aber dann vereinbare ich das nicht über [den Kolchosvorsitzenden], sondern direkt mit den Brigaden. Sie machen das dann, sie können das machen. Aber bei ihm [dem Kolchosvorsitzenden, E.M.] selbst, da hängt alles am Geld, das verstehen wir schon auch.«

(Leiterin der dörflichen Krankenstation, 14.8.2008)

Im Hinblick auf die angestrebte wirtschaftliche Primärorientierung des Betriebs erscheinen diese illegalen und informalen Erwartungsstrukturen als hochgradig widersprüchlich - fließen, wie die leitende Ökonomin beschrieben hat, dadurch doch finanzielle Mittel ab und beeinträchtigen die Zahlungsfähigkeit des Betriebs, ohne dass dies für die Organisation im Einzelnen zu beobachten und zu quantifizieren wäre. Zwei Gemeinderäte, die diese Lage registrieren und in besonders drastischen Worten beschreiben, sehen in der Fähigkeit, derartige Formen von Informalität zu unterdrücken oder zumindest in kontrollierbarem Rahmen zu halten, gar den wesentlichen Indikator für die Führungsstärke der Kolchosleitung und einen entscheidenden Faktor für die wirtschaftliche Reproduktionsfähigkeit eines Landwirtschaftsbetriebs:

[Gemeinderat]: Die [gemeint sind vor allem Kleinbauern, E.M.] bedienen sich doch alle beim Kolchos. Wenn nicht bei diesem Kolchos, dann bei einem anderen. [...] Er [ein Halter einer privaten Hoflandwirtschaft, E.M.] kauft etwas beim Kolchos und umgeht dabei die Kasse. 
Und auf diese Weise hält er sich über Wasser. [...] Nennen Sie mir einen Kleinbauern, der ehrlich lebt und trotzdem Gewinn macht - es gibt keinen. Alle leben auf Kosten des Kolchos nebenan, alle diese Bauern, sie hängen wie Parasiten am Kolchos - nur dadurch überleben sie. Das Getreide muss eingefahren werden, es muss getrocknet werden, all das muss gemacht werden. Und sie gehen zum Kolchos und verhandeln still und heimlich und nur deshalb können sie überleben. Aber im Resultat - der arme Kolchos akzeptiert das alles, dass alle ihn heimlich hintergehen. Und auf seiner Bilanz taucht das alles am Ende nicht auf. Und wir warnen davor, dass der Kolchos zugrunde geht. Wofür braucht man den Kolchos heute doch nur, um ihn zu bestehlen! Aber wenn all die Privaten sich ehrlich verhalten würden, dann würde es auch dem Kolchos besser gehen. Wenn alle, die sich Heu mähen lassen, an der Kolchoskasse bezahlen würden, und alle, die Brennholz gebracht kriegen, auch. Aber bei uns läuft das so: Der Traktorist liefert Brennholz und steckt das Geld in die eigene Tasche. Aber der Traktor gehört dem Kolchos. Wenn im Kolchos keine Ordnung herrscht, leidet der Kolchos - und die Privaten machen Gewinn.

[Frage]: Und der Kolchosvorsitzende erlaubt das?

[Gemeinderat]: Der Vorsitzende? Was heißt, er erlaubt das! Das heißt, dass er Mittel zur Kontrolle findet - das ist die Fähigkeit zu Wirtschaften, das macht einen wirtschaftlichen Menschen aus. Und wenn er nicht adäquat wirtschaftet, dann sickern die Einnahmen weg und er bleibt auf den Kosten sitzen.«

(Gemeinderat, 13.8.2008)

Lässt man hingegen den Organisationszweck außer Acht und rückt mit Blick auf den Betrieb stattdessen das System-Umwelt-Verhältnis in den Vordergrund, fällt das Urteil weniger eindeutig aus: Die zu beobachtende Kombination aus formalen, informalen und illegalen Erwartungsstrukturen kann nun gerade in ihrer Widersprüchlichkeit als eine mögliche Strategie der Umweltanpassung bewertet werden. In diesem Sinne ist der Strategiemix trotz illegaler Elemente durchaus brauchbar und zum Teil sogar für die betriebliche Reproduktionsfähigkeit notwendig. Die Organisation reagiert auf diese Weise auf diverse und einander widersprechende Umwelterwartungen, die gegensätzlicher kaum sein könnten. Während sich in den Darstellungen der Kolchosleitung globale Erwartungen spiegeln, die aus dem Transformationskontext und den daran geknüpften Normen resultieren und sich über die Einbindung in Marktstrukturen verfestigen, weisen die Erwartungen aus der unmittelbaren dörflichen Umwelt des Betriebs (also ausgehend von der Gemeindeverwaltung, anderen öffentlichen Einrichtungen im Dorf und den Haushalten) in eine entgegengesetzte Richtung: Zwar werden die angestrebte wirtschaftliche Primärorientierung und die daran geknüpften Anforderungen bezüglich der (Wieder-)Herstellung der Zahlungsfähigkeit des Betriebs nicht vollständig ignoriert, sie gelten jedoch allenfalls als sekundär. Stattdessen tritt die sowjetische Prägung überdeutlich hervor: Der ehemalige Kolchos wird als Wohlfahrtsorganisation beschrie- 
ben, die für das Dorf in umfassender Weise verantwortlich zeichnet. Beobachtet wird er dabei explizit im Horizont anderer, vermeintlich allein gewinnorientierter Unternehmen ohne >soziales< Verantwortungsbewusstsein. Die wirtschaftlich prekäre Lage des Betriebs mit allen Konsequenzen (besonders deutlich zu spüren sind die stark verzögerten Lohnzahlungen) wird zwar registriert, vor dem Hintergrund seiner umfassenden Leistungen im Dorf jedoch billigend in Kauf genommen. So kontrastiert eine Kolchosangestellte, die mit ihrer Familie erst vor einigen Monaten in die Gemeinde gezogen ist, die Situation im Dorf (>hier $<$ ) mit ihrem letzten Wohnort ( $>$ dort $<$ ) mit den Worten:

»Dort existiert der Betrieb nur für sich selbst, hier versucht er, gleichzeitig für sich selbst und für die Menschen da zu sein. Diese Position hat [der Kolchosvorsitzende] - für sich selbst, aber auch für die Menschen. Dort ist der Betrieb nur für sich selbst da, nur für das Wachstum seines Kapitals. Ich weiß nicht, wie ich es sagen soll, dass es für uns hier natürlich auch schwierig ist, wenn die Löhne ein bisschen verzögert bezahlt werden. Natürlich bekommt man jeden Monat etwas, aber von Monat zu Monat gibt es immer einen Rückstand. Dort wurde der Lohn natürlich pünktlich gezahlt. Dort war der Lohn aber auch etwas niedriger als hier, vielleicht hat es der Betrieb hier deshalb schwerer. Er versucht, die Löhne steigen zu lassen, aber die Umsätze steigen nicht mit.« (Kolchosangestellte, 22.2.2010)

Vor allem die Gemeindeverwaltung weigert sich vehement, an die wirtschaftliche Selbstdarstellung des Betriebs und die daran geknüpften Rationalitäten anzuschlieBen - auch wenn die damit verbundenen Schwierigkeiten im selben Atemzug explizit zur Sprache gebracht werden und diesbezüglich sogar Verständnis geäußert wird. Der Wunsch des ehemaligen Kollektivbetriebs, die Wasserleitung aus wirtschaftlichen Überlegungen heraus loszuwerden und an die Gemeinde zu übergeben, stößt auf diese Weise an den Umwelterwartungen an seine Grenzen - die Bürgermeisterin weigert sich schlicht, darauf einzugehen:

»[Bürgermeisterin]: Natürlich hat er [der Kolchosvorsitzende, E.M.] den großen Wunsch, die Wasserleitung an die Gemeinde zu übergeben. [...] Denn für ihn ist das ein Klotz am Bein.

[Frage]: Aber haben Sie auch den Wunsch, sie zu übernehmen? [lacht]

[Bürgermeisterin]: Diesen Wunsch hatte ich noch nie [lacht]. Er tut mir leid deswegen, weil er die Wasserleitung nicht abgeben kann, er tut mir einfach leid. [...] Im Prinzip liegt er mir damit jedes Jahr in den Ohren - wäre es nicht doch möglich, dass...? Und ich sage: Nein, das kommt nicht in Frage [lacht].

[Frage]: [lacht] Aber sind Sie als Gemeindeverwaltung nicht verpflichtet die Wasserleitung zu übernehmen?

[Bürgermeisterin]: Nein, so eine Verpflichtung besteht nicht. Nur wenn er offiziell den Unterhalt der Wasserleitung ablehnt, dann sind wir zur Übernahme verpflichtet. 
[Frage]: Und das hat er offiziell noch nicht vor?

[Bürgermeisterin]: Wie will er sie mir übergeben, wenn ich sie nicht nehmen will? [lacht] Als Gentleman bringt er das nicht übers Herz.«

(Bürgermeisterin, 12.3.2010)

Zurückzuführen ist das Scheitern der Selbstbeschreibung des Betriebs in Form nicht realisierter Anschlusskommunikation unter anderem offensichtlich auf die fehlende Differenzierung von Rolle und Person, die in der zitierten Passage zum Ausdruck kommt (zur Differenzierung zwischen Rolle und Person vgl. Luhmann 1984: 429ff; zum Begriff der Person auch Luhmann 2008 [1991]): Die Imperative, die mit den jeweiligen professionellen Rollen von Bürgermeisterin und Kolchosvorsitzendem verbunden sind und aus den Transformationsskripten sowie den daran geknüpften globalen Erwartungen resultieren - für die Bürgermeisterin: die Übernahme kommunaler Aufgaben, für den Kolchosvorsitzenden: die effiziente Betriebsführung scheitern an den lokalen Erwartungen, die jeweils an die beiden Personen gerichtet werden und von denen nicht abstrahiert wird. Die Bürgermeisterin und den Kolchosvorsitzenden verbindet nicht nur, dass sie in den frühen 1980er Jahren als $>$ Fremde< Stellen in Andreevka zugewiesen bekamen, sondern auch ihr durchweg enges Verhältnis auf professioneller und persönlicher Ebene. Seit dieser Zeit besetzten sie beide unterschiedliche Führungspositionen im Dorf - zunächst beide in der Kolchosleitung, bevor er Anfang der 1990er Jahre den Kolchosvorsitz übernahm und sie den Vorsitz der damaligen Gemeindeverwaltung -, arbeiteten durchweg entsprechend eng zusammen und sind zudem auch privat freundschaftlich verbunden. Dieses enge Verhältnis der beiden Personen zueinander schränkt das Verhaltensrepertoire in der skizzierten Situation offensichtlich dahingehend ein, dass für die Anschlusskommunikation die personenbezogenen Erwartungen ausschlaggebend sind. Dies führt nicht nur dazu, dass die Bürgermeisterin die Erwartungen der Gemeindeverwaltung mit besonderer Vehemenz an den Kolchosvorsitzenden herantragen kann, sondern auch, dass Letzterer und mit ihm die Kolchosleitung sich diesem Druck kaum entziehen und von den lokalen Erwartungen abstrahieren können. $\mathrm{Zu}$ unterstellen ist überdies, dass die formale Aufwertung der Rolle der Bürgermeisterin im Dorf und gegenüber dem Betrieb durch die Kommunalreform - im Gegensatz zur gängigen sowjetischen Praxis sind Gemeindeverwaltung und Kolchosleitung einander weder über- noch untergeordnet - ihre Verhandlungsposition zusätzlich untermauert. Rollenimperative, die sich im Kontext wirtschaftlicher Transformationsmaßnahmen an den Kolchosvorsitzenden richten und orthogonal zu den skizzierten personenbezogenen Erwartungen liegen, erscheinen hingegen im Dorf als nicht durchsetzbar, können gleichzeitig vom Unternehmen aber auch nicht verdrängt werden. 


\section{Latente Arrangements und alternative Stoppregeln}

Mit den widersprüchlichen Umwelterwartungen geht der Betrieb auf zweifache Weise um: Erstens - dies klang in den oben stehenden Interviewpassagen bereits an - kommen informale Arrangements sowohl mit den Haushalten als auch mit der Gemeindeverwaltung und anderen öffentlichen Einrichtungen im Dorf operativ vor. Die Kommunikation umgeht dabei jedoch die Betriebsleitung und setzt stattdessen - wie die Leiterin der Krankenstation, aber auch die Gemeinderäte beschreiben direkt an Stellen der unteren Hierarchieebenen an. Wie im sowjetischen Dorf kommen dabei Zugangsrechte anstelle von Eigentumsrechten zum Tragen, die an bestimmte Stellen in der Organisation geknüpft sind (der Traktorist sichert sich einen Nebenverdienst, indem er die Formalstrukturen umgeht und das Geld in die eigene Tasche steckt). Die Strukturen, die daraus für die Organisation resultieren, lassen sich mit Luhmann als »aufgedrängte Illegalität« beschreiben (Luhmann 1964: 306). Aufgedrängte Illegalität - als spezielle Form von »brauchbarer Illegalität« (Luhmann 1964: 304) - ergibt sich potenziell dann, wenn Umwelterwartungen den eigenen Normen der Organisation widersprechen oder durch Befolgen der eigenen Normen nicht zu erfüllen sind. Die Organisation muss stattdessen mit externen Erwartungen umgehen, die intern nicht oder nur unter großen Schwierigkeiten legitimierbar sind. Für die Betriebsleitung, um wieder zum Kolchos zurückzukommen, bleiben die entsprechenden Vereinbarungen (bewusst oder unbewusst) latent, ${ }^{16}$ d.h. direktes Anschließen daran kann vermieden und ungeachtet dieser Widersprüche können in der Selbstdarstellung die Formalstrukturen in den Vordergrund geschoben werden.

Noch auf eine zweite Weise geht der Betrieb auf die Ansprüche aus seiner lokalen Umwelt ein: Zwar werden Dienstleistungen gegenüber der Gemeinde und den Haushalten insoweit in das wirtschaftliche Selbstbild integriert, dass sie als eigener Geschäftsbereich beobachtet und geführt werden. Dieser Bereich wird jedoch gleichzeitig von der Maxime der Gewinnerwartung entbunden und entlang abweichender Logiken ausgerichtet, die sich am Besten mit moralischer Bindung oder einer Gemeinwohlverpflichtung beschreiben lassen. Die >Stoppregel<wird für diesen Bereich in jedem Fall nicht aus der Wirtschaft (außerhalb des Dorfes), sondern aus der (unmittelbaren) dörflichen Umwelt bezogen. Zum Tragen kommt - um mit Bourdieu (2011 [1994]: 221) zu sprechen - eine Logik des »öffentlichen Feldes«, die sich am Gemeinwohl und dem Dienst an der (lokalen) Öffentlichkeit orientiert

16 Luhmann verweist in diesem Zusammenhang auf die Etablierung von latenten Rollen, »die nicht ins Bewußtsein gebracht oder doch taktvoll ignoriert werden« (Luhmann 1964: 306). 
und explizit mit Marktlogiken kontrastiert und in Differenz dazu beobachtet wird ${ }^{17}$ (vgl. zum Beispiel öffentlicher Güter auch Schultheis 2012: 12ff). Diese Beobachtungsweise findet sich auch beim Kolchosvorsitzenden, obgleich dieser nicht müde wird, den temporären Charakter der entsprechenden Arrangements zu betonen und Änderung in Aussicht zu stellen, wobei er explizit auch auf die jüngste Kommunalreform verweist:

»[Diese Dienstleistungen, E.M.] tragen zu meinem Umsatz kaum etwas bei. Ich verwende keine große Mühe darauf, um mit diesen Dienstleistungen Geschäfte zu machen. Das läuft auf Grundlage der Selbstkosten dieser Dienstleistungen, plus drei bis fünf Prozent Gewinn. Ich verstehe, dass jetzt Gesetz 131 in Kraft ist, und vielleicht ist das auch richtig, dass sie den Gemeinden mehr Verantwortung übertragen haben, aber leider reicht das Geld nicht, um die Aufgaben, vor denen die Gemeinden jetzt stehen, auch in vollem Umfang zu bewältigen. Die Produktion muss also vorangetrieben werden, damit die Gemeinde von den Steuern dieser Produktion leben kann und die Bedürfnisse der Bevölkerung aus eigener Kraft befriedigen kann. Wenn das dann mal so ist, dann werden wir vermutlich auf einer konstruktiveren Ebene zusammenarbeiten, ich werde Gewinne erzielen und [die Bürgermeisterin] wird in der Lage sein, die Leistungen in vollem Umfang zu bezahlen, die ich erbringen werde. Im Moment reicht ihr das Geld nicht für alle diese Maßnahmen. Sie bezahlen zwar, aber das ist keine angemessene Form der Zusammenarbeit von Politik und Business. Ich mache mit diesen Dienstleistungen bis jetzt kein Geschäft. Aber der Idee nach sollte es so sein. Es sollte vertragliche Beziehungen zwischen der Politik und den Unternehmen geben. Es sollte einen Markt für diese Dienstleistungen geben.« (Kolchosvorsitzender, 20.2.2010)

In diesem Sinne betonen sowohl der Kolchosvorsitzende als auch die leitende Ökonomin mehrfach, dass der Inhalt der mit der Gemeindeverwaltung geschlossenen Verträge wirtschaftlicher Rationalität widerspreche. Zwar seien die vereinbarten Preise für die Gemeindeverwaltung und die Dorfbewohnerinnen und Dorfbewohner vorteilhaft. Mittelfristig schadeten sie jedoch der wirtschaftlichen Reproduktionsfähigkeit des Betriebs, da in Kenntnis der begrenzten Zahlungsfähigkeit der Kommune stets von vornherein nur ein Teil der tatsächlichen Kosten in Rechnung gestellt würde:

17 Bei Bourdieu heißt es dazu: »Und schließlich gibt es [...] die Analyse der Ökonomie der öffentlichen Güter und des bürokratischen Feldes, des Staates als eines Ortes, an dem die Ökonomie verneint wird. [...] Historisch entsteht die Ordnung des >öffentlichen Interessesく, der >öffentlichen Angelegenheiten<, durch die Bildung eines Feldes, in dem das Handeln im Interesse der Allgemeinheit, im Dienste der Öffentlichkeit, möglich sein und gefördert, erkannt, anerkannt und belohnt werden soll.« (Bourdieu 2011 [1994]: 221) 
»[Frage]: Und was ist mit der Wasserleitung?

[Ökonomin]: Die Wasserleitung ist unsere. Darauf entfällt auch ein großer Teil unserer Ausgaben. Mittlerweile wurde ansonsten alles der Gemeinde übergeben, aber ich weiß nicht - der Direktor will nicht oder [die Bürgermeisterin] will nicht - irgendwer will sie nicht abgeben, irgendwer will sie nicht nehmen.

[Frage]: Aber es gibt Gespräche darüber [mit der Gemeinde, E.M.]?

[Ökonomin]: Das kann ich nicht genau sagen. Für die Leute wird das sehr nachteilig. Das wird sehr teuer. Wir halten die Preise niedrig, einen Teil der Ausgaben tragen wir selbst. Der Preis pro Kubikmeter Wasser deckt nicht all unsere Kosten. Das heißt, einen Teil übernehmen wir selbst. Für die Gemeinde wird das schwierig, sie wird diese Kosten nicht selbst tragen können und alles in den Tarif einschließen, der dann natürlich steigen wird. Deshalb ist das bis jetzt so.«

(Kolchosökonomin, 19.2.2010)

Eine ähnlich generöse Preispolitik betreibt der Kolchosvorsitzende mit Blick auf die Bereitstellung von technischem Gerät für die Instandhaltung der Wege im Dorf, im Zuge derer auf die Abrechnung von Abschreibungen, Reparaturen und ähnlichem verzichtet wird:

»Die Preise sind für sie [die Gemeindeverwaltung, E.M.] akzeptabel, nicht besonders hoch. Wenn unser Traktor für die Säuberung der Wege eingesetzt wird, bezahlt [die Bürgermeisterin] nur den Lohn und den Treibstoff. Darüber hinaus fallen Ausgaben für den Unterhalt des Traktors an, die übernehmen wir selber. Moderate Preise - so dass sich unsere Vereinbarungen für uns beide lohnen.« (Kolchosvorsitzender, 20.2.2010)

Die Verluste bei Aufgaben im Bereich öffentlicher Infrastruktur können auf diese Weise zwar nicht vermieden werden, lassen sich organisationsintern jedoch zumindest als klar eingehegter - und damit kontrollierbarer - Bereich beobachten, an den sich im Zweifelsfall wirtschaftlich anschließen lässt.

\section{"Vom Kolchos sehen wir nicht eine Kopeke«}

Noch in einem zweiten Bereich treten Kolchos und Kommune zueinander in Beziehung: Steuern und kommunale Finanzen. Die jüngste Reform kommunaler Selbstverwaltung und die zeitgleich stattfindende Reform der russischen Haushaltsordnung legen für diesen Bereich fest, dass dem Budget ländlicher Gemeinden die Grund- und Vermögenssteuer in vollem Umfang sowie die (föderale) Steuer auf Agrarunternehmen anteilig zu 30 Prozent zufließen (vgl. Tabelle 5). Zum ebenfalls durch die Reform eingeführten erweiterten Verantwortungsbereich der Gemeinden verhalten sich die Budgetregelungen in zweifacher Weise komplementär: Erstens 
zielen sie darauf, Zahlungen in Richtung der Gemeindeverwaltungen zu initiieren und somit die von der Gemeinde geforderten öffentlichen Dienstleistungen durch eigene Einnahmen finanziell $\mathrm{zu}$ untermauern und Entscheidungsspielräume zu schaffen. Zweitens geht es erneut implizit um die Entflechtung von Gemeindeverwaltung und ehemaligem Kolchos: Zwar sind die Großbetriebe gerade in ländlichen Gemeinden die wichtigsten Steuerzahler im Dorf und als solche zweifellos unverzichtbar, die Kommunikation zwischen Kommune und Kolchos soll nun jedoch von informellen Arrangements, netzwerkartigen Verflechtungen und reziproken Leistungserwartungen auf Zahlungen umgestellt werden.

Wie bei sämtlichen Steuern, die dem Budget ländlicher Gemeinden ganz oder anteilig zufließen, gehört das Eintreiben der Steuern jedoch nicht zum Aufgabenbereich der Gemeindeverwaltung, sondern obliegt den Organen der (föderalen) Steuerbehörde, die auf übergeordneten administrativen Ebenen angesiedelt sind. In der Praxis führt diese Vorgehensweise dazu, dass der gesamte Prozess aus Sicht der Gemeindeverwaltung hochgradig intransparent ist und Durchgriffsmöglichkeiten ebenso wenig gegeben sind wie Ansatzpunkte für bloße Einblicke. Das Zustandekommen der eigenen Einnahmen beobachtet die Bürgermeisterin entsprechend als Blackbox:

»Was die finanziellen Mittel betrifft, tappen wir im Dunklen. Wir wissen nicht, wie viele Einnahmen wir bekommen. Wir wissen im Moment überhaupt nicht, wie viel wir bekommen nicht auf Eigentum, nicht auf Land. Wir leben im Moment vom Geld der Region, das dem Bezirk zugeteilt wurde. Das geben wir vollständig aus und was wir auch wollen - wir können es uns nicht leisten. Wir wissen, dass es am Ende des Jahres für die Gehälter nicht mehr reicht - nicht für die Kultur [Mitarbeiterinnen des Kulturhauses, E.M.], nicht für uns [Mitarbeiterinnen der Gemeindeverwaltung, E.M.]. [...] Unverständlich, wie die Grundsteuer berechnet wurde. Wir haben mehr erwartet und schließlich überhaupt nichts erhalten. Wie das mit der Vermögenssteuer läuft, wissen wir auch nicht. Und die Zusammenarbeit mit der Steuerinspektion ist sehr schwierig. Sie sagen uns nicht $>$ Wanja hat bezahlt, Manja nicht $<$. Sie sagen uns nur die Gesamtsumme, wie viel auf unserem Territorium bezahlt wurde - und das war's. [...] Und unter diesen hochgradig unsicheren Bedingungen müssen wir unser Budget verabschieden.« (Bürgermeisterin, 8.8.2008)

Als Konsequenz dessen, so beschreibt die Bürgermeisterin, steigt die Unsicherheit im Hinblick auf die eigene Budgetplanung. Die Autonomie, die durch die kommunalen Einnahmen geschaffen werden sollte, wird dadurch unterlaufen, dass die Gemeindeverwaltung sich selbst als in hohem Maße abhängig von übergeordneten Ebenen beobachtet, von deren Einfluss gerade nicht zu abstrahieren ist. Der eigene Handlungs- und Entscheidungsspielraum - zunächst unabhängig von der tatsächlichen Höhe der letztlich zugewiesenen Zahlungen - stellt sich als eng begrenzt dar: 
»Das heißt das Hauptproblem, das ist die Unbestimmtheit der Zahlen, die von oben kontrolliert werden und von denen wir abhängen und auf die wir keinen Einfluss nehmen können.« (Bürgermeisterin, 8.8.2008)

Die Gemeinde Andreevka scheint diesbezüglich kein Sonderfall zu sein: Mit Blick auf die Steuerproblematik, so die einhellige Meinung, die auch von anderen Bürgermeisterinnen und Bürgermeisterin im Bezirk vorgebracht wird, wäre es gerade für kleine ländliche Gemeinden erheblich einfacher und würde die Planungssicherheit erhöhen, wenn sie die ihnen zustehenden Steuern selbst berechnen und eintreiben dürften und sich nicht auf externe Institutionen verlassen müssten:

»Das Budget - für die großen Gemeinden läuft das sowieso. Was uns kleine Gemeinden betrifft, bin ich überzeugt, dass es einfacher wäre, wenn wir diese Steuern selber berechnen und eintreiben würden. Wir würden diese Steuern zu 100 Prozent eintreiben. Jetzt geht das über die Steuerbehörde und alle möglichen Registrierungskammern. Selbst [der Bezirksgouverneur] hat darauf keinen Einfluss - das sind föderale Strukturen.« (Bürgermeister, 11.8.2008)

Gewissheit besteht in der Gemeinde Andreevka hingegen darüber, dass der Kolchos keine Steuern zahlt - darüber sind sich die Mitglieder der Gemeindeverwaltung einig:

»Auf [den Kolchosvorsitzenden] waren im vergangenen Jahr fünf Unternehmen registriert, ${ }^{18}$ alle auf dem Territorium von Andreevka - und wir haben von ihm nicht eine Kopeke gesehen. Und sie [die Steuerbehörde, E.M.] haben uns die Löhne gezeigt, die Einnahmen vorgerechnet - alles theoretisch. Sie haben mir erklärt, dass nur die Daten der Steuerinspektion Aussagekraft darüber haben, welche Einnahmen wir faktisch erzielen. Aber für uns bleibt das Theorie - wie viel wir bekommen sollten. Aber tatsächlich bekommen haben wir nichts.« (Bürgermeisterin, 8.8.2008)

Und auch seitens des Betriebs werden die unterlassenen Steuerzahlungen offen eingeräumt:

»Unser Unternehmen ist ausreichend groß und die Steuern sind entsprechend hoch. Man kann schon sagen, dass wir eigentlich verpflichtet sind, Steuern zu zahlen, aber das gelingt nicht immer. Denn das Geld bewegt sich rasch [...], das ist ein ständiger Umlauf. Hier muss noch etwas gekauft werden - und dafür reicht es nicht.«(Kolchosökonomin, 19.2.2010)

18 Viele der landwirtschaftlichen Großbetriebe ändern häufig ihre Rechtsform, für die interne Struktur und die operative Geschäftstätigkeit bleibt dies jedoch meist folgenlos. 
Auf welche Weise schlagen sich diese augenscheinlich ausbleibenden Zahlungen im Verhältnis von Großbetrieb und Gemeinde nieder? Auf der semantischen Ebene tauchen die fehlenden Steuerzahlungen als Teil eines Tauscharrangements auf, bei dem Leistung und Gegenleistung aneinander gekoppelt werden: Die Gemeindeverwaltung gesteht dem Kolchos die ausbleibenden Zahlungen zu und der dadurch entstehende Verbindlichkeitsüberschuss wird durch die oben beschriebenen Kolchosleistungen gefüllt. Dieses Arrangement erwähnt die Bürgermeisterin offen und explizit bereits bei unserem ersten Treffen (7.8.2008): Der Kolchos, so äußert sie sich, zahle keine Steuern, »weil er nicht will«, leiste dafür jedoch praktische Unterstützung gegenüber der Gemeindeverwaltung. In ähnlicher Weise differenzieren auch zwei Gemeinderäte zwischen Steuern in monetärer Form und anderen Leistungen des Betriebs, die sich - so die implizite Aussage - miteinander verrechnen ließen:

»[Der Kolchos] könnte in monetärer Form Steuern zahlen, er könnte Zahlungen leisten. Er stellt Transportmittel zur Verfügung, Rentnern liefert er Brennholz. [...] Wenn jemand beerdigt werden muss, braucht man Transportmöglichkeiten. Und schon wieder ist dann der Kolchos zur Stelle.« (Gemeinderat, 13.8.2008)

Jedoch: Solche Beschreibungen verstellen den Blick darauf, dass damit nicht notwendigerweise ein Tausch auf der operativen Ebene einhergehen muss. Um diese Überlegung zu präzisieren, ist zunächst zu klären, was Tauschkommunikation operativ auszeichnet. Naheliegend sind drei Merkmale: Erstens werden reziproke Leistungserwartungen kommuniziert, eine Leistung ist also kommunikativ an eine in sachlicher, zeitlicher und sozialer Hinsicht klar definierte Gegenleistung gekoppelt. ${ }^{19}$ Zweitens treffen die beteiligten Systeme im Vorfeld des Tauschs individuelle Entscheidungen, die sich auf den Gegenstand - Leistung und Gegenleistung - richten, sich in den daran gekoppelten Erwartungen verschränken und damit wechselseitig aufeinander verweisen. Entscheidungen betreffen die Auswahl unter verschiedenen Möglichkeiten und setzen damit - unabhängig von sachlichen Kriterien >richtiger $<$ oder >rationaler $<$ Entscheidungen - voraus, dass überhaupt verschiedene Möglichkeiten als umsetzbar beobachtet werden. Mit Blick auf die Entscheidung divergieren Vergangenheit und Zukunft, und die Zukunft wird dabei als offen (und nicht vergangenheitsdeterminiert) beobachtet. Ist dies nicht der Fall, entfällt der

19 Die Bestimmtheit in allen drei Sinndimensionen unterscheidet die Kommunikation reziproker Leistungserwartungen im Rahmen eines Tauschs von einem Netzwerk. Folgt man dem Netzwerkverständnis von Bommes und Tacke (2006: 47f; auch Tacke 2009, 2011) reproduziert sich zwar auch das Netzwerk über die Kommunikation reziproker Leistungserwartungen, diese bleiben jedoch in allen drei Dimensionen bewusst unbestimmt, d.h. sie werden »in der Schwebe gehalten «. 
Entscheidungsspielraum und damit die Entscheidung an sich (Luhmann 2000b: 145). In der Folge variieren die Möglichkeiten, wie an die Entscheidung angeschlossen werden kann und welche Erwartungen daran geknüpft sind:

»Mit Hilfe der Orientierung an Erwartungen, die strukturell gesichert, also relativ beständig sind, überbrückt die Entscheidung ihre eigene Vorher/Nachher-Differenz. Die Entscheidung ist, wenn man das so formulieren darf, vor der Entscheidung eine andere als nach der Entscheidung.« (Luhmann 1984: 402)

Mit dem Vollzug (und Abschluss) der Tauschkommunikation heben sich drittens Erwartung und Gegenerwartung wechselseitig auf und erlöschen. Ein Verbindlichkeitsüberschuss in Form einer wie auch immer gearteten »übrig bleibende[n] Verpflichtung « (Luhmann 1997: 653) tritt nicht auf. ${ }^{20}$ Der Tausch ist in diesem Sinne ein Ereignis, das im Moment seines Vollzugs zerfällt. Zwar kann Tauschkommunikation wiederholt werden, sie reproduziert sich jedoch nicht aus sich heraus und weist somit für sich genommen keine Systemeigenschaften auf.

Was passiert diesbezüglich im vorliegenden Fall? Vordergründig scheinen mit Blick auf Gemeindeverwaltung und Kolchos alle Merkmale einer Tauschkommunikation erfüllt zu sein: Der ehemalige Kollektivbetrieb erbringt im Dorf bestimmte Leistungen und als Gegenleistung akzeptiert die Gemeindeverwaltung das Ausbleiben der Steuerzahlungen - oder vice versa. Wären Leistung und Gegenleistung dabei aber operativ (und nicht nur in der Darstellung) gekoppelt, würde dies implizieren, dass die Zahlungsverpflichtung mit dem Vollzug der Kommunikation auch tatsächlich erlischt. Genau dies geschieht jedoch nicht und die Bruchstelle sind dabei die (formalen) Entscheidungskompetenzen der Gemeindeverwaltung: Sie - und konkret die Bürgermeisterin - kann zwar darüber entscheiden, in welcher Form sie mit Blick auf die Steuerzahlungen an den Betrieb appelliert (oder auch nicht), bezüglich der zugrundeliegenden Zahlungsverpflichtung als Tauschobjekt ergibt sich aus dieser Entscheidung jedoch kein Unterscheid zwischen vorher und nachher. Unabhängig von den Erwartungen der Gemeinde an den Kolchos besteht für den Betrieb Steuerpflicht und beiden Beteiligten ist dies selbstverständlich auch bewusst.

Dass die kommunalen Entscheidungskompetenzen in ihrer Darstellung augenscheinlich wider besseres Wissen erheblich verzerrt werden, bedeutet jedoch nicht, dass keine Anschlussfähigkeit generiert würde - und an diesem Punkt wird die Funktion des Arrangements im Dorf deutlich: Für das Verhältnis von Gemeinde und Großbetrieb wird eine stabile Grundlage geschaffen, wobei die Gemeinde die

20 Beispielhaft ist dafür die ökonomische Transaktion: Gut gegen Geld. Tauschkommunikation ist jedoch nicht auf wirtschaftliche Kontexte beschränkt. 
erwünschten und benötigten Leistungen erhält und dabei die Steuerschulden des Betriebs ausgeklammert werden können. Die Steuerbehörde, die im Hinblick auf die Zahlungsverpflichtungen des Betriebs die eigentlich entscheidende Instanz ist, wird als Umwelt und ihre Handlungen werden als Gefahr beobachtet, die - im Gegensatz zum Risiko - unabhängig von den eigenen Entscheidungen ist. ${ }^{21}$ Durch sie ausgelöste potenzielle Nachteile - konkret: Steuerinspektion und Sanktionen müssen damit ebenfalls nicht den eigenen Entscheidungen zugerechnet werden. Maßgeblich für die Selektion von Anschlusskommunikation, so wird deutlich, ist unter diesen Umständen allein der unmittelbare lokale Kontext:

»Unsere Steuergrundlage, das sind die Privatunternehmen und der Kolchos. Und was den Zahlungseingang betrifft, so sollte der Löwenanteil vom Kolchos kommen. Das Jahr ist bald zu Ende, aber Eingänge haben wir von ihm nicht, aus unterschiedlichen Gründen. Er hat seine Schulden gegenüber dem Staat und so weiter, für uns heißt das, dass wir auf unsere Steuergrundlage nicht zugreifen können. Die Summe liegt so um 200.000, sogar weniger. [...] Er existiert hier bei uns. Wir versuchen ihn zu ermahnen und so weiter. Keine Möglichkeit. Also zumindest haben wir keine Möglichkeiten, aber es gibt ja noch die Steuerinspektion. Aber die kommt aus irgendwelchen Gründen nicht zu ihm, zu ihm gehen sie nicht. Vorgesehen sind eigentlich drei Kontrollen pro Jahr [...]. Das wäre wünschenswert, aber es passiert nicht.« (Gemeinderat, 13.8.2008)

Gleichzeitig sorgt die operative Trennung von Leistung und Gegenleistung für ein hohes Maß an Robustheit gegen Irritationen von außen: Von allen unerwartet wird Anfang des Jahres 2010 die Steuerbehörde doch auf den Betrieb aufmerksam und führt eine Überprüfung durch. Der ehemalige Kolchos, der über mehrere Jahre keine Steuern entrichtet hatte, wird zu umfangreichen Nachzahlungen und Strafen verpflichtet, so dass dem kommunalen Budget unerwartet 420.000 Rubel (etwa 12.000 Euro) und damit etwa ein Fünftel der ursprünglich geplanten Summe zusätzlich zufließen. ${ }^{22}$ Die Bürgermeisterin verkündet dies auf einer Gemeinderatssitzung:

21 »Als Gefahr kann man jede nicht allzu unwahrscheinliche negative Einwirkung auf den eigenen Lebenskreis bezeichnen, etwa die Gefahr, dass ein Blitz einschlägt und das Haus abbrennt. Von Risiko sollten man dagegen nur sprechen, wenn die Nachteile einer eigenen Entscheidung zugerechnet werden müssen.« (Luhmann 2008: 362).

22 Laut Angaben der Gemeindeverwaltung belief sich das Budget der Gemeinde Andreevka im Jahr 2008 auf knapp 2,3 Millionen Rubel und bewegte sich auch in den folgenden Jahren in diesem Rahmen (vgl. auch Tabelle 6 und Center for Fiscal Policy 2008: 40). 
»Wir haben eine Änderung im Budget. Auf dem Territorium der ländlichen Gemeinde Andreevka hat sich für uns alle eine angenehme Situation ergeben. Für [den Kolchosvorsitzenden] ist sie wahrscheinlich nicht so angenehm. Sie sind ja alle darüber informiert: Bei ihm hat es eine Kontensperrung gegeben. Es gab eine Kontrolle durch die Steuerinspektion, er ist gezwungen, Verzugszinsen, Strafen und die Steuern selbst nachzuzahlen und ein Teil dieser Steuern ist uns zugeflossen. Das hat niemand erwartet, deshalb haben wir diese Summe aus der Einkommenssteuer auch nicht eingeplant. [...] [überraschte Nachfragen der Gemeinderäte zu den eingegangenen Zahlungen] Und dann ist da noch die Landwirtschaftssteuer. Die haben wir auch nicht eingeplant, haben sie aber erhalten. 139.000 sind allein aus der Landwirtschaftssteuer eingegangen - wir haben das überhaupt nicht vorgesehen.« (Bürgermeisterin auf einer Gemeinderatssitzung, 11.3.2010)

Diese Irritation aus der Umwelt des Dorfes, die sich auf eine Seite des Arrangements - die Steuerzahlungen des Betriebs an die Gemeinde - richtet, führt jedoch weder dazu, dass das Verhältnis zwischen Kolchos und Gemeindeverwaltung nachhaltig gestört wird - die Bürgermeisterin spricht lediglich von einer »Änderung im Budget « und erwähnt, dass der Kolchosvorsitzende nun möglicherweise »schlechte Laune « habe -, noch bewirkt sie, dass der Kolchos von seiner Leistungsbereitschaft absieht oder die Gemeinde diesbezüglich von ihrer Anspruchshaltung abweicht.

\section{Horizontverschiebungen}

Dem Verhältnis von Gemeindeverwaltung und ehemaligem Kollektivbetrieb habe ich mich in dieser ersten empirischen Episode über das Beispiel kommunaler Dienstleistungen und Steuern angenähert und damit zwei Bereiche in den Blick genommen, die Teil der jüngsten Kommunalreform sind. Deutlich wurde, dass der ehemalige Kolchos im Dorf in sowjetischer Tradition als relevante Instanz beobachtet wird und in enger Kooperation mit der Gemeindeverwaltung eine Reihe von Leistungen im Bereich der öffentlichen Infrastruktur erbringt. Der Stoßrichtung der Transformationsmaßnahmen - sowohl im Bereich der Privatisierung als auch komplementär dazu in Form der Kommunalreform - scheint dies auf den ersten Blick zu widersprechen. Auf den zweiten Blick ist hingegen zu erkennen, dass diese Maßnahmen keineswegs folgenlos bleiben, sondern die dörflichen Strukturen zumindest nachhaltig irritieren und indirekt auf eine Entflechtung von Gemeindeverwaltung und Landwirtschaftsbetrieb und die Positionierung von Letzterem als Wirtschaftsorganisation hinwirken.

Insbesondere die an die Privatisierung geknüpften globalen Erwartungen werden überdeutlich in der Selbstbeschreibung des Betriebs aufgegriffen und auch operativ umzusetzen versucht. In der lokalen dörflichen Umwelt erweisen sie sich allerdings nur als begrenzt anschlussfähig. Zwar stellt sich die Kommunikation zwi- 
schen Gemeindeverwaltung und ehemaligem Kolchos zunehmend auf Verträge um, in denen der Auflösungsprozess der tradierten Hierarchie beispielhaft zum Ausdruck kommt. Kolchosinterne wie -externe Formalisierungsversuche mit Blick auf die sogenannten Kolchosleistungen in der Gemeinde laufen jedoch immer wieder ins Leere oder bleiben unvollständig. Dies geschieht nicht zuletzt aufgrund der fehlenden Differenzierung von Rolle und Person im Verhältnis von Bürgermeisterin und Kolchosvorsitzendem, in der sich die Kollision von globalen und lokalen Erwartungsstrukturen widerspiegelt: Wechselseitige Ansprüche werden stets im Horizont der persönlichen Beziehungen zwischen beiden formuliert und unterlaufen auf diese Wiese die Umsetzbarkeit der (Transformations-)Imperative, die an die jeweiligen Rollen geknüpft sind. Verwiesen wird auf den Kolchos als >umfassende Wohlfahrtsorganisation<, dem gerade keine Gewinnorientierung als primäre Handlungsmaxime zugeschrieben wird. Als vermeintliche Gegenleistung zu den Sachleistungen des Betriebs unterbleiben Steuerzahlungen, die dem Gemeindebudget zufließen würden. Der Tausch zwischen Gemeindeverwaltung und Betrieb hat dabei zwar auf der Darstellungsebene Bestand und generiert Anschlussfähigkeit, findet sich jedoch nicht auf der operativen Ebene wieder.

Die übergreifende Frage nach Kontinuität oder Wandel der dörflichen Strukturen lässt sich angesichts der Vielzahl und Heterogenität der Arrangements und Kommunikationsformen zwischen ehemaligem Kolchos und Gemeindeverwaltung, die allein im sachlichen Bereich der jüngsten Kommunalreform zu beobachten sind, mit Blick auf diese erste Episode nicht eindeutig beantworten: Mal schwingt das Pendel stärker in Richtung des Wandels - wie beispielsweise angesichts der Verträge oder der Selbstdarstellung des Betriebs als Wirtschaftsorganisation - und mal in Richtung Kontinuität - so beispielsweise angesichts der zahlreichen informalen Kommunikationsstrukturen, die die Formalstrukturen teils ergänzen, teils unterlaufen. Eine spezifische Entwicklung tritt ungeachtet dieses ambivalenten Befunds dennoch als Gemeinsamkeit und generelle Stoßrichtung hervor: So kommt in den Äußerungen der Beteiligten regelhaft zum Ausdruck, dass die gegenwärtigen Arrangements nicht mehr als alternativlos, sondern explizit im Horizont der entsprechenden Transformationsmaßnahmen beobachtet werden. Entsprechend hält der Kolchosvorsitzende die Leistungen, die er im Dorf erbringt und die in der Vergangenheit stets unhinterfragt blieben, nur noch für die zweitbeste Variante, die er im Horizont einer durch die Kommunalreform geschärften Vorstellung darüber beobachtet, wie die Verhältnisse im Dorf >eigentlich< sein sollten:

»Nehmen wir zum Beispiel die Wasserleitung. Um ehrlich zu sein bereitet sie mir Kopfschmerzen. Sie ist alt und ich überlege mir, wie es eigentlich sein müsste. Ich mache mir so meine Gedanken. Ich würde die Wasserleitung gerne abgeben, auf die Bilanz der Gemeindeverwaltung. Ich könnte bei der Reparatur helfen und die Gemeinde könnte die Wasserleitung 
dann an jemanden verpachten - entweder an uns oder noch lieber an einen anderen Privatunternehmer. [...] Dann würde sich ausgebildetes Personal um die Wasserleitung kümmern. Das Unternehmen würde dafür bezahlt werden, es würde Gewinn machen und könnte seine Mitarbeiter bezahlen. Im Moment kümmert sich eine meiner Brigaden um die Wasserleitung, die noch viele andere Dinge machen muss und die Effektivität dieser Arbeiten [an der Wasserleitung, E.M.] - sie ist gering.« (Kolchosvorsitzender, 20.2.2010)

Ganz ähnlich und komplementär dazu beobachtet auch die Bürgermeisterin die ausbleibenden Steuerzahlungen explizit im Horizont der neuen gesetzlichen Regelungen:

»Uns [der Gemeindeverwaltung, E.M.] geht es schlecht - wir erhalten keine Einnahmen. [Der Kolchosvorsitzende] zahlt uns keine Steuern, die uns eigentlich als Einnahmen zustehen. Wir kriegen nicht die Einnahmen, wie sie uns zustehen würden - im Grunde haben wir gar keine Einnahmen.«(Bürgermeisterin, 8.8.2008)

Obwohl die Umsetzung der angesprochenen gesetzlichen Regelungen allgemein als wünschenswert beurteilt wird, weichen die tatsächlichen Erwartungsstrukturen im Dorf von diesem Möglichkeitshorizont signifikant ab und zurren sich mit jeder Aktualisierung in dieser Distanz fest. Jede Abweichung eines der beteiligten Systeme, Gemeindeverwaltung und Kolchos, lässt sich dabei mit Verweis auf die Abweichung des jeweils anderen rechtfertigen. Der aufgespannte Horizont bleibt auf diese Weise buchstäblich Horizont, der sich mit jedem Annäherungsversuch weiter von den aktuellen Strukturen entfernt.

Anzumerken ist dabei, dass der vor allem von der Kolchosleitung als Gegenstruktur konstruierte Markt für kommunale Dienstleistungen nicht nur eine fixe Idee ist, sondern in der Umwelt des Dorfes tatsächlich existiert. So schreibt beispielsweise die Kommunalverwaltung im Bezirkszentrum anstehende Projekte wie die Renovierung des Kulturhauses oder den Ausbau des Wasserleitungsnetzes öffentlich aus und private Unternehmen - sowohl aus dem Bezirk selbst als auch überregional - bewerben sich um die Aufträge. Beobachtet werden damit nicht nur Preise, sondern auch die Konkurrenz. In Andreevka werden diese Marktstrukturen zwar registriert und im Dorf evoluieren mit Preisen und formalen Verträgen auch potenzielle Markrequisiten, doch etwaige Anschlusskommunikation bricht sich bislang an den Dorfgrenzen und kommt als Option (vorerst) augenscheinlich nicht in Frage. Im Hinblick auf Letzteres betont der Leiter eines Privatunternehmens für kommunale Dienstleistungen aus dem Bezirkszentrum seine grundsätzliche Bereitschaft zur Zusammenarbeit mit den ländlichen Gemeinden. Nach eigenem Bekunden sendet er auch entsprechende Signale in Richtung der Gemeindeverwaltungen aus, die jedoch bislang ins Leere laufen: 
»Mit den ländlichen Gemeinden arbeiten wir prinzipiell auch auf vertraglicher Basis, aber bis jetzt erbringen wir dort noch keine Leistungen. Zwar haben zwei ländliche Gemeinden angekündigt, dass sie auf uns zukommen wollen, [...], aber bis jetzt herrscht diesbezüglich Stille.« (Privatunternehmer, 9.3.2010)

Jedoch: Haben sich die genannten Marktrequisiten einmal etabliert, erfordert die Begrenzung ihrer Anschlussfähigkeit auch außerhalb des Dorfes zunehmend Mühe: Sobald Zahlungen fließen, lässt sich Geld immer auch anders verwenden, sobald Verträge geschlossen werden, können sie gekündigt und die Vertragspartner gewechselt werden. Die potenzielle Durchlässigkeit der Dorfgrenzen, so ist zu vermuten, zeichnet sich im Versuch ihrer Schließung bereits ab, auch wenn entsprechende Anschlusskommunikation (noch) ausgebremst wird. Auf welche Weise sich Öffnungen und eine sukzessive Umstellung der Kommunikation auf weltgesellschaftliche Strukturen in Folge konkreter Kommunikationsangebote aus der Umwelt ergeben, soll eine zweite Episode verdeutlichen: Der Anschluss der Gemeinden an das Gasnetz.

\section{DeR ANSChLUSS AN DAS GASNETZ - ODER: DIE »GASIFIZIERUNG DES GANZEN LANDES« ALS EINFALLSTOR DER WELtGeselLSChAFT}

Was bei meinem ersten Feldaufenthalt noch als vages Gerücht kursierte, war in den Folgejahren aus den dörflichen Diskussionen kaum mehr wegzudenken: der Anschluss des Bezirkes und damit auch >meiner Gemeinden an das Gasnetz oder um den russischen Begriff zu verwenden - die sogenannte Gasifizierung (»gazifikacija «). Nachdem das Bezirkszentrum und eine daran unmittelbar angrenzende Gemeinde bereits Mitte der 1980er Jahre an das Gasnetz angeschlossen worden waren, stimmte im Herbst 2008 die Regionalverwaltung in Perm dem Bau von zwei weiteren Hauptleitungen im Bezirk zu, die drei weitere ländliche Gemeinden Andreevka, Beljaevka und Dubrovo - an das Gasnetz anschließen sollten (vgl. »Ochanskaja Storona« vom 14.11.2008: 1).

Wurden mit Blick auf die Energieversorgung im Dorf vor allem während der Wintermonate bislang die stetig steigenden Preise von Brennholz diskutiert, die Zuverlässigkeit des neuen (privaten) Sägewerks mit der des kolchoseigenen Sägewerks verglichen oder die Frage geklärt, wer wem beim Holzhacken hilft, zog mit der anstehenden Gasifizierung ein neuer Aspekt der Energieversorgung in die dörflichen Debatten ein. Wie im gesamten ländlichen Raum Russlands galt Gas fortan nicht mehr nur als Garant nationalen Wirtschaftswachstums und damit als Symbol russischer Stärke, sondern auch und vor allem als Fortschrittsmetapher und Moder- 
nisierungsfaktor mit Blick auf die wirtschaftliche Entwicklung und den Lebensstandard in den Dörfern. So pries die Lokalzeitung die Vorteile der Gasifizierung bereits im Vorfeld mit Überschriften wie »Die Hausgeister müssen umlernen: Mit der günstigen und >sauberen $<$ Art zu heizen erreicht die Lebensqualität eine neues Niveau - das Gas verdrängt Brennholz und Kohle« (»Ochanskaja Storona« vom 28.7.2006: 6) und beschrieb den technischen Akt des Anschlusses des eigenen Hauses an das Gasnetz in blumigen Worten als einschneidendes Ereignis für jedes einzelne Gemeindemitglied, das es nicht zu verpassen gilt. ${ }^{23}$ Im Zusammenhang mit einem Aufruf an die Bürgerinnen und Bürger zur Teilnahme am Gasifizierungsprojekt zitierte dieselbe Zeitung einen Bürgermeister mit der Äußerung: »Die Dorfbewohner verstehen, dass es vorteilhafter und ökonomischer ist, sein Haus mit Erdgas zu heizen als mit Kohle oder Holz« (»Ochanskaja Storona« vom 25.7.2008: 6). Im Entwurf des »Programms für die soziale und wirtschaftliche Entwicklung der Gemeinde Andreevka in den Jahren 2007 bis 2010«, das vom Gemeinderat verabschiedet wurde und der Verwaltung als Orientierung und Entscheidungsgrundlage dienen soll, findet sich zur Gasifizierung die vielversprechende Passage:

»Der Bau des Gasleitungsnetzes im Hinblick auf das Ziel der Gasifizierung des Gemeindeterritoriums beeinflusst maßgeblich die soziale und wirtschaftliche Entwicklung des Dorfes, den Lebensstandard der Bevölkerung und die wirtschaftliche Lage. Sie ist einer der bedeutendsten Faktoren für die Erhöhung der Energieversorgungseffektivität.«

Die Erwünschtheit der Gasifizierung stand damit offiziell nicht mehr zur Diskussion und Politikerinnen und Politiker aller Ebenen waren sichtlich bemüht, aus dem Projekt Kapital zu schlagen und sich die ausschlaggebende Entscheidung möglichst in vollem Umfang selbst zuzuschreiben: So versah der Bezirksgouverneur unmittelbar nach der Zustimmung der Regionalverwaltung das Projekt ostentativ mit einem Namen und einem Zeitplan (»Ochanskaja Storona« vom 14.11.2008: 1) und bemühte sich, das Thema im Wahlkampf als wesentliche politische Errungenschaft in den Vordergrund zu rücken, so beispielsweise bei seinem Auftritt auf einer Gemeindeversammlung in Anderevka:

»Es war sehr schwierig, im Gasifizierungsprojekt zu bleiben. Zunächst waren zwölf Bezirke der Region Perm Teil des Projekts, heute sind davon noch vier Bezirke übrig geblieben. ${ }^{24}$ Die

23 In diese Kerbe schlug beispielsweise ein ganzseitiger Beitrag unter dem Titel »Ein Strauß Schneeglöckchen. Viktor Ivanovich war nicht in der Stadt, als sein Haus an das Gasnetz angeschlossen wurde, er lag im regionalen Krankenhaus« (»Ochanskaja Storona« vom 10.6.2005: 4).

24 Ingesamt umfasst die Region Perm 42 Bezirke. 
anderen Bezirke haben die Teilnahme nicht geschafft. Vier Bezirke und darunter unserer blieben im Projekt! [...] Wir leiten heute das Gas bis zu jedem Haus - das ist teuer für das Budget, aber wir setzen das um, um Ihnen das Leben zu erleichtern« (Bezirksgouverneur auf einer Gemeindeversammlung, 18.2.2010)

$\mathrm{Ob}$ im Einzelfall tatsächlich allein politischer Einfluss ausschlaggebend war oder ob, wie in den Gemeindeverwaltungen teils unter der Hand ebenfalls vermutet wird, nicht vielmehr die Zahlungsfähigkeit eines privaten Investors und seine Netzwerke auf regionaler Ebene ausschlaggebend waren (vgl. dazu die dritte Episode), kann an dieser Stelle nicht abschließend geklärt werden.

In jedem Fall aber schwappt mit der Gasifizierung ein Thema in die Dorfkommunikation, das auf der nationalen Ebene bereits seit mehreren Jahren für Diskussionsstoff sorgt. Dass Russland über enorme Ressourcen an Erdöl und -gas verfügt und sich außenpolitisch gern als Energiegroßmacht inszeniert, ist keine überraschende Beobachtung (für eine Analyse der russischen Energiepolitik gegenüber dem In- und Ausland vgl. z.B. Götz 2006a, 2006b, 2007; Grätz 2009; Milov 2008). Die westlichen Nachbarstaaten reagieren darauf teils mit offener Skepsis, teils mit reflexhafter Nervosität, teils explizit um Rationalität bemüht (für die Beziehungen zwischen Russland und der EU vgl. z.B. Finon/Locatelli 2008; Geden 2008; Noël 2008). Von der russischen Bevölkerung (auch) im ländlichen Raum werden derartige Versuche, gegenüber dem Ausland nationale Stärke zu demonstrieren, durchaus registriert und mal wohlwollend, mal skeptisch beäugt. Von den Ressourcen profitieren konnte insbesondere die Bevölkerung des ländlichen Raums bislang jedoch nur sehr begrenzt, da lange Zeit fast ausschließlich der Export politische Priorität genoss: »The gigantic gas pipeline network that delivers the >blue flame< to German, French, and Italian cities [...] leaves the Russian countryside, and even most of Russia's cityscapes, untouched « (Dienes 2002: 447). Dieses Ungleichgewicht blieb auch der Bevölkerung in den ländlichen Gemeinden nicht verborgen, wie eine Rentnerin kritisch bemerkt:

»Natürlich ist das teuer und wir wissen nicht, wie wir das alles bezahlen sollen [gemeint ist die anstehende Gasifizierung, E.M.]. Aber wir haben lange gewartet. Unser Land ist reich und vor unserer Haustür laufen die Gasleitungen vorbei, nur wir haben von alldem nichts.« (Rentnerin, 19.8.2008)

Zwar war bereits im Jahr 1970 mit der Gasifizierung der sowjetischen Städte und Dörfer begonnen worden - ein Schritt, der damals sowohl sozial- als auch umweltpolitisch begründet wurde (Dudek/Golub/Strukova 2006: 1664f). Doch Anfang der 1990er Jahre, also zum Zeitpunkt des Zusammenbruchs des sowjetischen Regimes, waren lediglich rund 40 Prozent der Gemeinden des Landes an das Gasnetz ange- 
schlossen (RIA Novosti 2008). Nach dem Regimewechsel wurde das Gasifizierungsprojekt im Jahr 1995 erneut in Angriff genommen, zeitigte jedoch nur sehr zögerliche Erfolge (Dienes 2002: 446). Eine wesentliche Änderung ergab sich erst nach der Jahrtausendwende unter der Regierung Putin. Während Lenin (1970 [1920]: 513) seinerzeit verkündet hatte »Kommunismus - das ist Sowjetmacht plus Elektrifizierung des ganzen Landes«, schrieb sich Präsident Putin (ob in intendierter Analogie oder nicht, sei dahingestellt) nun die Gasifizierung des ganzen Landes als großes innenpolitisches Ziel auf die Fahnen. Entsprechende Maßnahmen zum umfassenden Anschluss der russischen Regionen an das Gasnetz wurden 2006 zum nationalen Projekt ausgerufen, von Dmitrij Medvedev, dem damaligen VizePremierminister und späteren Präsidenten (2008 bis 2012) zur Chefsache erklärt und mit demonstrativer Entschiedenheit in Angriff genommen. Regionalen Gouverneuren, die den vom Zentralstaat auferlegten Verpflichtungen nicht nachkamen, wurde mit »administrativen und organisatorischen « Strafen gedroht (RIA Novosti 2008). Der Auftrag für den Gasleitungsbau zwischen den Gemeinden ging an das Unternehmen Gazprom, ${ }^{25}$ dessen Aufsichtsrat Medvedev zur selben Zeit ebenfalls vorsaß (Ballin 2006).

Seit dem Beginn dieses Ausbauprogramms hat sich der Anteil der Gemeinden mit Zugang zum Gasnetz deutlich erhöht: Im Jahr 2011 war er auf 63 Prozent im nationalen Durchschnitt angestiegen, wobei die Städte mit knapp 70 Prozent über diesem Durchschnittswert lagen, die ländlichen Regionen mit 47 Prozent hingegen deutlich darunter (Gazprom 2011: 2). In der Region Perm sind die Werte geringer, das Stadt-Land-Gefälle ist größer und vor allem der ländliche Raum hinkt dem nationalen Durchschnitt deutlich hinterher: Hier waren im Jahr 2010 durchschnittlich 60 Prozent aller Gemeinden an das Gasnetz angeschlossen, wobei die Städte wiederum mit 75 Prozent über und die ländlichen Regionen mit nur knapp 18 Prozent signifikant unter dem regionalen Durchschnitt lagen. ${ }^{26}$ Nicht zuletzt vor diesem Hintergrund wurde die Euphorie über das russische Wirtschaftswachstum nach der Jahrtausendwende (vgl. für viele Ahrend 2004; Beck/Kamps/Mileva 2007; Nestmann 2007) vor allem aus geographischer Perspektive gern mit dem Verweis auf räumliche Diskrepanzen innerhalb des Landes gebremst: Mit Blick auf seine wirtschaftliche Entwicklung gleiche Russland einem Archipel aus einigen wirtschaftlich stark integrierten Städten und städtischen Regionen, die von »dead

25 http://www.gazprom.com/press/news/2012/january/article128588/ (zuletzt eingesehen am 17.4.2015).

26 http://ankarlov.wordpress.com/2011/03/04/hello-world/(zuletzt eingesehen am 17.4.2015), vergleichbare Daten finden sich auch in Pressemitteilungen zum Kooperationsabkommen, das Gazprom mit der Verwaltung der Region Perm im März 2010 geschlossen hat. 
space« umgeben seien (Dienes 2002; für ähnliche Befunde vgl. Bradshaw/Vartapetov 2003; Buccellato/Mickiewicz 2009; Golubchikov 2007).

Bei der Umsetzung des Projekts sind die Aufgaben und Verantwortlichkeiten in räumlich-funktionaler Hinsicht auf verschiedenen Schultern verteilt: Um die Gasleitungen zwischen den Dörfern kümmert sich der Bezirk und faktisch das Unternehmen Gazprom. ${ }^{27}$ Die Leitungen in den Dörfern fallen in den Verantwortungsbereich der Gemeindeverwaltungen. Um die Anschlüsse der Häuser, d.h. die Leitungen auf den Privatgrundstücken und die technische Ausstattung der Gebäude, haben sich die Gemeindemitglieder selbst zu kümmern. Während der erste Gasifizierungsschritt somit außerhalb der Gemeinden stattfindet und sie - abgesehen von der grundsätzlichen Zustimmung zum Anschluss an das Gasnetz - faktisch kaum tangiert, sorgt die dorfinterne Umsetzung, an der die Haushalte und die Gemeindeverwaltung beteiligt sind und um die es im Folgenden gehen soll, in den Dörfern für erhebliche Aufregung. Aus soziologischer Perspektive und mit Blick auf die hier problematisierten Transformationen sind die damit verbundenen Prozesse in mehrfacher Hinsicht instruktiv: Der Anschluss an das Gasnetz bietet erstens ein weiteres Beispiel dafür, wie Politik in den Gemeinden funktioniert, auf welche Weise insbesondere die Bürgermeisterinnen und Bürgermeister Macht ausüben und an welchen Punkten Einflussgrenzen sichtbar werden. Darüber hinaus lässt sich zweitens am Beispiel der Gasifizierung beobachten, an welchen Stellen die tradierten Strukturen des Dorfes, die stark von den ehemaligen Kollektivbetrieben dominiert waren, zu weltgesellschaftlichen Strukturen in Widerspruch geraten, auf welche Weise speziell funktionale Differenzierung - hier vor allem in Form wirtschaftlicher Kommunikation - ins Dorf geleitet wird und welche Konsequenzen für die Anschlussfähigkeit der lokalen Strukturen - insbesondere der Modi der Inklusion - damit verbunden sind. Nicht zuletzt wird dabei sichtbar, auf welche Weise sich alte und neue Strukturen miteinander verzahnen und aufeinander einstellen.

\section{Die Gasifizierung als lokalpolitisches Steuerungsproblem}

Anders als die skizzierten lokalpolitischen Äußerungen möglicherweise suggerieren, bedeutet das Gasifizierungsprojekt für die Gemeindeverwaltungen bei Weitem nicht nur die Aussicht auf politisches Prestige. Vielmehr gilt es eine Reihe von Aufgaben zu bewältigen, die mit erheblichem politischem Druck verbunden sind, der aus der Position der Gemeindeverwaltung im administrativen Institutionengefüge resultiert. So verfügt die Verwaltung einer ländlichen Gemeinde in der Gasifizierungsfrage selbst über keine grundsätzliche Entscheidungskompetenz: $\mathrm{Ob}$ ein

27 http://www.gazprom.com/about/production/gasification/ (zuletzt eingesehen am 17.4.2015). 
Dorf an das Gasnetz angeschlossen wird oder nicht, wird auf den Ebenen des Bezirks und der Region entschieden. Auf der Gemeindeversammlung wird lediglich noch die Zustimmung der Bevölkerung eingeholt ${ }^{28}$ (für Andreevka vgl. »Ochanskaja Storona vom 25.7.2008: 2). Im Anschluss an diese Entscheidungen haben die Gemeinden dafür zu sorgen, dass die Umsetzung in den Dörfern gelingt - eine Aufgabe, die auch in den Regelungen von Gesetz Nr. 131 enthalten ist (vgl. Tabelle 4).

Konkret steht die Gemeindeverwaltung damit in erster Linie vor bürokratischadministrativen Aufgaben: Eine geeignete Karte des Dorfes muss erstellt werden, die häufig eine von wenigen professionellen Kartierungen seit dem Zusammenbruch der Sowjetunion darstellt. So beklagt die Sachbearbeiterin für Landnutzung und Eigentumsfragen der Gemeindeverwaltung in Andreevka, dass sie zuvor lediglich über ein einziges abgegriffenes Exemplar einer Karte des Gemeindeterritoriums verfügte, die im Rahmen der Kolchosprivatisierung Anfang der 1990er erstellt wurde und auf der die Landanteile eingetragen wurden. Die neu erstellte Karte dient als Entscheidungsgrundlage, entlang welcher Straßen die dorfinternen Hauptleitungen verlegt werden, wobei der Spielraum in dieser Frage faktisch ohnedies begrenzt ist: Zwar sorgen Verteilungsfragen im Zusammenhang mit der Gasifizierung durchaus für Diskussionen auf den Gemeindeversammlungen, in den meisten Dörfern ist die Anzahl an verfügbaren Kilometern jedoch bereits durch die Höhe der bewilligten Subventionen vorgegeben und beschränkt sich in der Regel auf den Kernbereich des Dorfes. Die Bewohnerinnen und Bewohner abseits gelegener Siedlungen werden auf spätere Runden des Projekts vertröstet.

Schließlich obliegt es der Gemeindeverwaltung, geeignete Bau- und Montageunternehmen auszuwählen und mit dem Verlegen der Hauptleitungen entlang der Straßen zu beauftragen, den zeitlichen Ablauf der Arbeiten zu koordinieren und dabei allen Beteiligten als Ansprechpartnerin zur Verfügung zu stehen. Bemerkenswert ist in diesem Zusammenhang und vor dem Hintergrund der in der ersten Episode beschriebenen Formen der Zusammenarbeit zwischen Gemeindeverwaltung und ehemaligem Kolchos, dass die Gasifizierung im Bezirk zumindest in Ansätzen die Herausbildung eines Marktes für entsprechende Dienstleistungen bewirkt hat, an dem auch die ländlichen Gemeinden partizipieren. Die Lokalzeitung berichtet nicht nur mit großem Optimismus von der Gründung eines neuen Unternehmens in

28 Trotz Nachfragen blieb mir der Stellenwert dieser Zustimmung im Entscheidungsprozess unklar, denn ungeachtet dessen wurde im Dorf im Zusammenhang mit der Gasifizierung stets auf >Druck von oben< und daraus resultierende Sachzwänge verwiesen. Dies könnte auf mangelnde Informationen und/oder unpräzise Erinnerungen der Befragten ebenso zurückzuführen sein wie darauf, dass es sich bei diesem Schritt um fassadenhafte Partizipation ohne faktische Mitsprache- und vor allem Einflussmöglichkeiten - kurz: um folgenloses Rauschen im System - handelte. 
diesem Bereich, dem sie ein umfangreiches Portrait widmet (»Gas in jedes Haus schnell und in guter Qualität«, »Ochanskaja Storona« vom 3.9.2004: 3). Sie versucht darüber hinaus, die Existenz von zwei konkurrierenden Unternehmen in diesem Marktsegment durch ihre Berichterstattung ins Bewusstsein der Bürgerinnen und Bürger zu rücken (vgl. beispielsweise »Ochanskaja Storona« vom 22.7.2005: 2 und vom 9.2.2007: 7). Zudem ist in Andreevka und Beljaevka geplant, dass die Gasleitungen unmittelbar nach ihrer Fertigstellung und Inbetriebnahme von Privatunternehmen übernommen werden, die zukünftig für Wartungs- und Reparaturarbeiten verantwortlich zeichnen. Neben Infrastrukturbereiche wie beispielsweise die Wasserleitungen, die entgegen der Reformintention weiterhin vom ehemaligen Kolchos getragen werden respektive deren Eigentumsverhältnisse in der Schwebe sind, tritt mit den Gasleitungen ein Bereich, in dem mit solchen Pfadabhängigkeiten gebrochen wird und der sich allem Anschein nach anschickt, ganz im Sinne der jüngsten Kommunalreform zu >funktionieren<. In diesem Sinne sieht auch der Leiter eines Privatunternehmens für kommunale Dienstleistungen in der Gasifizierung eine entscheidende Zukunftsperspektive für seinen Geschäftsbereich:

»Was haben wir für Perspektiven? Ich denke, dass die Gasifizierung die wichtigste Perspektive dafür ist, dass das private Business endlich dazu kommt, etwas zu bauen. Gasleitungen, Wasserleitungen, Energieversorgung - und fertig. Das wäre die Grundlage.« (Privatunternehmer, 9.3.2010)

Ich möchte mit Blick auf die Umsetzung der Gasifizierung in den Dörfern jedoch noch einen weiteren Aspekt hervorheben, bei dem die Gemeindeverwaltung indirekt, aber kaum weniger exponiert in der Verantwortung steht: Ihr obliegt die Koordination der Haushalte, denn die Aufnahme einer Gemeinde in das Gasifizierungsprogramm setzt die Zusage voraus, dass eine Mindestanzahl an Haushalten zur Teilnahme bereit ist. Diese Anforderung im Blick zogen die Bürgermeisterinnen und Bürgermeister und Mitglieder der Gemeindeverwaltungen im Vorfeld der Entscheidung von Haus zu Haus, informierten die Gemeindemitglieder über das Projekt und sammelten Unterschriften von Interessierten, die anschließend der Bezirksverwaltung vorgelegt wurden. Nicht wenige Haushalte zogen jedoch nach einer ersten Euphorie ihre Zusage wieder zurück. Was aus rechtlicher Sicht möglich ist - die Unterschriften stellen lediglich Interessensbekundungen ohne weitere rechtliche Verbindlichkeiten dar -, bedroht aus Gemeindesicht das gesamte Projekt:

»Um in das Programm aufgenommen zu werden, haben [der Bezirksgouverneur] und ich versprochen, dass in Dubrovo 320 Häuser angeschlossen werden. [...] Wenn wir am Anfang nur 100 Häuser angegeben hätten, würde das Gas mit Sicherheit nicht hierher kommen, man hätte uns gar nicht erst ins Programm aufgenommen. Das ist das ganze Schlamassel. Wir haben 
320 verkündet, also 80 Prozent der Häuser in Dubrovo. Das ist der Plan: 80 Prozent der Haushalte. Von diesem Ziel weichen wir nicht ab. Wenn das Gas erstmal hier in Dubrovo wäre, wenn die Fackel erstmal brennen würde, dann wär's das. Die Bevölkerung würde begreifen und die Sache wäre schnell erledigt.« (Bürgermeister auf einer Gemeindeversammlung, 26.3.2009)

»Als auch wir unter das Gesetz fielen, das vorgibt, wie viele bei uns [in der Gemeinde, E.M.] bei der Gasifizierung mitmachen müssen, damals sind wir, nicht ich allein, alle Sachbearbeiter [Mitarbeiterinnen der Gemeindeverwaltung, E.M.] von Haus zu Haus und von Straße zu Straße gegangen: Wer sagt zu, dass er an der Gasifizierung teilnimmt? Sie [die Gemeindemitglieder, E.M.] haben sich direkt auf Listen eingetragen, dass sie mit der Gasifizierung einverstanden sind, dass sie ihr Haus an das Gasnetz anschließen wollen. Aber jetzt, wie sich die Dinge entwickeln, haben viele von denen, die damals unterschrieben haben, schon wieder abgesagt.« (Sachbearbeiterin der Gemeindeverwaltung, 12.3.2009)

Was passiert, wenn das deklarierte Ziel verfehlt wird, konnte ich während meiner Feldaufenthalte nicht herausfinden und auch die Befürchtungen der Bürgermeisterinnen und Bürgermeister blieben diesbezüglich im Vagen. Daran, dass die Umsetzung der Gasifizierung von ihnen als politisch alternativlos beobachtet wurde, sobald eine Gemeinde einmal in das Programm aufgenommen worden war, ließ ihr Verhalten jedoch keinen Zweifel. Alles andere als die erfolgreiche Umsetzung des Projekts würde vor diesem Hintergrund als politisches Scheitern beobachtbar und als Widerstand der Kommune gegen Modernisierung und Fortschritt ausgelegt, was es um jeden Preis zu verhindern gilt. Erfolg oder Misserfolg werden letztlich in vollem Umfang der Gemeindeverwaltung - respektive den Bürgermeisterinnen und Bürgermeistern - zugerechnet.

»[Vorsitzende des Veteranenrats]: Das [der Verlauf der Vorbereitungsarbeiten, E.M.] hängt nicht nur von uns ab. Sie arbeiten nicht besonders schnell, die Gasarbeiter.

[Bürgermeister]: Sie haben viel zu tun, das muss man auch verstehen.

[Vorsitzende des Veteranenrats]: Aber dann schieben Sie es nicht auf uns, dann behaupten Sie nicht, dass wir schuld sind!

[Bürgermeister]: Verstehen Sie - in jedem Fall bin ich der Schuldige.

[Vorsitzende des Veteranenrats]: Warum?

[Bürgermeister]: Weil Sie niemandem etwas versprochen haben.

[Vorsitzende des Veteranenrats]: Aber was hätte ich denn versprechen sollen?

[Bürgermeister]: Nichts. Aber ich musste, damit das Gas überhaupt nach Dubrovo kommt, gegenüber der Regionalverwaltung versprechen, dass ich 186 Häuser anschließe. Wenn ich das nicht versprochen hätte, würde jetzt überhaupt nichts passieren. Das ist wie im Sport: Wenn die Mannschaft gewinnt, sind die Sportler verantwortlich, wenn die Mannschaft ver- 
liert, ist der Trainer schuld. So ist es auch jetzt. Wenn alles gut läuft, bekommen Sie Ehre und Lob. Wenn es schlecht läuft, heißt es, dass ich schuld bin. Ich habe keine Wahl. Das Gasifizierungsprojekt jetzt noch abzusagen ist sinnlos.«

(Bürgermeister und Vorsitzende des Veteranenrats, 27.8.2008)

Der Faktor, von dem die erfolgreiche Umsetzung der Gasifizierung letztlich abhängt, sind vor diesem Hintergrund die individuellen Entscheidungen der Haushalte als eigentliche Adressaten des Programms, die rekursiv auf die Entscheidungen der Gemeindeverwaltung verweisen und mit deren politischer Handlungsfähigkeit verschnitten sind. Gleichzeitig stellen die Haushaltsentscheidungen für die Gemeindeverwaltung jedoch Umweltereignisse dar, auf die sie nicht direkt zuzugreifen vermag und die entsprechend als Risiko beobachtet werden, das es möglichst durch gezielte Einflussnahme einzuhegen gilt. Um dieses Steuerungsproblem zu lösen, greifen die Bürgermeisterinnen und Bürgermeister auf die Gemeindeversammlungen zurück - und kehren die mit diesem Forum verbundene Intention dabei gleichsam um.

\section{Die Gemeindeversammlung als Instrument der Einflussnahme}

Gesetz Nr. 131 richtet die Gemeindeversammlungen als eine zentrale Schnittstelle zwischen der kommunalen Politik und ihrem Publikum ein. Mit ihnen soll ein Forum etabliert werden, um das Publikum in den politischen Entscheidungsprozess zu inkludieren und auf diese Weise Möglichkeiten der direkten Einflussnahme zu etablieren. Daneben werden die Versammlungen auch zur Vermittlung von Sachinformationen, hier etwa über den geplanten Ablauf der Gasifizierung und die technischen und rechtlich-bürokratischen Voraussetzungen, genutzt. Durch ihre Teilnahme an der Versammlung erhalten die Gemeindemitglieder Informationen, die andernfalls für sie kaum zugänglich wären. Dass das Projekt im Dorf als bedeutsam bewertet wird, illustriert die Anzahl der Teilnehmenden: Schwanken die Teilnehmerzahlen bei >gewöhnlichen< Gemeindeversammlungen, auf denen beispielsweise das Budget diskutiert wird oder die Gemeindeverwaltung Rechenschaft ablegt, meist zwischen 30 und 40 Personen (darunter überwiegend Rentnerinnen und Rentner), zogen die Versammlungen zur Gasifizierung, so die einhellige Beobachtung der Bürgermeisterinnen und Bürgermeister, durchweg erheblich mehr Gemeindemitglieder an (100 bis 150 Personen).

Mit Blick auf das Gasifizierungsprojekt wird auch deutlich, dass die Bürgermeisterinnen und Bürgermeister die Versammlungen zu einem dritten Zweck nutzten, der auf ihre Aufgabe der Hauhaltskoordination verweist: Sie bemühten sich, auf den Veranstaltungen politischen Druck aufzubauen und die Gemeindemitglieder zur >Kooperation<, also zur Entscheidung für den Anschluss an das Gasnetz, zu 
bewegen. Wie dies geschah, lässt sich anhand der folgenden Szene verdeutlichen, die sich auf einer Gemeindeversammlung in Dubrovo abspielte. Das Gasifizierungsprojekt befand sich in dieser Gemeinde zum Zeitpunkt der Versammlung in einem fortgeschrittenen Stadium, es hatten bereits mehrere Versammlungen zum Thema stattgefunden und ein Teil der Haushalte im Dorf erfüllte mittlerweile die technischen Voraussetzungen für den Anschluss an das Gasnetz. Die erforderliche Mindestanzahl war allerdings noch nicht erreicht:

»[Bürgermeister]: Ich begrüße Sie, liebe Mitbürger, lassen Sie uns unsere Gemeindeversammlung beginnen. Ich arbeite nun seit einem Jahr [an der Gasifizierung, E.M.] und auf die Gemeindeversammlung kommen immer nur diejenigen, die zuhause schon alles für die Gasifizierung vorbereitet haben - und die sind auch heute wieder da. Das Ziel der heutigen Versammlung besteht darin, die Bewohner von Dubrovo noch mal mit Blick auf die Gasifizierung anzutreiben. [Es folgen Ausführungen dazu, wie viele Haushalte ihre Verpflichtungen bereits erfüllt haben und wie viele noch ausstehen.] Dieses Resultat erfreut mich in vollem Maße. Ich sage, es erfreut mich, weil bedacht werden muss, dass es zwar um uns alle geht, aber ich Sie alle im Bezirk und in der Region repräsentiere. Wir alle haben Verpflichtungen gegenüber Gazprom und gegenüber Moskau. [...] Ich denke, dass alle, die hier sind, ihre Verpflichtungen erfüllt haben. Das heißt, mit den Leuten hier müsste ich eigentlich gar nicht über das alles sprechen. [...] Ich denke, dass das Gas zu allen kommen wird [...], auch zu denen, die es ablehnen. Warum? Weil es schon jetzt einige Präzedenzfälle gibt, bei denen die Leute eine schriftliche Erklärung abgegeben haben, abgesagt haben, geschrieben haben, dass sie kein Gas brauchen. Und als die Leitung dann in der Straße verlegt war, kamen sie zu mir $>$ Ich habe noch einmal darüber nachgedacht, ich brauche doch Gas $<$.

[Äußerung aus dem Publikum]: Müssen wir auf diese Leute jetzt etwa warten?

[Bürgermeister]: Das Gas wird angeschlossen werden, bei Ihnen wird das Gas angeschlossen werden. Das Ziel unserer Versammlung ist, die Leute anzutreiben, die noch nicht alles dafür erledigt haben. Wir versuchen jetzt alles, was in unserer Macht steht, um die Leute dazu zu bringen, ihre Häuser für den Gasanschluss vorzubereiten.

$[\ldots]$

[Leiter der Bezirksplanungsamtes]: Ich weiß nicht, vielleicht lohnt es sich, mit den Nachbarn zu sprechen. [Der Bürgermeister] geht von Tür zu Tür, redet auf die Leute ein, dass wir das [das Gas, E.M.] alle brauchen. Wenn wir die Auflagen des Programms nicht erfüllen, wird [der Bezirksgouverneur] es sehr schwer haben. In erster Linie er, weil der Gouverneur [der Region Perm, E.M.] sich stark auf ihn verlässt. [...] Am Ende heißt es dann im Fernsehen: >Es tut uns leid, aber der Bezirk [.] ist nicht bereit für die Gasifizierung, weil nicht genug Haushalte mitmachen<.

[...]

[Bürgermeister]: Gazprom hat seine Verpflichtungen, nämlich die Leitung OstrožkaDubrovo-Ponomary [die Hauptleitung zwischen den Gemeinden, E.M.] zu bauen. UralGaz- 
Service [ein regionales Gasunternehmen, E.M.] hat seine Verpflichtungen, nämlich das Leitungsnetz innerhalb des Dorfes Dubrovo zu verlegen. Jeder von uns hat seine Verpflichtungen - wir müssen unsere Häuser vorbereiten, damit sie an das Gasnetz angeschlossen werden können. Im Moment erfüllt Gazprom seine Verpflichtungen, UralGazService erfüllt seine Verpflichtungen. Wir erfüllen unsere Verpflichtungen noch nicht. Das ist die Situation. Ich denke, dass das alle verstehen.

$[\ldots]$

[Äußerung aus dem Publikum]: Sie haben am Anfang über die Gasifizierung gesprochen und da haben sie gesagt, dass wir, solange das letzte Haus in Osinovka [ein Ortsteil der Gemeinde, E.M.] nicht fertig ist, dass wir solange auch kein Gas kriegen. So verstehe ich das. Das heißt also, dass die, die hier sitzen, gar nicht hier sitzen müssten - stimmen Sie mir zu?

[Bürgermeister]: Darüber habe ich gesprochen.

[Äußerung aus dem Publikum]: Diese Leute müssen eigentlich gar nicht hier sein, sondern die, die ihre Häuser noch nicht vorbereitet haben.

[Bürgermeister]: Da stimme ich ihnen zu.

$[\ldots]$

[Äußerung aus dem Publikum]: Holen Sie die her, die nicht fertig geworden sind.

[Bürgermeister]: Wie? Geben Sie mir einen Rat. Ich bin zwar der Bürgermeister, aber ohne die Unterstützung der Bevölkerung kann ich nichts machen. Wir haben alle ein gemeinsames Ziel, Sie genauso wie ich. Allein kann ich nichts ausrichten. Wenn nicht alle Dorfbewohner wollen, kann ich nichts machen. Es geht nur mit Ihrer Hilfe. Oder habe ich nicht Recht? Keine Macht kann ohne das Volk etwas ausrichten.«

(Gemeindeversammlung, 26.3.2009)

In Ermangelung von (formalen) Sanktionsmöglichkeiten als lokalpolitische Machtgrundlage - niemand kann zur Teilnahme gezwungen werden, wie der Bürgermeister am Ende der Passage treffend einräumt -, zielt die Kommunikation darauf, die Haushalte möglichst wirkungsvoll zu beeinflussen, also die aus Sicht des Bürgermeisters erwünschten Selektionen herbeizuführen (vgl. zum Begriff des Einflusses Luhmann 2012: 52). Im Wesentlichen kommen dabei drei Strategien zum Einsatz: Moralkommunikation, Verbindlichkeitskommunikation und der Verweis auf die Einbindung in gemeindeübergreifende Hierarchien.

Erstens und wiederkehrend wird moralisch an das Publikum appelliert: »Eine Kommunikation tritt als moralisch auf, wenn sie suggeriert oder explizit macht, dass Selbstachtung und Achtung anderer von der Erfüllung bestimmter Bedingungen abhängen « (Luhmann 2008: 368f). In diesem Sinne versucht der Bürgermeister, zumindest auf der Darstellungsebene die Achtung der einzelnen Gemeindemitglieder innerhalb des Dorfes an die Teilnahme am Gasifizierungsprojekt zu koppeln. Die Gasifizierung wird als gemeinschaftliches Projekt dargestellt, das im Interesse aller ist - auch und besonders derjenigen, die die Teilnahme bislang verwei- 
gern - und dessen Gelingen durch die >Abweichler i im Dorf in Gefahr gebracht wird. Ohnedies wird die Ablehnung der Gasifizierung als irrationale Strategie dargestellt, die kaum durchzuhalten ist. Wiederholt wird das Dorf mittels entsprechender Semantiken in diejenigen, die mitmachen, und diejenigen, die sich verweigern, unterteilt (Letztere im vermeintlichen Irrglauben, sie könnten auf den Anschluss an das Gasnetz verzichten). Die Grenze läuft dabei - darauf komme ich auch im folgenden Abschnitt noch einmal zurück - entlang der Interaktion: Während sich die auf der Versammlung Anwesenden auf der kooperativen >richtigen< Seite befinden, sind die Abwesenden auf der widerständigen >falschen< Seite - und damit für die Kommunikation faktisch gar nicht erreichbar, die, so formuliert der Bürgermeister zumindest das Ziel der Veranstaltung, eigentlich an sie gerichtet werden müsste.

Zweitens werden Verbindlichkeiten respektive eine Bringschuld der Gemeindemitglieder - und dabei schließt sich der Bürgermeister selbst mit ein - konstruiert, die daraus resultieren, dass die >anderen< ihre Verpflichtungen bereits erfüllt haben. Während die moralischen Appelle eine Grenze innerhalb des Dorfes ziehen, wird nun auf die Unterscheidung zwischen dem Dorf und seiner Umwelt hin beobachtet: Die >anderen< respektive die Parteien, denen gegenüber Verpflichtungen bestehen, befinden sich jenseits der Außengrenzen des Dorfes. Zudem werden sie möglichst hochrangig verortet - Verpflichtungen bestehen gegenüber Gazprom, dem Bezirks- und dem Regionalgouverneur und sogar gegenüber »Moskau « - und damit eine Hierarchie konstruiert, an deren unterem Ende sich das Dorf befindet.

Schließlich wird dieser zweite Aspekt verstärkt, indem drittens explizit auf die übergeordnete Verwaltungsebene und konkret auf den Bezirksgouverneur, der allen im Dorf bekannt sein dürfte, verwiesen wird. Über die Anwesenheit eines Vertreters der Bezirksverwaltung auf der Versammlung wird zum einen politischer Druck weitergegeben. Zum anderen wird auf diese Weise die Begrenztheit des Handlungsund Entscheidungsspielraums der Gemeindeverwaltung gegenüber diesen übergeordneten Ebenen demonstriert und versucht, den Eindruck der Alternativlosigkeit an die Anwesenden weiterzugeben.

Operiert und Einfluss auszuüben versucht wird somit anhand von drei Unterscheidungen: Kooperation|Widerstand als moralische Unterscheidung und Grenze innerhalb des Dorfes, Dorf|Umwelt im Rahmen der Konstruktion von Verbindlichkeiten der gesamten Dorfgemeinschaft sowie oben|unten als Ausdruck hierarchischer Strukturen, in die die Gemeinde eingebunden ist.

\section{Grenzen lokaler Machtkommunikation}

Wie weit reichen diese Versuche der Einflussnahme? Zwei Grenzen lassen sich beobachten: Die erste resultiert aus den Eigenschaften von Interaktion als Systemtyp, 
die zweite verweist auf funktionale Differenzierung und die operative Geschlossenheit gesellschaftlicher Funktionssysteme.

In der beschriebenen Kommunikation auf der Gemeindeversammlung spiegeln sich die wesentlichen Merkmale von Interaktionssystemen wider - Inklusion und Grenzziehung gegenüber der Umwelt über Anwesenheit, die Nicht-Negierbarkeit der Kommunikation im Rahmen von Interaktion sowie die fehlenden Durchgriffsmöglichkeiten von Interaktionssystemen über die eigenen Grenzen hinaus - und markieren eine Grenze lokalpolitischer Einflussnahme: Vor dem Hintergrund des speziellen Typenprogramms der Versammlung kommt dem Bürgermeister oder der Bürgermeisterin eine hervorgehobene Stellung bei der Themensetzung zu, die durch die Anwesenden auch nicht in Frage gestellt wird (zu Typenprogrammen von Interaktion vgl. Kieserling 1999: 18). In der Konsequenz kann niemand der Anwesenden mit Blick auf die Gasifizierung und die mit einer Teilnahme verbundenen Anforderungen an die Haushalte auf Unwissenheit verweisen und sich auf diese Weise aus der Affäre ziehen. Gleichzeitig bleiben die Abwesenden unadressierbar, weil die Gemeindeversammlung allein die Anwesenden inkludiert und integriert, keinesfalls aber die Gemeinde und ihre Mitglieder insgesamt. Eine Differenz übrigens, die in den Interaktionen der Gemeindeversammlung bereits selbst in paradoxer Form reflektiert wird: Man versichert sich immer wieder, dass der Inhalt der Kommunikation nicht für die Anwesenden, sondern für die im Moment unerreichbaren Abwesenden bestimmt ist, zieht daraus aber nicht die Konsequenz, die Interaktion zu beenden. Dass diese Problematik der Nicht-Adressierbarkeit durch Abwesenheit zwar mit Blick auf die Gasifizierung in besonderer Weise virulent wird, jedoch den Gemeindeversammlungen im Allgemeinen anhängt, wird auf einem Treffen der Bürgermeisterinnen und Bürgermeister reflektiert:

»Wir legen gegenüber der Bevölkerung Rechenschaft ab. Wir versammeln sie im Kulturhaus - wir treiben sie zusammen, wäre die richtige Bezeichnung. Wir treiben so um die 15 Leute zusammen, wenn es 40 sind, ist das schon großes Glück. Sie kommen nicht, sie nehmen nicht teil. Aber außerhalb [der Versammlung, E.M.], wenn du als Bürgermeister nicht dabei bist, dann gibt es Diskussionen. Wenn sie wenigstens bei den öffentlichen Anhörungen auftauchen und dort mit ihren Fragen kommen würden - ja, dann könnten wir darüber reden. Aber das, was sie reden, so hinter dem Rücken - das wissen wir nicht einmal.« (Bürgermeister, 11.8.2008)

Deutlich wird: Die Kommunikation beabsichtigt zwar eine Wirkung über die Versammlung - die Interaktion - hinaus, kann diese Bindungskraft jedoch aus sich heraus nicht entfalten (solange ihr nicht vom politischen System die Kompetenz zugewiesen wird, diesbezüglich kollektiv bindende Entscheidungen zu treffen, die auch für die Abwesenden Gültigkeit haben - aber das ist dann erstens nicht mehr Sache 
der Interaktion und liegt zweitens im Fall der Gasifizierung ohnedies nicht vor). Diese Beschränkung besteht unabhängig davon, ob und in welcher Form in der Interaktion selbst Anschlussfähigkeit erzeugt wird. In der oben zitierten Passage wird an die vom Bürgermeister heraufbeschworene Teilung des Dorfes zwar im Versammlungskontext durchaus angeschlossen - man fragt nach, stimmt zu, zeigt sich empört -, die operative Durchschlagskraft dieser Semantiken nach dem Ende und außerhalb der Versammlung erwies sich jedoch als gering. Weder die Bildung von zwei >Lagern « noch Überzeugungsversuche der Dorfbewohner untereinander ließen sich beobachten, auch wenn das Thema auch abseits der Versammlung diskutiert wurde. Für die sogenannten >Abweichler<, also Gemeindemitglieder, die eine Teilnahme am Projekt (vorerst) ablehnten, wurde dabei unter Verweis auf finanzielle Argumente in der Regel sogar Verständnis geäußert - was auf eine zweite Grenze verweist, an die die kommunalpolitischen Einflussversuche stoßen: die spezifischen Operationsweisen der Funktionssysteme und deren Geschlossenheit.

Während die Gasifizierung für die Gemeindeverwaltung ein politisches Projekt ist und entsprechend im Horizont politischer Anschlusskommunikation beobachtet wird - in erster Linie das Durchsetzen von Entscheidungen, aber auch die Anhäufung von politischem Kapital in Form von Prestigegewinn, Erfolg bei den nächsten Wahlen und ähnlichem -, liegt den Beobachtungen durch die Gemeindemitglieder eine andere Unterscheidung zugrunde. Einen ersten Eindruck der daraus resultierenden Diskrepanzen vermittelt erneut ein Auszug aus einer Gemeindeversammlung zur Gasifizierung, die im März 2009 in Beljaevka stattfand. Im Unterschied zur oben erwähnten Versammlung in Dubrovo stand das Gasifizierungsprojekt dort zum Zeitpunkt der Versammlung noch am Anfang und die Veranstaltung sollte dazu dienen, erste Informationen darüber zu vermitteln. Die Bürgermeisterin eröffnete die Versammlung, führte kurz in das Thema ein und hob die Bedeutung sowohl des Gasifizierungsprojekts an sich als auch der finanziellen Unterstützung durch den landwirtschaftlichen Großbetrieb hervor. Nach wenigen Minuten übergab sie das Wort an die Vertreter von zwei Montageunternehmen, die den Rest der gut einstündigen Veranstaltung bestritten. Beide erläuterten detailliert die technischen Voraussetzungen für den Anschluss privater Wohnhäuser an das Gasnetz und gingen dabei in aller Ausführlichkeit auf die Vor- und Nachteile verschiedener Modelle von Boilern, Gasherden, Heizungssystemen und sonstigen Bestandteilen der technischen Infrastruktur ein. Ihre Ausführungen wurden schließlich von einer Frage aus dem Publikum, das bis zu diesem Zeitpunkt mehrheitlich geschwiegen hatte, nach den zu erwartenden Kosten unterbrochen: 
»[̈̈ußerung aus dem Publikum]: Wie hoch ist die Gesamtsumme? Mit der Ausstattung und allem drum und dran?

[Unternehmensvertreter ${ }_{1}$ ]: Lassen Sie uns gemeinsam rechnen. Sie fragen jetzt nach der Montage?

[Äußerung aus dem Publikum]: Ja, nach der Montage und den technischen Voraussetzungen und den Arbeitskosten.

[Unternehmensvertreter ${ }_{1}$ ]: Ich habe Ihnen gesagt, die technischen Voraussetzungen [gemeint sind die Gasleitungen auf dem Grundstück, E.M.] kosten 1.280. Die Sache ist die, dass es Montageunternehmen gibt, die für den laufenden Meter 3.000 nehmen und abmessen.

[ ̈̈̈ußerung aus dem Publikum]: Aber Sie wissen das doch.

[Unternehmensvertreter ${ }_{1}$ ]: UralGazService montiert für 18 bis 20 [Tausend, E.M.], andere Unternehmen für 30.000. Aber die Ausstattung ist da nicht eingeschlossen [...]. Verstehen Sie mich nicht falsch, aber ich kenne den Grundriss Ihres Hauses nicht.

[Äußerung aus dem Publikum]: So ungefähr?

[Unternehmensvertreter 2 ]: Dazu gibt es keine Zahlen.

[Stimmengewirr]

[Äußerung aus dem Publikum]: So ungefähr das Minimum?

[Unternehmensvertreter 2 ]: Das kann ich Ihnen nicht sagen.

[Äußerung aus dem Publikum]: Die Frage ist doch die - die technische Ausstattung für das Gas, welcher Herd, welcher Kessel, welche Heizkörper, welche Rohre - das hängt alles vom individuellen Auftraggeber ab, von demjenigen, der im Haus wohnt.

[Unternehmensvertreter ${ }_{1}$ ]: Völlig richtig.

[Äußerung aus dem Publikum]: Aber was kostet der Anschluss insgesamt? Wie machen die das - sie verlegen die Leitungen ins Haus, da gibt es ein Ventil und einen Zähler am Kessel oder, wenn es nur ein einzelner Wassererhitzer ist, direkt am Erhitzer. Das ist also das System von der Straße bis ins Haus - was kostet das, nur zur Orientierung?

[Unternehmensvertreter ${ }_{1}$ ]: Sie haben ganz richtig gesagt, dass das vom Haus abhängt. Lassen Sie mich die Situation erklären: Nehmen wir mal an, das ist Ihr Haus, der Ausgang ist hier. [...] [Erläuterung verschiedener technischer Varianten] Deshalb kann ich Ihnen das [den Preis, E.M.] nicht sagen.

[Stimmengewirr]

[Unternehmensvertreter ${ }_{1}$ ]: Deshalb bin ich zu Ihnen gekommen. Wenn ich Sie frage, wohin wollen Sie den Herd? Dann sagen Sie: >Hier an der Stelle ist bei mir schon alles voll, der Herd kommt hierhin. Wohin der Kessel kommt, weiß ich noch nicht, entweder hier gleich daneben, oder dorthin, wenn ich den Ofen abbaue<.

[Äußerung aus dem Publikum]: Das ist alles verständlich. Aber dann sagen Sie doch wenigstens, wie das in Andreevka [der Nachbargemeinde, E.M.] ist, nach Ihrer Erfahrung - wie viel kostet dort die Montage eines laufenden Meters?

[Unternehmensvertreter ${ }_{1}$ ]: Entschuldigen Sie, ich weiß es nicht. 
[Unternehmensvertreter ${ }_{2}$ ]: Wir stehen hier jetzt nicht vor Ihnen als Verkäufer, wir kennen diesen Markt nicht. Wir sind hergekommen, weil wir Ihnen helfen wollen.

[Themenwechsel, die Stimmung im Saal beruhigt sich langsam]

(Gemeindeversammlung, 25.3.2009)

Was ist passiert? Zunächst ist festzuhalten, dass sich mit der Frage nach dem Preis der Gasifizierung hörbar Aufregung im Saal verbreitet, die gesamte Sequenz ist untermalt von Stimmengewirr und Zwischenrufen, so dass die einzelnen Äußerungen teilweise nur schwer zu verstehen sind. Ganz offensichtlich wird mit dieser Frage ein Thema angesprochen, dass im Verlauf der Versammlung bis zu diesem Zeitpunkt noch nicht erwähnt wurde, dem Publikum aber unter den Nägeln brennt. Die Beobachtung der Gasifizierung durch die anwesenden Gemeindemitglieder läuft auf die Frage nach den zu erwartenden Kosten - und damit nach dem Preis hinaus, ohne deren Kenntnis alle anderen Informationen nicht eingeordnet werden können. Ebendiese Information wird nun jedoch auch auf wiederholtes Drängen und Nachfragen nicht preisgegeben - sei es, weil die beiden Ansprechpartner tatsächlich nicht über das entsprechende Wissen verfügen (wie sie behaupten), sei es, weil sie diese Information den Anwesenden aus anderen Gründen bewusst vorenthalten. Beide beginnen zu lavieren, weichen auf Nebenaspekte aus, konkretisieren mehrfach bereits bekannte Äußerungen. Im Effekt bleibt der Preis, auf den das Publikum hinaus will, jedoch unbeobachtbar, was die Situation eher verschärft als zu entspannen vermag. Der Wortwechsel kulminiert schließlich in einer Bemerkung, die augenscheinlich darauf zielt, die Situation durch die Klärung von Rollen und Zuständigkeiten zu lösen: »Wir stehen hier jetzt nicht vor Ihnen als Verkäufer« und stattdessen: »Wir sind hergekommen, weil wir Ihnen helfen wollen«. Diese Abschlussbemerkung reflektiert in aller Deutlichkeit die Diskrepanz zwischen der Beobachterposition der Gemeindeverwaltung auf der einen Seite (zu der die beiden Unternehmensvertreter in diesem Kontext hinzuzählen sind), für die die Um- und Durchsetzung des Gasifizierungsprojekts im Vordergrund steht, und den Gemeindemitgliedern auf der anderen Seite, für die die Entscheidung über die Teilnahme in erster Linie eine Zahlungsentscheidung ist: Es geht um potenzielle Zahlungen und damit den Preis, der auf beiden Seiten der Knappheitscodierung »nicht nur die Zahlungen, die erfolgen, sondern auch die Zahlungen, die nicht erfolgen«, reguliert (Luhmann 1988: 19). Zwar versucht die Gemeindeverwaltung durchaus, dem wirtschaftlichen Aspekt der Gasifizierungsentscheidung Rechnung zu tragen, gerade auch indem Unternehmensvertreter auf die Gemeindeversammlung eingeladen werden. Ohne Preisinformation bleibt der Markt für seine potenziellen Teilnehmerinnen und Teilnehmer jedoch unbeobachtbar und die intendierte »Hilfe« durch die anwesenden Unternehmensvertreter läuft an ihren Adressaten vorbei ins Leere. 
Die skizzierte Szene auf der Gemeindeversammlung, so lässt sich an dieser Stelle schlussfolgern, verweist auf wirtschaftliche Operationslogiken, die über die Gasifizierung in das Dorf hineinwirken. Wie sich dieses Hineinwirken im Einzelnen vollzieht, an welchen Stellen Anschlussmöglichkeiten generiert werden, wo Reibung mit tradierten dörflichen Erwartungsstrukturen entsteht und auf welche Weise sich die lokalpolitische Machtkommunikation in diesem Kontext bricht, wird deutlich, wenn man berücksichtigt, wie sich die angesprochene (Zahlungs-)Entscheidung für die Haushalte entfaltet.

\section{Eigentum, Zahlungen und Kredite als unwiderstehliche Kommunikationsangebote}

Für die Haushalte ist der Anschluss des eigenen Hauses an das Gasnetz mit einer Reihe von Voraussetzungen verbunden. Einige dieser Voraussetzungen mögen aus der Außensicht banal erscheinen, doch sie tangieren zentrale Strukturen der Dörfer und sind damit über den konkreten Anlass der Gasifizierung hinaus bedeutungsvoll. Die Grundvoraussetzung für die Installation der technischen Infrastruktur auf einem Grundstück und in den Gebäuden ist eine technische Dokumentation - der sogenannte technische Pass (»techničeskij pasport«) - von Haus und Grundstück. Dieses Dokument wird von einer Bezirksbehörde erstellt, jedoch nur unter der Bedingung, dass die Eigentumsverhältnisse mittels eines entsprechenden Nachweises eindeutig zu klären sind.

»Und das [die Möglichkeit der Gasifizierung, E.M.] ist nicht nur eine Frage des Geldes, sondern es gibt noch weitere Hindernisse. Die ergeben sich dann, wenn das Haus, in dem Sie leben, nicht Ihres ist, also nicht bei der Registrierungskammer registriert ist. Das muss nun dringend erledigt werden.« (Bürgermeisterin auf einer Gemeindeversammlung, 25.3.2009)

Die Tragweite dieser Forderung wird vor dem Hintergrund der sowjetischen und frühen postsowjetischen Vergangenheit der Gemeinden deutlich: Die Tatsache, dass die Kollektivbetriebe im sowjetischen Regime als umfassende Organisationen im Dorf fungierten und in sozialer wie in räumlicher Hinsicht kongruent mit den jeweiligen Gemeinden waren, impliziert auch, dass die Betriebe neben öffentlichen Gebäuden vielfach Wohnhäuser für ihre Mitglieder errichteten (dazu ausführlich S. 72ff). Während im Zuge der Privatisierung des Landwirtschaftsbetriebs in Andreevka in den frühen 1990er Jahren Anteilsscheine auf Agrarland und das betriebliche Sachvermögen ausgegeben wurden, geschah dies im Falle der Wohnhäuser nicht, obgleich auch sie formal zum Kollektiveigentum zählten. Stattdessen hatten die Bewohnerinnen und Bewohner die Möglichkeit, die Häuser, die sie bewohnten, zu kaufen - was in vielen, jedoch nicht in allen Fällen geschah. 
Für einen kleinen Anteil der Häuser hatte die Betriebsleitung den Verkauf abgelehnt, so dass im Sommer 2008 nach wie vor 38 Haushalte und damit etwa 10 Prozent der Gemeindehaushalte in Kolchoshäusern wohnten.

Der anstehende Anschluss an das Gasnetz induzierte nun eine zweite Privatisierungswelle: Da der Betrieb sich nicht in der Lage sah, die für den Anschluss notwendige technische Ausstattung der betriebseigenen Wohnhäuser zu finanzieren, hatte der Kolchosvorsitzende, wie die Bürgermeisterin beschreibt, mit Blick auf die Gasifizierung und auf das Betreiben der Gemeindeverwaltung hin seinen Widerstand gegen eine weitergehende Privatisierung aufgegeben und sich entschlossen, auch die bislang in seinem Eigentum verbliebenen Wohnhäuser den Bewohnern zum Kauf anzubieten:

»Den Bürgern, die in Kolchoswohnungen wohnen, hat [der Kolchosvorsitzende] bereits eindeutig signalisiert, dass er nichts unternehmen wird. Das heißt, sie müssen sich um die Vermessung durch das $\mathrm{BTI}^{29}$ selbstständig kümmern, so wie sie auch den Rest selbstständig machen müssen. Das einzige, wovon wir ihn überzeugen konnten - Sie wissen, dass er ein ausgesprochener Gegner des Verkaufs der Kolchoswohnungen war - wir haben ihn also davon überzeugt, dass es jetzt unvermeidlich ist, den Leuten die Möglichkeit zum Kaufen zu geben. Ihnen stehen jetzt kolossale Ausgaben für die Gasifizierung bevor. [...] Wir haben in partnerschaftlicher Weise mit ihm verhandelt - er gibt den Leuten das Recht, die Häuser zu kaufen, so wie wir sie [zu Beginn der 1990er, E.M.] gekauft haben. Wir haben auch mit Landanteilen bezahlt, uns hat niemand etwas >für einen Apfel und ein Ei< gegeben. Das heißt, er akzeptiert auch jetzt die Bezahlung mit Landanteilen und, bei wem das nicht ausreicht, mit Geld, auch auf Raten - aber er akzeptiert es, damit die Bewohner an der Gasifizierung teilnehmen können.«(Bürgermeisterin auf einer Versammlung des Veteranenrats, 13.8.2008)

Wie bereits im Zuge der ersten Privatisierungswelle in den frühen 1990er Jahren vollzog sich der Verkauf nicht über den Markt, sondern war mit einer Reihe von Einschränkungen versehen: Zum Kauf berechtigt waren ausschließlich die gegenwärtigen Bewohnerinnen und Bewohner und der Verkauf an Dritte war ausgeschlossen; die Preise wurden auf Vorschlag der Kolchosleitung festgelegt und richteten sich nach Parametern wie Alter, Zustand und Lage der Gebäude. Ebenfalls analog zur ersten Privatisierungswelle bot der Betrieb den Käuferinnen und Käufern erneut drei Finanzierungsvarianten an: Möglich waren erstens die Bezahlung auf Raten (»na rassročku«) über maximal ein Jahr, zweitens die Gewährung eines

29 BTI ist die Abkürzung für »bjuro techničeskoj inventarizacii«, wörtl.: »Büro für technische Inventarisierung «. Hinter dieser Bezeichnung steht eine staatliche Institution, die sich um die Erfassung des technischen Inventars sämtlicher Immobilien in Russland kümmert. 
ermäßigten Preises im Tausch gegen Landanteile und drittens die (Teil-)Finanzierung über Gehaltsabschläge.

Damit die Wohnhäuser, die auf diese Weise de facto in Privateigentum übergingen, auch de jure als solches galten, war die Transaktion mit dem ehemaligen Kollektivbetrieb jedoch nicht hinreichend. Notwendig war der Eintrag bei der Registrierungskammer des Justizministeriums, einer föderalen Institution, die Zweigstellen in den Regionen und Bezirken unterhält. In Andreevka war dieser Schritt jedoch bereits im Zuge der ersten Privatisierungswelle in den meisten Fällen unterblieben. Auf die Frage nach den individuellen Motiven für diese Praxis wurde mir regelmäßig ein ganzes Spektrum an Gründen präsentiert: die Nachlässigkeit der Eigentümer, mangelnde Informationen, bürokratischer Aufwand und damit verbundene Kosten, die Ablehnung von Privateigentum oder schlicht fehlendes Interesse. Darüber, ob es sich bei dieser Aufzählung um tatsächliche Beweggründe der einzelnen Haushalte oder um externe Zuschreibungen handelt, möchte ich an dieser Stelle nicht urteilen. Unabhängig von den individuellen Motiven bewirkte jedoch der Verzicht auf die rechtliche Formalisierung der Transaktion, dass die Grenze zwischen Eigentum und Nicht-Eigentum stets zu einem gewissen Grad latent gehalten werden konnte, sie bestand faktisch als Vereinbarung zwischen Kolchos und Haushalt ohne die Beteiligung eines Dritten. Die Funktion eines solchen Latenthaltens wird mit Blick auf die Bedeutung des Kollektivbetriebs im Dorf deutlich: Ungeachtet aller Privatisierungsversuche und ihrer neuen und oftmals häufig wechselnden Rechtsform hatten die Kollektivbetriebe über den Zusammenbruch des sowjetischen Regimes hinaus faktisch ihren Status als umfassende Organisationen in den Gemeinden behalten: Sie stellten Arbeitsplätze bereit, trugen große Teile der sozialen und technischen Infrastruktur, erbrachten öffentliche Dienstleistungen und sie unterstützten die Hoflandwirtschaften der Privathaushalte mit Inputfaktoren. Damit einher gingen nicht nur verschiedene Facetten der Kolchosmitgliedschaft als Arbeitnehmer, als Miteigentümer im Kolchoskollektiv und als Leistungsempfänger. Die Bindung an den Kolchos, die auch im Verzicht auf die Abgrenzung über (Privat-)Eigentum zum Ausdruck kam, war vor allem während der 1990er Jahre für die meisten Bewohnerinnen und Bewohner ländlicher Gemeinden gleichbedeutend mit materieller Versorgung, sozialer Sicherheit und einer funktionierenden lokalen Infrastruktur (Lindner/Moser 2009a: 13; auch z.B. Amelina 2000). Zwar dürfte sich die Versorgungssituation in vielen Gemeinden nach den turbulenten Anfangsjahren der Transformation entspannt haben, eine formale Klärung der Eigentumsverhältnisse hat in diesem Bereich jedoch nicht stattgefunden.

Die Beobachtung, dass den >Zettelchen< (»bumažki«) - so die umgangssprachliche und leicht abfällige Bezeichnung für Eigentumszertifikate - im Dorfkontext augenscheinlich wenig Bedeutung beigemessen wurde, bedeutet wohlgemerkt nicht, dass die Unterscheidung von Eigentum und Nicht-Eigentum abseits rechtlicher 
Kategorien keine Anschlussfähigkeit generiert hätte. ${ }^{30}$ Das Exklusionsmoment, das dem Eigentumscode immanent ist - alle Nicht-Eigentümer sind vom Zugriff auf ein Gut ausgeschlossen (und durch die Akzeptanz dieser Exklusion und die Möglichkeit des Kreuzens der Unterscheidung potenziell wiederum ins Wirtschaftssystem inkludiert) (Luhmann 1988: 189; auch Baecker 2006: 52f; Bohn 2009: 245) - entfaltete im Dorf durchaus operative Wirkung. Mit Blick auf die Häuser der Dorfbewohner war unumstritten, wem was gehört, welche Verantwortlichkeiten mit dem eigenen Eigentum verbunden sind und wer vom Zugriff (respektive der Nutzung) ausgeschlossen ist. Man verkaufte Gemüse aus dem Gemüsegarten und alljährlich wurden Auszeichnungen für die Gestaltung von Haus und Garten verliehen. Durch den Verzicht auf die rechtliche Formalisierung konnte der Eigentumscode im Dorf reibungslos seine Wirkung entfalten, ohne dass die Eigentümer ihren Ausschluss aus dem dörflichen, kolchos-dominierten Kommunikationszusammenhang formal vollziehen mussten - man behielt buchstäblich stets einen Fuß in der Tür des Kolchos.

Der Dorfkontext determiniert dabei jedoch auch die Grenzen der Anschlussfähigkeit: Zwar sind unter diesen Bedingungen Knappheitszugriffe möglich, Knappheitsübertragungen jedoch nicht. Der Verkauf der Häuser an Dritte steht den Eigentümern ohne Registrierung ebenso wenig zur Wahl wie beispielsweise die Belastung durch Hypotheken oder ähnliches (vgl. dazu auch Baecker 2006: 53). Aus Gemeindesicht rückt mit der jüngsten Kommunalreform noch eine weitere, ebenfalls blockierte Anschlussmöglichkeit in den Vordergrund: die Besteuerung. Die Gemeinden verfügen nun erstmals über ein eigenes Budget und eigene Einnahmen und damit über die Perspektive auf einen erweiterten Handlungs- und Entscheidungsspielraum - in diesem Sinne fasst der Bürgermeister von Dubrovo den aus seiner Sicht zentralen Effekt der Reform mit den Worten zusammen: »Der wesentliche Unterschied liegt darin: Es gab kein Budget. Und mit Blick auf das Budget gibt es eine gewisse Freiheit« (12.8.2008). Die Steuer auf privates Vermögen als eine von zwei vollumfänglich lokalen Steuern (vgl. Tabelle 5) kann jedoch nur erhoben werden, wenn das private Eigentum - und dies betrifft hier im Wesentlichen die Wohnhäuser - als solches registriert ist. Andernfalls bleibt den Gemeindeverwaltungen der Zugriff auf Einnahmen, die ihnen nicht nur zustehen, sondern auch buchstäblich vor ihrer Haustür liegen, verwehrt. Und ähnlich wie gegenüber dem Landwirtschaftsbetrieb als Steuerzahler sind die Durchgriffsmöglichkeiten beschränkt. Sowohl gegenüber der Steuerbehörde als föderaler Institution als auch gegenüber den Dorfbewohnerinnen und Dorfbewohnern kann die Gemeinde appel-

30 In ähnlicher Weise wie für die Wohnhäuser gilt dies auch für die Eigentumszertifikate auf Agrarland, das die Kolchosmitglieder ebenfalls im Zuge der Privatisierung in den frühen 1990er Jahren erhalten hatten (vgl. dazu ausführlich die dritte und vierte Episode). 
lieren und auf ihre Lage aufmerksam machen, die Registrierung erzwingen kann sie aus eigener Kraft nicht:

»Sie [die Gemeindemitglieder, E.M.] bezahlen weder für das Haus noch für das Grundstück. Sie leben hier, machen von allem Gebrauch - aber um keinen Preis sind sie bereit zu zahlen. Theoretisch müsste die Steuerinspektion die Sache nach drei Jahren vors Gericht bringen. Sie machen das nicht. Wir haben ihnen schon Listen zukommen lassen. Laut Gesetz sind sie dazu verpflichtet, aber sie haben das nicht nötig, denn diese Summen im Vergleich mit denen in den Städten - das ist nichts.« (Bürgermeisterin, 8.8.2008)

Mit der Gasifizierung als Kommunikationsangebot ergibt sich nun von unerwarteter Seite Änderungsdruck: Wer teilnehmen will, ist gezwungen, auch die Eigentumsfrage zu klären und rechtlich zu formalisieren, und muss sich fortan entsprechend besteuern lassen. Neben dem erfolgreichen Abschluss des Gasifizierungsprojekts, das es voranzutreiben gilt, beobachtet die Gemeinde den Prozess zunehmend auf Budgeteffekte hin - und damit auf Anschlussmöglichkeiten für Steuerzahlungen, die bislang nicht existierten und sich durch die Eigentumsregistrierung eröffnen. Die Steuerbasis erweitert sich als Begleiterscheinung der Gasifizierung und mit den kommunalen Einnahmen steigt auch die (Aussicht auf) finanzielle Autonomie der Kommunen:

»Die Arbeit an der Eigentumsregistrierung läuft kontinuierlich. Bei uns steht in diesem Jahr die Gasifizierung an. Die Leute sind auf Probleme gestoßen. Sie wohnen schon seit Jahren in den Häusern - seit den 60ern, 70ern - und zahlen keine Steuern. Wozu registrieren, wenn man dann Steuern zahlen muss? Jetzt ist aber das Problem: Ohne den >technischen Pass<, ohne die Registrierung können die Voraussetzungen für den Anschluss an das Gasnetz nicht erfüllt werden. Einige drängen wir, andere kommen von selbst. Dennoch geht es jetzt voran.« (Bürgermeister, 11.8.2008)

Dass vielen Gemeindemitgliedern der Registrierungsprozess angesichts einer undurchschaubaren Bürokratie als unüberwindliches Hindernis erscheint, bleibt aus Gemeindesicht weitgehend unbeobachtet und wird, ebenso wie Finanzierungsschwierigkeiten, mit Unwillen oder gar Widerstand der Dorfbewohner gegen das Gasifizierungsprojekt an sich gleichgesetzt. So wischt der Bürgermeister von Dubrovo die Klagen einer Rentnerin über den unerwartet langwierigen Registrierungsprozess schlicht vom Tisch. Der Verweis auf >externe< bürokratische Unwägbarkeiten, den die Gemeindeverwaltung gerade mit Blick auf die Steuern für sich selbst exzessiv in Anspruch nimmt, wird für die Haushalte betont nicht gelten gelassen: 
»[Bürgermeister]: Wenn wir bis November 186 Häuser schaffen, dann kommt das Gas noch in diesem Jahr.

[Vorsitzende des Veteranenrats]: Aber das hängt nicht nur von uns ab. Nehmen Sie zum Beispiel mich. Ich hatte keine Dokumente für das Haus. Dann sind vom Notar Dokumente aufgetaucht. Dann hatte ich einen Termin bei Valentina Viktorovna [in der Registrierungskammer, E.M.]. Ich habe alles eingereicht. Das hat dann ungefähr drei Wochen gedauert, einen Monat fast. Jetzt muss ich zum zweiten Mal Dokumente einreichen, das geht aber erst im Oktober. Die [in der Behörde, E.M.] ziehen das da auch in die Länge wie Gummi, das hängt nicht nur von uns ab.

[Bürgermeister]: Maria Sergeevna, wenn Sie denken, dass die Registrierung Ihres Hauses nicht von Ihnen abhängt...

[Vorsitzende des Veteranenrats]: Ja!

[Bürgermeister]: Dann irren Sie sich.«

(Bürgermeister und Vorsitzende des Veteranenrats, 27.8.2008)

Ist mit der Eigentumsregistrierung die formale und rechtliche Voraussetzung für die Teilnahme an der Gasifizierung geschaffen, gilt es für die Haushalte, eine Reihe von technischen Bedingungen für den Anschluss an das Gasnetz zu erfüllen: Leitungen müssen auf dem Grundstück verlegt werden, ein Gaskessel ist zu montieren und je nach geplantem Umfang sind gegebenenfalls ein neuer Herd, der den veränderten Sicherheitsvorgaben entspricht, oder der Einbau von Heizkörpern erforderlich. Die Kosten, so die Schätzung eines Bürgermeisters, belaufen sich pro Haushalt auf mindestens 25.000 Rubel (700 Euro), liegen in der Regel jedoch um 70.000 Rubel (2.000 Euro). Angesichts von Gehaltszahlungen, die im Dorf mehrheitlich zwischen 5.000 und 6.000 Rubel monatlich liegen (zwischen 140 und 170 Euro), ist diese Summe beachtlich und übersteigt deutlich die Dimension von Ausgaben, mit denen im Alltag kalkuliert wird.

Ermöglicht werden soll die Gasifizierung vor diesem Hintergrund durch ein spezielles Kreditprogramm (respektive die Erweiterung eines bereits bestehenden Programms): Bereits einige Jahre vor Beginn des Gasifizierungsprojekts wurde im ländlichen Raum ein Kredit angeboten, der im Kontext der Privatisierung und politisch angestrebten Umstrukturierung der Landwirtschaft ursprünglich ausschließlich auf die Förderung privater Hoflandwirtschaften zielte. Er konnte für den Kauf von Maschinen oder Vieh oder für Umbau- und Renovierungsmaßnahmen eingesetzt werden und sollte auf diese Weise die Entwicklung kleinbäuerlicher Produktionsformen fördern. Der Kredit ist in hohem Maße durch das Landwirtschaftsministerium subventioniert, so dass sich der Schuldner von den rund 15 Prozent Zinsen, die auf die Kreditsumme zu zahlen sind, 12 Prozent über das Landwirtschaftsministerium zurückerstatten lassen kann - also nur die verbleibenden 3 Prozent selber tragen muss. Mit dem Aufkommen der Gasifizierung wurde der Verwendungs- 
zweck ausgeweitet, sodass der Kredit nun auch zur Finanzierung der Gas-Infrastruktur in den Haushalten verwendet werden darf.

Mit dem Kredit wird ein Kommunikationsangebot geschaffen, das in den Dörfern buchstäblich auch als solches in Erscheinung tritt: Die Lokalzeitung schließt Information über Kredite in regelmäßigen Abständen und an prominenter Stelle in ihre Berichterstattung ein. So werden beispielsweise unter der Überschrift »Ein Kredit ist eine vorteilhafte Sache« (»Ochanskaja Storona« vom 25.4.2008: 4) die Konditionen der Kreditvergabe in aller Ausführlichkeit erläutert und wenige Monate später kann berichtet werden, dass »drei weitere Personen im vergangenen Monat einen Kredit für die Entwicklung ihrer privaten Hoflandwirtschaft erhalten haben« (»Ochanskaja Storona« vom 25.7.2008: 5). Daneben treten Repräsentantinnen und Repräsentanten der örtlichen Bankfiliale auf den Gemeindeversammlungen auf, um unter explizitem Verweis auf die Gasifizierung für den Kredit zu werben und seine Erreichbarkeit, d.h. die potenzielle Adressierbarkeit jedes Gemeindemitglieds als Kreditnehmer, zu betonen:

»Das ist eine völlig reale Möglichkeit, wenn man bereit ist [über seine finanziellen Verhältnisse, E.M.] Rechenschaft abzulegen. Das heißt, das ist ein erreichbarer, machbarer Kredit. $\mathrm{Zu}$ uns sind sie sogar in der Stadt in Massen gekommen - >Wir wollen diesen Kredit $<$. Nicht alle haben ihn bekommen. Was die Dorfbevölkerung betrifft, sofern Finanzierungsbedarf besteht - wir laden Sie alle dazu ein.« (Bankangestellte auf einer Gemeindeversammlung, 26.3.2009)

Die gesellschaftliche Bedeutung des Kredits erschließt sich mit Bohn (2009) über die Beobachtung multipler Inklusion in die Wirtschaft und ihre Subsysteme. Davon ausgehend, dass sich die Inklusion in das Wirtschaftssystem generell über den Gebrauch des Mediums Geld und der dadurch ermöglichten Adressierbarkeit im Rahmen wirtschaftlicher Kommunikation vollzieht, unterscheidet Bohn zwischen wirtschaftlicher Peripherie, Semiperipherie und Zentrum. Mit Blick auf diese drei Bereiche lässt sich eine graduierbare Inklusion (respektive Exklusion) beobachten: Alle drei rekurrieren zwar auf das Geldmedium und die Herstellung von Zahlungsfähigkeit, variieren jedoch hinsichtlich der darauf bezogenen Operationen und Praktiken (Bohn 2009: 247ff). Die Inklusion in die Peripherie (oder die Sphäre des Konsums) vollzieht sich über die Versorgung mit Gütern und Dienstleistungen und deren Bezahlung mit Geld, das dabei auf systemexterne Zwecke verweist. Die Inklusion in die Semiperipherie (oder die Sphäre von Produktion und Handel) erfolgt, wenn Zahlungsfähigkeit über Einkommen und Besitz hergestellt wird. Das Kriterium für die Inklusion ins Zentrum ist schließlich die Beteiligung am Prozess der Geldschöpfung, also die Möglichkeit, aus Zahlungsunfähigkeit Zahlungsfähigkeit zu generieren - in Form von Krediten (Bohn 2009: 249ff). 
Auf welche Weise wird im Dorf an den Kredit als Kommunikations- und Inklusionsangebot angeschlossen? Verlässliche Daten zur Kreditnachfrage sind schwer zu ermitteln, da es keine - zumindest keine mir zugänglich gemachten - Statistiken über die entsprechenden Entwicklungen in der jüngeren Vergangenheit gibt. Einige Anhaltspunkte bieten jedoch verschiedene Einzelaussagen aus meinen Interviews: Einer Mitarbeiterin in der Kreditabteilung der zuständigen Bankfiliale im Bezirkszentrum zufolge existiert das vergünstigte Kreditangebot seit den späten 1990er Jahren. Während sich das Interesse anfangs nur zögerlich entwickelte und lediglich einige größere Hoflandwirtschaften Kredite aufnahmen, sei die Nachfrage in den Dörfern, in denen der Anschluss an das Gasnetz anstehe, deutlich angestiegen. Konkrete Zahlen nennt sie jedoch nicht. Eine Bürgermeisterin meint zu wissen, dass im gesamten Bezirk, der rund 17.000 Einwohner umfasst, insgesamt etwa 120 Personen den vergünstigten Kredit in Anspruch genommen haben. Bei dieser Zahl ist allerdings unklar, inwiefern die Kreditaufnahme zum Zweck der Gasifizierung erfolgte. In Andreevka wurden der Bürgermeisterin zufolge insgesamt vier dieser Kredite in Anspruch genommen, davon zwei für Investitionen in private Hoflandwirtschaften und zwei explizit mit Blick auf die Gasifizierung. Zu berücksichtigen ist dabei, dass das Gasifizierungsprojekt in Andreevka zum Zeitpunkt dieser Angabe gerade erst begonnen hatte. In der Gemeinde Dubrovo, in der das Projekt während meiner Forschungsaufenthalte kurz vor dem Abschluss stand, nahmen nach Auskunft des Bürgermeisters zwischen zehn und fünfzehn Personen einen Kredit zur Finanzierung der technischen Infrastruktur auf. Vor dem Hintergrund, dass zuvor zwölf Personen den Kredit für landwirtschaftliche Zwecke in Anspruch genommen hatten, lässt sich in Zusammenhang mit der Gasifizierung eine Verdopplung der Kreditnehmer im Dorf beobachten - was der Bürgermeister als aktive Nachfrage bewertet: »Sobald man den Kredit für das Gas verwenden konnte, kamen die Leute und zahlten« (12.8.2008). Auch wenn die absoluten Zahlen gering erscheinen, scheint angesichts dieser Äußerungen nicht in Frage zu stehen, dass der Anschluss an das Gasnetz und die damit verbundenen Kosten eine nach lokalen Maßstäben signifikante Steigerung der Kreditnachfrage bewirkt haben.

Deutlich gewandelt hat sich darüber hinaus auch die Art und Weise, wie die Kreditnachfrage der Gemeindemitglieder durch die Gemeindeverwaltung beobachtet wird. Zum einen werden die Kredite nach dem Beginn des Gasifizierungsprojekts nicht mehr vornehmlich als wirtschaftliches Phänomen beobachtet, sondern rücken sukzessive in den Horizont der Politik und ihrer Steuerungsansprüche. Damit einher geht zum anderen ein Wechsel des Zuschreibungsmodus von Erleben auf Handeln, die Sinnselektion wird also nicht mehr der Umwelt, sondern den beobachteten Systemen selbst - den Gemeindemitgliedern respektive den Haushalten zugerechnet (für eine empirische Anwendung dieser Unterscheidung vgl. auch Goeke 2007): 
»Wird die Sinnselektion der Umwelt zugerechnet, gilt die Charakterisierung Erleben, und die Anknüpfung für weitere Maßnahmen wird in der Umwelt des Systems gesucht (obwohl das System als erlebend beteiligt war!). Wird dagegen die Sinnselektion dem System selbst zugerechnet, dann gilt die Charakterisierung Handeln (obwohl solches Handeln ohne Bezug auf die Umwelt gar nicht möglich ist).«(Luhmann 1984: 124)

Auf welche Weise kommen diese Verschiebungen zum Ausdruck? Mit Blick auf die Kreditnachfrage im Dorf zeigte sich die Bürgermeisterin von Andreevka kurz vor Beginn des Gasifizierungsprojekts ausgesprochen optimistisch und in Erwartung einer deutlichen Nachfragesteigerung: »Der Kredit wird [in Zusammenhang mit der Gasifizierung, E.M.] massenweise nachgefragt werden« (10.8.2008). Die Tatsache, dass bislang drei Kredite an Gemeindemitglieder für landwirtschaftliche Zwecke vergeben wurden, erachtete sie dabei angesichts der strikten Vergabebedingungen der Bank als durchaus beachtlich:

»[Frage]: Haben viele hier im Dorf einen Kredit für ihre Hoflandwirtschaft bekommen?

[Bürgermeisterin]: Drei Personen.

[Frage]: Drei - das ist wenig.

[Bürgermeisterin]: Warum soll das wenig sein? Man muss schließlich in der Lage sein, alles rechtzeitig zurückzuzahlen. Man muss sehr sorgfältig und verantwortungsvoll wirtschaften.« (Bürgermeisterin, 10.8.2008)

Die Kreditaufnahme ist aus Sicht der Bürgermeisterin positiv konnotiert und wird als erwünscht beobachtet. Mit Blick auf den Prozess der Kreditvergabe wird die Sinnselektion der (wirtschaftlichen) Umwelt zugerechnet: Es ist die Bank, die nach eigenen Kriterien über die Bewilligung entscheidet, während die einzelnen Gemeindemitglieder selbst an dieser Entscheidung lediglich erlebend beteiligt sind und die Konsequenzen zu tragen haben. Wird der Kredit verweigert, werden gute Gründe im Wirtschaftssystem unterstellt und die Schuld nicht den Dorfbewohnerinnen und Dorfbewohnern zugeschrieben.

Mit dem Beginn des Gasifizierungsprojekts wandelt sich diese Einschätzung in zweifacher Weise: In dem Maße, wie zahlreiche Haushalte im Dorf de facto auf Kredite (oder andere Finanzierungsmöglichkeiten) angewiesen sind, um an der Gasifizierung teilnehmen zu können, wird die Kreditnachfrage und -vergabe zum Bestandteil eines politischen Projekts, auf dessen Umsetzung die Bürgermeisterin mit allen Mitteln hinwirkt. Beobachtet wird nun im Horizont der Politik und die zögerliche Kreditnachfrage durch die Gemeindemitglieder wird für die Bürgermeisterin in erster Linie zum Hindernis bei der Umsetzung ihrer Pläne. Die Ursache sucht sie nun nicht mehr in den Entscheidungen der Bank, sondern in einer generellen >moralischen Haltung <, die in der Gemeinde verbreitet sei: »Es gilt als schlecht, 
wenn du einen Kredit aufnimmst« (5.3.2010). Die Bürgermeisterin verhehlt dabei nicht, dass sie selbst sich von dieser Haltung distanziert und sie als hochgradig irrational bewertet. Wird ein Kredit nicht bewilligt oder gar nicht erst beantragt, beobachtet sie dies nicht im Horizont wirtschaftlicher Anschlussfähigkeit, sondern unterstellt Trägheit oder gar den Widerstand der Bevölkerung gegen das Gasfizierungsprojekt an sich. Den Gemeindemitgliedern schreibt sie die entsprechende Sinnselektion - in Form einer Entscheidung gegen den Kredit und damit gegen die Gasifizierung - selbst zu: Sie erleben nicht, sondern handeln:

»Sie hören sich das auf der Versammlung an. Anschließend kommen sie zu mir und sagen: $>$ Wenn Geld da ist, werden wir das [die Gasifizierung, E.M.] machen, aber wenn nicht - weshalb sollen wir uns dann Gedanken machen? Das ist eine Besonderheit von Andreevka.« (Bürgermeisterin, 5.3.2010)

Mit der Verschiebung von Wirtschaft auf Politik und von Erleben auf Handeln legt sich die Bürgermeisterin (respektive die Gemeindeverwaltung) auf bestimmte Formen des Anschließens fest und schließt andere aus: Konnte sie zunächst den operativen Vollzug wirtschaftlicher Logiken ebenfalls erleben, sieht sie sich angesichts der vermeintlichen Widerständigkeit der Gemeindemitglieder, die in der Ablehnung der Kreditaufnahme zum Ausdruck kommt, zum eigenen Handeln motiviert. Den vermeintlichen Widerstand gilt es zu kontrollieren, wobei Kontrolle meint, »dass man einen Sachverhalt in seiner Eigenständigkeit akzeptiert, aber Operationen anschließt, die den Sachverhalt den Imperativen eines anderen Systems zu unterwerfen versuchen« (Stichweh 2003: 95). Akzeptiert werden in diesem Sinne zwar die Finanzierungsschwierigkeiten der Gemeindemitglieder im Kontext der Gasifizierung als wirtschaftliches Problem. Daran angeschlossen wird jedoch - wohl auch mangels Alternativen - mit politischer Überzeugungsarbeit. Dies kommt in den $>$ Beschwörungsformeln<, die im Kontext der Gemeindeversammlungen geäußert werden, ebenso zum Ausdruck wie in der Weigerung der Bürgermeisterin, individuelle Finanzierungsschwierigkeiten als Argument gegen die Teilnahme an der Gasifizierung gelten zu lassen:

»[Bürgermeisterin]: Heute waren zum Beispiel Vertreter von UralGazService ${ }^{31}$ bei mir. Sie haben darüber gesprochen, dass sie im ersten Schritt die Straßen >Centralnaja< [Zentralstraße], $>$ Školnaja< [Schulstraße] und >Pobeda< [Straße des Sieges] anschließen wollen. In der $>$ Ulica Centralnaja< mag das mit Blick auf die Vorbereitungen durch die Bewohner noch realistisch sein, aber in der >Ulica Pobeda< wohnen praktisch nur Kolchosarbeiter, für die die ganze Gasifizierung sowieso sehr schwierig ist. Sie ist dort zwar technisch möglich und ei-

31 Das Unternehmen verlegt die Hauptleitungen im Dorf. 
nige haben mit ihren Projekten auch begonnen, aber es gibt keinen einzigen Anschluss, das heißt, es ist unmöglich, sie an die Hauptleitung anzuschließen.

[Frage]: Warum ist das unmöglich?

[Bürgermeisterin]: Weil es keine Anschlussmöglichkeit gibt, sie haben das noch nicht erledigt. Wir werden die Bewohner deshalb noch mal zusammenrufen und mit ihnen reden.

[Frage]: Das heißt, sie haben es nicht rechtzeitig geschafft?

[Bürgermeisterin]: Sie können es sich nicht leisten.«

(Bürgermeisterin, 5.3.2010)

Jedoch: Dieser Beobachtungsmodus reibt sich an der Beobachtung durch die Gemeindemitglieder - also der (potenziellen) Kreditnehmerinnen und Kreditnehmer selbst, und dies sowohl mit Blick auf den Horizont als auch auf die Zuschreibung von Sinnselektion. Was aus der Politik heraus als Widerstand beobachtet wird, ist die Logik wirtschaftlicher Inklusion, an der die Gemeindemitglieder erlebend beteiligt sind und an der der Einfluss der Politik an seine Grenzen stößt. Der Grund des vermeintlichen Konflikts zwischen Bürgermeisterin und Gemeindemitgliedern, der unter anderem auf den Gemeindeversammlungen zum Ausdruck kommt, sind divergierende Attributionsmodi. Zwar wirkt die Gasifizierung für viele Haushalte durchaus als ein Impuls dahingehend, dass Kredite als Option verstärkt in den Fokus der Wahrnehmung rücken, bleibt dabei jedoch ebenso wie andere Finanzierungsobjekte Fremdreferenz - und nicht Fluchtpunkt - der Kommunikation. Interessanterweise findet diese Wahrnehmungsverschiebung auch dann statt, wenn aktuell kein Kredit aufgenommen wird, sondern andere Finanzierungsmöglichkeiten gefunden werden (der Zugriff auf Erspartes, die Unterstützung durch Verwandte, der Verkauf von Vieh oder ähnliches). Man beobachtet sich selbst und andere auf »Kreditwürdigkeit als unterstellte Zahlungsfähigkeit« hin (Bohn 2009: 247), versucht also die eigenen Möglichkeiten auszuloten, aus Zahlungsunfähigkeit Zahlungsfähigkeit zu erzeugen (Bohn 2009: 253) - und dies im positiven wie im negativen Fall. So berichtet Sergej, der als Dorfpolizist arbeitet und nebenbei eine verhältnismäßig große Hoflandwirtschaft führt, nicht ohne Stolz, dass ihm ein Kredit über 300.000 Rubel (rund 8.500 Euro) zur Finanzierung eines Traktors bewilligt wurde. Den Grund für die Bewilligung sieht er dabei in seiner Fähigkeit, seine eigene Zahlungsfähigkeit glaubhaft darzustellen, was, wie er einräumt, durchaus nicht allen gelinge:

»[Frage]: Aber die Bewilligung des Kredits hängt doch trotzdem davon ab, wo man arbeitet? Das ist ja eine solide Organisation, bei der Sie arbeiten.

[Sergej]: Einverstanden.

[Frage]: Und wie machen das die Leute, die nur eine Hoflandwirtschaft und keine feste Stelle haben? 
[Sergej]: Das beantworte ich Ihnen folgendermaßen. Da kommt ein Bankinspektor und schaut sich das an. Nehmen wir an, ich möchte 300.000 aufnehmen. Was hast du als Sicherheit zu bieten? Ich habe fünf Kühe, zehn Schweine. Falls, nehmen wir an, bei mir irgendetwas schiefgeht, schlachte ich sie und überlasse sie der Bank. Er schaut genau hin, ich gefalle ihm, mein Haus gefällt ihm, meine Schweine gefallen ihm und er fährt wieder weg. Und er sagt Ja. Ich weiß, dass es bei anderen auch andere Fälle gab. Ihnen [der Bank, E.M.] hat die Hoflandwirtschaft nicht gefallen, sie haben gesehen, dass es dort keine Sicherheiten gibt, sie haben den Kredit nicht bewilligt. [...] Man muss den Bankinspektor von seiner Zahlungsfähigkeit überzeugen. Ich hatte eine Bescheinigung von meinem Arbeitgeber, dass ich zahlen kann. Und das war's, ohne Probleme.«

(Dorfpolizist, 10.8.2008)

Weniger optimistisch, jedoch im Kern aus einer identischen Perspektive beurteilen Aleksej und Svetlana die Lage. Die beiden unterhalten ebenfalls eine große Hoflandwirtschaft, die unter anderem zehn Kühe und 23 Hektar Agrarland umfasst und von der sie - dies ist im Dorf eine große Ausnahme - ihren Lebensunterhalt bestreiten können. Svetlana war bis zum Jahr 2008 zusätzlich beim ehemaligen Kolchos beschäftigt. Ein Kredit wurde ihnen zu dieser Zeit trotz umfangreicher Bemühungen dennoch nicht bewilligt. Zum einen weigerte sich die Bank aufgrund der rückständigen Lohnzahlungen und der insgesamt als instabil beobachteten Lage des Betriebs, Svetlanas Anstellung als Sicherheit zu akzeptieren:

»Wir wollten einen vergünstigten Kredit aufnehmen, für 14 Prozent. Und das lief dann so ab: Das erste Mal kamen wir zur Bank um uns beraten zu lassen. Wir kamen rein - > wo arbeiten Sie?<->[beim Kolchos]<. Sie haben sofort abgewinkt: >Der Betrieb ist nicht stabil, mit Ihnen kommen wir nicht ins Geschäft.« (Privatbauer, 10.8.2008)

Zum anderen stand der Kreditaufnahme die Forderung der Bank nach zwei Bürgen im Wege - das Resultat beschreibt Aleksej als > verschlossenen Kreis< ohne Zutrittsmöglichkeit für ihn:

»Außerdem haben sie uns noch gesagt, dass wir zwei Bürgen brauchen. Die Kreditsumme war natürlich beachtlich, ungefähr 300.000. Ich habe dann mit einem anderen Unternehmer gesprochen und der sagte: >Im Prinzip wäre ich bereit, aber so eine Summe kann nicht jeder aufbringen $<$. Das ist ein verschlossener Kreis. Hier in Andreevka kenne ich eine Person, er wollte für uns bürgen. Aber er ist schon 80 Jahre alt. Ich habe ihn als Bürgen mit [zur Bank, E.M.] genommen - und sie haben zu ihm gesagt: >Großväterchen, was willst du? Verschwinde <. Dann hat sich noch jemand Jüngeres bereit erklärt für uns zu bürgen. Sie haben sich seine Dokumente angeschaut und gesagt: >Mein Lieber, Ihr Gehalt erlaubt das nicht $\prec$. 
Und das war's, wieder ein verschlossener Kreis. [...] Jetzt müssen wir wohl mit unseren eigenen Mitteln auskommen.« (Privatbauer, 22.3.2009)

In diesen Erzählungen spiegeln sich die Versuche der Bank, Kreditrisiken unter den speziellen Bedingungen des ländlichen Raums handhabbar zu machen. Aufgrund der geringen Preise und oft ungeklärten Eigentumsverhältnisse akzeptiert die Bank nach eigenem Bekunden in der Regel weder Agrarland noch Immobilien als Sicherheit. Auch ein regelmäßiges Einkommen gilt, wie das Beispiel von Svetlana und Aleksej zeigt, oftmals nicht als ausreichender Nachweis zukünftiger Zahlungsfähigkeit. Stattdessen verlässt sich die Bank nach Auskunft einer Mitarbeiterin der Kreditabteilung neben dem persönlichen Eindruck des potenziellen Kreditnehmers und seiner Hoflandwirtschaft im Wesentlichen auf die Zusammenarbeit mit den Gemeindeverwaltungen: Verlangt wird ein Auszug aus dem Haushaltsregister, das von der Gemeindeverwaltung geführt wird und in dem für jeden Haushalt die Anzahl der zugehörigen Personen, die Größe von Wohnhaus und Garten sowie das Eigentum an Vieh, technischen Geräten und ähnlichem vermerkt sind. Zudem stellen die Gemeinden auf Verlangen der Bank zur Risikoeinschätzung eine offizielle Bestätigung über die Kreditwürdigkeit einer Person aus (»chodatajstvo«), agieren also als eine Art dörfliche Ratingagentur. Umgekehrt führt für die Gemeindemitglieder der Weg zum Kredit zwingend über die Gemeindeverwaltung, was - wie eine Bürgermeisterin selbst einräumt - für viele ein Hindernis darstellt: »Man muss hierher $\mathrm{zu}$ uns kommen und um Erlaubnis fragen - das ist auch nicht so angenehm « (5.3.2010).

Die Tatsache, dass die Gemeindeverwaltung mit Blick auf Kredite an dieser Stelle erneut ins Spiel kommt, mag zunächst verwundern, bestärkt jedoch bei genauerem Hinsehen das hier vorgebrachte Argument zur Reichweite lokalpolitischer Machtkommunikation: Zwar ist die Gemeinde als Institution augenscheinlich involviert in die Entscheidung, wem »Selektionskompetenz, Verantwortlichkeit und Schuldfähigkeit« zugeschrieben werden (Bohn 2009: 256) - und wem nicht. Sie hat sich dabei jedoch wirtschaftlichen Logiken zu unterwerfen und für Machtkommunikation bleibt kein Platz. Was sie tun kann, ist, die Kreditwürdigkeit eines Gemeindemitglieds in möglichst gutem Licht darzustellen. Die Entscheidung, ob und wie an die von ihr bereitgestellten Informationen angeschlossen wird, richtet sich jedoch letztlich nach den Kriterien der Bank zur Beurteilung von Kreditwürdigkeit und liegt damit jenseits ihrer Durchgriffsmöglichkeiten. 


\section{»Und das alles nur, weil die Finanzkrise nun auch bei uns angekommen ist «}

Welche Schlussfolgerungen lassen sich aus den beschriebenen Ereignissen im Kontext der Gasifizierung mit Blick auf die Eigenarten der dörflichen Transformationsgesellschaft ziehen? Zunächst ist festzuhalten, dass die hier dargestellten Prozesse auf die Verschneidung von Technosphäre und Soziosphäre verweisen (vgl. zu dieser Unterscheidung Milsum 1968): Deutlich wird, dass die Technosphäre zwar untrennbar an Semantiken und Programme der Soziosphäre und damit an gesellschaftliche Evolution gekoppelt ist und nicht unabhängig von ihr evoluieren kann, sie aber dennoch in der Lage ist, Anpassungsdruck auf die und in der Soziosphäre zu erzeugen. Der Anschluss an das Gasnetz ist vor diesem Hintergrund in erster Linie ein technischer Akt, der in den Dörfern für Irritationen sorgt und strukturelle Veränderungen in der Soziosphäre anstößt. Als konkretes und von außen an das Dorf herangetragenes Kommunikationsangebot lässt die Gasifizierung die latent angelegten Reibungspunkte zwischen tradierten Dorfstrukturen einerseits und funktionaler Differenzierung als Eigenstruktur der Weltgesellschaft andererseits deutlich hervortreten. Insbesondere diejenigen Strukturen, deren Aufrechterhalten verhindert, dass an dieses Kommunikationsangebot angeschlossen wird, werden aufgebrochen oder zumindest in Frage gestellt. Es entsteht eine Art Transmissionsriemen - in diesem Fall für wirtschaftliche Kommunikation - und Anpassungsbedarf wird freigelegt, mit dem man im Dorf irgendwie umgehen muss.

Dies betrifft die Gemeindemitglieder, für die der technische Anschluss an das Gasnetz mit einer Reihe von gesellschaftlichen Anschlussmöglichkeiten respektive »Und-so-weiter«-Angeboten begleitet wird: die Klärung von Eigentumsverhältnissen, anstehende Zahlungen und die Option der Kreditaufnahme. Diese Angebote werden nun zumindest insoweit unhintergehbar, als dass die Auseinandersetzung damit unumgänglich wird - eine Entscheidung ist auch dann gefragt (und macht einen Unterschied), wenn man die Teilnahme an der Gasifizierung am Ende ablehnt. Fluchtpunkt der Entscheidung sind letztlich die zu erwartenden Kosten, so dass die Weltwirtschaft über die entsprechenden Preise buchstäblich in die dörfliche Kommunikation hineinwirkt.

Für die Gemeindeverwaltung, der sich das Gasifizierungsprojekt in erster Linie als Steuerungsproblem darstellt, kristallisiert sich mit diesem Hineinwirken eine funktionale Grenze heraus, an der sich ihre Einflussmöglichkeiten brechen. Unabhängig von den Versuchen der Gemeindeverwaltung, politischen Einfluss im Dorf geltend zu machen, wird diese Grenze im Verwaltungssystem durchaus registriert und reflektiert und die Erwartungen werden angepasst: 
»Unsere Arbeit verzögert sich nun wegen dieser Finanzkrise. Vorher, vor Neujahr, als die Gemeindeversammlung stattgefunden hat und als wir das erste Mal über die Gasifizierung geredet haben, da wurden den Leuten völlig andere Preise genannt. Uns [der Gemeindeverwaltung, E.M.] wurde das gesagt und wir haben das den Leuten erklärt, so und so, [...] dass der Preis für die Gasifizierung so um die 25.000 bis 30.000 [Rubel, E.M.] liegen wird. Und vor diesem Hintergrund kamen die Leute an, 25 bis 30 kriegen wir irgendwie zusammen, wenn das in dem Rahmen bleibt. Und jetzt sagen sie wieder ab, je länger das geht - mit 30 kommt man jetzt schon nicht mehr weit, mindestens 50 wird es jetzt auf jeden Fall kosten [...]. Und deshalb fangen die Leute jetzt an, die Gasifizierung abzulehnen. Das ist natürlich sehr schlecht, weil wir laut Gesetz - in der Region Perm gibt es ein Gesetz zur Gasifizierung unseres Territoriums -, in dem Gesetz steht geschrieben, dass wir, sagen wir, 240 Haushalte zusammenkriegen müssen. Und jetzt die Leute - die Preise steigen, aber was sie zum Leben brauchen wird nicht weniger. Und das alles nur, weil die Finanzkrise nun auch bei uns angekommen ist. [...] Wenn ein [Bezeichnung eines Gaskesseltyps, E.M.] vor Neujahr 25.000 gekostet hat, kostet er jetzt 31.000. Wenn man uns gesagt hat, dass die Arbeiten im Haus, die Montage innen, 7.000 bis 8.000 gekostet hat, reden sie jetzt schon von 17.000 bis 18.000 . Und die Leute schreckt das ab, sie fürchten sich den Antrag zu schreiben, dass sie an der Gasifizierung teilnehmen.« (Sachbearbeiterin der Gemeindeverwaltung, 12.3.2009)

Lässt sich das in der ersten Episode beschriebene Verhältnis von Gemeindeverwaltung und ehemaligem Kolchos dahingehend interpretieren, dass sich weltgesellschaftliche Strukturen an den Grenzen des Dorfes brechen, innerhalb derer eigene >Spielregeln< Anschlussfähigkeit generieren und zu dorfexternen Strukturen in Kontrast gesetzt werden, so verdeutlicht das Beispiel der Gasifizierung, auf welche Weise sich Durchlässigkeiten ergeben - ohne dass diese Bruchstellen explizit intendiert waren. Die Preise, die als zentraler Parameter der Gasifizierung im Dorf wirksam werden, bilden sich außerhalb des Dorfes und entziehen sich dem Einfluss der Gemeindeverwaltung. Als externe Zwänge, denen auch die Gemeindeverwaltung selbst unterworfen ist, lassen sie sich argumentativ ins Feld führen, um auf dieser Grundlage die eigene Machtlosigkeit mit Blick auf die eigenen Interessen einzusetzen. In diesem Sinne verweist beispielsweise ein Bürgermeister gegenüber der Vorsitzenden des Veteranenrats auf die kontinuierlich steigende Inflation, um die Gemeindemitglieder von der Dringlichkeit der anstehenden Entscheidungen und der Vorteilhaftigkeit möglichst zeitnaher Zahlungen zu überzeugen:

»[Bürgermeister]: Ich rede mit den Leuten und Sie müssen auch mit den Leuten reden. Warum? Weil, erstens, selbst wenn wir im nächsten Jahr Gas haben werden, dann werden die Preise im nächsten Jahr völlig andere sein als heute, das verspreche ich Ihnen. Denn die Inflation in Russland, denke ich, wird so um die 20 Prozent liegen. 15 Prozent mindestens. 
[Vorsitzende des Veteranenrats]: Wie soll ich das den Leuten sagen? Eine solche Inflation innerhalb von drei Wochen?

[Bürgermeister]: In den ersten sechs Monaten dieses Jahres - 9,6 Prozent in Russland.

[Vorsitzende Veteranenrat]: Auf unsere Kosten verdienen sie Geld...

[Bürgermeister]: Mir tut es leid für die Leute, die schon jetzt die Montage erledigt haben.

[Vorsitzende des Veteranenrats]: Warum?

[Bürgermeister]: Sie leiden darunter, wenn das Gas in diesem Jahr nicht mehr kommt. Schuld bin dann ich und sind die Leute, die in diesem Jahr nicht bei der Gasifizierung mitgemacht haben. Aber gleichzeitig gewinnen sie auch, weil sie alle Arbeiten zu den heutigen Preisen abgeschlossen haben. Und im nächsten Jahr kommt das Gas auf jeden Fall, es kommt garantiert.

[Vorsitzende des Veteranenrats]: Sicher?

[Bürgermeister]: Wenn nicht, dann trete ich zurück.

[Vorsitzende des Veteranenrats]: Damit wäre auch keinem geholfen.«

(Bürgermeister und Vorsitzende des Veteranenrats, 27.8.2008)

Neben dieser und ähnlichen Überzeugungsstrategien basiert die faktische Gestaltungsmacht der lokalen Politik im Kontext des Gasifizierungsprojekts jedoch in erster Linie auf der Koordination der Kommunikation. Vor allem die Bürgermeisterinnen und Bürgermeister werden zu kaum umgehbaren Fixpunkten, an denen sich eine Engführung oder Bündelung der dörflichen Kommunikation beobachten lässt. Dies gilt ungeachtet ihrer begrenzten Durchgriffsmöglichkeiten. Dabei ist die Gasifizierung ein - und möglicherweise seit Beginn des Regimewechsels das erste Großereignis im Dorf, an dem der ehemalige Kolchos respektive der Kolchosvorsitzende nicht unmittelbar beteiligt ist und das alleine dadurch die Verschiebung der dörflichen Hierarchien unterstreicht. Nicht nur das Verhältnis zwischen Gemeindeverwaltung und Kolchosleitung tritt mit Blick auf dieses Ereignis in den Hintergrund. Auch für die Handlungsmöglichkeiten der Gemeindemitglieder ist nun nicht mehr die Mitgliedschaft im ehemaligen Kollektivbetrieb ausschlaggebend, sondern letztlich die eigene Zahlungsfähigkeit respektive Kreditwürdigkeit, deren Einschätzung Dritten unterliegt und auf die sich die Bindung an den Betrieb sogar tendenziell negativ auswirkt. Das Anschließen an die durch die Gasifizierung induzierten Kommunikationsangebote bewirkt die Öffnung des dörflichen Kommunikationszusammenhangs nach außen und die sukzessive Überlagerung der Sozial- durch die Sachdimension.

Ironischerweise - und so lässt sich der Bogen zum Beginn dieser Episode schlagen - lassen sich ganz ähnliche Effekte, wie Lenin sie sich von der »Elektrifizierung des ganzen Landes « für die kommunistische Aufklärung der Bauernschaft erhoffte, nun am Beispiel der Gasifizierung beobachten - nur dass sich »Aufklärung «, Wissen und Anschlussfähigkeiten, die im Dorf potenziell generiert werden, 
nicht auf den kommunistischen Wirtschaftsaufbau richten, sondern auf globale Marktstrukturen und die Wirtschaft als weltgesellschaftliches Funktionssystem:

»Wir müssen es dahin bringen, dass jede Fabrik, jedes Kraftwerk zu einer Stätte der Aufklärung wird, und wenn Russland sich mit einem dichten Netz von elektrischen Kraftwerken und mächtigen technischen Anlagen bedeckt haben wird, dann wird unser kommunistischer Wirtschaftsaufbau zum Vorbild für das kommende sozialistische Europa und Asien werden. (Stürmischer, nicht enden wollender Beifall.)«(Lenin 1970 [1920]: 515)

\section{Das Auftauchen der Agroholding - oder: Neue SELBSTVERSTÄNDNISSE UND FORTGEFÜHRTE STRUKTUREN}

»Wir haben Glück gehabt - vielleicht etwas mehr als die anderen. Mir scheint, dass Beljaevka [...] wieder jung ist. Mir scheint, dass in der Biographie unseres Dorfes eine neue Seite aufgeschlagen wurde. ${ }^{32}$ Mit diesen Worten begrüßte im Sommer 2008 die Bürgermeisterin Gemeindemitglieder und Gäste zu den Feierlichkeiten anlässlich des 385jährigen Dorfjubiläums. Selbst wenn man von der üblichen Pathosgeladenheit derartiger Veranstaltungen abstrahiert, überrascht der Optimismus angesichts der sonst üblichen Klagen über Unterfinanzierung, Überalterung und den generellen Niedergang der ländlichen Gemeinden. Um die Gründe dieses Optimismus zu verstehen, lohnt sich ein Blick auf die wechselvolle jüngere Vergangenheit der Gemeinde.

Um das Jahr 2005 - und damit etwa zeitgleich mit dem Amtsantritt ebenjener Bürgermeisterin - tauchte ein bis dahin unbekannter »dačnik « ${ }^{33}$ im Dorf auf, der die Sommerwochenenden mit Fischen und Jagen in Beljaevka verbrachte. Einen Gefährten für seine Freizeitaktivitäten fand er bald in der Person des ehemaligen Bürgermeisters, der ihm von den wirtschaftlichen Problemen des Dorfes berichtete: Der ehemalige Kolchos in Beljaevka, der ebenso wie der landwirtschaftliche Großbetrieb im benachbarten Andreevka über den Regimewechsel hinaus Bestand gehabt hatte, stand seit längerem kurz vor der Zahlungsunfähigkeit und musste seine Geschäfte im Jahr 2005 schließlich ganz einstellen. Nach einiger Überzeugungsarbeit seines Jagdgefährten begann sich der Wochenendgast für den zerfallenden Betrieb zu interessieren:

32 Aus dem Bericht des lokalen Fernsehsenders zum Dorfjubiläum der Gemeinde Beljaevka im August 2008.

33 Wörtlich: Wochenendgast, Sommerfrischler; abgeleitet vom russischen Wort »dača«, das ein kleines Wochenendhaus (eine Datscha) auf dem Land bezeichnet. 
»Ich kann mich nicht genau erinnern, in welchem Jahr er hier auftauchte. Vermutlich 2006. [...] Er kam zuerst nur für die Jagd hierher. Und dann begann er sich zu interessieren. Mein Mann war mit ihm unterwegs, mein Mann ist auch Jäger, und er hat sich mit ihm unterhalten während der Jagd. Auf diese Weise werden die wichtigsten Entscheidungen getroffen - ist das nicht so?« (ehemalige Gemeinderätin, 24.3.2009)

Schon nach kurzer Zeit verbreiteten sich Spekulationen im Dorf. Nicht allein die Freizeitaktivitäten zögen den Wochenendgast nach Beljaevka, vielmehr verberge sich eine zahlungskräftige Investorengruppe hinter dem Mann. Etwa ein Jahr nach seinem Erscheinen setzt er den Gerüchten um seine Person ein Ende, indem er offiziell als Geschäftsmann auf einer Gemeindeversammlung auftrat und von seinen Plänen für das Dorf berichtete. Er beabsichtige, so erläuterte er den anwesenden Gemeindemitgliedern, den insolventen Betrieb zu übernehmen und an seine Stelle ein Unternehmen zu setzen, das sich auf Kartoffelanbau spezialisieren würde. Dazu wolle er die Insolvenzmasse des Betriebs ersteigern und das Agrarland erwerben, das formal den Anteilseignerinnen und Anteilseignern des zerfallenen Kolchos gehörte. Die anwesenden Gemeindemitglieder und auch die Bürgermeisterin begegneten ihm und seinem Vorhaben zunächst mit Misstrauen, das sich, wie die Bürgermeisterin rückblickend beschreibt, erst nach den ersten sichtbaren Erfolgen seines Vorhabens langsam legte:

»Dass er dieses Unternehmen aufbauen will, kam überhaupt sehr plötzlich - das ist jetzt zwei Jahre her. Im Herbst sind es zwei Jahre - wie schnell sind sie verflogen. Im Herbst fand eine Gemeindeversammlung statt und dabei haben wir ihn auch zum ersten Mal gesehen. Er ist dann schon ganz offiziell auf der Versammlung aufgetreten, hat von seinen Plänen erzählt, darüber, was hier alles sein wird. Natürlich gab es viele Zweifel. Man hat ihm nicht geglaubt. [...] Dann ist ein Jahr vergangen. Ein Jahr lang haben sie nur gearbeitet. Und dann, als die Landwirtschaftssaison vorbei war, im Oktober, und sie die ersten Ergebnisse präsentiert haben, da kam auf einmal irgendwie Hoffnung auf, dass dieser Mensch tatsächlich mit ernsthaften Absichten gekommen ist und langfristig bleibt und nicht gleich wieder verschwindet, wenn etwas nicht klappt.« (Bürgermeisterin, 25.8.2008)

Weder das Auftauchen eines privaten Investors in Beljaevka noch seine Pläne für den insolventen Kolchos sind im ländlichen Russland ein Sonderphänomen. Vielmehr stehen die Ereignisse im Dorf für eine Entwicklung, die in der russischen Landwirtschaft um die Jahrtausendwende einsetzte und seither zunehmend an Fahrt gewann. 


\section{Die »neue Gigantomanie« im russischen Dorf}

Betrachtet man die Entwicklung der russischen Landwirtschaft in ihrer Gesamtheit, treten vom Zusammenbruch des sowjetischen Regimes bis in die späten 1990er Jahre zwei Merkmale besonders hervor: Zum einen brach die Agrarproduktion im Nachgang des Zerfalls des sowjetischen Regimes in den 1990er Jahren signifikant ein, so dass landwirtschaftliche Produkte zunehmend importiert werden mussten, um den inländischen Bedarf zu decken (Wegren 2009a, 2011; vgl. auch die Daten in Rozelle/Swinnen 2004). Zum anderen richteten sich die politischen Transformationsmaßnahmen zunächst ausschließlich auf zwei Typen von Agrarunternehmen: Sie zielten einerseits auf die Überführung der ehemaligen Staats- und Kollektivbetriebe in private Unternehmensformen (vor allem in Genossenschaften) und andererseits auf die Förderung kleiner privatbäuerlicher Unternehmen, die sich aus den Hoflandwirtschaften der ländlichen Haushalte entwickeln sollten (Ryl'ko 2002: 72; Wegren 1998: 36ff). Die Prioritäten der Transformationsstrategen lagen dabei meist auf den >Großen< und nur vereinzelt auf den >Kleinen< (dazu kritisch z.B. Rylko/Jolly 2005: 125; Uzun 2005: 99), die politische Beobachtung blieb jedoch stets auf diese beiden Akteurstypen - ehemalige Staats- und Kollektivbetriebe einerseits und kleinbäuerliche Betriebe andererseits - beschränkt (vgl. z.B. Wegren 1998).

Mit der Jahrtausendwende nahm nicht nur die russische Agrarproduktion Fahrt auf, was sich an der steigenden Produktion ebenso ablesen lässt wie am zunehmenden Rückgang unprofitabler Landwirtschaftsbetriebe ${ }^{34}$ (vgl. die statistischen Daten der »Russlandanalysen« zu Lindner/Vorbrugg 2011; Wegren 2009a). Es trat auch ein neuer Unternehmenstyp auf den Plan, der sich zuvor dem Blick der Transformationspolitik entzogen hatte (Rylko/Jolly 2005: 116; Wandel 2011: 424ff): Um das Jahr 2000 herum begannen sich russische Öl- und Gasunternehmen als erste (landwirtschafts-)externe Investoren unter anderem zu Zwecken der Spekulation und Risikodiversifikation für den Agrarsektor zu interessieren (Visser/Spoor 2011: 301). Ausgehend von dieser Intitialzündung ließ sich ein deutliches Wachstum der Direktinvestitionen in die russische Landwirtschaft beobachten, das nur durch die globale Finanzkrise leicht gedämpft wurde: Waren im Jahr 2003 lediglich rund 90 sogenannte Agroholdings unter Beteiligung inländischer und/oder ausländischer Investoren in der russischen Landwirtschaft registriert, waren es nur drei Jahre später bereits 319 (Visser/Spoor 2011: 310). Inländische Direktinvestitionen stiegen von 12 Billionen Russische Rubel im Jahr 1998 auf 183 Billionen in 2010 (wobei

34 Galten nach offiziellen Statistiken im Jahr 1998 noch rund 90 Prozent aller landwirtschaftlichen Großunternehmen als unprofitabel, war dieser Anteil im Jahr 2000 auf die Hälfte und im Jahr 2010 auf ein Viertel gesunken (Wegren 2011: 9f). 
sie kurz vor Beginn der Finanzkrise im Jahr 2008 einen Zwischenhöchstwert von 235 Billionen erreichten). Ausländische Direktinvestitionen wuchsen von 12 Millionen US-Dollar im Jahr 1998 auf 446 Millionen US-Dollar in 2010, wobei die stärksten Wachstumsraten seit dem Jahr $2005 \mathrm{zu}$ beobachten waren (Wegren 2011: 10f; auch Ustinova 2010). Vor allem das Wachstum der ausländischen Investitionen erstaunt angesichts der Tatsache, dass das »Bodengesetz der Russischen Föderation« (Föderales Gesetz Nr. 136 vom 25. Oktober 2001) den Besitz von Agrarland durch ausländische Unternehmen oder Staatsangehörige ebenso wie durch russische Firmen, die zu mehr als 50 Prozent unter ausländischer Beteiligung stehen, verbietet. Um dieses Gesetz zu umgehen, wickeln ausländische Investoren seither den Landerwerb über sogenannte Stellvertreterfirmen ab, die formal als russische Unternehmen registriert sind. Ein Vorgehen, das, so lässt sich zumindest vermuten, die russische Regierung in der Praxis nicht nur toleriert, sondern hinterrücks aktiv fördert (Visser/Mamonova/Spoor 2012: 907).

Die Holdinggesellschaften, die sich im Zuge dieser Entwicklung in der russischen Landwirtschaft etablieren, sind für die Dörfer zweifach neu: Neu ist zum einen die Trennung zwischen Eigentum und Management, durch die sich die Organisationsstruktur von Holdinggesellschaften auszeichnet. Eine solche Trennung kam weder bei den überwiegend in Genossenschaften überführten ehemaligen Kollektivbetrieben noch bei kleinen privatbäuerlichen Betrieben vor. Jetzt aber sind die Eigentümer eines Betriebs, also beispielsweise Banken, Unternehmen, staatliche Institutionen, Kommunen oder andere, ${ }^{35}$ von der Geschäftsführung, die im Dorf mit den Betriebsabläufen und der landwirtschaftlichen Produktion befasst ist, räumlich getrennt und in der Sozialdimension distinkt (vgl. dazu Ryl'ko 2002: 74). Neuartig sind die Agroholdings zum anderen mit Blick auf die räumliche Ausdehnung der von ihnen bearbeiteten Flächen. Da dieser Unternehmenstyp nicht als eigene Kategorie in staatlichen Statistiken zur russischen Landwirtschaft auftaucht, sind offizielle Durchschnittswerte über Betriebsgrößen, Produktionsmengen oder das bearbeitete Agrarland zwar nicht verfügbar, doch die Werte, die sich aus verschiedenen Einzelstudien zusammentragen lassen, vermitteln einen Eindruck der Größendimensionen: Die 100 privaten russischen Agroholdings aus einer Studie von Uzun (2009b: 146ff) kontrollieren durchschnittlich jeweils knapp 33.000 Hektar Land. Visser, Mamonova und Spoor (2012: 906) verweisen auf Statistiken, denen zufolge 196 von den im Jahr 2008 in der russischen Landwirtschaft registrierten Agroholdings zusammen 11,5 Millionen Hektar Land besaßen (also im Durchschnitt knapp 60.000 Hektar pro Unternehmen), wobei auf die 32 größten jeweils mehr als

35 Je nach Art des Eigentümers - private oder staatliche, inländlische oder ausländische Akteure, juristische oder natürliche Personen - lassen sich verschiedene Typen von Holdinggesellschaften klassifizieren (vgl. z.B. Ryl'ko 2002: 74; Uzun 2009: 132ff). 
100.000 Hektar entfielen. Die zwölf Agroholdings, die Wandel (2011: 430) analysiert, bearbeiten zwischen 15.000 und 400.000 Hektar Land (vergleichbare Angaben finden sich in Rylko/Jolly 2005: 119f; Uzun 2009a: 26ff). Zum Vergleich: Die durchschnittliche Größe der sowjetischen Kollektivbetriebe belief sich im Jahr 1983 auf 6.500 Hektar, die der wesentlich größeren Staatsbetriebe auf 16.300 Hektar (Schinke/Wädekin 1985: 36), die meist genossenschaftlich verfassten Nachfolgeunternehmen beider bearbeiten gegenwärtig durchschnittlich 5.000 Hektar Land (Belaya/Hanf 2010: 58). Nikulin (2005) spricht angesichts dieser Entwicklung von einer »neuen Gigantomanie« im ländlichen Russland und Uzun (2009a: 24) stellt fest: »Feudal lords and noble landowners could not even dream of holdings as immense as those that owners of present-day agricultural holding companies have taken possession ${ }^{36}{ }^{36}$

Die steigende Nachfrage nach russischem Agrarland sowohl durch nationale als auch durch ausländische und oftmals global agierende Investoren lässt sich in einen weltweiten Trend einordnen. Richtete sich die Landnachfrage zunächst primär auf die afrikanischen Staaten, nahm sie - unter anderem getrieben durch rasant steigende Preise für Agrarprodukte um das Jahr 2007 - in der jüngeren Vergangenheit vermehrt globale Ausmaße an und begann im Zuge dessen auch in den postsowjetischen Raum hineinzuwirken (vgl. z.B. Deininger/Byerlee 2011; Deininger 2011; Kugelman/Levenstein 2009). Beobachtet wird diese Entwicklung generell und zunehmend auch mit Blick auf Russland ausgesprochen kontrovers: Was aus wirtschaftswissenschaftlicher Sicht als Katalysator für technische Weiterentwicklung, den Transfer westlicher Managementstrukturen, Produktivitätszuwächse und generell als überfälliger Modernisierungsschub für die russische Landwirtschaft gelobt wird (vgl. beispielsweise Belaya/Hanf 2010; Ustinova 2010), stößt vor allem in den (kritischen) Sozialwissenschaften auf Skepsis und Kritik. Politische Brisanz erhält

36 Die russische Regierung reagierte auf den Aufschwung der nationalen Landwirtschaft auf ihre Weise: Im Januar 2010 erließ der damalige Präsident Medvedev per Dekret eine »Doktrin zur Ernährungssicherheit der Russischen Föderation«, die Maßnahmen und Ziele festlegte, um die Lebensmittelversorgung der russischen Bevölkerung langfristig zu gewährleisten (Dekret des Präsidenten der Russischen Föderation Nr. 120 vom 30. Januar 2010 »Über die Bestätigung der Doktrin zur Ernährungssicherheit der Russischen Föderation«). Unter anderem versucht die Doktrin über Vorgaben darüber, welche Anteile des inländischen Bedarfs an bestimmten Agrarprodukten durch die russische Produktion zu decken sind, die Importabhängigkeit des Landes zu mindern (z.B. 95 Prozent an Getreide und Kartoffeln, 90 Prozent an Milchprodukten, 85 Prozent an Fleisch und 80 Prozent an Zucker, pflanzlichen Ölen und Fisch). Aus dem Ausland werden diese Regelungen primär als protektionistische Maßnahmen und Exportbeschränkungen beobachtet. 
die Debatte vor allem dadurch, dass die jüngsten Entwicklungen in der russischen Landwirtschaft zunehmend als Landraub (»land grabbing «) ${ }^{37}$ etikettiert werden. Die Kritik an den >Großen $<-$ den Investorengruppen und Holdinggesellschaften - und die Besorgnis um die Interessen der >Kleinen <- der lokalen Bevölkerung -, so fällt auf, wird dabei oftmals fast reflexhaft hervorgerufen ${ }^{38}$ (allgemein z.B. De Schutter 2011; für Russland Lindner/Vorbrugg 2011, 2012; Visser/Spoor 2011; Visser/Mamonova/Spoor 2012).

Ich möchte die inhaltliche Substanz dieser divergierenden Perspektiven auf das Phänomen der zunehmenden Nachfrage nach Agrarland und des Auftauchens neuartiger Unternehmensformen im russischen Dorf im Folgenden weder in Frage stellen noch mich von Vornherein auf die Seite eines der aufgezeigten Lager der Debatte schlagen. Dass das Auftauchen der Agroholding im Dorf Strukturänderungen (auch) im Hinblick auf die lokale Selbstverwaltung nach sich zieht, ist aber unabhängig von solchen politisch und/oder disziplinär motivierten Blickwinkeln nicht zuletzt auch mit Blick auf die fortdauernde Bedeutung der ehemaligen Kollektivbetriebe anzunehmen. Welche dies im Einzelnen sind, zeigt sich am Beispiel der Gemeinde Beljaevka.

\section{»Ja, das war erschreckend - bei uns kaufen sie Land!«}

Das Unternehmen, dessen Gründung und Niederlassen in der Gemeinde Beljaevka sich im Jahr 2006 abzeichnete, bewegte sich in seinen Anfangsjahren und bis zu meinem letzten Feldaufenthalt 2010 zwar noch nicht in oben beschriebenen GröBendimensionen, ist jedoch strukturell dem für die russische Landwirtschaft neuartigen Typus der Agroholding zuzuordnen. Nach Angaben der Betriebsleitung befanden sich im Jahr 2008 rund 4.000 Hektar Land im Eigentum des Unternehmens, wovon im Jahr 2010 auf rund 2.000 Hektar Kartoffeln angebaut wurden, während die übrige Fläche zum Teil von Wald überwuchert war und erst noch landwirt-

37 Was sich genau hinter dieser Bezeichnung verbirgt, bleibt häufig unscharf. So räumen Borras et al. (2012: 210) ein: »The phrase >global land grab $<$ has become a catch-all to describe and analyze the current explosion of large scale (trans)national commercial land transactions«. Wenn von »land grabbing « die Rede ist, sind damit meist implizit Prozesse des Erwerbs oder der anderweitigen Aneignung von Agrarland vornehmlich in sogenannten Entwicklungs- und Schwellenländern durch (meist multinationale) Großunternehmen gemeint, die auf Spekulations- und/oder Investitionsgewinne zielen (vgl. z.B. Visser/Mamonova/Spoor 2012: 900f).

38 Eine wissenssoziologische Analyse dieses und verwandter Argumentationsschemata am Beispiel der kritischen Geographie findet sich in Goeke (2013). 
schaftlich nutzbar gemacht werden musste. Zwar betonten sowohl der Investor ${ }^{39}$ selbst als auch die Betriebsleitung im Dorf regelmäßig die Expansionspläne der Agroholding, einer zügigen Realisierung stand jedoch nicht zuletzt der Prozess des Erwerbs von Agrarland im Wege, der sich für den Betrieb als zäh und umständlich erwies.

Wie die meisten sowjetischen Kollektivbetriebe war auch der Kolchos in Beljaevka im Zuge der Privatisierung in den frühen 1990er Jahren formal in einen genossenschaftlichen Betrieb umgewandelt worden. Dies geschah unter anderem, indem Anteilsscheine (»paj«) sowohl auf das Betriebsvermögen als auch auf das Agrarland an die damaligen Kolchosmitglieder (und faktisch die überwiegende Mehrheit der Dorfbewohnerinnen und Dorfbewohner) ausgegeben wurden, die so zu Anteilseignerinnen und Anteilseignern des Betriebs wurden (zur Gutscheinprivatisierung in Russland vgl. z.B. Appel 1997; Boycko/Shleifer/Vishny 1993; zur Privatisierung der russischen Landwirtschaft vgl. z.B. Barnes 1998; Wegren 2009b: 51ff). Seitens der russischen Regierung war diese Privatisierungsform mit der Erwartung verbunden, dass die Anteilseignerinnen und Anteilseigner sich ihr Stück Land als Grundlage für eigene privatbäuerliche Betriebe in Natur ausweisen ${ }^{40}$ und als Privateigentum registrieren lassen würden und sich die sowjetische Landwirtschaft damit > von unten< und ohne weiteres regulatives Eingreifen transformieren würde (zu diesen politischen Intentionen vgl. Appel 1997: 1434). In den meisten Fällen unterblieb dieser Schritt jedoch (Prosterman/Mitchell/Bradley 1997: 1384ff) und auch in Beljaevka hatten nur wenige Anteilseigner von dieser Möglichkeit der Landprivatisierung Gebrauch gemacht. Die Mehrheit hatte ihren Landanteil offiziell an den Kolchos verpachtet, was in der Praxis bedeutete, dass dem Betrieb das Land anfangs gegen eine geringe und aufgrund der zunehmenden finanziellen Schwierigkeiten des Unternehmens in späteren Jahren gegen gar keine Bezahlung

39 Wenn hier und im Folgenden von der Person des »Investors« die Rede ist, verwende ich diese Bezeichnung - ebenso wie zuvor die Bezeichnung »Kolchos« für den Landwirtschaftsbetrieb im Nachbardorf - aus Gründen der sprachlichen Einfachheit und in Anpassung an den lokalen Sprachgebrauch. Formal handelt es sich bei dieser Person um den Vorstandsvorsitzenden der Agroholding, der im Dorf jedoch stets als »unser Investor« und vereinzelt auch als »unser Oligarch « bezeichnet wurde.

40 In den Eigentumszertifikaten, die im Rahmen des Privatisierungsprozesses ausgegeben worden waren, war zwar die Größe der Parzelle festgelegt, auf die ein Eigentumsanspruch bestand (in den hier analysierten Fällen zwischen sieben und acht Hektar), die Lage und die materiellen Grenzen des Landanteils waren jedoch unbestimmt. Wollte ein Anteilseigner seine Parzelle als Privateigentum registrieren, mussten die Grenzen des Landstücks zunächst »in Natur« ausgewiesen werden (»vydelit' v nature«), um es sodann rechtlich und physisch aus dem Kolchos herauslösen zu können. 
zur Bewirtschaftung überlassen wurde. Das Eigentum bestand in diesem Fall nicht im Nutzungsrecht an einem konkreten Stück Land, sondern lediglich im > virtuellen< Anrecht darauf, sich eine Parzelle von sieben bis acht Hektar in Natur ausweisen zu lassen und aus dem Kolchos herauszulösen, deren Grenzen erst im Zuge dieses Prozesses auch räumlich-materiell bestimmt werden.

Eine Weiterführung der Pachtverhältnisse in der Tradition des Kolchos hatte der neue Investor unter Verweis auf die damit verbundenen Unsicherheiten von Vornherein abgelehnt:

»Laut dem Gesetz über die Bearbeitung von Agrarland können die Anteilseigner, die die Landanteile besitzen, dem Landwirtschaftsunternehmen diese Anteile verpachten - so, wie sie es auch schon beim Kolchos gemacht hatten. Aber pachten wollte er nicht. >Wozu soll ich pachten? sagte er. >Heute gehe ich in Produktion - und morgen überlegen sie [die Anteilseigner, E.M.] es sich anders mit der Pacht. Und ich sitze dann da ohne Land. Ich werde das Land kaufen.« (Sachbearbeiter der Gemeindeverwaltung, 24.3.2009)

Der Erwerb des Kolchoslandes war für den Investor indes nur möglich, indem den Eigentümerinnen und Eigentümern der insgesamt 651 Landanteile ihre Eigentumszertifikate abgekauft wurden und er das Land anschließend in Natur ausweisen und registrieren ließ - ein Schritt, der aufgrund des hohen bürokratischen Aufwands allgemein als eine wesentliche Zutrittsbarriere für externe Investoren in der russischen Landwirtschaft beobachtet wird (Atkin 2009: 112; Rylko/Jolly 2005: 118; Visser/ Spoor 2011: 316).

Hinzu kam in Beljaevka eine zweite Schwierigkeit: Da innerhalb der Gruppe der Anteilseignerinnen und Anteilseigner des Betriebs ein Vorkaufsrecht auf die Eigentumszertifikate bestand, war der Erwerb der Landanteile durch Außenstehende - und damit auch den Investor - rechtlich nicht möglich. Erst wenn eine Parzelle in Natur ausgewiesen und registriert ist, kann das Land frei verkauft werden. Um diese Restriktion zu umgehen, wurden die Transaktionen über einen Anteilseigner abgewickelt, den der Investor für seine Pläne gewinnen konnte - ebenjenen Jagdgefährten und ehemaligen Bürgermeister der Gemeinde, der kurze Zeit später als Geschäftsführer des Betriebs eingestellt wurde:

»Er hat 350 Landanteile gekauft, [der Investor]. Er selber hatte eigentlich gar nicht das Recht, sie zu kaufen, deshalb war [der ehemalige Bürgermeister] der Käufer, der ist selbst Anteilseigner. Laut Gesetz verfügen die anderen Anteilseigner über ein Vorkaufsrecht. Nur wenn alle Anteilseigner den Kauf ablehnen, darf anderweitig verkauft werden. Aber wie soll man die Ablehnung von allen bescheinigen? 650 Anteilseigner und von jedem eine schriftliche Ablehnung? Die Hälfte davon ist bereits verstorben.« (Sachbearbeiter der Gemeindeverwaltung, 24.3.2009) 
Das Kaufangebot, das den Anteilseignern schließlich im Rahmen einer Gemeindeversammlung unterbreitet wurde, sah einen Preis von 1.000 Rubel (rund 30 Euro) pro Hektar Land vor, also zwischen 7.000 und 8.000 Rubel pro Landanteil. Um auf die Kaufentscheidung hinzuwirken, bot der Investor den Anteilseignern seine Unterstützung bei der im Verkaufsprozess anfallenden Bürokratie an, die nach Aussage vieler Gemeindemitglieder besonders im Fall von geerbten Landanteilen beträchtlich ist, übernahm die mit dem bürokratischen Aufwand verbundenen Gebühren, stellte Transportmöglichkeiten zu den entsprechenden Ämtern bereit und ähnliches.

Der Preis von 1.000 Rubel pro Hektar sei zwar durchaus niedrig, so räumte ein Sachbearbeiter der Gemeindeverwaltung ein. Aufgrund dessen, dass sich dieser Betrag nicht auf bereits registrierte Landanteile beziehe, sondern es lediglich um den Erwerb von Anteilsscheinen ohne konkreten materiellen Bezug ginge, sei das Preisangebot aber gerechtfertigt und entspreche zudem dem amtlichen Katasterpreis. Andere im Dorf teilten diese Ansicht nicht, sprachen schlicht von »Betrug« oder angesichts des gebotenen Preises abfällig von »Kopeken«. Ganz unabhängig von der Höhe des Preises, so wird einstimmig berichtet, habe jedoch allein der Gedanke daran, das Kolchosland zu verkaufen, viele erschreckt:

»Es wurden Gemeindeversammlungen abgehalten, als sich das Unternehmen [Name des Unternehmens] gegründet hat, als sie hierher gekommen sind. Es gab viele Gegner. Einige waren für den Verkauf. Sehr viele hingen an ihrem Land. Sie interessierten sich dafür, was er machen wird.« (Direktor des Kulturhauses, 27.2.2010)

Zum Zeitpunkt des Auftauchens des Investors schien im Dorf Konsens darüber zu bestehen, dass die Landanteile aus der Knappheitskommunikation herauszunehmen waren: ${ }^{41}$ Man hatte sie in der Vergangenheit dem Kolchos zur (wirtschaftlichen) Verwendung zur Verfügung gestellt, bestand jedoch nicht auf Pachtzahlungen, die faktisch seit Jahren ausgeblieben waren. Ein Verkauf war darüber hinaus auch mangels Nachfrage nicht in Betracht gekommen und allein die Beobachtung der Eigentumszertifikate auf Zahlungsmöglichkeiten hin galt als impliziter Verstoß gegen die dörfliche Moral. ${ }^{42}$ Diese Grenze von Knappheitskommunikation wurde durch das Kaufangebot des Investors herausgefordert und verschob sich sodann

41 Vgl. dazu Baecker (2008: 114): »Gesellschaften lassen sich darin unterscheiden, was sie für knapp halten und was nicht.«

42 Die einzige alternative Verwendungsmöglichkeit, die nach anfänglicher Skepsis im Dorf akzeptiert und respektiert wurde, war das Registrieren des eigenen Landanteils für private kleinbäuerliche Nutzung, von der allerdings nur eine verschwindend geringe Minderheit der Anteilseignerinnen und Anteilseigner Gebrauch machte. 
sukzessive: Nachdem sich die erste Aufregung gelegt hatte, schlug die Stimmung langsam um und die Mehrheit entschloss sich zum Verkauf, so dass der Investor schlussendlich 350 der 651 Landanteile erwerben konnte. So eindeutig dieses Resultat beschrieben werden kann, so ambivalent oder gar gegensätzlich sind die rückblickenden Darstellungen der Verkaufsgründe und der mit der Entscheidung verbundenen Freiheitsgrade. Zwei Argumentationsstränge treten hervor: Ein Argumentationsstrang stellt die Verkaufsentscheidung als einen ökonomischen Läuterungsprozess dar, im Zuge dessen die anfängliche Skepsis wich und die Freiheitsgrade durch das Kaufangebot schlussendlich stiegen; der andere stellt auf den faktischen Zwang und die Beschränkung von Freiheitsgraden im Vorfeld sowie durch die Verkaufsentscheidung selbst ab.

Während im Kontext des ersten Argumentationsstrangs fast ein wenig verschämt eingeräumt wird, dass man die moralischen Bedenken und die allgemeine Skepsis zunächst geteilt habe, wird der Entscheidungsfindungsprozess im Nachhinein als eine Art >ökonomische Läuterung< beschrieben, wobei im Wesentlichen zwei Argumente zum Tragen kommen: Zum einen das Fehlen alternativer Verwendungsmöglichkeiten für die Landanteile (sofern man sich nicht im Stande sah, das Land selbst zu bearbeiten) und daran anschließend die Beobachtung der faktischen Nutzlosigkeit der Anteilsscheine nach der Kolchosinsolvenz; zum anderen die Tatsache, dass die Eigentumszertifikate durch das Angebot des Investors zum ersten Mal als transformierbar beobachtet wurden, wobei vor allem konkrete, meist langlebige Konsumgüter als Vergleichshorizont fungierten:

»Als der Investor hier auftauchte und das Land zusammengekauft hat, um es zu bewirtschaften, hat sich die Mehrheit entschlossen zu verkaufen. Das Geld wurde damals dringend gebraucht und der Preis war beachtlich. Für seinen Landanteil konnte man auf einmal eine moderne Waschmaschine kaufen. Sowas besaßen die Leute nicht, die Zeiten waren >geldlos $<$ der Kolchos ist zerfallen und wohin soll ich mit meinen acht Hektar Land? Was nützen sie mir, was soll ich damit machen? Ich habe einen Gemüsegarten und mehr kann ich nicht bearbeiten, mir fehlen die technischen Möglichkeiten. Die Leute haben entschieden - darüber, was realistisch ist [...]. Sie haben begriffen, welchen Nutzen sie von diesen acht Hektar haben, die da irgendwo hinter dem Wald liegen. Man muss sie irgendwie bearbeiten und selbst wenn ich am Ende Heu mähen kann, was mache ich dann mit dem Heu? Selber essen werde ich es nicht und meinem Kalb reicht die Mahd von einem Hektar. [...] Ja, das war erschreckend bei uns kaufen sie Land. Aber wenn du anfängst, darüber nachzudenken, was dir dieses Land nützt - wozu brauchst du das Land? [...] Was kannst du damit anfangen?« (Gemeinderätin/Leiterin der Krankenstation, 25.2.2010)

Verwiesen wurde im Kontext dieses ersten Argumentationsstrangs jedoch nicht nur auf die unmittelbar gestiegenen Freiheitsgrade durch die Zahlung des Kaufpreises, 
sondern auch auf indirekte Freiheitsgewinne in Folge des wirtschaftlichen Erfolgs des Unternehmens, den man durch die eigene Verkaufsentscheidung (mit-)ermöglicht hatte und der als Ex-post-Bestätigung für die Richtigkeit dieser Entscheidung gewertet wurde. Der ehemalige Kolchos habe bereits mehrere Jahre vor seiner Insolvenz keine Geldzahlungen mehr an seine Belegschaft geleistet und seine Angestellten stattdessen ausschließlich in Naturalien entlohnt - in diesem Sinne ist die Aussage der Gemeinderätin »wir waren geldlos« durchaus wörtlich zu nehmen. Der neue Betrieb habe somit nicht nur wirtschaftliche Stabilität ins Dorf gebracht, sondern einen regelrechten Monetarisierungsschub bewirkt. Letzterer, um mit Simmel (1989 [1900]: Kap. 4) zu sprechen, kam gerade in der Dualität von Freiheit und sozialer Entfremdung - in Form einer zunehmenden Distanz vom Kolchos und seinen Entscheidungen (beispielsweise über die Art der Naturalzahlungen) - zum Ausdruck, was beides gleichermaßen goutiert wurde:

»Die Leute haben keinen Lohn bekommen, und wenn doch, dann nur in Naturalien: Getreide, irgendwelche Dienstleistungen. Das heißt, das waren richtig schlechte Zeiten, das war nichts, kann man sagen. Jetzt hat das Unternehmen angefangen zu arbeiten, es gibt Geld, die Leute bekommen Geld. Und das ist das aller-aller-allergrößte Plus, dass die Leute jetzt ein Einkommen haben, ihre Familie ernähren können, und nicht abwandern, nicht mehr trinken müssen. Verstehen Sie, das ist die wesentliche Frage, die für das Dorf entschieden wurde: Den Leuten wurde Geld gegeben für ihre Mühen, für ihre Arbeit. Und nicht Getreide, Einstreu, Vieh, sondern richtiges Geld, das man im Geschäft für das eintauschen kann, was man will und nicht für das, was dir der Kolchos anstelle von Geld vorschlägt. Das ist ein riesiges Plus.« (Angestellter der Agroholding, 7.3.2010)

Auch wenn die Bedeutung des Betriebs für das Dorf kaum in Frage gestellt wird, findet sich mit Blick auf die Verkaufsentscheidung noch ein gegensätzliches zweites Argumentationsmuster: Während das Verpachten an den Kolchos oftmals weniger Resultat einer Entscheidung als vielmehr die schlichte Fortführung des Gewohnten war, stellte das Kaufangebot in Kombination mit der Insolvenz des Betriebs für viele Anteilseignerinnen und Anteilseigner einen Anlass dar, sich erstmals bewusst mit der Verwendung der Eigentumszertifikate auseinanderzusetzen. Tatsächlich eröffnete sich mit dem Kaufangebot eine Handlungsoption, die zuvor zwar rechtlich, aufgrund mangelnder Nachfrage jedoch nicht faktisch bestanden hatte. Auch die Möglichkeit, sich seine Parzelle als Privateigentum registrieren zu lassen, um sie selbst zu bearbeiten oder anderweitig zu verkaufen, so beschreibt beispielsweise der Direktor des Kulturhauses, hätten während der Umbruchphase, als der Investor im Dorf auftauchte, durchaus einige Gemeindemitglieder ernsthaft zu erwägen begonnen. Die Hoffnungen richteten sich in diesem Zusammenhang auf 
günstige Zugangsmöglichkeiten zum Sachvermögen des insolventen Kolchos, das als Grundstock für einen eigenen privatbäuerlichen Betrieb hätte dienen können:

»Einige sahen sich schon als Konkurrenten [des Investors, E.M.]. Einige dachten, dass ihnen der Staat entgegen kommt, dass ihnen die Technik [des insolventen Kolchos, E.M.] günstiger verkauft wird. Einige wollten das Land selber bearbeiten. Aber das kam nicht zustande und die Leute waren schließlich gezwungen zu verkaufen. Wenn jemand am Ende nicht verkauft hat, dann nimmt [der Investor] sich sowieso das Land, das am günstigsten gelegen ist. Und wenn eine Parzelle noch nicht registriert war, haben sie [die Agroholding, E.M.] die trotzdem belegt. Sie sind zu den entsprechenden Behörden gefahren, haben sich das alles schützen lassen und im Ergebnis haben sie alles abgegriffen.« (Direktor des Kulturhauses, 27.2.2010)

Wie in dieser Darstellung bereits anklingt, haben sich die Möglichkeiten der Registrierung der Landanteile mit dem Auftauchen des Investors im Dorf entscheidend geändert. Eine wesentliche Ursache dafür liegt im offiziellen dreistufigen Ablauf der Eigentumsregistrierung, die der Investor mit Blick auf seine Ziele wirkungsvoll durchkreuzte: Im ersten Schritt wird beim sogenannten Landkomitee (»zemelnyj komitet«), einer Behörde im Bezirkszentrum, ein Gesuch für die Eigentumsregistrierung eingereicht und gemeinsam mit dem zuständigen Sachbearbeiter der Gemeindeverwaltung die genaue Lage der gewünschten Parzelle bestimmt. Im zweiten Schritt wird das Vorhaben in der Lokalzeitung angezeigt. Für die übrigen Anteilseigner besteht dann eine 30tägige Einspruchsfrist. Erfolgt kein Einspruch, wird das Gesuch bestätigt. Im dritten Schritt wird die Registrierung - die eigentliche Privatisierung - durch die Registrierungskammer vollzogen und der Gesuchsteller offiziell als Eigentümer der Parzelle vermerkt. In den wenigen Fällen, in denen vor dem Auftauchen des Investors Landanteile auf diesem Weg registriert wurden, so betont ein Sachbearbeiter der Gemeindeverwaltung, sei dieser Prozess stets reibungslos und zügig verlaufen. Erst als die Registrierung der Landanteile für den Verkauf an den Investor begann, habe sich dies geändert: Über den Stellvertreter, der offiziell als Käufer der Eigentumszertifikate auftrat, habe das Unternehmen fortan gegen jeden weiteren Registrierungsversuch Einspruch erhoben. In diesem Fall muss die Registrierungskammer den Registrierungsprozess solange unterbrechen, bis die Beteiligten sich einigen und der Einspruch offiziell geklärt ist. Für Anteilseigner und Anteilseignerinnen, die fest mit einer Registrierung rechneten und bereits die anfallenden Gebühren bezahlt hatten, ist dies mit finanziellen Verlusten verbunden:

»Er [der Stellvertreter des Investors, E.M.] ist Anteilseigner, er hat das Recht dazu. Und dann stoppt alles. Viele haben bereits Geld hingelegt, für die Vermessungsarbeiten, um die Parzelle zu registrieren, um die 15.000 haben sie hingelegt - aber die Registrierungskammer hat die Registrierung verweigert.« (Sachbearbeiter der Gemeindeverwaltung, 24.3.2009) 
Ein Anteilseigner versuchte daraufhin, gegen die Entscheidung der Registrierungskammer zu klagen, bekam jedoch kein Recht. Die Behörde habe entsprechend der geltenden Gesetze gehandelt, so das Gerichtsurteil, geklagt werden müsse gegen denjenigen, der den Einspruch erhoben habe - falls man sich nicht anderweitig einigen könne:

»Einer von denen, die nicht registrieren konnten, ist gegen die Registrierungskammer vor Gericht gezogen, aber das Gericht hat ihm nicht Recht gegeben. >Wenden Sie sich an den, der den Einspruch [gegen die Registrierung, E.M.] erhoben hat $\iota$, so hieß es im Gerichtsurteil. $>$ Klagen Sie gegen ihn, aber nicht gegen die Registrierungskammer - sie hat im Einklang mit dem Gesetz gehandelt $<$ « (Sachbearbeiter der Gemeindeverwaltung, 24.3.2009)

In dem Moment, in dem sich die Beobachtung der eigenen Handlungsmöglichkeiten durch die Anteilseignerinnen und Anteilseigner geändert hatte, so lässt sich zusammenfassen, schlug das Zeitfenster für deren Umsetzung augenscheinlich bereits wieder zu und verengte sich auf die Beobachtung einer einzigen verbleibenden Option: den Verkauf an die Agroholding. Im Unterschied zu der Befürchtung, dass Anteilseignerinnen und Anteilseigner in Unkenntnis ihrer Rechte und/oder aufgrund fehlender Formalisierungen um ihre Eigentumszertifikate gebracht werden (Feifer 2003; Visser/Spoor 2011: 317), deuten die Rückblicke der Beteiligten in diesem Fall darauf hin, dass bekannte Rechte vor dem Hintergrund konfligierender Interessen an den Rändern einer dominanten Organisation nicht mehr durchsetzbar waren.

Der Kauf von Eigentumszertifikaten durch den Investor verweist auf einen Bereich der Transformationsforschung, der im Zusammenhang mit den Privatisierungsmaßnahmen zwar bereits anklang, bislang aber nicht explizit zur Sprache kam: Beobachtet - und aus wirtschaftswissenschaftlicher Perspektive zum Teil deutlich kritisiert - wird das Fehlen eines Marktes für Agrarland in Russland, auf dessen Herausbildung die Kolchosprivatisierung in Form der Ausgabe von Eigentumszertifikaten als politische Maßnahme ursprünglich zielte (Wegren 2008: 124; auch Lerman 2001; Lerman/Shagaida 2007: 14). Die Forderung nach Markt und die daran anschließende Kritik an den gegenwärtigen Verhältnissen wird dabei vorwiegend mit neoklassischen Argumenten begründet: Da letztlich nur ein marktförmiger Distributionsmechanismus sicherstellen könne, dass die Agrarflächen ihrer produktivsten Verwendung zugeführt werden, sei ein funktionierender Markt die Voraussetzung für landwirtschaftliche Produktivitäts- und Effizienzsteigerungen (vgl. für viele Deininger/Binswanger 1999: 252; Lerman/Shagaida 2007: 14). Die zögerliche oder ausbleibende Entwicklung eines Marktes wird dann meist auf unklare Eigentumsverhältnisse, fehlende Rechtssicherheit, persistente Abhängigkeitsbeziehungen und generell mangelhafte politische Rahmenbedingungen zurückgeführt 
(vgl. dazu empirisch mit leicht variierenden Foki und Fragestellungen Deininger/ Binswanger 1999; Lerman 2001; Lerman/Shagaida 2007; Shagaida 2005; Wegren 2008, 2009b: Kap. 3 und 4). Vor allem aus ethnologischer Sicht werden dieser Sichtweise alternative Begründungen entgegengesetzt: Herausgestellt werden hier die soziale Einbettung und »fuzziness « von Eigentum, die »moral economy « des Dorfes, traditionale Strukturen und die inadäquate Unterscheidung zwischen privaten und kollektiven Eigentumsformen (vgl. z.B. Hann 1998, 2003; Verdery 1999). Entsprechend divergieren auch die Eigenschaften, die den Akteuren - den individuellen Anteilseignerinnen und Anteilseigner - aus den Perspektiven der unterschiedlichen Disziplinen unterstellt werden: Während die (neoklassische) Ökonomie Rationalität und das Streben nach Nutzenmaximierung betont, die sich unter den gegebenen Umständen schlicht nicht entfalten können, tendieren die Ethnologie und verwandte Disziplinen oftmals zur Zuschreibung von Motiven wie der Ablehnung von Eigentum, die aus einem generellen Widerstand gegen > westliche< Institutionen rührt.

Welche Schlussfolgerungen lassen sich im Hinblick auf die Herausbildung eines Marktes für Agrarland vor diesem Hintergrund aus dem Kaufangebot des Investors und den Reaktionen darauf im Dorf ziehen? Ohne die Berechtigung der skizzierten Erklärungsversuche generell leugnen zu wollen, legt das Fallbeispiel wenngleich ein Einzelfall - eine alternative Erklärung nahe, die auf die Beobachtungsmodi der beteiligten Systeme sowie auf die Möglichkeiten für Anschlusskommunikation abstellt und damit ohne die Zuschreibung konkreter Motive und Akteursmerkmale auskommt. Ausgangspunkt ist dabei ein Verständnis von Markt als spezifische Beobachtungsstruktur: Käufer und Verkäufer beobachten primär die eigene Marktseite im Modus der Konkurrenz auf Preise und (erwartete) Zahlungen hin (vgl. dazu Goeke/Moser 2011). Die Beschreibungen der Anteilseignerinnen und Anteilseigner im Dorf deuten darauf hin, dass das Kaufangebot des Investors entsprechende marktförmige Beobachtungsformen ausgelöst hat. Das bis dahin (wirtschaftlich) >nutzlose < Eigentumszertifikat, dessen Wert einzig in der Bindung an die Dorfgemeinschaft bestand, erhielt erstmals einen Preis und wurde in andere Güter transformierbar. Daran anschließend wurde auch über alternative Verwendungsund Verkaufsmöglichkeiten, einschließlich der Spekulation auf steigende Preise, nachgedacht. Jedoch: Was sich für den Investor zur Konkurrenz hätte entwickeln können - das Auftauchen von anderen Käufern und Verkaufsmöglichkeiten im Beobachtungshorizont der Anteilseigner - wurde vom Unternehmen (und flankiert durch die Bürgermeisterin, die nach anfänglicher Skepsis Interesse daran entwickelte, dass sich der Betrieb im Dorf etablierte) buchstäblich im Moment ihres Aufscheinens wieder unterdrückt. Versuche, an die neuen Beobachtungsformen anzuschließen und die Landparzellen beispielsweise mit Blick auf erwartete Zahlungen zur eigenen Bearbeitung oder zu Spekulationszwecken zu registrieren, waren be- 
reits kurze Zeit nach dem Auftauchen des Investors faktisch nicht mehr möglich. Der Investor generierte sich letztlich erfolgreich als einziger potenzieller Käufer, der selbst von denjenigen, die einem Verkauf zunächst ablehnend gegenüberstanden, als alternativlos beobachtet wurde. Der Preis spiegelte dabei jedoch weder Erwartungserwartungen noch die Beobachtung von Knappheitskommunikation wider. Verwiesen wurde stattdessen primär auf die dominante Verhandlungsmacht des Investors gegenüber den Gemeindemitgliedern:

»Wie der Preis bestimmt wurde? Er [der Investor, E.M.] hat einen Preis genannt - und das war's. Er hat das selbst so gesehen - ein Hektar für Tausend -, dass das natürlich ein rein symbolischer Preis ist. Wir haben das sehr wohl verstanden.« (Bürgermeisterin, 25.8.2008)

Zwar fanden schlussendlich Transaktionen statt und die Eigentumszertifikate wurden in diesem Sinne zu Gütern, bewerten lässt sich dies jedoch bestenfalls als ein kurzes Aufflackern von Markt und kaum als nachhaltiges Etablieren marktförmiger Beobachtungsstrukturen mit Bezug auf die Landanteile.

\section{»In der Stadt kann ich von den sozialen Strukturen abstrahieren«}

Wie beobachtet der neue landwirtschaftliche Großbetrieb selbst seine Position im Dorf? Auf dem bereits erwähnten Dorfjubiläum trat nicht nur die Bürgermeisterin der Gemeinde auf, auch der Investor selbst sprach zu den Anwesenden:

$»$ Von unserer Seite tun wir alles, dass dieses Dorf lebt, dass es eine Zukunft hat. Als ich hierher kam, vor zwei oder drei Jahren - genau erinnere ich mich nicht mehr -, als >dačnik<, zur Erholung, habe ich einen Kolchos gesehen, der faktisch zerfallen war. Das war ein schmerzlicher Eindruck. Ich fing an darüber nachzudenken, was ich tun kann. Ich habe eine gewisse Erfahrung darin, Unternehmen aufzubauen - warum sollte ich es nicht versuchen? Ob es mir gelungen ist, müssen Sie selbst beurteilen. ${ }^{43}$

Seine Rede und die Auszeichnungen, die er im Anschluss daran an verdiente Betriebsangehörige und Gemeindemitglieder verlieh, waren nicht seine einzigen Festbeiträge: Für die Kinder stand eine Hüpfburg bereit, auf einem Segelboot konnten Runden auf dem nahegelegenen Fluss gedreht werden und einen besonders nachhaltigen Eindruck hinterließ ein Flugzeug, das über dem Dorf kreiste und Flugblätter mit Jubiläumsglückwünschen abwarf. Beobachtet man diese Aktivitäten im Horizont der ersten Äußerungen des Investors aus dem Jahr 2006, könnte der Kontrast

43 Aus der Aufzeichnung der Rede durch einen lokalen Fernsehsender, der im August 2008 einen Bericht zum Dorfjubiläum ausstrahlte. 
kaum größer sein. So erinnern sich die Bürgermeisterin ebenso wie ein Mitarbeiter der Gemeindeverwaltung:

»Das gegenseitige Verständnis hat sich erst schrittweise entwickelt. Am Anfang konnte davon keine Rede sein. Und er [der Investor, E.M.] sagte: >Ich habe nicht vor, solche Belastungen auf mich zu nehmen, Einbußen für Sie in Kauf zu nehmen<. Mittlerweile hat sich das gebessert.« (Bürgermeisterin, 25.8.2008)

»Er ist ein Unternehmer, er ist zweimal hergekommen, ist auf der Gemeindeversammlung aufgetreten, hat alles ehrlich erzählt. Sogar kritische Fragen hat er beantwortet. Er wurde zum Beispiel gefragt: >Wir als Gemeindeverwaltung - auf welche Weise werden Sie als NichtWohlfahrtsorganisation uns unterstützen?«>Unterstützung werde ich keine leisten - meine Unterstützung besteht darin, dass es hier bei Ihnen ein Unternehmen geben wird, dass Sie Steuerzahlungen erhalten werden, Abgaben, dass Geld in Ihr Budget fließt. Das ist meine Unterstützung.« (Sachbearbeiter der Gemeindeverwaltung, 24.3.2009)

Seine Unterstützung für das Dorf sah der Investor, wie er selbst einräumt, ursprünglich ausschließlich in den Begleitaspekten seiner Geschäftstätigkeit - der Schaffung von Arbeitsplätzen und Steuerzahlungen - und zog sich auf diese Weise allein auf den Standpunkt einer Wirtschaftsorganisation zurück: »Man könnte etwas vereinfacht formulieren: Wirtschaftsorganisationen sind Organisationen zur Zahlung von Steuern, Gewinnen und Löhnen« (Luhmann 2009: 466f).

Diese »Trias der Abführungen«, so stellt Luhmann (2009: 467) weiter fest, gilt in der modernen Gesellschaft als Standarderwartung an Wirtschaftsorganisationen. Sie löst die »moralische Maxime« stratifizierter Gesellschaften ab, »daß Reichtum zu angemessenem Gebrauch verpflichtet « und sich allein daraus die gesellschaftliche Existenzberechtigung eines Unternehmens speist. Im Profit des modernen Unternehmens vollzieht sich die Selbstreferenz der Wirtschaft - nur über Profit ist Kapitalbildung möglich. In Löhnen und Steuern schwingt hingegen einerseits die Fremdreferenz auf andere Funktionssysteme respektive die Umwelt der Wirtschaft mit: »Die Leistungen an den Staat und an die Arbeiter überführen das Wirtschaftsmedium Geld in nichtwirtschaftende Teilsysteme innerhalb der Gesellschaft bzw. an Personen als Umwelt des Gesellschaftssystems« (Luhmann 2009: 468). Da es sich bei beiden um Zahlungen handelt, verweisen sie aber andererseits auch indirekt wieder auf die Wirtschaft zurück. Die Tauschbeziehungen, die das Unternehmen auf diese Weise mit seiner Umwelt unterhält, erfolgen dabei jedoch keineswegs freiwillig, sondern auf Druck aus dieser Umwelt heraus: 
»Es bedarf, soweit man bisher sieht, externer Pressionen, um zu verhindern, daß die Organisationssysteme sich bei aller tauschmäßigen Verknüpfung mit ihrer Umwelt gleichwohl zielmäßig auf sich selbst beschränken und nur >für sich< existieren.« (Luhmann 2009: 468)

Allen drei Bereichen der Ressourcenabführung, so Luhmann, muss das Unternehmen im Sinne des Erhalts seiner Reproduktionsfähigkeit innerhalb der Gesellschaft stets gleichzeitig Rechnung tragen, ihre relative Bedeutung zueinander kann im Einzelfall jedoch variieren - was auch am Beispiel der Agroholding deutlich wird. So wird das Unternehmen zwar aus dem Dorf heraus in erster Linie als Arbeitgeber beobachtet:

»Das Verhältnis [zum Investor, E.M.] - das sind Arbeitsplätze.« (Bürgermeisterin, 25.8.2008)

»Die Leute haben gemerkt, dass man auch anders leben kann. Dass man nicht trinken muss, sondern Arbeit hat, noch dazu mit einem vernünftigen Lohn. Und dann gibt es noch Geschenke. In diesem Jahr wurden Prämien bezahlt. Der größte Teil der Bevölkerung steht ihm [dem Investor, E.M.] jetzt positiv gegenüber.«(Gemeinderätin, 24.3.2009)

Im Unterschied zum Fokus der Fremdbeobachtungen deutet die Selbstbeschreibung des Investors jedoch darauf hin, dass die Schaffung von Arbeitsplätzen samt Lohnzahlungen in der »Trias der Abführungen « gerade jener Bereich ist, dem nachrangige Bedeutung eingeräumt wird. Zwar erkennt der Betrieb seine Relevanz als Arbeitgeber, sieht sich im Unterschied zum Kolchos jedoch in der Sozialdimension keinesfalls als Organisation, die die Gemeindemitglieder umfassend integriert. Der neue Investor kündigt sogar Personalkürzungen an, die für ihn gleichermaßen Maßstab seines unternehmerischen Erfolgs und Abgrenzungskriterium gegenüber seinem Vorgänger, dem ehemaligen Kolchos, sind. Die (sowjetische) Kongruenz von Dorfgemeinschaft und Arbeitskollektiv schwindet auf diese Weise zunehmend:

»In Beljaevka arbeiten in unserem Unternehmen 80 Personen, aber wir haben die Fläche an Land, die wir bearbeiten, in diesem Jahr gesteigert. Wir sind jetzt fast bei der Hälfte der Fläche des Sovchos [eigentlich Kolchos, E.M.] >Traktor<. Der Sovchos >Traktor $<$ hat 9.000 Hektar Land bearbeitet und wir sind in diesem Jahr bei 4.000. Bei uns arbeiten 80 Personen, dort haben damals 660 gearbeitet. [...] Ich behaupte sogar, dass bei uns im Moment vergleichsweise viele Leute arbeiten, das heißt, ich setze eigentlich auf ein Geschäftsmodell, das mit 40 Leuten für die ganzen 9.000 Hektar auskommt. Wenn wir also dieses Jahr erfolgreich beenden, wenn alles gut läuft, werden bei mir nur noch 40 Personen arbeiten. Ich wähle die aus, die ich brauche und die vernünftig arbeiten, und ich biete diesen Leuten entsprechende Arbeitsbedingungen.« (Investor, 13.8.2008) 
Darüber hinaus führt der Mangel an qualifizierten Arbeitskräften im Dorf dazu, dass vor allem Führungskräfte extern rekrutiert werden. Auch dadurch werden die Belegschaft des Unternehmens und die (alteingesessene) Dorfbevölkerung zunehmend inkongruent:

»[Frage]: Sie haben gesagt, dass Sie in Beljaevka keine Arbeitskräfte finden. Hier wohnen doch ausreichend viele Leute. Warum? Weil sie trinken oder nicht arbeiten wollen? Warum können die Männer aus dem Dorf bei Ihnen nicht als Traktoristen arbeiten?

[Investor]: Nun, schauen Sie - 900 Personen sind hier ungefähr gemeldet, real leben hier $600 .^{44}$ [...] Von diesen 600 sind 279 Pensionäre. Bleiben 300, davon sind vielleicht 100 Kinder. Bleiben 200. Jetzt schauen wir die Struktur der Bevölkerung an. Ungefähr die Hälfte davon sind deklassierte Elemente. Bleiben 100. Von 900 Leuten im Dorf sind also 100 arbeitsfähig, mehr nicht. Dann gibt es die Schule, wo einige arbeiten. [...] Es gibt Lebensmittelgeschäfte, in denen einige arbeiten. In der Krankenstation arbeiten auch nicht wenige. Krankenstation, Bankfiliale, Gemeindeverwaltung, Apotheke. Das gibt es hier alles. Und da sitzen die Leute, da arbeiten sie. Von den 100 Leuten arbeiten dort ungefähr 40. Bleiben für uns 60. Bei uns arbeiten ungefähr 30 Personen vernünftig und 30 so lala - unter dem Regime des Alkoholismus.

[Frage]: Werden Sie für diese Personen Verwendung haben oder Personal aus anderen Orten einstellen?

[Investor]: Was soll ich mit denen machen? Soll ich mich mit denen streiten? Mit denen zu streiten ist sowieso schwierig.«

(Investor, 13.8.2008)

Nicht nur im Hinblick auf die Einbindung der Gemeindemitglieder zeigt sich im Selbstverständnis der Agroholding ein Bruch mit der sowjetischen Funktion der landwirtschaftlichen Großbetriebe. Auch Sondererwartungen aus seiner neuen unmittelbaren dörflichen Umwelt, die über die Zahlung von Gewinnen, Steuern und Löhnen hinausgehen, hatte der Investor nicht nur nicht einkalkuliert, er distanzierte sich sogar zunächst explizit davon. Augenscheinlich war diese Haltung jedoch nicht durchzuhalten: Bereits kurze Zeit nach seinen ersten Auftritten im Dorf lässt sich im Hinblick auf >nicht-wirtschaftliche < Umwelterwartungen ein Anpassungsprozess beobachten, der in den Leistungen des Betriebs für das Dorf sichtbar wird und den der Investor selbst detailliert reflektiert. Dreh- und Angelpunkt seiner Überlegun-

44 Die Differenz zwischen der Anzahl der offiziell gemeldeten Gemeindemitglieder und der tatsächlich im Dorf lebenden Personen ergibt sich daraus, dass in vielen Familien Großeltern und Enkel im Dorf leben, während die mittlere (erwerbstätige) Generation mindestens die Arbeitswoche außerhalb des Dorfes - meist in der Regionshauptstadt Perm verbringt und nur am Wochenende oder seltener tatsächlich in der Gemeinde wohnt. 
gen ist dabei der Kontrast zwischen städtischen und dörflichen Strukturen. Könne man in der Stadt als Unternehmen von den gegebenen sozialen Strukturen abstrahieren und sich - durch den Verweis auf Steuern - auf eine rein wirtschaftliche Position zurückziehen, sei dies im Dorf offenkundig nicht möglich:

»So versuchen wir also eine Beziehung zum Dorf zu unterhalten, eine partnerschaftliche Beziehung aufzubauen. Ich bin davon überzeugt, dass ich in der Stadt - ich habe noch ein groBes Unternehmen in der Stadt - vollständig von den sozialen Strukturen in der Stadt abstrahieren kann und dort eine vollkommen andere Position einnehme. [...] In der Stadt gibt es Steuern und die Möglichkeit, Leute aus irgendwelchen anderen Orten einzustellen - aber hier im Dorf ist die Situation eine andere.« (Investor, 13.8.2008)

Mittlerweile, so die Konsequenz, beschränkt sich das soziale Engagement des Betriebs nicht mehr auf einmalige Ereignisse wie das Dorfjubiläum, sondern umfasst kontinuierliche Sonderleistungen, die sich zudem beständig ausweiten. Neben gängigen Tätigkeiten wie der Bereitstellung von technischem Gerät für die Instandhaltung der Dorfwege oder für Reparaturen der öffentlichen Infrastruktur wurden am Schulgebäude umfangreiche Renovierungsarbeiten vorgenommen, wurde die zur Ruine zerfallene Dorfkirche wieder aufgebaut, die Stelle eines Geistlichen eingerichtet, das Kulturhaus mit neuen Kostümen und Requisiten ausgestattet, der Dorfpolizist finanziert und Schulkinder und Pensionäre werden an Feiertagen mit Geschenken bedacht:

»So kommt es, bitte schön, dass diese sozialen Funktionen immer weiter zunehmen. Jetzt heißt es, dass wir einen Polizisten hier im Dorf brauchen. Was meinen Sie, was mir die Bürgermeisterin sagt? Kommen Sie, lassen Sie uns das gemeinsam machen, dass wir einen Polizisten im Dorf haben. Warum ist das überhaupt meine Angelegenheit? Und jetzt brauche ich auf einmal einen Polizisten. Im Moment restaurieren wir die Kirche, wir haben viel Geld für die Kirche ausgegeben. Sehr aktiv haben wir nach einem Geistlichen gesucht, jetzt kommt einer. Sollen sie ihren Pfarrer haben. Ein ganzes Jahr hat das gedauert.«(Investor, 13.8.2008)

Wie begründet der Investor ein derartig breites Leistungsspektrum, gegen das er sich anfangs vehement gestemmt hatte? Einerseits, und darin stimmt seine Argumentation mit derjenigen des Kolchosvorsitzenden im Nachbardorf überein, erkennt er nolens volens die Alternativlosigkeit der von ihm letztlich erbrachten Leistungen im Hinblick auf eine funktionierende dörfliche Infrastruktur:

»[Investor]: Hier hat es sich so ergeben, dass die soziale Infrastruktur so oder so vom Unternehmen getragen wird. Andere Varianten gibt es nicht.

[Frage]: Und was heißt das für Sie? 
[Investor]: Für uns ist das schlecht.«

(Investor, 13.8.2008)

Andererseits tritt im weiteren Verlauf der Argumentation ein bedeutender Unterschied zwischen Investor und Kolchosvorsitzendem hervor. Während der Kolchosvorsitzende in seiner Selbstbeschreibung die Gemeinwohlorientierung seiner Person wie auch seines Unternehmens in den Vordergrund stellt und sich selbst ungeachtet potenzieller Widersprüche mit den eigentlichen Unternehmenszielen - der Profitgenerierung - eine Reihe von Verantwortlichkeiten zuschreibt, beobachtet der Investor die von ihm erbrachten Leistungen explizit im Horizont einer Zweck-Mittel-Beziehung und als Teil seiner Unternehmensstrategie: Wenn er sich in seiner lokalen Umwelt dauerhaft Legitimität für seine Geschäfte verschaffen möchte, so sein Argument, habe er bestimmte Erwartungen zu erfüllen, ganz gleich wie unsinnig oder überflüssig sie in seinen Augen auch sein mögen und wie wenig er von seiner diesbezüglichen Verantwortung überzeugt ist. Im Kontrast zum Kolchos werden die Leistungen für die Gemeinde also nicht als Dienst am Gemeinwohl und losgelöst von wirtschaftlichen Logiken und Gewinnorientierung beobachtet, sondern es geht letztlich um die Legitimität des Unternehmens in seiner Umwelt im Hinblick auf den Zugang zu (externen) Ressourcen - mit Pfeffer und Salancik (2003 [1978]: 32ff) also um die Effektivität der Organisation. Effektivität bezeichnet bei Pfeffer und Salancik als externes und damit beobachterabhängiges Maß »den Grad, in dem die Organisation den Erwartungen anderer Organisationen oder externer Anspruchsgruppen entspricht« (Bonazzi 2008: 327) und dies im Hinblick auf die Minimierung der Risiken, die mit der Ressourcenmobilisierung verbunden sind. Als Gegenbegriff zu Effektivität wird die Effizienz als internes und objektiv bestimmbares Leistungsmaß einer Organisation gesetzt, die sich in Einklang mit dem gängigen Verständnis auf Input-Output-Relationen bezieht. Die Reaktion des Investors und seiner Agroholding auf die Erwartungen und Ansprüche aus der lokalen dörflichen Umwelt erfolgt in diesem Sinne im Horizont konkreter eigener (Wirtschafts-)Interessen.

Dass diese Haltung ungeachtet aller Ansprüche und Erwartungen auch in der dörflichen Umwelt reflektiert wird, kommt in Fremdbeschreibungen des Betriebs zum Ausdruck: Mit Blick auf die Leistungen für die Gemeinde wird die Agroholding durchgängig als »Sponsor« bezeichnet. Es hält damit ein Begriff Einzug in den dörflichen Sprachgebrauch, mit dem auch der Investor selbst seine Unterstützungsleistungen beschreibt. Auch wenn die Verwendung in der dörflichen Kommunikation vermutlich kaum in einem explizit betriebswirtschaftlichen Horizont stattin- 
det, ${ }^{45}$ verweist die zugrundeliegende Intuition dennoch auf die Zuschreibung von Eigeninteresse und spezifischen Kommunikationsabsichten des Unternehmens als stets mitschwingende Motive. Die Agroholding wird damit kontrastiert mit der (unterstellten) Gemeinwohlorientierung des ehemaligen Kolchos, für den eine solche Bezeichnung nicht zur Anwendung kommt.

Um welche Art von Umweltressourcen geht es dem Unternehmen? Während dies mit Blick auf die Leistungen für die Gemeindeverwaltung verhältnismäßig diffus bleibt und in den Begründungen tendenziell allgemeine Mythen gesellschaftlicher Verantwortung zum Tragen kommen (vgl. dazu und unter Verweis auf »Corporate Social Responsibility« Bluhm 2008: 156), schärft sich das Bild, wenn man die Leistungen für die Hoflandwirtschaften der Gemeindemitglieder - ebenfalls eine übliche Kolchosaufgabe - berücksichtigt: Daraus, dass er die privaten Hoflandwirtschaften nicht nur für ökonomisch überflüssig hält, sondern auch einen unmittelbaren Interessenskonflikt mit seinem eigenen Unternehmen sieht, macht der Investor keinen Hehl. Insbesondere während der arbeitsintensiven Erntezeit stehe seinen Mitarbeitern die Zeit, die sie für die eigene Hoflandwirtschaft aufwenden, nicht für ihre Tätigkeit in seinem Unternehmen zur Verfügung:

»Meine Haltung dazu ist die folgende: Je weniger Hoflandwirtschaften es in diesem Dorf gibt, desto besser. Je mehr Leute in der betrieblichen Produktion arbeiten, desto interessanter ist das für mich. [...] Während der Erntezeit fahre ich sie für einen zwölfstündigen Arbeitstag raus und wenn sie nachhause kommen, müssen sie nur noch schlafen und kommen dann wieder zur Arbeit, schlafen und wieder zur Arbeit. Der Zyklus der maximalen Arbeitsbelastung ist dabei identisch: Ich brauche sie im August-September, wenn sie auch in ihren Hoflandwirtschaften gebraucht werden.« (Investor, 13.8.2008)

Allem Missfallen zum Trotz beobachtet er die Hoflandwirtschaften jedoch als eigenständigen Sachverhalt, der sich seinem Einfluss entzieht. Ein Verbot ist nicht möglich und Streit darüber hält er für sinnlos. Um dennoch Kontrolle ausüben und den Widerspruch zu seinen eigenen Zielen minimieren zu können, greift er auf eigene Mittel zurück:

45 Das Gabler Kompakt-Lexikon Wirtschaft definiert Sponsoring als Teil der Kommunikationspolitik eines Unternehmens, das Aktivitäten umfasst, »durch die Personen und Organisationen in den Bereichen Sport, Kultur, Soziales, Umwelt oder Medien gefördert werden. Durch S. soll v.a. der Bekanntheitsgrad eines Unternehmens gesteigert und sein Image verbessert werden« (Gabler Verlag 2010: 409). 
»[Investor]: Im vergangenen Jahr habe ich ihnen Kartoffeln gegeben. Sie sollen so viele Kartoffeln nehmen, wie sie wollen, damit sie in ihrem Gemüsegarten keine Kartoffeln setzen und dann mit dem Spaten ausgraben.

[Frage]: Und das hat funktioniert?

[Investor]: Im Prinzip - die Leute haben gearbeitet und Kartoffeln gab es sowieso so viele, dass wir nicht wussten, wohin damit. Das heißt, ich versuche, die Leute von ihrer Hoflandwirtschaft zu lösen, damit sie anfangen, in meinem Unternehmen zu arbeiten. Für mich ist das profitabel. Aber auf der anderen Seite - wenn die Leute eine Kuh halten, kann ich nicht hingehen und ihnen sagen, ihr müsst die Kuh schlachten, andernfalls kriegt ihr von mir keine Arbeit. Die Leute halten Kühe, das ist die Situation. Für mich gibt es zwei Möglichkeiten: Entweder streite ich mich mit ihnen wegen der Kuh oder ich sorge dafür, dass die Kuh so wenig Zeit wie möglich in Anspruch nimmt.«

(Investor, 13.8.2008)

Agiert der Vorsitzende des ehemaligen Kolchos in derselben Situation in der Überzeugung von der Wichtigkeit der Hoflandwirtschaften als dörfliche Institution, wird deren Bedeutung aus Sicht der Agroholding betont heruntergespielt und als verschwindendes und mittlerweile überwundenes Vergangenheitsphänomen dargestellt:

»[Agronom 1 ]: Man kann sagen, dass das [die Hoflandwirtschaften, E.M.] kein dominierender Faktor im Leben der Leute mehr ist, das ist keine wesentliche Einkommensquelle.

[Agronom 2 ]: Das ist mehr aus Gewohnheit.

[Agronom 1 ]: Das ist so was wie ein Hobby. Man zieht sich ein paar Pflanzen auf seinem Grundstück.«

(Angestellte der Agroholding, 7.3.2010)

Dass für die Hoflandwirtschaften letztlich identische Unterstützungsleistungen wie zuvor durch den Kolchos erbracht werden - die Bereitstellung von technischem Gerät, die vergünstigte oder kostenlose Abgabe von Futter, Heu und ähnlichem -, beobachtet der Investor als Notwendigkeit für die Schaffung, Sicherung und Erweiterung eigener Handlungsspielräume in Form von Arbeitskraft. Die für das sowjetische Dorf einst charakteristische Symbiose zwischen landwirtschaftlichen Großbetrieben und Hoflandwirtschaften, die in den Semantiken des Kolchosvorsitzenden noch leise anklingt (auch wenn auch hier längst kein operativer Austausch wie zu sowjetischer Zeit mehr stattfindet), wird aufgebrochen. Die Grenzen werden scharf und das Verhältnis auf Distanz gestellt.

Darüber hinausgehende Beziehungen zwischen dem Großbetrieb und den Hoflandwirtschaften finden sich zwar vereinzelt auch in Beljaevka, nehmen aber eine im Vergleich mit den (post)sowjetischen Strukturen kategorial andere Form an. 
Beispielsweise erwähnt der Investor ein vertragliches Arrangement mit privaten Bienenzüchtern, deren Honig er aufkauft, kommodifiziert - also Qualitätskontrollen durchführt, mit Zertifikaten versieht, industriell abfüllt - und über Supermärkte vertreibt. Anstoß für die Zusammenarbeit war seine Beobachtung, dass Honig zwar ein profitabler Produktionszweig für das Unternehmen sein könnte, die Massenproduktion aufgrund der speziellen Eigenschaften dieses Produkts jedoch mit einer Reihe von (Kontroll-)Problemen verbunden ist. Diese sollen umgangen werden, indem die zahlreichen privaten Bienenzüchter im Dorf, die aus eigener Kraft nur über sehr begrenzte Verkaufsmöglichkeiten verfügen, als Zulieferer in die Produktion integriert werden. Die Symbiose wird durch eine Wertschöpfungsbeziehung ersetzt:

»Wir kaufen jetzt eine Abfüllanlage, um diesen Honig abzufüllen, Zertifikate auf die Verpackung zu kleben, einen Strichcode, damit man ihn in normalen Geschäften verkaufen kann, damit er eine Ware wird. Denn der Honig, so wie er aus dem Bienenstock kommt, ist natürlich sehr lecker, aber er ist keine Ware, man kann ihn nicht verkaufen, außer vielleicht auf dem Wochenmarkt oder von Nachbar zu Nachbar. Und wir versuchen jetzt, Kooperationen aufzubauen. Allerdings ist Honig eine sehr eigenwillige Ware. [...] Es ist praktisch unmöglich, ein effektives Kontrollsystems aufzubauen. Wie lange habe ich gesessen und überlegt, wie kann man ein Kontrollsystem aufbauen, wie erfasst man ihn im Rechnungswesen, wie managt man diese Sache - und ich habe keine Lösung gefunden. Mal fliegen diese Bienen, mal fliegen sie nicht, zählen kannst du sie sowieso nicht. Und dann haben wir es auf andere Weise versucht: Private Bienenzüchter gibt es hier viele. Wir sagen: Verkauft uns den Honig, wir überprüfen seine Qualität, füllen ihn ab und verkaufen ihn weiter. Interessant ist für sie schon ein Preis von 120 bis 130 Rubel, dann lohnt es sich für sie schon, die Produktion komplett zu verkaufen. Denn sie können ihn für 150 bis 160 verkaufen, aber dann müssen sie ihn einzeln in Gläsern verkaufen. Und so verkaufen sie ihn für 120 oder 130 komplett und kriegen das Geld sofort und das war's. Und wir erzielen heute im Bezirk einen Preis von 220 Rubel. Sie wissen ja selbst - hübsche Etiketten sind der halbe Preis des Produkts.« (Investor, 13.8.2008)

\section{Professionalisierung, Formalisierung und Entflechtung zwischen Agroholding und Gemeinde}

Die Agroholding, so lässt sich die bisherige Argumentation zusammenfassen, erbringt für die Gemeindeverwaltung und die Dorfbewohnerinnen und Dorfbewohner eine Reihe von Leistungen, die inhaltlich den Kolchosleistungen gleichen. Im Unterschied zum ehemaligen Kolchos werden diese Leistungen jedoch von der Leitung der Agroholding nicht als altruistische Pflicht betrachtet, die vom eigentlichen Organisationszweck - dem Profit - ablenken. Vielmehr ist zu erkennen, dass die vielfältigen Leistungen als ein Mittel zur Herstellung von Legitimität in der lo- 
kalen Umwelt beobachtet und explizit in den Horizont der eigenen Geschäftstätigkeit gerückt werden. Vorbehalte aus dieser unmittelbaren Umwelt, dem Dorf, gegen die Agroholding beziehen sich weniger auf die Art der Produktion - gegen den Kartoffelanbau hat niemand etwas einzuwenden. Die Quelle (potenzieller) Illegimität ist vielmehr die schiere Existenz des Unternehmens als formal-strukturell und personell distinkter Nachfolgebetrieb des Kolchos sowie die Tatsache, dass Kolchosland gekauft wurde. Auf diese Vorbehalte reagiert die Agroholding durch Imitation der Kolchosleistungen bei geänderten Bewertungsmaßstäben. An diesen Befund schließt sich nun die Frage an, auf welche Weise diese Leistungen erbracht werden und welche Kommunikationsformen und Kooperationsstrukturen sich vor allem im Verhältnis zur Gemeindeverwaltung herausbilden.

Vergleicht man die Arrangements zwischen Betrieb und Gemeindeverwaltung mit den Vereinbarungen des ehemaligen Kolchos im Nachbardorf und der dortigen Gemeindeverwaltung, scheinen mit Blick auf den Formalisierungsgrad und die Preisbildung zunächst die Gemeinsamkeiten zu überwiegen. Wie im Fall des ehemaligen Kolchos bewegen sich die Vereinbarungen in einer Zone zwischen Formalität und Informalität. Laut den Beteiligten - Mitgliedern der Gemeindeverwaltung und Betriebsangehörigen - werden zumindest die regelmäßig vom Unternehmen erbrachten Leistungen schriftlich fixiert:

»[Frage]: Leistet das Landwirtschaftsunternehmen Unterstützung im Bereich kommunaler Dienstleistungen?

[Sachbearbeiterin]: Soweit ich weiß, sponsern sie Unterhaltungsveranstaltungen. Wir haben dafür praktisch kein Geld. Dabei helfen sie uns. Und für den Winter haben wir einen Vertrag über das Schneeräumen auf den Dorfwegen abgeschlossen. Wir selbst haben keinen Traktor. Wenn Not am Mann ist, wenden wir uns an sie und sie haben noch nie abgelehnt.

[Frage]: Verträge bedeutet, dass sie Zahlungen leisten?

[Sachbearbeiterin]: Über die Säuberung der Wege? [...] Darüber gibt es einen Vertrag und wir bezahlen für diese Leistungen.«

(Sachbearbeiterin der Gemeindeverwaltung, 3.3.2010)

Gleichzeitig schwingt in den Arrangements jedoch stets ein Moment der Gemeinwohlorientierung und Gemeinschaftlichkeit mit, das den vertraglichen Rahmen übersteigt. So hebt eine Gemeinderätin, die gleichzeitig die dörfliche Krankenstation leitet, hervor, dass sie die Unterstützung der Agroholding in fast allen Bereichen der kommunalen Infrastruktur gerade aufgrund ihres informalen Charakters (respektive ihrer unvollständigen Formalisierung) schätze. Auf diese Weise, so ihre Sichtweise, könne man sich stets auf die Solidargemeinschaft des Dorfes berufen, innerhalb derer eine Bitte kaum ausgeschlagen werden könne. Sobald vollständig formalisierte Verträge existierten und Zahlungen formal eingefordert würden, so 
ihre Befürchtung, werde sich diese Form der Gemeinschaftlichkeit auflösen und die Ablehnungswahrscheinlichkeit seitens des Betriebs zunehmen. Befürchtet wird, um die folgende Interviewpassage mit Blick auf Weber (1964: 29f) zu paraphrasieren, ein Übergang von Gemeinschaft als affektueller oder traditionaler Zusammengehörigkeit zu Gesellschaft als zweckrationaler Zusammengehörigkeit - der jedoch, folgt man den oben dargestellten Ausführungen des Investors, tatsächlich längst eingesetzt hat:

»[Frage]: Kommt es vor, dass das Landwirtschaftsunternehmen im Bereich kommunaler Dienstleistungen hilft?

[Gemeinderätin]: Ja ja, das ist unentbehrlich. Was sollten wir ohne unser Unternehmen machen? Im Winter den Schnee räumen - an wen wenden wir uns? Dorthin wenden wir uns und bitten um Hilfe. Jetzt kommt der Frühling, es wird >subbotniki< [freiwillige Arbeitseinsätze in der Gemeinde, E.M.] geben, wir werden das Gelände vom Unrat befreien, der sich im Herbst und Winter angesammelt hat. Wen bitten wir dafür um eine Karre? Wir werden sie [das Unternehmen, E.M.] anrufen: >Seien Sie so gut, stellen Sie uns eine zur Verfügung «. Bis jetzt machen sie das noch auf freiwilliger Basis. Aber im Prinzip könnten sie für jede dieser Leistungen Geld verlangen. Aber es heißt, dass wir hier im Dorf zusammen leben, jeder kennt jeden...

[Frage]: Das heißt, die Hilfe wird auf informaler Ebene erbracht?

[Gemeinderätin]: Auf informaler Ebene.

[Frage]: Und kommt es vor, dass Verträge geschlossen werden?

[Gemeinderätin]: Bis jetzt läuft alles auf informaler Ebene. Aber sie haben uns schon darauf hingewiesen, dass das alles eigentlich offiziell sein sollte. Ehrlich gesagt, macht mir dieses Offizielle Angst.

[Frage]: Warum?

[Gemeinderätin]: Wir können nicht plötzlich bezahlen. Wenn jemand freiwillig hilft: >Vielen Dank, kommen Sie gern wieder, ich werde Ihnen zuhören, ich messe Ihnen den Blutdruck, ich helfe Ihnen<. Wie du mir, so ich dir. Wir sind Nachbarn. Aber wenn ein Vertrag geschlossen wird: >Aha, du schuldest mir noch eine Kopeke, so und so viel hast du noch nicht bezahlt $<$. Die Beziehungen werden sich vollständig verändern.«

(Gemeinderätin/Leiterin der Krankenstation, 25.2.2010)

Zum Ausdruck kommt der Aspekt der Gemeinschaftlichkeit insbesondere in den vereinbarten Preisen: Ebenso wie der ehemalige Kolchos betonen auch Vertreter der Agroholding, dass sich die Preise für die einzelnen Leistungen allein auf die anfallenden Selbstkosten beziehen (also im Wesentlichen Treibstoff und Arbeitskosten). Die Preisbildung wird explizit aus der Marktlogik herausgenommen und in Distanz dazu beobachtet - und dies gilt für Leistungen an Betriebsangehörige ebenso wie an >Externe<, darunter auch die Gemeindeverwaltung: 
»[Agronom 1 ]: Das ist sozusagen rein symbolisch - für unsere Leute. Auch wenn es vielleicht Einbußen für den Betrieb bedeutet, aber wenn es für die eigenen Leute ist, dann ist das nicht der Rede wert, dass es ein Verlust ist. Das sind unsere Leute, die sich für uns abrackern, die ihre Seele in ihre Arbeit legen. Deshalb, so scheint es mir, ist das nur ein symbolischer Preis. [Frage]: Und die übrigen Dorfbewohner, die nicht hier arbeiten, wenden sich auch an Sie und bitten um Unterstützung - oder kommt das nicht vor?

[Agronom ${ }_{2}$ ]: Ja, das kommt vor, sie kommen auch. Ihnen helfen wir auch.

[Frage]: Sind die Preise für sie dann höher?

[Agronom $\mathrm{m}_{2}$ : Ja ja, die sind etwas höher, aber immer noch niedriger als auf dem Markt. Denn wir wohnen hier alle nebeneinander, man muss den Leuten helfen.«

(Angestellte der Agroholding, 7.3.2010)

Leistungen, die darüber hinaus in Einzelfällen und Notsituationen anfallen, werden ohnedies ganz ohne quasi-vertragliche Rahmung, jedoch auch gegen (symbolische) Bezahlung erbracht. Als während eines meiner Feldaufenthalte im Winter ein Schaden an der Wasserleitung für chaotische Verhältnisse im Dorf sorgte, beteiligte sich der Betrieb aktiv an den Reparaturarbeiten:

»[Frage]: Gibt es Formen der Zusammenarbeit zwischen ihrem Unternehmen und der Gemeindeverwaltung?

[Agronom]: Natürlich gibt es die. Gerade haben wir zum Beispiel den Traktor für die Reparatur der Wasserleitung zur Verfügung gestellt. [lacht] Und wir helfen mit Brennholz. Was noch? Unterhaltungsveranstaltungen führen wir durch, an Feiertagen. Das machen wir alles zusammen, wir arbeiten zusammen, soweit wir können.

[Frage]: Ist diese Zusammenarbeit mehr oder weniger informal oder schließen sie Verträge darüber $a b$ ?

[Agronom]: Verträge im strengen Sinne gibt es nicht, das ist alles von Fall zu Fall.

[Frage]: Einfach nur so?

[Agronom]: Natürlich, Papierkram gibt es auch, wenn Dienstleistungen bestellt werden. Aber zum großen Teil auch nur so.«

(Angestellter der Agroholding, 7.3.2010)

Die Reparatur der defekten Wasserleitung hat allerdings eine Vorgeschichte: Beim Gespräch mit dem Leiter eines privaten Unternehmens für kommunale Dienstleistungen (9.3.2010) erfuhr ich zufällig, dass ebenjener Privatunternehmer der Bürgermeisterin angeboten hatte, sich um die Reparatur der Wasserleitung zu kümmern. Voraussetzung dafür sei für ihn jedoch eine offizielle Auftragsvergabe sowie der Abschluss eines Vertrags, in dem die zu leistenden Zahlungen festgehalten werden sollen. In Sorge um die Tragfähigkeit des kommunalen Budgets und mit 
Blick auf die Unverhandelbarkeit der vertraglichen Vereinbarungen lehnte die Bürgermeisterin dies jedoch ab und bat stattdessen die Agroholding um Hilfe.

Immer wieder deuten die Äußerungen von Dorfbewohnerinnen und Dorfbewohnern, Mitgliedern der Gemeindeverwaltung oder Betriebsangehörigen vordergründig auf Gemeinsamkeiten zwischen den Leistungen des ehemaligen Kolchos und der Agroholding in Form und Inhalt hin. Bedeutende Unterschiede kommen bestenfalls in Randbemerkungen zum Vorschein: Die Kooperationsstrukturen zwischen Gemeindeverwaltung und landwirtschaftlichem Großbetrieb, über die die Leistungsarrangements ausgehandelt werden, werden im Fall der Agroholding formalisiert und professionalisiert - und dies in zweifacher Hinsicht. Erstens werden innerhalb des Unternehmens sowohl eigens Stellen für die Zusammenarbeit mit der Gemeinde geschaffen als auch Aufgaben, die im Zusammenhang mit dörflichen Leistungen anfallen, formal - d.h. ausgehend von der Unternehmensführung - delegiert und damit in die bestehende Organisations- oder genauer Stellenstruktur integriert. Entsprechend beschreibt die Bürgermeisterin rückblickend, dass sich das anfänglich schwierige Verhältnis zum Investor sukzessive verbessert und das Unternehmen sich der Dorfgemeinschaft »zugewendet« habe. Zwar sei der Investor selbst nur selten im Dorf und auch darüber hinaus kaum persönlich zu erreichen, doch angesichts seiner Rolle als »Geschäftsmann« wird dafür durchaus Verständnis geäußert. Überdies könne sie sich mit ihren Anliegen an eine eigens dafür vorgesehene Mitarbeiterin des Unternehmens wenden, zu der sie mittlerweile in regelmäßigem Kontakt stehe:

»Sie [das Unternehmen, E.M.] haben sich uns, den Leuten, zugewendet - so kann man das beschreiben. Sie helfen. Es gibt in seiner Belegschaft sogar eine Person, die für Öffentlichkeitsarbeit zuständig ist - also für die Zusammenarbeit mit uns. Wir arbeiten mittlerweile sehr eng mit dieser jungen Dame zusammen. Sie ist häufig hier bei uns. Er [der Investor, E.M.] ist weit weg, er ist ein vielbeschäftigter Mensch, nicht erreichbar. Zu ihm dringst du mit deinen Fragen, mit deinen Problemen nicht so leicht durch. Das läuft alles über sie. Auf diese Weise hilft er uns.« (Bürgermeisterin, 25.8.2008)

Zwar klingt in der Äußerung der Bürgermeisterin die Normalitätserwartung an, dass die Zusammenarbeit mit einem Betrieb üblicherweise über dessen Spitze laufen und sich durch einen enges Verhältnis zwischen Gemeindeverwaltung und Betriebsleitung auszeichnen solle. Doch durch die Abwesenheit und Nicht-Erreichbarkeit des Investors wurde diese Erwartung durchbrochen und augenscheinlich hat sich die Bürgermeisterin mit den neuen Verhältnissen arrangiert.

Ein weiteres Beispiel für die zunehmende Professionalisierung sind die bereits erwähnten Feierlichkeiten zum Dorfjubiläum. Hier beschreibt der Investor selbst nicht nur sein (finanzielles) Engagement als unumgänglich, sondern hebt auch die 
Delegation der entsprechenden Aufgaben an seine Markenmanager hervor, die auf derartige Tätigkeiten spezialisiert seien:

»Das Dorfjubiläum in Beljaevka musste ausgerichtet werden, für Unterhaltung musste gesorgt werden. Wir haben das mit fast 150.000 Rubel gesponsert, haben das Fest ausgerichtet. Das heißt nicht nur Geld, auch meine Markenmanager aus der Geschäftsstelle in Perm sind hierher gekommen, haben die Feierlichkeiten organisiert, den Ablauf und alles. Sie sind dazu in der Lage, das ist ihr Spezialgebiet.« (Investor, 13.8.2008)

Während, wie in der ersten Episode deutlich wurde, in den Äußerungen des Kolchosvorsitzenden stets er selbst Dreh- und Angelpunkt der Kooperation mit der Gemeinde ist, die Leistungserbringung tatsächlich aber oftmals scheinbar unkontrolliert über untere Hierarchieebenen und ohne das Wissen der Betriebsleitung abgewickelt wird, verweist der Investor - ohne dabei Zweifel an seiner eigenen Führungsposition aufkommen zu lassen - explizit auf entsprechende Formalstrukturen im Unternehmen und auf Stellen, an die Aufgaben delegiert werden. Für das Unternehmen sind die Leistungen gegenüber der Gemeinde ein klar definierter und abgrenzbarer Tätigkeitsbereich und die dabei anfallenden Entscheidungen einzelnen Stellen eindeutig zuzuordnen. Für die Gemeindeverwaltungen ergibt sich daraus eine personelle und räumliche Differenzierung der Ansprechpersonen. Dies ist relevant, da sämtliche Leistungen der Agroholding im Dorf mangels sowjetischer Kontinuitäten tatsächlich neu initiiert (d.h. angefragt und begründet) werden müssen: Wenn es um Leistungen des Großbetriebs geht, werden nunmehr Rollen - und nicht Personen - adressiert. Eine Verquickung von Rolle und Person, wie sie für das Verhältnis von Bürgermeisterin und Kolchosvorsitzendem charakteristisch und für die Rolle des ehemaligen Kolchos im Dorf maßgeblich ist, wird in der Beziehung von Bürgermeisterin und Investor von vornherein unterbunden.

Verstärkt werden diese Effekte, indem sich die Formalisierungen und Professionalisierungen nicht nur unternehmensintern zeigen, sondern noch in einem zweiten Bereich stattfinden: Bei den Gemeinderatswahlen im Herbst 2008 - rund zwei Jahre nach dem ersten Auftreten des Investors im Dorf - traten von den insgesamt 15 Kandidaten fünf explizit als Gruppe und im Namen der Agroholding an. Neben dem Investor selbst kandidierten der Geschäftsführer, der leitende Agronom und die Lagermeisterin des Unternehmens; kurzerhand assoziiert wurde die Ehefrau des Geschäftsführers, die als Lehrerin an der Dorfschule arbeitet. Der Wahlspruch dieses Kollektivs - »Gemeinsam verbessern wir das Leben im Dorf « - tauchte unter anderem in großformatigen Anzeigen in der Lokalzeitung auf (vgl. »Ochanskaja Storona« vom 9.10.2008: 5). Illustriert waren die Anzeigen mit einem Bild der Dorfkirche, die zu dieser Zeit gerade vom Betrieb restauriert wurde. Konkret verwiesen wurde darüber hinaus auf Bereiche wie die Straßenbeleuchtung, die Gasifi- 
zierung, den Bau von Straßen und Wohnungen sowie die Unterstützung von Schule und Kindergarten - allesamt Themen, die im Dorf als relevant und deren Bearbeitung vor allem aus finanziellen Gründen als problematisch beobachtet werden. Kurz vor den Wahlen wurde die Wahlanzeige zusätzlich buchstäblich gerahmt von einem ganzseitigem Bericht über die Agroholding, der unter der Überschrift »Die Probleme des Dorfes sind unsere Probleme« den Betrieb als >Retter der Gemeinde inszenierte, sein soziales Engagement hervorhob - wovon sich der Investor selbst nur wenige Wochen zuvor im Interview distanziert hatte - und die begeisterten Reaktionen der Dorfbevölkerung darlegte. Verfasst wurde der Bericht von der Presseabteilung des Unternehmens (»Ochanskaja Storona« vom 9.10.2008: 5).

Das Ergebnis der Gemeinderatswahlen spiegelt einen Effekt dieser und ähnlicher Maßnahmen partiell wider: Von den fünf Kandidaten der Agroholding zogen drei in den siebenköpfigen Gemeinderat ein, so dass der Betrieb dort zwar über keine absolute Mehrheit, aber doch eine hinreichend starke Fraktion verfügte. Die Ehefrau des Geschäftsführers (55 Prozent) konnte dabei ebenso wie der Investor selbst (53 Prozent) hohe Stimmenanteile von über 50 Prozent auf sich verbuchen, die nur vom Resultat der Bürgermeisterin überstiegen wurden (60 Prozent). In das Gremium gewählt wurden auch der leitende Agronom des Betriebs sowie die Schuldirektorin, eine Mitarbeiterin des Lebensmittelgeschäfts und die Leiterin der Krankenstation (alle mit Stimmenanteilen um 40 Prozent) ${ }^{46}$ (vgl. »Ochanskaja Storona « vom 5.9.2008: 7 und vom 17.10.2008: 6). Im Nachhinein durchaus skeptisch beobachtet wurde, dass der Investor selbst aufgrund seiner unregelmäßigen Anwesenheit im Dorf sein Amt auf »nichtständiger« Basis wahrnahm und sich bei den Sitzungen meist von seinem Geschäftsführer vertreten ließ.

Das politische Engagement des Investors und seiner Mannschaft zeitigte zwei Effekte: Zum einen wird durch das explizite Auftreten im Kollektiv nicht nur die Person des Investors, sondern das Unternehmen als Ganzes mit politischer Bedeutung und Macht versehen. Die Art und Weise, wie dies geschieht, unterstreicht gleichzeitig die distinkte Logik des politischen Systems: Einfluss auf politische Entscheidungen wird nicht etwa durch eine personenbezogene Beziehung zur Bürgermeisterin gesucht, sondern durch die Übernahme eines Amtes - also einer politischen Rolle. Zum anderen werden die Unterstützungsleistungen durch die Agroholding nun explizit als politisches Thema inszeniert und in den Bereich des Gemeinderats als kommunales und für die Öffentlichkeit beobachtbares Forum verlegt: Die Protokolle zu den Sitzungen sind öffentlich einsehbar und der Gemeinderat hat über seine Entscheidungen gegenüber den Gemeindemitgliedern Rechenschaft abzule-

46 Die Ergebnisse dieser und anderer kommunaler und regionaler Wahlen und Referenden in der Region Perm sind auf der Website der regionalen Wahlkommission online verfügbar: http://permkrai.izbirkom.ru/way/951908.html (zuletzt eingesehen am 22.4.2015). 
gen. Auch wenn dies von keinem der Beteiligten explizit im Rahmen dieser Unterscheidung beobachtet wurde, kontrastiert diese Form der Kommunikation mit der informalen und von außen weitgehend intransparenten Grauzone, die durch die Verbindung zwischen Bürgermeisterin und Kolchosvorsitzendem im Nachbardorf konstituiert wird.

Mit Blick auf die Bedeutung des Gemeinderats als Organ kommunaler Selbstverwaltung dürften die Folgen ambivalent sein: Einerseits wird er als lokalpolitische Institution aufgewertet, indem er - auch hier liegt ein Kontrast zum kolchosdominierten Nachbardorf vor - tatsächlich zum Ort relevanter Entscheidungen wird, andererseits steht er durch seine neue personelle Zusammensetzung stark unter dem Einfluss der Agroholding.

\section{Brüchige Kontinuitäten}

Wie lassen sich die Anpassungs- und Strukturänderungsprozesse zusammenfassen, die im Zusammenhang mit dem Auftauchen einer Agroholding als neuartigem und vom ehemaligen Kolchos distinkten Betriebstyp im Dorf zu beobachten sind? Betrachtet und bewertet man die Etablierung des Unternehmens im Dorf im Horizont der Herausbildung eines Marktes für Agrarland - und damit zugleich im Horizont eines der klassischen Themen der postsowjetischen Transformationsforschung -, sind die Effekte ambivalent: Durch den Verkauf der Landanteile hat zwar die reine Anzahl an Transaktionen ebenso wie der Anteil der registrierten (und somit vollständig privatisierten) Landparzellen zugenommen, was gemeinhin als Erfolg mit Blick auf die Marktentwicklung gewertet wird (eine solche Perspektive vertreten z.B. Lerman 2001; Lerman/Shagaida 2007; Shagaida 2005; Wegren 2008). Ein marktförmiger Beobachtungsmodus, also Konkurrenzbeobachtung auf Preise und Zahlungsmöglichkeiten hin, flackerte für die Anteilseignerinnen und Anteilseigner jedoch nur für einen kurzen Moment auf. Potenzielle Anschlussmöglichkeiten wurden durch das Verhalten der Agroholding unmittelbar wieder unterdrückt.

Ähnlich ambivalent ist auch die Änderung von Erwartungs- und Kommunikationsstrukturen mit Blick auf die Operationsweise kommunaler Selbstverwaltung im Dorf. Analog zur Situation in Andreevka stehen dabei die wechselseitigen Beobachtungen, Zuschreibungen und Formen der Kommunikation zwischen dem landwirtschaftlichem Großbetrieb auf der einen und Gemeindeverwaltung und Dorfgemeinschaft auf der anderen Seite im Fokus. Mit Blick auf die postsowjetische Vergangenheit der Gemeinde und auf die Imperative der jüngsten Kommunalreform lassen sich die Persistenz tradierter und die Umstellung auf neue Strukturen gleichermaßen beobachten.

Brüche mit tradierten Strukturen werden in der Art und Weise deutlich, wie der Landwirtschaftsbetrieb mit den Ansprüchen und Erwartungen umgeht, die aus der 
lokalen dörflichen Umwelt an ihn herangetragen werden. Erstens lässt sich mit Blick auf die Leistungen des Betriebs eine Tendenz zu Formalisierung und Monetarisierung beobachten, die nicht zuletzt darauf zurückzuführen ist, dass gegenüber dem Unternehmen in keinerlei Hinsicht an tradierte Verantwortlichkeiten angeknüpft werden kann, sondern jede einzelne Leistungserwartung formuliert und begründet werden muss. Zweitens erbringt der Landwirtschaftsbetrieb zwar trotz anfänglich anderweitiger Pläne letztlich inhaltlich identische Leistungen wie der ehemalige Kolchos. Beobachtet werden diese jedoch nicht als selbstverständlicher und unhinterfragter Beitrag des Unternehmens zum dörflichen Gemeinwohl, sondern als unverzichtbarer Schritt zur Herstellung von Umweltlegitimität und damit im Horizont der Gewinnorientierung. Die Rede vom Sponsoring spiegelt dies sowohl in der Selbst- wie auch in der Fremdbeschreibung des Betriebs wider. Und drittens vollzieht sich die Professionalisierung der Leistungserbringung durch den Betrieb in Form der Schaffung von Stellen, in deren Folge sich die Differenzierung von Rollen und Personen beobachten lässt. Zwar gilt auf der Seite der Gemeindeverwaltung nach wie vor die Bürgermeisterin als zentrale Person und Ansprechpartnerin für Probleme jeglicher Art, ihr fehlt jedoch ein analoger Gegenpart auf der Seite des Betriebs: Der Investor selbst steht an der Spitze des Betriebs und tritt im Dorf entsprechend in Erscheinung, ist jedoch persönlich kaum erreichbar; zur Ansprechpartnerin aus der Abteilung für Öffentlichkeit pflegt die Bürgermeisterin zwar nach eigenem Bekunden ein gutes Verhältnis, diese befindet sich jedoch ebenfalls außerhalb des Dorfes; die Mitglieder der Betriebsleitung, die ebenfalls als wichtige Ansprechpersonen für Unternehmensdienstleistungen fungieren, leben und arbeiten zwar im Dorf, wurden jedoch in der Regel extern rekrutiert und fluktuieren (zumindest im Vergleich mit der hohen, meist seit sowjetischer Zeit bestehenden personellen Konstanz der ehemaligen Kolchose) häufig. ${ }^{47}$ Eine dörfliche Elite aus Bürgermeisterin und Betriebsleiter, die beide umfassend im Dorf verankert sind, bricht mit dem Auftauchen der Agroholding nachhaltig auf.

Kontinuität mit Blick auf die postsowjetische Vergangenheit lässt sich ungeachtet dieser Änderungen vor allem mit Blick auf dorfinterne Abhängigkeiten und die Schließung des Dorfes als Kommunikationszusammenhang beobachten: Unabhängig davon, ob die aus Gemeindesicht unverzichtbaren Dienstleistungen vom ehemaligen Kolchos auf tendenziell informaler oder von der Agroholding auf stärker formalisierter und professionalisierter Basis erbracht werden, reproduziert sich durch die Inanspruchnahme dieser Leistungen die Abhängigkeit des Dorfes von einer dominanten Organisation. Potenziell aufzubrechen wären diese Abhängigkeits-

47 Beispielsweise wechselte der Geschäftsführer ein erstes Mal bereits während meiner Forschungsaufenthalte und im Juli 2012 porträtierte die Lokalzeitung erneut einen neuen Stelleninhaber (»Ochanskaja Storona« vom 16.7.2012). 
strukturen von zwei Seiten: Entweder innerhalb des Dorfes durch die Herausbildung neuer Betriebe an den Grenzen der Agroholding, die in der Lage sind vergleichbare Leistungen zu erbringen, oder durch die Teilnahme der Gemeinde am Markt für kommunale Dienstleistungen außerhalb des Dorfes, indem Geschäftsbeziehungen mit entsprechenden Spezialunternehmen eingegangen werden. Beide Optionen scheinen zwar am Horizont auf, verschwimmen jedoch zunehmend, je nachhaltiger sich die Agroholding im Dorf etabliert. Letztere, so formuliert Nikulin (2010) treffend, generiert sich in der Nachfolge des ehemaligen Kollektivbetriebs als »Oligarchos «, ${ }^{48}$ um den herum sich die dörfliche Kommunikation bündelt. Die Dorföffentlichkeit gewinnt dabei zwar scheinbar an Einfluss, indem die Entscheidungen über die Unternehmensleistungen (zumindest teilweise) durch die Wahl von Unternehmensvertretern in entsprechende politische Ämter in den Gemeinderat als öffentliches und gegenüber den Gemeindemitgliedern rechenschaftspflichtiges Gremium verlegt werden und damit nicht mehr allein Gegenstand privater Aushandlungsprozesse zwischen Bürgermeisterin und Kolchosvorsitzendem sind. An der faktischen Dominanz des Unternehmens und seiner Entscheidungen im Dorf dürfte dieser Szenenwechsel indes wenig ändern.

\section{Die VeRteidigung DES DORFES - ODER: WIE VERÄNDERUNGSSORGEN INDIVIDUALISIERUNG UND FUNKTIONALE DIFFERENZIERUNG BEFÖRDERN}

Derselbe Investor, der im Jahr 2006 in Beljaevka die Dorföffentlichkeit über seine Geschäftspläne informiert und in der Folge begonnen hatte, den Anteilseignerinnen und Anteilseignern des dortigen insolventen Kolchos ihre Landanteile abzukaufen, streckte auch andernorts seine Fühler nach Agrarland aus und forderte so auch jenseits der Dorfgrenzen Beljaevkas, im benachbarten Andreevka, die tradierte räumliche Kongruenz von Kolchos und Gemeinde heraus. Nicht nur die Ausgangssituation, sondern auch die Vorgehensweise der Agroholding und die Reaktionen darauf unterschieden sich in Andreevka jedoch signifikant von den Strukturen und Ereignissen in Beljaevka: Auch in Andreevka war der ehemalige Kollektivbetrieb formal, aber nicht faktisch privatisiert. Die Anteilseignerinnen und Anteilseigner überließen ihre virtuellen Landparzellen dem Betrieb zur Pacht und verrechneten in Abwesenheit von Pachtzahlungen - die umfangreichen Leistungen des Betriebs für die Hoflandwirtschaften und die Gemeindeverwaltung als eine Art kollektive

48 Eine Wortschöpfung, die sich aus »Oligarch«, unter Anspielung auf die in Russland aus der Phase der Perestroika und der frühen postsowjetischen Zeit hervorgegangenen Wirtschaftsoligarchen, und »Kolchos« zusammensetzt. 
Gegenleistung. Anders als in Beljaevka existierte der ehemalige Kollektivbetrieb, obwohl auch er mit wirtschaftlichen Problemen kämpfte und staatliche Subventionen beanspruchte, aber als zahlungsfähiges und stabiles Unternehmen, dessen Leitung zudem mit der Gemeindeverwaltung allgemein und der Bürgermeisterin im Speziellen ein enges Verhältnis pflegte.

Der Kauf der Eigentumszertifikate von den Anteilseignern durch den Investor stellte sich für den ehemaligen Kolchos in Andreevka einerseits als konkrete und unmittelbare, andererseits jedoch als kaum detailliert beobachtbare und diffuse Bedrohung der eigenen Existenz dar: Unmittelbar, weil die Verfügungsrechte an Agrarland als zentralem Produktionsfaktor des Unternehmens auf dem Spiel stehen; diffus, weil mit den Eigentumszertifikaten keine konkreten, räumlich definierten Landanteile, sondern nur die Rechte auf die Herauslösung von Parzellen einer bestimmten Größe erworben werden. Die Gefahr, dass diese Rechte geltend gemacht werden und dem ehemaligen Kolchos das entsprechende Agrarland entzogen wird, beginnt mit dem Abschluss der Transaktion zwischen den Anteilseignern und Anteilseignerinnen und dem Investor über dem Kolchos zu schweben - wohlgemerkt ohne dass er notwendigerweise Kenntnis von Vollzug und Umfang der dezentral abgewickelten Transaktionen hat oder in den Verlauf der Ereignisse eingreifen könnte. Wer sein Eigentumszertifikat veräußert, bleibt für den ehemaligen Kolchos ebenso wie für die Gemeindeverwaltung bis zum Moment der Registrierung der entsprechenden Parzelle offiziell unbeobachtbar und somit auch nicht quanitifizierbar. Letzteres - die Quantifizierung der Landanteile, die sich in den Händen des Investors befinden - ist aus Kolchossicht vor allem im Hinblick auf die Mehrheitsverhältnisse unter den Anteilseignerinnen und Anteilseignern relevant: Verfügt der Investor über mehr als 50 Prozent der Eigentumszertifikate, ist er rechtlich nicht nur in der Lage, eigenständig eine Anteilseignerversammlung einzuberufen, sondern kann beispielsweise auch Entscheidungen über die Registrierung der Parzellen treffen und umsetzen, ohne dass der Kolchosvorsitzende oder die (Minderheit der) verbleibenden Anteilseignerinnen und Anteilseignern dagegen Widerspruch einlegen könnten. Die einzige direkte Möglichkeit der Einflussnahme bestände darin, dass der Kolchosvorsitzende ebenfalls mit dem Erwerb von Eigentumszertifikaten beginnt. Doch dazu ist er finanziell nicht in der Lage.

Verstärkt wurde die Unbeobachtbarkeit des Verkaufsprozesses für den ehemaligen Kolchos und für die Gemeindeverwaltung durch die Vorgehensweise des Investors in Andreevka: Anders als im benachbarten Beljaevka wählte er in Andreevka nicht die Dorföffentlichkeit um seine Geschäftsidee zu präsentieren, etwa mittels eines Auftritts auf einer Gemeindeversammlung oder vor dem Gemeinderat. Stattdessen gingen Unternehmensvertreter im Dorf von Tür zu Tür und unterbreiteten den Anteilseignerinnen und Anteilseignern ihr Kaufangebot im persönlichen Gespräch. Auch die Kaufverträge wurden - sofern sie zustande kamen - räumlich au- 
Berhalb von Andreevka abgeschlossen, ohne dass dafür sachlich zwingende Gründe vorlagen: Käufer ${ }^{49}$ und Verkäufer trafen sich im Beisein eines Juristen in einer Nachbargemeinde und wickelten dort die erforderlichen Formalitäten ab. Dass mit diesem Vorgehen die Hoffnung des Investors verknüpft war, in Antizipation von Widerständen gegen sein Vorhaben die Einwilligung zum Verkauf möglichst reibungslos einzuholen, ohne dass dieser Akt für die Gemeindeverwaltung beobachtbar geworden wäre und eine öffentliche Thematisierung hätte einsetzen können, liegt nahe. Zumindest die Bürgermeisterin ließ keinen Zweifel daran, dass sie die Ereignisse nicht zuletzt aufgrund der unterstellten Heimlichtuerei als hinterhältigen Angriff auf das Dorf bewertet:

»Ich habe nicht verstanden, wieso sie sich verstecken und sich weigerten, hier aufzutauchen. Du hast die Entscheidung getroffen [die Landanteile zu kaufen, E.M.], dann komm auch und sag, was du vorhast! Warum machst du das? Aber von denen kam nichts [...]. Aber wir unterhalten uns doch mit denen aus Beljaevka. Und [der Kolchosvorsitzende] hat es [dem Investor] sehr übel genommen, dass er das alles still und heimlich gemacht hat. Erst in diesem Jahr [knapp drei Jahre nach den Landverkäufen, E.M.] haben sie sich getroffen, da haben sie sich überhaupt zum ersten Mal gesehen.« (Bürgermeisterin, 12.3.2010)

Von den Verkäufen der Eigentumszertifikate durch Anteilseigner und Anteilseignerinnen des Kolchos erfuhren der Kolchosvorsitzende und die Bürgermeisterin zunächst nur gerüchteweise - und reagierten nach eigenem Bekunden mit Unglauben: Allein die Möglichkeit des Verkaufs der Landanteile an Außenstehende, so räumt die Bürgermeisterin rückblickend ein, überstieg damals den Horizont dessen, was sie als möglich beobachtete. Sie selbst habe ebenso wie der Kolchosvorsitzende zunächst den Kopf in den Sand gesteckt und den Verkauf nicht wahrhaben wollen. Als sie ihren Beobachtungshorizont schließlich doch an die Ereignisse, die sich im Dorf bereits vollzogen und nicht mehr ignoriert werden konnten, anpasste, beschreibt sie diese Erkenntnis als einen Schock - aufgrund der Gefahr, die dem Kolchos durch die Landverkäufe drohte und die ihr schlagartig bewusst wurde, und aufgrund der moralischen Implikationen des Verkaufs, die für sie zutage traten:

49 Ebenso wie in Beljaevka gilt auch in Andreevka die Regelung, dass Anteilseignerinnen und Anteilseigner des Betriebs über ein Vorkaufsrecht auf die Eigentumszertifikate verfügen und der Verkauf an Außenstehende nicht ohne weiteres möglich ist. Wie in Beljaevka wurden die Transaktionen deshalb formal über einen >Strohmann<, d.h. einen Anteilseigner, den der Investor gegen Bezahlung für seine Pläne gewinnen konnte, abgewickelt. Wer diese Person war, blieb im Dorf lange unbekannt. Wenn die Rede vom Käufer der Landanteile war, fiel stets der Name des Investors. 
»Und [der Kolchosvorsitzende] hat das sowieso lange nicht geglaubt, dass bei uns Land verkauft wird. Ich habe ihm gesagt, was hier bei uns passiert, aber er hat gesagt: Nein. Aber das ist wirklich bei uns geschehen. [...] Damals war das schon allen klar, dass das kein Scherz war, was da läuft. In diesem Moment habe ich so eine Angst erlitten, dass ich praktisch gelähmt war - der Schreck ist mir wie ein Schmerz bis in die Beine gefahren. Für mich war das überhaupt ein Schock, dass die Leute bei uns so etwas machen. Dass man Land verkauft, das brachliegt, wenn der Kolchos schon nicht mehr existiert, das habe ich verstanden - aber von einem existierenden Betrieb? Das ist mir zum ersten Mal begegnet, dass das Land von einem existierenden Betrieb verkauft wird. Und ich habe sehr gelitten." (Bürgermeisterin, 12.3.2010)

Nachdem der Schreck überwunden war, erlangte das Ziel, den ehemaligen Kolchos vor dem >Angriff $<$ zu bewahren und in möglichst unveränderter Form zu erhalten, für Bürgermeisterin und Kolchosvorsitzenden höchste Priorität. Um in den Verlauf der Ereignisse eingreifen zu können, galt es jedoch zunächst, sie beobachtbar (und greifbar) zu machen. Da weder der Kolchosleitung noch der Gemeindeverwaltung Informationen darüber zugänglich waren, wer sein Eigentumszertifikat bereits verkauft hatte, wählten sie komplementär dazu die Pachtverhältnisse als Ansatzpunkt der $>$ Ermittlungen $<$ :

»Diese Information haben wir nicht, wie viele Landanteile die Bevölkerung bei uns schon verkauft hat. Wir haben nur die Informationen von der Versammlung, wir wissen, wer dort seine Landanteile dem Kolchos zur Pacht überlassen hat. Wir wissen, wie viele Landanteile und entsprechend wie viele Hektar dem Landwirtschaftsbetrieb verpachtet werden. Aber von denen, die ihr Landanteil nicht dem Kolchos überlassen haben, wie viele davon verkauft haben oder einfach gar nichts damit machen - diese Information haben wir nicht.« (Sachbearbeiterin der Gemeindeverwaltung, 12.3.2009)

Als Forum, um die Dorföffentlichkeit zu mobilisieren und die Anteilseignerinnen und Anteilseigner im Hinblick auf diese Frage direkt anzusprechen, wählte man eine Anteilseignerversammlung, an der mit 241 Personen ein im Vergleich mit normalen Versammlungen geradezu überwältigend hoher Anteil der Gemeindemitglieder teilnahm. ${ }^{50}$ Diese Versammlung fand im August 2007 statt und wird nicht nur von der Bürgermeisterin rückblickend in dramatischen Worten beschrieben. Auch die Äußerungen der Gemeindemitglieder verdeutlichen, dass sie als ein-

50 Was formal als Anteilseignerversammlung stattfand (»Obščee sobranie učastnikov dolevoj sobstvennosti na zemel'nie učastki«), wird von den Beteiligten rückblickend meist als Gemeindeversammlung (kurz: »schod «) beschrieben, worin wiederum die beobachtete Kongruenz von Gemeinde und Kolchos zum Ausdruck kommt. 
schneidendes Ereignis zu einem wesentlichen Bestandteil der kollektiven Erinnerung wurde, das in den meisten Gesprächen in der einen oder anderen Form Erwähnung fand:

»Es war für mich sehr schwer, [dem Kolchosvorsitzenden] zu erklären, dass sich dieser Prozess bei uns vollzog und dass er sich sehr aktiv vollzog. Schließlich hat er endlich verstanden, dass das wirklich geschieht. Dann haben wir sofort mit allen rechtlichen Mitteln, die uns zur Verfügung standen, die Versammlung der Anteilseigner einberufen. Wir haben die Versammlung durchgeführt, auf der wir den Leuten erklärt haben, dass es jetzt unterschiedliche Preise gibt für das Umgraben der Gemüsegärten - wenn man einen Landanteil hat oder wenn man keinen hat. Dass [der Kolchosvorsitzende] für die Pacht zahlen wird, wenn auch keine großen Summen. Dass sich alle Preise danach unterscheiden werden, ob man seinen Landanteil [dem Kolchosvorsitzenden] überlässt oder ob man keinen Landanteil besitzt. Und dass die Preise sich sehr stark unterscheiden werden.« (Bürgermeisterin, 12.3.2010)

Die Beschreibung der Bürgermeisterin verweist bereits auf eine Reihe von (Abwehr-)Maßnahmen gegen den Investor, die auf und im Zusammenhang mit der Versammlung beschlossen wurden (vgl. dazu auch Moser/Lindner 2011): Erstens sollten diejenigen Landparzellen, die dem ehemaligen Kolchos weiterhin zur Pacht überlassen werden, mit Blick auf ihre räumliche Lage und ihre Grenzen bestimmt werden. Zweitens sollten die Pachtverträge auf eine neue rechtliche Grundlage gestellt werden. Drittens wurde als Äquivalent zu den seit Jahren ausbleibenden Pachtzahlungen durch den ehemaligen Kolchos ein monetärer Rahmen für Leistungsansprüche gegenüber dem Betrieb festgelegt: Wer dem Betrieb sein Land zur Bearbeitung überließ, erhielt fortan den Anspruch auf Leistungen und Vergünstigungen im Wert von 500 Rubel (knapp 15 Euro) jährlich. Viertens führte man für die Leistungen des Kolchos gegenüber den Haushalten im Dorf unterschiedliche Preise für Anteilseigner und Nicht-Anteilseigner ein. Diejenigen, die kein Landanteil besaßen oder ihr Eigentumszertifikat verkauft hatten, verloren zwar nicht vollständig den Zugang zu solchen Dienstleistungen, mussten aber fortan höhere Preise zahlen.

Im Hinblick auf das Thema und die Problemstellung meiner Arbeit werfen die Ereignisse, die in der Anteilseignerversammlung und den dort beschlossenen Maßnahmen vorläufig kulminieren, eine Reihe von Fragen auf: Wie werden die Ereignisse im Dorf beobachtet und welche Implikationen ergeben sich für die Gemeindeverwaltung und die Position des ehemaligen Kolchos in der Gemeinde? Welche Bedeutung entfalten die beschlossenen Maßnahmen im Dorfkontext und wie wird daran angeschlossen? Oder polemisch gewendet: Bleibt die kämpferische Abwehrrhetorik von Bürgermeisterin und Kolchosvorsitzendem im Kontext des Kaufangebots und der Versammlung folgenlos oder setzt sie Impulse für die Änderungen von 
Erwartungsstrukturen - und wenn ja: welche? Im Hinblick auf diese Fragen sollen die skizzierten Ereignisse in den folgenden Abschnitten noch einmal reflektiert werden.

\section{Lokalpolitische Kontrollverluste}

Zwar vollzog sich der Verkauf der ersten Landanteile über die individuellen Entscheidungen der einzelnen Haushalte, denen Vertreterinnen und Vertreter der Agroholding ihr Kaufangebot individuell unterbreiteten, die dorföffentliche Deutungshoheit über den Prozess übernahmen jedoch schon bald die Bürgermeisterin und der Kolchosvorsitzende. Bemerkenswert ist, dass der Kolchosvorsitzende, dessen Unternehmen von den Verkäufen in ungleich direkterer Form betroffen war als die Gemeindeverwaltung, dabei deutlich zurückhaltender reagierte als die Bürgermeisterin. Nach Aussage der Bürgermeisterin war er nur mit großem Überzeugungsaufwand dazu zu bewegen, sich auf >Gegenmaßnahmen< einzulassen. In den Interviews äußerte er sich zu den Landverkäufen nur zögerlich und nebulös, wohingegen die Bürgermeisterin die Ereignisse in unseren Gesprächen wiederholt und ausführlich beschrieb. In der Gemeindeöffentlichkeit schien sie die dominante Sprecherrolle übernommen zu haben. ${ }^{51}$ Auch die besagte Versammlung wurde von der Bürgermeisterin einberufen und geleitet, obgleich es sich formal nicht um eine Gemeindeversammlung, sondern um eine Versammlung der Anteilseigner des ehemaligen Kolchos handelte, die Zuständigkeit also formal bei der Betriebsleitung gelegen hätte.

Das Kaufangebot des Investors, so wird aus den Beschreibungen der Bürgermeisterin deutlich, beobachtete sie als Angriff auf das Dorf aus dem Hinterhalt, der die Existenz von Kolchos und Gemeinde gleichermaßen in Frage stellte. Was als Bedrohung wahrgenommen wurde, lässt sich systemtheoretisch als ein Absinken der Wahrscheinlichkeit bestimmter gegebener dorfinterner Strukturen beschreiben, das durch ein Kommunikationsangebot aus der Umwelt des Dorfes bewirkt wird. Es hat zur Folge, dass ebenjene Strukturen abgelehnt werden und von tradierten Handlungsmustern abgewichen wird:

»Je unwahrscheinlicher die Struktur, um so eindeutiger läßt sich das, was sie ausschließt, innerhalb des Systems selbst artikulieren und um so wahrscheinlicher ist es, daß dann auch

51 Die Ereignisse im Zusammenhang mit den Landverkäufen spielten sich überwiegend in der zweiten Hälfte des Jahres 2007 ab. Mein erster Forschungsaufenthalt fand rund ein Jahr später im August 2008 statt. Meine Analyse stützt sich deshalb auf rückblickende Beschreibungen der Beteiligten, auf Protokolle und auf Zeitungsberichte, jedoch nicht auf eigene Beobachtungen der Ereignisse selbst. 
strukturwidriges und dadurch irritierendes Kommunizieren versucht wird und vorkommt.« (Kieserling 1999: 272)

In diesem Sinne wurde das selbstverständliche Verpachten der Landanteile an den ehemaligen Kolchos als Struktureigenschaft des Dorfes und Voraussetzung dafür, dass der Betrieb faktisch in sowjetischer Form fortbestand, durch das Kaufangebot des Investors herausgefordert: Die Tatsache, dass man sein Land ohne unmittelbare Gegenleistung dem Kolchos überlässt, war teils auf sowjetisch geprägte Gewohnheiten, teils schlicht auf das Fehlen alternativer Verwendungsmöglichkeiten zurückzuführen und nicht zwingend mit einer Entscheidung der Anteilseignerinnen und Anteilseigner >für< den Betrieb verknüpft. Durch das Kaufangebot des Investors erweiterte sich das Möglichkeitsspektrum für die Verwendung der Eigentumszertifikate. Aus der Sicht der meisten Anteilseignerinnen und Anteilseigner entstand nun erstmals Entscheidungsbedarf über die Verwendung des Eigentumszertifikats und die Wahrscheinlichkeit, dass die Wahl auf die Fortführung des Pachtverhältnisses fiel, sank angesichts der neuen Alternative.

Für die Bürgermeisterin kam der Verkauf der Eigentumszertifikate einem Kontrollverlust in mehrfacher Hinsicht gleich, wie sich aus dem Kontrollbegriff ableiten lässt, den Baecker (2012) unter Verweis auf Ashby und Luhmann formuliert:

» Kontrolle< heißt jetzt, dass der Beobachter seine Interaktion mit dem Gegenstand auf die Bestätigung und Enttäuschung von Erwartungen hin beobachtet, diese Bestätigung und Enttäuschung implizit oder explizit protokolliert und seine Interaktionsangebote derart variiert, dass neue wiederum protokollierbare Erfahrungen gemacht werden können. Der Begriff der $>$ Kontrolle< beschreibt die Arbeit am Gedächtnis der Interaktion.«(Baecker 2012: 160)

An dieses Verständnis anschließend bedeutet Kontrollverlust, dass Ereignisse nicht nur von den Erwartungsstrukturen eines Beobachters abweichen, sondern auch das Ausmaß dieser Abweichung für ihn unbeobachtbar ist. In diesem Sinne realisierte die Bürgermeisterin, dass sich in >ihrem< Dorf mit den ersten Landverkäufen Ereignisse vollzogen, über die sie nicht nur in Unkenntnis war, sondern die sie schlicht für unmöglich gehalten hatte. Dies gilt wohlgemerkt unabhängig davon, dass der Zertifikatsverkauf seit der formalen Kolchosprivatisierung in den frühen 1990er Jahren nicht nur rechtlich vorgesehen, sondern transformationsstrategisch mit Blick auf die Bildung von Marktstrukturen in der russischen Landwirtschaft sogar explizit erwünscht war. Zudem war der Verkauf der Landanteile - ob von ihr bemerkt oder unbemerkt - für sie ein Indikator dafür, dass ihre moralischen Standards darüber, was im Dorf möglich ist und was nicht - »das Land eines aktiven Unternehmens verkauft man nicht « -, plötzlich sichtbar in Widerspruch zur dörflichen Kommunikation traten. Den Moment, in dem sie aufgrund ihres Wissens um die Landver- 
käufe (wenn auch noch nicht um deren genaues Ausmaß) nicht mehr umhin konnte, diese Abweichung zu registrieren, beschreibt die Bürgermeisterin als einen Schock.

In der Folge galt es für sie zunächst, die Kontrolle wiederzuerlangen, indem sie die Abweichung von Tatsächlichem und Erwartetem - also die Differenz von Sollund Ist-Wert - beobachtbar machte, um anschließend steuernd in den Prozess einzugreifen und die Differenz zwischen den beiden Seiten der Unterscheidung tatsächlich|erwartet zu verringern. Denn mit Luhmann ist Steuerung nicht nur Beobachtung als Bezeichnung im Rahmen einer Unterscheidung, sondern »vielmehr eine ganz spezifische Verwendung von Unterscheidungen, nämlich das Bemühen um eine Verringerung der Differenz« (Luhmann 1988: 328). Angesichts der Abhängigkeit der Gemeindeverwaltung vom Kolchos - in den Worten der Bürgermeisterin: »Für mich ist das eine Katastrophe, wenn der Kolchos zerstört wird « (8.8.2008) - kam für sie als Ansatzpunkt zur Verringerung dieser Differenz nur das Faktische in Frage und nicht etwa die Anpassung der eigenen (normativen) Erwartungen. ${ }^{52}$ Es galt, die Landverkäufe zu unterbinden, um den Betrieb in seiner sowjetischen Form und die daran anschließenden Strukturen möglichst unverändert zu erhalten.

\section{Schwelende Ablehnung und internalisierte Gegnerschaft}

Hinsichtlich der Verwendung der Eigentumszertifikate durch die Anteilseignerinnen und Anteilseigner lagen die divergierenden Interessen der Beteiligten auf der Hand: Auf der einen Seite standen die Bürgermeisterin und der Kolchosvorsitzende, auf der anderen Seite der Investor. Die Bürgermeisterin verhehlt nicht, dass sie Letzterem ihre Ablehnung des Kaufangebots gerne persönlich und möglichst in der Dorföffentlichkeit kundgetan hätte. Sowohl die Ablehnung als auch daran anschließend der Aufbau eines Konfliktsystems scheiterten jedoch daran, dass der Investor aufgrund seiner Abwesenheit im Dorf für diese Art der Kommunikation unadressierbar war - was die Bürgermeisterin ihm auch explizit vorwirft. Zu beobachten war die Figur des Investors aus der Dorfperspektive lediglich schemenhaft. Das Nein der Bürgermeisterin zum Kaufangebot schwelte, fand jedoch keinen Anhaltspunkt um sich zu entzünden und kommunikativ sichtbar oder gar zum Thema weiterer Kommunikation werden zu können. Letzteres aber wäre die Voraussetzung für einen Konflikt oder gar ein Konfliktsystem (vgl. auch Luhmann 2003 [1975]: 5; Schneider 1994: 202f): »Um einen Konflikt handelt es sich nur

52 Die fehlende Anpassung an das Faktische ist für Luhmann (1997: 638) wesentliches Merkmal normativer Erwartungen: »Normative Erwartungen sind ja kontrafaktische Erwartungen, sind Erwartungen, die etwaigen Enttäuschungen nicht angepaßt, sondern aufrecht erhalten werden.« 
dann, wenn die eine Kommunikation der anderen widerspricht und diesen Widerspruch in ihrer Selbstsinngebung reflektiert. Konflikte sind negierte Kommunikation, die als solche zum Thema weiterer Kommunikation werden« (Kieserling 1999: 266f). Divergierende Interessen allein reichen dagegen aus systemtheoretischer Perspektive nicht für die Konstitution eines Konflikts hin: »Man mag anderer Meinung sein, ein Angebot für unannehmbar halten, Interessenlagen können unvereinbar sein - solange daraus kein >Nein< in der Kommunikation entsteht, besteht sozial kein Konflikt« (Schneider 1994: 201). Zumindest in der von der Bürgermeisterin erwünschten Form kam genau dies also nicht zustande und ihre Ablehnung lief an dieser Stelle ins Leere.

Ein adressierbares Nein tauchte im Zusammenhang mit dem Kaufangebot des Investors jedoch noch an anderer Stelle auf, gleichwohl es auch hier nicht zum Konflikt kam: Mit Blick auf die eigentliche Verkaufsentscheidung war die Haltung von Bürgermeisterin und Kolchosvorsitzendem rechtlich ohnedies nicht unmittelbar relevant. Über das Zustandekommen der Transaktion entschieden dezentral die einzelnen Anteilseignerinnen und Anteilseigner und im Hinblick auf das aggregierte Resultat dieser Einzelentscheidungen wird deutlich, dass das Kaufangebot weit häufiger abgelehnt als angenommen wurde. Während die Vertreterinnen und Vertreter der Agroholding der überwiegenden Mehrheit der Anteilseignerinnen und Anteilseigner ihr Kaufangebot unterbreitet hatten, wechselten letztlich nur 15 Prozent der Eigentumszertifikate tatsächlich ihren Besitzer. Das Nein der Anteilseigner, das in diesen Fällen direkt in der Interaktion mit den Vertretern der Agroholding ausgesprochen wurde und somit im Unterschied zur Ablehnung durch die Bürgermeisterin adressierbar war, wurde jedoch ohne Umschweife akzeptiert und nicht weiter auf den Verkauf insistiert:

»[Frage]: Haben Sie jemals darüber nachgedacht, Ihr Eigentumszertifikat zu verkaufen?

[Rentnerin]: [...] Nein, ich habe nicht daran gedacht zu verkaufen. Bei uns haben viele verkauft, aber ich nicht. Sie sind auch zu mir gekommen und haben gefragt: >Wozu brauchst du den Landanteil?<. Und ich habe gesagt: >Der soll ruhig beim Kolchos bleiben<.

[Frage]: Wer hat das gefragt?

[Rentnerin]: Leute sind gekommen, irgendjemand von den Buchhaltern [des Investors]. Was hast du mit dem Landanteil vor - willst du es verkaufen oder willst du, dass es beim Kolchos bleibt? Nun, und ich habe gesagt: >Es soll ruhig beim Kolchos bleiben, ich werde nicht verkaufen $<$ So kann ich hingehen [zum Kolchos, E.M.], bitte um etwas und er [der Kolchosvorsitzende, E.M.] gibt seine Zustimmung.«

(Rentnerin, 22.2.2010)

Die Aufregung, die sich im Dorf an das Bekanntwerden des Kaufangebots und der Landverkäufe anschloss, lässt sich also weder als Konflikt zwischen Bürgermeiste- 
rin und Investor verstehen noch zwischen Anteilseignern und Investor. Im ersten Fall lagen lediglich divergierende Interessen beider Parteien vor, im zweiten Fall fand zwar eine direkte Ablehnung von Kommunikation statt, aber diese wurde nicht weiter thematisiert. In den Ereignissen, die sich in der Folge im Dorf vollzogen, spiegelt sich jedoch die Kombination dieser beiden möglichen, aber nicht realisierten Konflikte wider: Zwar kam der >Angriff< auf das Dorf in Form des Kaufangebots zunächst von außen. Die konfliktkonstituierende Gegnerschaft wurde in der Folge jedoch in das Dorf hineinverlagert, indem die individuellen Entscheidungen für den Verkauf des Eigentumszertifikats den Anteilseignerinnen und Anteilseignern als Ablehnung der dörflichen Strukturen zugerechnet und diese Ablehnung thematisiert wurde. Der Investor blieb auf diese Weise der Auslöser der Ereignisse, dem man zu schaden und dessen Interessen man zu durchkreuzen versuchte. Der Gegner des Dorfes, an dem man sich abarbeiteten konnte, wurde durch diese Verschiebung ins Dorf hineingeholt: Das Nein zum Verkaufsangebot wurde als ein Ja zur Gemeinde gewertet und vice versa - und beides wurde ausführlich thematisiert. Die daran anknüpfende Gegnerschaft zwischen Anteilseignern und Nicht-MehrAnteilseignern, die durch das Betreiben von Bürgermeisterin und Kolchosvorsitzendem angestoßen wurde, fand unmittelbar Anschluss unter den Dorfbewohnern und Dorfbewohnerinnen selbst. So beobachtete die Bürgermeisterin, dass sich das Dorf beinahe schlagartig in »Rote« und »Weiße« geteilt habe (12.3.2010) und spielt mit dieser Formulierung auf die (bolschewistische) Rote und die (konservative) Weiße Armee an, die sich im russischen Bürgerkrieg im Anschluss an die Oktoberrevolution von 1917 bis 1920 gegenüberstanden. Der genaue >Frontverlauf< zwischen beiden Seiten blieb im Dorf zunächst jedoch diffus: Zwar wusste man, dass sich diverse Landverkäufe und damit die vermeintliche Ablehnung der tradierten dörflichen Strukturen vollzogen hatten und weiterhin vollzogen, wer sein Eigentumszertifikat verkauft und sich damit auf die >gegnerische< Seite des Investors gestellt hatte, bleibt jedoch lange Zeit unklar. ${ }^{53}$

53 Als ironischer Nachtrag zu den Ereignissen lässt sich an dieser Stelle ergänzen, dass im Jahr 2010, also rund drei Jahre später, der Zufall der Gemeindeverwaltung eine Liste des Bezirkskatasteramts in die Hände spielte, auf der sämtliche Verkäufe der Eigentumszertifikate vermerkt waren und die somit ebenjene Informationen enthielt, die die Gemeindeverwaltung zuvor mühsam zusammenzutragen versucht hatte. Deren Inhalt wurde von der Bürgermeisterin zwar immer noch mit großem Interesse zur Kenntnis genommen, die allgemeine Aufregung hatte sich zu diesem Zeitpunkt jedoch längst gelegt. 


\section{Lokalpolitische Steuerungskonstruktionen}

Das Mittel der Wahl, zu dem die Bürgermeisterin in dieser Situation griff, waren zunächst moralische Appelle, mit denen die >Verbündeten< in ihrer Entscheidung bestärkt und potenzielle >Gegner $<$ rechtzeitig, also vor einer Verkaufsentscheidung, auf den >richtigen< Weg zurückverwiesen werden sollen. Dass sowohl die Bürgermeisterin als auch der Kolchosvorsitzende diese Appelle als >Aufklärungsarbeit< bezeichneten, verweist auf ihre Form der Beobachtung der eigenen Position im Dorf. Im Begriff der Aufklärung schwingt erstens der Anspruch auf exklusive Gültigkeit der eigenen moralischen Standards mit (»Kolchosland verkauft man nicht«) und daran anschließend auch auf die uneingeschränkte Deutungshoheit über die Verkaufsmotive: Die Verkaufsentscheidungen wurden einzig auf die Unterscheidung vernünftig|unvernünftig bezogen, hinter der sich das vermeintliche dörfliche Gemeinwohl verbirgt. Davon abweichende Motive, also beispielsweise wirtschaftliche Argumente und der Verweis auf finanzielle Probleme, wurden hingegen auf Kurzsichtigkeit und Unwissenheit über die > wahre < Bedeutung des eigenen Handelns zurückgeführt und somit auf die Seite der Unvernunft platziert:

»Wenn die Summe für einen Landanteil in irgendeiner Form solide gewesen wäre, dann hätte man unsere Bürger ja verstehen können. Aber sie haben 8.000 [Rubel, E.M.] bekommen und ich habe sie gefragt: >Was wollt ihr mit den 8.000 anfangen? Vor zwei Jahren habe ich für 8.000 noch nicht mal einen Mantel gekriegt. Wieso habt ihr euch entschieden, für $8.000 \mathrm{zu}$ verkaufen? Wofür - für eine Antenne? ${ }^{54}$ Das ist alles, was man damit machen kann.< Eine Antenne, aber kein Landanteil mehr. Was war euer Problem? [...] Ihr musstet dafür nicht mal Steuern zahlen.« (Bürgermeisterin, 12.3.2010)

Etwaige Reue als Indikator für die erfolgreiche Aufklärung der Gemeindemitglieder rechneten Bürgermeisterin und Kolchosvorsitzender unumwunden ihren eigenen Einflussversuchen zu. Die Entscheidung gegen den Verkauf wurde nicht als Resultat einer wirtschaftlichen Entscheidung beobachtet, sondern als gestiegenes Verantwortungsbewusstsein im Umgang mit den Landanteilen - also als Vernunft im Sinne des dörflichen Gemeinwohls. Dies geht aus den Beschreibungen der Bürgermeisterin hervor:

»Eine Babuschka kommt und sagt: >Verstehst du, ich habe das Geld in dem Moment für dieses und jenes gebraucht. $<$ Ich verstehe das natürlich nicht. Da kam zum Beispiel Tamara Ivanovna. Sie hat ihr ganzes Leben beim Kolchos gearbeitet, so eine ruhige, ausgeglichene Frau.

$54 \mathrm{Zu}$ dieser Zeit hatten sich einige Haushalte Antennen für besseren Fernsehempfang gekauft. 
Sie hat keine Kinder und niemanden. Sie hat so darüber geweint, dass sie ihren Landanteil verkauft hat $->$ Verzeihen Sie mir, dass ich das getan habe $<$. Sie hat mir erklärt, wozu sie das Geld in dem Moment gebraucht hat, was sie in dem Moment machen musste. Wenn das nicht so gewesen wäre, hat sie gesagt, dann hätte ich niemals verkauft.« (Bürgermeisterin, 12.3.2010)

Und eine vergleichbare Sichtweise kommt auch in der Darstellung durch den Kolchosvorsitzenden zum Ausdruck:

»Die Leute verhalten sich jetzt viel verantwortungsvoller bei dieser Frage [der Landverkaufs, E.M.]. [...] Wenn du heute verkaufst, dann kann es sein, dass es den Betrieb morgen nicht mehr gibt. Das ganze Dorf nimmt doch Kolchosdienstleistungen in Anspruch. Wahrscheinlich haben sie das aus patriotischer Überzeugung gemacht - wir brauchen den Betrieb, er braucht uns - und deshalb haben die Verkäufe aufgehört.« (Kolchosvorsitzender, 20.2.2010)

Unabhängig von konkreten Inhalten klingt zweitens in den Äußerungen die Überzeugung von der dorfweiten Geltung und Durchschlagskraft des eigenen Handelns und von einer Art >Monopol< auf Sinnselektion an (Sinnselektionen der Umwelt - der Gemeindemitglieder selbst - werden im Umkehrschluss nicht zugelassen). Dass überhaupt Verkäufe stattgefunden hätten, sei letztlich allein darauf zurückzuführen, dass sowohl die Bürgermeisterin als auch der Kolchosvorsitzende es versäumt hätten, in ausreichendem Maße Aufklärungsarbeit zu leisten. Dies sei jedoch nachgeholt worden und die Landverkäufe hätten daraufhin geendet.

»[Frage]: Wie viel Land wurde von den Leuten ungefähr verkauft?

[Kolchosvorsitzender]: Keine große Fläche. So ungefähr ein Fünftel, ein Viertel. Aber jetzt hat das sowieso aufgehört.

[Frage]: Und wieso haben sich die Leute entschieden, ihren Landanteil zu verkaufen?

[Kolchosvorsitzender]: Das ist in erster Linie meine Schuld. Ich habe nicht rechtzeitig Aufklärungsarbeit geleistet. Nachdem wir die Versammlung dann durchgeführt haben, habe ich Aufklärungsarbeit geleistet und die Verkäufe der Landanteile haben praktisch aufgehört.« (Kolchosvorsitzender, 20.2.2010)

Und erneut schlägt die Bürgermeisterin mit ihrer Beschreibung in eine ähnliche Kerbe:

»Was nützt dir das? Ich denke, dass die Leute das in der Mehrheit der Fälle unbewusst gemacht haben oder es gab irgendeine materielle Notwendigkeit, irgendwelche Ausgaben, die zu decken waren. [...] Wenn du anfängst, mit ihnen zu sprechen, dann wird ihnen das in dem Moment klar und sie erröten - warum haben wir das gemacht? Aber in dem Moment haben 
sie es schon getan. Deshalb haben wir den Leuten auf der Versammlung erklärt, wohin das alles führen kann, das kann zum Verschwinden der Arbeitsplätze ihrer eigenen Kinder führen, ihrer Verwandten, ihrer Bekannten. Und wohin wendet ihr euch? Wer wird euch euren Gemüsegarten umgraben? Macht das [der Investor]? Nein, der kommt nicht. Ihr geht [zum Kolchosvorsitzenden]? Er wird sich weigern. Ihr habt den Landanteil verkauft, ihr habt ihn ihm weggenommen und verkauft. Und dann wurde den Leuten das bewusst.« (Bürgermeisterin, 12.3.2010)

Das Bemühen darum, die Ereignisse in der Gemeinde den eigenen Handlungen und Entscheidungen zuzurechnen, lässt sich mit Luhmann als Versuch beobachten, die Gefahr der Landverkäufe in ein Risiko zu transformieren (Luhmann 2008: 362ff). Wenn dies gelingt und entsprechende Kausalitäten zwischen dem eigenen politischen Handeln - der Aufklärungsarbeit - und den Ereignissen im Dorf - den Verkäufen - hergestellt werden, stützt dies die Konstruktion der eigenen politischen Handlungsfähigkeit (zu Kausalschemata in der Politik vgl. auch Kusche 2008: insb. 95ff). Gelingt dies nicht, bleibt der Bürgermeisterin und dem Kolchosvorsitzendem nur, die Ereignisse im Dorf und die Entscheidungen der Anteilseigner zu erleben.

Ob diese Form der Selbstbeobachtung als zentrale, mit Deutungshoheit ausgestattete Instanz im Dorf zutreffend ist und die Appelle über die semantische Ebene hinaus Wirkung entfalten, gerät mit Blick auf die Äußerungen der Gemeindemitglieder selbst in Frage. Deren Beschreibungen ihrer Motive für oder gegen einen Verkauf schließen nicht reibungslos an die Appelle an, stattdessen treten drei Begründungszusammenhänge gleichermaßen hervor, die nicht nur auf den Kolchos, sondern gerade auch auf wirtschaftliche Rationalitäten verweisen: Zentral ist erstens eine instrumentelle Argumentation mit Blick auf die praktischen Leistungen des ehemaligen Kolchos für die öffentliche Infrastruktur und gegenüber den Hoflandwirtschaften der Gemeindemitglieder. Würde der Kolchos verschwinden, so die Überlegung, entstünde eine Lücke, die aufgrund der chronischen Unterfinanzierung der Gemeindeverwaltung nicht geschlossen werden könnte. Weiter geht ein zweiter Argumentationsstrang, der darüber hinaus die kollektive Bedeutung des Betriebs in den Vordergrund rückt. Der Kolchos gilt als Indikator und Garant für die Existenz und (Über-)Lebensfähigkeit von Dorf und Dorfgemeinschaft nicht nur in rein instrumenteller, sondern auch in ideeller Hinsicht: Der Betrieb stellt Arbeitsplätze bereit, verleiht dem dörflichen Leben dadurch >Sinn $<$ und generiert Solidarität und Zusammenhalt innerhalb der Gemeinde. In diesen Strang fügen sich Äußerungen wie »Der Kolchos ist dorfkonstituierend « (Privatbauer, 21.2.2010), »Wir sind aneinander gebunden« (Bürgermeisterin, 11.8.2008) oder auch: 
»Ohne den Kolchos gäbe es kein Dorf. Das Dorf würde zugrunde gehen, man könnte hier nirgends arbeiten, nichts, womit man sich beschäftigen könnte - die Leute würden förmlich davonlaufen.«(Kolchosangestellte, 19.2.2010)

Drittens spielen ökonomische Überlegungen nicht nur bei der Entscheidung für, sondern auch gegen den Verkauf eine Rolle: Nicht das Kaufangebot als solches, sondern die Höhe des Kaufpreises und Spekulationen auf Preissteigerungen haben zur Ablehnung des Kaufangebots geführt. Hintergrund der Spekulationen sind Pläne für den Bau einer Brücke über den nahegelegenen Fluss, der die Transportmöglichkeiten in die Regionshauptstadt Perm deutlich verbessern und die Attraktivität der Gemeinde als Standort für Wochenendhäuser wohlhabender Städter erhöhen könnte:

»Mein Mann hat mich überzeugt, dass es sich nicht lohnt, jetzt zu verkaufen, dass das Land später teurer sein wird.«(Kolchosangestellte, 22.2.2010)

Ohne die Bedeutung dieser Argumente im Einzelnen gegeneinander aufrechnen zu wollen (und zu können), wird deutlich, dass die >Aufklärungsarbeit< von Bürgermeisterin und Kolchosvorsitzendem zumindest nicht vollumfänglich verfing. Stattdessen wurde die Verkaufsentscheidung von den Anteilseignerinnen und Anteilseignern auch auf >unerwünschte< wirtschaftliche Aspekte hin beobachtet und die Landanteile wurden somit sukzessive in den Gegenstandsbereich von Knappheitskommunikation hineingezogen. Dass moralpolitische Appelle allein nicht ausreichten, um in den Verkaufsprozess steuernd einzugreifen und die Ereignisse in die gewünschten Bahnen zu lenken, wurde von Bürgermeisterin und Kolchosvorsitzendem zwar nicht direkt eingeräumt. Diese Erkenntnis kommt jedoch implizit in einer Parallelstrategie zum Ausdruck, die ebenjenem Sachverhalt Rechnung trägt und die Verkaufsentscheidung in ihrer (wirtschaftlichen) Eigenständigkeit akzeptiert, ohne auf das Moment der Einflussnahme zu verzichten. Sichtbar wird diese Strategie in Form jener Maßnahmen, die auf und im Kontext der eingangs bereits erwähnten Anteilseignerversammlung beschlossen wurden und den lokalen Kommunikationszusammenhang maßgeblich irritierten.

\section{Formalisierung und Monetarisierung der Pachtverhältnisse}

Die erste Maßnahme, die auf der Anteilseignerversammlung festgelegt wurde, knüpfte unmittelbar und in materieller Form an das Sichtbarkeits- und Beobachtbarkeitsproblem der Landverkäufe an und bestand in der räumlichen Spezifikation der gepachteten Landanteile. Wenn nicht eindeutig zu klären war, wer sein Eigentumszertifikat bereits verkauft hatte, galt es zumindest die Eigentumsverhältnisse des 
ehemaligen Kolchos zu visibilisieren und abzusichern. Nach Auskunft einer Sachbearbeiterin der Gemeindeverwaltung verfügte der ehemalige Kolchos zum Zeitpunkt des Kaufangebots des Investors über 283 Landanteile von je 7,5 Hektar, bearbeitete also eine Fläche von rund 2.100 Hektar Agrarland. Um zu verhindern, dass der Investor die von ihm erworbenen Eigentumszertifikate für Teile dieser Fläche geltend machte und sich entsprechend Parzellen herauslösen und als Eigentum registrieren ließ, initiierte der Kolchosvorsitzende - mit Zustimmung der Anteilseignerinnen und Anteilseigner - seinerseits einen Registrierungsschritt: Die Lage und Grenzen der bislang unspezifizierten Landanteile, die dem Betrieb überlassen wurden, sollten nun erstmals bestimmt und amtlich registriert werden. Dafür kamen juristische Instrumente zum Einsatz, die in den dörflichen Eigentumspraktiken bislang keine Rolle gespielt hatten. Der Registrierungsprozess spielte sich weitgehend außerhalb des Dorfes ab, war für die Beteiligten aus dem Dorf nur schwer einzusehen und mit hohem bürokratischem Aufwand verbunden. Die Dokumentation im Dorf übernahm eine Sachbearbeiterin der Gemeindeverwaltung, die auf einer Karte des Gemeindeterritoriums die entsprechenden Flächen einzeichnete und markierte:

»Bei uns - das Protokoll habe ich Ihnen ja schon gegeben - bei uns gab es im Jahr 2007 eine allgemeine Anteilseignerversammlung, auf der wir festgelegt haben, welche Felder der Kolchos pachtet. Und welche Felder in erster Linie für die Herauslösung der Landanteile vorgesehen sind. Das heißt, wenn jetzt jemand seinen Landanteil herauslösen will, dann steht das Feld dafür schon fest. [...] Bei uns möchte [der Kolchosvorsitzende] das Herauslösen dieser Landanteile nun juristisch bestätigen lassen, dass läuft alles über die Registrierungskammer. Im Moment verfügt der Kolchos über 283 Landanteile. Wenn wir 283 Landanteile mit 7,5 Hektar multiplizieren, dann ist das eine ganz schöne Masse. Die möchte er jetzt herauslösen, vermessen und abgrenzen, sich in Natur ausweisen lassen und die Pacht dieser Landanteile vertraglich regeln. Bis jetzt ist die Sache offensichtlich noch nicht so weit gediehen, ihm fehlen die Fachkräfte und er hat in seinem Unternehmen niemanden, der sich damit beschäftigt. Er hat keinen Agronom, niemanden, er muss überall selbst hinlaufen und hat eigentlich keine Zeit, sich damit zu beschäftigen.« (Sachbearbeiterin der Gemeindeverwaltung, 12.3.2009)

Wie hier bereits anklingt wurde im Zuge dieses Prozesses nicht nur das Kolchosland spezifiziert, sondern komplementär dazu auch diejenigen Flächen, auf die der Betrieb keinen Anspruch erhob und innerhalb derer das Herauslösen und Registrieren von Landparzellen - durch den Investor ebenso wie durch andere Anteilseignerinnen und Anteilseigner - fortan möglich war. Auf diese Weise wurde die Grenze von Eigentum|Nichteigentum (im Sinne von Nutzungsrechten des Betriebs) räumlich-materiell beobachtbar und Unsicherheit reduziert, indem die Selektionsmög- 
lichkeiten für künftige Registrierungen eingeschränkt wurden. Rechtlich möglich war dieser Schritt, da der Kolchosvorsitzende und seine >Anhänger< (noch) über mehr als die Hälfte der Landanteile und damit die erforderlichen Entscheidungskompetenzen verfügten - Mehrheitsverhältnisse, die durch die Kaufaktivitäten des Investors allerdings leicht hätten kippen können:

»Bei uns ist es im Moment so, dass der Landwirtschaftsbetrieb über mehr als 50 Prozent der Landanteile verfügt und deshalb haben wir auf der Versammlung entschieden, welche das sind. Und natürlich hat er die am günstigsten gelegenen Felder genommen. Wenn sich jetzt jemand seinen Landanteil herauslösen will, dann kommen dafür nur noch die weit entfernten Felder in Frage. [...] Das ist schon eine Einschränkung, aber, noch einmal, der Hauptanteil der Parzellen wurde dem Kolchos überlassen, und auf dieser Grundlage wurde auf der Versammlung entschieden, dass die Felder dem Kolchos gehören, die ihm auch früher schon gehört haben - und die übrigen sind freigegeben zur Registrierung. Ich denke, dass es jetzt schon höchste Zeit ist, dass es dringend nötig ist, die Landanteile jetzt zu registrieren, offiziell zu beurkunden, was ihm gehört.« (Sachbearbeiterin der Gemeindeverwaltung, 12.3.2009)

Mit Blick auf den Investor, so zeigte sich die Sachbearbeiterin überzeugt, sei diese Strategie letztlich aufgegangen. Im Wissen um die Abwehrstrategien von Kolchos und Anteilseignern habe er bislang davon abgesehen, seine Eigentumsansprüche in Andreevka geltend zu machen, das Land zu bewirtschaften und somit dem Kolchos zu entziehen:

»Er beansprucht das Land im Moment noch nicht, weil er weiß, dass gemäß der Entscheidung der Anteilseignerversammlung alle nahegelegenen Felder dem Kolchos gehören. Und wenn er jetzt auf seine Anteile Anspruch erhebt, dann wird er nur die weit entfernten Felder kriegen.« (Sachbearbeiterin der Gemeindeverwaltung, 12.3.2009)

In der Darstellung der Agroholding selbst findet sich zum vorläufigen Verzicht auf die besagten Landanteile in Andreevka freilich eine andere Lesart: Im Vordergrund stand nicht das Erleben der Abwehrstrategie des Nachbardorfes, vor der man kapituliert, sondern das eigene Handeln im Horizont wirtschaftlich rationaler Entscheidungen. Für die Bearbeitung der Landanteile in Andreevka, so bemerkte der leitende Agronom der Agroholding, fehle es gegenwärtig schlicht an personellen und technischen Kapazitäten:

»[Frage]: Kaufen Sie im Moment noch zusätzlich Landanteile oder ist das beendet?

[Agronom]: Nein, das gesamte Territorium, das erworben werden konnte, haben wir gekauft. Davon ist allerdings die Hälfte mit Wald überwuchert. Wir sind im Moment dabei, die Felder zu säubern und die Anbaufläche zu vergrößern. 
[Frage]: Und das Land, das Sie beispielsweise nebenan in Andreevka gekauft haben, das bearbeiten Sie bis jetzt noch nicht?

[Agronom]: Lassen Sie mich das so sagen: Nach Andreevka zieht es uns bis jetzt noch nicht. [lacht] Im Moment sind wir mit dem Land hier ringsum beschäftigt.«

(leitender Agronom der Agroholding, 7.3.2010)

Für die (vermeintliche) Kontrolle von Landregistrierungen, die trotz dieser Maßnahmen potenziell stattfinden könnten, sollte außerdem die Wahl einer kommunalen Sachbearbeiterin zur Sprecherin der Anteilseigner sorgen, die auf diese Weise an die Schnittstelle zwischen Kommune und Kolchos rückt. Durch ihre Hände lief fortan jeder Registrierungsprozess, der damit für alle Beteiligten beobachtbar wurde - ohne dass sie selbst allerdings dabei über nennenswerte Entscheidungskompetenzen oder Eingriffsmöglichkeiten verfügen würde.

Im Zusammenhang mit der räumlichen Spezifikation der Landanteile stand auch eine zweite Maßnahme, die im Rahmen der Gemeindeversammlung beschlossen wurde: die Neuregistrierung der Pachtverträge. Diesbezüglich ist anzumerken, dass die Rede von der Registrierung oder gar Neuregistrierung den tatsächlichen Formalisierungsgrad der Pachtverträge vor der Anteilseignerversammlung verkennt. In vielen und vermutlich den meisten Fällen beruhte das Pachtverhältnis auf einer Absprache zwischen Anteilseignern und Kolchosvorsitzendem, die nicht schriftlich fixiert worden war. Im Zuge der Anteilseignerversammlung wurden diese Pachtverhältnisse folglich nicht erneut registriert, sondern überwiegend überhaupt erstmals vertraglich geregelt:

»[Anteilseigner]: Gleich nach der Gemeindeversammlung und noch vor der Anteilseignerversammlung $^{55}$ sind die Leute [der Kolchosleitung, E.M.] gekommen und haben einen Vertrag mit uns geschlossen, das heißt schon auf diese Weise wurde ausgefiltert, wer sein Land verkauft hatte und wer nicht. Das heißt, zu uns ist jemand gekommen, hat erklärt, weshalb er da ist und wie und was. >Haben Sie Ihr Land nicht verkauft?<>Nein, haben wir nicht.< >Zeigen Sie das Eigentumszertifikat.<Wir haben das Zertifikat gezeigt und dann gab es sofort fertige Vertragsformulare und das war's. Und im Vertrag war festgehalten, zu welchen Bedingungen wir dem Kolchos unser Land überlassen. Wir haben diese Verträge unterschrieben. Und als dann die Anteilseignerversammlung stattfand, hat der Kolchos diese Verträge an sich genommen.

[...]

[Frage]: Gab es solche Verträge über das Pachtverhältnis auch vor der Versammlung schon?

55 Unmittelbar vor der besagten Anteilseignerversammlung fand eine Gemeindeversammlung statt. 
[Anteilseigner]: Nun, in manchen Fällen wahrscheinlich schon, als diese ganze Aufteilung [im Rahmen der Kolchosprivatisierung, E.M.] war, als diese ganzen Papiere verteilt wurden. Aber meistens gab es wohl keine Verträge, sondern nur die Entscheidung der Anteilseignerversammlung über bestimmte Kolchosleistungen. Aber inwiefern diese Entscheidung umgesetzt und erfüllt wurde, das kann ich schlicht und einfach nicht sagen.«

(Anteilseigner, 21.2.2010)

In den Fällen, in denen auch vor besagter Anteilseignerversammlung Verträge mit der Kolchosleitung existierten, war deren rechtliche Gültigkeit ohnedies in der Regel auf den Dorfkontext begrenzt und hatte keine Bindungskraft darüber hinaus:

»[Der Kolchosvorsitzende] muss jetzt einen Vertrag abschließen, einen Einheitsvertrag - wie ist noch mal die Bezeichnung? Jetzt sage ich es Ihnen richtig: >Vertrag über die Pacht von Landanteilen gegenüber einer Vielzahl an Personen auf Seite der Verpächter ${ }^{56}{ }^{56}$ Und das heißt: Jeder Eigentümer eines Landanteils überlässt [dem Kolchosvorsitzenden] seinen Anteil. Sie haben unter sich schon vorher Verträge geschlossen, aber die wurden juristisch nirgends dokumentiert. Sie haben das nur unter sich ausgemacht. Zwischen dem Generaldirektor und, sagen wir, einem Anteilseigner, dass er seinen Landanteil dem Kolchos überlasst.« (Sachbearbeiterin der Gemeindeverwaltung, 12.3.2009)

Darauf, dass die fehlende Formalisierung der Pachtverhältnisse in Andreevka kein Sonderfall ist, verweist auch der Kommentar des Bürgermeisters einer Nachbargemeinde zur zaghaften Institutionalisierung von Pachtverträgen im dortigen Kontext:

»Jetzt wurde ein Vertrag geschlossen. Das ist eine Neuheit - mit Blick auf die Eigentumsverhältnisse an Land haben wir keine juristischen Praktiken, keine gerichtlichen Praktiken.« (Bürgermeister, 12.8.2008)

Als grundsätzlich zu behebendes Desiderat wurde die nicht vorhandene Formalisierung jedoch erst beobachtet, als der Investor sich mit seinem Kaufangebot an die Anteilseignerinnen und Anteilseigner wandte. Die informalen oder semiformalen Vereinbarungen, an denen sich die lokalen Normalitätserwartungen bislang ausgerichtet hatten und die die dörfliche Kommunikation in ausreichendem Maße struk-

56 Die offizielle Bezeichnung des Vertragswerks auf Russisch lautet: »Dogovor arendy zemel'noj doli (pri množestve lic na storone arendodatelej)«. Verbindliche Bestandteile des Einheitsvertrags sind unter anderem die Festlegung von Pachtzahlungen sowie der räumlichen Grenzen der Landanteile. Eine Vertragsvorlage findet sich online beispielsweise unter http://docstandard.com/obrazcy/tomlu/obrazec-n1m90y.htm (zuletzt eingesehen am 22.4.2015). 
turierten, gerieten durch dieses Angebot unter unmittelbaren Druck. Um die Kontinuität der dörflichen Strukturen - also eine Fortführung des Verhältnisses zwischen Kolchos und Anteilseignern in der gewohnten Form - zu sichern, ist nun Anpassung erforderlich: Die Formalisierungslücke ist zu schließen und die Grundlagen der Pachtverhältnisse sind an Regeln und Logiken anzupassen, die zwar bereits in der Umwelt des Dorfes Gültigkeit hatten, an die innerhalb der Dorfgrenzen jedoch bislang nicht angeschlossen wurde. Dieser Anpassungsprozess, über den die Dorfgemeinschaft - Gemeindeverwaltung, Kolchos und Gemeindemitglieder - mit Blick auf die Wahrung der tradierten dörflichen Strukturen entschied, schlug seinerseits auf die lokalen Strukturen zurück und setzte innerhalb der Gemeinde neue Logiken in Gang. Maßnahmen zur eigentlichen Stärkung der Dorfgrenzen trugen im Zuge dessen paradoxerweise zur vermehrten Durchlässigkeit dieser Grenzen bei. Dies wird deutlich, wenn zusätzlich zum Fakt der Vertragsabschlüsse der Inhalt der Pachtverträge berücksichtigt wird, auf den sich die verbleibenden beiden Maßnahmen, die auf der Versammlung beschlossen wurden, direkt und indirekt bezogen.

\section{Ein aufbrechendes Kollektiv und die sedimentierte Teilung des Dorfes}

So wie es bis zum Kaufangebot des Investors im Dorf als Selbstverständlichkeit gegolten hatte, sein Landanteil dem ehemaligen Kollektivbetrieb zu überlassen und alternative Verwendungsmöglichkeiten gar nicht erst im Horizont des Möglichen zu beobachten, hatte der Verzicht auf eine unmittelbare Gegenleistung - der wohlgemerkt nicht als solcher markiert wurde - ebenfalls der dörflichen Normalitätserwartung entsprochen. Die Pachtverhältnisse waren Bestandteil einer stillschweigenden und unhinterfragten Vereinbarung zwischen den Anteilseignerinnen und Anteilseignern - und damit faktisch allen Gemeindemitgliedern, denn nur eine kleine Minderheit besaß keinen Landanteil - und dem ehemaligem Kollektivbetrieb (vgl. auch Prosterman/Mitchell/Bradley 1997: 1389): Die Gemeinschaft (>alle<) stellte kollektiv ihr Land zur Verfügung und sicherte dem Betrieb auf diese Weise die Existenzgrundlage. Im Gegenzug erbrachte der Betrieb Leistungen gegenüber der Gemeinschaft, worunter sowohl die Unterstützung der Gemeindeverwaltung als auch der privaten Haushalte fiel. Die Kolchosleistungen wurden weder individuell $z u$ gerechnet noch im Vergleich zueinander aufgerechnet und hatten somit ebenfalls kollektiven Charakter.

Das Kaufangebot und die Eröffnung einer alternativen, aufgrund mangelnder Nachfrage bislang nicht unmittelbar zugänglichen Verwendungsmöglichkeit für die Eigentumszertifikate setzte dieses Arrangement unter Druck: Im Zuge des Abschlusses respektive der Neuregistrierung der Pachtverträge nach allgemeinen juristischen Standards formierte sich die Forderung nach Pachtzahlungen respektive 
einer konkreten und zurechenbaren Gegenleistung, der der Kolchos schließlich auch entsprach. Auf der Anteilseignerversammlung wurde zwar nicht die Einführung monetärer Zahlungen beschlossen - dazu war der Betrieb nach eigenem Bekunden nicht in der Lage -, wohl aber als dritte Maßnahme die Festlegung von klar definierten Zahlungsäquivalenten: Anteilseignerinnen und Anteilseigner, die einen Pachtvertrag abschlossen, erhielten fortan das Recht auf Sach- und/oder Dienstleistungen des Kolchos im Wert von 500 Rubel pro Jahr. Diese Regelung hatte sich ein Jahr nach der Anteilseignerversammlung als selbstverständliches Moment der Pachtverhältnisse etabliert:

»[Frage]: Was steht Ihnen pro Landanteil zu? Irgendwelche Dienstleistungen? Was bietet der Betrieb?

[Anteilseignerin]: 500 Rubel pro Landanteil. In diesem Rahmen kriegt man kostenlos, was man möchte - Einstreu, Leistungen des Traktors, Heu, was auch immer.

[Frage]: Das heißt innerhalb von einem Jahr?

[Anteilseignerin]: Ja.

[Frage]: Bei drei Landanteilen sind das für Sie 1.500?

[Anteilseignerin]: Ja. Futter, Traktor, was man möchte.«

(Anteilseigner, 10.8.2008)

Dass die Beobachtung von Kolchosleistungen gegenüber den privaten Haushalten im Horizont von Zahlungen keine bloße Formalität ist, sondern zum festen Bestandteil der dörflichen Kommunikation geworden ist und beispielsweise in Form entsprechender Abrechnungspraktiken zum Ausdruck kommt, verdeutlichen auch die Äußerungen der Betriebsleitung:

»[Frage]: Es gibt in Andreevka die Anteilseigner. Welche Leistungen bekommen die von Ihnen?

[Kolchosvorsitzender]: Brennholz, Fisch, Getreide.

[Frage]: Kostenlos?

[Kolchosvorsitzender]: Kostenlos - wieso kostenlos? Für die Bereitstellung des Landanteils. Für jeden Landanteil liefern wir Brennholz, ein Kubikmeter kostet 300 Rubel. ${ }^{57}$ [...] Für jeden Landanteil haben wir das gemacht. Wir führen darüber Buch.

[Frage]: Und die Leute, die keinen Landanteil besitzen, bezahlen für diese Leistungen?

[Kolchosvorsitzender]: Ja.«

(Kolchosvorsitzender, 20.2.2010)

57 Hierbei handelt es sich um einen vergünstigten Preis für Anteilseigner. Zum Vergleich: Das private Sägewerk im Dorf verlangt 500 Rubel pro Kubikmeter. 
Zwei Differenzierungslinien treten hervor, die die Position des ehemaligen Kollektivbetriebs im Dorf und sein Verhältnis zur Dorfgemeinschaft strukturieren: Erstens wurden Leistung und Gegenleistung durch die vertragliche Festlegung eines Zahlungsäquivalents sachlich klar definiert und konditional fest miteinander verkoppelt. Sie wurden damit, auch darauf verweisen die Äußerungen des Kolchosvorsitzenden, unterscheidbar von Leistungen des Betriebs gegenüber der Gemeinde, bei denen stets der Verweis auf das Gemeinwohl mitschwingt und die Kopplung an eine Gegenleistung diffus gehalten wird. Zweitens tritt in der Formalisierung der Pachtverhältnisse eine Differenzierungslinie in der Sozialdimension zutage, die vorher bestenfalls vage existierte. Leistung und Gegenleistung waren nun sozialen Adressen eindeutig zurechenbar. Vice versa bedeutete dies, dass das Resultat der individuellen Verkaufsentscheidung nun zur relevanten Komponente der sozialen Adressen im Dorf wurde (zum Begriff der Adresse vgl. Fuchs 1997; Stichweh 2000a): Hatte der Kolchos vormals die Unterstützung durch die Dorfgemeinschaft als kollektives, undifferenziertes und nicht weiter auflösbares Phänomen beobachtet - was auch hinreichend war, um daran anzuschließen -, wurde nun beobachtbar und zurechenbar, wer sein Land bereitstellte und damit bestimmte Ansprüche erwarb und wer nicht, wer also in die Leistungskommunikation des Kolchos inkludiert wurde und wer außen vor blieb. Die kollektive Tauschbeziehung zwischen Kolchos und Dorfgemeinschaft wandelte sich in individuelle Geschäftsbeziehungen mit einzelnen Anteilseignerinnen und Anteilseignern, die - wie die Landverkäufe zeigten - auch individuell aufkündbar sind.

Verschärft wird dieser Umstand durch die vierte Maßnahme, die unter dem Eindruck der Landverkäufe beschlossen wurde: die Einführung von unterschiedlichen Preisen für Kolchosleistungen. Denjenigen Gemeindemitgliedern, die ihr Eigentumszertifikat verkauft haben oder die sich aus anderen Gründen gegen den Abschluss eines Pachtvertrags entschieden haben, war der Zugang zu Kolchosleistungen nicht vollständig verwehrt, sie mussten dafür jedoch fortan höhere Preise entrichten als die Verpächter des Betriebs. Der Kolchosvorsitzende, so beschreibt die Bürgermeisterin, sei von diesem Schritt nur schwer zu überzeugen gewesen, habe ihrem Vorschlag aber letztlich zugestimmt. Die leitende Ökonomin des Kolchos verweist in diesem Zusammenhang auf die Unterscheidung von drei Preiskategorien: Mitarbeiter, Rentner und Anteilseigner (Verpächter) des Kolchos fallen in die günstigste Kategorie, einer zweiten höheren Preiskategorie werden die übrigen Dorfbewohnerinnen und Dorfbewohner zugeordnet und der dritten und höchsten Kategorie gehören schließlich andere Unternehmen und Organisationen an. ${ }^{58}$

58 Auf ähnliche Strategien ehemaliger Kollektivbetriebe, die sich an die Bereitstellung von Land durch die Anteilseigner knüpften, sind Kalugina und Fadeeva (2009: 64f) in ihrer Feldforschung gestoßen. 
Bemerkenswert ist, dass das Preisniveau dabei selbst nach lokalen Maßstäben durchweg niedrig ist und die Preisdifferenzen für die meisten Leistungen insbesondere zwischen den beiden erstgenannten Kategorien mit rund fünf Prozent nicht besonders groß sind. Der Beobachtung und der einschneidenden Wirkung der Maßnahme tut dies jedoch keinen Abbruch. Ungeachtet ihrer Höhe wurden die Preisdifferenzen und das dahinterstehende Exklusionsmoment übereinstimmend als ein wesentliches Argument gegen den Verkauf des eigenen Landanteils genannt:

»[Frage]: Haben Sie jemals darüber nachgedacht, Ihr Eigentumszertifikat zu verkaufen? [Rentnerin]: Nein.

[Frage]: Und warum?

[Rentnerin]: Wozu verkaufen? Ich brauche immer mal wieder was vom Kolchos und dann sagen sie mir: >Du hast deinen Landanteil verkauft, geh und kauf dir deine Sachen woanders Ich gehe zum Kolchos, bitte ihn um Getreide, und der Kolchosvorsitzende stellt mir eine Erlaubnis aus. Man muss nur dieses Papier [das Eigentumszertifikat, E.M.] dabei haben. Und das war's.«

(Rentnerin, 22.2.2010)

In ähnlicher Weise beobachten auch andere im Dorf die Nachteile, die aus dem Verkauf der Landanteile resultieren. Die leitende Technikerin des ehemaligen Kolchos geht sogar noch einen Schritt weiter und beschreibt nicht nur, dass viele derjenigen, die ihr Eigentumszertifikat verkauft haben, dies später aufgrund der höheren Preise für Kolchosleistungen bereut hätten. Sie bilanziert darüber hinaus, dass die Landanteile aufgrund der mit ihrem Besitz einhergehenden Bindung an den ehemaligen Kollektivbetrieb ein Gut darstellen, dessen Wert der vom Investor gebotene Kaufpreis nicht gerecht werde:

»Wenn wir verkaufen, was soll dann aus dem Unternehmen werden? Aber viele haben natürlich verkauft. Und jetzt sind sie eingeschnappt, wenn ihnen der Kolchos das Kalb nicht für 40 Rubel verkauft, sondern für 60. Jetzt sind sie beleidigt, aber vorher darüber nachgedacht haben sie nicht. Sie haben Geld gebraucht und verkauft, Geld braucht man immer, aber es gibt noch etwas Höheres - Land.« (leitende Technikerin des Kolchos, 22.2.2010)

Durch die Preisdiskriminierungen, die übereinstimmend als signifikanter Einschnitt im Verhältnis von Kolchos und Dorfgemeinschaft beobachtet wurden, wurde die moralische Teilung des Dorfes, auf die die Bürgermeisterin verweist, nicht nur in wirtschaftlicher Form beobachtbar, sondern letztlich vollständig in einen wirtschaftlichen Sachverhalt transformiert: 
»[Frage]: Und wie war das Verhältnis zwischen denjenigen, die verkauft haben, und denjenigen, die nicht verkauft haben?

[Bürgermeisterin]: Am Anfang hat sich das Dorf in >Rote $<$ und $>$ Weiße< geteilt.

[Frage]: Oh! [lacht] Das klingt nach Krieg?

[Bürgermeisterin]: Krieg gab es nicht, aber eine Teilung schon. Und ich selbst war wahrscheinlich die erste, die dazu beigetragen hat und klar und offen gesagt hat, dass mir das nicht gefällt und dass ich das nicht verstehe. Aber die Zeit heilt und heute...

[Frage]: Die Zeit heilt - heißt das, dass sich die Leute jetzt schon nicht mehr daran erinnern?

[Bürgermeisterin]: Doch, sie erinnern sich. Wenn diese Leute [zum Kolchosvorsitzenden, E.M.] kommen und um irgendwelche Leistungen bitten. Die Preise sind jetzt andere.

[Frage]: Das heißt, er weiß tatsächlich und nicht nur in der Theorie, wer verkauft hat?

[Bürgermeisterin]: Wenn jemand kein Landanteil hält. Wie zum Beispiel diese Ljubov Aleksandrovna, die zu ihm gekommen ist. Er hat eine Liste mit den Bürgern, die ihm ihr Land verpachten. Er schaut dort nach - und dann gibt es einen anderen Preis. Das geschieht schon automatisch. Und es streitet auch niemand mehr darüber, weil ja jeder selbst diese Entscheidung [zu verkaufen, E.M.] getroffen hat. Natürlich haben die Leute das am Anfang alles gar nicht verstanden. Ich frage mich: >Haben sie selbst eigentlich verstanden, was sie da gemacht haben, oder nicht?<. Die Mehrheit, 70 Prozent, haben das nicht bewusst gemacht. Denn dieses Dokument [das Eigentumszertifikat, E.M.] lag fünfzehn Jahre lang regungslos bei ihnen zuhause.«

(Bürgermeisterin, 12.3.2010)

In den Preisdifferenzen für Kolchosleistungen sedimentierte sich die Teilung des Dorfes. Aus der anfänglichen semantischen und moralischen Differenz, die sich als Reaktion auf das Kaufangebot des Investors entwickelte, war eine strukturelle Differenz geworden. Sie wurde mit Blick auf den Zugang zu Kolchosleistungen stets aufs Neue aktualisiert und damit gleichermaßen visibilisiert und auf Dauer gestellt. Die Preisunterschiede fungierten als eine Art >Speicher $<$ und das Eigentumszertifikat wurde durch sie - darauf deuten die zitierten Äußerungen hin - mit neuer Bedeutung aufgeladen und im buchstäblichen wie im übertragenen Sinne zur Eintrittskarte: Es war fortan in materieller Form vorzuweisen, um bestimmte Zugangsrechte geltend zu machen, und sicherte als Symbol für den Status als Anteilseigner des Betriebs darüber hinaus die Adressierbarkeit im Kommunikationszusammenhang derjenigen, die angesichts des >Angriffs $<$ auf das Dorf die >richtige< Entscheidung getroffen hatten.

Gleichzeitig sorgte die Differenzierung von Preiskategorien und Zugangsmöglichkeiten jedoch auch für eine Entlastung der dörflichen Alltagskommunikation: Der Verweis der Bürgermeisterin darauf, dass »die Zeit heilt« und man über bestimmte Dinge nicht mehr streite, deutet darauf hin, dass die Alltagskommunikation von den individuellen Verkaufsentscheidungen absehen kann. Im Wissen darum, 
dass der Verkauf des Landanteils an klar definierte Konsequenzen gekoppelt ist, verschwimmt die erwähnte Front zwischen >Roten $<$ und >Weißen<. Die Verkaufsentscheidung an sich bleibt außen vor, kann (rückblickend) erlebt werden und zieht kein weiteres Handeln nach sich. Das einst zugerechnete Nein zur Dorfgemeinschaft zeitigt strukturelle Konsequenzen und bedarf daher keiner weiteren Thematisierung.

\section{»Wir haben die Sache zum Halten gebracht «}

Wie ging die Geschichte um den Verkauf der Eigentumszertifikate aus? Die Tatsache, dass letztendlich rund 15 Prozent der Landanteile verkauft wurden, erscheint bedeutsam angesichts dessen, dass die Möglichkeit des Verkaufs der Eigentumszertifikate seit der formalen Privatisierung des Kolchos im Jahr 1992 nie als Handlungsoption beobachtet wurde. Weniger bedeutsam ist dieses Resultat hingegen nicht nur im Hinblick auf die Mehrheitsverhältnisse auf der Anteilseignerversammlung - von den kritischen 50 Prozent ist der Investor weit entfernt -, sondern auch hinsichtlich des weiteren Bestands des Unternehmens: Ungeachtet aller Aufregung fand eine Registrierung und Herauslösung von Agrarland durch den Investor zunächst nicht statt und war auch bei meinem letzten Feldaufenthalt nicht abzusehen, so dass der ehemalige Kolchos vorerst unverändert bestehen blieb.

Im Dorf und vor allem aus der Perspektive der Gemeindeverwaltung werden der Verlauf der Ereignisse und die Folgen der eigenen Interventionen als erfolgreiche Abwehr des Angriffs auf Kolchos und Dorfgemeinschaft beobachtet. Wir haben alles richtig gemacht - so rückblickend der Tenor der Bürgermeisterin:

»Wir dachten, sie [der Investor und sein Unternehmen, E.M.] kommen zu uns, spielen mit offenen Karten, weshalb sie das Land kaufen. Aber sie sind nicht zu uns auf die Versammlung gekommen. Wir haben sie juristisch korrekt durchgeführt - wir haben [dem Kolchosvorsitzenden] die Landanteile zugeteilt und räumlich bestimmt, die die Leute ihm verpachten. Es waren Juristen mit dabei. Wir haben die Sache zum Halten gebracht. [Der Kolchosvorsitzende] hat seine Haltung geändert und ist von seiner übermäßigen Güte abgekommen. Jetzt gibt es unterschiedliche Preise für die Leute, die ihm ihr Land überlassen, und für diejenigen, die es [dem Investor] geben.« (Bürgermeisterin, 8.8.2008)

Dass sich trotz dieses Erfolgs Strukturen quasi unter der Hand gewandelt haben, schwingt in der Äußerung der Bürgermeisterin nur in Zwischentönen mit, wenn sie auf die >Güte< des Kolchosvorsitzenden verweist, die vor den Ereignissen als selbstverständlich galt und nun als >übermäßig< eingestuft wird. Ungeachtet dessen, dass die Selbstbeschreibungen im Dorf den Erhalt des ehemaligen Kolchos und damit Kontinuität in den Vordergrund stellen, deuten die beschriebenen Ereignisse 
darauf hin, dass sich die dörflichen Erwartungsstrukturen gerade auch mit Blick auf den Betrieb und seine Position im Dorf verschoben haben und im Zuge dessen neue Differenzierungslinien zutage treten. Ironischerweise, so lässt sich resümieren, setzte sich letztlich gerade die Form von Kommunikation durch und generierte Anschlussfähigkeit, die die Bürgermeisterin und der Kolchosvorsitzende abzuwehren versuchten, und diejenigen Struktureigenschaften, die erhalten werden sollten, beginnen aufzubrechen. In formal-rechtlicher Hinsicht wirken juristische Praktiken, die bislang im Dorf nicht zur Anwendung kamen, in das Verhältnis von ehemaligem Kolchos und seinen Anteilseignerinnen und Anteilseignern hinein und lokale Idiosynkrasien lassen sich nicht länger aufrechterhalten: An die Stelle informaler Vereinbarungen treten Verträge, die wiederum allgemeinen Rechtsnormen entsprechen müssen und von dorfexternen Institutionen registriert werden. Die Gemeinwohlorientierung des ehemaligen Kollektivbetriebs transformiert sich gegenüber den Haushalten als den Empfängern von Kolchosleistungen in wirtschaftliches Kalkül: Klar definierte Leistungen werden an ebenso klar definierte Gegenleistungen gekoppelt. Das stillschweigende Arrangement zwischen Kolchos und dem Kollektiv der Dorfgemeinschaft löst sich auf und wird durch eine Vielzahl individueller Vereinbarungen ersetzt. Die Kongruenz von Dorfgemeinschaft und Kolchoskollektiv verschwindet, unterschieden wird nun zwischen Anteilseignern und Nicht-Anteilseignern und diese Unterscheidung manifestiert sich unter anderem in unterschiedlichen Preisen für Kolchosleistungen.

Indem auf Zahlungen mit Zahlungen reagiert wird, bewirken die Bürgermeisterin und der Kolchosvorsitzende das, was sie zu verhindern versuchten: Wirtschaftliche Kommunikation beginnt über die Landanteile im Dorf Anschlussfähigkeit zu generieren. Ausgerechnet die Landanteile werden von den Gemeindemitgliedern im Horizont von Knappheit beobachtet - und dies gilt selbst für jene Anteilseignerinnen und Anteilseigner, die einen Verkauf ursprünglich gar nicht in Erwägung gezogen hatten. Die Eigentumszertifikate verlieren an identitätsstiftendem Wert und gewinnen an ökonomischem Wert, indem ihr Besitz mit bestimmten Gegenleistungen verbunden wird und wirtschaftliche Argumente für und gegen den Verkauf an Bedeutung zunehmen.

Die Position der Bürgermeisterin scheint sich dabei im Kontext der Ereignisse zu festigen und aufzuwerten: Wie bereits im Rahmen des Gasifizierungsprozesses etabliert sie sich als Fixpunkt der dörflichen Kommunikation, der kaum zu umgehen ist - und dies ungeachtet dessen, dass nicht sie (respektive die Gemeindeverwaltung), sondern der ehemalige Kollektivbetrieb in erster Linie vom Kaufangebot des Investors betroffen ist und die Bürgermeisterin zudem weder im Hinblick auf die Verkaufsentscheidung noch bezüglich der diskutierten Maßnahmen über formale Entscheidungskompetenzen verfügt. Letztere liegen allein beim ehemaligen Kolchos und bei den Anteilseignern und Anteilseignerinnen selbst. Die Bürger- 
meisterin übernimmt jedoch im Verlauf der Ereignisse die zentrale Sprecherrolle (teilweise auch anstelle des Kolchosvorsitzenden). Sie rückt die Landverkäufe als Thema in die Dorföffentlichkeit, steckt die Foren ab, in denen Kommunikation stattfindet, und sie beeinflusst maßgeblich, welche Art von Entscheidung konstruiert wird und welche Optionen dabei zur Auswahl stehen. Dass nicht sie, sondern der Kolchosvorsitzende und die Anteilseignerinnen und Anteilseigner diejenigen sind, die diese Entscheidungen letztlich treffen, scheint angesichts ihrer Gestaltungsmacht über die Entscheidungsprämissen kaum ins Gewicht zu fallen. Folglich wird der >Sieg < über den Investor am Ende auch in erster Linie ihr zugeschrieben.

Die Bedeutung des ehemaligen Kolchos scheint demgegenüber tendenziell abzunehmen. Zwar spielt er gerade mit Blick auf die öffentliche Infrastruktur nach wie vor eine wichtige Rolle, sein Status als umfassende Organisation bröckelt jedoch - nicht ausschließlich, aber auch in Folge der Landverkäufe und der damit verbundenen Ereignisse - und die interne Heterogenität des Dorfes steigt. Letzteres lässt sich an zwei Beispielen von privatem Unternehmertum anekdotisch illustrieren: Erstens setzten die Anteilseignerversammlung und die dort diskutierten Maßnahmen für eine Familie den Impuls, sich ihre Landanteile herauszulösen und als Eigentum registrieren zu lassen:

»Jetzt sehe ich mal im Protokoll [der Versammlung, E.M.] nach. [...] Es gab diese Anteilseignerversammlung. Dort wurde entschieden, welche Flächen vom Betrieb bearbeitet werden und welche in erster Linie zum Herauslösen und Registrieren vorgesehen sind. Als das alles so passierte, haben wir einfach beschlossen, dass wir unsere Landanteile herauslösen. Wir sind zur >zemel'naja palata< [Behörde für die Landregistrierung, E.M.] gegangen, haben dort die Situation erklärt und man hat uns gesagt >Nehmen Sie sich Land, wo Sie wollen $<-$ das heißt, vor uns hat das niemand gemacht, wir waren die ersten. Wir haben das Land markiert, das uns am nächsten liegt, und haben gezeigt, wo das ist.« (Privatbauer, 20.8.2008)

Statt das Land an den Kolchos zu verpachten, beschloss die Familie, ihre drei Landanteile (insgesamt 23 Hektar) als Grundlage für den Aufbau eines eigenen privatbäuerlichen Betriebs zu nehmen. Sie spezialisierte sich auf Milchwirtschaft und im Herbst 2008, als ich das erste Interview mit Aleksej und Svetlana führte, besaßen sie mit sieben Kühen bereits deutlich mehr Vieh als die anderen Hoflandwirtschaften im Dorf. Bei meinem dritten und letzten Besuch im Frühjahr 2010 war der Bestand auf zehn Tiere angewachsen. Aleksej und Svetlana hatten ihre Stellen beim Kolchos gekündigt und die Familie stand nicht nur wirtschaftlich auf eigenen Füßen, sondern hatte sich gemessen an den Verhältnissen im Dorf einen gewissen Wohlstand erarbeitet: Stolz wurde mir der neu angeschaffte Computer präsentiert und erzählt, dass man dem ältesten der drei Söhne ein Hochschulstudium in Perm ermöglichen könne. Verkauft wurden die Milchprodukte zu einem kleinen Teil im 
Dorf, überwiegend jedoch an einen mittlerweile etablierten Kundenstamm im Bezirkszentrum, den sie sich über die Zeit erschlossen hatten und täglich belieferten. Dafür pendelten sie mit dem eigenen Auto täglich ins rund 40 Kilometer entfernte Bezirkszentrum - ein Grad an (selbstverständlicher) Mobilität, der im Vergleich zur Mehrheit der übrigen Gemeindemitglieder eine Besonderheit darstellt.

Ein zweites Beispiel für die zunehmende Heterogenität und Komplexität der dörflichen Strukturen ist ein privates Sägewerk, das als Ableger eines größeren Unternehmens aus Perm seit dem Jahr 2009 im Dorf existiert und unter anderem Brennholz an private Haushalte liefert. Nun wäre es zweifellos überzogen, die Entstehung des Sägewerks analog zum privatbäuerlichen Betrieb auf die Ereignisse rund um den Verkauf der Eigentumszertifikate zurückzuführen. Festzustellen ist jedoch, dass das Angebot des Sägewerks in Folge der Thematisierung der Preise für Kolchosdienstleistungen mittlerweile im Vergleich mit und als potenzielle Alternative zur Brennholzlieferung durch den Kolchos beobachtet wird, der ebenfalls ein Sägewerk unterhält. Zumindest für diesen kleinen Ausschnitt verliert der ehemalige Kolchos sein altes Monopol und die Beobachtung auf Preise und im Modus der Konkurrenz beginnt sich zaghaft zu etablieren.

\section{EPILOG: FORMEN, FOREN UND FIXPUNKTE DER DÖRFLICHEN KOMMUNIKATION}

Welche übergreifenden Schlussfolgerungen zum Wandel gesellschaftlicher Strukturen im postsowjetischen ländlichen Russland lassen sich aus der empirischen Analyse ziehen? Es sind vor allem zwei Prozesse, die durch die vier Episoden hindurch sichtbar werden: $\mathrm{Zu}$ beobachten ist erstens, dass sich die Politik auf der lokalen Ebene sowohl intern als auch gegenüber anderen Funktionssystemen - speziell der Wirtschaft - ausdifferenziert, d.h. sich als eigenständiger Kommunikationszusammenhang etabliert und im Hinblick auf ihre Zuständigkeitsbereiche und Steuerungsanprüche expandiert. Sie erzeugt zunehmend eigene Komplexität, diversifiziert die Teilnahmemöglichkeiten und beginnt, sich an anderen Funktionssystemen zu reiben. Zweitens und damit eng verzahnt zeigt sich, dass und wie die Grenzen des dörflichen Kommunikationszusammenhangs schrittweise aufbrechen und die Durchlässigkeit für weltgesellschaftliche Strukturmuster steigt, die in den lokalen Kontext hineinzuwirken beginnen und dort Anschlussfähigkeit generieren. Beide Prozesse spiegeln sich mit besonderer Deutlichkeit in der Verschiebung von Inklusionsmodi, in der Herausbildung spezifischer Parameter und Institutionen der lokalen Politik und im Zusammenspiel von formalen, informalen und illegalen Strukturen in und zwischen den beteiligten Organisationen. Auf diese drei Aspekte möchte ich abschließend nochmals eingehen. 


\section{Globale und lokale Inklusionsmodi}

In allen vier Episoden findet sich Evidenz dafür, dass dörfliche Kommunikationsformen nach Anlehnungskontexten in global operierenden Funktionssystemen suchen und die partikularen Logiken des dörflichen Kommunikationszusammenhangs im Zuge dieses Suchprozesses zunehmend mit den universalen Logiken global operierender Funktionssysteme konkurrieren. Ein unmittelbarer Ausdruck dieser Konkurrenz ist das Verhältnis verschiedener Inklusionsformen: Die Adressierbarkeit in gesellschaftlichen Funktionssystemen, die Anschlussmöglichkeiten über die Dorfgrenzen hinweg eröffnet, gewinnt gegenüber der vormals dominierenden Kolchosmitgliedschaft an Bedeutung. Letztere stellt sich in der Folge auf Kompatibilität mit den Inklusionsmodi der Funktionssysteme um. Das bedeutet: Anders als noch bis in die späten 1990er Jahre hinein ergeben sich die Freiheitsgrade einzelner Gemeindemitglieder und die Handlungsspielräume der Gemeindeverwaltung nun nicht mehr primär aus dem Verhältnis zur und der Integration in die umfassende Organisation Kolchos, sondern resultieren aus der Möglichkeit zur Oszillation zwischen beiden Inklusionsmodi - Kolchos und Funktionssysteme -, deren Verhältnis (vorläufig) in der Schwebe gehalten wird.

In diesem Sinne illustriert die Episode um den Anschluss der Gemeinden und ihrer Haushalte an das Gasnetz, wie ein per se technischer Akt zum Transmissionsriemen für weltgesellschaftliche Strukturen wird, die im Dorf zunehmend alternativlos erscheinen und Anschlussfähigkeit generieren. Lokale Kommunikationsformen werden sukzessive überlagert und teilweise außer Kraft gesetzt, was besonders deutlich mit Blick auf Eigentum und Kredite zum Ausdruck kommt: Die Gasifizierung löste nicht nur eine zweite Privatisierungswelle der sogenannten Kolchoshäuser aus, sondern auch einen Formalisierungsschub, der die Unterscheidung Eigentum|Nicht-Eigentum in eine auch außerhalb des Dorfes anschlussfähige Form brachte und Möglichkeiten für die Zweitcodierung von Eigentum durch Zahlungen eröffnete. Im Hinblick auf Kredite als Mechanismus der Inklusion ins Zentrum des Wirtschaftssystems induzierte der Gasifizierungsprozess im Dorf die Beobachtung auf Kreditwürdigkeit hin, also auf die Möglichkeit, aus Zahlungsunfähigkeit Zahlungsfähigkeit zu generieren. Damit gewinnen die Modi wirtschaftlicher Inklusion an Bedeutung, während die Inklusion in den ehemaligen Kolchos gegenläufig an Relevanz verliert: Die Bindung an den Betrieb erwies sich für den Anschluss an das Gasnetz als Handlungsoption im Einzelfall als nutzlos oder sogar hinderlich und wurde mit der Privatisierung der Kolchoshäuser für die entsprechenden Gemeindemitglieder in zumindest einer Dimension gelockert. Dabei scheinen die Freiheitsgrade, die in dieser Situation durch die Inklusion in das Wirtschaftssystem gewonnen werden, den potenziellen Verlust an Freiheitsgraden durch die (zumindest partielle) Ablösung vom ehemaligen Kolchos auszugleichen. 
Ganz ähnlich schlossen auch die Abwehrmechanismen gegen das Kaufangebot des Investors, die im Zuge der Ereignisse rund um den Verkauf von Eigentumszertifikaten in der Gemeinde Andreevka beschlossen wurden, an funktionssystemspezifische Kommunikationsformen an, die zuvor an den Dorfgrenzen abgeprallt waren. In der Spezifikation und Neuregistrierung der Pachtverträge zwischen dem ehemaligen Kolchos und den Anteilseignern spiegeln sich beispielsweise mit Einheitsverträgen oder Beglaubigungen juristische Praktiken wider, die man in der dörflichen Kommunikation bislang schlicht nicht > gebraucht< hatte. Um angesichts des Drucks, der vom Kaufangebot der Agroholding auf die tradierten dörflichen Strukturen ausgeht und dem sich die dorfinternen und überwiegend informalen Arrangements kaum wirkungsvoll entgegensetzen lassen, Unsicherheit zu reduzieren und zukünftige Selektionen mit Blick auf die Verwendung der Landanteile zu binden, wird nun erstmals auf ebenjene >fremden< Praktiken zurückgegriffen. Die Einführung von Zahlungsäquivalenten und Preisdiskriminierungen in die Pachtverhältnisse trägt zudem wirtschaftliche Kommunikation in den lokalen Kontext hinein. Eine Kommunikationsform, die im Hinblick auf die Eigentumszertifikate bislang unterdrückt wurde. Die Landanteile werden zunehmend im Knappheitshorizont von Leistung und Gegenleistung beobachtet und die Individualisierung der Pachtverhältnisse bricht das tradierte kollektive Arrangement zwischen ehemaligem Kolchos und Dorfgemeinschaft durch eine Individualisierung der Pachtverhältnisse auf. Die lokale Moralordnung über die Verwendung der Eigentumszertifikate verliert ihre Bindekraft und wird an dieser Stelle schrittweise durch ökonomische Rationalitäten ersetzt. Die Ironie dieser Episode liegt darin, dass die gravierenden Änderungen der Erwartungsstrukturen durch Maßnahmen eingeleitet wurden, die eigentlich auf den Erhalt der tradierten Strukturen - insbesondere des Kolchos in seiner >sowjetischen< Form - zielten und man sich zudem im Dorf rückblickend von der erfolgreichen Abwehr des Angriffs auf diese Strukturen in Form des Kaufangebots überzeugt gab.

Eigentum und Zahlungen als wirtschaftliche Inklusionsmodi markieren damit eine wesentliche Dimension eines einsetzenden Wandlungs- und Anpassungsprozesses: Eine Klärung der Eigentumsverhältnisse mit Blick auf Agrarland und teilweise auch auf Wohngebäude und damit das Scharfstellen der Grenze zwischen Eigentum und Nicht-Eigentum, worauf bislang im Dorf verzichtet wurde, wird angesichts von Umweltirritationen wie dem Gasifizierungsprojekt oder dem Kaufangebot des Investors unumgänglich. Dies induziert erstens die Neubestimmung der Grenzen der landwirtschaftlichen Großbetriebe innerhalb des Dorfes und des Verhältnisses der Betriebe zur Dorfgemeinschaft. Letztere, so wird deutlich, wird als Kollektiv rissig und es tritt eine Bruchstelle hervor, an der die soziale Kongruenz von landwirtschaftlichem Großbetrieb und Gemeinde zu erodieren beginnt. Durch den Verweis auf Zahlungen und Kredite werden zweitens Beobachtungsformen 
forciert und Anschlussmöglichkeiten eröffnet, die über die Dorfgrenzen hinaus weisen und innerhalb der Gemeinde zwar thematisiert, aber nicht mehr politisch kontrolliert werden können.

>Sowjetische< Strukturen (beispielsweise in Form der umfassenden Ansprüche an den Kolchos, der personellen Kontinuität in der Kolchosleitung und der Kommunalverwaltung sowie in Form des engen Verhältnisses zwischen Kolchosvorsitzendem und Bürgermeisterin) verschwinden nicht notwendigerweise, verlieren aber angesichts steigender Komplexität der dörflichen Umwelt relativ an Bedeutung. Auch wenn weiterhin daran angeschlossen wird, steht dies den skizzierten Wandlungsprozessen nicht zwingend im Wege, die sich mit diesen >sowjetischen Nischen $<$ arrangieren. Dies wird in besonderem Maße mit Blick auf neu hinzukommende Akteure wie die Agroholding deutlich, um die herum sich neue Differenzierungslinien herausbilden, ohne dass die alten vollständig verloren gehen. Es wird aber auch deutlich mit Blick auf den ehemaligen Kolchos, der im dörflichen Kontext zunehmend weniger als umfassende Organisation und damit auch nicht mehr als alleiniger Schlüssel fungiert, um die veränderte >Logik < der Gemeinde zu verstehen. Im Unterschied zum sowjetischen und frühen postsowjetischen Dorf bestimmt sich für das einzelne Gemeindemitglied die Summe seiner Handlungsmöglichkeiten längst nicht mehr über die Frage der Kolchoszugehörigkeit und den individuellen Status innerhalb der Organisationsstrukturen, sondern aus der Adressierbarkeit in funktionssystemspezifischen Kommunikationszusammenhängen, welche die Dorfgrenzen überschreiten. Die Zugehörigkeit|Nicht-Zugehörigkeit zum Kolchos, die aufgrund der >sowjetischen<Kongruenz von Betrieb und Gemeinde mit der Unterscheidung innen|außen hinsichtlich der Dorfgrenzen gleichzusetzen war und an der sich in der Vergangenheit die Erwartungen ausrichteten, wird durch funktionale Differenzierung abgelöst. Will sich der ehemalige Kolchos unter diesen Bedingungen reproduzieren, müssen die Formen der Kolchosmitgliedschaft nun mit den Inklusionsmodi gesellschaftlicher Funktionssysteme wechselseitig kompatibel werden. Im Gegensatz dazu galt bis in die späten 1990er Jahre hinein für die Gemeindemitglieder im Hinblick auf die Kolchosmitgliedschaft zwar nicht in allen, aber doch in vielen Bereichen des alltäglichen Lebens ein Entweder-oder.

Nicht nur die (weitgehend) hermetische Schließung des dörflichen Kommunikationszusammenhangs in sowjetischer Tradition ist mit dieser Umstellung auf funktionale Differenzierung und der steigenden Komplexität der dörflichen Strukturen unvereinbar, sondern auch die Existenz einer dorfweiten Machtinstanz, wie sie im sowjetischen Regime die Kolchosleitung (respektive die Person des Kolchosvorsitzenden) darstellte - ein Sachverhalt, der zu einem zweiten zentralen Ergebnis überleitet: den Parametern lokaler Politik. 


\section{Die Parameter und Institutionen lokaler Politik}

Über alle vier Episoden hinweg ist zu beobachten, wie sich die lokale Politik sukzessive als eigenständiger Kommunikationszusammenhang im Dorf herausbildet, d.h. sich gegenüber anderen Funktionssystemen profiliert und nach eigenen Maßgaben zu operieren beginnt. Die lokale Politik gewinnt nicht nur formal (wie durch die Kommunalreform eingefordert), sondern auch faktisch an Autonomie. In der Sozialdimension kommt dies vor allem darin zum Ausdruck, dass sich die Möglichkeiten zur Teilnahme an politischer Kommunikation vervielfältigen und nach eigenem Ermessen der Politik modelliert werden. In der Sachdimension wird deutlich, dass und wie in den lokalpolitischen Institutionen für bestimmte, sachlich abgegrenzte Bereiche Entscheidungen mit kollektiver Bindungswirkung getroffen werden. Diese Entscheidungen schließen auch diejenigen Gemeindemitglieder als Teil des Kollektivs ein, die in konkreten Fällen nicht überzeugt worden sind. Hinzu kommt, dass es im Dorf weder eine außerpolitische Instanz gibt, die diese Bindung generell auflösen könnte, noch eine vorpolitische Sozialordnung existiert (z.B. in Form der Kolchosmitgliedschaft), die einzelne aus dieser Bindung entlassen und ungebrochen in die politische Kommunikation hineinwirken könnte. Diese Beobachtungen laufen quer zum üblichen Tenor der Debatte über die russische Lokalverwaltung, die von Sorgen über die Subventionsabhängigkeit der Gemeinden und unterschwellige Rezentralisierung geprägt ist. Drei Merkmale des lokalpolitischen Kommunikationszusammenhang treten in der empirischen Analyse besonders deutlich hervor: Erstens die Bürgermeisterinnen und Bürgermeister, die sich als Fixpunkte lokaler Machtkommunikation verankern, zweitens das hohe Maß an Interaktionsgebundenheit lokaler Politik sowie drittens die sich herauskristallisierende lokalpolitische Rolle der landwirtschaftlichen Großbetriebe.

Die Bürgermeisterinnen und Bürgermeister, so wurde über alle Episoden hinweg deutlich, verfügen nicht über die umfassende Stellung, welche die Kolchosvorsitzenden im sowjetischen Dorf innehatten, positionieren sich jedoch als zentrale Figuren in einem zunehmend komplexen Kommunikationszusammenhang. Ihre Gestaltungsmacht gewinnen sie weniger aus ihren unmittelbaren Entscheidungskompetenzen, sondern in erster Linie aus den Möglichkeiten zur Koordination und Kanalisation von Kommunikation, die mit ihrem Amt verknüpft sind. Besondere Bedeutung hat dies mit Blick auf jene Interaktionen, die auf ihre Entscheidung hin zustande kommen oder auch verhindert werden. Es sind die Bürgermeister und Bürgermeisterinnen, nicht die Kolchosvorsitzenden, die die Gemeindeversammlungen als zentrale politische Kommunikationsforen einberufen, Themenrelevanzen maßgeblich gestalten und damit eine politische Öffentlichkeit schaffen und moderieren, in der sie die zentrale Sprecherrolle übernehmen. 
Über diese Interaktionsforen konstituiert sich maßgeblich das lokale Publikum, mit allen Möglichkeiten und Grenzen, die mit diesem Systemtyp verbunden sind. Entsprechend ist Anwesenheit eine wesentliche Inklusionsform in politische Entscheidungsprozesse, wohingegen andere Modi politischer Inklusion (beispielsweise Wahlen oder die öffentliche Meinung) zumindest für das lokalpolitische Alltagsgeschehen in den Hintergrund treten. So lässt sich beispielsweise sowohl im Kontext der durch das Kaufangebot des Investors ausgelösten >Eigentumsfrage< als auch mit Blick auf den Gasifizierungsprozess verfolgen, wie politische Kommunikation generiert wird, die sich in erster Linie über Gemeindeversammlungen vollzieht, an denen diejenigen teilnehmen, die sich auf die durch die Ereignisse aufgeworfenen Klärungsprozesse einlassen. Auf der anderen Seite scheinen der Verzicht auf Eigentum (respektive auf die Klärung der Eigentumsverhältnisse), Zahlungsunfähigkeit und die Vermeidung politischer Adressierbarkeit (über Abwesenheit auf den Gemeindeversammlungen) an dieser Stelle Hand in Hand zu gehen. Andersherum wird auch für die Politik Interaktion zum zentralen Anhaltspunkt der Publikumsbeobachtung und damit der Beobachtung der maximalen Reichweite lokaler Machtkommunikation. Letztere stößt nicht nur an den spezifischen Logiken gesellschaftlicher Funktionssysteme (vor allem der Wirtschaft) an ihre Grenzen, deren Eigendynamiken es gerade mit Blick auf ihre politische Kontrollierbarkeit politikintern zu reflektieren und akzeptieren gilt, sondern auch an den Eigenheiten der Interaktionssysteme.

Im Hinblick auf diese beiden Merkmale lokaler Politik - die Schlüsselposition der Bürgermeisterinnen und Bürgermeister und die Interaktionsgebundenheit - aufschlussreich ist beispielsweise das Fungieren der Gemeindeverwaltung als eine Art dörfliche Ratingagentur im Kontext der Gasifizierung, entscheidet sie doch über die Kreditwürdigkeit einzelner Gemeindemitglieder mit. Dabei erweist sich die Kommunalverwaltung - und die Bürgermeister und Bürgermeisterinnen sind hier zweifellos die treibenden Kräfte - nicht nur als Vermittlerin, sondern im Hinblick auf den beschriebenen Ablösungsprozess einzelner Gemeindemitglieder vom Kolchos und die damit einhergehende Verschiebung von Inklusionsmodi sogar als Katalysator. Indirekt lässt sich dies als ein Effekt der Kommunalreform verbuchen, die unter anderem den Gasifizierungsprozess in den Verantwortungsbereich der Bürgermeisterinnen und Bürgermeister stellt, so dass sowohl Erfolg als auch Scheitern ihnen respektive der Kommunalverwaltung zugeschrieben werden. Entsprechend erwiesen sich sämtliche Maßnahmen im Sinne der Gemeindeverwaltung, die eine Teilnahme der Haushalte an der Gasifizierung ermöglichen und bewirken, darunter auch die Privatisierung und Registrierung von privatem Wohnraum und die Aufnahme von Krediten zur Finanzierung der technischen Infrastruktur. Darüber hinaus ist die offizielle Registrierung der Wohnhäuser als Privateigentum die Voraussetzung dafür, dass sich in Form von Steuerzahlungen daran anschließen lässt. Grund- 
und Vermögenssteuern fließen gemäß den Regelungen der Kommunalreform in vollem Umfang dem lokalen Budget zu, so dass sich als Nebeneffekt der Gasifizierung die Steuergrundlage und damit die finanziellen Freiheitsgrade der Verwaltung erweitern. Die Appelle, anhand derer vor allem die Bürgermeisterinnen und Bürgermeister angesichts möglicher Vorteile auf die individuellen Haushaltsentscheidungen einzuwirken versuchen, werden in erster Linie im Rahmen von Gemeindeversammlungen - als Interaktionssysteme - an die Gemeindemitglieder gerichtet. Die Reichweite dieser Form der Machtkommunikation erweist sich allerdings als zweifach begrenzt: Zum einen durch Anwesenheit als konstitutives Merkmal von Interaktion - mangels formaler Sanktionsmöglichkeiten kann man sich durch Abwesenheit den Appellen leicht entziehen -, zum anderen aufgrund der divergierenden Logiken der Funktionssysteme, die in das Gasifizierungsprojekt hineinspielen. Während die Bürgermeisterinnen und Bürgermeister das Projekt als politische Angelegenheit beobachten, die es durch- und umzusetzen gilt, sehen sich die Haushalte primär mit einer wirtschaftlichen Entscheidung konfrontiert, die für sie auf Preise und Zahlungen hinausläuft. Weder die Gemeindeverwaltung noch ein anderes der beteiligten Systeme ist in der Lage, sich - in der Tradition des umfassenden sowjetischen Kolchos - in dieser Situation als zentrale Entscheidungsinstanz aufzuführen. Stattdessen sind Politik und Wirtschaft in ihrer Eigenständigkeit zu akzeptieren. Der politische Erfolg des Gasifizierungsprojekts hängt letztlich erheblich davon ab, inwieweit im Dorf an wirtschaftliche Kommunikationsangebote - konkret: den Kredit - angeschlossen wird.

In ähnlicher Weise instruktiv sind die Ereignisse rund um das Kaufangebot des Investors in der Gemeinde Andreevka, die nur auf den ersten Blick wenig mit lokaler Politik zu tun haben. Bei genauerem Hinsehen bieten sie Einsichten, wie kollektiv bindende Entscheidungen im Dorf getroffen werden und wer auf welche Weise in den Entscheidungsprozess eingebunden wird. Vom Kaufangebot demonstrativ betroffen und für den weiteren Verlauf der Dinge verantwortlich fühlt sich in erster Linie die Gemeindeverwaltung - und nicht der ehemalige Kolchos, auf den der >Angriff $<$ in Form des Kaufangebots des Investors eigentlich gerichtet war. Die Bürgermeisterin übernimmt hier eine dominante und unangefochtene Sprecherrolle im Dorf. Allerdings scheitern sämtliche Versuche ihrerseits, machtvoll auf den Verlauf der Ereignisse einzuwirken, oder verfangen nicht in der von ihr erhofften Weise: Der Investor als Person entzieht sich von Vornherein einem Konflikt, im Rahmen dessen gegebenenfalls ein Rückzug des Kaufangebots hätte bewirkt werden können, und an die an die Anteilseignerinnen und Anteilseigner gerichtete Aufklärung durch die Bürgermeisterin und den Kolchosvorsitzenden wird nur bedingt angeschlossen (auch wenn sich beide von ihrer moralischen Deutungshoheit im Dorf überzeugt zeigen). Um in dieser Lage politischen Einfluss auszuüben, erscheint es ähnlich wie im Gasifizierungsprozess unumgänglich, mit der Gemeinde- 
versammlung ein öffentliches Forum zu wählen und damit die Beteiligung aller Gemeindemitglieder am Entscheidungsprozess zu ermöglichen. Zudem muss - und auch hier tritt eine Parallele zur Gasifizierung hervor - die Verkaufsentscheidung in ihrer Eigenständigkeit als dezentrale und individuelle Entscheidung der Haushalte akzeptiert werden, deren Resultat zwar relevant für die Dorfstrukturen ist, auf die sich aber weder kollektiv noch politisch unmittelbar einwirken lässt. Letzteres spiegelt sich in den Maßnahmen wider, die schließlich beschlossen werden und zu verfangen scheinen. In ihnen und der Art und Weise, wie daran angeschlossen wird, kommt darüber hinaus zum Ausdruck, dass durch den politischen Entscheidungsprozess offensichtlich ein Modus gefunden wurde, wie - dies wurde bereits erwähnt - die Kolchosinklusion und wirtschaftliche Inklusion nicht gegeneinander in Stellung gebracht, sondern wechselseitig kompatibel gemacht werden.

Landwirtschaftliche Großbetriebe, dies ist als drittes Merkmal hervorzuheben, erweisen sich (weiterhin) als zentraler Bezugspunkt lokaler Politik - sei es in Form des >sowjetischen< Modells wie im Falle des ehemaligen Kolchos, sei es in expliziter Abgrenzung davon wie im Falle der Agroholding. In vordergründig sowjetischer Kontinuität sind sie nach wie vor unverzichtbar für die lokale Infrastruktur. Anders als im sowjetischen und frühen postsowjetischen Dorf treten sie nun jedoch zunehmend nicht mehr als superrelevante Umwelt der Gemeindeverwaltung in Erscheinung, sondern als ihr Kooperationspartner. Die Zusammenarbeit zeichnet sich durch ein zunehmendes Maß an Formalisierung und Professionalisierung aus, wodurch trotz aller praktischen Abhängigkeiten immer auch die Grenzen und unterschiedlichen Rationalitäten beider Organisationen betont werden. Die Retraktion des Kolchos aus dem Politischen kommt auch darin zum Ausdruck, dass Entscheidungen der Kolchosleitung per se keine gemeindeumfassende kollektive Bindungswirkung mehr aufweisen und, sofern eine solche Wirkung erreicht werden soll, die Unterstützung durch die Politik und das Einlassen auf die Logiken Letzterer unumgänglich wird. Hinzu kommt, dass sich für die Gemeindemitglieder die Adressierbarkeit als Leistungsempfänger an verschiedenen Stellen von der Kolchosmitgliedschaft entkoppelt: Zum einen treten Zahlungen und Verträge mit einzelnen Dorfbewohnerinnen und -bewohnern (samt entsprechend individueller und vor allem individuell verhandelbarer Konditionen) an die Stelle, die vorher durch Mitgliedschaftsprogramme geregelt war. Zum anderen und parallel dazu vollziehen sich Formalisierungsprozesse und die schrittweise Umstellung auf wirtschaftliche Kommunikation auch im Kontext der Kooperation mit der Gemeindeverwaltung, die hinsichtlich der entsprechenden Leistungen und Leistungserwartungen als vermittelnde Instanz und mit zunehmender Verhandlungsmacht auftritt.

Mit Blick auf die politische Rolle der Großbetriebe ließ sich am Beispiel kommunaler Aufgaben zeigen, dass der ehemalige Kolchos in der Gemeinde Andreevka weiterhin einen erheblichen Teil derjenigen Leistungen erbringt, die er auch schon 
im sowjetischen Regime übernommen hatte. Und auch die Agroholding, die sich seit dem Jahr 2006 in der Gemeinde Beljaevka etablierte, rückt sukzessive von ihrer anfänglichen, stark von der Haltung des Investors geprägten Intention ab, sich im Dorf auf eine rein wirtschaftliche Position zu beschränken. Stattdessen erbringt das Unternehmen bereits nach kurzer Zeit ein breites Spektrum an Aufgaben, die in Inhalt und Umfang den klassischen Kolchosleistungen stark ähneln. Auf diese Weise scheinen das Auftauchen des Investors und die dadurch in der Gemeinde angestoBenen Entwicklungen, die auf den ersten Blick als radikaler Bruch mit den tradierten Strukturen gedeutet werden können (und von den Beteiligten tendenziell auch so beobachtet werden), auf Strukturen hinauszulaufen, die den Strukturen um den ehemaligen Kolchos stark ähneln. Betrachtet man zudem die mit der jüngsten Kommunalreform verbundene Erwartung bezüglich der Herausbildung marktförmiger Strukturen im Bereich kommunaler Dienstleistungen, in die auch und gerade kleine ländliche Gemeinden eingebunden sein sollen, wird deutlich, dass die Leistungen der Agroholding für die Gemeindeverwaltung einen ähnlichen Effekt haben wie diejenigen des ehemaligen Kolchos: Obwohl entsprechende Kommunikationsangebote durch Privatunternehmen außerhalb des Dorfes durchaus registriert werden, lehnt die Gemeindeverwaltung es selbst in dringenden Fällen ab, entsprechende vertraglich gerahmte Geschäftsbeziehungen einzugehen.

Ungeachtet dessen zeichnen sich bei genauerem Hinsehen jedoch Verschiebungen in der Form und Ausgestaltung der zugrundeliegenden Arrangements zwischen den Betrieben und den Gemeindeverwaltungen ab. Brüche mit tradierten Strukturen, durch die neuartige und sich wandelnde Differenzierungsformen durchscheinen, lassen sich vor allem in der Art und Weise beobachten, wie die Agroholding in ihre dörfliche Umwelt eingebunden wird. In der direkten Kommunikation mit der Gemeindeverwaltung fällt zunächst der hohe Professionalisierungsgrad auf: Organisationsintern wird die Kooperation über eigens eingerichtete Stellen im Betrieb abgewickelt und Aufgaben werden entsprechend delegiert, so dass die Unternehmensleitung kaum davon berührt wird. Für eine Verflechtung von Rolle und Person, die das Verhältnis zwischen Bürgermeisterin und Kolchosvorsitzendem im Nachbardorf prägt und die sich erheblich auf das Zustandekommen und die Ausgestaltung der Arrangements zwischen Kommune und Kolchos auswirkt, ergeben sich daher keine Anhaltspunkte.

Eine Professionalisierung tritt darüber hinaus auch organisationsextern im Hinblick auf das lokalpolitische Engagement der Agroholding hervor: Die (erfolgreiche) Kandidatur des Investors und einer Gruppe Betriebsangehöriger für den Gemeinderat und ihr Auftreten als geschlossene Fraktion mit eigenem Wahlprogramm deuten darauf hin, dass der lokalpolitische Einfluss des Unternehmens anders als im Falle des ehemaligen Kolchos nicht historisch gewachsen ist, sondern politisch erarbeitet wird. Ebenfalls im Unterschied zum ehemaligen Kollektivbetrieb, der für 
sich mit großer Selbstverständlichkeit und ohne formales Mandat beansprucht, die Interessen aller Gemeindemitglieder zu vertreten, generiert sich die Agroholding respektive einzelne Mitglieder, die sich in der Dorföffentlichkeit betont als solche präsentieren - in einer Art >Nebenrolle< als intermediäre Organisation der lokalpolitischen Peripherie. Vertreten und im Vorfeld der Wahl explizit benannt werden spezifische Interessen, die potenziell in Konkurrenz zu anderen, divergierenden Interessen stehen und - dies lässt sich zumindest ansatzweise aufzeigen - eine organisationale Bündelung provozieren. So erwähnt eine kommunale Sachbearbeiterin (3.3.2010) auf die Frage nach den Themen, die auf den Gemeindeversammlungen diskutiert werden, als aktuelles Beispiel die Initiative zur Gründung einer ImkerVereinigung im Dorf: In den vergangenen beiden Jahren habe sich die Bienenpopulation in Beljaevka erheblich verringert. Die zahlreichen Imker im Dorf führen dies darauf zurück, dass die Kartoffelfelder der Agroholding per Flugzeug chemisch gedüngt werden. Zwar sei ein Mindestabstand zu bewohnten Gebieten eingehalten worden, doch vermutlich haben sich die Düngemittel über eine weitaus größere Fläche verteilt und so den Bienen geschadet. Nachdem Beschwerden einzelner Gemeindemitglieder beim Unternehmen kein Gehör gefunden hätten, habe man im Rahmen einer Gemeindeversammlung beschlossen, eine offizielle Imkervereinigung (»obščestvo pčelovodov«) zu gründen, um auf das Problem aufmerksam zu machen und die eigenen Interessen insbesondere gegenüber der Agroholding besser artikulieren zu können.

Diese Einzelbeachtungen verweisen immer wieder darauf, dass die wirtschaftliche Stärke des Unternehmens nicht als Grundlage eines umfassenden dorfweiten Status taugt - und dies unabhängig von der Frage, ob dies überhaupt angestrebt wird. Zwar scheinen angesichts der zentralen Stellung der Agroholding in der Gemeinde Analogien zu sowjetischen Strukturmustern naheliegend. Wiederholt wird jedoch deutlich, dass sie ihre Zahlungsfähigkeit zwar in verschiedenen Bereichen ausspielen kann und damit zweifellos über Einfluss im Dorf verfügt, sich jedoch gleichzeitig zur Anpassung an die Logik der Politik gezwungen sieht, um auf kollektiv bindende Entscheidungen einzuwirken. In der Folge treten in einem wechselseitigen Modellierungsprozess des Unternehmens und seiner lokalen dörflichen Umwelt Differenzierungslinien zutage, die zuvor keine oder bestenfalls eine untergeordnete Rolle gespielt haben - zwischen Rolle und Person, Wirtschaft und Politik, (lokal-)politischem Zentrum und Peripherie.

Was in Bezug auf Brüche und Wandlungsprozesse für die Agroholding gilt, lässt sich in Ansätzen auch für den ehemaligen Kolchos beobachten, wenngleich in abgeschwächter und weniger klar konturierter Form: Mit der Einführung von Verträgen und Preisverhandlungen in die Zusammenarbeit zwischen dem ehemaligem Kolchos und der Gemeindeverwaltung und vor allem über entsprechende Relevanzbekundungen aller Beteiligten wird abermals die Grenze zwischen beiden Or- 
ganisationen betont. Beide, so die Stoßrichtung der vom Gesetz ermöglichten und nahegelegten und jetzt vom Kolchos durchgesetzten Formalisierungen, sind ungeachtet ihrer engen Kooperation füreinander Umwelt und orientieren sich in ihren Operationen an differenten Rationalitäten. Ihre jeweiligen Entscheidungen stellen sich aufeinander ein und treffen sich in Verträgen und Preisen, ohne dabei jedoch ineinander zu greifen, beispielsweise in Form füreinander zwingender Prämissen. Und ähnliches lässt sich schließlich auch in der Beziehung zwischen dem ehemaligem Kolchos und den Gemeindemitgliedern beobachten. Im Zuge der Episode um den (potenziellen) Verkauf der Eigentumszertifikate an Agrarland in der Gemeinde Andreevka setzt ein Prozess der Verrechtlichung, Ökonomisierung und Individualisierung im Verhältnis des Betriebs zu seinen Anteilseigerinnen und Anteilseignern ein. Die Beziehung zwischen Betrieb und Anteilseignern wird, wie auch die daran anknüpfenden Strukturen im Dorf, grundlegend verändert, ohne dass sich dies zwingend in den formalen Eigentumsverhältnissen widerspiegeln würde.

\section{Formalität, Informalität und IIlegalität im Horizont postsowjetischer Transformationsimperative}

Als letztes Resultat hervorzuheben sind schließlich die Konfigurationen von Formalität, Informalität und Illegalität innerhalb der und zwischen den beteiligten Organisationen, die in allen vier Episoden wiederholt und auf unterschiedliche Weise aufscheinen. Mit Blick auf die Formalstrukturen wird deutlich, dass vor allem über sie neue globale Kommunikationsformen »eintröpfeln«. Gleichzeitig lässt sich auch beobachten - und dieser Sachverhalt an sich ist aus Sicht der Organisationsforschung wenig überraschend -, dass sich die Bedeutung dieser veränderten Formalstrukturen erst aus dem Zusammenspiel mit informalen und illegalen Strukturen ergibt. Bemerkenswert ist jedoch, auf welche Weise sich dieses Zusammenspiel in den konkreten Beispielen entfaltet und welche Implikationen sich für die Strukturierung des lokalen Kommunikationszusammenhangs ergeben. Die vier Episoden verweisen hier auf ein generelles Muster: Das Auseinanderdriften lokaler und globaler Erwartungen wird von den Betrieben bearbeitet, indem Letztere tendenziell in die Formalstruktur der Organisationen übernommen und Erstere über die informale Ebene absorbiert werden. Auf diese Weise sichern die Betriebe nicht nur ihre Reproduktionsfähigkeit angesichts sich widersprechender Umweltansprüche, sondern es wird auch deutlich, dass und wie sich der ehemalige Kolchos und die Agroholding hinsichtlich ihrer Selbstbeschreibungen und deren strukturellen Folgen aufeinander zu bewegen.

Mit aller Macht, so scheint es, ist der ehemalige Kolchos darum bemüht, sich als Wirtschaftsorganisation $\mathrm{zu}$ generieren und an seiner Primärorientierung an Zahlungen und Zahlungsfähigkeiten sowie am Profit als alleinigem Erfolgskrite- 
rium keinen Zweifel aufkommen zu lassen. Fast lehrbuchartig spiegeln sich in den Äußerungen vor allem von Mitgliedern der Kolchosleitung jene Erwartungen, die im Zusammenhang mit Wirtschaftsreformen und Privatisierungsmaßnahmen, aber auch im Kontext der jüngsten Kommunalreform aus der globalen Umwelt an den Betrieb gerichtet werden. Dieses Bemühen schlägt sich besonders sichtbar in den Formalstrukturen des Betriebs nieder. Sie sollen >sowjetisch < anmutendes Engagement im Bereich der technischen und sozialen Infrastruktur, das von diesen proklamierten wirtschaftlichen Zielen abweicht oder sogar im Widerspruch dazu steht, über eigens konzipierte Buchhaltungs- und Abrechnungsinstrumente, die Einführung von Verträgen gegenüber der Gemeindeverwaltung und den Verweis auf Preisverhandlungen ausdrücklich in den Horizont ökonomischer Logiken rücken.

Die lokale Umwelt des Betriebs - die Gemeindemitglieder ebenso wie die Kommunalverwaltung - zeigt sich indes nur bedingt bereit, diese veränderten und an globale Erwartungen angepassten Selbstbeschreibungen samt der strukturellen Implikationen zu akzeptieren und an die entsprechenden Formalstrukturen anzuschließen. Deutlich wird stattdessen die lokale Persistenz von umfassenden >sowjetischen< Erwartungen. Vor allem die fehlende Differenzierung von Rolle und Person sorgt im Verhältnis von Bürgermeisterin und Kolchosvorsitzendem dafür, dass eine Vielzahl von Erwartungen mit besonderer Vehemenz an den Kolchosvorsitzenden als Person herangetragen werden, denen sich die Kolchosleitung als Ganzes kaum entziehen kann und die der Umsetzung rollenbezogener Transformationsimperative im Wege stehen. Auf den Anpassungsdruck, der aus den divergierenden Umwelterwartungen an den Betrieb erwächst, reagiert die Organisation Kolchos mit einer doppelten Strategie aus aufgedrängter Illegalität und der Einführung alternativer Stoppregeln für einzelne Tätigkeitsbereiche: Erstere führt zu informalen Leistungsarrangements, die im Widerspruch zu den Formalregelungen stehen und primär über untere Hierarchieebenen des Betriebs abgewickelt werden. Letztere bewirken, dass bestimmte Aktivitäten explizit dem wirtschaftlichem Kalkül entzogen und einer am Gemeinwohl ausgerichteten Logik des öffentlichen Feldes unterstellt werden. Diese starke interne Differenzierung mag angesichts des vielfältigen Engagements westlicher Wirtschaftsunternehmen unter dem Schlagwort der sozialen Verantwortung nicht allzu sehr überraschen, stellt jedoch für den ehemaligen Kolchos einen signifikanten Bruch mit sowjetischen Strukturen dar.

Eine analoge Tendenz findet sich erneut mit Blick auf die Agroholding: So passt sich der Investor, der seine Präsenz im Dorf ursprünglich als ein Mittel für wirtschaftliche Zwecke betrachtete, vor allem im Hinblick auf notwendige technische und soziale Leistungen für die Gemeindeverwaltung sukzessive an jene lokalen Erwartungen an, die in identischer Form auch an den ehemaligen Kolchos gerichtet werden. Wenngleich die Unternehmensleitung diesen Anpassungsprozess allein wirtschaftlich begründet, so ist nicht zu verkennen, dass die Arrangements 
des Unternehmens mit der Gemeindeverwaltung eine >kolchosähnliche< Mischung aus Formalstrukturen, in denen sich ökonomische Rationalitäten spiegeln, und informalen Strukturen, die davon explizit abgegrenzt und am Gemeinwohl des Dorfes ausgerichtet werden, aufweisen. Ein Muster, das folglich keineswegs allein auf personelle Kontinuitäten und eingespielte Praktiken zurückzuführen ist und das sich nicht quasi-automatisch auflöst, sobald neue Akteure ins Spiel kommen.

Führt man das Spektrum von Wohlfahrts- und Wirtschaftsorientierung ins Feld, das im Transformationskontext als Positionierungsaufforderung vor allem an die ehemaligen Kolchose herangetragen wird und eine Reaktion erfordert, wird deutlich, dass sich der ehemalige Kollektivbetrieb und die Agroholding hinsichtlich ihrer Selbstbeschreibungen und der im Dorf erbrachten Leistungen anähneln. Ersterer als >sowjetische< Organisation passt nicht nur seine Selbstbeschreibungen an globale Erwartungsstrukturen an, die aus Wirtschaftsreformen und Privatisierungsmaßnahmen erwachsen, sondern ist darum bemüht, sich auch strukturell als Wirtschaftsorganisation zu generieren. Letztere, mit der eine Organisation in den lokalen Kommunikationszusammenhang eintritt, die primär von (globalen) Marktstrukturen geprägt ist und sich an diesen zum Zeitpunkt ihres Auftauchens in der Gemeinde explizit orientiert, kommt spiegelbildlich nicht umhin, auf die lokalen Erwartungen mit einem zunehmenden Maß an Gemeinwohlorientierung zu reagieren. In beiden Fällen ist es die Gemeindeverwaltung respektive die Person der Bürgermeisterin, über die diese Erwartungen an die Großbetriebe herangetragen werden. In beiden Fällen werden soziale Leistungen, wenn auch mit divergierenden Begründungen, in das wirtschaftliche Selbstbild des Unternehmens zu integrieren versucht. Und in beiden Fällen erweisen sich dieses Vorhaben und die daran anschließenden Formalisierungsbestrebungen im Dorfkontext als nur bedingt umsetzbar und bleiben entsprechend unvollständig.

Ein Resultat ist eine innere Spaltung der Organisationen, die durch das Zusammenspiel von Formalität und Informalität stabilisiert wird, wobei das $>$ Neue $<$ regelhaft in Formalstrukturen einfließt, während das >Alte< auf die Ebene der Informalität ausgelagert wird. Die informalen Strukturen verweisen in erster Linie auf die lokale Umwelt. Sie ermöglichen es den beteiligten Organisationen, lokale und größtenteils im Widerspruch zu den globalen Transformationsimperativen stehende Erwartungen zu absorbieren und zu kanalisieren, diese mit den jeweils eigenen Zwecken kompatibel zu machen und die eigene Reproduktionsfähigkeit zu erhalten. Die landwirtschaftlichen Großbetriebe fungieren auf diese Weise als Projektionsflächen lokaler Erwartungen, so dass andere Bezugspunkte dafür (vorläufig) nicht nötig sind.

Die veränderten Formalstrukturen, dies wird an vielen Stellen deutlich, sind im postsowjetischen Dorf weit mehr als nur kosmetische Fassade: Unter Verweis auf sie verschieben sich Beobachtungshorizonte, Normalitätserwartungen und Kontin- 
genzbezüge. Und über die formale Ebene wirken weltgesellschaftliche Strukturen, die zuvor an den Dorfgrenzen förmlich abgeprallt sind, in den dörflichen Kommunikationszusammenhang hinein. Innerhalb der Dörfer etablieren sich Differenzierungsformen, die orthogonal zu den tradierten Strukturen verlaufen und an denen sich die lokalen Erwartungen auszurichten beginnen. Auch das Formale erweist sich dabei jedoch als anpassungsfähig. So wurde an verschiedenen Stellen der Analyse deutlich, dass über die Formalstruktur nicht Globales starr in die Gemeinde hineingespiegelt wird, sondern sich auf lokale Gegebenheiten einstellt.

Die formal-rechtlichen Transformationsmaßnahmen selbst bilden bei diesem Prozess einen losen Rahmen, durch den neue Möglichkeitsräume aufgespannt werden. Was im Dorf letztlich aber irritiert und Gewohntes zur Disposition stellt, sind konkrete Kommunikationsangebote, die eine Reaktion - und das heißt: eine Entscheidung - erfordern; und sei es die Entscheidung, sie zu ignorieren und (vermeintlich) weiterzumachen wie gewohnt. So kann die stabile Reproduktion des in weiten Teilen sowjetisch anmutenden Arrangements zwischen der Gemeindeverwaltung und dem ehemaligem Kollektivbetrieb nicht darüber hinwegtäuschen, dass sich die Sinnhorizonte der beteiligten Systeme auch infolge der Kommunalreform verschoben haben: Ungeachtet der faktischen Kontinuität der Kolchosleistungen orientieren sich die diesbezüglichen Normalitätserwartungen der Betriebsleitung zunehmend an den Reformregelungen, welche die entsprechenden Aufgaben klar der Gemeindeverwaltung zuweisen. Der Kolchosvorsitzende wird vor diesem Hintergrund nicht müde, auf den temporären Charakter der gegenwärtigen Arrangements zu verweisen. Und auch die Bürgermeisterin betont wiederholt, dass die ausbleibenden Steuerzahlungen zwar gegenwärtig akzeptiert werden, sie für die $\mathrm{Zu}$ kunft jedoch auf eine Anpassung an die Reformregelungen hofft. Inwieweit mit einer Erfüllung dieser Erwartungen zu rechnen ist, lässt sich angesichts der dargestellten Kooperationsstrukturen nur schwer beurteilen. Einerseits pausen sich in den skizzierten Formalisierungen weltgesellschaftliche Strukturen, allen voran des Wirtschaftssystems, durch und es werden insbesondere durch die Schaffung sogenannter Marktrequisiten in Form von Preisen und Verträgen Anschlussmöglichkeiten über die Dorfgrenzen hinweg generiert. Auch wenn diese gegenwärtig nicht realisiert werden, stehen sie gleichwohl im Raum und rücken einen Anschluss beispielsweise an Marktkommunikation im Bereich kommunaler Dienstleistungen in den Horizont des Möglichen, was wiederum unweigerlich auf die bestehenden Kooperationsformen von Kolchos und Kommune zurückschlagen und eine Öffnung des dörflichen Kommunikationszusammenhangs implizieren würde. Andererseits ist zu erkennen, dass sich das momentane Kooperationsverhältnis von Kolchos und Kommune mit jeder neuen Operation gleichsam in sich selbst festzurrt. Angeschlossen wird an der Formalstruktur vorbei auf der informalen Ebene, was eine 
sich selbst verstärkende Verflechtung beider Organisationen bewirkt und jegliche Kommunikationsangebote von außen abprallen lässt.

\section{Das postsowjetische Dorf als verschwindende Nische in der Weltgesellschaft}

Die Suche nach und zunehmende Orientierung dörflicher Kommunikation an globalen Anlehnungskontexten, die Verschiebung von Inklusionsmodi, die Etablierung von Bürgermeisterinnen und Bürgermeistern, Interaktionsforen und landwirtschaftlichen Großbetrieben als zentralen Parametern lokaler Politik, die Emergenz spezifischer Konfigurationen von formalen und informalen Strukturen welche Funktion erfüllen diese Phänomene und die dahinterstehenden Wandlungsprozesse im Kontext des ländlichen Russland? Nimmt man die Strukturen der sowjetischen Organisationsgesellschaft zum Ausgangspunkt, so führte der Zusammenbruch des sowjetischen Regimes und mit ihm der Wegfall zentralistischer Planung und umfassender, politisch kontrollierter gesellschaftlicher Organisation aus Sicht der ländlichen Gemeinden zu einem plötzlichen und signifikanten Anstieg an Umweltkomplexität. Zwar blieben ungeachtet dessen sowjetische Strukturen (vorerst) erhalten, da sich zentrale Institutionen wie der ehemalige Kolchos weiterhin reproduzierten. Die hermetische Schließung des dörflichen Kommunikationszusammenhangs war jedoch angesichts steigender Umweltkomplexität nicht mehr aufrechtzuerhalten. Trug der Fokus auf eine dominierende Umweltschnittstelle die Kolchosleitung - den Logiken der hierarchisierten Organisationsgesellschaft angemessen Rechnung, erscheint er im Transformationskontext zunehmend unterkomplex. Um sich in dieser Lage reproduzieren zu können, gerät der dörfliche Kommunikationszusammenhang unter Anpassungsdruck: Die innere Komplexität ist zu erhöhen, um die eigene Komplexitätsverarbeitungskapazität zu steigern oder die eigene Geschlossenheit ist aufzugeben. An dieser Stelle erschließt sich die Funktion der beschriebenen Phänomene und Wandlungsprozesse, die auf unterschiedliche Weise dazu beitragen, dass funktionale Differenzierung in den Dorfkontext hineinzuwirken beginnt. Dies wiederum impliziert die zunehmende Durchlässigkeit der vormals hermetischen Dorfgrenzen und letztlich das Auflösen des Dorfes als Nische - im Sinne eines geschlossenen und selbstreferenziellen Kommunikationszusammenhangs, der sich der gesellschaftlichen Evolution entzieht - in der Weltgesellschaft. Ein Prozess, der sich nicht schlagartig, friktionslos und quasi-automatisch vollzieht, sondern schrittweise und unter zum Teil erheblicher Reibung, die dazu führt, dass einerseits das $>$ Alte $<$ an das $>$ Neue $<$ angepasst wird, sich Letzteres aber andererseits auch kontinuierlich auf lokale Besonderheiten, Erwartungen und Praktiken einlässt. 
Das >Postsowjetische< wird auf diese Weise zur Simulation des Sowjetischen, das darin erkennbar wird, dass sich einzelne sowjetische Phänomene vordergründig und inhaltlich reproduzieren, sich unter der Oberfläche des Gewohnten der Modus der Reproduktion jedoch um- und auf die Logiken funktionaler Differenzierung einstellt. Hintergründig eröffnen sich so neue Anschlussmöglichkeiten, während das >Alte< stets im >Neuen< sichtbar bleibt. Dieser verbleibende Rest des Gewohnten, auch dies ist freilich einzuräumen, verliert offenkundig mit jeder neuen Irritation an Bindungskraft. Mit Blick auf die sowjetischen Strukturen entsteht eine Ironie: Wurden in der sowjetischen Organisationsgesellschaft per se regimefremde Strukturen marxistisch-leninistischen Prinzipien entsprechend ideologisch »bemäntelt« (Pollack 1998: 121, Fn. 32), lässt sich für die beschriebenen Merkmale des postsowjetischen Dorfes die These formulieren, dass hier erneut eine Bemäntelung stattfindet, indem die nun offiziell erwünschten funktionsspezifischen Kommunikationsformen mit einem sowjetischen Schleier versehen werden. Die Überlegenheit gegenüber anderen Modellen wird dabei nicht mehr über das moralische Pathos des Sozialismus und seiner Rede von einer besseren Gesellschaft und besseren Menschen hergestellt, sondern durch den Verweis auf und die Bindung an Marktrationalitäten und die Idee der Demokratie.

Mit dem Verweis auf Funktionssysteme, Organisationen und Inklusionsmodi lassen sich die vielfältigen Wandlungsprozesse auf diese Weise nicht nur auf einen gemeinsamen theoretischen Nenner bringen, sondern zudem anhand von Kategorien beschreiben, die zwar auf den speziellen Kontext postsowjetischer Transformation anwendbar, jedoch nicht zwingend daran gekoppelt sind. En passant entfällt damit auch die Notwendigkeit einer spezifischen postsowjetischen Transformationstheorie: Für die wissenschaftliche Beobachtung spielt es keine Rolle, ob die beschriebenen Wandlungsprozesse als Teil postsowjetischer Transformation beobachtet werden oder schlicht als Herausbildung einer regionalen Differenzierungsform in der Weltgesellschaft - und als solche als Momentaufnahme gesellschaftlicher Evolution. Dies eröffnet nicht nur Anschlussmöglichkeiten für zahlreiche empirische Befunde - dichte Beschreibungen, die mit Blick auf die postsowjetische Transformationsgesellschaft entstanden sind - und die Möglichkeit, diese theoretisch zu rahmen, ohne sie teleologischen oder normativen Imperativen zu unterwerfen. Der Fokus auf gesellschaftliche Differenzierungsformen und die Vermutung, dass strukturelle Verschiebungen im Verhältnis von Organisationen und Funktionssystemen sowie die jeweils daran gekoppelten Inklusions- und Exklusionsmuster sich potenziell auch in anderen Regionen der Weltgesellschaft und anderen historischen Kontexten beobachten lassen, erweitert zudem den Vergleichshorizont in räumlicher, sachlicher und zeitlicher Hinsicht: Instruktiv dürfte der Vergleich mit strukturell ähnlichen wirtschaftlichen und politischen Wandlungsprozessen in anderen Regionen der Weltgesellschaft, die nicht unter dem Etikett (postsowjetischer) 
Transformation subsumiert werden, ebenso sein wie der Blick auf Inklusions- und Exklusionsmodi in anderen Funktionssystemen der postsowjetischen Transformationsgesellschaft. 



\section{Von der Organisations- zur Transformationsgesellschaft}

Die vorliegende Studie fragte nach den Logiken und Mustern postsowjetischer Transformationen, nach Prozessen sozialen Wandels, die sich im Kontext der Privatisierung landwirtschaftlicher Großbetriebe und der Einführung einer kommunalen Selbstverwaltung im ländlichen Russland beobachten lassen. Die beiden in den Blick genommenen Reformen markieren eine Schnittstelle der sogenannten wirtschaftlichen und politischen Transformationen und versuchen besonders umfassend und tiefgreifend Strukturen zu verändern, die sich in den ländlichen Gemeinden Russlands während der über knapp 70 Jahre andauernden sowjetischen Gesellschaftsordnung herausgebildet hatten. Der empirische Fokus auf das ländliche Russland ist doppelt relevant und instruktiv, um die genannten Transformationen und ihre Auswirkungen zu studieren: Quantitativ stehen die Fallbeispiele der vorliegenden Analyse für einen Gebietstyp, auf dem zum Zeitpunkt der sowjetischen Zwangskollektivierung der Landwirtschaft in den späten 1920er und frühen 1930er Jahren mehr als zwei Drittel der russischen Bevölkerung lebten. Bis zum Zusammenbruch der Sowjetunion war dieser Anteil auf etwa ein Viertel zurückgegangen und blieb seither mehr oder weniger konstant. Die Studie rückt also ein spezifisches soziales Umfeld und eine Lebensweise in den Mittelpunkt, die zusammen und in mannigfaltigen Variationen gegenwärtig den Alltag von etwas mehr als 38 Millionen Menschen prägen. Qualitativ ist dieser Gegenstand bedeutungsvoll, weil das Dorf und die umfassende Fixierung des dörflichen Lebens auf die Staats- und Kollektivbetriebe als eine Art Miniaturausgabe der sowjetischen Gesellschaft verstanden werden kann. Im Sinne eines »Abbild[s] der Gesellschaft im Ganzen« (Oswald/ Ernst/Dittrich 2005: 242; vgl. auch Oswald 2007) fanden sich hier generelle und wesentliche Merkmale der gesellschaftlichen Ordnung wieder, steigerten sich teilweise und waren daher in kondensierter Form zu beobachten.

Wie diese Ordnung theoretisch zu greifen ist, wurde im ersten Argumentationsschritt präzisiert. Ansetzend am umfassenden Gesamtbild der sowjetischen Organisationsgesellschaft verengte sich der Blick dabei sukzessive auf das sowjetische 
Dorf als spezifischen Kommunikationszusammenhang. Die sowjetische Variante gesellschaftlicher Differenzierung, so lässt sich zusammenfassen, erschließt sich zum einen über die analytische Unterscheidung von Codes und Programmen gesellschaftlicher Funktionssysteme und die Frage nach deren Zusammenspiel und zum anderen über die Merkmale von Organisationen, auf welche die Prozesse gesellschaftlicher Reproduktion in der Sowjetunion kontinuierlich verweisen. Der Sozialismus - dies gilt zumindest für seine sowjetische Variante - lässt sich als ein Gesellschaftsprogramm mit umfassendem Geltungsanspruch begreifen. Es zielte darauf ab, die sowjetische Gesellschaft unter der Führung der kommunistischen Einheitspartei entlang von zwei Leitunterscheidungen einzurichten und zu strukturieren: Erstens die Unterscheidung von (Voll-)Inklusion im Sinne einer umfassenden politischen Adressierbarkeit und Exklusion, zweitens die Unterscheidung von Hierarchie (oder Planwirtschaft) und Markt respektive von Kollektiv- und Privateigentum. Innerhalb dieser Unterscheidungen sollte, so der Anspruch des Programms, jeweils die erstgenannte Seite markiert und präferiert werden - also (Voll-)Inklusion und Hierarchie respektive Kollektiveigentum -, während die jeweils letztgenannte als >kapitalistisches Anderes< mitlief - also Exklusion und Markt respektive Privateigentum. Als zentrales Instrument zur Programmumsetzung kamen Organisationen zum Einsatz. Sämtliche gesellschaftlichen Operationen sollten in einen von zwei Organisationstypen integriert werden: Entweder in die Partei, die sich selbst an die Spitze der Gesellschaft setzte und auf die interne Entdifferenzierung des politischen Systems hinwirkte, oder in eine Vielzahl von Massenorganisationen, die für faktisch jeden gesellschaftlichen Bereich eingerichtet wurden und für die umfassende Inklusion aller Gesellschaftsmitglieder zuständig waren.

Wohlgemerkt bedeutet die Identifikation der genannten Leitunterscheidungen und des zentralen Implementationsmechanismus keineswegs, dass die sozialistische Programmierung der Gesellschaft in der von der politischen Führung beabsichtigten Weise gelang. Aber diese Elemente des sowjetischen Regimes fungierten in der Selbst- wie in der Fremdbeobachtung als Referenzpunkte, über die sich sowohl die Konformität mit als auch die Abweichung von den politischen Idealen und den daraus abgeleiteten Maßnahmen erschließen und veranschaulichen lassen. Dies kommt exemplarisch mit Blick auf die Strukturmerkmale sowjetischer ländlicher Gemeinden zum Ausdruck: An den landwirtschaftlichen Kollektivbetrieben als dörfliche Massenorganisationen lässt sich illustrieren, wie sowohl die Hierarchisierung wirtschaftlicher Kommunikation und die Eigentumskollektivierung als auch die umfassende Inklusion der Dorfbevölkerung in Organisationsstrukturen wirkungsvoll umgesetzt wurden und das sozialistische Programm auf diese Weise auf die Codeebene des Wirtschaftssystems durchgriff. Der Kolchos generierte sich innerhalb des Dorfes als Machtinstanz, wohingegen der Gemeindeverwaltung eine untergeordnete Position zukam. Gleichzeitig führte die Dominanz der Kollektivbe- 
triebe dazu, dass sich die ländlichen Gemeinden als weitgehend abgeschlossene Kommunikationszusammenhänge oder Nischen innerhalb der staatlich gelenkten Hierarchie etablierten, in denen sich bemerkenswerte Spielräume für abweichende und regimefremde Kommunikationsformen - beispielsweise in Form privater Hoflandwirtschaften und wirtschaftlicher Kommunikation - eröffneten. Deutlich wird, dass und wie regimekonforme und abweichende Strukturen in den sowjetischen Dörfern nicht nur koexistierten, sondern sich durch kontinuierliche Verweise aufeinander reproduzierten und ineinander verzahnten.

Ein weiteres Einfallstor funktionaler Differenzierung, das auch, aber nicht ausschließlich die Landwirtschaft tangierte, resultierte aus dem Erfolgsdruck, den sich das sowjetische Regime durch seinen betont zu Schau gestellten Überlegenheitsanspruch gegenüber anderen Gesellschaftsordnungen, allen voran dem Kapitalismus, einhandelte respektive selbst auferlegte: Auch das um deutliche Abgrenzung innerhalb der Weltgesellschaft bemühte sowjetische Regime konnte (und wollte) sich weltgesellschaftlicher Kommunikation nicht vollständig entziehen. Um die eigene Überlegenheit nach innen und außen zu demonstrieren und sich so zu legitimieren, erwies sich die Einbindung in globale, funktional differenzierte Kommunikationszusammenhänge als unverzichtbar. Wollte man hier adressierbar sein, war es jedoch unumgänglich, sich auf die entsprechenden Eigenlogiken einzulassen, die in der Folge unweigerlich auch auf das sowjetische Regime selbst durchschlugen (wenn auch die politische Führung kontinuierlich darum bemüht war, diese Durchschlagskraft zu mindern und ein Anschließen daran zu kontrollieren und möglichst zu unterbinden).

Solche Nischen und Einfallstore funktionaler Differenzierung samt der - aus Sicht der politischen Führung - damit verbundenen Unwägbarkeiten stehen nicht im Widerspruch zu dem Argument, dass die politische Organisation der Gesellschaft in der Sowjetunion zumindest insoweit gelang, als dass Organisationsstrukturen funktionssystemspezifische Kommunikationszusammenhänge weitgehend dominierten. Letztere waren stets an Erstere gekoppelt (und nicht umgekehrt), was nicht allein in der Omnipräsenz politisch kontrollierter Organisationen zum Ausdruck kam, sondern auch in den Determinanten der Freiheitsgrade, dem Spektrum an Handlungs- und Entscheidungsmöglichkeiten jedes einzelnen Mitgliedes der sowjetischen Gesellschaft. Diese wiederum verweisen unweigerlich auf die Mitgliedschaft in ebenjenen Organisationen als zentralem Zutrittsmechanismus (auch) $\mathrm{zu}$ funktionssystemspezifischen Kommunikationszusammenhängen mit gesellschaftsweiter Geltungskraft. Organisationsmitgliedschaft vermittelte, ganz gleich ob politisch gewollt oder nicht, die Adressierbarkeit in gesellschaftlichen Funktionssystemen. Andersherum konnte über die entsprechenden Organisationen diese Adressierbarkeit auch wieder unterbunden oder zumindest mit signifikanten Hürden versehen werden. Im sowjetischen Dorf kam dies unter anderem darin zum 
Ausdruck, dass der Zugang zu den beschriebenen kleinbäuerlichen Produktionsformen und die Ausrichtung an Knappheitskommunikation mit allen damit verbundenen Freiheitsgraden unauflöslich an die Mitgliedschaft im Kollektivbetrieb gekoppelt blieb. Die Auflösung dieser Kopplung in den späten 1980er Jahren durch die rechtliche Möglichkeit, Land auch unabhängig von der Kolchosmitgliedschaft zu pachten, erwies sich im Nachhinein als eine frühe Auflösungserscheinung des Regimes. Die Logik der sowjetischen Organisationsgesellschaft erschließt sich über die Formen organisationaler Inklusion und Exklusion, während (regimefremde) funktionale Differenzierung zwar im Hinblick auf die zumindest temporäre Stabilität und Reproduktionsfähigkeit der sowjetischen Gesellschaft unverzichtbar war, ihr aber ungeachtet dessen eine im oben skizzierten Sinne nachrangige Bedeutung zukam.

Die ländlichen Gemeinden stehen nicht nur beispielhaft für die Funktionslogik der sowjetischen Gesellschaft, weil hier die Reichweite und die Bruchstellen des sozialistischen Programms gleichermaßen hervortreten. Sie sind auch ein instruktiver Ansatzpunkt, um die durch den Regimewechsel induzierten Wandlungsprozesse zu beobachten, im Zuge derer speziell die wirtschaftlichen Privatisierungs- und politischen Dezentralisierungsmaßnahmen wesentliche Kontrapunkte zu den zentralen Strukturmerkmalen des sowjetischen Dorfes setzten. Besonders deutlich tritt dieser Gegensatz vor dem Hintergrund eines soziologischen Verständnisses von politischer Dezentralisierung und lokaler Selbstverwaltung hervor, das im zweiten Argumentationsschritt entwickelt wurde. Im Vergleich zu den gängigen Definitionen der beiden Konzepte, die größtenteils politischen Zielsetzungen folgen und im politischen System selbst anschlussfähig sind - was einerseits bestimmte Einsichten vermittelt, andererseits aber auch mit eigenen blinden Flecken verbunden ist -, führt ein solches Verständnis zu einem dazu inkongruenten Bild und lässt drei Aspekte der inneren Ordnung des politischen Systems in den Vordergrund treten: Erstens seine interne Differenzierung durch die Einführung einer relativ autonomen lokalen Verwaltungsebene im Zentrum des politischen Systems; zweitens die Einrichtung von relativ autonomen Organisationen auf dieser Ebene und im Zuge dessen die Institutionalisierung politischer Leistungsrollen; drittens die Konstitution eines lokalen Publikums, die spezifische Formen der Publikumsinklusion hervortreten lässt. Im postsowjetischen Kontext wird darüber hinaus ein vierter Aspekt relevant, der das Umweltverhältnis der Politik betrifft: Die Entflechtung von Gemeindeverwaltung und (ehemaligen) Kollektivbetrieben durch die Zuweisung von verschiedenen Organisationszwecken und damit der impliziten Forderung nach Primärorientierung am politischen respektive wirtschaftlichen Funktionssystem. Ein Aspekt, der die Komplementarität und wechselseitige Gebundenheit der beiden Reformbereiche Kommunalverwaltung und Privatisierung unterstreicht. 
Die genannten Aspekte sind auch im föderalen Gesetz »Über allgemeine Prinzipien der Organisation lokaler Selbstverwaltung in der Russischen Föderation « aus dem Jahr 2003 enthalten, das seit 2006 (respektive verbindlich seit 2009) in Kraft ist und als jüngster Reformversuch im Bereich der politischen Dezentralisierung die Analyse meines eigenen empirischen Materials rahmte. Das Gesetz regelt im Wesentlichen fünf Bereiche neu: Erstens die territoriale Neugliederung der Verwaltungseinheiten als Maßnahme zur Kontrolle des Raums, die die ländlichen Gemeinden auf der unteren von zwei lokalen Verwaltungsebenen verortet; zweitens die Einführung obligatorischer Organe, Ämter und Stellen lokaler Verwaltung als Fixpunkte der Machtkommunikation; drittens die Einrichtung von interaktionsbasierten Foren der Publikumsinklusion in Form von Gemeindeversammlungen und öffentlichen Anhörungen; viertens die Festlegung kommunaler Aufgabenbereiche, in denen sich der Anspruch auf funktionale Zuordnung der Lokalverwaltung und Entflechtung von Kommune und ehemaligem Kolchos spiegelt; und fünftens die Einrichtung eines eigenen Budgets auch kleiner ländlicher Gemeinden (inklusive eigener Steuern), was deren Akteursstatus unterstreichen soll, in der Praxis jedoch meist zu einer hohen Subventionsabhängigkeit führt.

Angesichts des Wissens um die Strukturen des sowjetischen Dorfes und eines soziologischen Verständnisses von Dezentralisierung und Kommunalverwaltung lässt sich der Blick für Bruchstellen schärfen, die mit der Implementation der jüngsten Kommunalreform in ländlichen Gemeinden potenziell verbunden sind. Empirisch rückt auf diese Weise insbesondere das Verhältnis von Gemeindeverwaltungen und ehemaligen Kollektivbetrieben als dominanten dörflichen Organisationen in den Fokus und theoretisch abermals die Beziehung von Organisationen und gesellschaftlichen Funktionssystemen. Daran anschließend und konkretisierend lassen sich vier Leitaspekte für die Beobachtung von Kommunikation in diesem Transformationsbereich ableiten: Formen der Inklusion und Exklusion, Entscheidungen und Lernprozesse von Organisationen, der Umgang mit Formalität und Informalität sowie die Frage der Integration der Dörfer in weltgesellschaftliche Strukturen.

Die Irritationen und Wandlungsprozesse, die vor diesem Hintergrund in ausgewählten postsowjetischen ländlichen Gemeinden zu beobachten sind, wurden im dritten Argumentationsschritt anhand von vier empirischen Episoden nachgezeichnet. In der ersten Episode, ausgelöst durch ein Flugblatt, das im Zusammenhang mit der Kandidatur des Kolchosvorsitzenden für ein politisches Amt im Dorf verteilt wurde, ging es um Kommunikations- und Kooperationsformen zwischen Gemeindeverwaltung und ehemaligem Kollektivbetrieb primär im Bereich der lokalen Infrastruktur. Die zweite Episode setzte am Prozess des Anschlusses der Gemeinden an das Gasnetz an, der sich während meiner Forschungsaufenthalte in meinem Forschungsgebiet vollzog. Im Fokus der dritten Episode stand eine Agroholding als ein für das ländliche Russland neuartiger Betriebstyp, die sich ebenfalls während mei- 
ner Aufenthalte in einem der Dörfer zu etablieren begann. Die vierte Episode drehte sich schließlich um eine Reihe von Ereignissen, die durch das Kaufinteresse ebenjener Agroholding an Agrarland ausgelöst wurden. Die empirische Analyse lässt sich auf drei zentrale Ergebnisse hin zuspitzen - Wandlungsprozesse, auf die die inhaltlich sehr unterschiedlichen Episoden gleichermaßen, wenn auch in unterschiedlicher Intensität verweisen. Zu beobachten ist erstens eine Verschiebung der Inklusionsmodi. Die empirischen Beispiele verdeutlichen, auf welche Weise sich dörfliche Kommunikationsformen Anlehnungskontexte in global operierenden Funktionssystemen suchen. Im Zuge dessen konkurrieren die partikularen Logiken des lokalen Kommunikationszusammenhangs zunehmend mit den universalen Logiken der Weltgesellschaft, was unmittelbar im wechselseitigen Verhältnis verschiedener Inklusionsformen zum Ausdruck kommt: Die Adressierbarkeit in gesellschaftlichen Funktionssystemen, die nun Anschlussmöglichkeiten über die Dorfgrenzen hinaus eröffnen, gewinnt gegenüber der vormals dominierenden Kolchosmitgliedschaft an Bedeutung. Letztere stellt sich in der Folge auf Kompatibilität mit den Inklusionsmodi der Funktionssysteme um. Anders als noch bis in die späten 1990er Jahre hinein ergeben sich die Freiheitsgrade einzelner Gemeindemitglieder und die Handlungsspielräume der Gemeindeverwaltung damit nun nicht mehr aus dem Verhältnis zu und der Integration in die umfassende Organisation des (ehemaligen) Kolchos, sondern sie erwachsen aus der Möglichkeit zur Oszillation zwischen beiden Inklusionsmodi - Organisation und Funktionssysteme -, deren Verhältnis zumindest temporär in der Schwebe gehalten wird.

Daran schließt sich unmittelbar eine zweite zentrale Erkenntnis an, die die Institutionen und zentralen Parameter der lokalen Politik betrifft: Anhand des untersuchten Materials lässt sich nachzeichnen, wie sich die lokale Politik sukzessive als eigenständiger und relevanter Kommunikationszusammenhang herausbildet und Autonomie erlangt. Sie profiliert sich gegenüber anderen Funktionssystemen, beginnt nach eigenen Maßgaben zu operieren und es werden Entscheidungen mit kollektiver Bindungswirkung getroffen, die nicht mehr ohne Weiteres von nichtpolitischen Instanzen im Dorf überrollt werden können. Als Fixpunkte der lokalpolitischen Kommunikationszusammenhänge erweisen sich die Bürgermeisterinnen und Bürgermeister. Zwar verfügen sie nicht mehr über die umfassende Stellung der sowjetischen Kolchosvorsitzenden, aber sie positionieren sich erfolgreich als zentrale Figuren in zunehmend komplexen Kommunikationszusammenhängen und verstehen es, die beobachteten Prozesse sichtbar zu kanalisieren und zu moderieren. Darüber hinaus wurde die Interaktionsgebundenheit der lokalen Politik deutlich. Das lokale Publikum konstituiert sich maßgeblich in Interaktionen, mit allen Möglichkeiten und Grenzen, die mit diesem Systemtyp verbunden sind. Entsprechend wird Anwesenheit zur wesentlichen Inklusionsform in politische Entscheidungsprozesse, während andere Formen der Einbindung (wie Wahlen, die öffentliche Mei- 
nung und ähnliche) zumindest für die lokale Politik in den Hintergrund treten. Spiegelbildlich dazu wird Interaktion für die Politik zum zentralen Mechanismus der Publikumsbeobachtung. Zuletzt sind die landwirtschaftlichen Großbetriebe als zentrales Moment lokaler Politik zu nennen, sei es in der sowjetischen Form wie im Fall des ehemaligen Kolchos oder in expliziter Abgrenzung davon wie im Fall der Agroholding. Beide sind, in scheinbar sowjetischer Kontinuität, nach wie vor unverzichtbar für die lokale Infrastruktur. Aber sie sind dies nicht mehr als superrelevante Umwelt der Gemeindeverwaltung, sondern als Kooperationspartner. Die Zusammenarbeit zeichnet sich durch ein zunehmendes Maß an Formalisierung und Professionalisierung aus, wodurch trotz aller praktischen Abhängigkeiten immer auch die Grenzen und unterschiedlichen Rationalitäten beider Organisationen betont werden. An diesem Punkt finden Verschiebungen statt, auch wenn es vordergründig nicht so aussehen mag.

Der Verweis auf Formalstrukturen führt zum dritten Ergebnis: Mit Blick auf die formale Ebene der beteiligten Organisationen wird deutlich, dass hier neue globale Kommunikationsformen eintröpfeln, deren Bedeutung sich jedoch erst im Zusammenspiel mit informalen und illegalen Strukturen ergibt. Informale Strukturen verweisen dabei auf die lokale Umwelt: Sie wirken als ein Faktor, der lokale und den Transformationsimperativen teils zuwiderlaufende Erwartungen absorbiert, der für die Organisationen die Vereinbarkeit globaler und lokaler Erwartungen herstellt und der die Logiken der Funktionssysteme auf lokale Besonderheiten einstellt. Die veränderten Formalstrukturen, auch dies wird deutlich, sind dabei weder ausschließlich Fassade noch in Stein gemeißelt: Einerseits verschieben sich unter Verweis auf sie Beobachtungshorizonte, Normalitätserwartungen und Kontingenzbezüge, andererseits stellen sie sich immer wieder auch auf orthogonal verlaufende lokale Erwartungsstrukturen ein. Die formal-rechtlichen Transformationsmaßnahmen selbst bilden bei diesem Prozess einen losen Rahmen, durch den neue Möglichkeitsräume eröffnet werden. Was im Dorf letztlich aber für Irritation sorgt und Gewohntes zur Disposition stellt, sind konkrete Kommunikationsangebote, die eine Reaktion - und das heißt: eine Entscheidung - erfordern, und sei es die Entscheidung, sie zu ignorieren und in gewohnter, vermeintlich unveränderter Manier weiterzumachen.

Die Suche nach und die zunehmende Orientierung dörflicher Kommunikation an globalen Anlehnungskontexten und die Verschiebung von Inklusionsformen, die Etablierung von Bürgermeistern und Bürgermeisterinnen, Interaktionsforen und landwirtschaftlichen Großbetrieben als zentralen Parametern lokaler Politik, die Emergenz spezifischer Konfigurationen von formalen und informalen Strukturen all diesen beobachteten Wandlungsprozessen und Transformationsmustern liegt eine gemeinsame Stoßrichtung zugrunde: Mal unter Verursachung großer Aufregung, mal kleinteilig und fast unbemerkt wirken sie alle auf die Öffnung des vor- 
mals hermetisch geschlossenen dörflichen Kommunikationszusammenhangs hin, auf die zunehmende Durchlässigkeit seiner Grenzen für weltgesellschaftliche Strukturen und Kommunikationsformen und speziell: für funktionale Differenzierung. Sie tragen einer im Transformationskontext schlagartig gestiegenen Umweltkomplexität Rechnung, die nach neuen Modi des Umgangs und der Verarbeitung verlangt. Rückblickend wird damit das Potenzial einer funktionalen Analyse deutlich, Ungleiches und auf den ersten Blick Verschiedenartiges aufeinander zu beziehen und mit Blick auf seine Funktion - im Sinne einer Problembearbeitung - vergleichbar und nachvollziehbar zu machen (Luhmann 1984: 83ff; vgl. auch Nassehi 2008). An diese Überlegungen anschließend und in diese Logik einhakend möchte ich abschließend den empirischen Horizont im Hinblick auf die hier beobachteten Muster und Mechanismen postsowjetischer Transformationen erweitern. Die Einsichten und Resultate, die unmittelbar aus den untersuchten Fallbeispielen folgen, beobachte ich dazu vor dem Hintergrund des Forschungsstands zu Transformationsprozessen im ländlichen Russland, um dort nach Spuren und Anknüpfungspunkten zu suchen.

\section{Dynamiken der wirtschaftlichen Lage privater Haushalte als Indikatoren für Inklusionsverschiebungen}

Dass sich die dörfliche Kommunikation neue Anlehnungskontexte sucht und dass sich Inklusionsformen verschieben, ist auch anderen Arbeiten zu entnehmen, wenngleich diese Transformationen nicht immer explizit auf einen Begriff gebracht wurden. Spuren finden sich vor allem in Studien zu Wirtschaftsreformen und Privatisierungen, einem Forschungsfeld, auf dem sich Anthropologen, Geographen, Ökonomen und vereinzelt auch Politikwissenschaftler und Soziologen tummeln - auffällig häufig jedoch scheinbar ohne voneinander Notiz zu nehmen. Als Vergleichshorizont erscheint dieses Feld fragmentiert und befindet sich durch seinen expliziten und meist exklusiven Fokus auf die Privatisierung landwirtschaftlicher Großbetriebe und damit verbundene Eigentumsrechte, -formen und -praktiken in thematischer Distanz zum Gegenstand der vorliegenden Analyse. Hinzu kommt, dass die lokale Politik und die kommunale Verwaltung bestenfalls am Rande und in unmittelbarem Bezug zu den beobachteten Wirtschaftsreformen und Privatisierungsmaßnahmen eine Rolle spielen. Auch wenn gerade im Zusammenhang mit der sozialen Funktion der ehemaligen Kollektivbetriebe immer wieder auf die Beziehung zur Gemeindeverwaltung und auf die Komplementarität von Privatisierungsmaßnahmen und Kommunalreformen hingewiesen wird, die unumgänglich sind, um die beobachteten Strukturen und Prozesse zu verstehen, bleibt es diesbezüglich meist bei Lippenbekenntnissen und vertieft werden diese Aspekte selten. Ungeachtet dessen bieten sich jedoch auch hier diverse Ansatzpunkte für die Identifikation allge- 
meiner Muster postsowjetischer Transformation. Augenfällig sind vor allem zwei Forschungsbereiche, in denen sich Prozesse der Verschiebung von Inklusionsmodi und des Hervortretens globaler Anlehnungskontexte erkennen lassen, auch wenn dies nicht explizit benannt wird: zum einen Analysen zur wirtschaftlichen Lage privater Haushalte in ländlichen Regionen und zum anderen Studien zu verschiedenen Formen dörflicher Knappheitskommunikation, vor allem mit Blick auf die Relation von Naturaltausch und Zahlungen.

Ein zentrales Anliegen Ersterer - Studien zur wirtschaftlichen Lage ländlicher Privathaushalte - ist die Ermittlung von mittel- und langfristigen Effekten wirtschaftlicher Transformationsmaßnahmen. Hervorzuheben sind in dieser Hinsicht die Analysen von O'Brien, Patsiorkovski und Wegren (O'Brien/Patsiorkovski 2006; Patsiorkovski 2002; Wegren 2008, 2009b, 2012) sowie von Spoor und Visser (2004), die allesamt aufgrund ihrer breiten quantitativen Datenbasis auffallen. In verschiedenen Varianten thematisieren sie Anpassungsstrategien von Haushalten und landwirtschaftlichen Großunternehmen im Reformkontext und stützen sich teils auf statistische Aggregatdaten (zu Produktionsanteilen verschiedener Unternehmenstypen, zu Haushaltseinkommen, zu Landeigentum und -transaktionen und ähnlichem), teils auf breit angelegte eigene Erhebungen in verschiedenen Regionen Russlands. ${ }^{1}$ Instruktiv im Hinblick auf die Konkurrenz zwischen und die Verschiebung von Inklusionsformen sind in erster Linie die Beobachtungen zu Beschäftigungsformen, Einkommensquellen und Einkommensverteilungen in ländlichen Gemeinden.

Hinsichtlich der Beschäftigungsformen wird mit Blick auf ländliche Privathaushalte deutlich, dass die dominierende Position der ehemaligen Kollektivbetriebe als Arbeitgeber gegenüber anderen Beschäftigungsformen stetig abnimmt. Waren in den drei Gemeinden, in denen O'Brien und Patsiorkovski umfangreiche Interviewdaten erhoben haben, im Jahr 1991 noch 86 Prozent der Befragten bei einem landwirtschaftlichen Großbetrieb beschäftigt, so sank dieser Anteil auf 34 Prozent im Jahr 2003. Im Gegenzug stiegen die Anteile der Beschäftigten im öffentlichen

1 Die genannten Analysen von O'Brien, Patsiorkovski und Wegren (O'Brien/Patsiorkovski 2006; Patsiorkovski 2002; Wegren 2008, 2009, 2012) basieren auf zwei umfangreichen Haushaltsumfragen: Einem Panel von rund 500 Haushalten aus sieben ländlichen Regionen Russlands (Novgorod, Tver, Belgorod, Čuvašija, Krasnodar, Rostov, Volgograd), die in den Jahren 1991, 1993, 1995, 1997, 1999 und 2003 befragt wurden sowie einer Umfrage unter 800 Haushalten in fünf Regionen im Jahr 2001. Teilweise werden in den Analysen ergänzend dazu statistische Daten zur makroökonomischen Entwicklung berücksichtigt. Spoor und Visser (2004) stützen sich auf eine eigene Umfrage unter den Führungskräften von 43 ehemaligen Kollektivbetrieben in den Regionen Pskov und Rostov. 
Dienst (von 14 auf 24 Prozent), der Privatbauern (von 0 auf 10 Prozent) und der >informal Selbstständigen ${ }^{2}$ (von 0 auf 21 Prozent) (O’Brien/Patsiorkovski 2006: 58; vgl. auch Patsiorkovski 2002: 124). Angesichts dessen, dass im sowjetischen Dorf die Anstellung beim Kolchos der zentrale Schlüssel für den Zugang zu faktisch allen öffentlichen Dienstleistungen war - Gesundheitsversorgung, Schule, Kolchosdienstleistungen und andere -, ist zu vermuten, dass diese Verschiebungen nicht allein eine Strukturänderung des Arbeitsmarktes anzeigen, sondern sich hier ein tiefgreifender sozialer Wandel in den Gemeinden abspielt: »Thus, any shift away from employment in the collective enterprise is an indicator of an important institutional and organizational shift in the basic character of village life that goes beyond employment « (O’Brien/Patsiorkovski 2006: 58).

In eine ähnliche Richtung weisen die Veränderungen der relativen Bedeutung verschiedener Einkommensquellen und -formen, wobei sich zwei unterschiedliche Verschiebungen beobachten lassen: Der relative Rückgang nicht-monetären Einkommens gegenüber monetären Einkommensanteilen und die allmähliche Verdrängung des Einkommens aus der Beschäftigung beim ehemaligen Kolchos durch andere Quellen. Belief sich der nicht-monetäre Anteil am Haushaltseinkommen im Jahr 1995 noch auf rund 40 Prozent, war dieser Anteil im Jahr 2003 auf 23 Prozent gesunken. Im selben Zeitraum stieg das monetäre Einkommen aus der eigenen Produktion der Haushalte von durchschnittlich 20 Prozent auf rund 40 Prozent (O’Brien/Patsiorkovski 2006). Die Autoren der Studie identifizieren private Haushalte vor diesem Hintergrund als Keimzellen des Unternehmergeistes im ländlichen Russland (O’Brien/Patsiorkovski 2006: 61f). Komplementär dazu lässt sich beobachten, wie sich die Anpassungs- und Reproduktionsstrategien der Haushalte seltener an sowjetischen Institutionen wie dem ehemaligen Kolchos orientieren (Wegren 2012; vgl. auch O'Brien/Wegren/Patsiorkovski 2007: 42f). Stattdessen gewinnen eigenständige wirtschaftliche Aktivitäten, unter anderem auf der Basis von privatisiertem Land, an Bedeutung und mausern sich zur Erfolgsstrategie, deren Bezugspunkte nicht mehr zwingend auf den Dorfkontext beschränkt sind. Das >reine Überleben< weicht ambitionierteren, meist wirtschaftlichen Zielen:

»Thus, those who could adapt to survive, did so, and they did so by decreasing reliance on traditional survival strategies of rural households: household labour and informal networks. The transition to new survival strategies is measurable in two ways. First, the effects of household labour and the effects of rental land on household income exerted influence inde-

2 D.h. faktische Selbstständigkeit, ohne dass die Betreffenden offiziell ein Gewerbe angemeldet haben. Im ländlichen Raum fallen darunter beispielsweise Familien, die vom Verkauf der eigenen landwirtschaftlichen Produktion leben, aber nicht als privatbäuerlicher Betrieb registriert sind. 
pendently as time went on, that is, the effect were separate effects could be quantified, showing the property holdings became more and more important. Second, during 1995-2006 the percentage of household income that was non-monetized declined, that is, reliance on food produced and consumed by the household declined, thus indicating that as time passed the quest for survival was replaced by a pursuit of growth.« (Wegren 2012: 196)

Das ehemals klar geregelte Verhältnis zwischen den Haushalten und den Großbetrieben werde vor allem durch den Wegfall der obligatorischen Arbeitsverhältnisse »schwieriger und komplizierter « und unterliege zunehmend dörflichen Aushandlungsprozessen (Patsiorkovski 2002: 124). Parallel dazu gewinnen für die Haushalte neue Beziehungen - etwa zur Gemeindeverwaltung oder zu anderen Haushalten - an Bedeutung, so dass sich das soziale Gefüge insgesamt wie auch ihre Einbettung darin wandeln.

Die daraus resultierende Pluralisierung und Individualisierung von Handlungsund Entscheidungsmöglichkeiten, mit der die einzelnen Haushalte konfrontiert sind und die sie in unterschiedlicher Weise - und das heißt auch: mit unterschiedlichem wirtschaftlichem Erfolg - nutzen, werden im zunehmenden Einkommensgefälle sichtbar (vgl. Lindner 2007; Wegren 2008: 141, 2009b: 160ff). An die Stelle der Kolchosmitgliedschaft, von der eine deutlich homogenisierende Wirkung ausgegangen war, treten nun verstärkt alternative, kolchosunabhängige Faktoren, die auf die Umwelt des Dorfes und/oder die Verschiebung tradierter Beziehungsmuster verweisen. So wird beispielsweise die Einbindung in die ehemals sowjetische Hierarchie - primär über die Position im Organisationsgefüge des landwirtschaftlichen Großbetriebs - als Garant für ein hohes Haushaltseinkommen von marktgebundenen Formen der Erwerbstätigkeit abgelöst. Entfielen die höchsten Einkommen im Jahr 1991 noch mit deutlichem Abstand auf das Kolchosmanagement, traten im Jahr 2003 die Privatbauern an die Spitze der dörflichen Einkommenspyramide (O’Brien/Patsiorkovski 2006: 101). Auch die räumliche Entfernung des Dorfes zum Bezirkszentrum spielt, wie Wegren (2008) zeigt, eine zunehmend wichtige Rolle für die wirtschaftliche Lage der Haushalte. So weisen Haushalte in Dörfern, die maximal zehn Kilometer vom Bezirkszentrum entfernt liegen, aufgrund von leichter anzubahnenden individuellen Geschäftsaktivitäten eine höhere Produktion, höhere Einkommen und größeren Landbesitz auf als Haushalte in entlegeneren Gemeinden (mehr als 35 Kilometer vom Bezirkszentrum). Erneut wird deutlich, dass der ehemalige Kolchos seinen Status als Fixpunkt und umfassende Determinante der individuellen Lebensführung verliert. Schließen lässt sich außerdem, dass individuelle Mobilität und Kontakte über die Dorfgrenzen hinweg an Bedeutung gewinnen dürften, was die Unterschiede in der Einkommensverteilung weiter verstärkt. 
Eine Reihe von Studien widmet sich der Frage, inwieweit sich Sozialkapital respektive die Vernetzung privater Haushalte im Dorf und über die Dorfgrenzen hinweg auf ihre wirtschaftliche Lage auswirkt (O'Brien/Patsiorkovski 2006: 81ff; Shubin 2007; für Russland allgemein Rose 2000). Leistungen und Leistungserwartungen, um die es in den untersuchten Netzwerken geht, umfassen in Anlehnung an das klassische Verständnis von Sozialkapital die Unterstützung in verschiedenen Bereichen der alltäglichen Lebensführung, darunter das Leihen von Geld, Hilfe bei der landwirtschaftlichen Arbeit (z.B. bei der Ernte) und beim Verkauf der eigenen Produktion, Einspringen im Krankheitsfall, Beratung in Alltagsfragen und ähnliches. Vorhandene Netzwerkbeziehungen eines Haushalts, so ein kaum überraschendes Resultat, wirken sich per se positiv auf die Einkommenslage aus. Darüber hinaus beobachten O'Brien und Patsiorkovski (2006: 83f) im Zeitverlauf jedoch einen interessanten Wandel der Netzwerkeigenschaften, die besonders förderlich für individuellen Wohlstand zu sein scheinen: 1995 ist ein positiver Zusammenhang zwischen kleinen Netzwerken (aus weniger als acht Personen) und der landwirtschaftlichen Produktivität eines Haushalts zu erkennen, für größere Netzwerke wird der Zusammenhang negativ. Nur wenige Jahre später hat sich dies gewandelt und im Jahr 2003 ist der Zusammenhang von Netzwerkgröße und Produktivität durchweg positiv. Ähnliches zeigt sich für das »community involvement«, die Einbindung eines Haushalts in das allgemeine Sozialleben der Gemeinde in Form der Teilnahme an Dorffesten, Hochzeiten und Geburtstagsfeiern (O'Brien/Patsiorkovski 2006: 85). Verbindungen, die als »weak ties« im Sinne Granovetters (1973) ebenfalls relevant für den individuellen wirtschaftlichen Erfolg sein müssten. Auch hier zeigt sich für das Jahr 1995 ein leicht negativer Zusammenhang mit Haushaltsproduktion und -einkommen, für das Jahr 2003 hingegen ein deutlich positiver. Die Ergebnisse dieser Netzwerkanalyse sollten, auch im Hinblick auf ihre Verallgemeinerbarkeit, nicht überstrapaziert werden. Sie verweisen aber doch darauf, dass eingespielte Strukturen innerhalb des Dorfes sukzessive aufbrechen und sich verschieben, der ehemalige Kolchos im Dorf an Zentralität verliert und die Einbindung in diese neuen, von den Großbetrieben tendenziell entkoppelten Strukturen - dies gilt zumindest in wirtschaftlicher Hinsicht - nach und nach zur zentralen Reproduktionsbedingung für die Haushalte wird: »In short, houdeholds that were more successful were beginning to look outward in order to expand their economic opportunities « (O’Brien/Patsiorkovski 2006: 87). Dieser Befund fügt sich in das Bild, das Rose (2000) in einer vielzitierten Studie mit Blick auf individuelle Netzwerke und Sozialkapital als Determinanten individuellen Wohlstands in Russland zeichnet. Der theoretische Kern der Analyse ist hier die Unterscheidung von modernen, anti-modernen und vormodernen Netzwerken: Moderne Netzwerke, so lässt sich in Anlehnung an Rose formulieren, bestehen aus formalisierten Verbindungen zwischen Individuen, die sich an gesellschaftlichen Funktionssystemen ausrichten (also z.B. 
Markt oder Verwaltungsbürokratie). Anti-moderne Netzwerke laufen modernen Netzwerken zuwider und untergraben diese potenziell (also z.B. Korruption, Klientelismus, mafiöse Strukturen). Als vormoderne Netzwerke werden jene Verbindungen bezeichnet, die Relikte aus vormodernen Gesellschaftsformen darstellen (also z.B. Verwandtschaftsbeziehungen, die Zugehörigkeit zu einer Dorfgemeinschaft oder einem Klan). Ungeachtet dessen, dass man diese Klassifikation für ihre geringe analytische Trennschärfe kritisieren könnte, sind die Resultate bemerkenswert: Erstens beobachtet Rose, dass für die untersuchten Explananda (Lebensmittelversorgung, Sicherheitsgefühl im öffentlichen Raum, Einkommenssicherheit) die Bedeutung vormoderner Netzwerke durchweg gering ist, während moderne und vielmehr noch anti-moderne Netzwerke dominieren. Zweitens kommt er zu dem Ergebnis, dass sich gerade die Diversifikation der Netzwerkverbindung in Form der parallelen Einbindung in alle drei Netzwerktypen positiv auf das individuelle Wohlergehen auswirkt.

\section{Das ländliche Russland zwischen De- und Remonetarisierung}

Weitere Aspekte postsowjetischer Transformationen, in denen die schwindende Zentralität der landwirtschaftlichen Großbetriebe und das Hineinwirken funktionsspezifischer Logiken in den lokalen Kontext zum Ausdruck kommen, sind der Monetarisierungsgrad wirtschaftlicher Aktivitäten und die spezifischen Formen von Knappheitskommunikation. Wurde in der sowjetischen Organisationsgesellschaft die wirtschaftliche Funktion des Geldmediums ausgehöhlt, weil auch bei Geldtransfers eher Macht als Knappheit kommuniziert wurde, so verschwand das Medium Geld in den 1990er Jahren fast gänzlich: Die russische Wirtschaft und vor allem die Produktion waren im ersten Jahrzehnt nach dem Zusammenbruch der Sowjetunion zu großen Teilen entmonetarisiert. Genau betrachtet teilte sich die Wirtschaft in eine auf Geldzahlungen basierende »reale« Ökonomie und in eine vom Naturaltausch dominierte »virtuelle« Wirtschaft (vgl. die Beiträge in Seabright 2000; auch Gambold Miller 2002; Kitching 1998). Die Unternehmen entwickelten in diesem Umfeld zum Teil ausgefeilte Strategien, um ihre Einbindung in beide Bereiche sicherzustellen und operierten oftmals parallel in Form von Zahlungen und (nichtmonetären) Tauscharrangements. Systematische Daten, geschweige denn offizielle Statistiken zur sogenannten virtuellen oder fiktiven Wirtschaft existieren nicht. Es finden sich jedoch punktuell Erhebungen und Einschätzungen einzelner Autorinnen und Autoren. So scheinen beispielsweise im Jahr 1998 über alle bedeutenden Industrien Russlands hinweg 44 Prozent des Verkaufs in Form von Tauschgeschäften abgewickelt worden zu sein (Guriev/Ickes 2000: 151). In der Landwirtschaft lag der Anteil im Jahr 1999 sogar bei etwas mehr als 50 Prozent (Carlin et al. 2000: 243). Einsicht in die ausgefeilten und komplexe Arrangements und Netzwerke hinter die- 
sen Zahlen bietet beispielsweise Kitching (1998: 8f), der einen vielstufigen Tauschprozess beschreibt: Der Tausch beginnt mit dem Bedarf des ehemaligen Kolchos nach Dieseltreibstoff und endet damit, dass der Leiter eines Tanklagers ein einjähriges Anrecht auf die Versorgung der gesamten Belegschaft mit Backwaren erhält (ähnlich auch die Empirie in Gambold Miller 2002).

Der geringe Monetarisierungsgrad betraf nicht allein Unternehmen, sondern auch private Haushalte. Auch hier fehlen verlässliche Daten, doch es ist allgemein bekannt, dass die Löhne in der Landwirtschaft zumindest in den frühen 1990er Jahren ganz oder teilweise in Naturalien ausgezahlt wurden (vgl. z.B. Clarke 2000: 192; Gambold Miller 2002: 228) und ein signifikanter nicht-monetärer Anteil am Haushaltseinkommen gilt für diese Periode als normal (siehe oben und vgl. z.B. O’Brien/Patsiorkovski 2006: 62). Während derartige Tauschpraktiken den Unternehmen durchaus gelegen kamen, weil sie das Verschleiern von Gewinnen, das Umgehen von Steuerzahlungen und die intransparente Gestaltung der Preisstrukturen zum eigenen Vorteil ermöglichten (Carlin et al. 2000; Guriev/Ickes 2000; Kitching 1998), gerieten die privaten Haushalte dadurch oftmals in Schwierigkeiten, zumal der geringe Monetarisierungsgrad nicht alle wirtschaftlichen Bereiche gleichermaßen betraf. Konsumgüter, Steuern, Fahrkarten für den öffentlichen Nahverkehr und ähnliches mussten auch weiterhin mit Geld bezahlt werden. Entsprechend beschränkte das Ausbleiben von monetären Lohnzahlungen massiv die Freiheitsgrade der betroffenen Haushalte. Um überhaupt die eigene Existenz zu sichern, blieb meist nur der Rückzug vom Markt und die Umstellung auf Subsistenz (Clarke 2000: 194). Entscheidend für die individuelle Lage war dabei (erneut) der unmittelbare dörfliche Kontext und allen voran der ehemalige Kolchos als zentraler Tauschpartner, von dessen Entscheidungen die einzelnen Gemeindemitglieder umso abhängiger waren. Umgekehrt blieb auch den Betrieben oftmals wenig Wahl. Sie mussten sich mit ihren Operationen primär auf ihre unmittelbare Umwelt beziehen und zulassen, dass der Organisationszweck von lokalen Erwartungen gekapert wurde:

»The lack of cash earning and financial profits is apparently secondary to the informal social obligations traditionally upheld in the village. A 1997 study found that farm managers reported maintaining unprofitable operations for spreservation of jobs, the need to secure foodstuffs for farm residents and hope for future subsidies. $<$ While suffering from the absence of subsidies and a negative cash flow, farm directors were forced to develop their agricultural operations and maintain current job levels.« (Gambold Miller 2002: 228)

Dass solche Strukturen als ein wesentliches Hinderniss bei der Implementation von Wirtschaftsreformen galten, liegt auf der Hand und wurde an verschiedenen Stellen konstatiert: »With the demonetarization of rural Russia paving the way for an ela- 
borate system of barter, it is difficult to assert that the market economy is being developed under the current reforms « (Gambold Miller 2002: 229; vgl. auch Kitching 1998; Ledeneva/Seabright 2000). In den 1990er Jahren sahen folglich zahlreiche Autorinnen und Autoren die Praktiken des Naturaltauschs als wesentlichen Schlüssel, um die gegenwärtigen und zukünftigen Entwicklungen vor allem des ländlichen Raums zu verstehen. Mit der Jahrtausendwende scheint sich das Blatt jedoch entgegen solcher Erwartungen langsam in Richtung Monetarisierung zu wenden. Der nicht-monetäre Anteil an den Haushaltseinkommen sank Umfragen zufolge bis zum Jahr 2003 auf 23 Prozent (O’Brien/Patsiorkovski 2006: 62). Zudem scheinen die landwirtschaftlichen Großbetriebe ihre Geschäftspraktiken schrittweise umzustellen. Spoor und Visser (2004) beobachten bei ihrer Analyse von Bewältigungsstrategien landwirtschaftlicher Großbetriebe mit Blick auf die geforderte Marktanpassung ein Nebeneinander von monetären und nicht-monetären Formen wirtschaftlicher Kommunikation. Sie stellen jedoch eine leichte Neigung in Richtung Ersterer fest und leiten daraus die Vermutung ab, dass tradierte und auf den Dorfkontext begrenzte Operations- und Reproduktionsformen gegenüber den Logiken global operierender Funktionssysteme sukzessive an Anschlussfähigkeit verlieren:

»Another indication of change away from a (partially) virtual economy is the decline in barter transactions within agriculture. [...] For these reasons, it is a positive sign that half of the LFEs do not conduct barter (any more), and that in two-thirds of the LFEs that do still engage in barter, the number of such transactions has declined with the recovery of agricultural economy since 1999. LFEs with a large share of barter deals are less profitable than those that conduct more business through cash.«(Spoor/Visser 2004: 543f)

Während Privatisierungsmaßnahmen und Kommunalreformen auf ein Zurückdrängen der ehemaligen und vormals umfassenden Kollektivbetriebe in die Wirtschaft hinwirken, erscheint die Position der Betriebe aus Sicht der lokalen Umwelt heraus nun auch innerhalb der Wirtschaft in Folge steigender Komplexität zunehmend kontingent. Fungierte die Kolchosmitgliedschaft im sowjetischen Regime als eine Art universaler Schlüssel zur Inklusion in faktisch alle anderen gesellschaftlichen Bereiche, löst sich diese klare und eindeutige Konditionalität nun auf und an ihre Stelle tritt ein Bündel an Inklusionsformen. Dies heißt im Umkehrschluss, dass einzelne Exklusionen aus bestimmten Zusammenhängen nicht mehr aneinander gekoppelt sind und sich in der polyzentrischen Kontextur funktionaler Differenzierung potenziell durch andere Inklusionen kompensieren lassen. Darüber vermag auch nicht hinwegzutäuschen, dass die Kolchosvorsitzenden oftmals weiterhin als zentrale Figuren im Dorf respektvoll beobachtet werden und sich selbst gerne als zentrale Macher inszenieren - strukturell ist ihnen ihr dorfweiter Status längst abhanden gekommen. Der zunehmende Anwendungsbereich des Geldmediums im 
Dorf birgt für die Beteiligten in erster Linie einen Anstieg an individuellen Freiheitsgraden - und dies gilt für Privathaushalte, landwirtschaftliche Großbetriebe und Gemeindeverwaltungen gleichermaßen. Im Unterschied zum Naturaltausch sind die Verwendungsmöglichkeiten von Geld sachlich nahezu unbegrenzt und verweisen auf einen Welthorizont, der fortan auch in der dörflichen Knappheitskommunikation mitzuschwingen beginnt. Die ehemals fest verkoppelten tradierten Strukturen werden damit notwendigerweise in ihrer Kontingenz sichtbar: Es kann, aber es muss nicht an ihnen festgehalten werden.

Die Umstellung von einem über mehrere Jahre durchaus funktionierenden Naturaltausch auf Zahlungen ist nicht auf einen Beschluss einer zentralen Instanz zurückzuführen, sondern als evolutionärer und kleinteiliger Prozess zu verstehen. Entsprechend spiegelt sich die zunehmende Bedeutung und Unausweichlichkeit des Geldmediums im Dorf auch an zahlreichen Stellen der vorliegenden Analyse: Wenn die Gemeinden und die landwirtschaftlichen Großbetriebe kooperieren - sei es der ehemalige Kolchos oder die Agroholding -, wird zunehmend auf Zahlungen und Zahlungsfähigkeit hin beobachtet, wobei die wirtschaftliche Kommunikation vertraglich gerahmt und auf diese Weise an das Rechtssystem angelehnt wird (zu Recht als zunehmend sichtbarer Anlehnungskontext bei Landtransaktionen vgl. auch Wegren 2009b: 119). Im Kontext der Gasifizierung gewinnen Kredite als wirtschaftlicher Inklusionsmodus und als Möglichkeit, Zahlungsfähigkeit aus Zahlungsunfähigkeit zu generieren, an Bedeutung und treten in direkte Konkurrenz zur Kolchosinklusion. Im Zusammenhang mit der Etablierung der Agroholding wurden in beiden Dörfern die Eigentumszertifikate auf Landanteile als wirtschaftliches Gut beobachtet (und zum Teil wurden sie auch Gegenstand wirtschaftlicher Transaktionen) und sogenannte Kolchosleistungen sichtbar kommodifiziert, d.h. aus sozialen Verflechtungen gelöst und in den Horizont von Zahlungen und Reziprozität gerückt. Deutlich wird dabei einerseits die sich selbstverstärkende Form des Monetarisierungsprozesses: Die zunehmende Bedeutung des Erfolgsmediums Geld schlägt sich in bestimmten Bereichen nieder und wirkt dort katalytisch, was abermals zu einem Bedeutungszuwachs von Geld führt. Andererseits lässt sich beobachten, dass Monetarisierungsprozesse keine Selbstläufer sind, sondern in vielfältiger Weise Reibung verursachen. Dies trägt letztendlich zur Entstehung neuer lokaler Arrangements bei, in denen globale und lokale Inklusionsformen aufeinander eingestellt und kompatibel gemacht werden (vgl. dazu mit Blick auf Landanteile auch Verdery 2004). Die >Nahaufnahme< konkreter Einzelbeispiele gewährt dabei Einblicke in Mechanismen und Prozesse, die den quantitativ beobachtbaren Wandlungsprozessen zugrunde liegen, jedoch nicht mitbeobachtet werden. So werden in den zuletzt erwähnten Studien zwar am Rande immer wieder »kreative Anpassungsprozesse« der Beteiligten unterstellt, diese jedoch nicht expliziert. Das bedeutet nicht, dass sich die von mir aufgezeigten Phänomene in allen Details und Besonderheiten un- 
begrenzt verallgemeinern lassen, verweist aber auf eine Variante, wie sich sozialer Strukturwandel vollzieht.

\section{Konfigurationen von Formalität und Informalität}

Wie wirken sich diese Entwicklungen auf das organisatorische Gefüge der landwirtschaftlichen Großbetriebe aus? Meine Beobachtungen zur Entstehung spezifischer Konfigurationen formaler und informaler Strukturen sprechen zwei generelle Mechanismen an, die in meinen Beispielen ineinanderspielen, die aber auch - das legt der Forschungsstand nahe - getrennt voneinander wirken. Gemeint sind zum einen der Umgang der Betriebe mit divergierenden Erwartungen, die im Transformationskontext aus der lokalen (dörflichen) und globalen Umwelt an sie herangetragen werden, und zum anderen die wechselseitigen Wirkungen und Abhängigkeiten zwischen formalen und informalen Strukturen, die es den Betrieben ermöglichen, sich als globale Marktakteure und lokale Wohlfahrtsorganisationen gleichzeitig zu reproduzieren.

Mit Blick auf den Umgang mit Transformationsimperativen als globale Erwartungen findet sich wiederholt Evidenz dafür, dass diese nicht nur auf den jeweiligen Führungsebenen aufgegriffen werden, sondern auch in den Selbstbeschreibungen der landwirtschaftlichen Großbetriebe an eine zentrale Stelle rücken. So stößt beispielsweise Wegren (2005: 82ff) auf Anzeichen eines aufkommenden Unternehmergeistes und den ausdrücklichen Wunsch der Leitungspersonen nach Freiheit und Unabhängigkeit. Die Reformen werden auf den Führungsebenen durchaus nicht nur als Bedrohung der alten Pfründe wahrgenommen, sondern in den Maßnahmen werden neue Möglichkeiten, Entscheidungsräume, Freiheitsgrade erkannt und als Chance bewertet: »Empirically, survey research has shown that farm managers of privatized and non-privatized farms prioritize farm profitability as their most important objective (though managers on privatized farms are somewhat more profit-oriented)«(Wegren 2005: 88). Damit einher gehen die generelle Akzeptanz von staatlichen Reformmaßnahmen (Wegren 2005: 88ff) und eine generell zustimmende Haltung gegenüber privatem Landbesitz und der Umstrukturierung der Eigentumsverhältnisse (Wegren 2005: 96ff). Dass es sich dabei nicht allein um semantische Verschiebungen handelt, davon zeugen diverse Änderungen von Organisationsstrukturen wie beispielsweise die zunehmende Stratifizierung der Löhne, die vermehrte Abgabe von Landanteilen und Dividendenzahlungen, der Wegfall von Kündigungsschutz, das Streben nach der Erschließung neuer Absatzmärkte, die Aufnahme von direktem Kontakt zu potenziellen Käufern, der Aufbau von Beziehungen $\mathrm{zu}$ anderen (Privat-)Unternehmen oder die tendenziell nachlassende Bedeutung staatlicher Subventionen (Wegren 2005: 79ff). 
Angesichts dieser semantischen und strukturellen Veränderungen könnte man eine steigende Indifferenz gegenüber lokalen Erwartungen vermuten, stehen diese doch mehrheitlich im Widerspruch zu den Transformationsimperativen und appellieren an die soziale Verantwortung der Betriebe gegenüber der Dorfgemeinschaft respektive an die Bereitschaft, Gewinnerwartungen hintanzustellen oder sogar ganz auf Gewinne zu verzichten. Doch für die Großbetriebe scheint eine indifferente Haltung nicht in Frage zu kommen (vgl. z.B. Gambold Miller 2002; Lindner 2008; Spoor/Visser 2004). Sogenannte Kolchosleistungen, so wird beobachtet, werden in aller Regel bis heute in beträchtlichem Umfang erbracht. Um in dieser Situation wirtschaftlich zu überleben und die soziale Legitimation zu erhalten, müssen die Betriebe strategisch handeln und widersprüchliche Umweltansprüche und daran geknüpfte Imperative kompatibel gestalten. Wie ihnen das gelingt, wird unter anderem bei Spoor und Visser (2004) deutlich. Bei ihren Untersuchungen zu den Anpassungsstrategien landwirtschaftlicher Großbetriebe zeigt sich, dass gerade in der Verbindung dieser Ansprüche ein Teil der wirtschaftlichen Erfolgsbedingungen zu liegen scheint:

»Few of the LFEs [large farm enterprises, E.M.] that do relatively well have restructured in terms of their labour force. They maintain to a certain extent their former socialist role as rural >social units $<$. [...] However, managers of even the most unprofitable enterprises are trying hard to keep intact the most essential social services, like running water, gas and schools. The profitable LSEs continue a wider range of social services.« (Spoor/Visser 2004: 519)

Die Hierarchie, in die die unterschiedlichen Umwelterwartungen durch die Betriebe gebracht und entsprechend organisationsintern prozessiert werden, erscheint den Autoren dabei keinesfalls eindeutig. Wirtschaftliche Ambitionen kommen als Triebfeder für das Streben nach Marktintegration ebenso in Frage wie das Bewusstsein für soziale Verantwortung - oder auch eine Mischung aus beidem:

»The decision to integrate [in agribusiness chains, E.M.] can also be (partially) motivated by a desire to continue the social role of the enterprise, such as by providing food from own processing units to workers and shouldering responsibility for providing employment for village residents. [...] This behaviour confirms that the heirs of the Soviet kolkhozy and sovkhozy retain an important social function. Therefore, as part and parcel of the >coping with the market< strategy, integration can originate from economic arguments or from attempts to maintain some of the LFE's social functions.« (Spoor/Visser 2004: 523)

Meine eigene Empirie stützt diesen Befund: Selbst die Agroholding, die zunächst keinen Zweifel an ihrer marktorientierten Strategie ließ, erbringt bereits nach kurzer Zeit sachlich vergleichbare Leistungen wie der ehemalige Kolchos im Nachbardorf. 
Darauf, dass dieses >Investorenmodell< inklusive des sozialen Engagements der Betriebe und ihres signifikanten Einflusses auf die lokale Politik vor allem in Südrussland verbreitet ist, verweist Oswald (2007: 223).

Organisationsintern vollzieht sich das Erwartungsmanagement, das zeigt die vorliegende Studie, in Form von spezifischen Konfigurationen formaler und informaler Strukturen - damit komme ich zum zweiten der genannten Mechanismen. Globale Erwartungen, die auf Marktintegration und Primärorientierung an wirtschaftlicher Rationalität - Zahlungsfähigkeit und Profit - zielen, werden über die formale Ebene absorbiert, während die informale Ebene lokale Erwartungen auffängt. Auf den ersten Blick eröffnen sich damit Anschlussmöglichkeiten an den breiten Forschungsstand zu Informalität, die in verschiedenen Spielarten disziplinübergreifend ein beliebtes Thema in der postsowjetischen Transformationsforschung ist. Assoziiert mit Phänomenen wie Korruption, Klientelismus, Schattenwirtschaft und ähnlichen sind informale Strukturen vor allem in der Politikwissenschaft und den Wirtschaftswissenschaften tendenziell negativ konnotiert. Besteht die Gemeinsamkeit der genannten Phänomene doch darin, dass sie bei der Transition hin zu Markt und Demokratie der erwünschten Umsetzung und der Effektivität formaler Institutionen im Wege stehen und deren Funktionsweisen untergraben. Ganz anders konnotieren anthropologische Studien informale Strukturen, stellen sie doch heraus, dass sich hinter dem Begriff der Informalität in der Regel persönliche (Vertrauens-)Netzwerke verbergen, die auf der Zugehörigkeit zu einer Gemeinschaft (Dorf, Stamm etc.) oder auf Verwandtschaftsbeziehungen beruhen. Informale Strukturen gelten ihnen als begrüßenswerte Alternative zu unpersönlichen Marktbeziehungen und/oder aufoktroyierten (staatlichen) Formalstrukturen, die lokalen Idiosynkrasien nicht gerecht werden. Nicht selten werden informale Strukturen uno actu mit Widerstand gegen formal-rechtliche Vorgaben gleichgesetzt. Lokale Lösungen auf der Basis von Informalität (»to get things done«) opponieren in dieser Lesart gegen zentralstaatliche Maßnahmen (»one-size-fits-all«) und werden meist schon a priori präferiert. Eine Variante dieser Lesart betont anstelle der Dysfunktionalität gerade die Funktionalität informaler Strukturen im Hinblick auf bestimmte gesellschaftliche Zielvorstellungen (für einen Überblick zur Verwendung des Informalitätsbegriffs vgl. auch Hayoz 2013; Helmke/Levitsky 2004).

Der starke normative Tenor derartiger Betrachtungen, und dies gilt ungeachtet der Frage welche Seite der Unterscheidung Formalität|Informalität asymmetrisch bevorzugt wird, steht meist einer auffälligen analytischen Unbestimmtheit gegenüber: Informalität fungiert gemeinhin vor allem als Residualkategorie für alles, was von formalen Vorgaben (im politisch-rechtlichen Sinne) abweicht, und beobachtet wird folglich in der nicht weiter aufgelösten Kontextur generalisiert|partikularistisch. Ein systemtheoretischer Blick vermag diese analytische Asymmetrie nicht vollständig aufzuheben, denn auch hier steht die scharf abgrenzbare und beobacht- 
bare Formalität der »theoretischen Sperrigkeit« des Informalen gegenüber (Tacke 2015: 86), das stets auf das >Andere < verweist, organisationsintern meist geheim gehalten wird und dessen Dynamiken schwer fassbar sind. Die zwingende Referenz des Informalitätsverständnisses auf Formalität besteht also auch in der systemtheoretischen Lesart fort. Das Problem der Offenheit dieses Begriffs wird jedoch theoretisch zumindest handhabbar gemacht durch die konsequente Bindung an den Systemtyp Organisation und den systematischen Bezug von Formalität und Informalität auf den Systembegriff, also die Unterscheidung von System und Umwelt (vgl. dazu auch Tacke 2015). Die normative Dimension tritt so explizit in den Hintergrund und stattdessen geht es um die Funktion formaler und informaler Strukturen und Praktiken, also um Fragen der Abgrenzung und Selbstreferenz und des Umgangs mit Kontingenz und Komplexität durch das Organisationssystem. Beide Seiten der Unterscheidung werden symmetrisch gehandhabt: Formale und informale Strukturen sind per se weder $>$ gut $<$ noch $>$ schlecht $<$, sondern sie reproduzieren sich einfach (oder nicht) und treten als Lösungen für spezifische Probleme hervor, die es zu ermitteln gilt - wie beispielsweise den Umgang mit divergierenden Umwelterwartungen in landwirtschaftlichen Großbetrieben. Die vorliegende Analyse griff damit eine analytische Perspektive auf, mit der die Organisationsforschung operiert (siehe z.B. Kühl 2007, 2011: Kap. 3; für einen detaillierten und kenntnisreichen Überblick vgl. Tacke 2015), dabei jedoch Phänomene postsowjetischer Transformation weitgehend ausspart. Andersherum verzichten Studien, die sich selbst als Beitrag zur Transformationsforschung verstehen, meist auf einen theoretisch präzisen Blick auf den organisationellen Wandel (eine Ausnahme bilden die netzwerkanalytischen Studien zu Transformationsprozessen ungarischer Unternehmen von Stark und anderen, vgl. Stark 1996; Grabher/Stark 1997; Stark/Vedres 2006).

Deutlichere Anschlussfähigkeit meiner Beobachtungen zu den Wechselwirkungen und Bedingungszusammenhängen formaler und informaler (und teils illegaler) Strukturen besteht hingegen im Hinblick auf die Frage, ob und wie sich sozialer Wandel im postsowjetischen Kontext über spezifische Konfigurationen von Formalität und Informalität und die Interdependenzen zwischen beidem vollzieht. Eine vor allem in der Politikwissenschaft vielzitierte Klassifikation zur Unterscheidung von formalen und informalen Institutionen ist jene von Helmke und Levitsky (2004). Sie bestimmt Informalität über das Verhältnis zu formalen Institutionen mittels eines zweidimensionalen Schemas: Konvergierende vs. divergierende Outcomes von formalen und informalen Institutionen in der ersten und Effektivität vs. Ineffektivität der Formalstruktur in der zweiten Dimension. Die resultierenden vier Typen informaler Institutionen - komplementär oder substitutiv für konvergierende Outcomes, akkommodierend oder konkurrierend für divergierende Outcomes (Helmke/Levitsky 2004: 728ff) - weisen eine Asymmetrie in Richtung Formalität auf, die sich mit Blick auf die daran anschließenden Implikationen für soziale 
Wandlungsprozesse noch verstärkt: ${ }^{3}$ Als wesentlicher Katalysator für Verschiebungen auf der informalen Ebene gelten Änderungen der Formalstruktur, die sich auf die Kosten- und Nutzenstrukturen der beteiligten Akteure niederschlagen. Informale Institutionen werden in Folge formaler Änderungen unter Umständen kostenintensiver und dadurch schwieriger aufrechtzuerhalten (Helmke/Levitsky 2004: 732). Die Vermutung, dass dieses Schema zu kurz greift und die unterstellten Mechanismen der gesellschaftlichen Komplexität nicht gerecht werden, wurde verschiedentlich geäußert. So erwarten beispielsweise Van Assche et al. (2013) Wechselwirkungen zwischen formalen und informalen Institutionen, die sie als Dialektik bezeichnen, und gehen davon aus, dass Formalität und Informalität nicht nur in der Gegenwart interdependent sind, sondern zudem als pfadabhängigkeit zu betrachten sind (Van Assche/Shtaltovna/Hornidge 2013: 89ff). Wheatley (2013) betont unter anderem am Beispiel von Änderungen der georgischen Verfassung im Kontext der sogenannten Rosenrevolution im Jahr 2003 die Möglichkeit, dass sich auch die Formalstrukturen allmählich an informale Strukturen anpassen. Ein Mechanismus, der gerade für den sowjetischen Kontext charakteristisch sei: »The adaption of formal rules in order to ensure that they are compatible with informal rules, or in order to make informal institutions more effective, is precisely what we have seen in the Soviet Union« (Wheatley 2013: 330).

Dieser Prozess lässt sich in meinen Episoden im Kleinen beobachten: Mit Blick auf das Erwartungsmanagement der landwirtschaftlichen Großbetriebe - des ehemaligen Kolchos und der Agroholding - zeigt sich nicht so sehr eine Persistenz von Strukturen, die bereits im sowjetischen System als informal galten, sondern vielmehr eine Verschiebung sowjetischer Formalstrukturen (z.B. in Form der Verpflichtung des Kolchos zu bestimmten Leistungen gegenüber der Gemeinde und den Dorfbewohnerinnen und Dorfbewohnern) auf die Ebene des Informalen. Dort werden sie zum einen mitprozessiert, schlagen sich parallel dazu jedoch auch in Anpassungen der organisationalen Formalstrukturen nieder (in Form von Verträgen, betriebsinternen Abrechnungssystemen, der Schaffung von Stellen und ähnlichem). Andersherum werden auch informale Strukturen von der formalen Ebene ir-

3 Die Typologie informaler Strukturen nach Helmke und Levitsky (2004):

\begin{tabular}{l|c|c} 
& $\begin{array}{c}\text { Effektive formale } \\
\text { Institutionen }\end{array}$ & $\begin{array}{c}\text { Ineffektive formale } \\
\text { Institutionen }\end{array}$ \\
\hline Konvergierende & Komplementäre & $\begin{array}{c}\text { Substitutive } \\
\text { Outcomes }\end{array}$ \\
\hline Informalität & Informalität \\
\hline Outcomes & Akkommodierende & Konkurrierende \\
& Informalität & Informalität
\end{tabular}


ritiert und unter Druck gesetzt, was in der Verschiebung von Normalitätserwartungen und Beobachtungshorizonten mit Blick auf die lokalen Ansprüche zum Ausdruck kommt.

\section{Die latente Macht lokaler Politik}

Empirische Analysen, die ihren Fokus explizit auf die Logiken kommunaler Politik und die Strukturen lokaler Machtkommunikation legen und durch Einblicke in die lokalpolitische Praxis den Rahmen füllen könnten, der durch verwaltungswissenschaftliche Studien zum formal-rechtlichen Kontext kommunaler Verwaltung aufgespannt wird, sind selten. Sofern die lokale Politik in der postsozialistischen Transformationsforschung beobachtet wird, geschieht dies in aller Regel mit Blick auf ihre Rolle bei der Umsetzung von Wirtschaftsreformen und insbesondere bei der Privatisierung der landwirtschaftlichen Großbetriebe (vgl. für Rumänien Verdery 2002, 2003; für Russland und die Ukraine Allina-Pisano 2004, 2008; für Russland Lindner 2008). Die Annäherung an den Gegenstand erfolgt > von außen<, das heißt nicht aus den Logiken der Politik selbst heraus, sondern über die Frage, welche Spuren, Reflexionen und Grenzen lokalpolitischer Macht sich in anderen gesellschaftlichen Kontexten einprägen, hier vor allem in der Wirtschaft. Ungeachtet dieser spezifischen Perspektive und der damit einhergehenden Einschränkungen sind die jeweiligen Resultate jedoch auch im Hinblick auf die Frage nach allgemeinen Mustern und Merkmalen lokaler Politik instruktiv, lassen sie doch Aspekte hervortreten, die sich auch in den empirischen Episoden der vorliegenden Studie widerspiegeln.

Das Ausmaß der Einflussnahme vor allem der Bürgermeisterinnen und Bürgermeister, das sowohl Verdery $(2002,2003)$ als auch Allina-Pisano $(2004,2008)$ anhand ihres empirischen Materials sichtbar machen, lässt auf deren klare formale und informale Machtposition in den Gemeinden schließen. Dies kontrastiert nicht nur mit der Verwaltungsatrophie des sowjetischen Dorfes, sondern überrascht auch angesichts der Sichtbarkeit und Persistenz sowjetischer Trajektorien in zahlreichen Bereichen des dörflichen Lebens. Eine Form kommunaler Autonomie gewinnt so an Kontur und tritt jenseits der Frage hervor, inwieweit der lokalen Verwaltungsebene innerhalb der formalen Verwaltungshierarchie Eigenständigkeit eingeräumt und Freiheitsgrade des Entscheidens zugestanden werden.

Land, so lässt sich ein gemeinsamer Tenor beschreiben, erscheint in den untersuchten Fällen als »political asset«, an das zahlreiche und vielfältige soziale Beziehungen gekoppelt sind, über die die Gemeindeverwaltung sich ihre Machtposition sichert. Die Transformation in ein »economic asset« beinhaltet damit neben einem komplexen bürokratischen Prozess auch das Kappen dieser Verbindungen und die Monetarisierung von Beziehungsstrukturen, was dem Interesse der Gemeindever- 
waltungen potenziell widerspricht und entsprechend zögerlich in Angriff genommen wird. Im Kontext dieser Verzögerungsstrategie scheinen zwei wiederkehrende Muster auf: Erstens das Kanalisieren von Kommunikation auf Grundlage von und in Kombination mit der Nutzung von exklusivem Wissen durch die Gemeindeverwaltung und zweitens das Spiel mit Interaktion respektive Interaktionsvermeidung, um Entscheidungen in die gewünschten Bahnen zu lenken. Diese Mechanismen entfalten sich, indem beispielsweise Personen, die rechtmäßig Anspruch auf bestimmte Parzellen erheben, systematisch gemieden werden, wenn sie versuchen, mit ihrem Anliegen zum Bürgermeister oder der Bürgermeisterin vorzudringen. Wissen über den formalen Ablauf des Privatisierungsprozesses wird von der Gemeindeverwaltung exklusiv gehalten und Anteilseigner und Anteilseignerinnen werden im Unklaren darüber gelassen, welche Schritte sie unternehmen müssen, um ihre virtuellen Eigentumsrechte wirksam werden zu lassen. Informationen über Eigentumsverhältnisse, die der Gemeindeverwaltung vorliegen, bleiben unter Verschluss. Die Vermessung von Land als erster Schritt im Privatisierungsprozess wird immer wieder verschoben und/oder fehlerhaft durchgeführt. Und in Interaktionen auf Versammlungen oder in persönlichen Gesprächen setzen die Gemeindeverwaltungen respektive die Bürgermeisterinnen und Bürgermeister alles daran, die Fäden in der Hand zu behalten. Dazu laden sie selektiv zu Anteilseignerversammlungen ein und betreiben ein strategisches Agenda Setting, um für ihnen genehme Entscheidungen unter dem Deckmantel umfassender Partizipation Mehrheiten zu gewinnen.

Deutlich wird aber auch: Die augenscheinlich durchschlagende Wirkung all dieser Mechanismen, d.h. die wirksame Unterdrückung von Wirtschaft durch die Politik, ist wesentlich auf den hohen Grad der Schließung der dörflichen Kommunikationszusammenhänge in den jeweiligen Beobachtungszeiträumen zurückzuführen. Sie entfalten sich im Schatten der vielleicht nicht formalen, aber doch faktischen Abwesenheit von Kontrollmöglichkeiten und vor allem von Sanktionen durch den Zentralstaat und übergeordnete Verwaltungsebenen. Hinzu kommt die weitgehende Abwesenheit von Zahlungen und von globalen Normen wie formale Eigentumsrechten, die wirksam durchbrochen werden respektive an den Dorfgrenzen buchstäblich abprallen. Diese Schließung, so verdeutlichen die empirischen Episoden der vorliegenden Studie, erodiert jedoch zunehmend. Parallel zu den genannten Studien lokaler Politik wurde gezeigt, dass sich die Bürgermeisterinnen und Bürgermeister als Fixpunkte der dörflichen Kommunikation etablieren, indem sie Kommunikation kanalisieren und Entscheidungsagenden setzen, und lokale Politik findet maßgeblich in Form von Interaktion respektive deren gezielter Vermeidung (wie beispielsweise im Falle des Investors in der Gemeinde Andreevka) statt. Es wurde darüber hinaus aber auch gezeigt, dass und wie die lokale Politik zunehmend mit den Eigendynamiken anderer Funktionssysteme rechnet, die sichtbar auf das 
Dorf ausstrahlen und dort selbst zum Katalysator für die steigende Durchlässigkeit der Dorfgrenzen werden.

\section{Postsowjetische Transformationen als Formen der Genese von Weltgesellschaft}

Die sowjetische Organisationsgesellschaft präsentierte sich als eine Gesellschaftsform, in der funktionale Differenzierung zwar nicht vollständig ausgeschaltet, ihr aber zumindest das Primat entzogen werden konnte. Welche Strukturen vor diesem Hintergrund entstanden, illustrieren ländliche Gemeinden, die zum einen politisch und zum anderen wirtschaftlich strikt in eine hierarchisierte gesellschaftliche Umwelt eingebunden waren. Die Beschränkung auf die Kolchosleitung als zentralem Berührungspunkt mit dieser Umwelt sorgte dabei jedoch dafür, dass das Geschehen im Dorf aus der Umwelt heraus nur begrenzt beobachtbar war. Im Schatten politischer Kontrolle und unter kontinuierlichem Verweis auf die gesellschaftliche Hierarchie öffneten sich so Möglichkeitsräume, in denen dorfinterne Zonen begrenzter Autonomie entstehen und regimefremde Kommunikationsformen und Erwartungsstrukturen Fuß fassen konnten.

Verschiedene Transformationsmaßnahmen wollten diese Grundlogik durchbrechen, umkehren und den Primat der Funktionssysteme (wieder-)herstellen. In den russischen Dörfern lässt sich beobachten, dass Strukturen, die unter den Bedingungen des sowjetischen Regimes entstanden sind, zum Teil unter den Bedingungen funktionaler Differenzierung fortbestehen oder gar einrasten. Bei der genauen Bewertung der Persistenzen oder Veränderungen kommt es aber auf Feinheiten an. So präsentieren sich manche Strukturen äußerlich unverändert, doch in dem Maß, wie sie sich auf neue Kontexte einstellen, verschieben sich ihre Sinnhorizonte. Mitgliedschaften - vor allem in den ehemaligen Kollektivbetrieben - bestehen zwar fort, doch (neue) funktionssystemspezifische Kommunikationsangebote lassen sich mit Verweis auf die Mitgliedschaft kaum mehr ablehnen. Dazu spiegelbildlich sind die landwirtschaftlichen Großbetriebe, die ihre dorfkonstituierende Rolle in wirtschaftlicher Hinsicht behalten haben, kaum mehr in der Lage, die Folgen etwaiger Ablehnung aufzufangen, geschweige denn zu kompensieren. Ein Prozess, den die zahlreichen kleinteiligen und oftmals auf den ersten Blick unauffälligen Beobachtungen dieser und anderer Studien verfolgen und nachzeichnen.

Wo aber liegt die theoretische Relevanz dieser Ergebnisse? Für den Geltungsanspruch der Theorie funktionaler Differenzierung, so lässt sich ein wenig provokativ einwenden, spielt es nur eine nachrangige Rolle, ob und wie sich die von ihr postulierten Logiken in einer - zumindest nach alltäglichem Verständnis - peripheren Region wie dem ländlichen Russland durchsetzen und welche Strukturen daraus entstehen. Diesem Einwand lässt sich erstens mit dem Verweis darauf begegnen, 
dass sich die vorliegende Analyse wie jede empirische Anwendung auch als Bewährungsprobe für die zugrundeliegende Theorie lesen lässt. Zweitens lässt sich das empirische Überraschungsmoment der Ergebnisse als Effekt dieses spezifischen analytischen Blickwinkels ins Feld führen, der die Wandlungsprozesse im ländlichen Russland nicht nur in einem neuen Licht erscheinen lässt, sondern - fügt man einen weiteren Abstraktionsschritt an - als Mechanismen der Genese von Weltgesellschaft erkennbar macht. Dies öffnet Vergleichshorizonte und Lernchancen, die anderen theoretischen Zugängen verschlossen bleiben oder für sie nur auf Umwegen zu erreichen sind.

Will die Theorie funktionaler Differenzierung an ihrem universalen und das heißt auch: an ihrem globalen Geltungsanspruch festhalten und ihm gerecht werden, dann wird sie dies kaum mit dem exklusiven Blick auf sogenannte westliche Gesellschaften und damit auf ihren eigenen Entstehungskontext tun können. Zu schwer wiegt der nicht abzuweisende Vorwurf der Zirkularität von Entstehungskontext, empirischem Blick und theoretischer Leistungsfähigkeit. Zu bewähren und dabei vielleicht zu verändern hat sie sich vielmehr auch und gerade in der Anwendung auf >nicht-westliche< und >fremde< Phänomene, denen ein oberflächlicher Blick intuitiv völlig andersartige Operationsweisen und Rationalitäten unterstellen mag. Eine solche Bewährungsprobe des systemtheoretischen Geltungsbereichs und seiner Grenzen schwingt als offene Frage bei der Wahl eines - zumindest aus der Perspektive der sogenannten westlichen Soziologie - peripheren und in vielerlei Hinsicht marginal anmutenden Gegenstandsbereichs wie dem ländlichen Russland stets mit. Sie darf als bestanden gelten, wenn es gelingt, anhand der bereitstehenden Theoriemittel schlüssige Beschreibungen anzufertigen, die dazu beitragen, die beobachteten Phänomene differenzierter zu verstehen respektive in bekannten und bewährten analytischen Kategorien aufzulösen. Hinzu kommt, dass Pollacks (2003: 11) Kritik an der DDR-Forschung im Kern auch für die postsowjetische Transformationsforschung zutrifft: Eine Verbindung zur Soziologie besteht faktisch nicht, obwohl beide Forschungsfelder beanspruchen, gesellschaftliche Strukturen zu verstehen und zu erklären. Ein Weg zur Überwindung dieser Trennung ist, die traditionellen Gegenstände der Transformations- oder Osteuropaforschung in das Blickfeld einer allgemeinen gesellschaftstheoretischen Perspektive zu rücken. Dies nicht allein, um den Anwendungsbereich von gesellschaftstheoretischen Ansätzen auszuweiten, sondern auch und vor allem um über eine Basis für den Vergleich von postsowjetischen mit anderen Gesellschaften zu verfügen.

Die vorliegende Studie schlägt in dieser Hinsicht Brücken. Die empirischen Überraschungsmomente, die unmittelbar auf den theoretischen Zugang - die Theorie funktionaler Differenzierung und die Theorie der Weltgesellschaft - zurückzuführen sind, zeigen deutlich, dass Wandlungsprozesse auch und gerade in peripheren Bereichen stattfinden, obwohl der Mainstream der Transformationsforschung 
alles im Zeichen der Kontinuität sieht und Änderungen angesichts der als unüberwindbar bewerteten Diskrepanz zwischen universalen politischen Intentionen und Umstrukturierungsplänen und dem >Lokalen< kaum (noch) erwartet. Hinzu kommt, dass Wandlungsprozesse nicht nur beobachtbar werden, sondern deutlich die Logiken funktionaler Differenzierung widerspiegeln. Dies wird insbesondere bei einem Blick unter die Oberfläche makroökonomischer Kennzahlen und formal-rechtlicher Regelungen deutlich, die oftmals Kontinuität suggerieren, wo sich tatsächlich signifikante Verschiebungen vollziehen. Auf welche Weise dies geschieht und sich sowjetische mit neuen Strukturen verschränken, lässt sich erst mit Blick auf die Kommunikationen und Sinnhorizonte beobachten und verstehen, die sich um vermeintlich eindeutige und unveränderte Praktiken ranken. Das Überraschungsmoment verschiebt sich dann von der Verwunderung darüber, dass sich die Dinge nicht in der (politisch) erwünschten und erwarteten Form ändern, hin zu der Überlegung, warum Wandel stattfindet und dabei spezifischen und benennbaren Pfaden folgt, wenn doch auch alles so bleiben könnte, wie es ist.

Schließt man an diese Überlegung einen letzten Abstraktionsschritt an und fragt nach den Mechanismen, über die sich die beobachteten Wandlungsprozesse Anlehnungskontexte, Inklusionsmodi, Politikparameter, formale und informale Organisationsstrukturen - vollziehen, so tritt ein weiterer Aspekt hervor: In den sichtbar und nachvollziehbar gemachten Mustern postsowjetischer Transformation werden Mechanismen der Genese von Weltgesellschaft im Sinne Stichwehs (vor allem 2000 [1999], 2009b) erkennbar, die durch die empirischen Beobachtungen dieser und der referierten Studien hindurchscheinen.

Es sind Organisationen und nicht Funktionssysteme, die über Strukturen für machtvolles Entscheiden verfügen und damit für politische Steuerungsversuche vielversprechende Adressaten sind. Dies illustriert nicht nur die sowjetische Organisationsgesellschaft, sondern auch die postsowjetische Transformationsgesellschaft. Entsprechend sind es die landwirtschaftlichen Großbetriebe und die Gemeindeverwaltungen als relevante dörfliche Organisationen, auf die sich staatliche Maßnahmen der Privatisierung und Kommunalreform richten. Für die Weltgesellschaftstheorie systemtheoretischer und auch neoinstitutionalistischer Lesart stellen formale Organisationen Innovationen dar, denen durch ihre Leistungen spezielle Bedeutung für die Genese und Strukturbildung der Weltgesellschaft zukommt (vgl. Stichweh 2000 [1999]: 251f, 2006: 244ff, 2009b: 15f). Konkret ermöglichen und fördern die beiden genannten Organisationen vor allem den Transfer von Marktund Verwaltungswissen. Globale Kategorien diffundieren auf diesem Weg in den Dorfkontext hinein und sorgen für die Verschiebung sachlicher und zeitlicher Beobachtungshorizonte. In der Sachdimension beobachten sich die Betriebe - vor allem die vermeintlich traditionellen Kollektivbetriebe, aber auch die Agroholding und die wenigen Privatbauern - als Wirtschaftsunternehmen und vergleichen sich 
im Horizont anderer Betriebe der Region und darüber hinaus. Die Gemeindeverwaltungen beobachten sich als politisch-administrative Akteure und positionieren sich im Horizont anderer Gemeindeverwaltungen. Letzteres wird durch Institutionen wie die regelmäßigen Treffen der Bürgermeisterinnen und Bürgermeister auf Bezirksebene zusätzlich und kontinuierlich gefördert. Das Wissen um die anderen wächst, der Detaillierungsgrad der Beobachtungen steigt und es schälen sich neue Orientierungspunkte für das eigene Entscheiden heraus. In der Zeitdimension etabliert sich ein Horizont des zukünftig Erwünschten und Angestrebten, in dem aktuelle Handlungen und Entscheidungen verglichen und bewertet werden können. Sichtbar gemacht werden so die vorläufigen, aber unvermeidbaren Abweichungen des Aktuellen von Soll-Zuständen, deren Eintreten für die Zukunft erwartet wird. Einmal beobachtbar, lassen sich diese Abweichungen je nach Lage und Bedarf heranziehen, um konkrete Entscheidungen unter Verweis auf Sachzwänge zu legitimieren oder zu kritisieren. In diesem Sinne wäre es verkürzt, die beinahe lehrbuchartigen (Selbst-)Darstellungen von Landwirtschaftsbetrieben und Kommunalverwaltungen als folgenlose Semantiken abzuwerten, zeigen sich in ihnen doch signifikante Verschiebungen der Beobachtungshorizonte und Orientierungspunkte mit strukturellen Konsequenzen. Dabei kommt es auf die »Umschaltfähigkeit« der Organisationen an, das heißt auf ihre Fähigkeit, zwischen »lokaler und globaler Selbstauffassung [zu] oszillieren und beide Seiten dieser Unterscheidung gleichzeitig betonen « (Stichweh 2009b: 16). Durch den kreativen Einsatz von formalen, informalen und illegalen Strukturen werden globale Kategorien nicht stur übernommen, sondern vielfach an lokale Spezifika angepasst und >dorfgerecht< modelliert, wobei sich wiederum die lokalen Eigenheiten (mit-)wandeln.

Aber auch außerhalb formaler Organisationen pausen sich Mechanismen der Genese von Weltgesellschaft auf den dörflichen Kommunikationszusammenhang durch. Wenn lokale Kommunikationen nach globalen Anlehnungskontexten suchen und dörfliche Erwartungsstrukturen darin einrasten, wird dies durch verschiedene parallel ablaufende und einander bedingende Nahwirkungsmechanismen erzeugt (im Kontrast zur Fernwirkung globaler Diffusion). Nahwirkungen in Form entstehender Interrelationen oder Vernetzungen zeigen sich besonders deutlich in der Entwicklung des Geflechts wirtschaftlicher Beziehungen. War Knappheitskommunikation im sowjetischen Dorf und - in Folge der temporären Demonetarisierung auch noch in den 1990er Jahren faktisch auf den Dorfkontext beschränkt, entstehen nun vielfältige Vernetzungen über die Dorfgrenzen hinweg: Dies betrifft marktförmige Tauschbeziehungen wie im Fall des Verkaufs eigener Produktion oder Landanteile. Es betrifft auch Anstellungsverhältnisse, bei denen die Bedeutung des ehemaligen Kolchos schrittweise zugunsten anderer Beschäftigungsverhältnisse abnimmt, oder die Aufnahme von Krediten, die das Hinaustreten aus dem Dorfkontext in Form von Zahlungen aber auch physisch impliziert. Sie alle verweisen auf 
ein »Und-so-weiter« (Stichweh 2000 [1999]: 257) und öffnen Türen zu »small worlds«, ohne dass diese Zusammenhänge den Beteiligten notwendigerweise bewusst sind und/oder ihre »provinzielle Mentalität« (Stichweh 2009b: 24) irritieren, denn »[e]s genügt, daß man Teil einer small world ist, die das, was man tut, stabil in einer über Vernetzungen erschlossenen Welt verankert, die man selbst erlebensmäßig vielleicht nicht realisiert« (Stichweh 2009b: 24).

Ko-evolutiv mit Prozessen der Vernetzung ziehen globale Selektionshorizonte in der dörflichen Kommunikation auf. Globale Selektivität als Mikromechanismus wirkt, sofern »einer Entscheidung und der zugehörigen kommunikativen Äußerung, die diese Entscheidung mitteilt, ein Auswahlbereich zugrunde liegt, der sich dadurch auszeichnet, daß die berücksichtigten Alternativen weltweite Möglichkeitsräume ausschöpfen« (Stichweh 2009b: 23). Vordergründige strukturelle Kontinuität schließt dann Wandlungsprozesse nicht aus, denn »auch bei scheinbarer Persistenz dieser Strukturen [kann] gleichzeitig ein Umbruch in den subjektiv erschlossenen Möglichkeitsräumen stattgefunden haben [.], der alles, was geschieht, unter die Konkurrenz alternativer Möglichkeiten setzt, die davor nicht vorhanden waren « (Stichweh 2009b: 23). Ausschlaggebend ist nicht in erster Linie, welche Handlungsmöglichkeit selektiert wird, sondern in welchem Horizont alternativer Möglichkeiten sich die Selektion vollzieht. Und diesen Horizont gilt es empirisch aufzuspüren. Ebenso wenig wie die Wahl eines Schulfreundes als Ehepartner notwendigerweise auf eine primär oder ausschließlich lokale Orientierung der Betreffenden schließen lässt (Beispiel aus Stichweh 2009b: 23), erlauben Phänomene wie die schiere Existenz landwirtschaftlicher Großbetriebe samt ihrer Position als bedeutende Arbeitgeber im Dorf und ihrer starken Einbindung in die Bereitstellung öffentlicher Dienstleistungen, persönliche Beziehungen zwischen Mitgliedern der Betriebsleitung und den Bürgermeistern und Bürgermeisterinnen oder der Verzicht auf den Verkauf von Eigentumszertifikaten durch die Anteilseigner und Anteilseignerinnen direkte Rückschlüsse auf die Persistenz sowjetischer Verhältnisse. Dass in den zugrundeliegenden Selektionen ein Welthorizont mitschwingt, drücken nicht nur die Äußerungen und Selbstbeschreibungen der Beteiligten aus, sondern wird auch über Formalisierungen - vor allem in Form von Verträgen, die immer auch auf ihre Kündbarkeit, andere mögliche Vertragspartner und andere Verwendungsmöglichkeiten von Geld verweisen - institutionalisiert und verstetigt. Globale Selektivität und Vernetzungen wirken dabei unablässig aufeinander ein. Einerseits weiten sich Selektionshorizonte durch die Beobachtung von aktuellen Vernetzungen im Dorf aus: Wenn einige Gemeindemitglieder Kredite aufnehmen, ihre Landanteile verkaufen oder wenn eine Gemeindeverwaltung Aufträge an dorfexterne Dienstleister vergibt, beginnt dies der Kommunikation als Möglichkeit anzuhaften, auch wenn die jeweiligen Optionen im konkreten Fall abgelehnt werden. Andererseits steigt die Wahrscheinlichkeit, dass sich diese Möglichkeiten aktualisieren, sobald sie im 
Horizont mitbeobachteter Alternativen aufscheinen. Welcher der beiden Mechanismen den Startpunkt der >Verweltgesellschaftlichung < setzte, wird dann zum Henne-Ei-Problem.

Für die Unterscheidung global|lokal erkennt Stichweh ein wechselseitiges Steigerungsverhältnis beider Seiten, denn »[i]n globalen Systemen, in denen sich eine zunehmende Zahl von globalen Interrelationen beobachten läßt, kann man zugleich eine intensivierte Artikulation lokaler Spezifitäten feststellen« (Stichweh 2000 [1999]: 264). Die ländlichen Gemeinden Russlands, dies als Schlusswort, erwiesen sich vor diesem Hintergrund in der Vergangenheit als Miniatur der sowjetischen Organisationsgesellschaft, die umfassend darauf ausgerichtet war, sich innerhalb der Weltgesellschaft abzusondern, und der dies in vielerlei Hinsicht auch gelang. In der Gegenwart erscheinen die in der dörflichen Transformationsgesellschaft beobachtbaren Wandlungsprozesse als eine Miniatur der Genese von Weltgesellschaft, anhand derer sich ihre Mechanismen studieren, darüber Vergleichshorizonte aufspannen und das wechselseitige Steigerungsverhältnis der Unterscheidung global|lokal illustrieren lassen. 



\section{Literatur}

Abers, Rebecca (1998): »From Clientilism to Cooperation: Local Government, Participatory Policy, and Civic Organizing in Porto Alegre, Brazil«, in: Politics and Society 26 (4), S. 511-537.

Agar, Michael H. (1996): The Professional Stranger. An Informal Introduction to Ethnography, San Diego: Academic Press.

Ahrend, Rudiger (2004): Accounting for Russia's Post-Crisis Growth, OECD Economics Department Working Papers Nr. 404, Paris: OECD Publishing.

Allina-Pisano, Jessica (2004): »Sub Rosa Resistance and the Politics of Economic Reform. Land Redistribution in Post-Soviet Ukraine«, in: World Politics 56 (4), S. 554-581.

Allina-Pisano, Jessica (2008): The Post-Soviet Potemkin Village. Politics and Property Rights in the Black Earth, Cambridge: Cambridge University Press.

Altrichter, Helmut (1984): Die Bauern von Tver: Vom Leben auf dem russischen Dorfe zwischen Revolution und Kollektivierung, München: Oldenbourg.

Altrichter, Helmut (Hg.) (1986): Die Sowjetunion: Von der Oktoberrevolution bis zu Stalins Tod. Band 1: Staat und Partei, München: Deutscher Taschenbuch Verlag.

Altrichter, Helmut und Heiko Haumann (Hg.) (1986): Die Sowjetunion: Von der Oktoberrevolution bis zu Stalins Tod. Band 2: Wirtschaft und Gesellschaft, München: Deutscher Taschenbuch Verlag.

Altvater, Elmar (1998): »Theoretical Deliberations on Time and Space in Postsocialist Transformation«, in: Regional Studies 32 (7), S. 591-605.

Amann, Klaus und Stefan Hirschauer (1997): »Die Befremdung der eigenen Kultur. Ein Programm«. In: Stefan Hirschauer und Klaus Amann (Hg.), Die Befremdung der eigenen Kultur. Zur ethnographischen Herausforderung soziologischer Empirie, Frankfurt a.M.: Suhrkamp, S. 7-52.

Amelina, Maria (2000): »Why Russian Peasants Remain in Collective Farms: A Household Perspective on Agricultural Restructuring«, in: Post-Soviet Geography and Economics 41 (7), S. 483-511. 
Amelina, Maria (2001): False Transformations. From Stalin's Peasants to Yeltsin's Collective Farmers, unveröffentlichte Dissertation, Fletcher School of Law and Diplomacy.

Amelina, Maria (2002): »What Turns the Kolkhoz into a Firm? Regional Policies and the Elasticity of Budget Constraints«. In: David J. O’Brien und Stephen K. Wegren (Hg.), Rural Reform in Post-Soviet Russia, Baltimore: John Hopkins University Press, S. 264-297.

Appel, Hilary (1997): »Voucher Privatisation in Russia: Structural Consequences and Mass Response in the Second Period of Reform«, in: Europe-Asia Studies 49 (8), S. 1433-1449.

Applebaum, Anne (2003): Der Gulag, Berlin: Siedler Verlag.

Ashby, Ross W. (1974): Einführung in die Kybernetik, Frankfurt a.M.: Suhrkamp.

Atkin, Carl (2009): »Investment in Farmland and Farming in Central and Eastern Europe and the Former Soviet Union - Current Trends and Issues«. In: Michael Kugelman und Susan L. Levenstein (Hg.), Land Grab? The Race for the World's Farmland, Washington D.C.: Woodrow Wilson International Center for Scholars, S. 109-119.

Atkinson, Dorothy (1982): »The zemstvo and the peasantry«. In: Terence Emmons und Wayne S. Vucinich (Hg.), The zemstvo in Russia. An experiment in local self-government, Cambridge: Cambridge University Press, S. 79-132.

Avritzer, Leonardo (2002): Democracy and the Public Space in Latin America, Princeton: Princeton University Press.

Baecker, Dirk (1998 [1995]): »Nichttriviale Transformation«. In: Dirk Baecker

(Hg.), Poker im Osten: Probleme der Transformationsgesellschaft, Berlin: Merve, S. 39-69.

Baecker, Dirk (1998 [1997]-a): »Geld und Unternehmertum«. In: Dirk Baecker

(Hg.), Poker im Osten: Probleme der Transformationsgesellschaft, Berlin: Merve, S. 79-97.

Baecker, Dirk (1998 [1997]-b): »Organisation und Gedächtnis in der Tranformationsgesellschaft«. In: Dirk Baecker (Hg.), Poker im Osten: Probleme der Transformationsgesellschaft, Berlin: Merve, S. 99-130.

Baecker, Dirk (1999): Organisation als System, Frankfurt a.M.: Suhrkamp.

Baecker, Dirk (2002): »Die Form der Zahlung«. In: Christoph Deutschmann (Hg.),

Die gesellschaftliche Macht des Geldes (= Leviathan - Zeitschrift für Sozialwissenschaft, Sonderheft 21/2002), Wiesbaden: Westdeutscher Verlag, S. 73-82.

Baecker, Dirk (2003): »Einleitung«. In: Dirk Baecker (Hg.), Kapitalismus als Religion, Berlin: Kadmos, S. 7-13.

Baecker, Dirk (2006): Wirtschaftssoziologie, Bielefeld: transcript.

Baecker, Dirk (2007): Form und Formen der Kommunikation, Frankfurt a.M.:

Suhrkamp. 
Baecker, Dirk (2008): »Wirtschaft als funktionales Teilsystem«. In: Andrea Maurer (Hg.), Handbuch der Wirtschaftssoziologie, Wiesbaden: VS Verlag für Sozialwissenschaften, S. 109-123.

Baecker, Dirk (2012): »Die Texte der Systemtheorie«. In: Matthias Ochs und Jochen Schweitzer (Hg.), Handbuch Forschung für Systemiker, Göttingen: Vandenhoeck \& Ruprecht, S. 153-186.

Bahl, Roy W. (1999): »Fiscal Decentralization as Development Policy«, in: Public Budgeting \& Finance 19 (2), S. 59-75.

Bahl, Roy W. und Jorge Martines-Vazquez (2006): Sequencing Fiscal Decentralization, Policy Research Working Paper 3914, Washington D.C.: The World Bank.

Ballin, André (2006): »Putins Wesir leitet die >Gasifizierung««, Spiegel Online: http://www.spiegel.de/wirtschaft/0,1518,427096,00.html vom 15.4.2015.

Baraldi, Claudio, Giancarlo Corsi und Elena Esposito (1997): GLU - Glossar zu Niklas Luhmanns Theorie sozialer Systeme, Frankfurt a.M.: Suhrkamp.

Barnard, Chester I. (1971 [1938]): The Functions of the Executive, Cambridge, MA: Harvard University Press.

Barnes, Andrew (1998): »What's the Difference? Industrial Privatisation and Agricultural Land Reform in Russia, 1990-1996«, in: Europe-Asia Studies 50 (5), S. 843-857.

Bartlett, Will und Julian Le Grand (1993): »The Theory of Quasi-Markets«. In: Will Bartlett und Julian Le Grand (Hg.), Quasi-Markets and Social Policy, Houndmills: Macmillan, S. 13-34.

Beck, Roland, Annette Kamps und Elitza Mileva (2007): Long-Term Growth Prospects for the Russian Economy, Occasional Paper Series Nr. 58, März 2007, Frankfurt a.M.: European Central Bank.

Belaya, Vera und Jon Henrich Hanf (2010): »Foreign direct investment as an agent of change in Russian agrifood business - consequences of the export of chain management concepts by foreign investors «, in: Post-Communist Economies 22 (1), S. 55-72.

Benhabib, Seyla (1996): »Toward a Deliberative Model of Democratic Legitimacy«. In: Seyla Benhabib (Hg.), Democracy and Difference, Princeton: Princeton University Press, S. 67-94.

Benjamin, Walter (2009 [1985]): »Kapitalismus als Religion«. In: Dirk Baecker (Hg.), Kapitalismus als Religion, Berlin: Kadmos, S. 15-18.

Berliner, Joseph S. (1952): »The Informal Organization of the Soviet Firm«, in: The Quarterly Journal of Economics 66 (3), S. 342-365.

Berliner, Joseph S. (1957): Factory and Manager in the USSR, Cambridge, MA: Harvard University Press.

Bikbov, Alexander (2005): »Fragliche Autonomie. Zur Lage der Soziologie im heutigen Russland«, in: Berliner Journal für Soziologie 15 (3), S. 309-330. 
Bilinsky, Yaroslav (1999): »Was the Ukrainian Famine of 1932-1933 Genocide?«, in: Journal of Genocide Research 1 (2), S. 147-156.

Birman, Igor (1978): »)From the Achieved Level««, in: Soviet Studies 30 (2), S. 153-172.

Bjalkina, Tatjana M. (2001): »Die örtliche Selbstverwaltung in der Russischen Föderation: Lage, Probleme, Perspektiven«, in: Osteuropa Recht 47 (1-2), S. 1534.

Blair, Harry (2000): »Participation and Accountability at the Periphery: Democratic Local Governance in Six Countries«, in: World Development 28 (1), S. 21-39.

Blanchard, Olivier (1996): »Theoretical Aspects of Transition«, in: The American Economic Review 86 (2), S. 117-122.

Blanchard, Olivier und Michael Kremer (1997): »Disorganziation«, in: The Quarterly Journal of Economics 112 (4), S. 1091-1126.

Blomley, Nicholas (2005): »Remember property?«, in: Progress in Human Geography 29 (2), S. 125-127.

Blomley, Nicholas und Janet C. Sturgeon (2009): »Property as Abstraction«, in: International Journal of Urban and Regional Research 33 (2), S. 564-566.

Blühdorn, Ingolfur (2013): Simulative Demokratie. Neue Politik nach der postdemokratischen Wende, Berlin: Suhrkamp.

Bluhm, Katharina (2008): »Corporate Social Responsibility - Zur Moralisierung von Unternehmen aus soziologischer Perspektive«. In: Andrea Maurer und Uwe Schimank (Hg.), Die Gesellschaft der Unternehmen - Die Unternehmen der Gesellschaft: Gesellschaftstheoretische Zugänge zum Wirtschaftsgeschehen, Wiesbaden: VS Verlag für Sozialwissenschaften, S. 144-162.

Bohman, James und William Rehg (1997): »Introduction«. In: James Bohman und William Rehg (Hg.), Deliberative Democracy. Essays on Reason and Politics, Cambridge, MA: MIT Press, S. ix-xxx.

Bohn, Cornelia (2005): »Eine Welt-Gesellschaft. Operative Gesellschaftskonzepte in den Sozialtheorien Luhmanns und Bourdieus«. In: Catherine Colliot-Thélène, Etienne Francois und Gunter Gebauer (Hg.), Pierre Bourdieu: Deutschfranzösische Perspektiven, Frankfurt a.M.: Suhrkamp, S. 43-78.

Bohn, Cornelia (2009): »Geld und Eigentum - Inkludierende und exkludierende Mechanismen in der Wirtschaft«. In: Rudolf Stichweh und Paul Windolf (Hg.), Inklusion und Exklusion. Analysen zur Sozialstruktur und sozialen Ungleichheit, Wiesbaden: VS Verlag für Sozialwissenschaften, S. 241-258.

Bohnsack, Ralf (2003): »Dokumentarische Methode und sozialwissenschaftliche Hermeneutik«, in: Zeitschrift für Erziehungswissenschaft 6 (4), S. 550-570.

Bohnsack, Ralf (2008): Rekonstruktive Sozialforschung. Einführung in qualitative Methoden, Opladen: Verlag Barbara Budrich. 
Bohnsack, Ralf (2010): »Dokumentarische Methode und Typenbildung - Bezüge zur Systemtheorie«. In: René John, Anna Henkel und Jana Rückert-John (Hg.), Die Methodologien des Systems. Wie kommt man zum Fall und wie dahinter?, Wiesbaden: VS Verlag für Sozialwissenschaften, S. 291-320.

Bohnsack, Ralf, Iris Nentwig-Gesemann und Arnd-Michael Nohl (Hg.) (2007a): Die dokumentarische Methode und ihre Forschungspraxis. Grundlagen qualitativer Sozialforschung, Wiesbaden: VS Verlag für Sozialwissenschaften.

Bohnsack, Ralf, Iris Nentwig-Gesemann und Arnd-Michael Nohl (2007b): »Einleitung: Die dokumentarische Methode und ihre Forschungspraxis«. In: Ralf Bohnsack, Iris Nentwig-Gesemann und Arnd-Michael Nohl (Hg.), Die dokumentarische Methode und ihre Forschungspraxis. Grundlagen qualitativer Sozialforschung, Wiesbaden: VS Verlag für Sozialwissenschaften, S. 9-27.

Bommes, Michael und Veronika Tacke (2006): »Das Allgemeine und das Besondere des Netzwerks«. In: Betina Hollstein und Florian Straus (Hg.), Qualitative Netzwerkanalyse. Konzepte, Methoden, Anwendungen, Wiesbaden: VS Verlag für Sozialwissenschaften, S. 37-62.

Bonazzi, Giuseppe (2008): Geschichte des organisatorischen Denkens, Wiesbaden: VS Verlag für Sozialwissenschaften.

Bornstein, Morris (1962): »The Soviet Price System«, in: The American Economic Review 52 (1), S. 64-103.

Bornstein, Morris (1978): »The Administration of the Soviet Price System«, in: Soviet Studies 30 (4), S. 466-490.

Borras, Saturnino M. et al. (2012): »Towards a better understanding of global land grabbing: an editorial introduction«, in: Journal of Peasant Studies 38 (2), S. 209-216.

Bourdieu, Pierre (2011 [1994]): »Die Ökonomie der symbolischen Güter«. In: Pierre Bourdieu (Hg.), Kunst und Kultur. Zur Ökonomie symbolischer Güter. Schriften zur Kultursoziologie 4, Konstanz: UVK, S. 187-223.

Boycko, Maxim, Andrei Shleifer und Robert W. Vishny (1993): »Privatizing Russia«, in: Brookings Papers on Economic Activity (2), S. 139-192.

Bradshaw, Michael J. und Karen Vartapetov (2003): »A New Perspective on Regional Inequalities in Russia«, in: Eurasian Geography and Economics 44 (6), S. 403-429.

Brodocz, André (1996): »Strukturelle Kopplung durch Verbände«, in: Soziale Systeme 2 (2), S. 361-387.

Brummer, Klaus (2008): Der Europarat: Eine Einführung, Wiesbaden: VS Verlag für Sozialwissenschaften.

Brunner, Georg und Klaus Westen (1970): Die sowjetische Kolchosordnung (mit Dokumenten): Von den Anfängen zum 3. Musterstatut 1969, Stuttgart: W. Kohlhammer.

Brunsson, Nils und Johan P. Olsen (1997): The Reforming Organization, BergenSandviken: Fagbokforlaget. 
Brunsson, Nils und Kerstin Sahlin-Andersson (2000): »Constructing Organizations: The Example of Public Sector Reform«, in: Organization Studies 21 (4), S. 721746.

Buccellato, Tullio und Tomasz Mickiewicz (2009): »Oil and Gas: A Blessing for the Few. Hydrocarbons and Inequality within Regions in Russia«, in: EuropeAsia Studies 61 (3), S. 385-407.

Buhr, Petra und Stephan Leibfried (2009): »Ist die Armutsbevölkerung in Deutschland exkludiert?«. In: Rudolf Stichweh und Paul Windolf (Hg.), Inklusion und Exklusion: Analysen zur Sozialstruktur und sozialen Ungleichheit, Wiesbaden: VS Verlag für Sozialwissenschaften, S. 103-122.

Burawoy, Michael und Katherine Verdery (1999): »Introduction«. In: Michael Burawoy und Katherine Verdery (Hg.), Uncertain Transition: Ethnographies of Change in the Postsocialist World, Lanham: Rowman \& Littlefield, S. 1-17.

Cai, Hongbin und Daniel Treisman (2006): »Did Government Decentralization Cause China's Economic Miracle?«, in: World Politics 58 (4), S. 505-535.

Campbell, Adrian (1995): »Regional Power in the Russian Federation«. In: Andrew Coulson (Hg.), Local Government in Eastern Europe: Establishing Democracy at the Grassroots, Hants: Edward Elgar Publishing, S. 145-167.

Campbell, Adrian (2006): »State vs. Society? Local Government and the Reconstruction of the Russian State«, in: Local Government Studies 32 (5), S. 659676.

Campbell, Adrian (2009): »Vertical or triangle? Local, regional and federal government in the Russian Federation after Law 131«. In: Cameron Ross und Adrian Campbell (Hg.), Federalism and Local Politics in Russia, London: Routledge, S. 263-283.

Carlin, Wendy et al. (2000): »Barter and non-monetary transactions in transition economies: evidence from a cross-country survey«. In: Paul Seabright (Hg.), The Vanishing Rouble. Barter Networks and Non-Monetary Transactions in Post-Soviet Societies, Cambridge: Cambridge University Press, S. 236-256.

Carothers, Thomas (2002): »The End of the Transition Paradigm«, in: Journal of Democracy 13 (1), S. 5-21.

Carruthers, Bruce G. und Laura Ariovich (2004): »The Sociology of Property Rights«, in: Annual Review of Sociology 30, S. 23-46.

Center for Fiscal Policy (2008): Analytical Work on Indicators of Effectiveness of Local Governments in the Context of Decentralization Reform in Russia, unveröffentlichtes Gutachten, Moskau: Center for Fiscal Policy.

Chernyavsky, Andrei und Karen Vartapetov (2004): »Municipal Finance Reform and Local Self-governance in Russia«, in: Post-Communist Economies 16 (3), S. 251-264.

Churchward, Lloyd G. (1983): »Public Participation in the USSR«. In: Everett M. Jacobs (Hg.), Soviet Local Politics and Government, London: George Allen \& Unwin, S. 34-47. 
Clark, Katerina (2011): Moscow, the Fourth Rome: Stalinism, Cosmopolitanism, and the Evolution of Soviet Culture, 1931-1941, Cambridge, MA: Harvard University Press.

Clarke, John und Janet Newman (1997): The Managerial State, London: Sage.

Clarke, Simon (2000): »The household in a non-monetary market economy«. In: Paul Seabright (Hg.), The Vanishing Rouble. Barter Networks and Non-Monetary Transactions in Post-Soviet Societies, Cambridge: Cambridge University Press, S. 176-206.

Coleman, James S. (1994): Foundations of Social Theory, Cambridge, MA: The Belknap Press of Harvard University Press.

Collier, David und Steven Levitsky (1997): »Democracy with Adjectives: Conceptual Innovation in Comparative Research«, in: World Politics 49 (3), S. 430451.

Coly, Annette und Elke Brecker (2004): »Dezentralisierung und Stärkung kommunaler Selbstverwaltung zur Förderung von Good Governance«, in: Aus Politik und Zeitgeschichte B 15-16 (5.4.2004), S. 3-11.

Craig, Gary und Marjorie Mayo (1995): »Community Participation and Empowerment: The Human Face of Structural Adjustment or Tools for Democratic Transformation?«. In: Gary Craig und Marjorie Mayo (Hg.), Community Empowerment: A Reader in Participation and Development, London: Zed, S. 1-11.

Craumer, Peter R. (1994): »Regional Patterns of Agricultural Reform in Russia«, in: Post-Soviet Geography 35 (6), S. 329-351.

Cunningham, Kathleen Gallagher (2014): Inside the Politics of Self-Determination, Oxford: Oxford University Press.

Davies, Robert W. und Stephen G. Wheatcroft (2002): »The Soviet Famine of 1932-33 and the Crisis in Agriculture«. In: Stephen G. Wheatcroft (Hg.), Challenging Traditional Views of Russian History, Palgrave: Macmillan, S. 6991.

Davydova, Irina und J. R. Franks (2006): »Responses to agrarian reforms in Russia: Evidence from Novosibirsk oblast«, in: Journal of Rural Studies 22 (1), S. 3954.

De Melo, Martha, Cevdet Denizer und Alan Gelb (1996): From Plan to Market. Patterns of Transition, Policy Research Working Paper 1564, Washington D.C.: The World Bank.

De Schutter, Olivier (2011): »How not to think of land-grabbing: three critiques of large-scale investments in farmland«, in: Journal of Peasant Studies 38 (2), S. 249-279.

Deininger, Klaus (2011): »Challenges posed by the new wave of farmland investment«, in: Journal of Peasant Studies 38 (2), S. 217-247.

Deininger, Klaus und Hans Binswanger (1999): »The Evolution of the World Bank's Land Policy: Principles, Experience, and Future Challenges«, in: The World Bank Research Observer 14 (2), S. 247-276. 
Deininger, Klaus und Derek Byerlee (2011): Rising Global Interest in Farmland: Can It Yield Sustainable and Equitable Benefits?, Washington D.C.: The World Bank.

Denzin, Norman K. (2009 [1970]): The Research Act. A Theoretical Introduction to Sociological Methods, New Brunswick: Aldine Transaction.

Der Spiegel (1984): »Feilschen erlaubt«. In: Der Spiegel (H. 24), 11.6.1984. S. 114-119.

Desai, Vandana und Rob Imrie (1998): »The new managerialism in local governance: North-South dimensions«, in: Third World Quarterly 19 (4), S. 635-650.

Deutschmann, Christoph (2009a): »Die Verheißung absoluten Reichtums: Kapitalismus als Religion?«. In: Dirk Baecker (Hg.), Kapitalismus als Religion, Berlin: Kadmos, S. 145-174.

Deutschmann, Christoph (2009b): »Geld als universales Inklusionsmedium moderner Gesellschaften«. In: Rudolf Stichweh und Paul Windolf (Hg.), Inklusion und Exklusion. Analysen zur Sozialstruktur und sozialen Ungleichheit, Wiesbaden: VS Verlag für Sozialwissenschaften, S. 223-239.

Diamond, Larry (1999): Developing Democracy: Towards Consolidation, Baltimore: John Hopkins University Press.

Dienes, Leslie (2002): »Reflections on a Geographic Dichotomy: Archipelago Russia«, in: Eurasian Geography and Economics 43 (6), S. 443-458.

DiMaggio, Paul J. und Walter W. Powell (1983): »The Iron Cage Revisited: Institutional Isomorphism and Collective Rationality in Organizational Fields«, in: American Sociological Review 48 (2), S. 147-160.

Donahoe, Brian und Joachim Otto Habeck (Hg.) (2011): Reconstructing the House of Culture: Community, Self, and the Makings of Culture in Russia and Beyond, New York: Berghahn.

Dubois, Hans F. W. und Giovanni Fattore (2009): »Definitions and Typologies in Public Administration Research: The Case of Decentralization«, in: International Journal of Public Administration 32 (8), S. 704-727.

Dudek, Daniel J., Alexander A. Golub und Elena B. Strukova (2006): »Should Russia increase domestic prices for natural gas?«, in: Energy Policy 34 (13), S. 1659-1670.

EBRD (2014): Transition Report 2014: The Many Faces of Innovation, Transition Report, London: European Bank for Reconstruction and Development (EBRD).

Efendiev, Azer G. und I. A. Bolotina (2002): »Sovremennoe rossijskoe selo: na perelome epoch i reform. Opyt institutzional'nogo analyza [Das gegenwärtige russische Dorf: Am Wendepunkt der Epochen und Reformen. Ergebnisse einer Institutionenanalyse]«, in: Mir Rossii [Universe of Russia] 11 (4), S. 83-125.

Egner, Heike (2010): Theoretische Geographie, Darmstadt: WBG.

Ellman, Michael (2005): »The Role of Leadership Perceptions and of Intent in the Soviet Famine of 1931-1934«, in: Europe-Asia Studies 57 (6), S. 823-841. 
Ellman, Michael (2007): »Stalin and the Soviet Famine of 1932-33 Revisited , in: Europe-Asia Studies 59 (4), S. 663-693.

Elster, Jon (1997): »The Market and the Forum: Three Varieties of Political Theory«. In: James Bohman und William Rehg (Hg.), Deliberative Democracy. Essays on Reason and Politics, Cambridge, MA: MIT Press, S. 3-34.

Ericson, Richard E. (2006): »Command Versus >Shadow $<$ : The Conflicted Soul of the Soviet Economy«, in: Comparative Economic Studies 48 (1), S. 50-76.

Esposito, Elena (2008): »Die normale Unwahrscheinlichkeit der Medien: der Fall des Geldes«. In: Stefan Münker und Alexander Roesler (Hg.), Was ist ein Medium?, Frankfurt a.M.: Suhrkamp, S. 112-130.

Ettrich, Frank (2003): »Differenzierung und Eliten im Staatssozialismus«, in: Historical Social Research 28 (1-2), S. 31-56.

Etzioni, Amitai (1975): A Comparative Analysis of Complex Organizations. On Power, Involvement, and Their Correlates, New York: The Free Press.

Europarat (1985): Europäische Charta der kommunalen Selbstverwaltung, Straßburg: Europarat.

Evans, Alfred B. (2004): »Contemporary Russian Scholars' Changing Views on Local Government in Late Tsarist Russia«. In: Alfred B. Evans und Vladimir Gelman (Hg.), The Politics of Local Government in Russia, Lanham: Rowman \& Littlefield, S. 68-81.

Faguet, Jean-Paul (2001): Does Decentralization Increase Government Responsiveness to Local Needs? Evidence from Bolivia, Policy Research Working Paper 2516, Washington D.C.: The World Bank.

Faguet, Jean-Paul (2003): Decentralization and Local Government in Bolivia: an overview from the bottom up, Crisis States Programme Working Paper 29, London: London School of Economics, Development Research Centre

Faguet, Jean-Paul (2008): »Decentralisation's Effects on Public Investment: Evidence and Policy Lessons from Bolivia and Colombia«, in: Journal of Development Studies 44 (8), S. 1100-1121.

Feifer, Gregory (2003): »Russia: Farmland Reform May Prove Putin's Lasting Legacy«, Radio Free Europe/Radio Liberty: http://www.rferl.org/content/ article/1102228.html vom 15.4.2015.

Ferguson, James (1990): The Anti-Politics Machine. `Development<, Depoliticization and Bureaucratic Power in Lesotho, Cambridge: Cambridge University Press.

Figes, Orlando (1986): »Collective Farming and the 19th-Century Russian Land Commune: A Research Note«, in: Soviet Studies 38 (1), S. 89-97.

Figes, Orlando (2012): Just Send Me Word. A True Story of Love and Survival in the Gulag, London: Allen Lane.

Finon, Dominique und Catherine Locatelli (2008): »Russian and European gas interdependence: Could contractual trade channel geopolitics?«, in: Energy Policy 36 (1), S. 423-442. 
Fischer, Stanley, Ratna Sahay und Carlos A. Végh (1996): »Stabilization and Growth in Transition Economies: The Early Experience«, in: The Journal of Economic Perspectives 10 (2), S. 45-66.

Fitch Ratings (2006): Reform of Local Self-Governance in Russia, Russian Federation Special Report, 16.10.2006, New York: Fitch Ratings.

Flick, Uwe (2009): Qualitative Sozialforschung: Eine Einführung, Reinbek: Rowohlt.

Fligstein, Neil (2001): The Architecture of Markets: An Economic Sociology of Twenty-First-Century Capitalist Societies, Princeton: Princeton University Press.

Föderales Gesetz 131 (2005 [2003]): »Russian Federation Federal Law >On the General Principles of the Organization of Local Self-Government in the Russian Federation< No. 131-FZ of October 6, 2003«, in: Statutes and Decisions: The Laws of the USSR and its Successor States 41 (5), S. 21-112.

Frey, Bruno S. (2005): »Functional, Overlapping, Competing Jurisdictions: Redrawing the Geographic Borders of Administration«, in: European Journal of Law Reform V (3-4), S. 534-555.

Frey, Bruno S. und Reiner Eichenberger (1996): »FOCJ: Competitive Governments for Europe«, in: International Review of Law and Economics 16 (3), S. 315327.

Friedmann, John (1996): »Rethinking poverty: empowerment and citizen rights«, in: International Social Science Journal 48 (2), S. 161-172.

Fuchs, Dieter und Edeltraud Roller (2006): »Learned Democracy? Support of Democracy in Central and Eastern Europe«, in: International Journal of Sociology 36 (3), S. 70-96.

Fuchs, Peter (1997): »Adressabilität als Grundbegriff der soziologischen Systemtheorie«, in: Soziale Systeme 3 (1), S. 56-79.

Fuhse, Jan (2003): »Das widerständige Publikum. Zur Relevanz von alltagsweltlichen Kommunikationsstrukturen für die politische Meinungsbildung«. In: KaiUwe Hellmann, Karsten Fischer und Harald Bluhm (Hg.), Das System der Politik. Niklas Luhmanns politische Theorie, Wiesbaden: Westdeutscher Verlag, S. 136-149.

Fuhse, Jan (2005): Theorien des politischen Systems: David Easton und Niklas Luhmann. Eine Einführung, Wiesbaden: VS Verlag für Sozialwissenschaften.

Fung, Archon und Erik Olin Wright (2001): »Deepening Democracy: Innovations in Empowered Participatory Governance«, in: Politics and Society 29 (1), S. 541.

Gabler Verlag (Hg.) (2010): Gabler Kompakt-Lexikon Wirtschaft, Wiesbaden: Gabler Verlag.

Gambold Miller, Liesl L. (2002): »Communal Coherence and Barriers to Reform«. In: David J. O’Brien und Stephen K. Wegren (Hg.), Rural Reforms in PostSoviet Russia, Baltimore: John Hopkins University Press, S. 221-242. 
García-López, Gustavo und Nancy Arizpe (2010): »Participatory processes in the soy conflicts in Paraguay and Argentina«, in: Ecological Economics (70), S. 196-206.

Gaventa, John (2002): »Towards Participatory Local Governance: Six Propositions for Discussion«, in: Currents 28 (August), S. 29-35.

Gaventa, John (2004): »Strengthening Participatory Approaches to Local Governance: Learning the Lessons from Abroad«, in: National Civic Review 93 (4), S. 16-27.

Gazprom (2011): Postavki gaza na vnutrennyj rynok. Realizacija programmy gazifikacii rossijskich regionov [Gaslieferungen auf dem Binnenmarkt. Die Umsetzung des Programms zur Gasifizierung der russischen Regionen], Pressebericht, 27.6.2011, Moskau: Gazprom.

Gebauer, Ronald (2003): »War die DDR eine entdifferenzierte Gesellschaft? Ergebnisse einer Ereignisanalyse auf Basis der Daten des Zentralen Kaderdatenspeichers des Ministerrates der DDR «, in: Historical Social Research 28 (1-2), S. 216-246.

Geden, Oliver (2008): Mehr Pragmatismus, weniger Geopolitik. Effiziente Ansätze für die Energieversorgungssicherheit der EU, SWP-Aktuell 83, November 2008, Berlin: Stiftung Wissenschaft und Politik.

Gel'man, Vladimir (2002): »The Politics of Local Government In Russia: The Neglected Side Of The Story«, in: Perspectives on European Politics and Society 3 (3), S. 495-508.

Gel'man, Vladimir (2009): »Leviathan's return. The policy of recentralization in contemporary Russia«. In: Cameron Ross und Adrian Campbell (Hg.), Federalism and Local Politics in Russia, London: Routledge, S. 1-24.

Gelb, Alan (1997): »Assessing the Transition from Plan to Market: What Have We Learned - about Policies and Economic Theory?«, in: Journal of International Development 9 (4), S. 449-458.

Gelman, Vladimir (2004): »Federal Politics toward Local Government in Russia: The Process of Institution Building «. In: Alfred B. Evans und Vladimir Gelman (Hg.), The Politics of Local Government in Russia, Lanham: Rowman \& Littlefield, S. 85-103.

Gelman, Vladimir (2007): »Von der lokalen Selbstverwaltung zur >Machtvertikale ««, in: Russlandanalysen (135), S. 2-7.

Georgieva, Kristalina (2007): »Foreword«. In: The World Bank (Hg.), Local Governance and Civic Engagement in Rural Russia. A World Bank Project, Moskau: The World Bank, S. 5-6.

Gerhards, Jürgen (2001): »Der Aufstand des Publikums. Eine systemtheoretische Interpretation des Kulturwandels in Deutschland zwischen 1960 und 1989«, in: Zeitschrift für Soziologie 30 (3), S. 163-184.

Gershon, Ilana (2005): »Seeing like a system: Luhmann for anthropologists«, in: Anthropological Theory 5 (2), S. 99-116. 
Gill, Graeme (1988): The Rules of the Communist Party of the Soviet Union, Armonk: M. E. Sharpe, Inc.

Goehrke, Carsten (2005): Russischer Alltag: Eine Zeitgeschichte in neun Zeitbildern. Band 3: Sowjetische Moderne und Umbruch, Zürich: Chronos.

Goeke, Pascal (2007): »Handeln und Erleben im Krieg. Die Differenz zwischen Hier und Dort als legitimierende und entfremdende Sinndimension«. In: Christian Berndt und Robert Pütz (Hg.), Kulturelle Geographien. Zur Beschäftigung mit Raum und Ort nach dem Cultural Turn, Bielefeld: transcript, S. 53-82.

Goeke, Pascal (2013): »Grenzenlose Konflikte. Programme kritischer Geographien und ihre Folgen«, in: Geographische Zeitschrift 101 (1), S. 1-19.

Goeke, Pascal und Evelyn Moser (2011): »Der Markt der Wirtschaft«, in: Zeitschrift für Wirtschaftsgeographie 55 (4), S. 226-238.

Golubchikov, Oleg (2007): »Re-scaling the Debate on Russian Economic Growth: Regional Restructuring and Developmental Asynchronies«, in: Europe-Asia Studies 59 (2), S. 191-215.

Götz, Roland (2006a): Nach dem Gaskonflikt. Wirtschaftliche Konsequenzen für Russland, die Ukraine und die EU, SWP-Aktuell 3, Januar 2006, Berlin: Stiftung Wissenschaft und Politik.

Götz, Roland (2006b): Russische Energiepolitik, Diskussionspapier FG 5 2006/01, Februar 2006, Berlin: Stiftung Wissenschaft und Politik.

Götz, Roland (2007): Russlands Erdgas und Europas Energiesicherheit, SWP-Studie S 21, August 2007, Berlin: Stiftung Wissenschaft und Politik.

Götz, Roland (2012): »Aussterbende Gattung. Deutschlands Russlandökonomen«, in: Osteuropa 62 (9), S. 113-116.

Grabher, Gernot und David Stark (1997): »Organizing Diversity: Evolutionary Theory, Network Analysis, and Post-Socialism«, in: Regional Studies 31 (5), S. 533-544.

Graham, Loren R. (1994): Science in Russia and the Soviet Union. A Short History, Cambridge: Cambridge University Press.

Granovetter, Mark (1973): »The Strength of Weak Ties«, in: The American Journal of Sociology 78 (6), S. 1360-1380.

Grätz, Jonas (2009): »Energy Relations with Russia and Gas Market Liberalization«, in: International Politics and Society (3), S. 66-80.

Grossman, Gregory (1962): »The Structure and Organization of the Soviet Economy«, in: Slavic Review 21 (2), S. 203-222.

Grossman, Gregory (1977): »The >Second Economy< of the USSR «, in: Problems of Communism 26 (5), S. 25-40.

Grossman, Gregory (1981): »La seconde économie et la planification économique soviétique«, in: Revue d'études comparatives Est-Ouest 12 (2), S. 5-24. 
Guriev, Sergei und Barry W. Ickes (2000): »Barter in Russia«. In: Paul Seabright (Hg.), The Vanishing Rouble. Barter Networks and Non-Monetary Transactions in Post-Soviet Societies, Cambridge: Cambridge University Press, S. 147-175.

Habermas, Jürgen (1997): »Popular Sovereignty as Procedure «. In: James Bohman und William Rehg (Hg.), Deliberative Democracy. Essays on Reason and Politics, Cambridge, MA: MIT Press, S. 35-66.

Haerpfer, Christian W. (2002): Democracy and Enlargement in Post-Communist Europe, London: Routledge.

Hahn, Jeffrey W. (1988): Soviet Grassroots. Citizen Participation in Local Soviet Government, Princeton: Princeton University Press.

Hahn, Jeffrey W. (1989): »Power to the Soviets?«, in: Problems of Communism 38 (1), S. 34-46.

Halton, Eugene (1992): »The Cultic Roots of Culture«. In: Richard Münch und Neil J. Smelser (Hg.), Theory of Culture, Berkeley: University of California Press, S. 29-63.

Hammersley, Martyn und Paul Atkinson (2007): Ethnography. Principles in Practice, London: Routledge.

Hann, Chris (1998): »Introduction: the embeddedness of property«. In: Chris Hann (Hg.), Property Relations: Renewing the Anthropoligical Tradition, Cambridge: Cambridge University Press, S. 1-47.

Hann, Chris (2003): »Introduction: Decollectivisation and the Moral Economy«. In: Chris Hann (Hg.), The Postsocialist Agrarian Question. Property Relations and the Rural Condition, Münster: Lit, S. 1-46.

Hann, Chris (2006): >Not the Horse We Wanted!<: Postsocialism, Neoliberalism, and Eurasia (= Halle Studies in the Anthropology of Eurasia; 10), Münster: Lit.

Hard, Gerhard (2003 [1987]): »Die Störche und die Kinder, die Orchideen und die Sonne«. In: Gerhard Hard (Hg.), Dimensionen geographischen Denkens. Aufsätze zur Theorie der Geographie, Band 2 (= Osnabrücker Studien zur Geographie; 23), Osnabrück: V\&R unipress, S. 315-327.

Hardin, Garrett (1968): »The Tragedy of the Commons«, in: Science 162 (3859), S. 1243-1248.

Harriss, John (2002): Depoliticizing Development. The World Bank and Social Capital, London: Anthem Press.

Hasse, Raimund und Georg Krücken (2005): Neo-Institutionalismus, Bielefeld: transcript.

Häussermann, Hartmut und Martin Kronauer (2009): »Räumliche Segregation und innerstädtisches Ghetto«. In: Rudolf Stichweh und Paul Windolf (Hg.), Inklusion und Exklusion: Analysen zur Sozialstruktur und sozialen Ungleichheit, Wiesbaden: VS Verlag für Sozialwissenschaften, S. 157-173. 
Hayoz, Nicolas (1995): »Dédifférenciations régionales et différences foncionelles universelles. Aspects de l'instrumentalisation politique de domaines fonctionnels au sein de la >société organisé< du socialisme soviétique«, in: Soziale Systeme 1 (2), S. 261-282.

Hayoz, Nicolas (1997): L'étreinte soviétique. Aspects sociologiques de l'effondrement programmé de l'URSS, Genf: Droz.

Hayoz, Nicolas (2007): »Regionale >organisierte Gesellschaften< und ihre Schwierigkeiten mit der Realität der funktionalen Differenzierung«, in: Soziale Systeme 13 (1-2), S. 160-172.

Hayoz, Nicolas (2013): »Observations on the Changing Meanings of Informality«. In: Christian Giordano und Nicolas Hayoz (Hg.), Informality in Eastern Europe. Structures, Political Cultures and Social Practices, Bern: Peter Lang, S. 47-65.

Held, David (2006): Models of Democracy, Stanford: Stanford University Press.

Heller, Patrick (2001): »Moving the State: The Politics of Democratic Decentralization in Kerala, South Africa, and Porto Alegre«, in: Politics and Society 29 (1), S. 131-163.

Helmke, Gretchen und Steven Levitsky (2004): »Informal Institutions and Comparative Politics: A Research Agenda«, in: Perspectives on Politics 2 (4), S. 725240.

Henke, Norbert und Willy Wirantaprawira (1981): Verfassung (Grundgesetz) der Union der Sozialistischen Sowjetrepubliken vom 7.10.1977. Eine einführende, erläuternde und vergleichende Studie, Frankfurt a.M.: Peter Lang.

Hill, Roland J. (1983): »The Development of Soviet Local Government since Stalin's Death«. In: Everett M. Jacobs (Hg.), Soviet Local Politics and Government, London: George Allen \& Unwin, S. 18-33.

Hirschman, Albert O. (1970): Exit, Voice, and Loyalty. Responses to Decline in Firms, Organizations, and States, Cambridge, MA: Harvard University Press.

Hirschman, Albert O. (1993): »Exit, Voice, and the Fate of the German Democratic Republic: An Essay in Conceptual History«, in: World Politics 45 (2), S. 173202.

Hofmann, Tessa (1983): Das Bauernthema in der sowjetrussischen Prosa der 20er Jahre. Konzeptionen, Konflikte und Figuren, München: Verlag Otto Sagner.

Holzer, Boris (2006): »Spielräume der Weltgesellschaft: Formale Strukturen und Zonen der Informalität«. In: Thomas Schwinn (Hg.), Die Vielfalt und Einheit der Moderne. Kultur- und strukturvergleichende Analysen, Wiesbaden: VS Verlag für Sozialwissenschaften, S. 259-279.

Hooghe, Liesbet, Gary Marks und Arjan H. Schakel (2010): The Rise of Regional Authority. A Comparative Study of 42 Democracies, London: Routledge.

Hough, Jerry F. und Merle Fainsod (1982): How the Soviet Union Is Governed, Cambridge, MA: Harvard University Press. 
Humphrey, Caroline (1998): Marx Went Away - But Karl Stayed Behind, Ann Arbor: University of Michigan Press.

Humphrey, Caroline (2002): »Subsistence Farming and the Peasantry as an Idea in Contemporary Russia«. In: Pamela Leonard und Deema Kaneff (Hg.), PostSocialist Peasant? Rural and Urban Constructions of Identity in Eastern Europe, East Asia and the former Soviet Union, Houndmills: Palgrave, S. 136-159.

Humphrey, Caroline und Katherine Verdery (2004): »Introduction: Raising Questions about Property«. In: Caroline Humphrey und Katherine Verdery (Hg.), Property in Question: Value Transformation in the Global Economy, Oxford: Berg, S. 1-28.

Huntington, Samuel P. (1991): »Democracy's Third Wave«, in: Journal of Democracy 2 (2), S. 12-34.

Huntington, Samuel P. (1993): The Third Wave: Democratization in the Late Twentieth Century, Norman: University of Oklahoma Press.

Inman, Robert P. und Daniel L. Rubinfeld (1997): »Rethinking Federalism«, in: The Journal of Economic Perspectives 11 (4), S. 43-64.

Ioffe, Grigory und Tatyana Nefedova (1997): Continuity and Change in Rural Russia: A Geographical Perspective, Boulder: Westview Press.

Ioffe, Grigory, Tatyana Nefedova und Ilya Zaslavski (2006): The End of Peasantry? The Disintegration of Rural Russia, Pittsburgh: University of Pittsburgh Press.

Jacobs, Everett M. (1983): »Introduction: The Organizational Framework of Soviet Local Government«. In: Everett M. Jacobs (Hg.), Soviet Local Politics and Government, London: George Allen \& Unwin, S. 3-17.

Jaglin, Sylvy, Clément Repussard und Anne Belbéoch (2011): »Decentralisation and governance of drinking water services in small West African towns and villages (Benin, Mali, Senegal): the arduous process of building local governments«, in: Canadian Journal of Development Studies/Revue canadienne d'études du développement 32 (2), S. 119-138.

Jahn, Egbert (2004): »Der Holodomor im Vergleich. Zur Phänomenologie der Massenvernichtung«, in: Osteuropa 54 (12), S. 13-32.

Japp, Klaus P. (2007): »Regionen und Differenzierung«, in: Soziale Systeme 13 (12), S. 185-195.

Japp, Klaus P. (2010): »Zur Bedeutung von Vertrauensnetzwerken für die Ausdifferenzierung politischer Kommunikation«. In: Michael Bommes und Veronika Tacke (Hg.), Netzwerke in der funktional differenzierten Gesellschaft, Wiesbaden: VS Verlag für Sozialwissenschaften, S. 261-286.

Japp, Klaus P. und Isabel Kusche (2004): »Die Kommunikation des politischen Systems: Zur Differenz von Herstellung und Darstellung im politischen System«, in: Zeitschrift für Soziologie 33 (6), S. 511-531.

John, Peter (2001): Local Governance in Western Europe, London: Sage.

Joo, Hyung-min (2004): »Voices of Freedom: Samizdat«, in: Europe-Asia Studies 56 (4), S. 571-594. 
Kalugina, Zemfira I. und Olga P. Fadeeva (2009): Rossijskaja derevnja v labirinte reform: Sociologičeskie zarisovki [Das russische Dorf im Labyrinth der Reformen: Soziologische Skizzen], Novosibirsk: Rossijskaja Akademija Nauk - Sibirskoe Otdelenie [Russische Akademie der Wissenschaften - Sibirische Abteilung].

Kasuga, Junichi (1987): »Die Beobachtung des Marktes: asymmetrische Strukturen und generalisierte Erwartungen«. In: Dirk Baecker u.a. (Hg.), Theorie als Passion, Frankfurt a.M.: Suhrkamp, S. 547-569.

Kegelmann, Jürgen (2007): New Public Management: Möglichkeiten und Grenzen des Neuen Steuerungsmodells, Wiesbaden: VS Verlag für Sozialwissenschaften.

Kieserling, André (1999): Kommunikation unter Anwesenden. Studien über Interaktionssysteme, Frankfurt a.M.: Suhrkamp.

Kieserling, André (2008): »Interaktion«. In: Sina Farzin und Stefan Jordan (Hg.), Lexikon Soziologie und Sozialtheorie. Hundert Grundbegriffe, Stuttgart: Reclam, S. 129-131.

Kitching, Gavin (1998): »The Development of Agrarian Capitalism in Russia 199197: Some Observations from Fieldwork«, in: Journal of Peasant Studies 25 (3), S. 1-30.

Kitching, Gavin (2001): »The Concept of Sebestoimost' in Russian Farm Accounting: A Very Unmagical Mystery Tour«, in: Journal of Agrarian Change 1 (1), S. 57-80.

Kneer, Georg und Armin Nassehi (2000): Niklas Luhmanns Theorie sozialer Systeme: Eine Einführung, München: Fink.

Knobloch, Jörn (2006): Hybride Systeme. Politische Praxis und Theorie am Beispiel Rußlands, Münster: Lit.

Knorr Cetina, Karin (1992): »Zur Unterkomplexität der Differenzierungstheorie. Empirische Anfragen an die Systemtheorie«, in: Zeitschrift für Soziologie 21 (6), S. 406-419.

Koch, Thomas (2002): »Die Parteien und Massenorganisationen der DDR als Sozialisationsinstanzen«. In: Gerd-Rüdiger Stephan u.a. (Hg.), Die Parteien und Organisationen der DDR: Ein Handbuch, Berlin: Dietz, S. 116-142.

Koenen, Gerd (2012): »Weil es Stalin gefiel? Zu Jörg Baberowskis Deutung des Stalinismus«, in: Osteuropa 62 (4), S. 81-88.

Kolodko, Grzegorz W. (1999): »Transition to a market economy and sustained growth. Implications for the post-Washington consensus«, in: Communist and Post-Communist Studies 32 (3), S. 233-261.

Komaromi, Ann (2004): »The Material Existence of Soviet Samizdat«, in: Slavic Review 63 (3), S. 597-618.

Kornai, János (1986): »The Soft Budget Constraint«, in: Kyklos 39 (1), S. 3-30.

Kornai, János (1992): The Socialist System. The Political Economy of Communism, Oxford: Clarendon Press. 
Kornai, János (2000): »What the Change of System From Socialism to Capitalism Does and Does Not Mean«, in: The Journal of Economic Perspectives 14 (1), S. 27-42.

Kourliandskaja, Galina, Yelena Nikolayenko und Natalia Golovanova (2001): »Local Government in the Russian Federation«. In: Victor Popa und Igor Muntaenu (Hg.), Developing New Rules in the Old Environment: Local Governments in Eastern Europe, Caucasus, and Central Asia, Budapest: Local Government Initiative of the Open Society Institute, S. 161-264.

Kronauer, Martin (2000): »Armut, Ausgrenzung, Unterklasse«. In: Hartmut Häußermann (Hg.), Großstadt. Soziologische Stichworte, Opladen: Leske + Budrich, S. 13-27.

Kugelman, Michael und Susan L. Levenstein (Hg.) (2009): Land Grab? The Race for the World's Farmland, Washington D.C.: Woodrow Wilson International Center for Scholars.

Kühl, Stefan (2007): »Formalität, Informalität und Illegalität in der Organisationsberatung. Systemtheoretische Analyse eines Beratungsprozesses«, in: Soziale Welt 58 (3), S. 271-293.

Kühl, Stefan (2011): Organisationen. Eine sehr kurze Einführung, Wiesbaden: VS Verlag für Sozialwissenschaften.

Kul'čyc'kyj, Stanislav (2004): »Terror als Methode. Der Hungergenozid in der Ukraine 1933«, in: Osteuropa 54 (12), S. 57-71.

Kusche, Isabel (2008): Politikberatung und die Herstellung von Entscheidungssicherheit im politischen System, Wiesbaden: VS Verlag für Sozialwissenschaften.

Lamnek, Siegfried (2005): Qualitative Sozialforschung, Weinheim: Beltz.

Lankina, Tomila V. (2003): »Federal, Regional Interests Shape Local Reforms «, in: Russian Regional Report 8 (18), S. 4-9.

Lankina, Tomila V. (2005): »President Putin's Local Government Reforms «. In: Peter Reddaway und Robert W. Orttung (Hg.), The Dynamics of Russian Politics: Putin's Reform of Federal-Regional Relations, Lanham: Rowman \& Littlefield, S. 145-177.

Le Grand, Julian (1991): »Quasi-Markets and Social Policy«, in: The Economic Journal 101 (408), S. 1256-1267.

Le Grand, Julian und Will Bartlett (Hg.) (1993): Quasi-markets and Social Policy, Basingstoke: Macmillan.

Ledeneva, Alena und Paul Seabright (2000): »Barter in post-Soviet societies: whwat does it look like and why does it matter?«. In: Paul Seabright (Hg.), The Vanishing Rouble. Barter Networks and Non-Monetary Transactions in PostSoviet Societies, Cambridge: Cambridge University Press, S.

Ledeneva, Alena V. (1998): Russia's Economy of Favours: Blat, Networking and Informal Exchange, Cambridge: Cambridge University Press. 
Ledeneva, Alena V. (1999): »Practices of Exchange and Networking in Russia«, in: Journal of Financial Crime 6 (3), S. 218-233.

Lee, Daniel B. (2007): »Observing Communication: Niklas Luhmann and the Problem of Ethnography«, in: Soziale Systeme 13 (1-2), S. 456-467.

Lee, Daniel B. und Achim Brosziewski (2007): »Participant Observation and Systems Theory: Theorizing the Ground«, in: Soziale Welt 58 (3), S. 255-269.

Lenin, Wladimir Iljitsch (1970 [1917]): Werke. Band 25, Berlin: Dietz.

Lenin, Wladimir Iljitsch (1970 [1920]): Werke. Band 31, Berlin: Dietz.

Lerman, Zvi (2001): »A Decade of land reform and farm restructuring: What

Russia can learn from the world experience«, in: Quarterly Journal of International Agriculture 40 (1), S. 5-28.

Lerman, Zvi (2002): »The Impact of Land Reform on the Rural Population«. In:

David J. O'Brien und Stephen K. Wegren (Hg.), Rural Reforms in Post-Soviet Russia, Baltimore: John Hopkins University Press, S. 42-67.

Lerman, Zvi und Natalya Shagaida (2007): »Land policies and agricultural land markets in Russia«, in: Land Use Policy 24 (1), S. 14-23.

Lessenich, Stephan (2008): Die Neuerfindung des Sozialen. Der Sozialstaat im flexiblen Kapitalismus, Bielefeld: transcript.

Lewis, Carol W. (1983): »The Economic Functions of Local Soviets«. In: Everett

M. Jacobs (Hg.), Soviet Local Politics and Government, London: George Allen \& Unwin, S. 48-66.

Lewytzkyi, Borys (1967): Die Kommunistische Partei der Sowjetunion. Porträt eines Ordens, Stuttgart: Ernst Klett Verlag.

Lindner, Peter (2003): »Kleinbäuerliche Landwirtschaft oder Kolchos-Archipel?

Der ländliche Raum in Russland zehn Jahre nach der Privatisierung der Kollektivbetriebe«, in: Geographische Rundschau 55 (12), S. 18-24.

Lindner, Peter (2007): »Localising Privatisation, Disconnecting Locales Mechanisms of Disintegration in Post-socialist Rural Russia«, in: Geoforum 38 (3), S. 494-504.

Lindner, Peter (2008): Der Kolchoz-Archipel im Privatisierungsprozess: Wege und Umwege der russischen Landwirtschaft in die globale Marktwirtschaft, Bielefeld: transcript.

Lindner, Peter und Evelyn Moser (2009a): »(De-)Centralizing Rural Russia: Local Self-Governance and the >Power Vertical««, in: Geographische Rundschau International Edition 5 (3), S. 12-18.

Lindner, Peter und Evelyn Moser (2009b): »Landwirtschaft und ländlicher Raum Der lange Weg von der Privatisierung zum Markt«, in: Russlandanalysen (178), S. 6-9.

Lindner, Peter und Alexander Vorbrugg (2011): »Die russische Landwirtschaft im Privatisierungsprozess: Vom Kolchos- zum Investorenarchipel?«, in: Russlandanalysen (229), S. 2-6. 
Lindner, Peter und Alexander Vorbrugg (2012): »Wiederkehr der Landfrage. Großinvestitionen in Russlands Landwirtschaft«, in: Osteuropa 62 (6-8), S. 325-342.

Linz, Juan J. und Alfred Stepan (1996): Problems of Democratic Transition and Consolidation: Southern Europe, South America, and Post-Communist Europe, Baltimore: John Hopkins University Press.

Lovering, John (2011): »The new regional governance and the hegemony of neoliberalism«. In: Andy Pike, Andrés Rodriguez-Pose und John Tomaney (Hg.), Handbook of Local and Regional Development, London: Routledge, S. 581-594.

Lüders, Christian (2008): »Beobachten im Feld und Ethnographie«. In: Uwe Flick, Ernst von Kardorff und Ines Steinke (Hg.), Qualitative Forschung: Ein Handbuch, Reinbek: Rowohlt, S. 384-401.

Luhmann, Niklas (1964): Funktionen und Folgen formaler Organisation, Berlin: Duncker \& Humblot.

Luhmann, Niklas (1984): Soziale Systeme. Grundriß einer allgemeinen Theorie, Frankfurt a.M.: Suhrkamp.

Luhmann, Niklas (1985): »Zum Begriff der sozialen Klasse«. In: Niklas Luhmann

(Hg.), Soziale Differenzierung, Opladen: Westdeutscher Verlag, S. 119-162.

Luhmann, Niklas (1988): Die Wirtschaft der Gesellschaft, Frankfurt a.M.: Suhrkamp.

Luhmann, Niklas (1989): »Politische Steuerung: Ein Diskussionsbeitrag«, in: Politische Vierteljahresschrift 30 (1), S. 4-9.

Luhmann, Niklas (1990): Die Wissenschaft der Gesellschaft, Frankfurt a.M.: Suhrkamp.

Luhmann, Niklas (1995): Die Kunst der Gesellschaft, Frankfurt a.M.: Suhrkamp.

Luhmann, Niklas (1995 [1984]): Social Systems, Stanford: Stanford University Press.

Luhmann, Niklas (1997): Die Gesellschaft der Gesellschaft, Frankfurt a.M.: Suhrkamp.

Luhmann, Niklas (2000a): Organisation und Entscheidung, Wiesbaden: Westdeutscher Verlag.

Luhmann, Niklas (2000b): Die Politik der Gesellschaft, Frankfurt a.M.: Suhrkamp.

Luhmann, Niklas (2003 [1975]): Macht, Stuttgart: Lucius \& Lucius.

Luhmann, Niklas (2004): Einführung in die Systemtheorie, Heidelberg: Carl-Auer.

Luhmann, Niklas (2007 [1971]): Politische Planung. Aufsätze zur Soziologie von

Politik und Verwaltung, Wiesbaden: VS Verlag für Sozialwissenschaften.

Luhmann, Niklas (2008): Die Moral der Gesellschaft, Frankfurt a.M.: Suhrkamp.

Luhmann, Niklas (2008 [1986]): Ökologische Kommunikation. Kann die moderne

Gesellschaft sich auf ökologische Gefährdungen einstellen?, Wiesbaden: VS

Verlag für Sozialwissenschaften. 
Luhmann, Niklas (2008 [1991]): »Die Form >Person««. In: Niklas Luhmann (Hg.), Soziologische Aufklärung 6. Die Soziologie und der Mensch, Wiesbaden: VS Verlag für Sozialwissenschaften, S. 137-148.

Luhmann, Niklas (2009): »Organisationen im Wirtschaftssystem«. In: Niklas Luhmann (Hg.), Soziologische Aufklärung 3. Soziales System, Gesellschaft, Organisation, Opladen: Westdeutscher Verlag, S. 451-478.

Luhmann, Niklas (2009 [1962]): »Funktion und Kausalität«. In: Niklas Luhmann (Hg.), Soziologische Aufklärung 1. Aufsätze zur Theorie sozialer Systeme, Wiesbaden: VS Verlag für Sozialwissenschaften, S. 11-38.

Luhmann, Niklas (2009 [1964]): »Funktionale Methode und Systemtheorie«. In: Niklas Luhmann (Hg.), Soziologische Aufklärung 1. Aufsätze zur Theorie sozialer Systeme, Wiesbaden: VS Verlag für Sozialwissenschaften, S. 39-67.

Luhmann, Niklas (2009 [1978]): »Organisation und Entscheidung«. In: Niklas Luhmann (Hg.), Soziologische Aufklärung 3. Soziales System, Gesellschaft, Organisation, Opladen: Westdeutscher Verlag, S. 389-450.

Luhmann, Niklas (2010): Politische Soziologie, Frankfurt a.M.: Suhrkamp.

Luhmann, Niklas (2012): Macht im System, Berlin: Suhrkamp.

Mählert, Ulrich (2002): »Die Massenorganisationen im politischen System der DDR «. In: Gerd-Rüdiger Stephan u.a. (Hg.), Die Parteien und Organisationen der DDR: Ein Handbuch, Berlin: Dietz, S. 103-115.

Mangott, Gerhard (2002): Zur Demokratisierung Russlands. Band 1: Russland als defekte Demokratie, Baden-Baden: Nomos.

Mark, Rudolf A. und Gerhard Simon (2004): »Die Hungersnot in der Ukraine und anderen Regionen der UdSSR 1932 und 1933«, in: Osteuropa 54 (12), S. 5-12.

Martinez-Vazquez, Jorge und Andrey Timofeev (2008): »Regional-local dimension of Russia's fiscal equalization«, in: Journal of Comparative Economics 36 (1), S. 157-176.

Marx, Karl und Friedrich Engels (1997 [1848]): Manifest der Kommunistischen Partei, Stuttgart: Reclam.

McKenzie, Kermit E. (1982): »Zemstvo organization and role within the administrative structure«. In: Terence Emmons und Wayne S. Vucinich (Hg.), The zemstvo in Russia. An experiment in local self-government, Cambridge: Cambridge University Press, S. 31-78.

Mehnert, Klaus (1971): Der Sowjetmensch: Versuch eines Porträts nach dreizehn Reisen in die Sowjetunion, 1929-1959, Stuttgart: Deutsche Verlagsanstalt.

Meissner, Boris (1965): Das Parteiprogramm der KPdSU 1903-1961, Köln: Verlag Wissenschaft und Politik.

Meissner, Boris (1985): Partei, Staat und Nation in der Sowjetunion: ausgewählte Beiträge, Berlin: Duncker \& Humblot. 
Melzer, Olaf (2008): »Der Europarat, Russland und Tschetschenien: Dialog ohne Konsequenzen?«. In: Martin Malek und Anna Schor-Tschudnowskaja (Hg.), Europa im Tschetschenienkrieg. Zwischen politischer Ohnmacht und Gleichgültigkeit, Stuttgart: Ibidem-Verlag, S. 297-339.

Melzer, Olaf (2012): Der Europarat und Russland 1992-2006, Wiesbaden: VS Verlag für Sozialwissenschaften.

Merkel, Wolfgang (2010): Systemtransformation. Eine Einführung in die Theorie und Empirie der Transformationsforschung, Wiesbaden: VS Verlag für Sozialwissenschaften.

Merkel, Wolfgang et al. (2003): Defekte Demokratien. Band 1: Theorien und Probleme, Opladen: Leske + Budrich.

Merl, Stephan (1985): Die Anfänge der Kollektivierung in der Sowjetunion. Der Übergang zur staatlichen Reglementierung der Produktions- und Marktbeziehungen im Dorf (1928-1930) (= Veröffentlichungen des Osteuropa-Institutes München: Reihe Geschichte), Wiesbaden: Otto Harrassowitz.

Merl, Stephan (1990): Bauern unter Stalin: Die Formierung des sowjetischen Kolchossystems 1930-1941 (= Osteuropastudien der Hochschulen des Landes Hessen), Berlin: Duncker \& Humblot.

Merton, Robert K. (1995 [1949]): »Manifeste und latente Funktionen«. In: Robert K. Merton (Hg.), Soziologische Theorie und soziale Struktur, Berlin: de Gruyter, S. 17-81.

Meuschel, Sigrid (1992): Legitimation und Parteiherrschaft. Zum Paradox von Stabilität und Revolution in der DDR 1945-1989, Frankfurt a.M.: Suhrkamp.

Meuschel, Sigrid (1993): »Überlegungen zu einer Herrschafts- und Gesellschaftsgeschichte der DDR«, in: Geschichte und Gesellschaft: Zeitschrift für historische Sozialwissenschaft 19 (1), S. 5-14.

Meyer, John W., John Boli und George M. Thomas (2005 [1987]): »Ontologie und Rationalisierung im Zurechnungssystem der westlichen Kultur«. In: John W. Meyer (Hg.), Weltkultur. Wie die westlichen Prinzipien die Welt durchdringen, Frankfurt a.M.: Suhrkamp, S. 17-46.

Meyer, John W. et al. (2005 [1997]): »Die Weltgesellschaft und der Nationalstaat«. In: John W. Meyer (Hg.), Weltkultur. Wie die westlichen Prinzipien die Welt durchdringen, Frankfurt a.M.: Suhrkamp, S. 85-123.

Meyer, John W. und Ronald L. Jepperson (2005 [2000]): »Die >Akteure< der modernen Gesellschaft: Die kulturelle Konstruktion sozialer Agentschaft«. In: John W. Meyer (Hg.), Weltkultur. Wie die westlichen Prinzipien die Welt durchdringen, Frankfurt a.M.: Suhrkamp, S. 47-84.

Mill, John Stuart (2010 [1861]): Considerations on representative government, New York: Cambridge University Press.

Milov, Vladimir (2008): Russia and the West: The Energy Factor, Working Paper Juli 2008, Washington D.C.: Center for Strategic and International Studies (CSIS) und Institut Francais des Relations Internationales (IFRI). 
Milsum, J. H. (1968): »The Technosphere, the Biosphere, the Sociosphere. Their Systems Modeling and Optimization«, in: IEEE Spectrum 5 (6), S. 76-82.

Miraftab, Faranak (2004): »Making Neo-liberal Governance: The Disempowering Work of Empowerment«, in: International Planning Studies 9 (4), S. 239-259.

Miraftab, Faranak (2008): »Decentralization and entrepreneurial planning «. In: Victoria A. Beard, Faranak Miraftab und Christopher Silver (Hg.), Planning and Decentralization: Contested Spaces for Public Action in the Global South, New York: Routledge, S. 21-35.

Mohan, Giles und Kristian Stokke (2000): »Participatory development and empowerment: the dangers of localism«, in: Third World Quarterly 21 (2), S. 247268.

Mohan, Giles und Kristian Stokke (2008): »The Politics of Localization: From Depoliticizing Development to Politicizing Democracy«. In: Kevin R. Cox, Murray Low und Jennifer Robinson (Hg.), The SAGE Handbook of Political Geography, London: Sage, S. 545-562.

Mommsen, Margareta (2013): »Paradigmenwechsel. 60 Jahre politikwissenschaftliche Osteuropaforschung«, in: Osteuropa 63 (2-3), S. 119-136.

Moser, Evelyn und Peter Lindner (2011): »Dezentralisierung im Zeichen der Machtvertikale: Paradoxien der Einführung einer lokalen Selbstverwaltung im ländlichen Russland«, in: Geographische Rundschau 63 (1), S. 28-35.

Müller, Klaus (2001): »Post-Washingtoner Consensus und Comprehensive Development Framework. Neue Perspektiven für Transformationsforschung und Transformationstheorie«, in: Berliner Osteuropa-Info (16), S. 5-13.

Musgrave, Richard A. (1959): The Theory of Public Finance. A Study in Public Economy, New York: McGraw-Hill.

Nassehi, Armin (1997): »Kommunikation verstehen. Einige Überlegungen zur empirischen Anwendbarkeit einer systemtheoretisch informierten Hermeneutik«. In: Tilmann Sutter (Hg.), Beobachtung verstehen, Verstehen beobachten. Perpektiven einer konstruktivistischen Hermeneutik, Opladen: Westdeutscher Verlag, S. 134-163.

Nassehi, Armin (2004): »Die Theorie funktionaler Differenzierung im Horizont ihrer Kritik«, in: Zeitschrift für Soziologie 33 (2), S. 98-118.

Nassehi, Armin (2008): »Rethinking Functionalism. Zur Empiriefähigkeit systemtheoretischer Soziologie«. In: Herbert Kalthoff, Stefan Hirschauer und Gesa Lindemann (Hg.), Theoretische Empirie. Zur Relevanz qualitativer Forschung, Frankfurt a.M.: Suhrkamp, S. 79-106.

Nassehi, Armin (2012): »Soziologie«. In: Oliver Jahraus u.a. (Hg.), LuhmannHandbuch. Leben - Werk - Wirkung, Stuttgart: J.B. Metzler, S. 399-407.

Nassehi, Armin und Irmhild Saake (2002): »Kontingenz: Methodisch verhindert oder beobachtet? «, in: Zeitschrift für Soziologie 31 (1), S. 66-86.

Nestmann, Thorsten (2007): Russland: Der Bär ist momentan in voller Fahrt, Aktueller Kommentar, 17.12.2007, Frankfurt a.M.: Deutsche Bank Research. 
Nikulin, Aleksandr (2005): »Novejshaja gigantomanija [Neue Gigantomanie]«, in: Politicheskij Zhurnal [Politisches Journal] 63 (12), S.

Nikulin, Aleksandr (2010): »Oligarchoz kak preemnik postkolchoza [Der Oligarchos als Nachfolger des Postkolchos]«, in: Ekonomičeskaja Sociologija [Wirtschaftssoziologie] 11 (1), S. 17-33.

Noël, Pierre (2008): Beyond Dependence: How to Deal With Russian Gas, Policy Brief ECFR/09, November 2008, London: European Council on Foreign Relations (ECFR).

Nohl, Arnd-Michael (2009): Interview und dokumentarische Methode: Anleitungen für die Forschungspraxis, Wiesbaden: VS Verlag für Sozialwissenschaften.

Norsworthy, L. Alexander und Olga Paluba (2000): »Impact of the Transition: Approaches and Findings«. In: L. Alexander Norsworthy (Hg.), Russian Views of the Transition in the Rural Sector: Structures, Policy Outcomes, and Adaptive Responses, Washington D.C.: The World Bank, S. 1-15.

Nove, Alec (1980): The Soviet Economic System, London: George Allen \& Unwin.

O'Brien, David J. und Valeri V. Patsiorkovski (2006): Measuring Social and Economic Change in Rural Russia. Surveys from 1991 to 2003, Lanham, Boulder, New York, Oxford: Lexington Books.

O’Brien, David J. und Valeri V. Patsiorkovski (2006): Measuring Social and Economic Change in Rural Russia. Surveys from 1991 to 2003, Lanham: Lexington Books.

O’Brien, David J., Stephen K. Wegren und Valeri V. Patsiorkovski (2007): »Income Stratification in Russian Villages: From Profession to Property«, in: Problems of Post-Communism 54 (1), S. 37-46.

O’Donnell, Guillermo und Philippe C. Schmitter (1991): Transitions from Authoritarian Rule. Tentative Conclusions About Uncertain Democracies, Baltimore: John Hopkins University Press.

Oates, Wallace E. (1972): Fiscal Federalism, New York: Harcourt Brace Jovanovic. Oates, Wallace E. (1981): »On Local Finance and the Tiebout Model«, in: The American Economic Review 71 (2), S. 93-98.

Oates, Wallace E. (1999): »An Essay on Fiscal Federalism«, in: Journal of Economic Literature 37 (3), S. 1120-1149.

OECD (2011): Ten Years of Water Sector Reform in Eastern Europe, Caucasus and Central Asia, OECD Studies on Water, Paris: OECD.

Offe, Claus (2007): »Staat, Demokratie und Krieg«. In: Hans Joas (Hg.), Lehrbuch der Soziologie, Frankfurt a.M.: Campus, S. 505-540.

Olson, Mancur (1969): »The Principle of >Fiscal Equivalence<: The Division of Responsibilities among Different Levels of Government«, in: The American Economic Review 59 (2), S. 479-487.

Olson, Mancur (2000): Power and Prosperity: Outgrowing Communist and Capitalist Dictatorships, New York: Basic Books. 
Osteuropa-Archiv (1989): »Zur Lage in der sowjetischen Landwirtschaft«, in: Osteuropa (Osteuropa-Archiv) 39 (6), S. A 266-A 289.

Ostrom, Elinor (1990): Governing the Commons: The Evolution of Institutions for Collective Action, Cambridge: Cambridge University Press.

Ostrom, Elinor (1999): Die Verfassung der Allmende: Jenseits von Staat und Markt, Tübingen: Mohr.

Oswald, Ingrid (2007): »>Industrialized Villages < in Post-Socialist Countries Steps towards an Integrative Research Concept«. In: Maarit Heinonen u.a. (Hg.), Reflecting Transformation in Post-Socialist Rural Areas, Newcastle: Cambridge Scholars Publishing, S. 215-235.

Oswald, Ingrid, Frank Ernst und Eckart Dittrich (2005): »Das industrialisierte Dorf: Zur Transformation ländlicher Lebensweisen in postsozialistischen Gesellschaften«. In: Raj Kollmorgen (Hg.), Transformation als Typ sozialen Wandels. Postsozialistische Lektionen, historische und interkulturelle Vergleiche, Münster: Lit, S. 241-257.

Oyugi, Walter O. (2000): »Editorial Introduction: Decentralization for Good Governance and Development. The Unending Debate«, in: Regional Development Dialogue 21 (1), S. iii-xix.

Palla, Gergely et al. (2005): »Uncovering the overlapping community structure of complex networks in nature and society«, in: Nature 435, S. 814-818.

Patsiorkovski, Valeri V. (2002): »Rural Household Behavior, 1991-2001«. In: David J. O’Brien und Stephen K. Wegren (Hg.), Rural Reforms in Post-Soviet Russia, Baltimore: John Hopkins University Press, S. 116-134.

Pelizzari, Alessandro (2001): Die Ökonomisierung des Politischen: New Public Management und der neoliberale Angriff auf die öffentlichen Dienste, Konstanz: UVK.

Pender, John (2001): »From >Structural Adjustment< to >Comprehensive Development Framework $<$ conditionality transformed?«, in: Third World Quarterly 22 (3), S. 397-411.

Perovic, Jeronim (2001): Die Regionen Russlands als neue politische Kraft. Chancen und Gefahren des Regionalismus für Russland, Bern: Peter Lang.

Pfeffer, Jeffrey und Gerald R. Salancik (2003 [1978]): The External Control of Organizations. A Resource Dependence Perspective, Stanford: Stanford University Press.

Phillips, Anne (1996): »Why does Local Democracy Matter?«. In: Lawrence Pratchett und David Wilson (Hg.), Local Democracy and Local Government, New York: St. Martin's Press, S. 20-37.

Pike, Andy und John Tomaney (2004): »Subnational governance and economic and social development«, in: Environment and Planning A 36 (12), S. 2091-2096.

Polanyi, Karl (1978 [1944]): The Great Transformation. Politische und ökonomische Ursprünge von Gesellschaften und Wirtschaftssystemen, Frankfurt a.M.: Suhrkamp. 
Pollack, Detlef (1990): »Das Ende einer Organisationsgesellschaft. Systemtheoretische Überlegungen zum gesellschaftlichen Umbruch in der DDR«, in: Zeitschrift für Soziologie 19 (4), S. 292-307.

Pollack, Detlef (1994): Kirche in der Organisationsgesellschaft: Zum Wandel der gesellschaftlichen Lage in der evangelischen Kirche in der DDR, Stuttgart: Kohlhammer.

Pollack, Detlef (1998): »Die konstitutive Widersprüchlichkeit der DDR. Oder: War die DDR-Gesellschaft homogen?«, in: Geschichte und Gesellschaft: Zeitschrift für historische Sozialwissenschaft 24 (1), S. 110-131.

Pollack, Detlef (2000): »Die offene Gesellschaft und ihre Freunde«, in: Geschichte und Gesellschaft: Zeitschrift für historische Sozialwissenschaft 26 (1), S. 184196.

Pollack, Detlef (2003): »Auf dem Weg zu einer Theorie des Staatssozialismus«, in: Historical Social Research 28 (1-2), S. 10-30.

Popov, Vladimir (2000): »Shock Therapy Versus Gradualism: The End Of The Debate (Explaining The Magnitude Of Transformational Recession)«, in: Comparative Economic Studies 42 (1), S. 1-57.

Porter, Thomas Earl und Scott Seregny (2004): »The Zemstvo Reconsidered«. In: Alfred B. Evans und Vladimir Gelman (Hg.), The Politics of Local Government in Russia, Lanham: Rowman \& Littlefield, S. 19-44.

Pratchett, Lawrence (2004): »Local Autonomy, Local Democracy and the $>$ New Localism««, in: Political Studies 52 (2), S. 358-375.

Prosterman, Roy L., Robert G. Mitchell und J. Rorem Bradley (1997): »Prospects for Family Farming in Russia«, in: Europe-Asia Studies 49 (8), S. 1383-1407.

Przeworski, Adam (1991): Democracy and the Market: Political and Economic Reforms in Eastern Europe and Latin America, Cambridge: Cambridge University Press.

Przyborski, Aglaja und Monika Wohlrab-Sahr (2009): Qualitative Sozialforschung. Ein Arbeitsbuch, München: Oldenbourg.

Reynolds, Sarah J. (2005): »Editor's Introduction: School for Democracy: What Will Be Learned in the Reform of Local Government in the Russian Federation?«, in: Statutes and Decisions: The Laws of the USSR and its Successor States 41 (5), S. 3-7.

RIA Novosti (2008): »Russlands Gasifizierung: Präsident droht nachlässigen Gouverneuren mit Strafen«, RIA Novosti: http://de.rian.ru/russia/20080721/ 114537019.html vom 31.3.2013.

Richter, Peter (2009): Ökonomisierung als gesellschaftliche Entdifferenzierung. Eine Soziologie zum Wandel des öffentlichen Sektors, Konstanz: UVK.

Richter, Peter (2012): »Die Organisation öffentlicher Verwaltung«. In: Maja Apelt und Veronika Tacke (Hg.), Handbuch Organisationstypen, Wiesbaden: Springer VS, S. 91-112. 
Rodden, Jonathan (2002): »The Dilemma of Fiscal Federalism: Grants and Fiscal Performance around the World«, in: American Journal of Political Science 46 (3), S. 670-687.

Rodden, Jonathan (2004): »Comparative Federalism and Decentralization: On Meaning and Measurement «, in: Comparative Politics 36 (4), S. 481-500.

Rodríguez-Pose, Andrés und Nicholas Gill (2003): »The global trend towards devolution and its implications«, in: Environment and Planning C: Government and Policy 21 (3), S. 333-351.

Rodrik, Dani (2000): Institutions for high-quality growth: What they are and how to acquire them, NBER Working Paper 7540, Cambridge, MA: National Bureau of Economic Research.

Rodríquez-Pose, Andrés und Richard Sandall (2008): »From identity to the economy: analysing the evolution of the decentralisation discourse Environment and Planning C: Government and Policy 26 (1), S. 54-72.

Roland, Gerard (2002): »The Political Economy of Transition«, in: The Journal of Economic Perspectives 16 (1), S. 29-50.

Rondinelli, Dennis A. (1981): »Government Decentralisation in Comparative Perspective: Theory and Practice in Developing Countries«, in: International Review of Administrative Science 47 (2), S. 133-145.

Rondinelli, Dennis A., James S. McCullough und Ronald W. Johnson (1989): $\gg$ Analysing Decentralization Policies in Developing-Countries - a PoliticalEconomy Framework«, in: Development and Change 20 (1), S. 57-87.

Rondinelli, Dennis A., John R. Nellis und G. Shabbir Cheema (1983): Decentralization in Developing Countries. A Review of Recent Experience, World Bank Staff Working Papers 581, Washington D.C.: The World Bank.

Rose, Nikolas und Peter Miller (1992): »Political power beyond the State: problematics of government«, in: The British Journal of Sociology 43 (2), S. 173-205.

Rose, Richard (2000): »Uses of social capital in Russia: modern, pre-modern, and anti-modern«, in: Post-Soviet Affairs 16 (1), S. 33-57.

Ross, Cameron (1987): Local Government in the Soviet Union, Kent: Croom Helm Ltd.

Ross, Cameron (2006): »The Tortuous Path of Local Government Reform in the Russian Federation «, in: Local Government Studies 32 (5), S. 639-658.

Ross, Cameron (2009): Local Politics and Democratization in Russia, New York: Routledge.

Rossijskaja Gazeta (2003): »Mesto mestnoj vlasti. Zamestitel' rukovoditelja administracii Prezidenta RF Dmitrij Kozak o reforme mestnogo samoupravlenija [Der Ort der lokalen Macht. Der stellvertretende Verwaltungschef des Präsidenten der Russischen Föderation Dmitrij Kozak über die Reform kommunaler Selbstverwaltung]«. In: Rossijskaja Gazeta 3176, 30.9.2003. S. 1 . 
Rossijskaja Gazeta (2005 [2003]): »The Place of Local Power. Interview with Dmitry Kozak«, in: Statutes and Decisions: The Laws of the USSR and its Successor States 41 (5), S. 8-16.

Rosstat (2006): Maloe i srednee predprinimatel'stvo v Rossii - 2006g. [Kleine und mittlere Unternehmen in Russland - 2006], Moskau: Rosstat [Föderaler statistischer Dienst der Russischen Föderation].

Rosstat (2012): Russia in Figures, Moskau: Rosstat [Föderaler statistischer Dienst der Russischen Föderation].

Rozelle, Scott und Johan F. M. Swinnen (2004): »Success and Failure of Reform: Insights from the Transition of Agriculture«, in: Journal of Economic Literature 42 (2), S. 404-456.

Ryl'ko, Dmitri (2002): »New Operators in Russian Agriculture «, in: Russian Politics and Law 40 (2), S. 72-84.

Rylko, Dmitri und Robert W. Jolly (2005): »Russia's New Agricultural Operators: Their Emergence, Growth and Impact«, in: Comparative Economic Studies 47 (1), S. 115-126.

Sachs, Jeffrey D. (1996): »The Transition at Mid Decade«, in: The American Economic Review 86 (2), S. 128-133.

Sachs, Jeffrey D., Wing Thye Woo und Xiaokai Yang (2000): Economic Reforms and Constitutional Transition, CID Working Paper 43, Harvard: Center for International Development at Harvard University (CID).

Sakwa, Richard (1998): Soviet Politics in Perspective, London: Routledge.

Sapper, Manfred (2012): »Niedergang und Neuanfang. Die Krise der deutschen Russlandexpertise«, in: Osteuropa 62 (6-8), S. 505-520.

Sartori, Giovanni (1991): »Rethinking democracy: bad polity and bad politics«, in: International Social Science Journal (129), S. 437-450.

Schedler, Kuno und Isabella Proeller (2009): New Public Management, Bern: Haupt.

Schimank, Uwe und Ute Volkmann (2008): »Ökonomisierung der Gesellschaft«. In: Andrea Maurer (Hg.), Handbuch der Wirtschaftssoziologie, Wiesbaden: VS Verlag für Sozialwissenschaften, S. 382-393.

Schinke, Eberhard (1989): Reformen in der sowjetischen Landwirtschaft (= Berichte des Bundesinstituts für ostwissenschaftliche und internationale Studien), Köln: Bundesinstitut für ostwissenschaftliche und internationale Studien.

Schinke, Eberhard und Karl-Eugen Wädekin (1985): Die sowjetische Landwirtschaft an der Wende zum 12. Planjahrfünft: Produktion, Verbrauch, Außenwirtschaft (= Berichte des Bundesinstituts für ostwissenschaftliche und internationale Studien), Köln: Bundesinstitut für ostwissenschaftliche und internationale Studien.

Schmidt, Manfred G. (2010): Demokratietheorien. Eine Einführung, Wiesbaden: VS Verlag für Sozialwissenschaften. 
Schneider, Eberhard (2001): Das politische System der Russischen Föderation. Eine Einführung., Wiesbaden: Westdeutscher Verlag.

Schneider, Hans K. (1992): »Tempo und Schrittfolge des Transformationsprozesses«. In: Bernhard Gahlen, Helmut Hesse und Hans J. Ramser (Hg.), Von der Plan- zur Marktwirtschaft. Eine Zwischenbilanz, Tübingen: Mohr, S. 8-21.

Schneider, Wolfgang Ludwig (1994): Die Beobachtung von Kommunikation. Zur kommunikativen Konstruktion sozialen Handelns, Opladen: Westdeutscher Verlag.

Schouten, Greetje, Pieter Leroy und Pieter Glasbergen (2012): »On the deliberative capacity of private multi-stakeholder governance: The Roundtables on Responsible Soy and Sustainable Palm Oil«, in: Ecological Economics 83, S. 42-50.

Schröder, Hans-Henning (2003): »Politisches System und politischer Prozess «, in: Informationen zur politischen Bildung (281), S. 16-23.

Schultheis, Franz (2012): »Im Dienste öffentlicher Güter«, in: Mittelweg 3621 (5), S. 9-21.

Scott, James C. (1998): Seeing Like a State. How Certain Schemes to Improve the Human Condition Have Failed., New Haven: Yale University Press.

Seabright, Paul (Hg.) (2000): The Vanishing Rouble. Barter Networks and NonMonetary Transactions in Post-Soviet Societies, Cambridge: Cambridge University Press.

Segbers, Klaus (2013): »Fehlgedeutete Mängelliste. Zur angeblichen Krise der Russlandexpertise«, in: Osteuropa 63 (1), S. 171-172.

Selowsky, Marcelo und Ricardo Martin (1997): »Policy Performance and Output Growth in the Transition Economies«, in: The American Economic Review 87 (2), S. 349-353.

Selzer, Dmitrij G. (2009): »Wer regiert? Die Transformation der kommunalen politischen Regimes in Russland 1991-2009. Eine Elitenstudie«, in: Russlandanalysen (191), S. 6-9.

Selznick, Philip (1952): The Organizational Weapon. A Study of Bolshevik Strategy and Tactics, New York: McGraw-Hill.

Shagaida, Natalya (2005): »Agricultural Land Market in Russia: Living with Constraints«, in: Comparative Economic Studies 47 (1), S. 127-140.

Shah, Anwar (2006a): »A Comparative Institutional Framework for Responsive, Responsible, and Accountable Local Governance«. In: Anwar Shah (Hg.), Local Governance in Industrial Countries, Washington D.C.: World Bank Publications, S. 1-39.

Shah, Anwar (Hg.) (2006b): Local Governance in Developing Countries (= Public Sector Governance and Accountability Series), Washington D.C.: The World Bank. 
Shah, Anwar (2006c): »The New Vision of Local Governance and the Evolving Role of Local Governments«. In: Anwar Shah (Hg.), Local Governance in Developing Countries, Washington D.C.: World Bank Publications, S. 1-46.

Shah, Anwar (2007a): »A Comparative Institutional Framework for Responsive, Responsible, and Accountable Local Governance«. In: The World Bank (Hg.), Local Governance and Civic Engagement in Rural Russia. A World Bank Project, Moskau: The World Bank, S. 10-15.

Shah, Anwar (Hg.) (2007b): Local Budgeting (= Public Sector Governance and Accountability Series), Washington D.C.: The World Bank.

Shah, Anwar (Hg.) (2007c): Participatory Budgeting (= Public Sector Governance and Accountability Series), Washington D.C.: The World Bank.

Sharman, Jason C. (1998): »Vorhersage und Vergleich. Zur Osteuropaforschung in den USA , in: Osteuropa 48 (8-9), S. 821-836.

Shipov, Vitalii (2003): »Foreword: Perspectives on the Development of Local SelfGovernance«. In: The World Bank (Hg.), Collection of Articles: Local SelfGovernment and Civic Engagement in Rural Russia, Washington D.C.: The World Bank, S. 6-7.

Shubin, Sergej (2007): »Networked Poverty in Rural Russia«, in: Europe-Asia Studies 59 (4), S. 591-620.

Simis, Konstantin M. (1982): USSR: The Corrupt Society, New York: Simon \& Schuster.

Simmel, Georg (1989 [1900]): Philosophie des Geldes. Gesamtausgabe, Band 6, Frankfurt a.M.: Suhrkamp.

Simon, Gerhard (2004): »Holodomor als Waffe. Stalinismus, Hunger und der ukrainische Nationalismus«, in: Osteuropa 54 (12), S. 37-56.

Sisk, Timothy D. (Hg.) (2001): Democracy at the Local Level: The International IDEA Handbook on Participation, Representation, Conflict Management and Governance, Stockholm: International IDEA.

Slater, David (1989): »Territorial Power and the Peripheral State: The Issue of Decentralization«, in: Development and Change 20 (3), S. 501-531.

Solga, Heike (1994): »>Systemloyalität< als Bedingung sozialer Mobilität im Staatssozialismus, am Beispiel der DDR , in: Berliner Journal für Soziologie 4 (4), S. 523-542.

Soós, Gábor und Violetta Zentai (2005): »Introduction«. In: Gábor Soós und Violetta Zentai (Hg.), Faces of Local Democracy: Comparative Papers from Central and Eastern Europe, Budapest: Local Government Initiative and Public Service Reform Initiative, Open Society Institute, S. 1-12.

Sørensen, Eva und Jacob Torfing (2005): »Network Governance and Post-liberal Democracy«, in: Administrative Theory \& Praxis 27 (2), S. 197-237.

Sovetskaja sociologiceskaja associacija [Sowjetischer Soziologenverband] (1989 [1988]): »Professional'nyj kodeks sociologa [Der Berufskodex des Soziologen]«, in: Osteuropa (Osteuropa-Archiv) 39 (5), S. A 242-A 243. 
Spoor, Max und Oane Visser (2004): »Restructuring Postponed? Large Russian Farm Enterprises >Coping with the Market««, in: Journal of Peasant Studies 31 (3), S. 515-551.

Staehr, Karsten (2003): Reforms and economic growth in transition economies: Complementarity, sequencing and speed, BOFIT Discussion Papers 2003, 1, Helsinki: Bank of Finland, Institute for Economies in Transition (BOFIT).

Stark, David (1996): »Recombinant Property in East European Capitalism«, in: The American Journal of Sociology 101 (4), S. 993-1027.

Stark, David und László Bruszt (1998): Postsocialist Pathways: Transforming Politics and Property in East Central Europe, Cambridge: Cambridge University Press.

Stark, David und László Bruszt (2001): »One Way or Multiple Paths: For a Comparative Sociology of East European Capitalism«, in: The American Journal of Sociology 106 (4), S. 1129-1137.

Stark, David und Balazs Vedres (2006): »Social Times of Network Spaces: Network Sequences and Foreign Investment in Hungary«, in: The American Journal of Sociology 111 (5), S. 1367-1411.

Steimann, Bernd (2010): Making a Living in Uncertainty. Agro-Pastoral Livelihoods and Institutional Transformations in Post-Socialist Rural Kyrgyzstan (= Schriftenreihe Humangeographie; 26), Zürich: Geographisches Institut, Universität Zürich.

Steinbrink, Malte et al. (2012): »Netzwerk(analys)e in der deutschen Humangeographie«, in: Berichte zur deutschen Landeskunde 86 (4), S. 309-358.

Stephan, Gerd-Rüdiger et al. (Hg.) (2002): Die Parteien und Organisationen der DDR: Ein Handbuch, Berlin: Dietz.

Stichweh, Rudolf (1995): »Zur Theorie der Weltgesellschaft«, in: Soziale Systeme 1 (1), S. 29-45.

Stichweh, Rudolf (2000a): »Adresse und Lokalisierung in einem globalen Kommunikationssystem«. In: Rudolf Stichweh (Hg.), Die Weltgesellschaft, Frankfurt a.M.: Suhrkamp, S. 220-231.

Stichweh, Rudolf (2000b): »Soziologie des Vereins. Strukturbildung zwischen Lokalität und Globalität«. In: Emil Brix und Rudolf Richter (Hg.), Organisierte Privatinteressen. Vereine in Österreich, Wien: Passagen, S. 19-31.

Stichweh, Rudolf (2000c): »Systemtheorie der Exklusion. Zum Konflikt von Wohlfahrtsstaatlichkeit und Globalisierung der Funktionssysteme«. In: Rudolf Stichweh (Hg.), Die Weltgesellschaft, Frankfurt a.M.: Suhrkamp, S. 85-102.

Stichweh, Rudolf (2000 [1999]): »Zur Genese der Weltgesellschaft. Innovation und Mechanismen«. In: Rudolf Stichweh (Hg.), Die Weltgesellschaft, Frankfurt a.M.: Suhrkamp, S. 245-267. 
Stichweh, Rudolf (2003): »Raum und modeme Gesellschaft. Aspekte der sozialen Kontrolle des Raums«. In: Thomas Krämer-Badoni und Klaus Kuhm (Hg.), Die Gesellschaft und ihr Raum. Raum als Gegenstand der Soziologie, Opladen: Leske + Budrich, S. 93-102.

Stichweh, Rudolf (2005): Inklusion und Exklusion. Studien zur Gesellschaftstheorie, Bielefeld: transcript.

Stichweh, Rudolf (2005 [1988]): »Inklusion in Funktionssysteme der modernen Gesellschaft«. In: Rudolf Stichweh (Hg.), Inklusion und Exklusion. Studien zur Gesellschaftstheorie, Bielefeld: transcript, S. 13-44.

Stichweh, Rudolf (2005 [1998]): »Zur Theorie der politischen Inklusion«. In: Rudolf Stichweh (Hg.), Inklusion und Exklusion. Studien zur Gesellschaftstheorie, Bielefeld: transcript, S. 67-81.

Stichweh, Rudolf (2006): »Strukturbildung in der Weltgesellschaft - Die Eigenstrukturen der Weltgesellschaft und die Regionalkulturen der Welt«. In: Thomas Schwinn (Hg.), Die Vielfalt und Einheit der Moderne. Kultur- und strukturvergleichende Analysen, Wiesbaden: VS Verlag für Sozialwissenschaften, S. 239-258.

Stichweh, Rudolf (2008): »Kontrolle und Organisation des Raumes durch Funktionssysteme der Weltgesellschaft«. In: Jörg Döring und Tristan Thielmann (Hg.), Spatial Turn. Das Raumparadigma in den Kultur- und Sozialwissenschaften, Bielefeld: transcript, S. 149-164.

Stichweh, Rudolf (2009a): »Autonomie der Universitäten in Europa und Nordamerika: Historische und systematische Überlegungen«. In: Jürgen Kaube (Hg.), Die Illusion der Exzellenz. Lebenslügen der Wissenschaftspolitik, Berlin: Wagenbach, S. 38-49.

Stichweh, Rudolf (2009b): Das Konzept der Weltgesellschaft. Genese und Strukturbildung eines globalen Gesellschaftssystems, Workingpaper WB 01/09, Luzern: Soziologisches Seminar der Universität Luzern.

Stichweh, Rudolf (2009c): »Leitgesichtspunkte einer Soziologie der Inklusion und Exklusion«. In: Rudolf Stichweh und Paul Windolf (Hg.), Inklusion und Exklusion: Analysen zur Sozialstruktur und sozialen Ungleichheit, Wiesbaden: VS Verlag für Sozialwissenschaften, S. 29-42.

Stichweh, Rudolf (2010): »Theorie und Methode in der Systemtheorie«. In: René John, Anna Henkel und Jana Rückert-John (Hg.), Die Methodologien des Systems. Wie kommt man zum Fall und wie dahinter?, Wiesbaden: VS Verlag für Sozialwissenschaften, S. 15-28.

Stichweh, Rudolf (2012): Regionale Diversifikation und funktionale Differenzierung der Weltgesellschaft. Zum Arbeitsprogramm des >Forum Internationale Wissenschaft Bonn $<$ Eröffnung des >Forum Internationale Wissenschaft $<$ der Universität Bonn, 13.11.2012, Bonn.

Stiglitz, Joseph (2002): Die Schatten der Globalisierung, Berlin: Siedler Verlag. 
Stoker, Gerry (1996): »Redefining Local Democracy«. In: Lawrence Pratchett und David Wilson (Hg.), Local Democracy and Local Government, New York: St. Martin's Press, S. 188-209.

Stoker, Gerry (2006): Why Politics Matters. Making Democracy Work, Houndmills: Palgrave Macmillan.

Stökl, Günther (1997): Russische Geschichte, Stuttgart: Kröner.

Sturgeon, Janet C. und Thomas Sikor (2004): »Post-socialist Property in Europe and Asia: Variations on >Fuzziness««, in: Conservation and Society 1 (2), S. 117.

Tacke, Veronika (2001): »Funktionale Differenzierung als Schema der Beobachtung von Organisationen. Zum theoretischen Problem und empirischen Wert von Organisationstypologien«. In: Veronika Tacke (Hg.), Organisation und gesellschaftliche Differenzierung, Wiesbaden: Westdeutscher Verlag, S. 141-169.

Tacke, Veronika (2009): »Differenzierung und/oder Vernetzung? Über Spannungen, Annäherungspotentiale und systemtheoretische Fortsetzungsmöglichkeiten der Netzwerkdiskussion«, in: Soziale Systeme 15 (2), S. 243-270.

Tacke, Veronika (2011): »Systeme und Netzwerke - oder: Was man an sozialen Netzwerken zu sehen bekommt, wenn man sie systemtheoretisch beschreibt«, in: Journal der dgssa (Deutsche Gesellschaft für Systemische Soziale Arbeit) 2 (2-3), S. 6-24.

Tacke, Veronika (2015): »Formalität und Informalität. Zu einer klassischen Unterscheidung der Organisationssoziologie«. In: Victoria von Groddeck und Sylvia Marlene Wilz (Hg.), Formalität und Informalität in Organisationen, Wiesbaden: Springer VS, S. 37-92.

Teubner, Gunther (1992): »Die vielköpfige Hydra: Netzwerke als kollektive Akteure höherer Ordnung«. In: Wolfgang Krohn und Günter Küppers (Hg.), Emergenz: Die Entstehung von Ordnung, Organisation und Bedeutung, Frankfurt a.M.: Suhrkamp, S. 189-216.

The World Bank (1992): Food and Agricultural Policy Reforms in the Former USSR: An Agenda for the Transition, Studies in Economies in Transformation 1, Washington D.C.: The World Bank.

The World Bank (1997): From plan to market. World Development Report 1997, Washington D.C.: Oxford University Press for the World Bank.

The World Bank (Hg.) (2003): Collection of Articles: Local Self Government and Civic Engagement in Rural Russia, Moskau: The World Bank.

The World Bank (Hg.) (2007a): Local Governance and Civic Engagement in Rural Russia: A World Bank Project, Moskau: The World Bank und CAF Russia.

The World Bank (2007b): Local Government Discretion and Accountability: A Local Governance Framework, Report 40153, Juni 2007, Washington D.C.: The World Bank, Social Development Department in collaboration with the Finance, Economics and Urban Department (FEU) and the Social Protection Team (HDNSP). 
Thom, Norbert und Adrian Ritz (2008): Public Management: innovative Konzepte zur Führung im öffentlichen Sektor, Wiesbaden: Gabler Verlag.

Thompson Manning, Roberta (1982): »The zemstvo and politics, 1864-1914«. In: Terence Emmons und Wayne S. Vucinich (Hg.), The zemstvo in Russia. An experiment in local self-government, Cambridge: Cambridge University Press, S. 133-175.

Tiebout, Charles M. (1956): »A Pure Theory of Local Expenditures«, in: The Journal of Political Economy 64 (5), S. 416-424.

Tocqueville, Alexis de (2006 [1835]): Über die Demokratie in Amerika, Stuttgart: Reclam.

Treisman, Daniel (2000): »Decentralization and Inflation: Commitment, Collective Action, or Continuity«, in: The American Political Science Review 94 (4), S. 837-857.

Treisman, Daniel (2006): »Fiscal Decentralization, Governance, and Economic Performance: A Reconsideration«, in: Economics \& Politics 18 (2), S. 219-235.

Treisman, Daniel (2007): The Architecture of Government: Rethinking Political Decentralization, Cambridge: Cambridge University Press.

UNDESA (2008): People Matter - Civic Engagement in Public Governance. World Public Sector Report 2008, New York: United Nations.

USAID (2000): Decentralization and Democratic Local Governance Programming Handbook, Technical Publication Series, Mai 2000, Washington D.C.: U.S. Agency for International Development (USAID), Office of Democracy and Governance.

Ustinova, Jenia (2010): »Time Is Ripe to Develop Agribusiness«. In: The St. Petersburg Times 1609 (70), 14.9.2010. S. 10.

Uzun, Vasilii J. (2005): »Large and Small Business in Russian Agriculture: Adaption to Market«, in: Comparative Economic Studies 47 (1), S. 85-100.

Uzun, Vasilii J. (2009a): »Agrarian Reform in Russia. Myths and Reality«, in: Problems of Economic Transition 51 (12), S. 18-41.

Uzun, Vasilii J. (2009b): Tendencii pazvitija i mechanizmy vzaimodejstvija krupnogo i malogo biznesa $\mathrm{v}$ agropromyšlennom komplekse [Entwicklungstendenzen und Mechanismen der Zusammenarbeit von Klein- und Großunternehmen im agroindustriellen Komplex], Moskau: Rossijskaja Akademija Sel'skochozjajstvennych Nauk [Russische Akademie für Agrarwissenschaften].

Van Assche, Kristof, Anastasiya Shtaltovna und Anna-Katharina Hornidge (2013): »Visible and Invisible Informalities and Institutional Transformation in the Transition Countries of Georgia, Romania, and Uzbekistan«. In: Christian Giordano und Nicolas Hayoz (Hg.), Informality in Eastern Europe. Structures, Political Cultures and Social Practices, Bern: Peter Lang, S. 89-118.

Vanhanen, Tatu (2006): Democratization. A comparative analysis of 170 countries, London: Routledge. 
Verdery, Katherine (1996): What Was Socialism, and What Comes next?, Princeton: Princeton University Press.

Verdery, Katherine (1998): »Property and Power in Transylvania's Decollectivization«. In: Chris Hann (Hg.), Property Relations. Renewing the Anthropological Tradition, Cambridge: Cambridge University Press, S. 160-180.

Verdery, Katherine (1999): »Fuzzy Property: Rights, Power and Identity in Transylvania’s Decollectivization «. In: Michael Burawoy und Katherine Verdery (Hg.), Uncertain Transition: Ethnographies of Change in the Postsocialist World, Lanham: Rowman \& Littlefield, S. 53-81.

Verdery, Katherine (2002): »Seeing like a mayor: Or, how local officials obstructed Romanian land restitution«, in: Ethnography 3 (1), S. 5-33.

Verdery, Katherine (2003): The Vanishing Hectare: Property and Value in Postsocialist Transylvania, Ithaca: Cornell University Press.

Verdery, Katherine (2004): »The Obligations of Ownership: Restoring Land Rights to Land in Postsocialist Transylvania«. In: Caroline Humphrey und Katherine Verdery (Hg.), Property in Question: Value Transformation in the Global Economy, Oxford: Berg, S. 139-160.

Visser, Oane, Natalia Mamonova und Max Spoor (2012): »Oligarchs, megafarms and land reserves: Understanding land grabbing in Russia«, in: Journal of Peasant Studies 39 (3-4), S. 899-931.

Visser, Oane und Max Spoor (2011): »Land grabbing in post-Soviet Eurasia: the world's largest agricultural land reserves at stake«, in: Journal of Peasant Studies 38 (2), S. 299-323.

Vogd, Werner (2007): »Empirie oder Theorie? Systemtheoretische Forschung jenseits einer vermeintlichen Alternative«, in: Soziale Welt 58 (3), S. 295-321.

Vogd, Werner (2009): »Systemtheorie und Methode? Zum komplexen Verhältnis von Theoriearbeit und Empirie in der Organisationsforschung «, in: Soziale Systeme 15 (1), S. 98-137.

Vogd, Werner (2010): »Methodologie und Verfahrensweise der dokumentarischen Methode und ihre Kompatibilität zur Systemtheorie«. In: René John, Anna Henkel und Jana Rückert-John (Hg.), Die Methodologien des Systems. Wie kommt man zum Fall und wie dahinter?, Wiesbaden: VS Verlag für Sozialwissenschaften, S. 121-140.

von Beyme, Klaus (1994a): AaSystemwechsel in Osteuropa, Frankfurt a.M.: Suhrkamp.

von Beyme, Klaus (1994b): »Ansätze zu einer Theorie der Transformation der exsozialistischen Länder Osteuropas«. In: Wolfgang Merkel (Hg.), Systemwechsel. Theorien, Ansätze und Konzeptionen, Opladen: Leske + Budrich, S. 141-171.

von Beyme, Klaus (1999): »Osteuropaforschung nach dem Systemwechsel. Der Paradigmawandel in der >Transitologie ««, in: Osteuropa 49 (3), S. 285-304. 
von Zsolnay, Vilmos (1968): »Der >neue Mensch< in Osteuropa«, in: Aus Politik und Zeitgeschichte B 12-68 (20.3.1968), S. 20-32.

Wädekin, Karl-Eugen (1969): Führungskräfte im sowjetischen Dorf. Ihre politischsoziale Situation und Funktion in der Ära Chruščev, Berlin: Duncker \& Humblot.

Wädekin, Karl-Eugen (1973): The Private Sector in Soviet Agriculture, Berkeley: University of California Press.

Wädekin, Karl-Eugen (1989a): »Die sowjetische Landwirtschaft zu Beginn der achtziger Jahre«, in: Osteuropa 31 (5), S. 375-391.

Wädekin, Karl-Eugen (1989b): »Sowjetische Agrarproduktion unter Gorbatschow«, in: Osteuropa 39 (6), S. 536-550.

Wampler, Brian (2007): »A Guide to Participatory Budgeting«. In: Anwar Shah (Hg.), Participatory Budgeting, Washington D.C.: The World Bank, S. 21-54.

Wandel, Jürgen (2011): »Business groups and competition in post-Soviet transition economies: The case of Russian >agroholdings ««, in: The Review of Austrian Economics 24 (4), S. 403-450.

Weber, Max (1964): Wirtschaft und Gesellschaft. Grundriß der verstehenden Soziologie, Köln: Kiepenheuer \& Witsch.

Wegren, Stephen K. (1998): Agriculture and the State in Soviet and Post-Soviet Russia, Pittsburgh: University of Pittsburgh Press.

Wegren, Stephen K. (2005): The Moral Economy Reconsidered. Russia's Search for Agrarian Capitalism, New York: Palgrave Macmillan.

Wegren, Stephen K. (2008): »Land Reform in Russia: What Went Wrong?«, in: Post-Soviet Affairs 24 (2), S. 121-148.

Wegren, Stephen K. (2009a): »Das Lebensmittelproblem der russischen Landwirtschaft«, in: Russlandanalysen (178), S. 10-12.

Wegren, Stephen K. (2009b): Land Reform in Russia. Institutional Design and Behavioural Responses, New Haven: Yale University Press.

Wegren, Stephen K. (2011): »Investment Trends in Russian Agriculture«, in: Russian Analytical Digest (99), S. 9-11.

Wegren, Stephen K. (2012): »Institutional Impact and Agricultural Change in Russia«, in: Journal of Eurasian Studies 3 (2), S. 193-202.

Wegren, Stephen K., David J. O’Brien und Valery V. Patsiorkovsky (2008): »The economics of rural households in Russia: Impact of village location«, in: Eurasian Geography and Economics 49 (2), S. 200-214.

Weick, Karl E. (1979): The Social Psychology of Organizing, Reading: AddisonWesley.

Weick, Karl E. (1995 [1979]): Der Prozeß des Organisierens, Frankfurt a.M.: Suhrkamp.

Weingast, Barry (1995): »The Economic Role of Political Institutions: Market-Preserving Federalism and Economic Development«, in: The Journal of Law, Economics, and Organization 11 (1), S. 1-31. 
Werron, Tobias (2010): »Direkte Konflikte, indirekte Konkurrenzen. Unterscheidung und Vergleich zweier Formen des Kampfes«, in: Zeitschrift für Soziologie 39 (4), S. 302-318.

Wheatley, Jonathan (2013): »Informal and Formal Institutions in the Former Soviet Union«. In: Christian Giordano und Nicolas Hayoz ( $\mathrm{Hg}$.), Informality in Eastern Europe. Structures, Political Cultures and Social Practices, Bern: Peter Lang, S. 319-335.

Williamson, John (1990): »What Washington means by policy reform«. In: John Williamson (Hg.), Latin American adjustment: how much has happened?, Washington D.C.: Institute for International Economics, S. 5-20.

Williamson, John (2004): A Short History of the Washington Consensus, Konferenz >From the Washington Consensus towards a new Global Governance<, 24.25.9.2004, Barcelona.

Willke, Helmut (1987): »Strategien der Intervention in autonome Systeme«. In:

Dirk Baecker u.a. (Hg.), Theorie als Passion, Frankfurt a.M.: Suhrkamp, S. 333361.

Willke, Helmut (2005): Systemtheorie II: Interventionstheorie, Stuttgart: UTB.

Witzel, Andreas (1982): Verfahren der qualitativen Sozialforschung. Überblick und Alternativen, Frankfurt a.M.: Campus.

Wolfensohn, James D. (1998): The other crisis, Adress to the Board of Governors, 6.10.1998, Washington D.C.: The World Bank.

Wolford, Wendy (2007): »Land Reform in the Time of Neoliberalism: A ManySplendored Thing «, in: Antipode 39 (3), S. 550-570.

Wollmann, Hellmut (2010): »Das deutsche Kommunalsystem im europäischen Vergleich - Zwischen kommunaler Autonomie und >Verstaatlichung «? . In: Jörg Bogumil und Sabine Kuhlmann (Hg.), Kommunale Aufgabenwahrnehmung im Wandel. Kommunalisierung, Regionalisierung und Territorialformen in Deutschland und Europa, Wiesbaden: VS Verlag für Sozialwissenschaften, S. 223-252.

Wollmann, Hellmut (2013): »Stadt im Blick der Kommunalwissenschaft «. In: Harald A. Mieg und Christoph Heyl (Hg.), Stadt. Ein interdisziplinäres Handbuch, Stuttgart: J.B. Metzler, S. 174-184.

Wollmann, Hellmut und Elena Gritsenko (2009): »Local self-government in Russia. Between decentralization and recentralization«. In: Cameron Ross und Adrian Campbell (Hg.), Federalism and Local Politics in Russia, London: Routledge, S. 227-247.

Yilmaz, Serdar, Beris Yakup und Rodrigo Serrano-Berthet (2008): Local Government Discretion and Accountability: A Diagnostic Framework for Local Governance, Social Development Working Papers, Local Governance \& Accountability Series, Paper 113, Juli 2008, Washington D.C.: The World Bank. 
Young, John F. und Gary N. Wilson (2007): »The View from Below: Local Government and Putin's Reforms«, in: Europe-Asia Studies 59 (7), S. 10711088.

Zaslavsky, Victor (1982): In geschlossener Gesellschaft. Gleichgewicht und Widerspruch im sowjetischen Alltag, Berlin: Wagenbach.

Zürn, Michael (2002): »Regime/Regimeanalyse«. In: Dieter Nohlen und RainerOlaf Schultze (Hg.), Lexikon der Politikwissenschaft, Band 2, München: C.H. Beck, S. 798-799. 



\section{Dank}

Mein Dank gilt zuallererst den Mitgliedern >meiner< Gemeinden in Russland, die sich mir als Interviewpartnerinnen und Interviewpartner zur Verfügung stellten und mir unverzichtbare Ansprechpartnerinnen und Ansprechpartner während meiner Forschungsaufenthalte waren. Ohne ihre Offenheit und Kooperationsbereitschaft wäre die Arbeit in dieser Form nicht möglich gewesen. Der herzliche Empfang, den sie mir immer wieder bereiteten, und die Einblicke in ihren Alltag, die sie mir trotz vielleicht mancher Verwunderung über mein Tun gewährten, sorgten dafür, dass meine Feldaufenthalte nicht nur zu wissenschaftlich erkenntnisreichen, sondern auch zu persönlich unvergesslichen Erfahrungen wurden. Danken möchte ich auch meinen beiden Gutachtern Rudolf Stichweh und Raimund Hasse für das Interesse, das sie meiner Arbeit entgegenbrachten, und für ihre konstruktiven und kritischen Anmerkungen, die den Entstehungsprozess begleiteten. Darüber hinaus gilt mein Dank allen Kolleginnen und Kollegen des Soziologischen Seminars sowie allen Mitdoktorandinnen und -doktoranden der Graduate School of Humanities and Social Sciences (GSL) der Universität Luzern, die durch ihre Diskussionsfreude und ihr kritisches Zuhören in zahlreichen Kolloquien und darüber hinaus wesentlich zum Gelingen der Dissertation beigetragen haben. Finanziert wurde die Dissertation maßgeblich durch ein Promotionsstipendium der GSL, die auch durch vielfältige organisatorische Unterstützung zum Erfolg des Projekts beigetragen hat. Für ihren ideellen Beistand und die organisatorische Unterstützung bei meinen Feldforschungsaufenthalten bin ich Peter Lindner und Aleksandr Nikulin verbunden, die mir in Russland viele Türen geöffnet haben. Teile meiner Feldforschung wurden finanziell unterstützt durch die Vereinigung von Freunden und Förderern der GoetheUniversität Frankfurt am Main. Ein großer Dank richtet sich an meine Mutter - für ihr Verständnis, für ihr Vertrauen in mich und in meine Arbeit und für das Aufspüren kleiner und großer Fehler im Manuskript. Und schließlich danke ich Pascal Goeke, dessen kluge und kritische Kommentare zu zahllosen unfertigen und (vermeintlich) fertigen Gedanken für mich eine unverzichtbare Quelle der Inspiration und Motivation waren und sind. 
